\title{
When Informed Decision Making Meets Health Promotion
}

Citation for published version (APA):

Gültzow, T. (2022). When Informed Decision Making Meets Health Promotion: An Integration of Both Areas Based on the Example of Smoking Cessation. [Doctoral Thesis, Maastricht University]. ProefschriftMaken. https://doi.org/10.26481/dis.20220308tg

Document status and date:

Published: 01/01/2022

DOI:

10.26481/dis.20220308tg

Document Version:

Publisher's PDF, also known as Version of record

\section{Please check the document version of this publication:}

- A submitted manuscript is the version of the article upon submission and before peer-review. There can be important differences between the submitted version and the official published version of record.

People interested in the research are advised to contact the author for the final version of the publication, or visit the DOI to the publisher's website.

- The final author version and the galley proof are versions of the publication after peer review.

- The final published version features the final layout of the paper including the volume, issue and page numbers.

Link to publication

\footnotetext{
General rights rights.

- You may freely distribute the URL identifying the publication in the public portal. please follow below link for the End User Agreement:

www.umlib.nl/taverne-license

Take down policy

If you believe that this document breaches copyright please contact us at:

repository@maastrichtuniversity.nl

providing details and we will investigate your claim.
}

Copyright and moral rights for the publications made accessible in the public portal are retained by the authors and/or other copyright owners and it is a condition of accessing publications that users recognise and abide by the legal requirements associated with these

- Users may download and print one copy of any publication from the public portal for the purpose of private study or research.

- You may not further distribute the material or use it for any profit-making activity or commercial gain

If the publication is distributed under the terms of Article $25 \mathrm{fa}$ of the Dutch Copyright Act, indicated by the "Taverne" license above, 


\section{WHEN INFORMED DECISION MAKING MEETS HEALTH PROMOTION:}

AN INTEGRATION OF BOTH AREAS BASED ON THE EXAMPLE OF SMOKING CESSATION

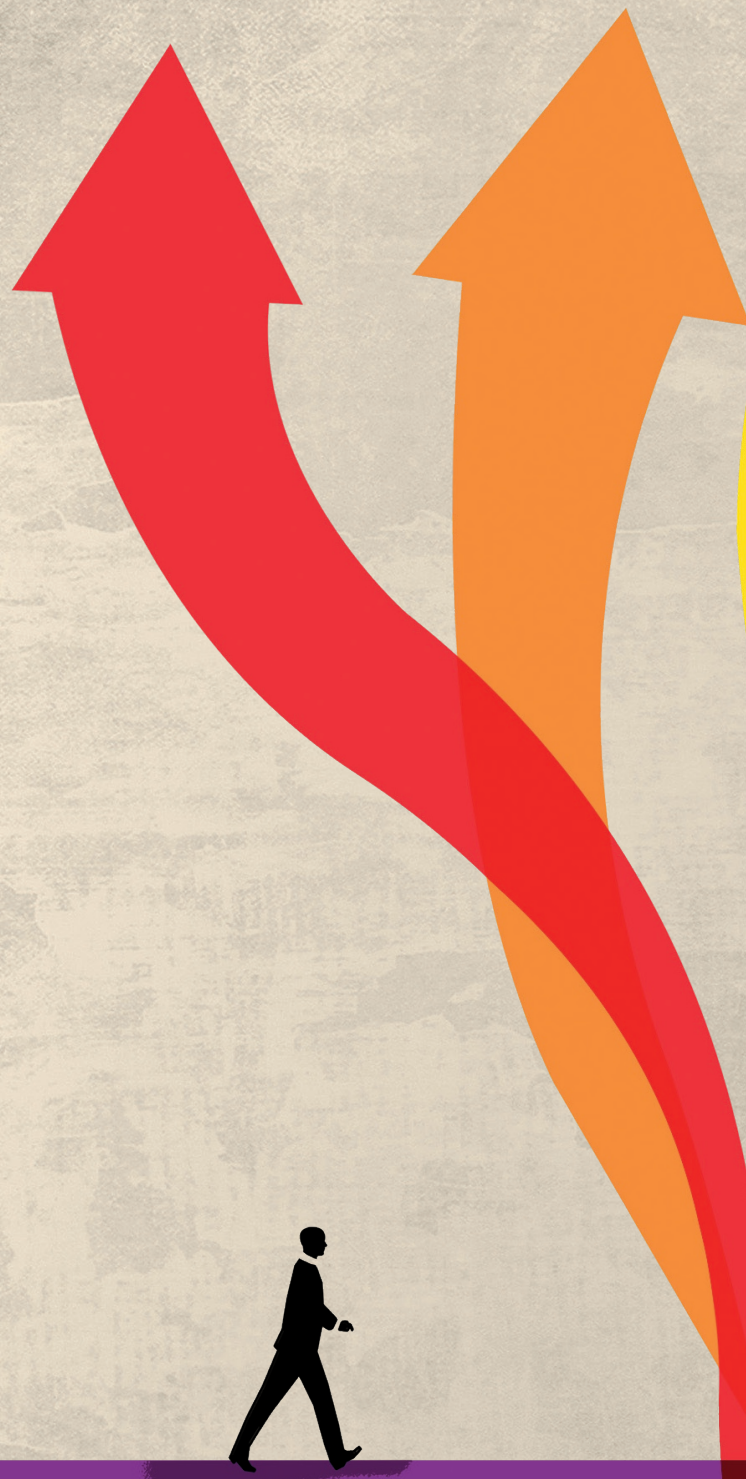



When Informed Decision Making Meets Health Promotion

An Integration of Both Areas Based on the Example of Smoking Cessation 
Cover: $\quad$ SIMONE GOLOB ILLUSTRATIE \& VORMGEVING

Layout: ProefschriftMaken

Printing: ProefschriftMaken

ISBN: $\quad$ 978-94-6423-659-0

(C) Thomas Gültzow, Maastricht 2022

The research presented in this dissertation was conducted at the Care and Public Health Research Institute (CAPHRI), Department of Health Promotion at Maastricht University. CAPHRI participates in the Netherlands School of Public Health and Care Research (CaRe). The research was funded by the Dutch Cancer Society (KWF Kankerbestrijding). The research described in Chapter 2 was additionally funded by the EU-funded Erasmus+ program.

All rights reserved. No part of this publication may be reproduced, stored in a retrieval system, or transmitted in any form or by any means, electronic, mechanical, photocopying, recording or otherwise, without prior permission of the author, or the copyright-owning journals for previous published chapters. 


\title{
When Informed Decision Making Meets Health Promotion
}

\author{
An Integration of Both Areas Based on the Example of Smoking \\ Cessation
}

Proefschrift

ter verkrijging van de graad van doctor aan de Universiteit Maastricht, op gezag van de Rector Magnificus, Prof. dr. Pamela Habibović volgens het besluit van het College van

Decanen, in het openbaar te verdedigen op dinsdag 8 maart 2022 om 16.00 uur

$$
\text { door }
$$

Thomas Gültzow

geboren op 27 september 1990 te Bergheim 


\section{Promotor}

Prof. dr. C. D. Dirksen

\section{Copromotores}

Dr. E. S. Smit (Universiteit van Amsterdam)

Dr. C. Hoving

\section{Beoordelingscommissie}

Prof. dr. T. van der Weijden (voorzitter)

Dr. E. Croes (Trimbos-instituut, Utrecht)

Prof. dr. B. van den Putte (Universiteit van Amsterdam)

Prof. dr. M. C. Willemsen 


\section{table of contents}

chapter 1 - general introduction 7

chapter 2 - integrating behavior change interventions and patient decision aids: 21

how to accomplish synergistic effects?

chapter 3 - decision aids to facilitate decision making around behavior change in 33

the field of health promotion: a scoping review

chapter 4 - smoker profiles and their influence on smokers' intention to use a 79

digital decision aid aimed at the uptake of evidence-based smoking cessation tools:

an explorative study

chapter 5 - informed decision making on the uptake of evidence-based smoking

cessation assistance by individuals motivated to quit smoking: a needs assessment among potential end users and experts to inform decision aid development

chapter $\mathbf{6}$ - an autonomy-supportive online decision aid to assist smokers in

choosing evidence-based cessation assistance: development process and protocol of a randomized controlled trial

chapter 7 - does an explicit value clarification method with computer-tailored advice increase the effectiveness of a web-based decision aid to assist smokers in choosing evidence-based cessation assistance? findings from a randomized controlled trial

chapter 8 - general discussion

impact paragraph

references

summary

samenvatting

zusammenfassung

dankwoord

publication list

curriculum vitae 


$$
1
$$




\section{chapter 1}

general introduction 


\section{Informed Decision Making}

Decision making is an integral part of people's daily living and includes health-related decision making [1]. Examples of such health-related decisions include relatively simple decisions as whether to brush one's teeth in the morning [2], as well as more far-reaching decisions such as which cancer treatment to choose together with one's healthcare provider [3]. Especially those more complex decisions are often made under uncertainty and can have a great impact on people's lives [4,5]; those decisions are therefore often described as difficult decisions (e.g., [6]). While decisional uncertainty (i.e., being unsure about one's decision) is seen as inherent in decision making, it is presumed that several factors can exacerbate this perceived uncertainty and difficulty associated with a decision [4,7]. It is, for instance, expected that decisional conflict (as decisional uncertainty is commonly called in the scientific literature [4]) is more likely to occur when individuals (1) feel they are not sufficiently informed about possible options, including their benefits and risks, (2) do not know what is important to them personally (i.e., when individuals are unclear about their personal values), and (3) do not feel supported in their decision making or are under pressure to choose one option over another $[4,7]$. In other words: Decisional conflict is more likely to arise when individuals fail to make what is described as an informed decision [1]. The concept of informed decision making therefore, provides us with a useful framework for understanding how decisions can be made in order to limit the amount of decisional conflict. This is especially important because decisions that are made under high decisional conflict are more likely to be delayed, discontinued and regretted $[4,8,9]$ and are likely to result in other emotional outcomes such as nervousness [9], and blaming external parties [10].

Informed decision making has become particularly important in healthcare since the 1990s and is seen as a direct result of a shift within the healthcare system from a paternalistic model in which healthcare providers were the dominant partners in decision-making processes to a system in which patients are seen as (at least) equal partners in these decision-making processes [11]. Yet, despite this general shift within healthcare from a paternalistic model to one in which decisions are at least partially shared between healthcare providers and patients, some authors (e.g., [12]) argue that individuals should only be guided towards making an informed decision if there is not one optional path to be taken (e.g., if there is not one 'best' treatment based on the available evidence) and that otherwise they should be steered towards the best option available. These types of decisions are commonly referred to as preference-sensitive decisions, which means that individuals must weigh known benefits and harms in order to make decisions based on their own preferences $[12,13]$. Interestingly, informed decision making (and related concepts) have become particularly prominent regarding treatment decisions (e.g., [14]) - which are often made together with healthcare providers (often referred to as shared decision making [15]) - and decisions to take part in population screening programs (e.g., [16]), a form of secondary 
prevention. This is also reflected in studies about decisional conflict, which have mainly focused on treatment and testing (including screening) decisions in the 20-year period after the development of the most widely-used scale to assess decisional conflict [17].

Therefore, it is noticeable that most of the decisions related to health promotion and primary disease prevention (hereinafter both referred to as health promotion) have been neglected in the informed decision-making literature so far. To a certain extent, this is understandable given that the aim of health promotion is primarily aimed at promoting behaviors that are considered healthier by a broad group of experts or that are known to yield greater health benefits than other behaviors (e.g., smoking cessation as opposed to smoking). In other words, there is one best 'treatment' based on the available evidence and people should be steered towards this option. This is in contrast to many clinical treatments, where the decision for a specific treatment in the vast majority of cases includes a certain degree of uncertainty [13]. Consequently, health promotion's goal is not to increase understanding of the multiple available options or to explore one's values, but rather to promote a single best option to which end personal values are less relevant. So-called nudges are probably the most fitting type of health promotion intervention to illustrate this. Nudges are defined as changes to how a decision is framed so that the uptake of one particular option is more likely to occur without changing the accessibility of the other options [18]. Therefore, although nudges are designed to preserve people's decisional autonomy, their ultimate goal is to influence people's behavior in a pre-specified manner. As such, and as opposed to informed decision making, nudges are not designed to facilitate people's understanding of the decision and to help them to choose something that aligns with what is personally important to them.

Having said all that, the lack of health promotion in the informed decision-making literature is still notable as health promotion decisions have characteristics that also make them prone to inducing decisional conflict and can also be preference sensitive. In the following section, I will illustrate this on the basis of smoking cessation.

\section{Smoking Cessation}

Smoking is still one of the most important topics within the field of health promotion. This is also reflected in the Dutch National Prevention Agreement (in Dutch: "Nationaal Preventieakkoord") which has been signed by the Dutch government and more than 70 Dutch organizations with the goal of reducing smoking, overweight, and problematic alcohol consumption [19]. Of those three, smoking continues to be the leading cause of preventable diseases and premature death $[20,21]$, and accounts for $9.4 \%$ of the disease burden in the Netherlands-leading to $€ 2.4$ billion in healthcare costs and 20,000 smoking-related deaths annually [21]. The aim of the Dutch Prevention Agreement is twofold (regarding smoking): prevention of smoking (e.g., by establishing a smoke-free generation) and prevention of 
long-term consequences of smoking by facilitating smoking cessation (treatment) [19]. The prevention of smoking clearly falls outside the definition of a preference-sensitive decision, as abstinence from smoking clearly outperforms smoking-particularly in terms of morbidity and mortality $[20,22]$. This is exactly why in the case of smoking prevention, interventions that aim to influence behavior are often relied on as opposed to interventions that promote informed decision making. In contrast, when it comes to the prevention of smoking-related consequences through smoking cessation, decisions around how to quit smoking, i.e., using which cessation assistance, do not imply such a definitive exclusion from the definition of a preference-sensitive decision as will be illustrated in the following.

In the agreement it is stated that approximately $80 \%$ of the three million individuals that smoke in the Netherlands want to quit, but that only a small group uses evidence-based cessation assistance to quit [19]. This is noteworthy in that evidence-based support for smoking cessation can at least double the likelihood of smoking cessation [23]. Logically, one of the aims of the agreement is that more individuals should be encouraged to use such evidence-based cessation assistance [19]. Evidence-based cessation assistance can be divided into behavioral support (e.g., counseling [24]) and pharmacological support. The latter refers to both nicotine replacement therapy (NRT), such as transdermal nicotine patches [25], as well as prescription medications that have been shown to aid smoking cessation efforts (e.g., varenicline [26]). All these different forms of support have been shown to increase an individual's chances to achieve smoking abstinence in scientific studies. However, next to these evidence-based support tools, there are a number of cessation assistance tools that are used by individuals without clear evidence that they lead to an increase in smoking cessation success compared to stopping unassisted (e.g., acupuncture [27]). It should also be noted that while several cessation assistance tools have been shown to be effective, some of these clearly outperform other tools in terms of effectiveness. Varenicline for example has been shown to be more effective than single forms of NRT [26]. That being said, varenicline does not perform better than combinations of different NRT [26] and all forms of pharmacological support can cause side effects [28], an aspect which behavioral cessation assistance clearly lacks-while still being effective. Individuals motivated to quit smoking therefore need to trade-off between cessation tools' benefits (e.g., effectiveness) and their disadvantages (e.g., side effects). In other words, the decision between different cessation tools is preference sensitive as there is not one option that outperforms all other options overall and, consequently, personal preferences come into play. To illustrate, imagine two smokers that want to use evidence-based cessation assistance during their next quit attempt. The first person considers the lack of side effects of a cessation assistance tool to be important and is therefore likely to prefer behavioral support (e.g., counseling) over the other options. The second person, on the other hand, highly values the lack of interpersonal contact that may make NRT (e.g., nicotine patches) more attractive for them. So, although both would opt for an evidence-based tool, their 
personal preferences determine their different choices in this context. This example can also be used to show why a health promoting decision can induce decisional conflict: Individuals motivated to quit smoking, like the two just described, often lack the required information to make this decision [29], which in addition limits their ability to know what is personally relevant to them in relation to the decision at hand. Individuals motivated to quit smoking also often report to experience some form of social pressure (e.g., [30]), which could potentially translate into experienced pressure to make a certain decision regarding cessation assistance. People who are motivated to quit can therefore potentially benefit from support to make an informed (rather than an uninformed or pressured) decision.

\section{Supporting Informed Decision Making}

The most commonly described and used interventions to support informed decision making in both health(care) and the scientific literature are referred to as decision aids (DAs, often also called patient decisions aids [PtDAs]) [13]. DAs can be delivered in various formats, such as videos, booklets, or (interactive) websites [13]. In contrast to other broadly used health education materials, DAs are evidence-based interventions that are specifically designed to make health(care) decisions explicit in order to facilitate deliberate decision making [13], i.e., decisions that are made very consciously as opposed to more intuitive decisions. Broader health education materials, on the other hand, generally do not specifically focus on decision making and also provide other types of health(-related) information that is not directly linked to decisions and/or decision making itself [13]. To qualify as DAs, decision support interventions have to (at least) explicitly describe (1) the relevant health problem; (2) as well as the decision at hand; (3) including all available options; (4) with all positive; (5) and negative features; (6) in such a way that users can imagine the physical, social, and/or psychological effects of all options [31]. There is robust evidence that DAs improve knowledge and perceived value clarification when used to facilitate treatment and screening decisions, while some evidence also points towards a beneficial effect on value-consistent choosing [13]. In other words, DAs can be used to support informed decision making and help DA-users to make informed choices. In order to do so, DAs often include two essential elements [32]: (1) balanced information provision [33] and so-called (2) value clarification methods (VCMs; previously referred to as value clarification exercises [VCEs]) [34,35].

VCMs refer to a number of various methods that are employed in DAs to help users assess the desirability of different options or options' characteristics in order to identify which option they prefer [34,35]. VCMs support users in clarifying their values (i.e., how (un)desirable the characteristics of certain options are to them personally [36]-however, the term is used increasingly broadly, for example by including contextual factors [35]) and identifying personal preferences (i.e., how (un)desirable options themselves are when taking these values into account [36]). To illustrate, a VCM could be employed to assist individuals 
in making a decision about whether to participate in a clinical trial, i.e., individuals could choose between two options: (1) participate in the clinical trial or (2) receive standard treatment [32]. Those two options have certain characteristics. For example, participation in the clinical trial allows participants to receive an innovative treatment with potential new benefits, while receiving standard treatment does not come with the burden of additional monitoring [32]. The VCM could then help individuals to identify their personal values and preferences, e.g., the VCM might help someone to identify that they highly value the potential benefits which would reveal their overall preference for the clinical trial as opposed to standard treatment [32].

Generally speaking, VCMs tend to be divided into implicit VCMs that lack interactive elements (e.g., tables that make options' characteristics explicit and leave the actual value clarification to the user), and explicit VCMs that include interactive elements for value clarification [34]. In general, explicit VCMs are much more studied [34] and several studies have found promising effects compared to implicit VCMs. In a study by Abhyankar et al. [30], no VCM was compared with both an implicit VCM and an explicit VCM in the previously described decision-making context (i.e., whether to participate in a clinical trial). It was found that the decisions of participants in the explicit VCM group showed more characteristics of informed decision making, that participants felt less ambivalent about the options, and less uncertain about the decision and their values overall [32]. Other evidence especially points to positive long-term effects: In a study by Feldman-Stewart et al. [37] it was shown that decisional conflict was unaffected by their explicit VCM immediately after having used the DA. On the other hand, positive effects emerged on feeling prepared for decision making after the decision had been made, while positive effects on decisional regret only emerged after a year [37].

Explicit VCMs can be further subdivided and range from methods such as rating scales (e.g., by making use of Likert scales) to math model-based methods [38]. Recent evidence has shown that the potential effect of explicit VCMs on value-consistent decision making is moderated by whether users are supported in understanding the implications of their values $[39,40]$. To illustrate, Witteman et al. have shown in a series of studies that the most effective VCMs in terms of value-consistent decision making show VCM-users to what extent options (fail to) align with their identified values [40]. For example, in one of their studies, participants were given an online application consisting of dynamic web sliders representing both values and preferences. Those web sliders were then linked, meaning that for participants who indicated that a particular value is very important to them, the slider representing the preference moved equally [40]. Another possible way to support users in understanding the implications of their values would be to provide tailored advice based on these values that shows users which options match their values and can thus be considered preferred options. However, all of this has been tested in a healthcare context only and up 
until this moment it remains unknown whether these ideas are as promising with regard to health promotion decision making.

Integration of Interventions to Support Informed Decision Making in Health Promotion

As described earlier, both research into decision conflict [17] and DAs [13] were so far mainly aimed at treatment and testing decisions, despite the fact that a number of health promotion decisions have characteristics that make them very susceptible to informed decision-making support. This is probably why some DAs in this area have been developed as well. For example, Koelewijn-van Loon et al. [41] developed an intervention (which included a DA) with the aim of including patients in nurse-led preventive cardiovascular risk management related to lifestyle adherence and cardiovascular risk, while Willemsen et al. [42] developed a DA aimed at supporting individuals in choosing an evidence-based smoking cessation assistance tool. To elaborate, Koelewijn-van Loon et al. [41] developed a comprehensive nurse-led intervention to improve adherence to lifestyle advice that consisted of (1) a risk assessment, (2) risk communication, (3) a DA, and (4) adapted motivational interviewing. Their DA was delivered as a brochure that was handed to patients by their nurses and contained information about patients' cardiovascular risks and on how (i.e., by using which options) to reduce these risks [43]. Willemsen et al. [42] similarly developed a largely non-digital DA, but with a focus on smoking cessation. Their DA consisted out of (1) a booklet describing all cessation assistance tools available in the Netherlands; (2) a video showing famous and unknown ex-smokers who described their smoking cessation process using specific smoking cessation tools, as well as a tobacco control expert objectively describing various smoking cessation tools; and (3) samples of different effective smoking cessation tools. Noticeably, both interventions aimed to influence health behavior (i.e., adherence to lifestyle advice and uptake of smoking cessation assistance as well as smoking cessation) which places them into the area of health promotion.

As alluded to earlier, health promotion has so far mainly focused on interventions aimed at changing behavioral patterns at the population level, as opposed to supporting informed decision making. As such, these interventions' main aim is changing of behavior into a prespecified direction and not to increase understanding of the decision itself and to clarify one's values. Next to nudges an example of such interventions is computer-tailored interventions. Tailoring means that users receive information based on personal data, for example data entered prior to the actual intervention $[44,45]$; meaning that they will only receive information that is relevant to them and their personal situation (e.g., [46]). Often tailoring is used to change behavior in a prespecified manner $[44,45]$. For example, if a person shows an unfavorable attitude regarding the desired behavior (e.g., "I do not like cessation assistance."), they will receive tailored information to influence their attitude (e.g., a positive statement regarding cessation assistance) with the assumption that this will lead 
to a change in attitude. If this change in attitude occurs, the likelihood of a subsequent change in behavior is higher [47]. This firmly places most computer-tailored interventions in the area of behavior change. Environmental interventions, another example, target environments and/or systems (e.g., the childcare setting) to support desired behavioral change (e.g., [48]). And while these behavior change interventions have a long history of showing that they can be effective (e.g., [49-51]), it remains unclear exactly how they differ from DAs. The existence of DAs, such as from Willemsen et al. [42], makes it even more complicated to differentiate between DAs and behavior change interventions because these interventions are presented and regarded as DAs, but are also aimed at influencing behavior. This ambiguity is unfortunate from several points of view. First, it can hinder scientific understanding, as terms, such as DA, are unclear and are applied differently by different researchers. Second, both approaches (i.e., health promotion and informed decision making) have long scientific histories, which have led to the development of effective interventions, and these can help to provide a more thorough understanding of health behaviors and decision making. Integrating insights from both approaches could therefore potentially be used to further increase the effectiveness of interventions and achieve the greatest possible health benefits for individuals and-ultimately-society. Unclear differentiations and terminology, however, hinder this integration potential. This leads to the first research question (RQ) that will be addressed in this thesis:

RQ1: What are similarities and differences between behavior change interventions and DAs; and how can synergistic effects be accomplished?

In addition to these more theoretical ambiguities about what constitutes a DA and what constitutes a behavior change intervention, there are other unclear aspects that relate to the integration of the two fields that warrant further investigation. For example, we know quite a bit about characteristics of DAs (e.g., intervention elements, theoretical foundations and (cost-)effectiveness) that are applied to treatment and screening decisions (e.g., [13]), but we do not know how DAs that focus on health promotion decision making are characterized. Single studies provide insights into this, of course, but broad overviews in this area are lacking. To make this clearer using the example of the characteristic 'effectiveness', Koelewijn-van Loon et al. did not find an overall positive effect of their intervention on lifestyle-associated behavior [41]. Willemsen et al. [42] on the other hand found that their DA had positive effects on informed decision-making outcomes (e.g., knowledge), as well as on some behavior (i.e., smoking cessation attempts and smoking cessation success), but not on the uptake of evidence-based smoking cessation assistance. Further work in the form of a systematic review has since shown that DAs in the field of smoking cessation probably have an effect on quitting attempts, but that the effects on smoking abstinence are still unclear [52]. It may thus be concluded that health promotion DAs' impact on various outcomes related to informed decision making and behavior change seem inconsistent. This clearly 
shows that research is lacking regarding health promotion DAs overall. Again, this is unfortunate for a number of reasons. First, the lack of a broad overview hinders scientific understanding and scientific progress as progress cannot be achieved by single studies alone. Second, this lack of scientific evidence means that no specific practical advice or guidelines can be developed, as those require synthesized data. Therefore, the second broad RQ that will be addressed in this thesis will be:

RQ2: What is known about DAs that support informed decision making about health promotion decisions, focusing in particular on their characteristics, intervention elements, theoretical foundations, and (cost-)effectiveness?

\section{VISOR}

In addition to these more fundamental research questions, the project presented in this thesis was also aimed at developing a DA intended to support individuals who are motivated to quit smoking in choosing an evidence-based cessation assistance tool, combining insights from the fields of informed decision making and health promotion. Whereas some smoking cessation DAs have been developed and tested in other countries (e.g., [53]), the only other DA developed for this purpose in a Dutch setting was developed by Willemsen et al. in 2006 [42]. Although a very nice first attempt to improve informed decision making in a smoking cessation context, this DA has a number of shortcomings. First, the largely non-digital nature of the DA limits its possible dissemination, which is problematic as an intervention's public health impact is not only depended on their effectiveness, but also on how many individuals are reached with an intervention [54]. Second, the largely non-digital nature prevents (or at least hinders) the adoption of an interactive and flexible design. This is unfortunate because interactive designs can probably improve comprehension in general [55], while also improving certain aspects of informed decision making, such as knowledge acquisition [56]. Third, the DA did not entail an explicit VCM which may have hindered its effects, as explained earlier. The lack of an impact on cessation assistance uptake is unfortunate, as an increased uptake would likely even further increase the rate of smoking cessation overall [23].

Addressing the first shortcoming (i.e., the largely non-digital nature that limits possible dissemination) is crucial as the public health impact of interventions also depends on their potential reach [54]. Therefore, it makes sense to develop an intervention that is optimally designed to be adopted by a large group of people. Two approaches that may go hand in hand to achieve this, is to design a fully digital (instead of largely non-digital) DA and first of all target a group that would use such a digital intervention early on $[57,58]$. Such early adopters can influence the adoption of a DA by later adopters through two possible mechanisms: (1) by disseminating their (positive) experiences with a DA through their own network, and (2) by initiating an imitation effect by letting others know that they have 
already used the DA [58]. Both mechanisms would lead to more adopters in general if more early adopters were reached in the beginning. This would mean that it could be wise, especially in the beginning, to focus on a group that is already interested in using such a (smoking cessation) DA. Having said this, it is relatively unknown which profiles (or types) of individuals show an interest in (smoking cessation) DAs, and which do not. This leads to the third $R Q$ addressed in this thesis:

RQ3: What characterizes early adopters of a smoking cessation DA?

In addition to the aforementioned approach to ensure sufficient reach, it is also important to ensure end user acceptance by incorporating their needs and wishes early in the development process. This is also reflected in the development process advocated by the International Patient Decision Aid Standards (IPDAS) Collaboration [59]. However, to the best of our knowledge, as far as smoking cessation DAs are concerned, this development process has never been followed. This is of course not a problem in itself if DAs still are developed through a systematic development process that includes a(n) (early) needs assessment, but as far as we know this is also not the case. In the project described in this thesis, it was therefore decided to follow the IPDAS development process that consists out of seven steps, three of which are further broken down into multiple steps (also see Figure 1). After having established scope and purpose of the DA (Step 1), a steering group is set up (Step 2), which is directly followed by a needs assessment among potential end users (described as patients) and clinicians (Step 3). Here again, the clear focus on clinical decisions (i.e., treatment and testing decisions) becomes apparent as only needs assessments among end users and clinicians are considered. It could be argued that, in the field of health promotion, other stakeholder groups are also important. To illustrate, in the Netherlands smoking cessation is often attempted without consulting clinicians [60], thus, one might conclude that other smoking cessation experts (e.g., scientists with a focus on smoking cessation) should be involved in this phase in addition to the clinicians involved in smoking cessation counselling. This leads to the fourth $R Q$ addressed in this thesis:

RQ4: What are the needs and opinions regarding smoking cessation decision making (support) of individuals motivated to quit smoking and smoking cessation experts? 


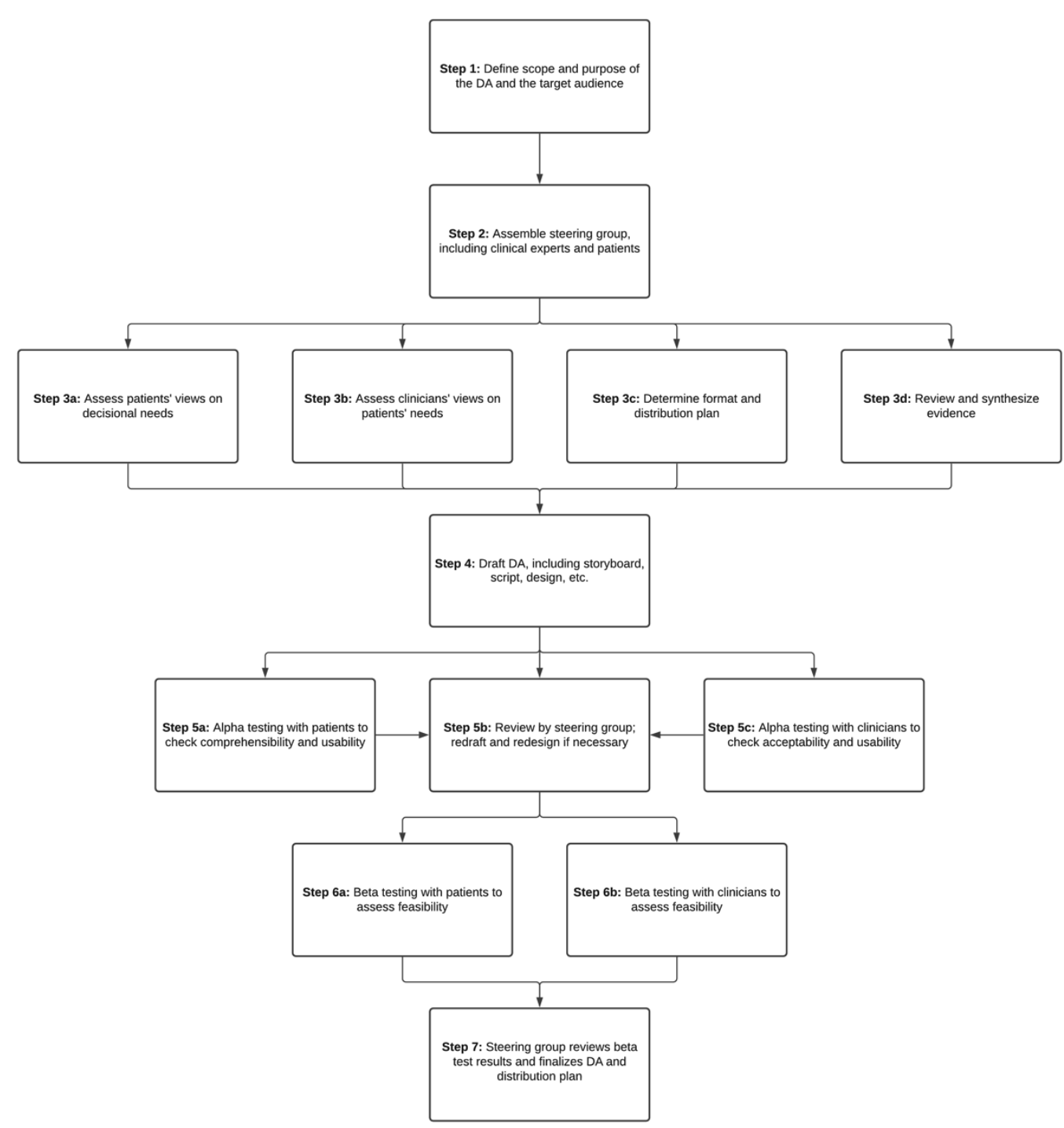

Figure 1. IPDAS Development Process Adapted by Coulter et al. [59]; DA = decision aid. 
In the remainder of the IPDAS steps, a fully functional DA is developed. After the needs of end users and the views of experts have been assessed, this information is combined with evidence-based information to develop a draft DA in Step 4. In Step 5, this first version of the DA is alpha-tested by both end users and experts and their feedback is incorporated (if necessary) in a new version of the DA. This version is then beta-tested, again with all stakeholder groups, in Step 6. Subsequently, the results from the beta-testing are reviewed and the DA is completed by incorporating this information in Step 7. This process was followed while the DA described in this thesis was developed. Along the way, this DA was given the name "VISOR". For the entirety of the development process of VISOR, I would like to refer to Chapter 6, but a brief description is provided in the following.

VISOR was designed with input from all three relevant stakeholder groups, i.e., end users, clinicians, and scientific experts, as well as based on relevant and emerging evidence. To counter the aforementioned shortcomings of the DA developed by Willemsen et al. [42] VISOR was also designed as a fully digital DA from the start. This not only allows for a possible broader dissemination in the future, but also allowed us to include interactive elements. Of these interactive elements, explicit VCMs paired with computer-tailored advice are perhaps the most interesting, as other studies have shown that this particular combination of explicit VCMs and support for understanding the implications of one's clarified values can have very positive effects on decision-making processes $[39,40]$ (see Supporting Informed Decision Making). As also explained earlier, the combination of explicit VCMs paired with computertailored advice has never been tested in the field of health promotion (as far as we are aware) and particularly not in relation to smoking (cessation). Theoretically, helping DA-users to choose a cessation assistance tool that is more in line with their values, could positively impact on decisional outcomes as well as smoking cessation outcomes, as the inclusion of preferences has been shown to positively influence the effectiveness and efficiency of healthcare interventions in other contexts [61]. However, again, this has never been tested. This leads to the final $R Q$ addressed in this thesis:

RQ5: Can adding an explicit VCM paired with computer-tailored advice to a fully digital smoking cessation DA facilitate the decision-making process of people who want to quit smoking and increase both the uptake of smoking cessation assistance as well as smoking cessation rates?

\section{This Thesis}

The overall aim of this thesis was twofold: (1) to provide a thorough understanding of DAs applied to health promotion and (2) to describe the work that was undertaken to develop and test the smoking cessation DA VISOR. This aim has been achieved by answering the five 
RQs listed throughout the introduction, that were identified based on existing gaps in the literature. To do so, this thesis is structured as follows:

In Chapter 2 theoretical work is presented to show the similarities and differences between behavior change interventions and DAs, while also highlighting room for synergetic effects (addressing RQ1). In Chapter 3 a scoping review is presented that was undertaken to find out what is known about health promotion DAs that support informed decision making about health promotion decisions, focusing in particular on their characteristics, intervention elements, theoretical foundations, and (cost-)effectiveness (addressing RQ2). In Chapter 4 a cross-sectional study is presented that was conducted to identify potential early adopters of a digital smoking cessation DA (addressing RQ3). In Chapter 5 a needs analysis among people motivated to quit smoking and smoking cessation experts is presented that was used to inform the development of the smoking cessation DA VISOR (addressing RQ4). In Chapter 6 the development process of VISOR is presented, as well as the study protocol that was followed in order to address RQ5. In Chapter 7 the effects (in terms of decision making and smoking (cessation) behavior) of adding an explicit VCM paired with computer-tailored advice to a smoking cessation DA are presented (addressing RQ5). Finally, in Chapter 8 the results presented in the different chapters are integrated, discussed, and put into a broader context.

\section{Abbreviations}

DA - decision aid

IPDAS — International Patient Decision Aid Standards

NRT - nicotine replacement therapy

PtDA - patient decision aid

$\mathrm{RQ}$ - research question

VCE - value clarification exercise

VCM - value clarification method 


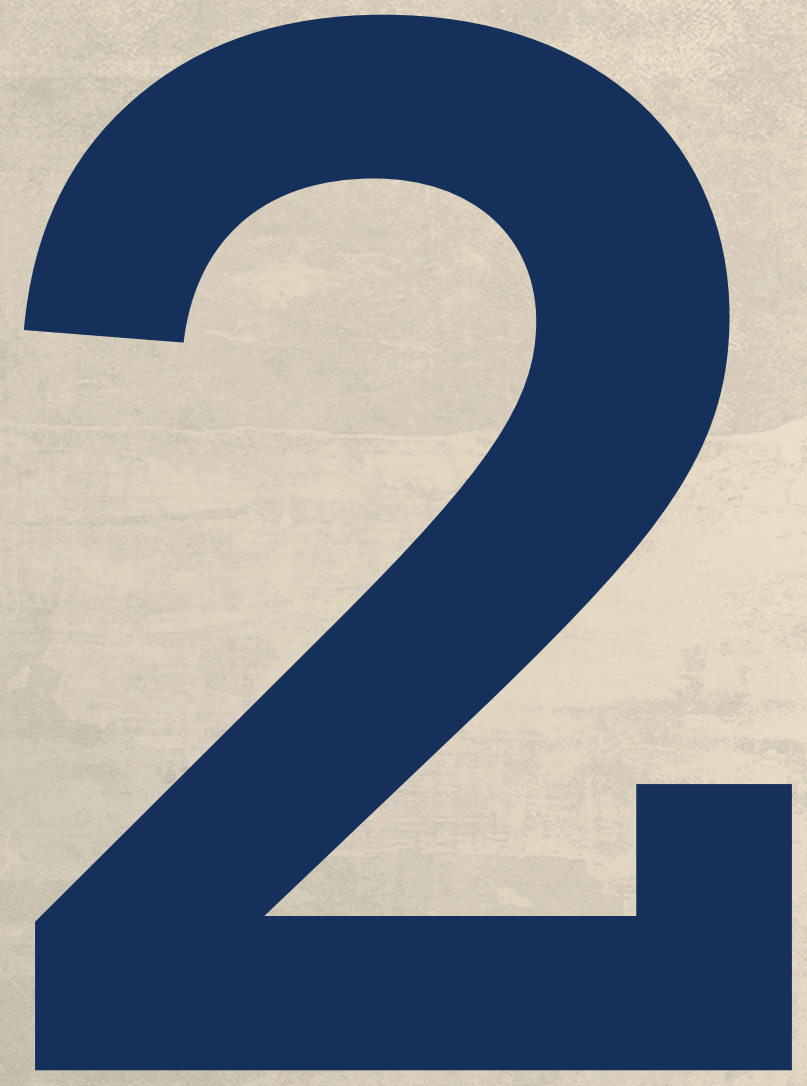




\section{chapter 2}

\section{integrating behavior change interventions and patient decision aids: how to accomplish synergistic effects?}

This chapter has been published as:

Gültzow, T., Hoving, C., Smit, E. S., \& Bekker, H. L. (2021). Integrating behaviour change interventions and patient decision aids: How to accomplish synergistic effects? Patient Education and Counseling, 104(12), 3104-3108. https://doi.org/10.1016/j.pec.2021.04.007 


\section{Abstract}

People make numerous health-related choices each day: for example, deciding to brush one's teeth or to eat well and healthy-or not to do these activities. To support complex decisions and subsequent behavior change, both Behavior Change Interventions (BCls) and Patient Decision Aids (PtDAs) have been developed and evolved independently to support people in health-related decision making. In this paper, we critically review BCls and PtDAs, examine their similarities and differences, and identify potential for integration of expertise to increase the benefits for people engaging with healthcare and health behaviors. The two approaches appear to mainly differ in terms of their (1) goals and foci, (2) theoretical basis, (3) development frameworks, (4) active ingredients, and (5) effect evaluation. To facilitate the integration of scientific insights from these two fields, we recommend to (1) bring both fields together and promote interprofessional discussions, (2) train (health) professionals to recognize strengths of both approaches, (3) investigate the synergy of the two fields, and (4) be prepared for and try to mitigate a culture shock when the fields start to interact. Knowledge generated by researching PtDAs could be used to facilitate decisional processes that enable patients to choose goals that are in line with their values and preferences, while insights from researching $\mathrm{BCls}$ could be used to facilitate engagement with, and implementation of those goals. This integration could allow researchers and intervention providers to increase the benefits for people engaging with healthcare and health behaviors. 


\section{Introduction}

People face a multitude of daily health-related decisions, for example whether to brush one's teeth [2] or to eat healthily [62]. While these decisions seem rather easy to make, and are often carried out automatically, some decisions seem harder to make and require much more conscious deliberation and effort [1]. Examples of such decisions are: deciding which kind of cancer treatment aftercare trajectory one wishes to follow [63] or which diabetes mellitus treatment one prefers [64]. Decisions of the first type are often habitual, wellbeingrelated, and/or daily choices, while decisions of the second type are often unfamiliar, illnessrelated, and/or one-off choices. Moreover, decisions of the first type are often associated with health and illness prevention, while the second with treatment and illnessmanagement. And while the latter often require engagement with health professionals [65], the former do not. That being said, health professionals provide guidance and information to support people to make informed, values-based decision in both contexts; e.g., by providing nutrition care in general practices [66] in order to help people to adjust their nutrition-related decision making in daily life or illness-related dietary modifications to manage some health problems.

Two types of complex intervention(s) (fields) [67] have been developed and evolved independently to support people in health-related decision making: Behavior Change Interventions ( $\mathrm{BCls}$ ) and Patient Decision Aids (PtDAs). BCls tend to target decisions associated with health and illness prevention, PtDAs with treatment and illness management. Both $\mathrm{BCls}$ and PtDAs can successfully support patients in health-related decision making to improve both personal and public well-being; e.g., by helping people to improve their behavior by being more physically active [68] (BCls) or by reducing the number of undecided people [13] (PtDAs). However, both types of interventions have been operating largely independently from each other so far. Yet, it could be argued that the distinction between the two types of decisions that tend to be targeted by the two types of complex interventions is rather arbitrary. In fact, some decisions require that individuals both (1) make a one-off choice and (2) change their habitual and/or daily behavior to act on that decision. An example to illustrate this idea is smoking cessation: Patients who decide to quit smoking subsequently have to choose from the plethora of smoking cessation options available (e.g., pharmacological support [69], behavioral counselling [24], self-help, or cessation without cessation assistance), after which they also have to adopt a new behavior (i.e., using the chosen option when quitting). Some other 'overlapping' contexts are immunization and screening, which often also are one-off choices with a clear focus on health and illness prevention as opposed to treatment and illness-management. Integrating insights from both $\mathrm{BCl}$ and PtDA research could therefore be potentially beneficial to optimally support patients in this and similar health (behavior) choices and changes. 
This paper provides a critical review of $\mathrm{BCl}$ s and PtDAs in order to examine their similarities and differences and identify integration potential. We provide practical recommendations to enable researchers and healthcare professionals to offer hybrid BCI-PtDA interventions in order to potentially increase the impact on patients' decisions and alignment with behavior change and to increase the benefits for people engaging with healthcare and health behaviors.

\section{Describing Behavior Change Interventions (BCls)}

$\mathrm{BCls}$ are defined as "coordinated sets of activities designed to change specified behavior patterns" with the goal to improve public health by targeting individuals' behavior [70]. $\mathrm{BCls}$ can be applied in different contexts, e.g., to change modifiable risk factors (e.g.,[71]), but also, for example, to ensure that patients are more likely to adhere to the advice given by healthcare providers [72]. BCl-development is often driven by health behavior change theories, the most often applied being the Theory of Planned Behavior $[73,74]$. As such, $\mathrm{BCls}$ often focus on the determinants of behaviors, thereby indirectly influencing behavioral performance. Examples of such determinants on the individual level are knowledge, awareness, risk perception, automaticity, attitudes, outcomes expectations, social influences, skills, capability, self-efficacy, and barriers $[75,76]$. In the field of BCls, those determinants reflect individuals' beliefs (e.g., "For me smoking is pleasurable", a belief related to the concept of attitude) and are expected to lead to a change in behavior by influencing individuals' intention to change [47]. Designers of $\mathrm{BCls}$ therefore need to identify the most important determinants that they need to target to change a particular behavior(al intention) in a desired direction-which is the ultimate goal of $\mathrm{BCls}$. As such, the focus of $\mathrm{BCls}$ is clearly on the outcome: Interventions are designed to stimulate people to engage in a predefined target behavior and their efficacy is most often assessed by adoption of the target behavior (e.g., smoking cessation [77]), and changes in the aforementioned behavioral determinants.

\section{Describing Patient Decision Aids (PtDAs)}

PtDAs are defined as "evidence-based tools designed to help patients make specific and deliberated choices among healthcare options" [13] and focus on proactively enhancing the decision-making or reasoning process [78]. Their goal is to present accurate information of all options and their consequences, and encourage people to evaluate this information with their own values, and trade-off these evaluations to reach a decision that is right for them $[13,79]$. PtDAs can be used in a wide range of contexts ranging from screening decisions (e.g., [80]), to treatment decisions (e.g., [64]) to aftercare decisions (e.g., [63]). PtDAs are underpinned by theories explaining how we make decisions under risk and uncertainty, and the factors influencing our judgments and choices, such as the Conflict Model of Decision 
Making [81]. That being said, it is reported that theories are underused in PtDAs [82]. The content and development of PtDAs are informed by evidence-informed guidance from the International Patient Decision Aid Standards (IPDAS) Collaboration guidelines $[59,79]$. Some argue PtDAs are particularly useful for decisions with clinical equipoise, where one option does not clearly outperform all other options in terms of benefits and harms according to the clinical evidence $[13,83]$. PtDAs' efficacy is therefore most often assessed by measures of informed decision making and decisional quality (such as decisional conflict [4]), understanding of the health problems and options, and engagement with health services [13].

\section{Differences Between BCls and PtDAs}

While BCls and PtDAs can both have an impact on health(care) decision making they differ regarding five domains: (1) goals and foci, (2) theoretical basis, (3) active ingredients or components, (4) development frameworks, and (5) effect evaluation approaches. A short overview of the main differences between $\mathrm{BCl}$ and PtDAs is presented in Table 1.

Table 1. Overview of the Main Differences Between Behavior Change Interventions (BCls) and Patient Decision Aids (PtDAs).

\begin{tabular}{|c|c|c|}
\hline & $\mathrm{BCls}$ & PtDAs \\
\hline Intervention goal and focus & $\begin{array}{l}\text { Behavior change/behavioral } \\
\text { outcomes }\end{array}$ & $\begin{array}{l}\text { Deliberate value-based } \\
\text { decision making, focus is on } \\
\text { the (shared) decision-making } \\
\text { process }\end{array}$ \\
\hline Theoretical basis & Explain behavior (change) & Explain decisional processes \\
\hline $\begin{array}{l}\text { Active } \\
\text { ingredients/components }\end{array}$ & $\begin{array}{l}\text { Elements encouraging behavior } \\
\text { change by changing behavioral } \\
\text { determinants }\end{array}$ & $\begin{array}{l}\text { Elements de-biasing } \\
\text { information and boosting } \\
\text { reasoning }\end{array}$ \\
\hline Development frameworks & $\begin{array}{l}\text { Encourage behavior change, } \\
\text { e.g., Intervention Mapping }\end{array}$ & $\begin{array}{l}\text { Encourage informed decision } \\
\text { making, e.g., International } \\
\text { Patient Decision Aid } \\
\text { Standards (IPDAS) }\end{array}$ \\
\hline Effect evaluation approach & $\begin{array}{l}\text { The extent to which they can } \\
\text { change people's behavior or } \\
\text { antecedent determinants }\end{array}$ & $\begin{array}{l}\text { The extent to which they can } \\
\text { support people in making } \\
\text { informed, value-based } \\
\text { health(care) decisions (with } \\
\text { health professionals) }\end{array}$ \\
\hline
\end{tabular}




\section{Goals and Foci}

Regarding their respective goals, $\mathrm{BCl}$-developers' focus is more likely to be behavior change, while PtDA-developers are more likely to focus on informed or shared decision making. Development frameworks used to develop $\mathrm{BCls}$ often start by identifying a behavior that needs to be adapted or implemented to improve health [76], e.g., a BCl designed to maintain a healthy workforce can focus on reducing working while being sick (i.e., presenteeism) [84]. PtDAs often start with identifying the decision problem, options, and consequences in the context of healthcare delivery [13], e.g., a PtDA designed to support patients in breast cancer aftercare decision making is designed to enable patients to identify what is important to them about care options, and share their preference when planning care with professionals in order to support autonomous decision making [63]. To conclude, $\mathrm{BCl}$-developers tend to focus their efforts on behavioral outcomes (such as presenteeism [84] or smoking cessation [77]), while PtDA-developers tend to focus their efforts on (decisional) processes, such as decision quality [85]. Most, if not all, other differences stem from this.

\section{Theoretical Basis}

Theoretical underpinnings of $\mathrm{BCl}$ s tend to explain behavior (change) and its antecedent factors and/or explain how motivation to perform a certain behavior is formed, whereas theoretical frameworks underpinning PtDAs are usually designed to either explain decisional biases or factors boosting reasoning. The aforementioned Theory of Planned Behavior for example posits that people's intentions are formed depending on their attitude, perceived behavioral control, and subjective norms [73]. Another well-known theory, the SelfDetermination Theory [86], describes different qualities of motivation that are formed through the fulfilment of different psychological needs, such as the need for autonomy [81,87]. The Conflict Model of Decision Making, on the other hand, describes decision making as a process in which decision makers have to choose one option from a set of alternatives (all with inherent (dis)advantages) following a stress-inducing event [88].

\section{Development Frameworks}

$\mathrm{BCl}$ - and PtDA-developers tend to use different development frameworks. For $\mathrm{BCls}$, frameworks such as Intervention Mapping [76] or the Behaviour Change Wheel [70] are often applied. Intervention Mapping [76] is an approach that can be used to design health promoting programs through an iterative step-based approach which starts by identifying what needs to be changed to realize an improved health status in populations. Interestingly, PtDAs are often developed by following the IPDAS systematic development process [59] which also advocates an iterative step-based approach to identify evidence to design the interventions. However, whereas frameworks such as Intervention Mapping [76] advocate 
the use of needs assessments to find out which determinants are associated with certain behaviors so that they can be specifically influenced in order to change behavioral patterns, the IPDAS development process emphasizes that individuals' decisional needs should be explored to facilitate choices for options that fit into their lives.

\section{Active Ingredients}

Both $\mathrm{BCls}$ and PtDAs include different types of active ingredients or components in order to achieve their goals: $\mathrm{BCls}$ make use of methods associated with adopting a behavior such as arguments and goal setting [75], PtDAs use methods to de-bias the information presented and boost reasoning such as removing value-terms [33] and rating advantages and disadvantages of all options [34]. One well known method to change attitudes are arguments for example [75] which present the message receiver with new information or information that challenges existing attitudes. Value clarification methods [34] on the other hand are often applied within PtDAs to support users in understanding what is important to them personally and cover a range of different applications, such as the rating of different attributes [38].

\section{Effect Evaluation}

In line with their respective goals, $\mathrm{BCl}$ s are often evaluated by the extent to which they can change individuals' behavior (e.g., to which extent smoking cessation interventions enable individuals to cease smoking [24]) or antecedent determinants (e.g., by also monitoring the impact on determinants such as attitude [89]), while PtDAs are often evaluated by the extent to which they can support people in making informed, value-based health(care) decisions (with health professionals) [13].

\section{How Can $\mathrm{BCl}$ - and PtDA-Expertise Be Integrated?}

We found that $\mathrm{BCl}$ and PtDAs are inherently different and valuable in their own right, both underpinned by methods to develop research-informed, complex interventions [90]. We can see a way forward for developers of both types of interventions to recognize their strengths and differences, to enable different questions to be addressed about their efficacy in different contexts, and ensure best practice integrated effectively in healthcare settings to increase the benefits for people engaging with healthcare and health behaviors. In the following, we will provide practical examples on how this could be accomplished. 
Integrating PtDA-Insights Into $B C l s$

$\mathrm{BCls}$ tend to focus on predefined outcomes without taking individual decisional outcomes into account, although of course this need not always be the case. However, given the fact that the inclusion of individuals' preferences can positively influence the effectiveness and efficiency of healthcare interventions [61] providing one predefined behavioral option for every patient could negatively influence the possible outcome of the intervention for some individuals. This is especially interesting, in light of Self-Determination Theory [86] which posits that we can increase the chances of behavioral maintenance if we increase individuals' perceived autonomy [91]. We propose that integrating insights from PtDA-research could be used to increase individuals' perceived autonomy. An established technique for increasing the autonomy of individuals is the provision of choice [92,93], a function that PtDAs contain explicitly by providing balanced and neutrally worded information about the different options available (i.e., not clearly emphasizing one predefined option). Example 1 shows one way of integrating PtDA-insights into a $\mathrm{BCl}$ in order to offer a hybrid BCI-PtDA intervention.

Example 1. Providing Balanced and Neutrally Worded Information in a Behavior Change Intervention $(B C l)$.

$\mathrm{BCls}$ often contain explicitly or implicitly persuasive elements to convince recipients to adopt behaviors that are considered superior (i.e., health-promoting or -sustaining) to other behaviors. To increase patients' perceived autonomy, $\mathrm{BCl}$ developers could include balanced, neutrally worded information that does not contain elements that convince patients to adopt certain behaviors. To provide concrete examples, $\mathrm{BCl}$ developers could use the IPDAS background papers on providing information [94] and balanced presentation [33] to develop a smoking cessation intervention that includes information on all available smoking cessation options without naming a superior option (i.e., providing actual choices) and neutrally describing the different options' effectiveness and possible consequences.

\section{Integrating $\mathrm{BCl}$-Insights Into PtDAs}

The PtDA-field, on the other hand, has developed in a direction were the focus is primarily on the process of decision making and engagement with healthcare, while patients and healthcare providers are interested in tangible outcomes as well, such as life expectancy and quality of life [94]. However, PtDAs often do not offer techniques to help people to actually implement their choices, even though it is known that a positive intention to change or to do something does not always translate into actual behavior [95]. Including behavior change techniques - such as the making of plans [95] - to overcome this so-called 'intentionbehavior gap' could potentially lead to a higher percentage of patients actually executing the decision made. Example 2 shows one way of integrating $\mathrm{BCl}$-insights into a PtDA. 
Example 2. Integrating Implementation Intentions Into a Patient Decision Aid (PtDA).

PtDAs often end after patients have made a decision-seemingly assuming that all patients will actually implement the option they decided to adopt. To improve the chances that patients convert their preferences into actual behavior, developers could use implementation intentions [75] which have been proven effective for various health behaviors [97,98], in other words: the making of ifthen plans. For example, a PtDA designed to support patients with diabetes mellitus in choosing an option to improve their HbA1c could (after elements that supported patients' decision-making processes) end with prompts to make if-then plans that link situational cues to a preferred response (that was revealed by making use of the PtDA). Through the use of the PtDA, users could for instance decide they want to incorporate more physical activity into their daily life, as opposed to changing their medication regimen. The PtDA could then end with the making of concrete plans, such as "If I travel to work on a working day, I will use my bicycle."

\section{Practical Recommendations}

Both fields may benefit from collaboration and integration of knowledge and expertise. In fact, hybrid interventions could potentially help patients achieve goals that reflect what they find personally important. Knowledge generated by researching PtDAs could be used to facilitate decisional processes that enable patients to choose goals that are in line with their values and preferences, while insights from researching $\mathrm{BCls}$ could be used to facilitate engagement with, and implementation of those goals. However, it may be that different intervention types are more effective at different points in healthcare (e.g., choosing between options, versus management). To achieve integration, the fields need to recognize their similarities and differences in order to develop more effective interventions. We propose four recommendations to achieve this goal:

\section{(1) Bring both fields together and promote interprofessional discussions}

Because the fields operate largely independently of each other, it is important to create awareness for the two fields, but also to create space for integrative discussions. For example, expert meetings could be held as part of ongoing projects where both fields may be able to contribute important expertise (e.g., [96]) or at scientific conferences that are of interest to both fields (e.g., the annual conference of the European Health Psychology Society [97]).

\section{(2) Train (health) professionals to recognize strengths of both approaches}

In order to increase joint activity and fully integrate insights, it could be helpful to train people, such as future health services researchers and practitioners, in the intersection between behavior change and medical decision making. For example, doctoral projects 
supervised by professionals from both fields could be set up that fall within this area, resulting in a new generation of researchers benefitting from this combined expertise. Such projects should be both theoretical (e.g., focused on how integration could actually take place) and practical (e.g., developing hybrid interventions for different health decisions) - or a combination of the two.

\section{(3) Investigate the synergy of the two fields}

At present, theories reflect the independence of both fields, with behavior change theories not making explicit how patients make decisions and theories of decision making not making explicit how patients can be supported in adopting an option they prefer. New theories that integrate insights from both fields are therefore very important to clarify how the one process relates to the other. Integrated theories could for example be used to support understanding how informed and reasoned decision making could lead to actual behavior change by making use of behavior change techniques, as illustrated in Example 2, but also could be used to inform intervention design (guidelines).

\section{(4) Be prepared for and try to mitigate a culture shock when the fields start to interact}

As we have made clear that the two intervention types and their backgrounds are different, it would not be surprising if the integration can evoke a kind of 'culture shock' which could impact on direct collaborative efforts. Various strategies might be used to mitigate this culture shock. For example, by hosting 'dual-field' expert meetings. A recent project in which a smoking cessation DA was developed [96], proved this approach to be successful. Another interesting avenue to reduce friction could be to establish a common ontology that includes definitions, but also what contexts are required to ensure effectiveness. We hope this article helps researchers and healthcare providers reflect on the insights gained through decades of valuable research into $\mathrm{BCls}$ and PtDAs and develop interventions to increase the benefits for people engaging with healthcare and health behaviors across pathways of care, as health states change.

\section{Abbreviations}

$\mathrm{BCl}$ - Behavior Change Intervention

IPDAS - International Patient Decision Aid Standards

PtDA - Patient Decision Aid 


$$
3
$$




\section{chapter 3}

\section{decision aids to facilitate decision making around behavior change in the field of health promotion: a scoping review}

This chapter has been published as:

Gültzow, T., Zijlstra, D. N., Bolman, C., de Vries, H., Dirksen, C. D., Muris, J. W. M., Smit, E. S., \& Hoving, C. (2021). Decision aids to facilitate decision making around behavior change in the field of health promotion: A scoping review. Patient Education and Counseling, 104(6), 1266-1285. https://doi.org/10.1016/j.pec.2021.01.015 
Abstract

Objective: To broadly synthesize literature regarding decision aids (DAs) supporting decision making about diet, physical activity, sleeping, and substance use a scoping review was performed.

Methods: Multiple sources were used: (1) scientific literature searches, (2) excluded references from a Cochrane review regarding DAs for treatments and screenings, and (3) results from additional searches. Interventions had to (1) support informed decision making and (2) provide information and help to choose between at least two options. Two researchers screened titles and abstracts. Relevant information was extracted descriptively.

Results: Thirty-five scientific articles and four DAs (grey literature) were included. Results were heterogeneous. Twenty-nine (94\%) studies described substance use DAs. All DAs offered information and value and/or preference clarification. Many other elements were included (e.g., goal setting). DAs' effects were mixed. Few studies used standardized measures, e.g., decisional conflict ( $n=4,13 \%)$. Some positive behavioral effects were reported: e.g., smoking abstinence $(n=1)$.

Conclusions: This research shows only some positive behavioral effects of DAs. However, studies reported heterogeneous results/outcomes, impeding knowledge synthesis. Areas of improvement were identified, e.g., establishing which intervention elements are effective regarding health behavior decision making.

Practice Implications: DAs can potentially be beneficial in supporting people to change health behaviors-especially regarding smoking. 


\section{Introduction}

Noncommunicable diseases continue to be the leading cause of deaths worldwide, inflicting heavy economic burden [98]. These diseases' main modifiable risk factors (i.e., blood pressure, blood glucose, cholesterol, and weight) are heavily influenced by individual health behaviors, e.g., tobacco use, physical activity (PA), diet, alcohol use, and sleep (duration) [99-102]. The occurrence of noncommunicable diseases can therefore be greatly reduced by changing these preventive health-related behaviors (for the sake of readability, we will use this term when referring to tobacco use, PA, diet, alcohol use, and sleep (duration) together).

In all these areas people face decision-making situations, such as deciding to check how well one is meeting behavioral recommendations or deciding whether to engage in actions to change an unhealthy behavior. In addition to these decisions, people are confronted with decisions between different possible actions to change their behavior, e.g., people wishing to stop smoking can choose between several effective cessation aids [26,103-105].

When multiple options exist and persons need to identify their own values (i.e., how (un)desirable certain options' characteristics are [36]) and preferences (i.e., how (un)desirable certain options themselves are taking values into account [36]), decisions are referred to as "preference-sensitive" [13]. This type of decision requires that people weigh the benefits and harms of each option on basis of their own values and preferences, since no option is objectively better than others [13]. In practice, it requires lay persons to gather available evidence, evaluate its quality and incorporate this information to assess which options fit their values and preferences best-tasks which can be difficult [106].

People facing such preference-sensitive decisions about preventive health-related behaviors may profit from support in their decision-making process, for instance by using decision aids (DAs). DAs are typically used to inform users about available options and their respective characteristics (e.g., effect, time investment, and availability) in a balanced manner and help users to choose options that are value- and preference-concordant $[13,107]$, in other words they help users to make informed decisions [1]. DAs structure the decision-making process with the help of value clarification methods (VCMs, previously also referred to as value clarification exercises or VCEs) [34] - which can be implicit (i.e., not including overt activity) or explicit (i.e., including overt activity) [37]. Such DAs, when applied to treatment or screening decisions (e.g., decisions about cancer treatment options), have shown to have a positive impact on knowledge, accuracy of risk perception, values-concordant choices, decisional conflict, feelings of being undecided, costs, and the number of people making a decision [13]. However, it is unclear whether this promising approach to decision support 
can also help individuals make informed decisions about preventive health-related behaviors.

The most comprehensive knowledge synthesis in the field of DAs excluded studies conducted around DAs focusing on lifestyle [13]. However, a systematic review by Moyo et al. [52] has shown that DAs could be a promising approach to smoking cessation, as have individual studies (e.g., [42]). Currently, there is a lack of concrete knowledge of DAs in the broader area of preventive health-related behaviors. To the best of our knowledge, no knowledge synthesis of any kind has been carried out to fill this knowledge gap. We therefore do not know for which preventive health-related behaviors DAs actually exist. In the recent past, studies have been carried out to examine intervention elements [38] of DAs in general and the theoretical basis [82] of treatment and screening DAs in more detail. Effects of DAs focused on treatment and screening decisions are also routinely synthesized in the aforementioned comprehensive knowledge synthesis in the form of a Cochrane review [13] and at least one systematic review has investigated DAs' cost-effectiveness in general [108]. However, all of this information is not available regarding DAs aimed at making decisions about changing preventive health-related behaviors specifically.

Consequently, our aim was to broadly synthesize existing literature in the form of a scoping review by reviewing information regarding DAs supporting informed decision making about these behaviors, focusing on their characteristics, intervention elements, theoretical foundations, and (cost-)effectiveness. The synthesized knowledge will be of value to guide future research directions, but also to inform (clinical) practice and to better understand the usefulness of DAs that focus on preventive health-related behavior change.

\section{Methods}

The methodological framework developed by Arksey \& O'Malley [109], the Joanna Briggs Institute Reviewers' Manual [110], and the Preferred Reporting Items for Systematic Reviews and Meta-Analyses Extension for Scoping Reviews (PRISMA-SCR) [111] guided the study protocol-which can be found on the Open Science Framework (https://osf.io/9xkbv/) [112]. However, one change was made: We decided to gather descriptive data instead of quantitative data as the heterogeneity of the results hindered us to conduct quantitative analyses. This made it impossible to calculate Cohen's kappa (however, other measures were taken to ensure reliability, see Article and DA Selection). Consequently, the data are therefore presented descriptively in text and/or tabular form. The completed PRISMA-ScR checklist can be found in Appendix A. 


\section{Information Sources}

Multiple sources were used to gather data: (1) scientific literature search results, (2) the excluded publications from the Cochrane review on DAs for people facing health treatment or screening decisions [13] (mentioned in the introduction), and (3) results from additional searches, such as a literature search on Google Scholar and a grey literature search on the Ottawa Hospital Research Institute Decision Aid Library Inventory (DALI) [113].

\section{Scientific Literature Searches}

Systematic literature searches were conducted in three relevant databases (i.e., PubMed, PsycINFO, and CINAHL) with search strings related to the aforementioned behaviors combined with "decision aid" (for the full overview see Appendix B and Appendix $C$ ) in October 2018. Terms were included to exclude papers that focus on policy decision making as the focus of this scoping review was on individual decision making. Searches were restricted to publications pertaining to humans (again, due to the focus on individual human decision making) between January 2008 and October 2018 (to synthesize the most recent literature). Search strategies were specified to each database and discussed with a scientific information specialist.

\section{Excluded Publications From the Cochrane Review}

As noted in the introduction, the most comprehensive knowledge synthesis in the field of DAs (the systematic review by Stacey et al. [13]) excluded articles describing DAs focusing on lifestyle-hence, those which were of interest for this scoping review. Therefore, all of those excluded publications were retrieved.

\section{Additional Searches}

Using Google Scholar, we applied a systematic search (see Appendix D for the search strings). Publications within the first 50 hits were screened for each search string. Again, this search was limited to the last 10 years (between January 2008 and October 2018). We also created a Google Scholar Alert to inform us of any other relevant publications. Subsequently, we searched through the DALI [113] using all the search terms described above.

Finally, we tried to identify any DAs (in development) that were missed. For this purpose additional strategies were: (1) cross-referencing included articles and articles only selected for full text screening (see Article and DA Selection, e.g., [52]), (2) checking the publications from first authors of included articles, (3) using Google Scholar's "related articles"-function, and (4) using our existing professional network (e.g., by making use of newsletters of 
professional associations) and contacting authors of known DAs in development or with currently unpublished findings.

\section{Article and DA Selection}

Retrieved titles and abstracts were screened by TG and DZ by using the following inclusion criteria: Articles had to describe interventions that (1) supported informed decision making in relation to preventive health-related behaviors and (2) provided information about the decision at hand and helped to choose between at least two options (e.g., by including VCMs) $[79,114]$. Articles describing (clinical) treatment DAs were excluded. Inconsistencies between the two reviewers were resolved by discussion. If an agreement could not be reached, $\mathrm{CH}$ helped to come to a conclusion. The selected full articles were assessed by DZ and TG, after which TG extracted all relevant information descriptively which was charted within an Excel spreadsheet developed a priori. After completion of the data extraction by TG, DZ reviewed $10 \%$ of the articles to ensure reliability. Inconsistencies were discussed between TG and DZ. The same procedure was applied to the DAs not found in scientific literature (i.e., grey literature), except for a change in author responsibilities, i.e., DZ initially abstracting the data and TG reviewing $10 \%$. The charting of the information was based on the Cochrane review on treatment and screening DAs (see Appendix E) [13]. Authors of the included articles were not contacted to clarify or add information.

Results

Scientific Literature

Descriptives and Study Characteristics

Through this scoping review 35 articles [115-122,43,41,123-131,53,132-146] were identified, including four study protocols $[43,126,128,131]$. It was not possible to determine the exact number of DAs described in the 35 articles due to a lack of clear identification of DAs by name or other distinguishing characteristic in the majority of the articles. Therefore, the units of analysis for this scoping review were individual studies (not DAs) with the exception of protocol papers which were analyzed together with their associated effect papers. More than half of the studies were of American origin ( $n=16,52 \%$ ) $[116,126,127,132-138,140-146]$. The main focus was on substance use ( $n=29,94 \%)$ [41,43,53,117-146] with 11 DAs solely focusing on smoking (35\%) [53,131-137,139141,146]. All studies described DAs that included both information provision and value clarification or described such DA-content without explicitly using the terms. All developed DAs contained a multitude of other intervention elements, such as personal stories [134] or encouragement to set a quit date $[53,131]$. For an overview of the included articles see Table 
1, for an overview of intervention elements see Table 2, and for a flow diagram depicting the selection process see Figure 1. 


\begin{tabular}{|c|c|c|}
\hline 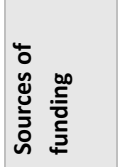 & 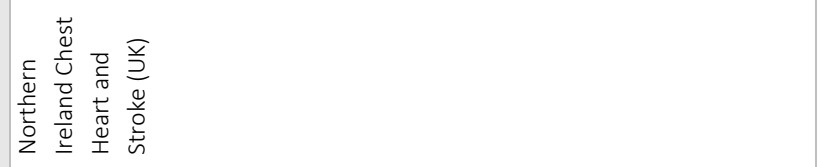 & 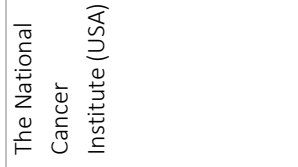 \\
\hline 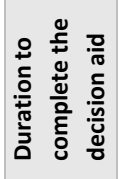 & 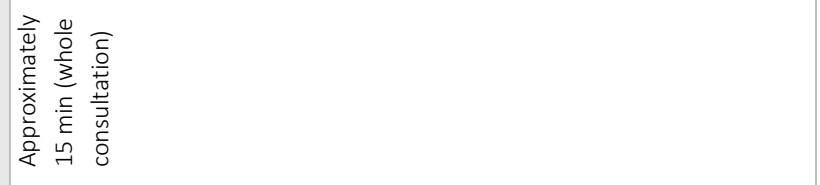 & $\begin{array}{l}\frac{c}{\bar{\varepsilon}} \\
o \\
0 \\
0 \\
0 \\
m\end{array}$ \\
\hline 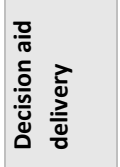 & 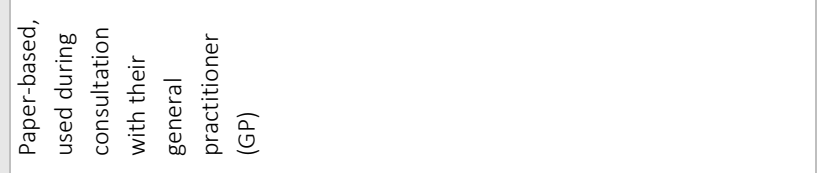 & 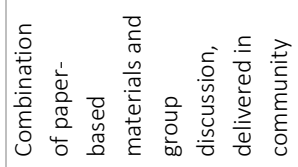 \\
\hline 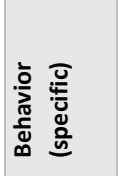 & 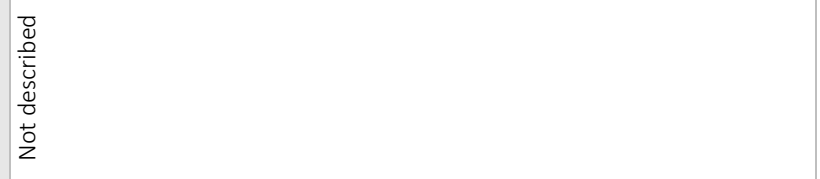 & 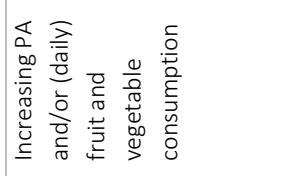 \\
\hline 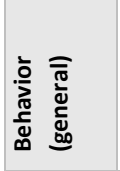 & 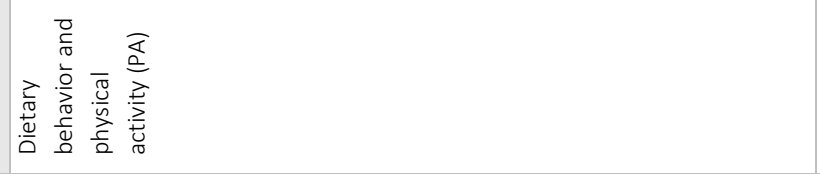 & 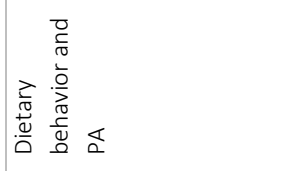 \\
\hline 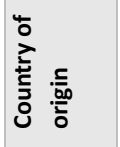 & 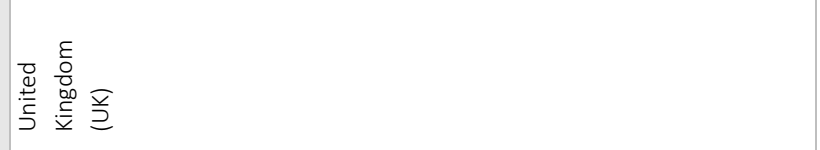 & 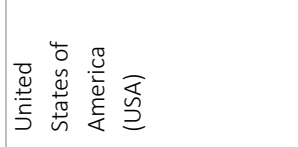 \\
\hline 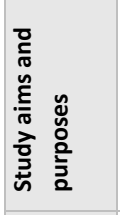 & 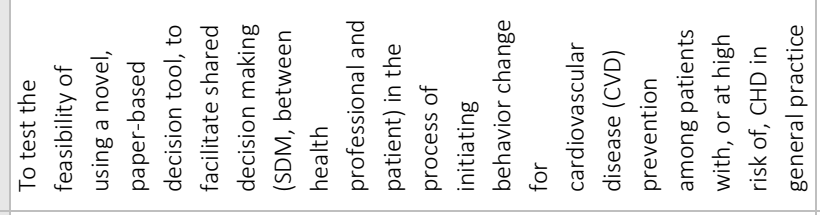 & 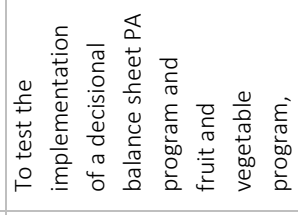 \\
\hline 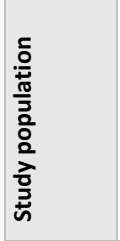 & 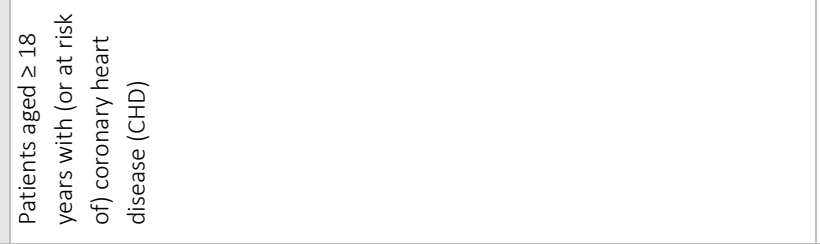 & 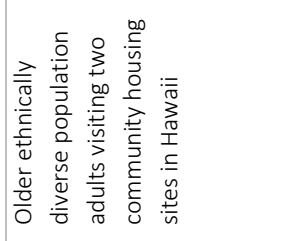 \\
\hline 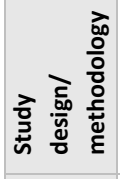 & 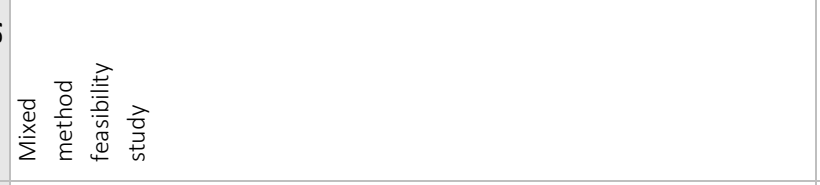 & 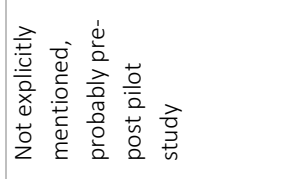 \\
\hline 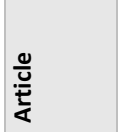 & 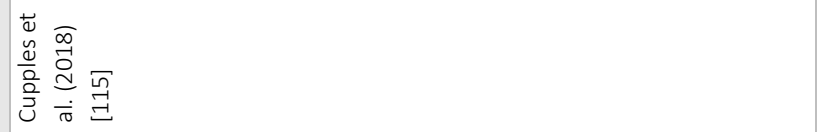 & 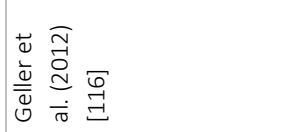 \\
\hline
\end{tabular}




\begin{tabular}{|c|c|c|}
\hline & 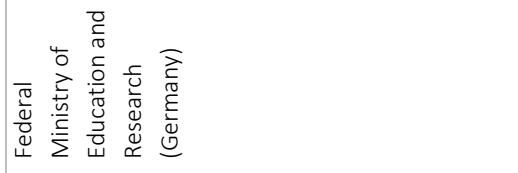 & 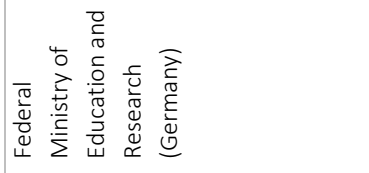 \\
\hline & 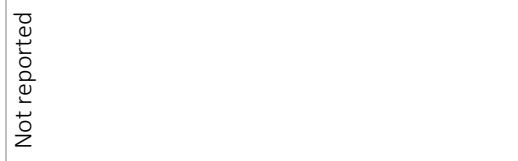 & 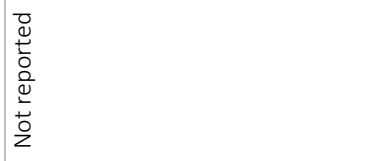 \\
\hline \multirow[t]{4}{*}{ 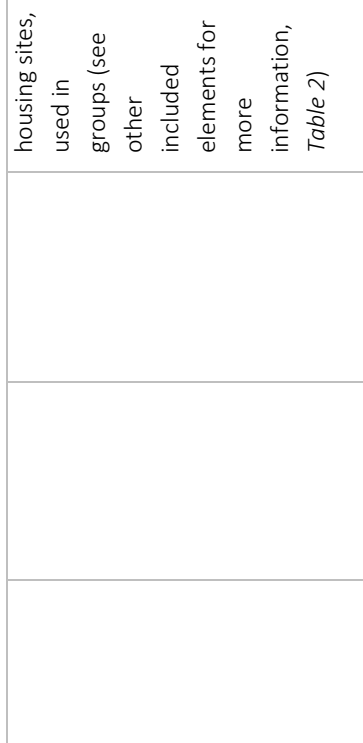 } & 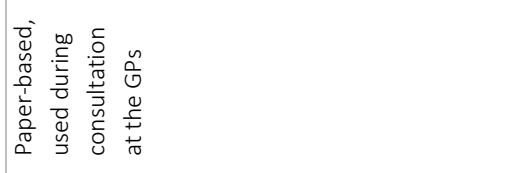 & 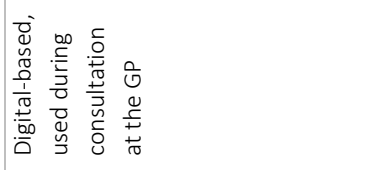 \\
\hline & 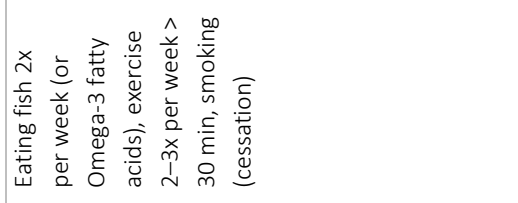 & 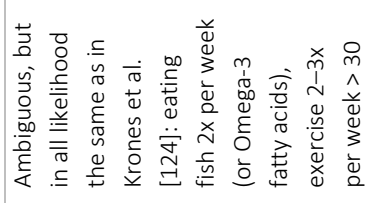 \\
\hline & 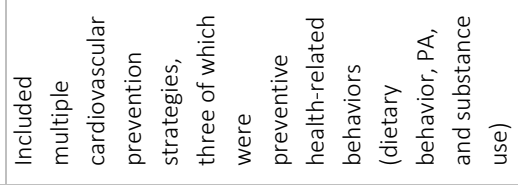 & 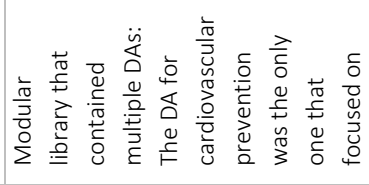 \\
\hline & 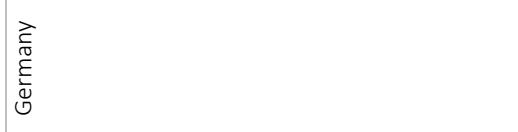 & 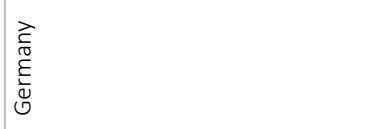 \\
\hline \multirow[t]{4}{*}{ 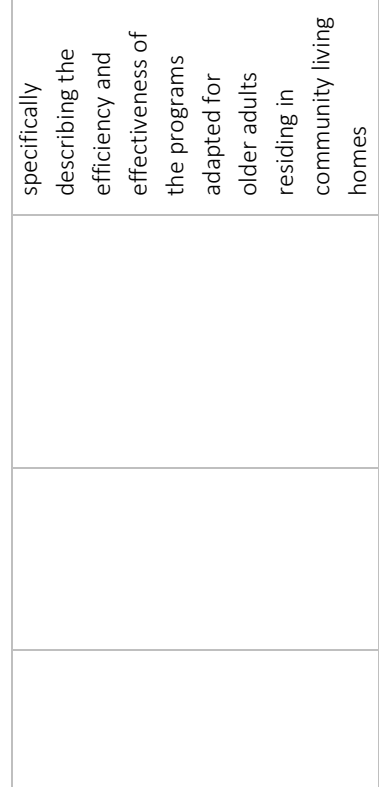 } & 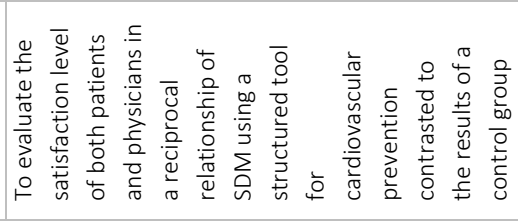 & 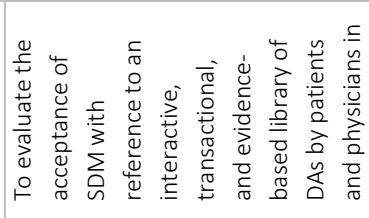 \\
\hline & 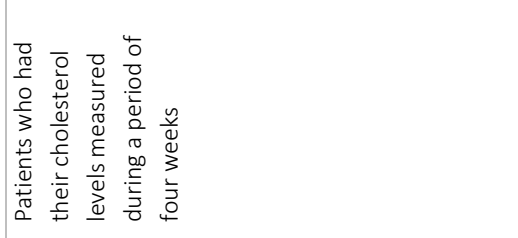 & 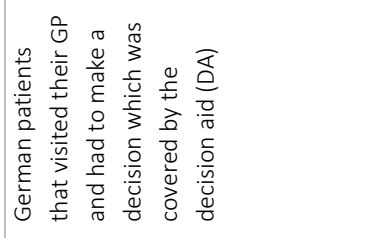 \\
\hline & 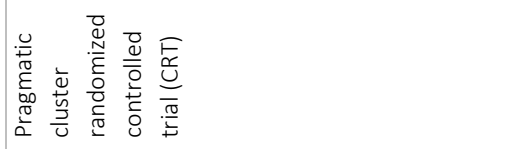 & 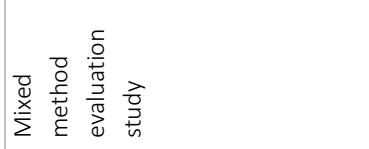 \\
\hline & 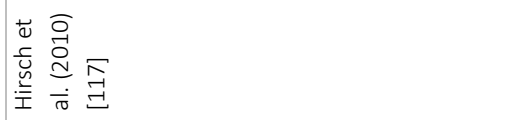 & 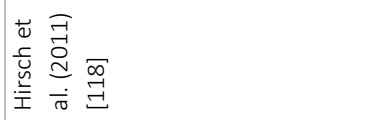 \\
\hline
\end{tabular}




\begin{tabular}{|c|c|c|}
\hline & 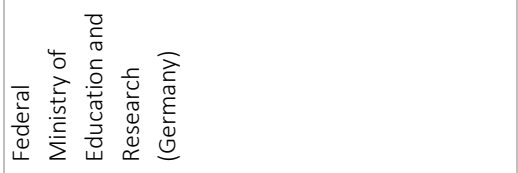 & 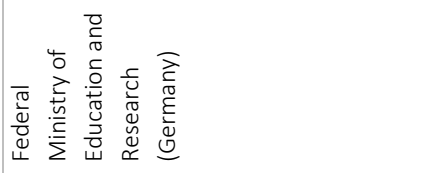 \\
\hline & 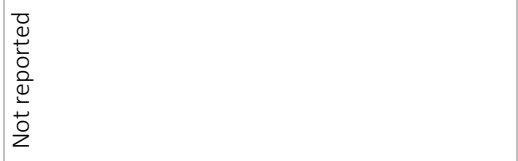 & \\
\hline & 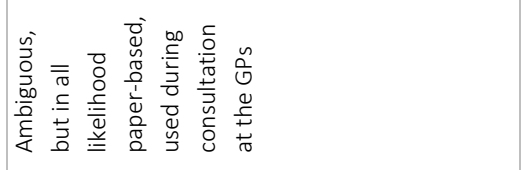 & 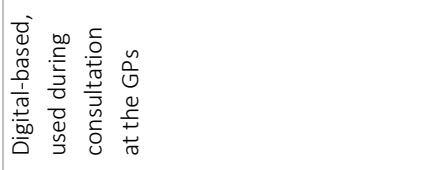 \\
\hline 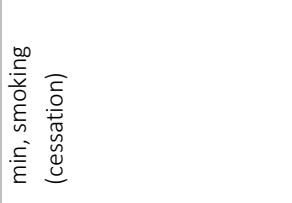 & 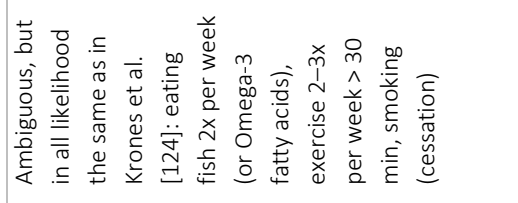 & 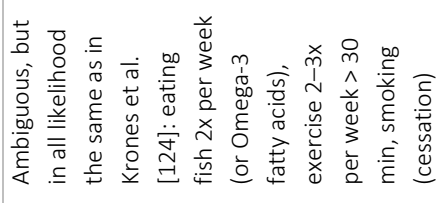 \\
\hline 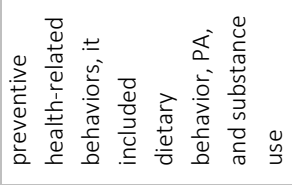 & 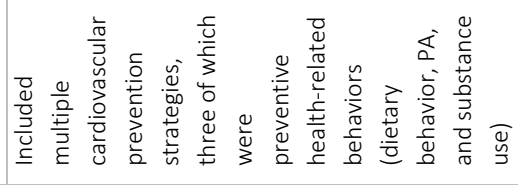 & 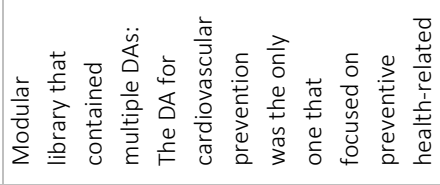 \\
\hline & 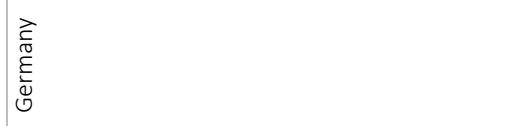 & 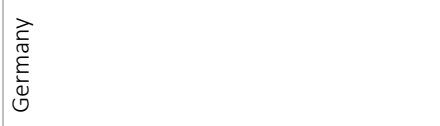 \\
\hline \multirow[t]{4}{*}{ 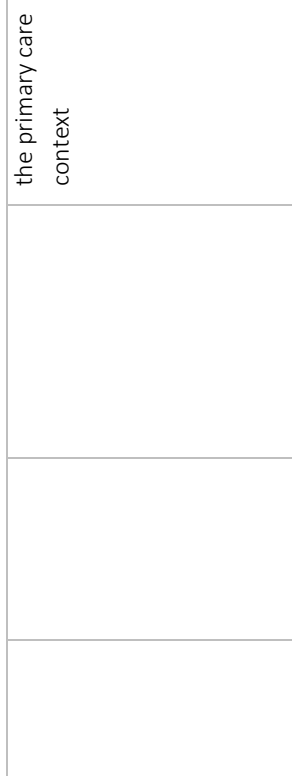 } & 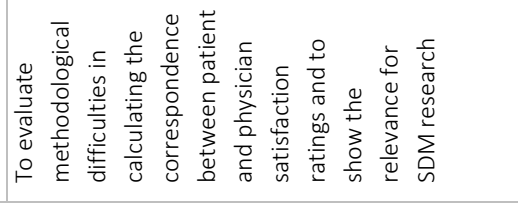 & 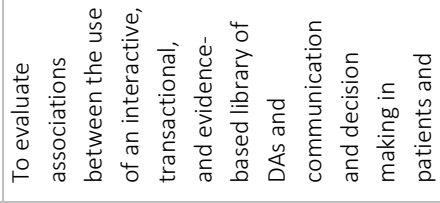 \\
\hline & 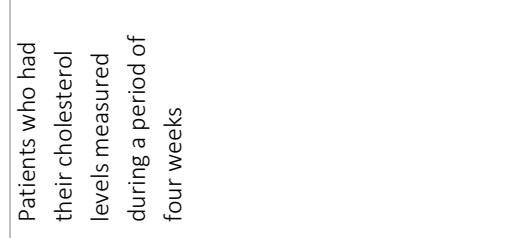 & 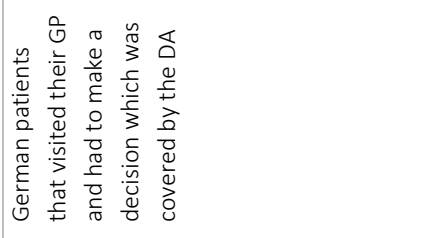 \\
\hline & 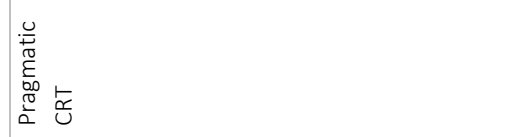 & 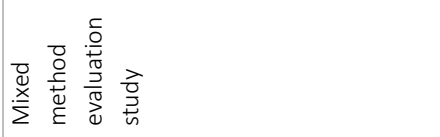 \\
\hline & 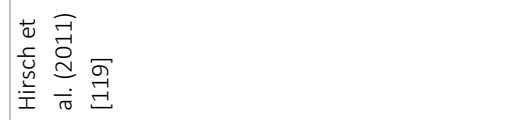 & 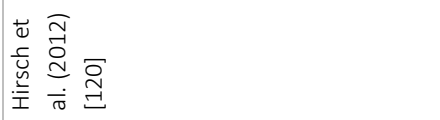 \\
\hline
\end{tabular}




\begin{tabular}{|c|c|c|}
\hline & 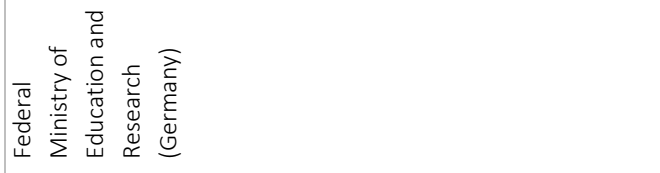 & 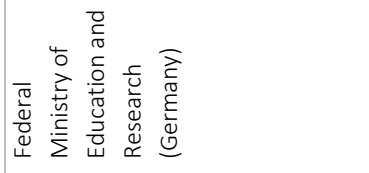 \\
\hline & 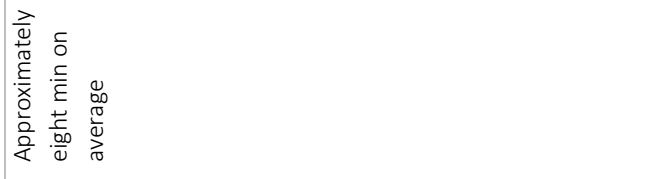 & 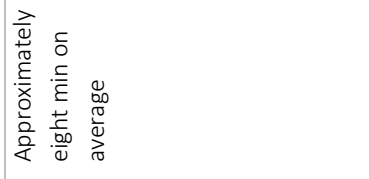 \\
\hline & 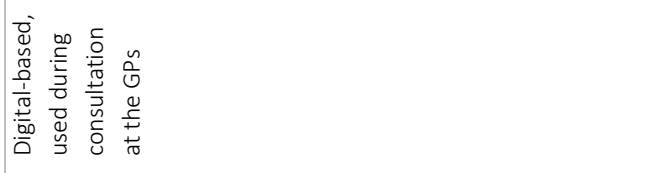 & 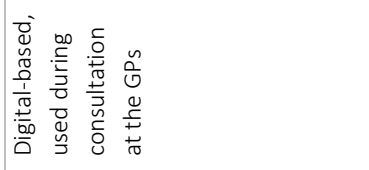 \\
\hline & 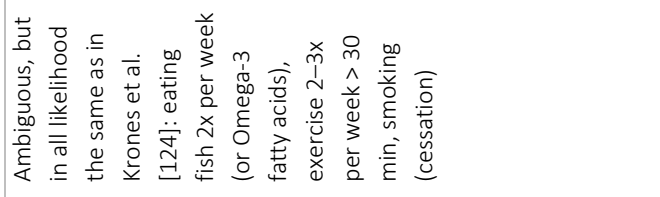 & 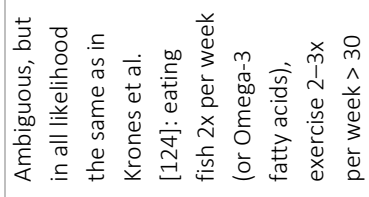 \\
\hline 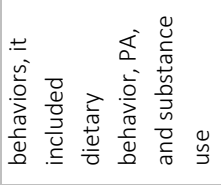 & 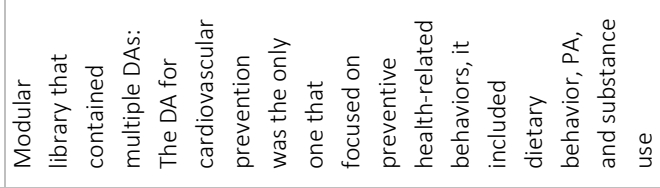 & 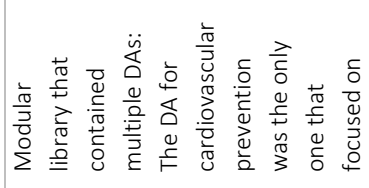 \\
\hline & 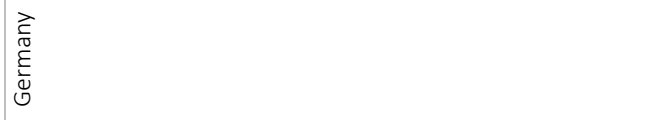 & 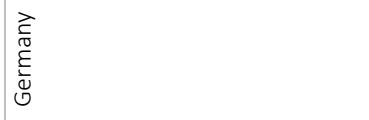 \\
\hline \multirow[t]{4}{*}{ 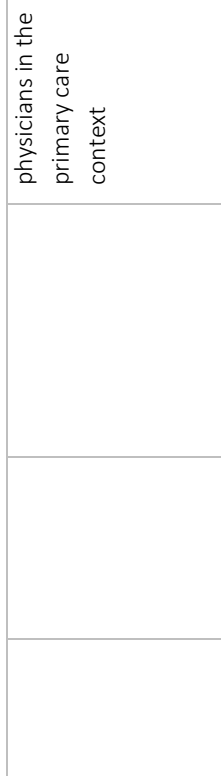 } & 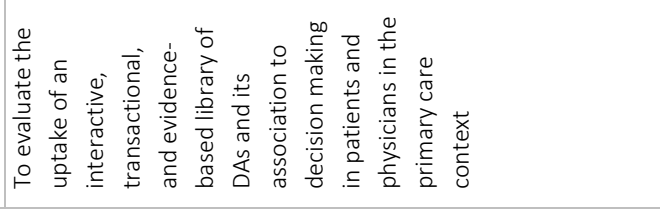 & 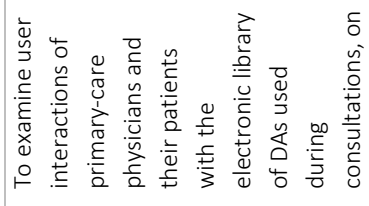 \\
\hline & 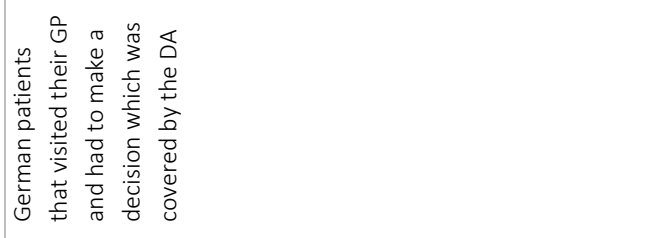 & 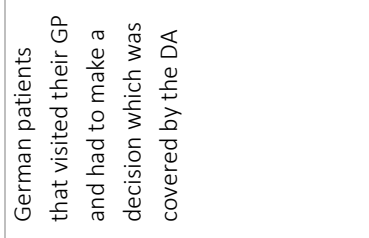 \\
\hline & 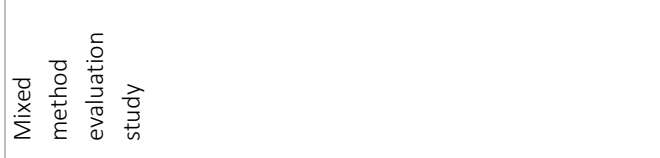 & 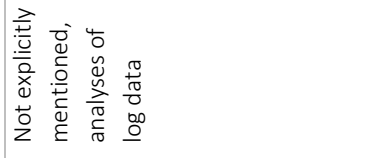 \\
\hline & 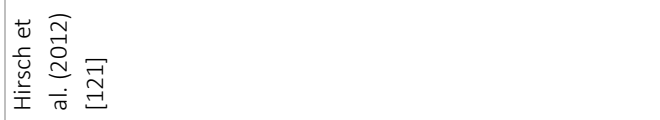 & 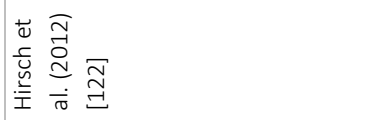 \\
\hline
\end{tabular}




\begin{tabular}{|c|c|}
\hline & 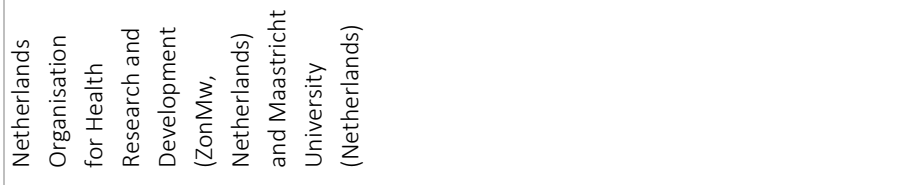 \\
\hline & 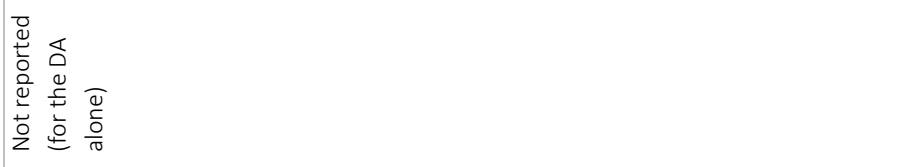 \\
\hline & 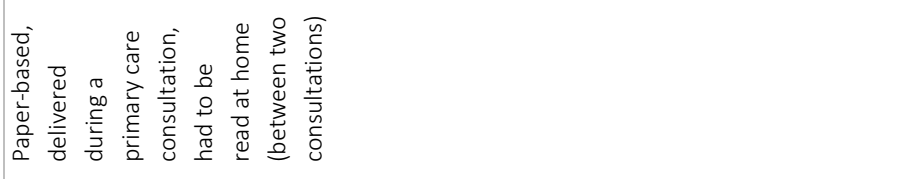 \\
\hline 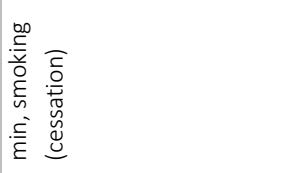 & 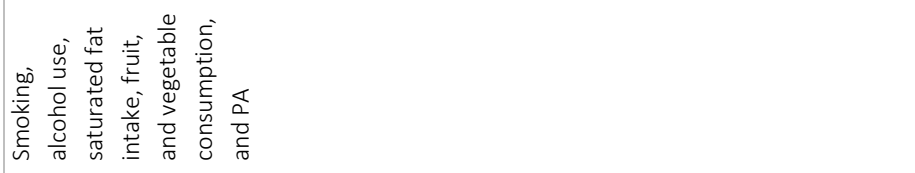 \\
\hline \multirow[t]{2}{*}{ 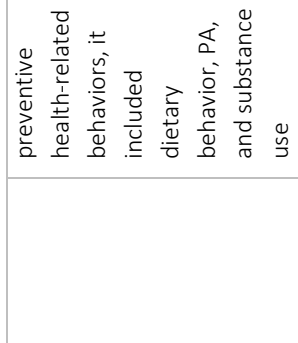 } & 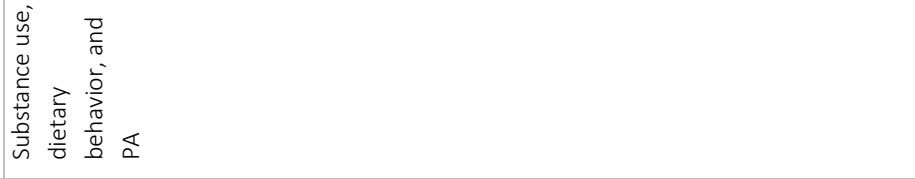 \\
\hline & 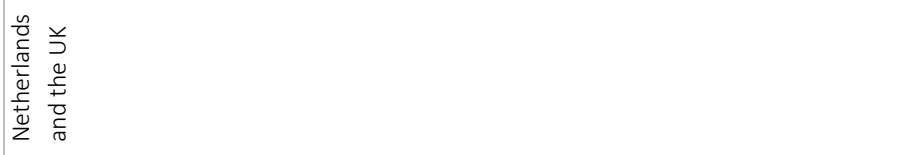 \\
\hline \multirow[t]{4}{*}{ 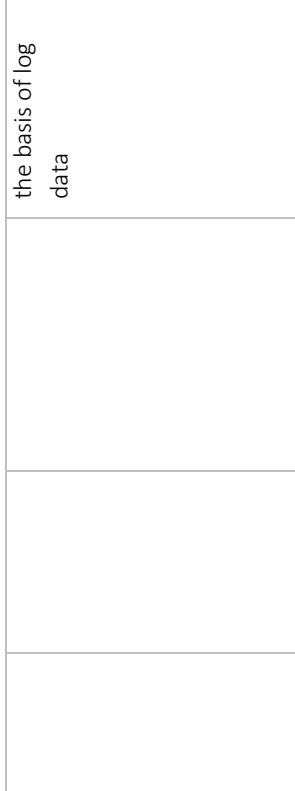 } & 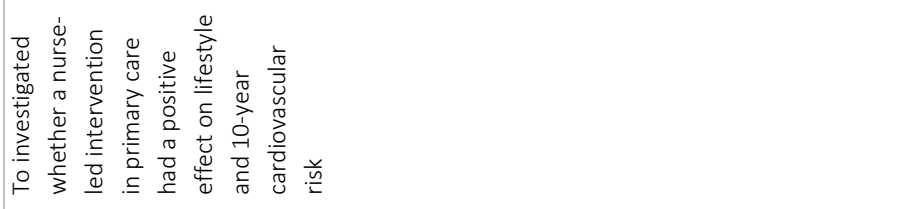 \\
\hline & 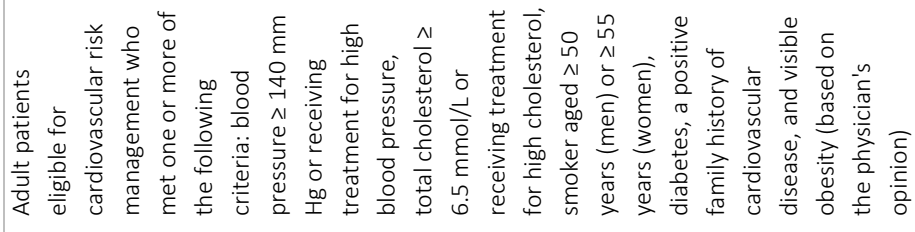 \\
\hline & 官 \\
\hline & 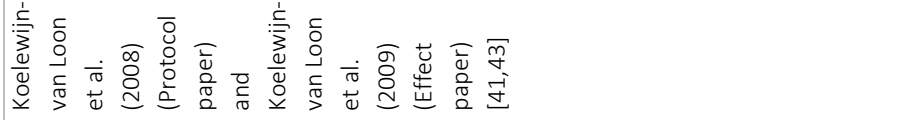 \\
\hline
\end{tabular}




\begin{tabular}{|c|c|}
\hline 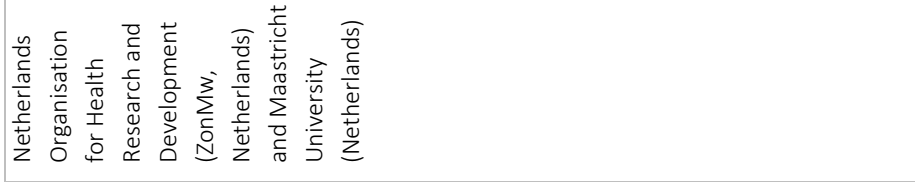 & 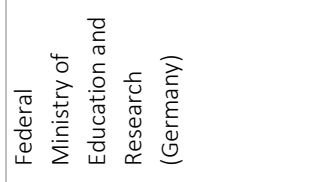 \\
\hline 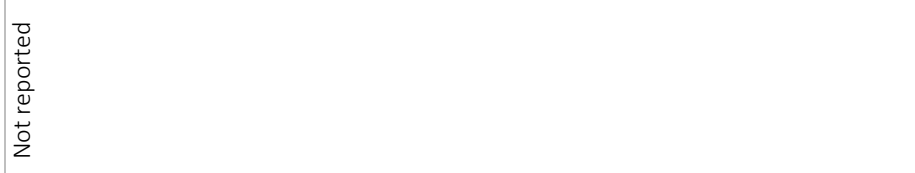 & 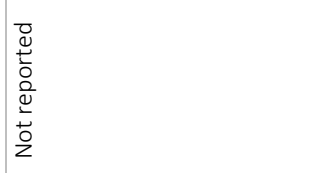 \\
\hline 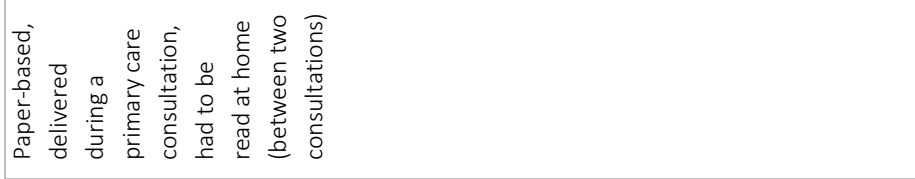 & 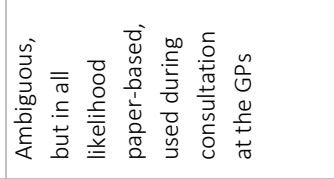 \\
\hline 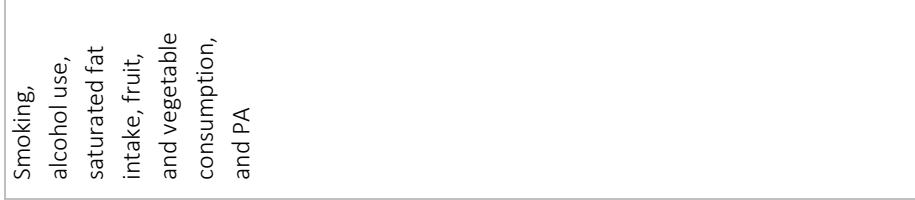 & 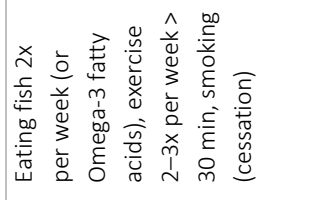 \\
\hline 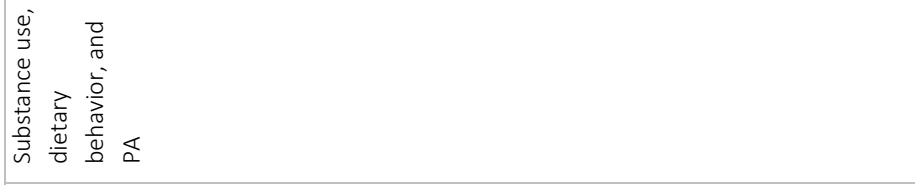 & 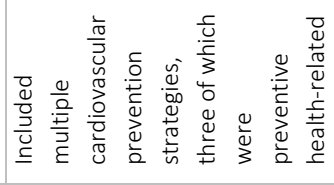 \\
\hline 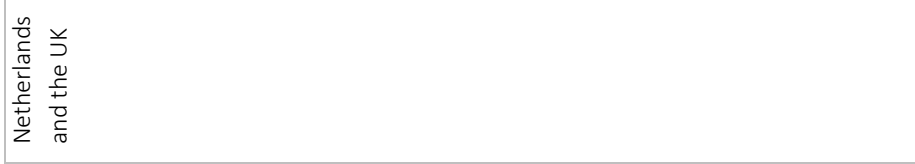 & 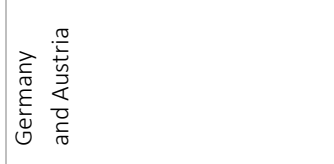 \\
\hline 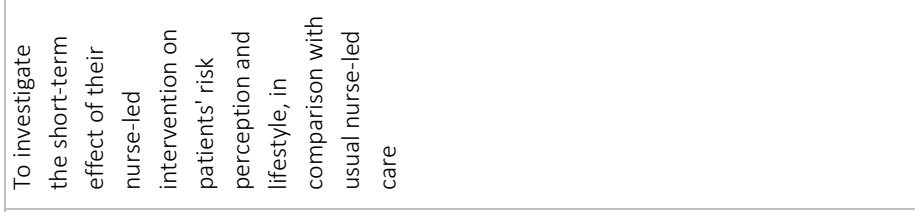 & 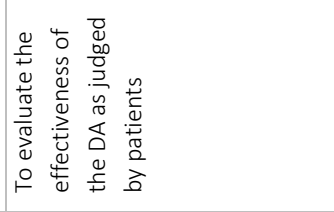 \\
\hline 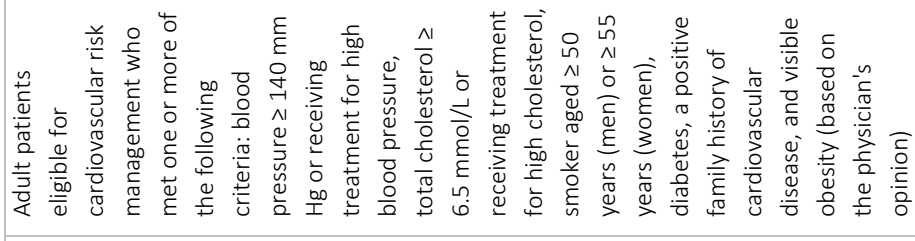 & 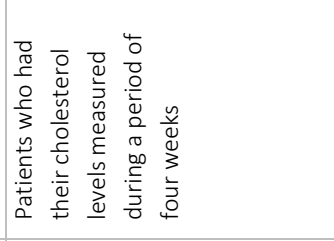 \\
\hline tr & 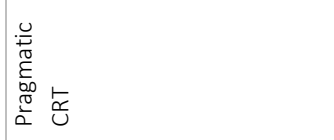 \\
\hline 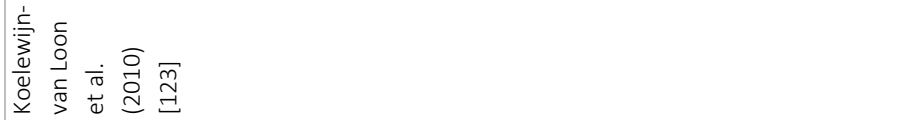 & 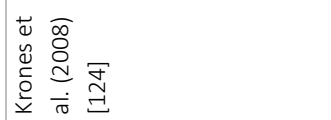 \\
\hline
\end{tabular}




\begin{tabular}{|c|c|c|}
\hline & 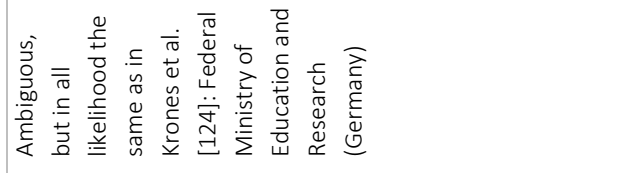 & 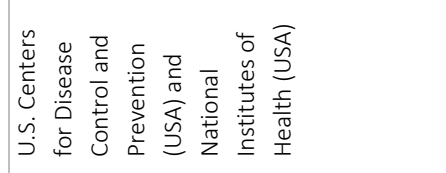 \\
\hline & 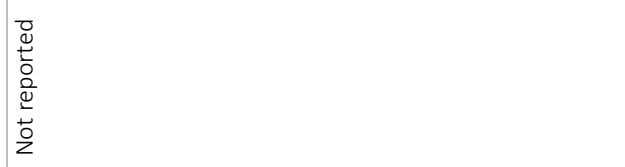 & 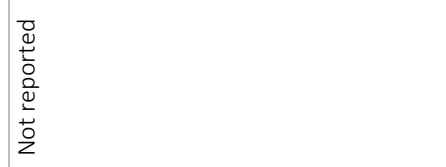 \\
\hline & 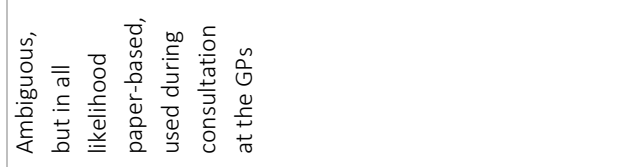 & 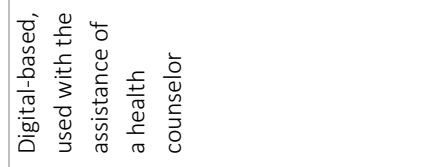 \\
\hline & 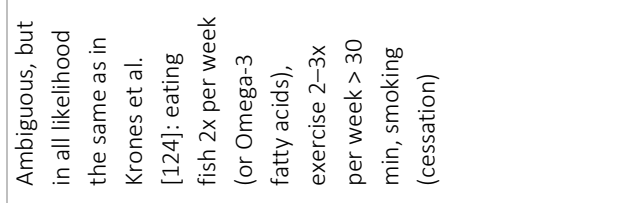 & 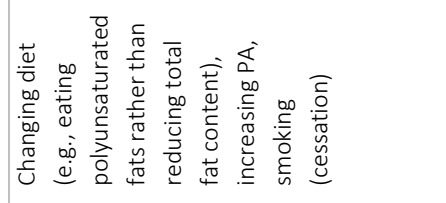 \\
\hline \multirow[t]{6}{*}{ 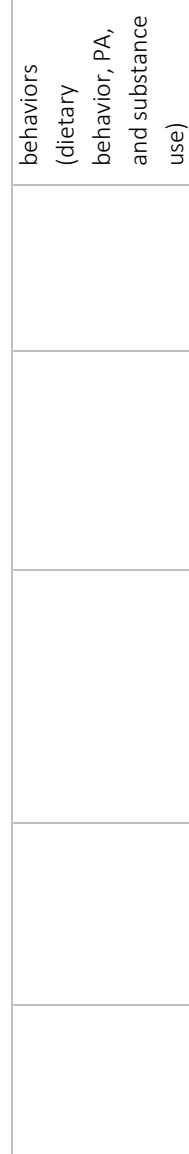 } & 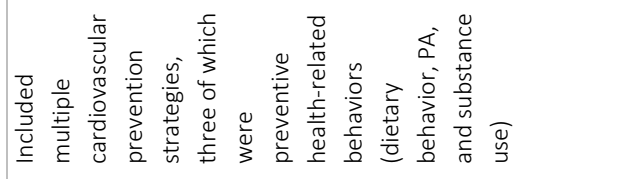 & 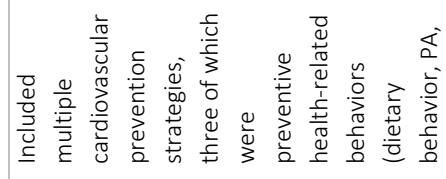 \\
\hline & 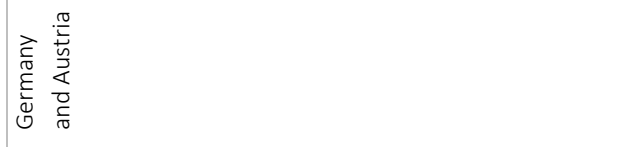 & 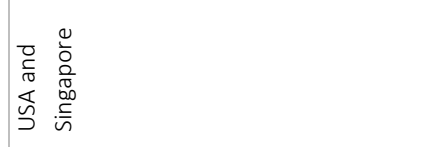 \\
\hline & 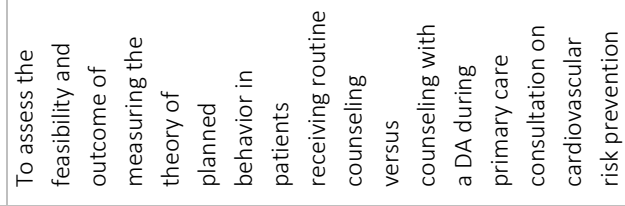 & 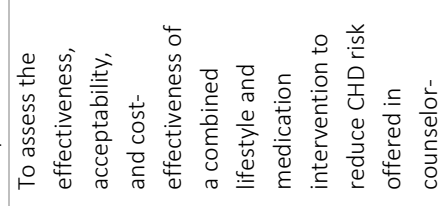 \\
\hline & 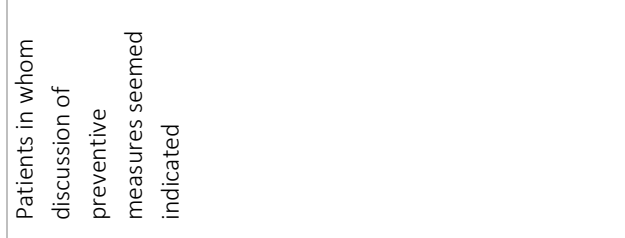 & 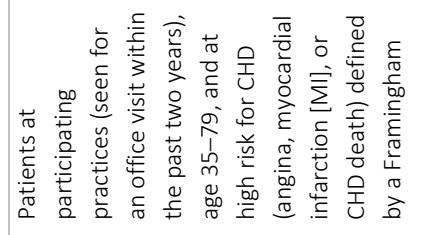 \\
\hline & 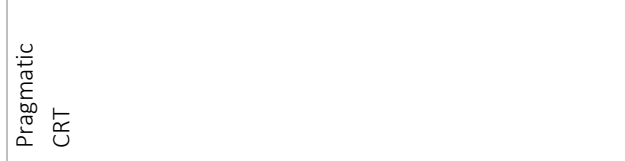 & 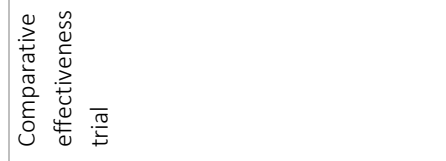 \\
\hline & 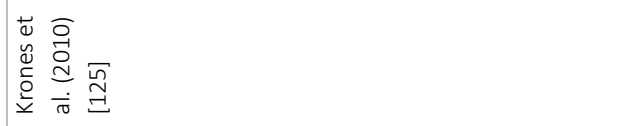 & 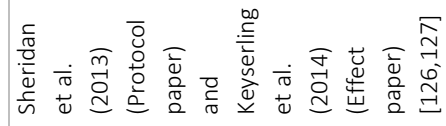 \\
\hline
\end{tabular}




\begin{tabular}{|c|c|c|}
\hline & 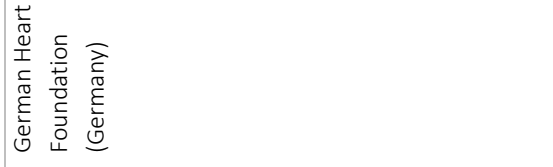 & 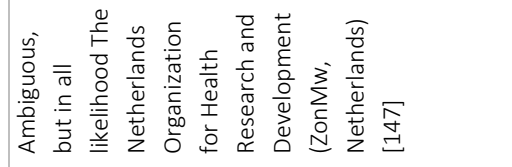 \\
\hline & & 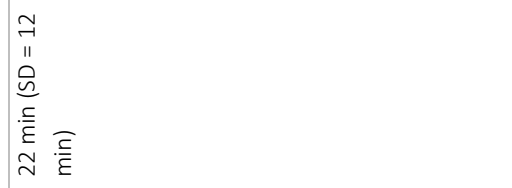 \\
\hline & 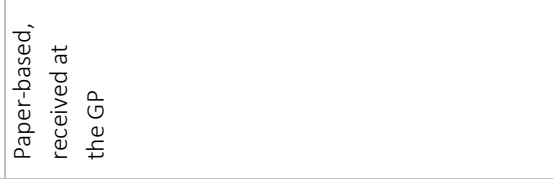 & 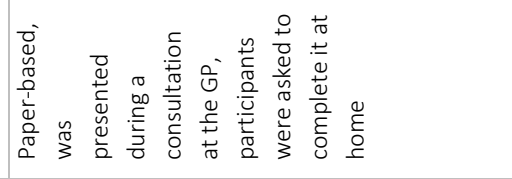 \\
\hline & 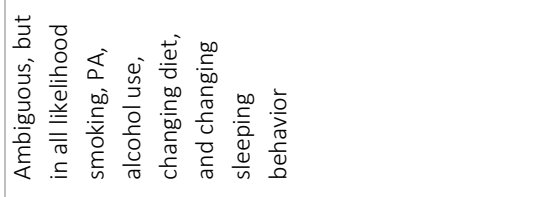 & 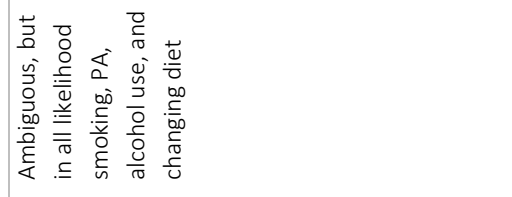 \\
\hline \multirow[t]{2}{*}{ 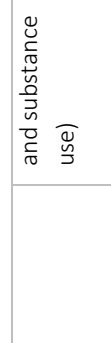 } & 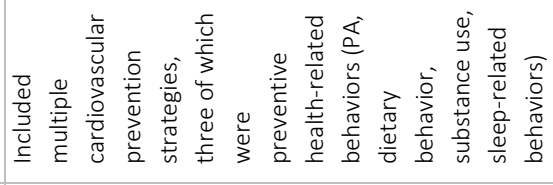 & 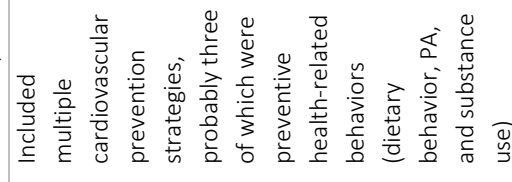 \\
\hline & & 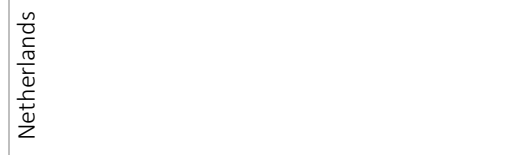 \\
\hline 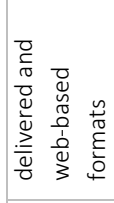 & 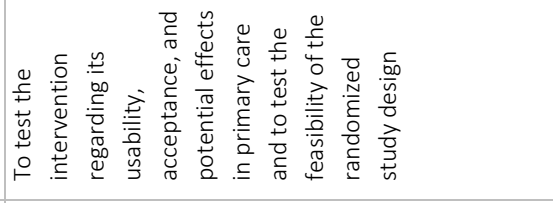 & 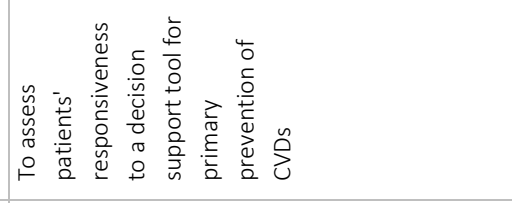 \\
\hline \multirow[t]{3}{*}{ 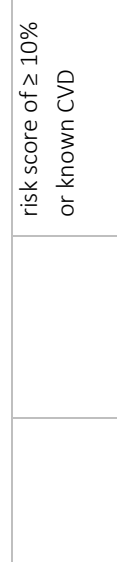 } & 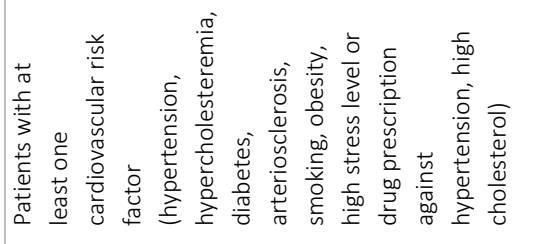 & 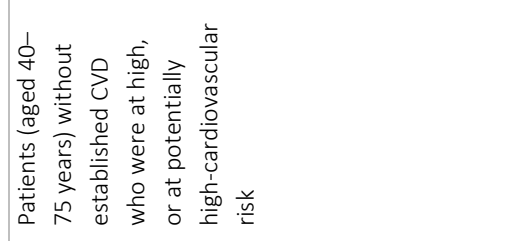 \\
\hline & 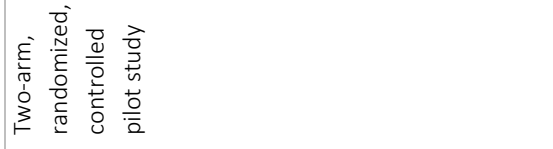 & 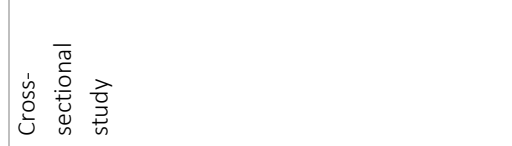 \\
\hline & 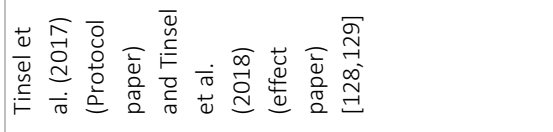 & 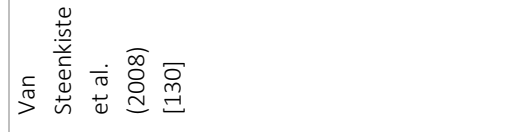 \\
\hline
\end{tabular}




\begin{tabular}{|c|c|c|}
\hline 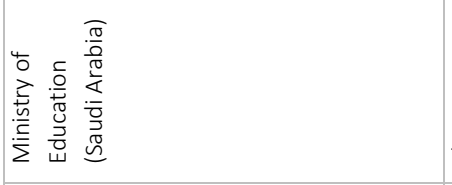 & 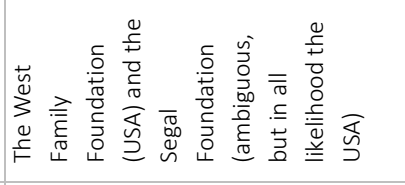 & 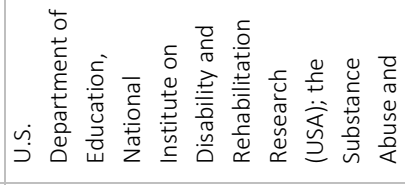 \\
\hline 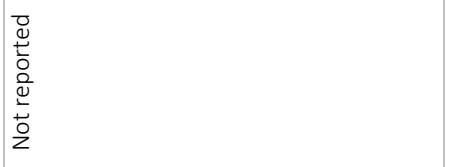 & $\begin{array}{l}\frac{c}{c} \\
\bar{c} \\
\varnothing \\
\phi \\
1 \\
0\end{array}$ & 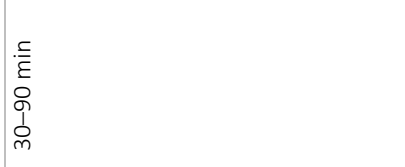 \\
\hline 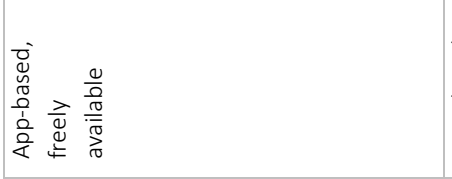 & 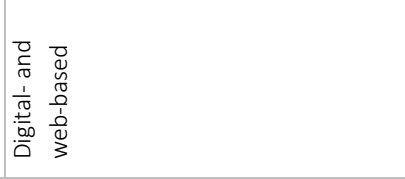 & 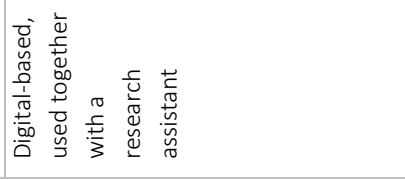 \\
\hline 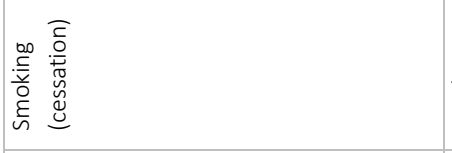 & 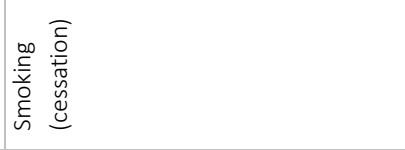 & 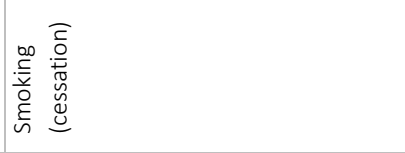 \\
\hline & 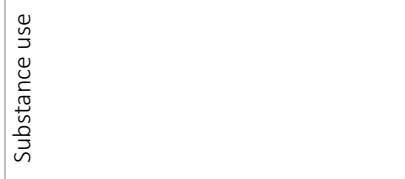 & 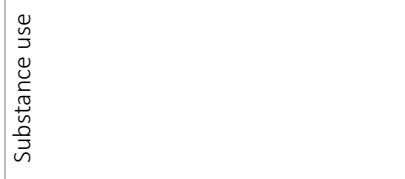 \\
\hline 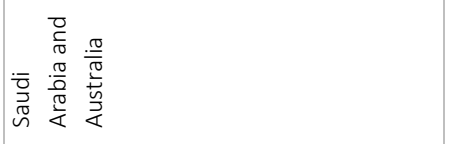 & 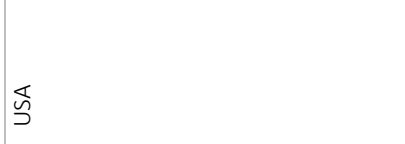 & گิ \\
\hline 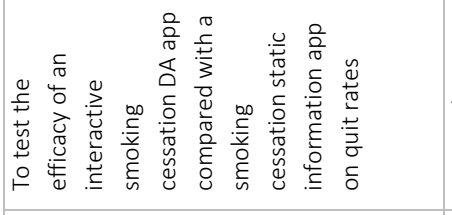 & 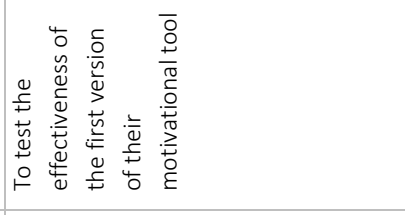 & 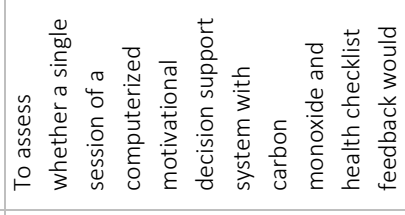 \\
\hline 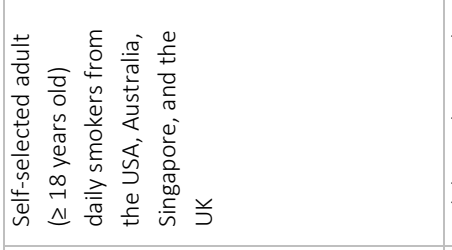 & 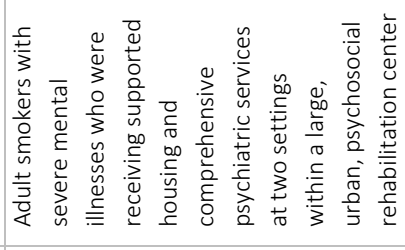 & 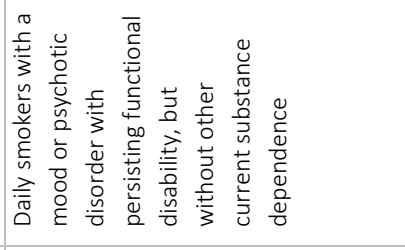 \\
\hline 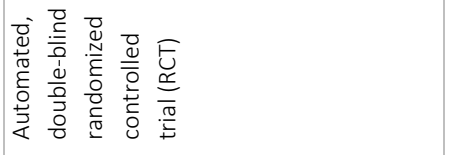 & 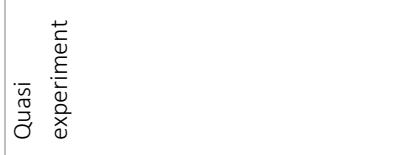 & $\underset{\check{c}}{\mathrm{t}}$ \\
\hline 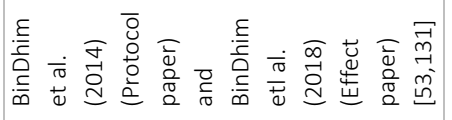 & 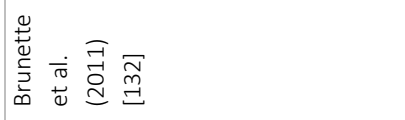 & 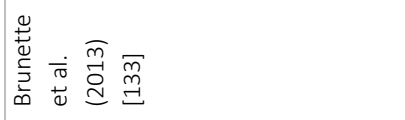 \\
\hline
\end{tabular}




\begin{tabular}{|c|c|c|}
\hline 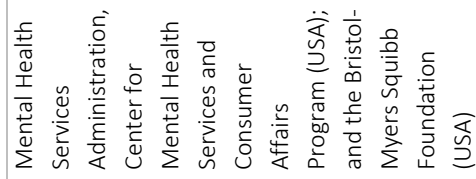 & 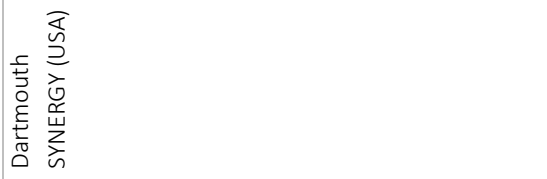 & 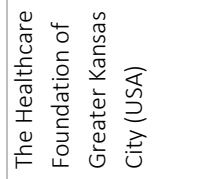 \\
\hline & 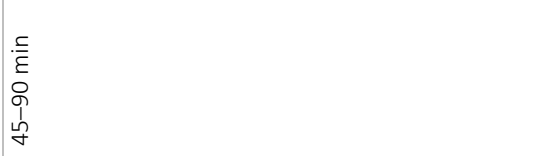 & 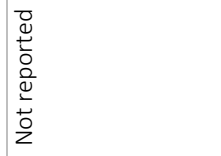 \\
\hline & 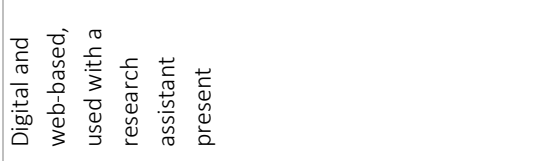 & 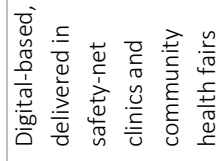 \\
\hline & 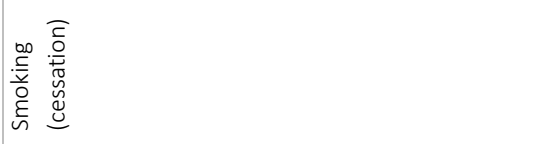 & 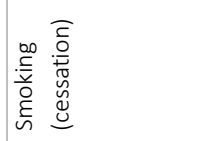 \\
\hline & 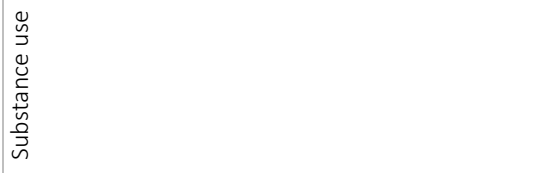 & 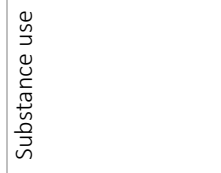 \\
\hline & 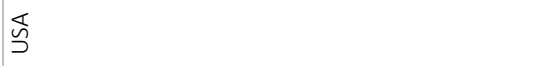 & ک \\
\hline \multirow[t]{4}{*}{ 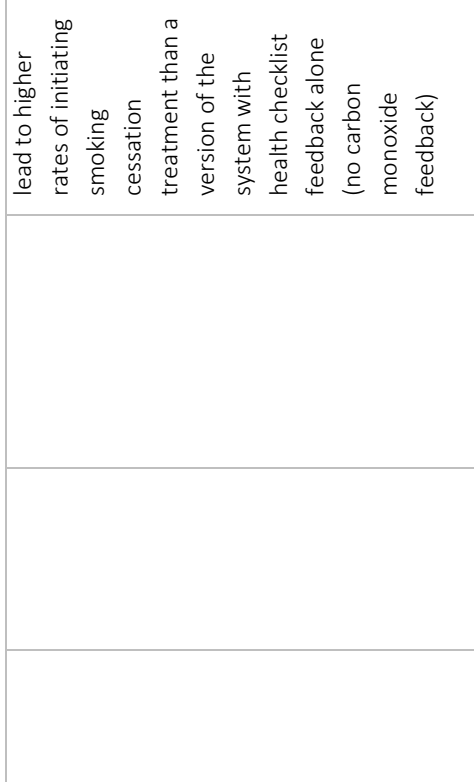 } & 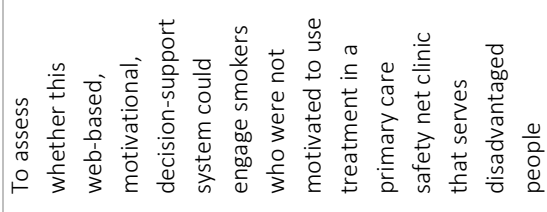 & 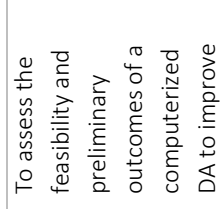 \\
\hline & 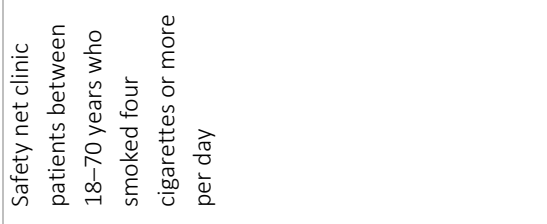 & 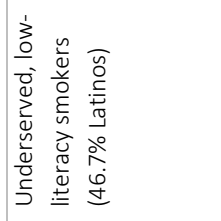 \\
\hline & 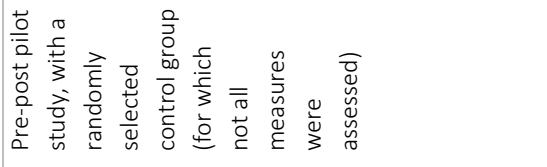 & 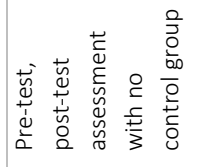 \\
\hline & 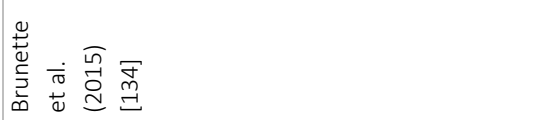 & 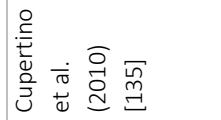 \\
\hline
\end{tabular}




\begin{tabular}{|c|c|c|}
\hline & 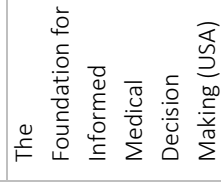 & 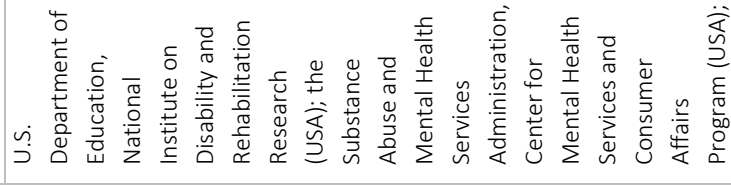 \\
\hline & 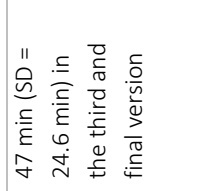 & 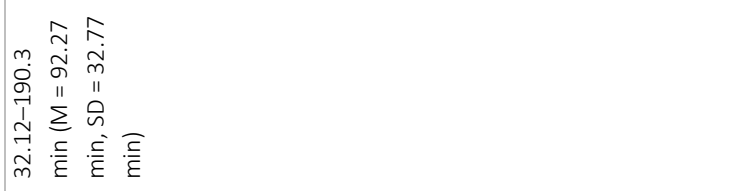 \\
\hline & 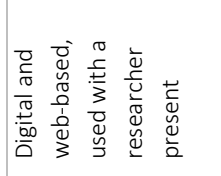 & 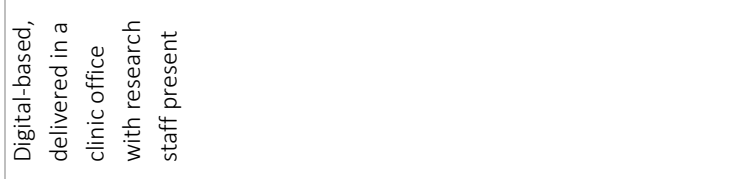 \\
\hline & 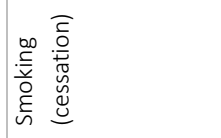 & 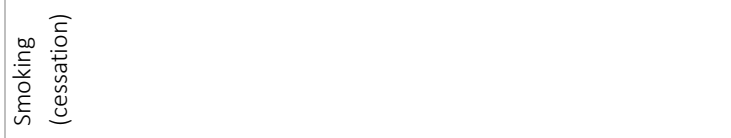 \\
\hline & 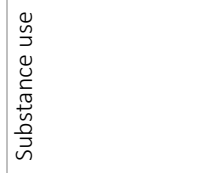 & 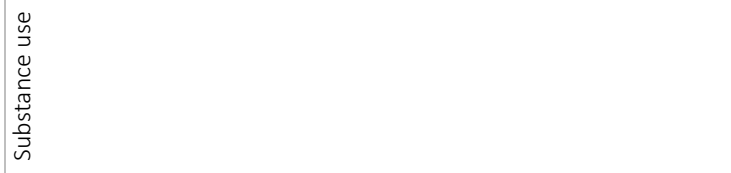 \\
\hline & گ̆ & گે \\
\hline \multirow[t]{4}{*}{ 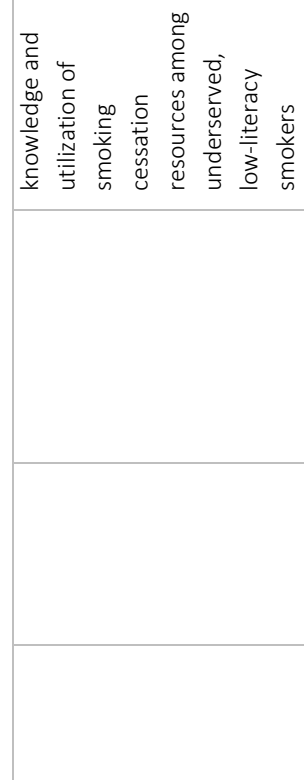 } & 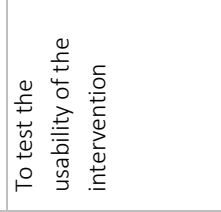 & 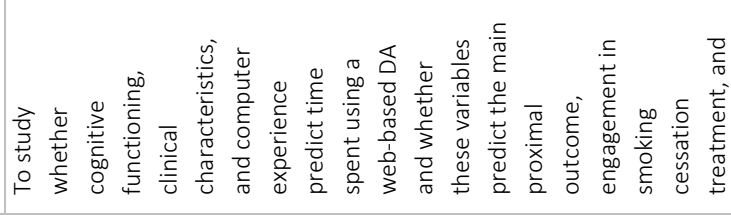 \\
\hline & 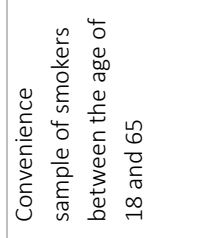 & 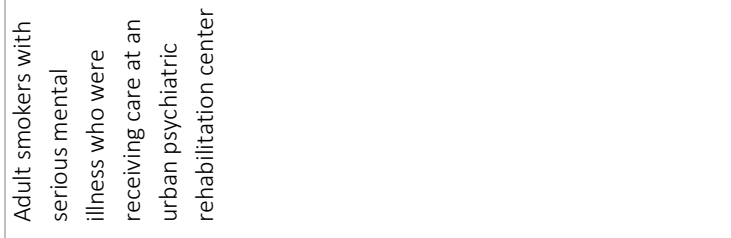 \\
\hline & 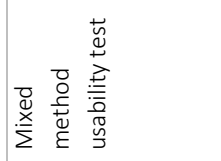 & 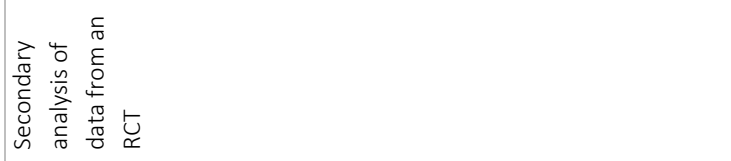 \\
\hline & 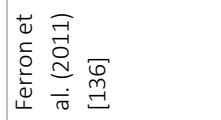 & 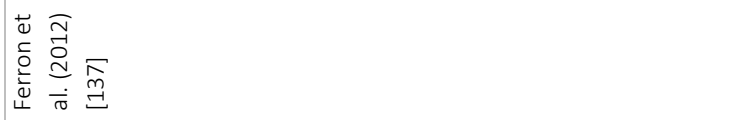 \\
\hline
\end{tabular}




\begin{tabular}{|c|c|c|c|}
\hline 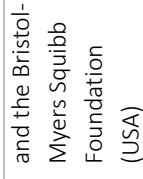 & 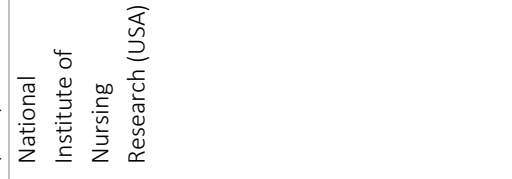 & 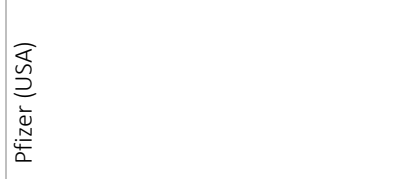 & 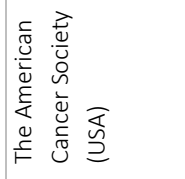 \\
\hline & 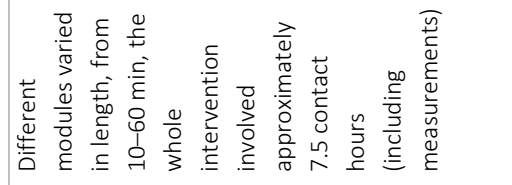 & 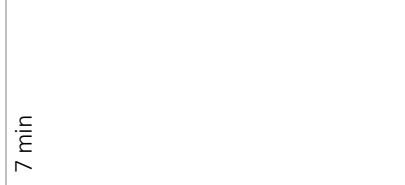 & 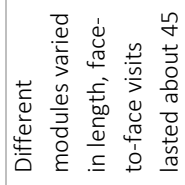 \\
\hline & 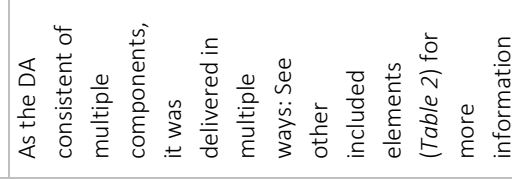 & 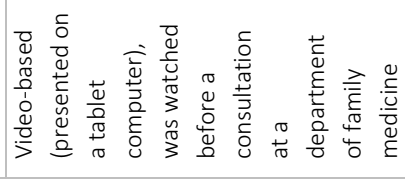 & 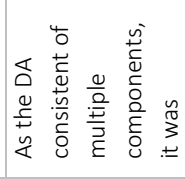 \\
\hline & 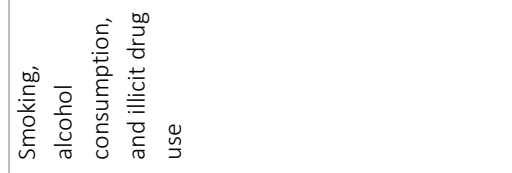 & 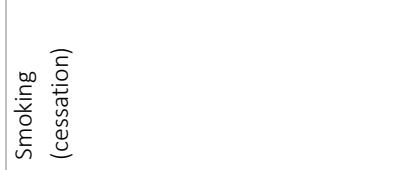 & 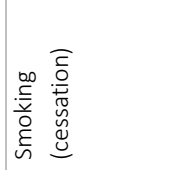 \\
\hline & 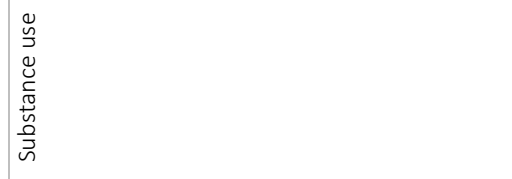 & 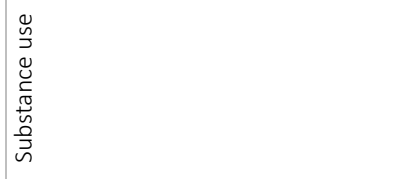 & 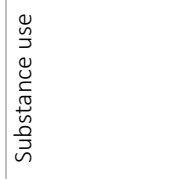 \\
\hline & ๕̊ & 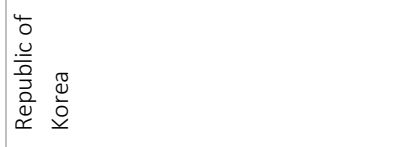 & گิ \\
\hline \multirow[t]{4}{*}{ 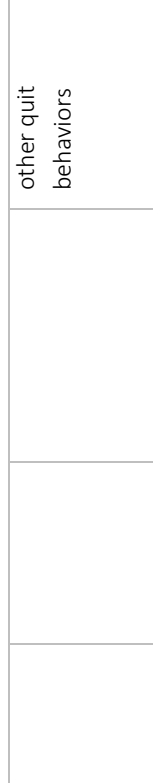 } & 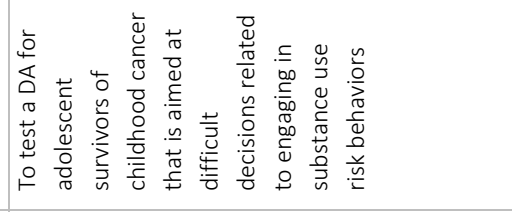 & 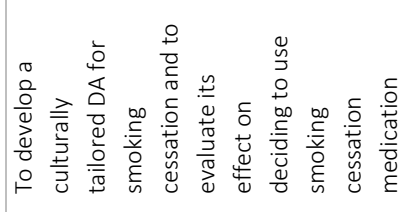 & 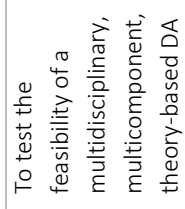 \\
\hline & 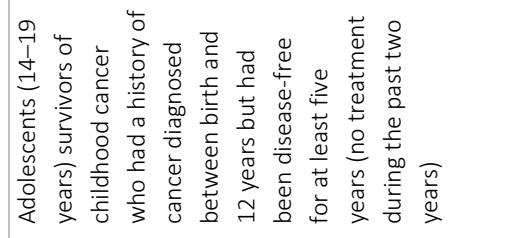 & 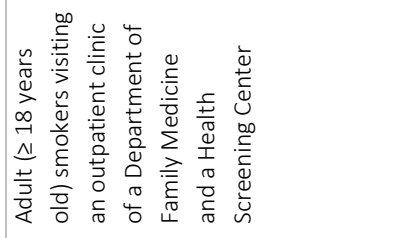 & 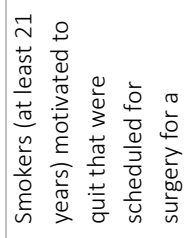 \\
\hline & 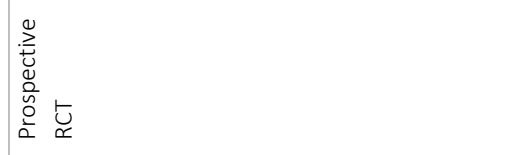 & 딴 & 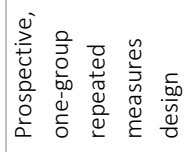 \\
\hline & 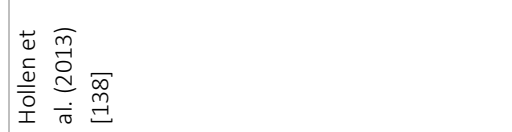 & 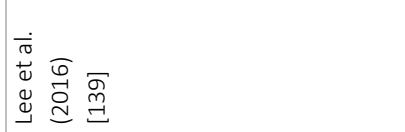 & 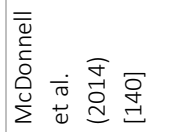 \\
\hline
\end{tabular}




\begin{tabular}{|c|c|c|}
\hline & 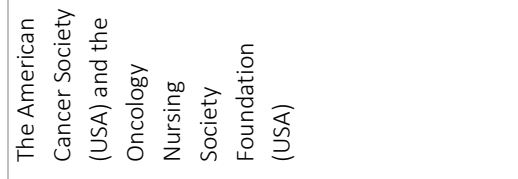 & 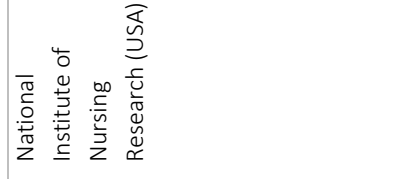 \\
\hline 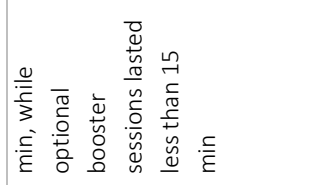 & 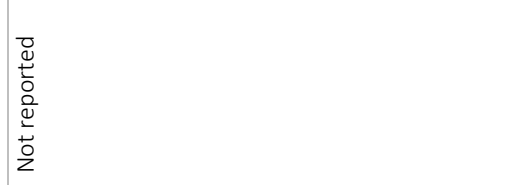 & 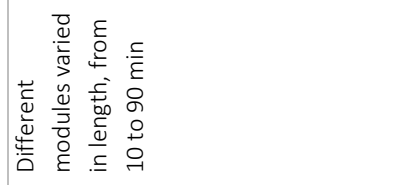 \\
\hline \multirow[t]{5}{*}{ 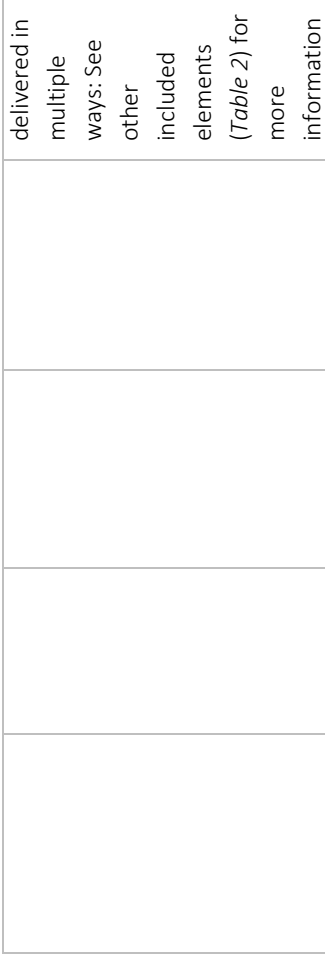 } & 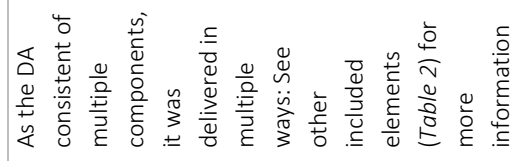 & 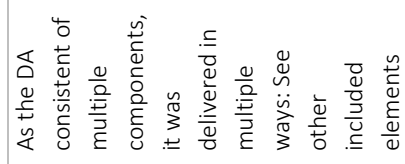 \\
\hline & 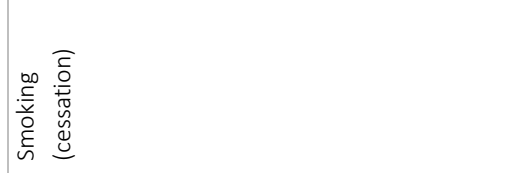 & 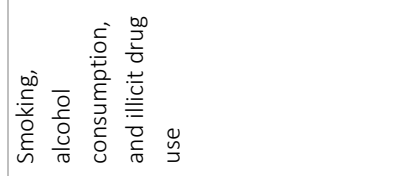 \\
\hline & 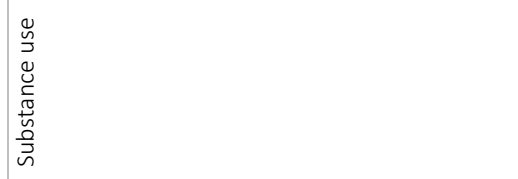 & 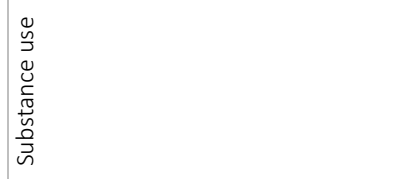 \\
\hline & $\overleftarrow{s}$ & $\overleftrightarrow{s}$ \\
\hline & 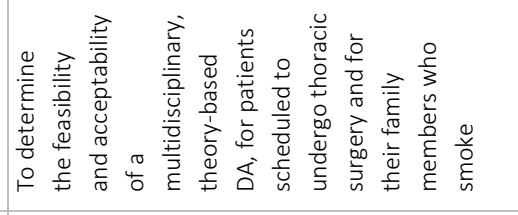 & 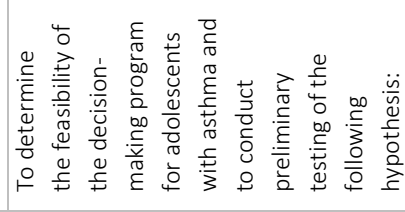 \\
\hline \multirow[t]{3}{*}{ 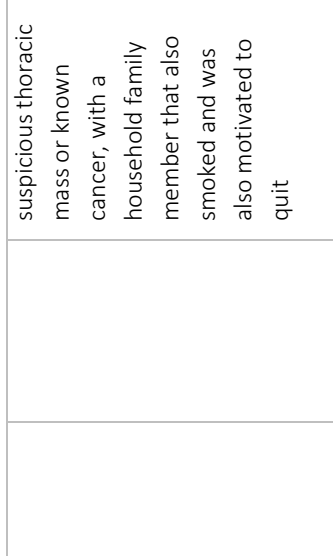 } & 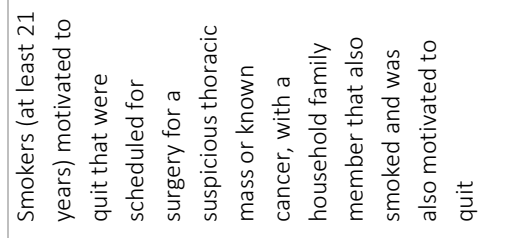 & 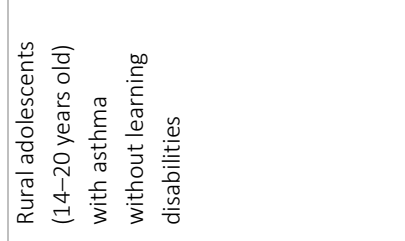 \\
\hline & 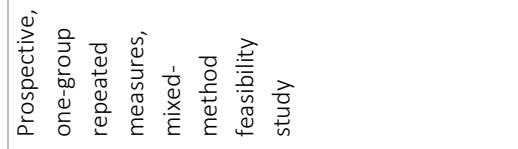 & 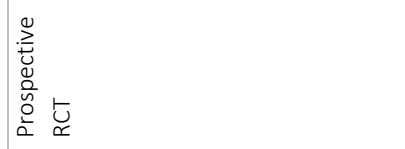 \\
\hline & 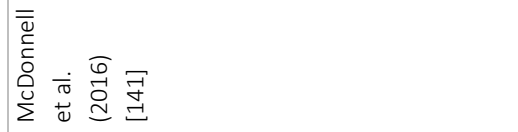 & 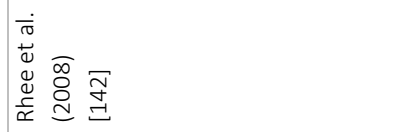 \\
\hline
\end{tabular}




\begin{tabular}{|c|c|}
\hline & 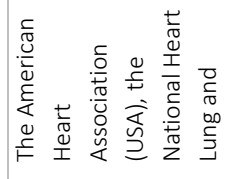 \\
\hline & 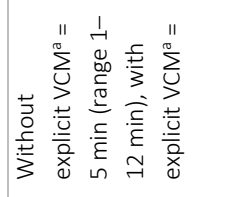 \\
\hline \multirow[t]{4}{*}{ 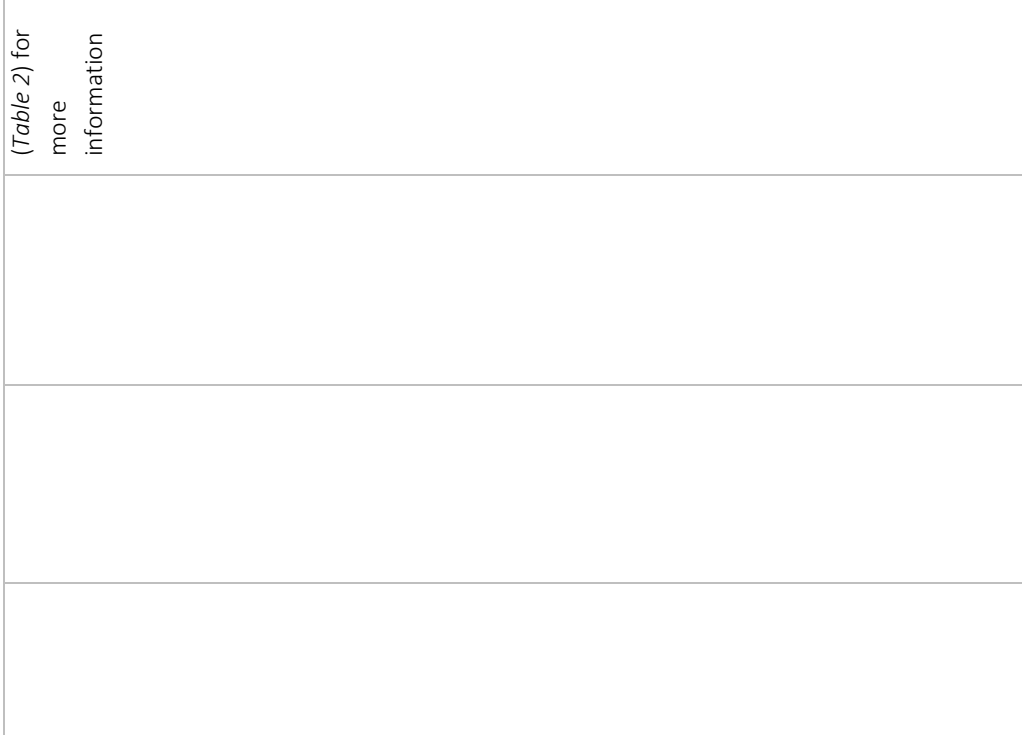 } & 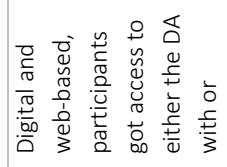 \\
\hline & 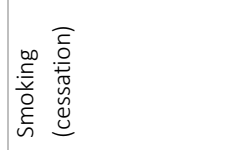 \\
\hline & 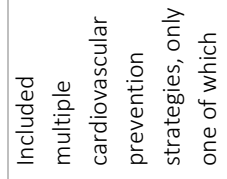 \\
\hline & \\
\hline \multirow[t]{4}{*}{ 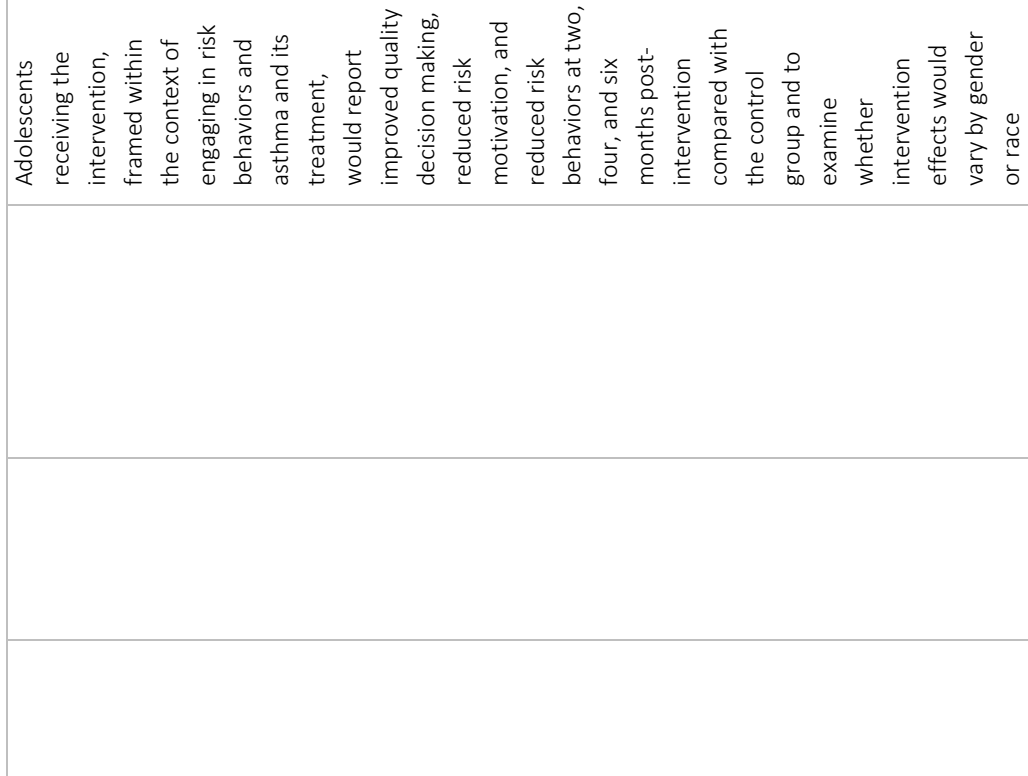 } & 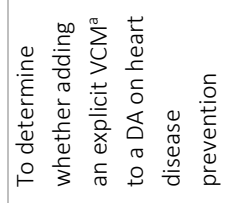 \\
\hline & 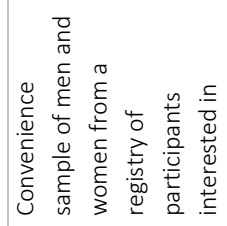 \\
\hline & 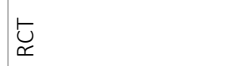 \\
\hline & 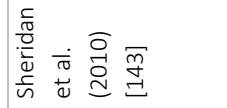 \\
\hline
\end{tabular}




\begin{tabular}{|c|c|c|}
\hline 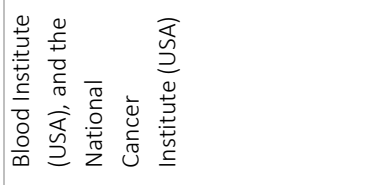 & 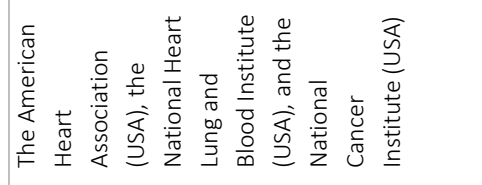 & 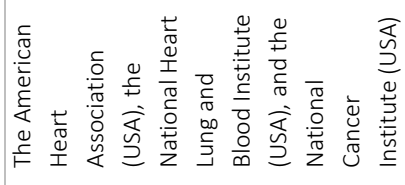 \\
\hline 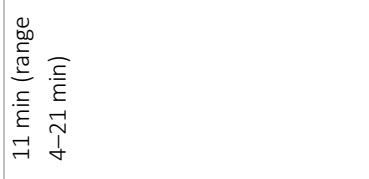 & 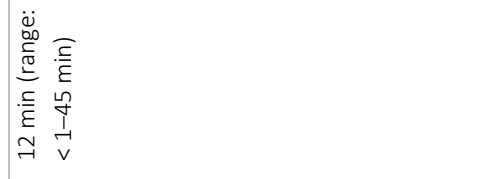 & 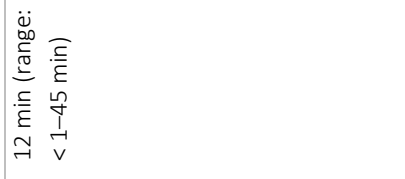 \\
\hline 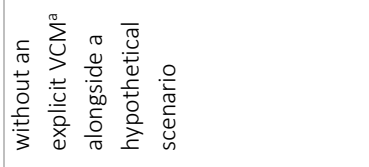 & 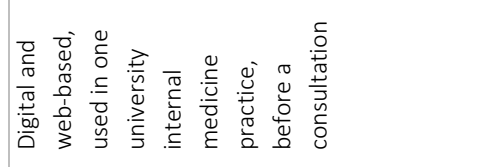 & 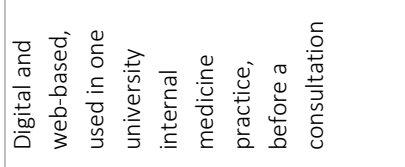 \\
\hline & 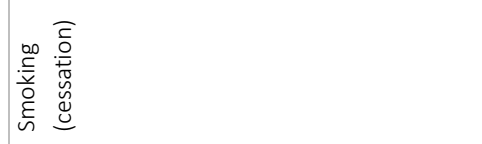 & 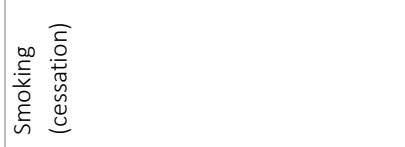 \\
\hline 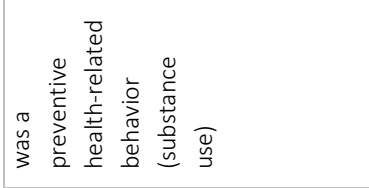 & 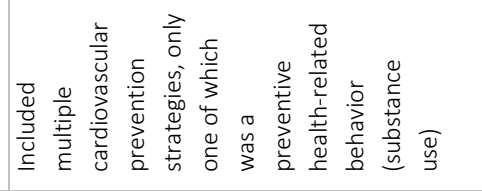 & 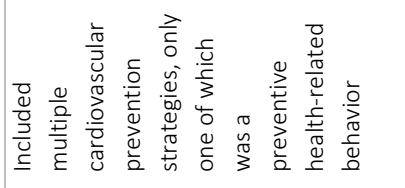 \\
\hline & گ્ & $\begin{array}{l}\overleftarrow{J} \\
\end{array}$ \\
\hline 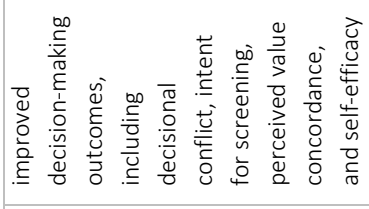 & 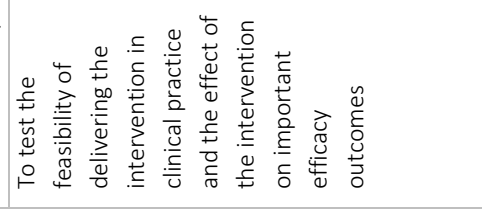 & 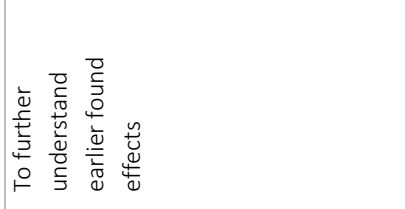 \\
\hline 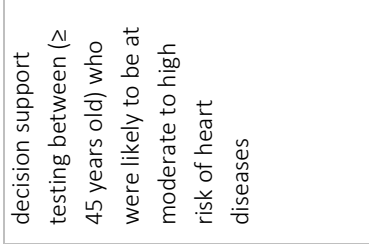 & 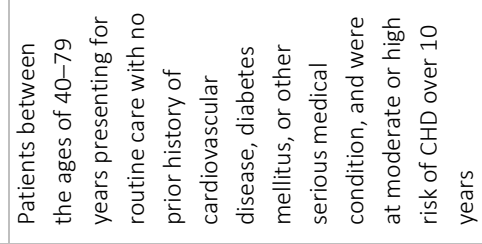 & 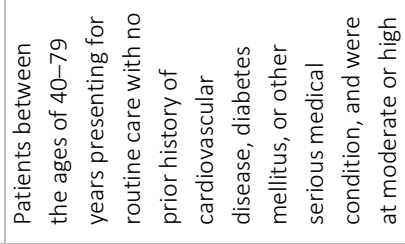 \\
\hline & $\underset{\check{x}}{\mathfrak{y}}$ & $\underset{\simeq}{\check{x}}$ \\
\hline & 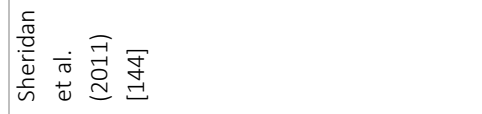 & 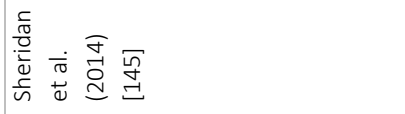 \\
\hline
\end{tabular}




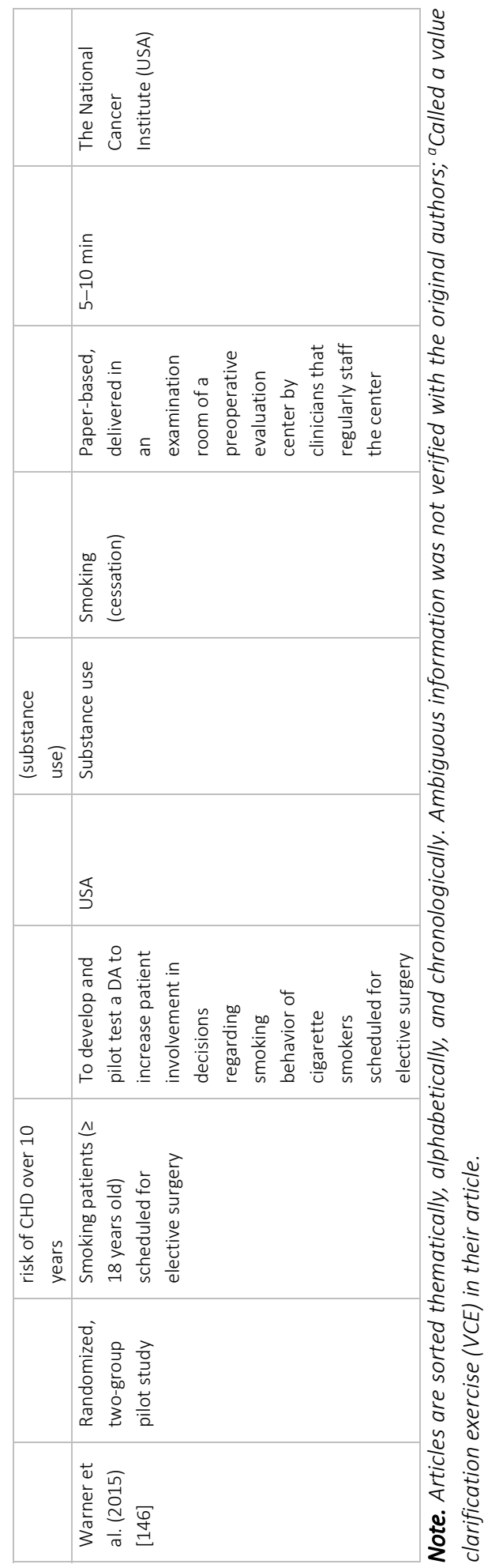




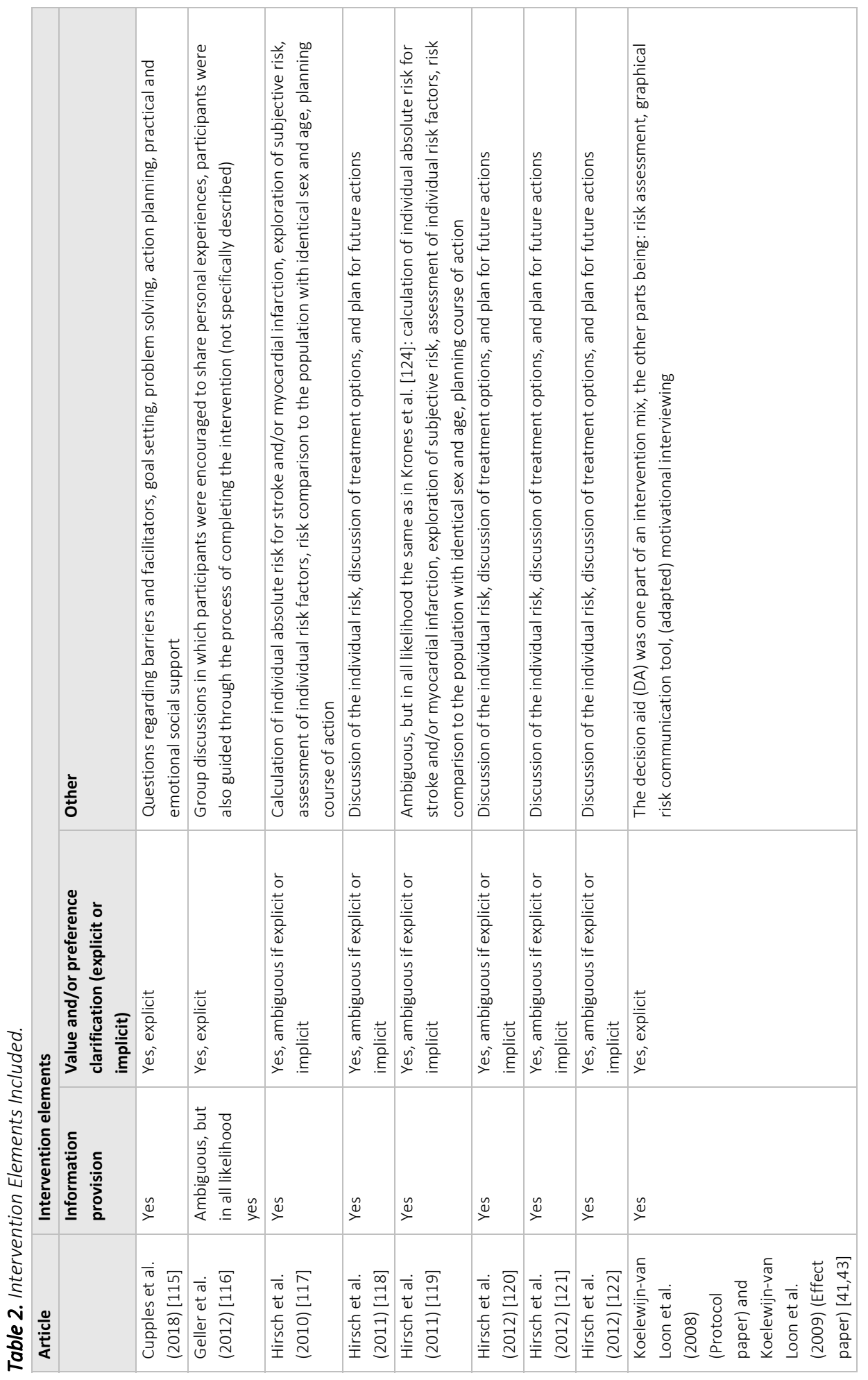




\begin{tabular}{|c|c|c|c|c|}
\hline 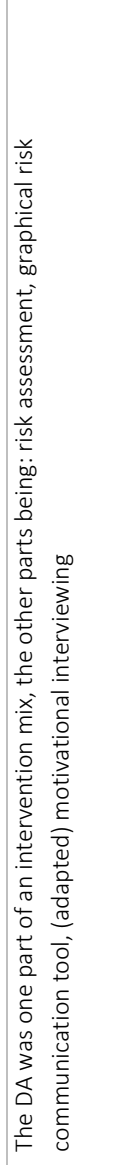 & 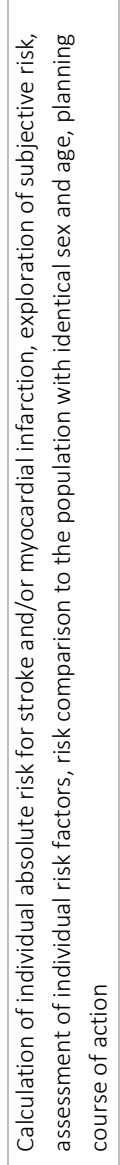 & 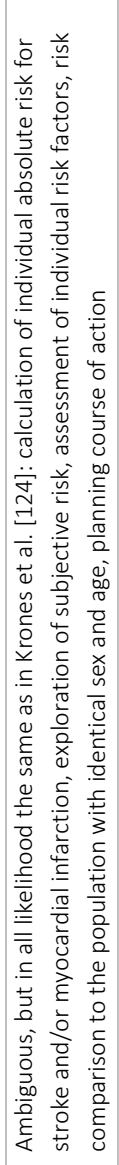 & 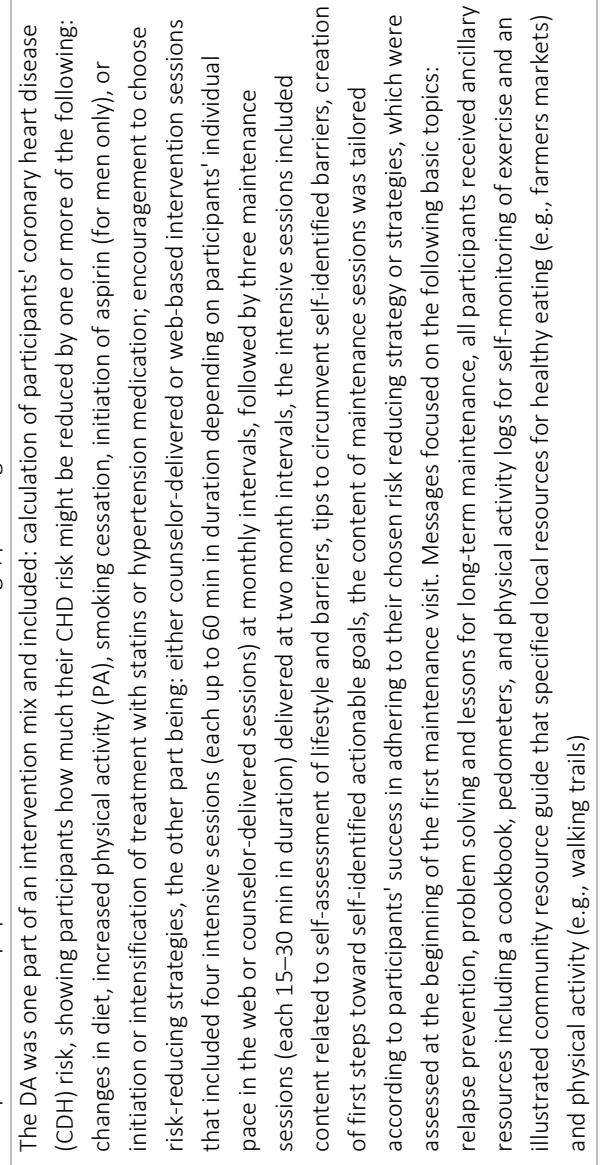 & 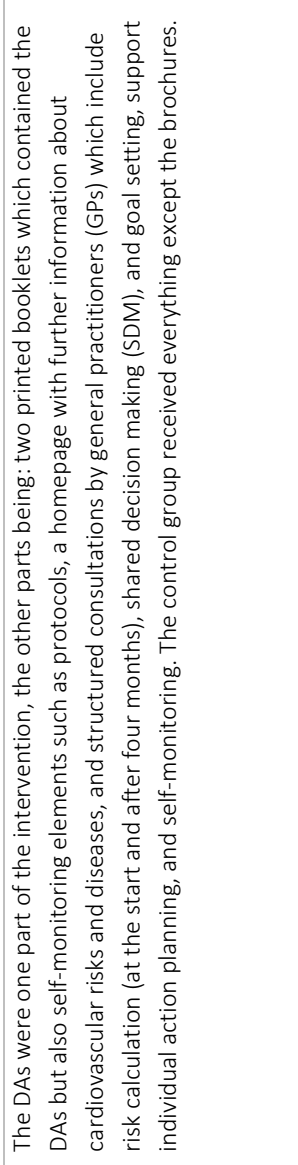 \\
\hline 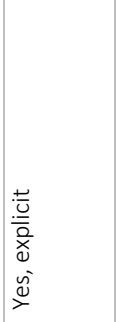 & 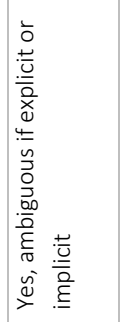 & 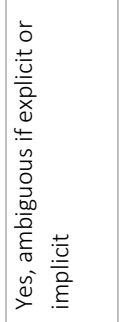 & 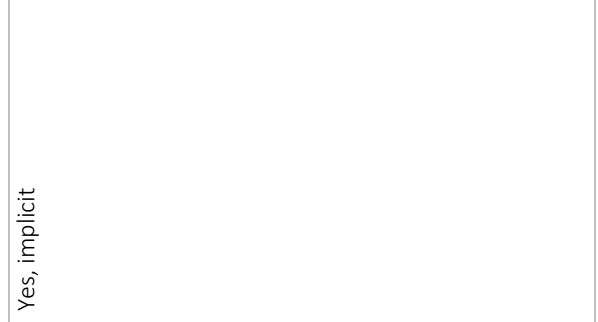 & 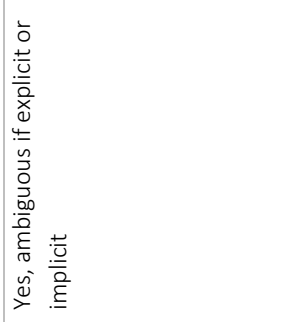 \\
\hline$\stackrel{\tilde{\Perp}}{\nu}$ & $\stackrel{\Perp}{\nu}$ & $\stackrel{\check{\Perp}}{>}$ & $\stackrel{\Perp}{\rightleftharpoons}$ & $\stackrel{\nu}{\nu}$ \\
\hline 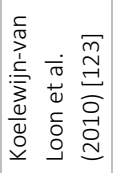 & 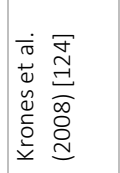 & 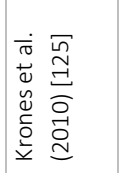 & 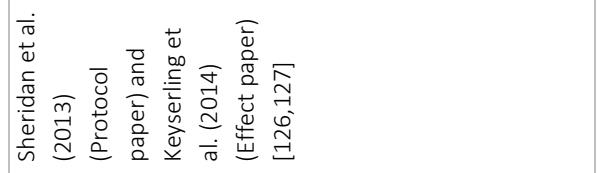 & 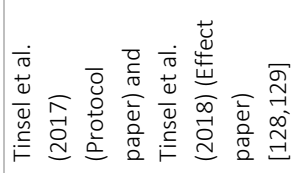 \\
\hline
\end{tabular}




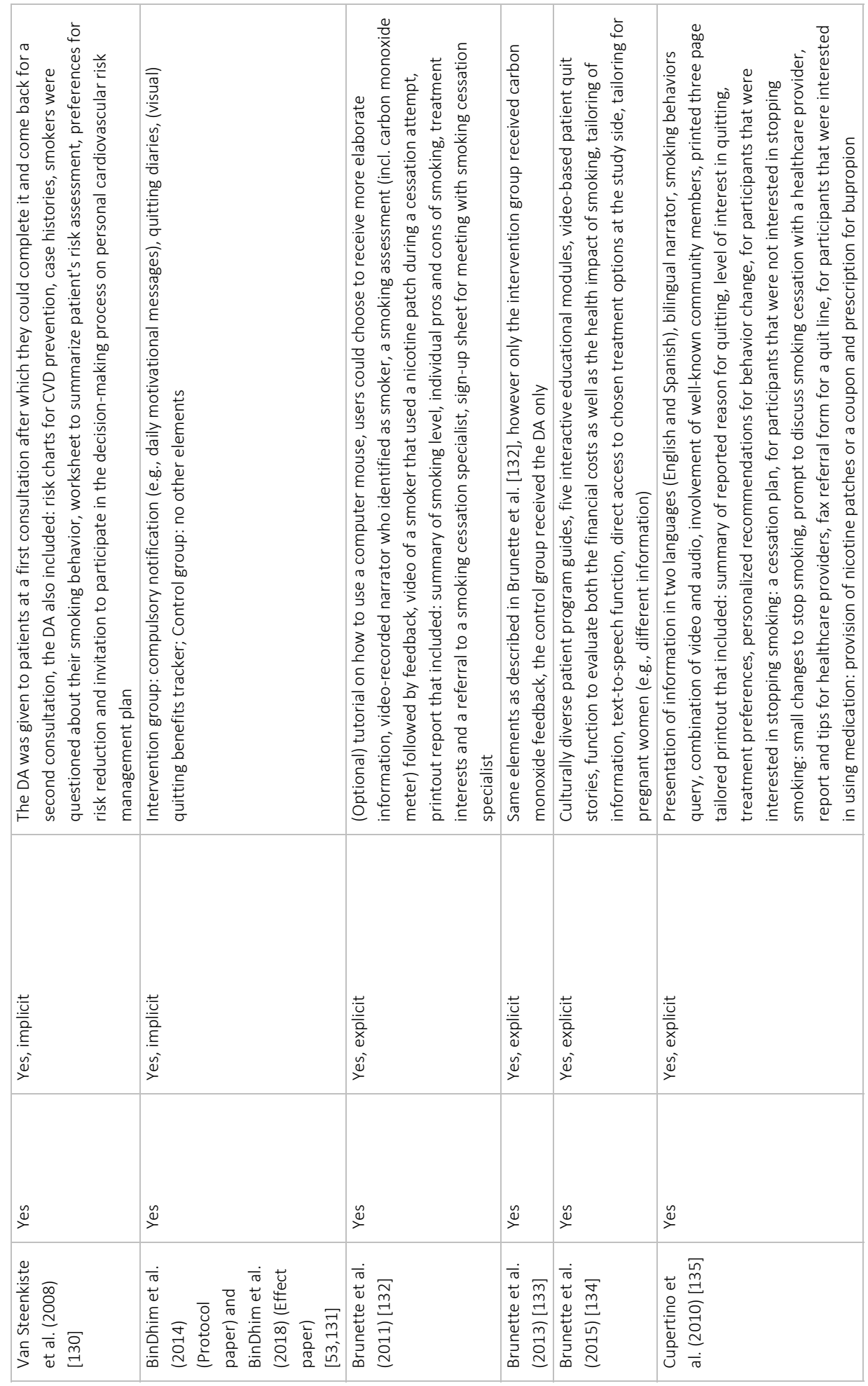




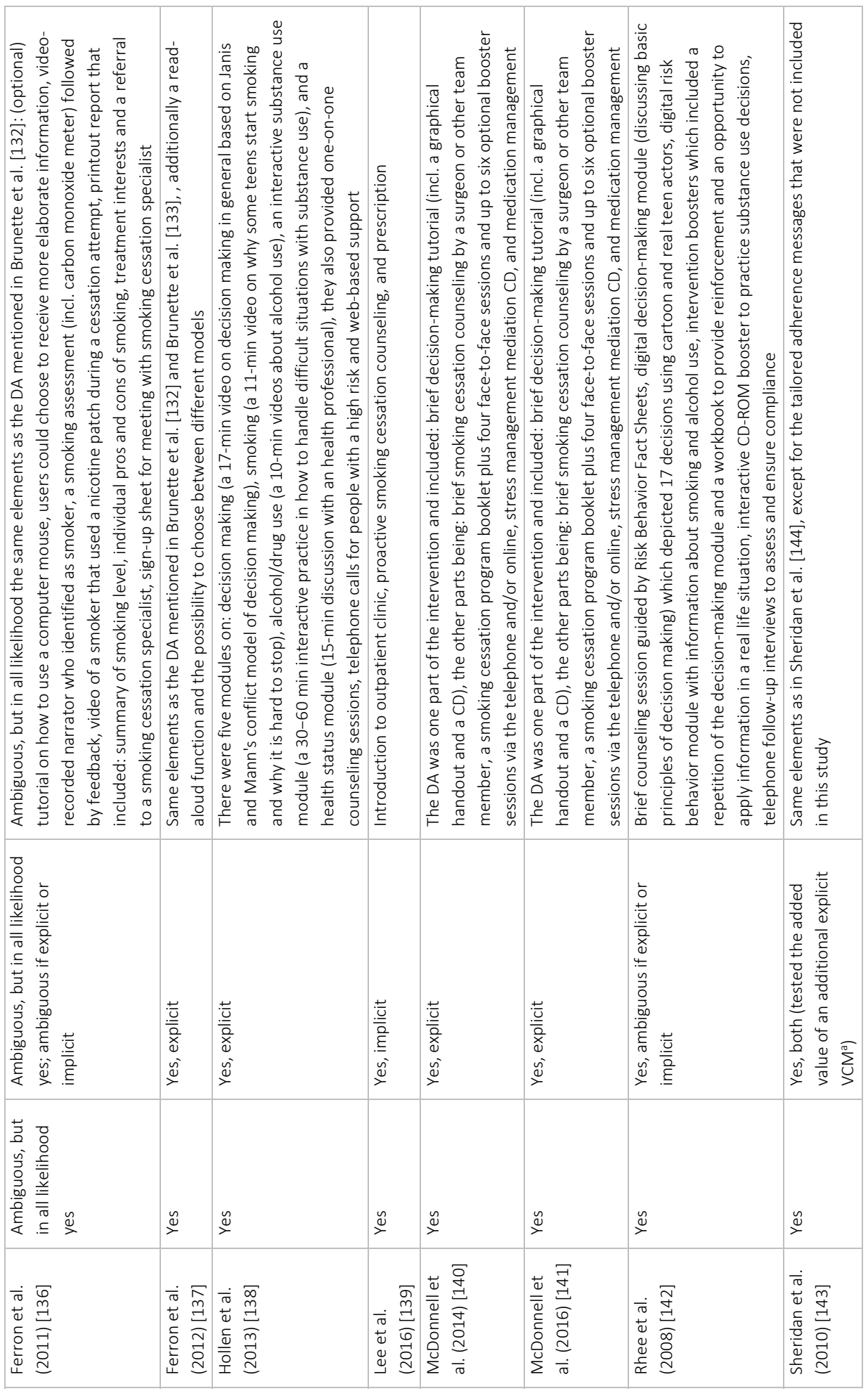




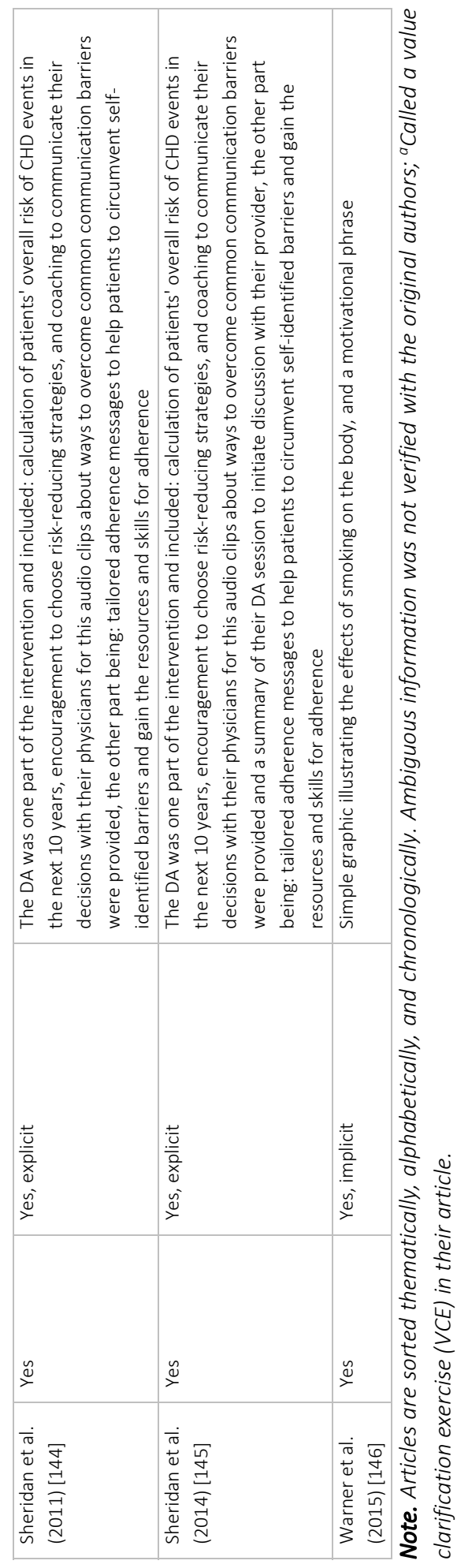




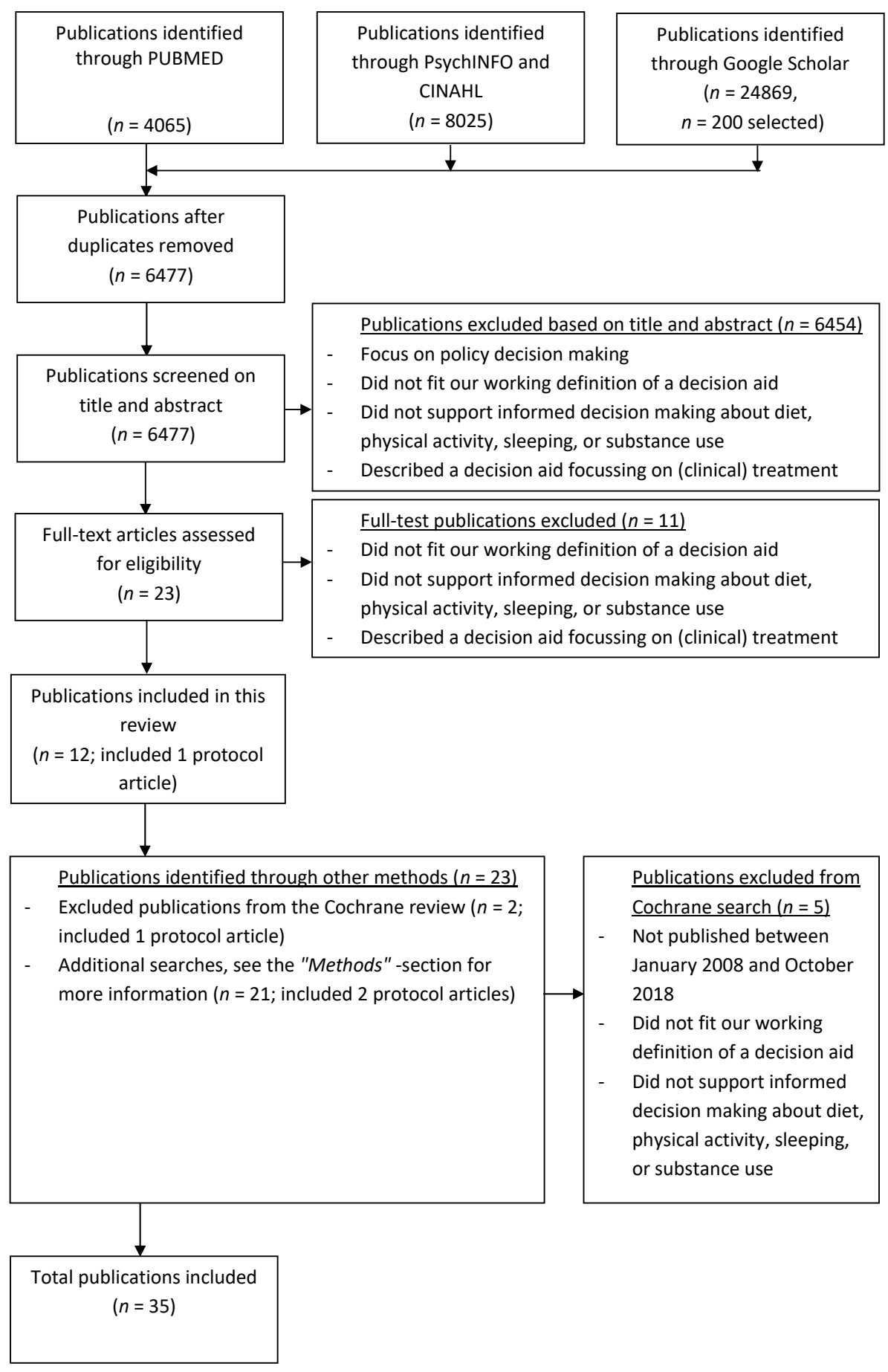

Figure 1. Flow Diagram. 
Theoretical Foundations

Twenty-two studies (71\%) [53,115-118,120,124,125,128-134,137-145] reported using theoretical frameworks, most commonly to identify relevant outcome measures $(n=15$, 48\%) [53,117,118,120,124,125,130-133,137,138,142-145]. Janis' and Mann's Conflict Theory of Decision Making was used most often ( $n=6,19 \%)$ [138,140-143,145]-however, largely the same researchers were involved. An overview over the theoretical foundations can be seen in Table 3 .

Table 3. Use of Theories.

\begin{tabular}{|c|c|c|}
\hline \multirow[t]{2}{*}{ Has a theory been used at all? } & Yes & $n=22$ \\
\hline & Not reported & $n=9$ \\
\hline \multirow[t]{15}{*}{ Specific theories/frameworks used ${ }^{a}$} & Conflict Theory of Decision Making & $n=6$ \\
\hline & Social Cognitive Theory & $n=5$ \\
\hline & Transtheoretical Model of Change & $n=5$ \\
\hline & $\begin{array}{l}\text { Glyn Elwyn's Model of Shared Decision } \\
\text { Making }\end{array}$ & $n=5$ \\
\hline & Theory of Planned Behavior & $n=4$ \\
\hline & Self-Determination Theory & $n=2$ \\
\hline & $\begin{array}{l}\text { Ottawa Decision Support Framework } \\
\text { (ODSF) }\end{array}$ & $n=1$ \\
\hline & Integrative Theory & $n=1$ \\
\hline & Protection Motivation Theory & $n=1$ \\
\hline & Hersey-Blanchard Model & $n=1$ \\
\hline & Behaviour Change Wheel & $n=1$ \\
\hline & Prospect Theory & $n=1$ \\
\hline & Expectancy Value Theory & $n=1$ \\
\hline & Health Action Process Approach (HAPA) & $n=1$ \\
\hline & $\begin{array}{l}\text { Other/model developed by authors for } \\
\text { the study }\end{array}$ & $n=1$ \\
\hline \multirow[t]{5}{*}{ Ways theories were used ${ }^{a}$} & $\begin{array}{l}\text { Theories' concepts used as outcome } \\
\text { measure }\end{array}$ & $n=15$ \\
\hline & To guide content development & $n=14$ \\
\hline & Part of the decision aid (DA) & $n=3$ \\
\hline & To guide study design & $n=1$ \\
\hline & To compare study groups at baseline & $n=1$ \\
\hline
\end{tabular}

Note. ${ }^{a}$ In some studies, multiple theories have been used for multiple purposes. Therefore, the absolute amount exceeds 31 . 
Effectiveness and Cost-Effectiveness of the Identified DAs

Most effects where tested in either a cluster $(n=7,23 \%)[41,43,117,119,123-125,139]$ or a randomized controlled trial (RCT) ( $n=8,26 \%)$ [53,131,133,137,138,142-145]. In this result section null effects are defined as insignificant findings that reflect neither an increase nor a decrease.

Effects on the Attributes of the Choice Made

In six studies, knowledge (19\%) was assessed [53,124,129,133,145,146], but only one [145] reported a significant increase in knowledge as compared to baseline measurement. In three studies (10\%), null effects were reported regarding knowledge $[124,133,146]$. All other studies examined knowledge only as part of another overarching concept $[53,129]$, e.g., informed choice. Effects on risk perception were examined in two studies (6\%) [123,145], both found an increase in appropriateness of risk perceptions, however in one study the effects disappeared after correction for baseline characteristics [123] and in the other effects were not compared to a control group [145]. Value-congruency was tested in four studies $(13 \%)[53,125,143,145]$. In one of those studies value-congruency was not examined in isolation [53]. Sheridan et al. [143] found that adding an explicit VCM (called a VCE in their article) did not increase value-consistency. In one study an increased attitude towards the chosen option (i.e., 'actual' value-consistency) was reported that was compared to a control group [125], while in another study positive effects on perceived value-consistency that were not compared to a control group were reported [145]. The one article that reported on the measurement of regret reported a significant positive effect (i.e., a decrease in regret) [124].

Effects on the Attributes of the Decision-Making Process

The most commonly investigated attribute was patient-practitioner communication $(n=12$, 39\%) [118-120,123,130,132,133,135,137,140,145,146]. However, mixed effects were found: decrease in communication ( $n=1$ ) [135], increase in communication ( $n=2,6 \%)$ $[132,145]$, increase in satisfaction with the communication $(n=1)$ [123], and null effects ( $n$ = 1) [146]. Other studies mainly reported descriptive characteristics, e.g., that most of the exposed patients were satisfied [118]. In four (13\%) out of seven (23\%) studies in which participation in decision making was investigated positive effects compared to a control group were found $[117,118,124,146]$ (the majority came from similar researchers), in one study null effects were reported [145]. Positive effects were found regarding decisional conflict, assessed in four studies (13\%) $[53,143,145,146]$; null effects were only reported in one study [143] on the added value of an explicit VCM. Positive effects were both observed compared to a control group $(n=2,6 \%)[53,146]$, and not compared to a control group $(n=$ 1) [145]. While the proportion of undecided people was reported in six studies (19\%) 
$[115,118,120,121,125,146]$ (again, the majority came from similar researchers), only in one the effect was tested [125]. They found a positive effect compared to a control group [125]. No study reported effects on decisional satisfaction.

Effects on Behavior

In 18 (58\%) articles an assessment on the impact of the DA on behavior was reported $[41,53,115,116,118,120,123,127,129,132-135,137,139,140,142,146]$.

\section{Dietary Behavior}

In one study in which differences between two study groups were tested, positive effects due to the interventions on fat and vegetable intake were found [41]. However, effects on fat and vegetable intake were not replicated in multilevel analyses [41]. In another study with a control group (same researchers) null effects for fat, fruit, and vegetable consumption were found [123], while in another study negative effects on overall diet were reported [129]. In one study mixed effects in terms of fruit and vegetable intake were reported [116] which were not compared to a control group. In this study two different versions of a DA were tested: one targeting PA, the other fruit and vegetable intake [116]. Interestingly, only the version targeting PA resulted in an increase in fruit and vegetable consumption, the fruit and vegetable version resulted in a small decrease in fruit and vegetable consumption [116]. In another study the same DA was compared alongside counseling or a web-based lifestyle intervention (thus, both study arms received the same DA): Positive effects were found for fat quality, fruit and vegetable intake [127]. One article simply reported that diet changed without providing much detail [115].

Physical Activity (PA)

In two of the three studies (6\%) [41,123] comparing effects to a control group no effects on PA were found (same researchers), the one that did [129] was a pilot study that only reported descriptive analyses. Within the study that tested two different versions of the same DA (one for PA, one for fruit and vegetables) positive effects regarding PA were found in both groups [116]. Strikingly, the effects were stronger in the non-PA version. In another study without control group positive effects on weekly PA time and sedentary behavior were found [115], negative effects were found for minutes of PA and daily number of steps [115]. The study that compared the effects of the DA alongside counseling or a web-based lifestyle intervention found positive effects for weekly walking time and daily number of steps. However, the effect for weekly walking time was only observed in the counselor group [127]. 
In studies including a control group positive effects on smoking cessation aid uptake $(n=3$, $10 \%)[53,132,134]$ and smoking abstinence $(n=1)$ [53] were found, while null effects were found on perioperative smoking behavior $(n=1)$ [146], smoking cessation medication $(n=1)$ [139], smoking abstinence $(n=3,10 \%)[41,123,139]$, and smoking, alcohol and illicit drug uptake $(n=1)$ [142]. Only in one study that included a control group negative effects regarding smoking were found, however positive effects on alcohol consumption were found as well [129]. Interestingly, in one study both an effect on smoking cessation aid uptake and abstinence was found, but the researchers did not find that the DA's effect on abstinence was mediated by the quitting method [53]. In another study [41] a difference between intervention and control group was found, however the difference was already present at baseline. In the one study without control group, positive effects were found on smoking cessation aid uptake and number of cigarettes, while negative effects were found on planning of a quit date and talking to healthcare providers about smoking cessation [135]. Other effects that were found: adding carbon monoxide feedback to a DA did not make it more effective ( $n=1$ ) [133], a DA for dyads (patient plus family member) seemed to be more effective for patients' quitting behavior than family members' quitting behavior $(n=1)$ [140] and in the study [127] that compared the effects of the DA alongside counseling or a webbased lifestyle intervention positive effects for smoking were found in both groups.

Effects on Adherence to the Chosen Option

Adherence was assessed in four (13\%) studies $[53,115,144,146]$. Three $(10 \%)$ compared the effects to a control group; one reported null effects [146], one positive effects (i.e., increased adherence) [144], and one reported that $97.7 \%$ adhered to their chosen option regardless of the assigned group [53].

Effects on Economic Impact

Cost-effectiveness was assessed in one study, however not the cost-effectiveness of the DA itself was tested but rather of a counseling or a web-based intervention used next to the DA [127].

Effects on Health Outcomes

Health status was assessed in five studies (16\%) $[41,124,127,129,144]$, both null $(n=3,10 \%)$ $[41,124,129]$ and positive effects $(n=2,6 \%)[127,144]$ (both from similar research teams) were found. Quality of life [127] and anxiety [123] were only assessed once, in both cases 
significant improvements were found. No study reported effects on depression and emotional distress.

\section{Results Grey Literature}

The initial search into the DALI resulted in 10 DAs (dietary behavior $n=5$ and substance use $n=5)$. Only four DAs were still available online at the time of the search [148-151]. All DAs stemmed from the same developer (www.healthwise.org), a nonprofit organization aimed at providing digital health education. All DAs shared a similar design. Theory application was not described.

All DAs made use of information provision and explicit elements to clarify values and preferences. Other elements were personal stories, a knowledge quiz, and a summary. Duration to complete the DAs was not reported.

The DAs were not reported in any scientific publications. No effects were reported. An overview of currently online accessible DAs can be seen in Table 4.

Table 4. Decision Aid Characteristics Grey Literature.

\begin{tabular}{|l|l|l|}
\hline Name & $\begin{array}{l}\text { Behavior } \\
\text { (general) }\end{array}$ & Behavior (specially) \\
\hline $\begin{array}{l}\text { Healthwise: Quitting Smoking: Should I Use } \\
\text { Medicine? [148] }\end{array}$ & Substance use & Smoking (cessation) \\
\hline $\begin{array}{l}\text { Healthwise: Obesity: Should I Use a Diet Plan to Lose } \\
\text { Weight? [149] }\end{array}$ & Dietary behavior & Diet \\
\hline $\begin{array}{l}\text { Healthwise: Weight Management: Should I Use } \\
\text { Over-the-Counter Diet Aids? [150] }\end{array}$ & Dietary behavior & Use of diet aids \\
\hline $\begin{array}{l}\text { Healthwise: Sleep Apnea: Should I Have a Sleep } \\
\text { Study? [151] }\end{array}$ & $\begin{array}{l}\text { Sleep-related } \\
\text { behaviors }\end{array}$ & $\begin{array}{l}\text { General sleep } \\
\text { management }\end{array}$ \\
\hline
\end{tabular}

\section{Discussion and Conclusion}

Discussion

With this scoping review we aimed to synthesize the literature on DAs that focus on preventive health-related behaviors by reviewing available information regarding their characteristics, intervention elements, theoretical foundations, and (cost-)effectiveness. We identified 35 scientific papers describing DA-development and/or evaluation and four DAs that focus on preventive health-related behaviors in the grey literature. We will focus on three key areas in this discussion: (1) characteristics and intervention elements of identified 
DAs, (2) theoretical foundations of the identified DAs, and (3) effectiveness of the identified DAs.

Characteristics and Intervention Elements of the Identified DAs

Identified DAs focused most often on substance use, primarily smoking. This could be due to the fact that smoking cessation trajectories show similarities with clinical treatment and screening trajectories, which is where the majority of DAs traditionally have been applied [13]. For example, one of the options that is regularly named in smoking cessation DAs is pharmacological support (e.g., [53]).

DAs were often combined with additional intervention elements. Therefore, it was difficult to ascertain the impact of the DA independent from these other components, as the additional components often had their basis in behavioral change theories, rather than informed decision making. Consequently, tested outcomes varied widely among studies, limiting the current evidence base for any behavior- or decision-related outcome.

Future studies should examine which intervention elements are effective regarding informed decision making in the area of preventive health-related behaviors. Furthermore, studies should be conducted to disentangle which intervention elements can be deployed to support which processes. To this end, however, consensus should be reached on which outcomes are relevant to be tested in studies investigating DAs that focus on preventive health-related behaviors. This would not only allow different intervention elements to be tested using the same criteria but would also enable developers of DAs that focus on preventive healthrelated behaviors to develop DAs that are even more rooted in evidence than current DAs. Ultimately, this could result in a taxonomy as used in behavior change [75] which clearly describes the purpose of most often applied intervention elements. Theoretical work to understand VCMs' effects and how those effects can be accomplished have recently been undertaken $[39,40]$.

Theoretical Foundations of the Identified DAs

Around $70 \%$ of the studies reported that they used a theory, most commonly to identify relevant outcome measures. Multiple studies used theories such as the Self-Determination Theory [86] or the Theory of Planned Behavior [73]; theories meant to explore motivation or behavior (change). We also found studies that used decision-making-focused theories, such as the Conflict Theory of Decision Making [81], however these are not explicitly designed to support people in changing behavior. Given the dual purpose of DAs that focus on preventive health-related behaviors, insights from multiple theories should be used to develop these DAs. 
There are two possible approaches to integrate insights from both areas when developing DAs that focus on preventive health-related behaviors: (1) Developers could flexibly integrate insights from multiple theories on respectively behavioral change and informed decision making as proposed by Peters \& Crutzen [152] (2) or attempts could be made to establish an integrative framework that can be applied in multiple (unrelated) DAdevelopment projects. The second approach could be particularly helpful for developers that are not familiar with both research fields.

Effectiveness of the Identified DAs

Studies reported positive effects such as uptake of effective smoking cessation aids and smoking abstinence, however interpretation is somewhat difficult as not all studies followed an RCT protocol and as we could not synthesize the effects quantitatively. Also, a formal analysis of the quality of the evidence has not taken place in this scoping review as this form of knowledge synthesis (often) does not include quality assessments in the same form as systematic reviews [111]. However, our findings are in line with a systematic review [52] in which it was found that smoking cessation DAs can be effective, but that there was major heterogeneity within studies and DAs. Beneficial effects were also identified regarding PA and nutritional behavior, however, due to the relatively low numbers of studies and the mixed findings found in the included studies, no clear conclusions can be drawn at this time.

Interestingly, the majority of the identified studies failed to report effects on decisional outcomes. Future studies should investigate how DAs that focus on preventive healthrelated behaviors affect those decisional outcomes as well and how these outcomes relate to behavior (change). Insights from Self-Determination Theory [86], for example, would suggest that the offering of choices (i.e., what DAs do inherently) can support individuals in becoming autonomously motivated towards self-chosen options, which in turn can lead to greater behavioral maintenance [91,92].

\section{Limitations}

A possible limitation was the focus on studies as the units of analysis rather than individual DAs. However, not all studies that referred to similar DAs clearly described how they related to one another, which made it impossible to report results per DA. To minimize the impact of this on our results, we highlighted if studies were conducted by similar author(s). Another possible limitation would be that we decided to exclude all treatment DAs, including those aimed at preventing secondary diseases or complications (e.g., cardiovascular diseases due to diabetes mellitus). However, our working definition of DAs that focus on preventive health-related behaviors has only focused on primary disease prevention and we are 
convinced that DAs aimed at primary, secondary, and tertiary prevention should be explored separately. Hence, the focus on DAs that focus on primary prevention.

\section{Future Research Directions}

Based on the discussion above, we have identified three main areas of interest for further research: (1) establishing which intervention elements are effective regarding decision making in the domain of preventive health-related behaviors, and for which processes, (2) strengthening the integration between theoretical insights from behavior change and informed decision making, by either adopting a flexible approach or by establishing an integrative framework, and (3) conducting more randomized trials to enable systematic reviews and meta-analyses in order to draw stronger conclusions regarding behavioral and decisional outcomes and how those relate to one another.

\section{Practice Implications}

While scoping reviews do not allow for strong conclusions to be drawn (compared to other forms of knowledge syntheses), our results show that DAs can potentially be beneficial in supporting people to change preventive health-related behaviors-especially regarding smoking (particularly when taken together with other evidence [52]). As such, DAs might be one potential approach to counteract the rise of noncommunicable diseases. However, further research is needed to substantiate this.

\section{Conclusions}

This study was the first attempt to broadly synthesize knowledge regarding DAs aimed at preventive health-related behavioral decisions. Findings regarding the effects on behavior were potentially promising, especially regarding smoking (particularly when taken together with other evidence [52]). However, while certain beneficial effects could be identified, interpretation was hindered by heterogenous reporting. Certain areas of improvement were identified, such as establishing which intervention elements are effective regarding decision making in the domain of preventive health-related behaviors.

\section{Abbreviations}

$\mathrm{CHD}$ - coronary heart disease

CRT - cluster randomized controlled trial

CVD - cardiovascular disease

DA - decision aid 
DALI - Ottawa Hospital Research Institute Decision Aid Library Inventory

GP - general practitioner

HAPA - Health Action Process Approach

$\mathrm{MI}$ - myocardial infarction

ODSF - Ottawa Decision Support Framework

PA - physical activity

PRISMA-SCR - Preferred Reporting Items for Systematic Reviews and Meta-Analyses

Extension for Scoping Reviews

$\mathrm{RCT}$ - randomized controlled trial

SDM - shared decision making

UK - United Kingdom

USA - United States of America

VCE - value clarification exercise

VCM - value clarification method 
Appendix A - Preferred Reporting Items for Systematic Reviews and Meta-Analyses Extension for Scoping Reviews (PRISMA-ScR) Checklist

\begin{tabular}{|c|c|c|c|}
\hline Section & Item & PRISMA-ScR Checklist item & REPORTED ON PAGE \# \\
\hline \multicolumn{4}{|l|}{ Title } \\
\hline Title & 1 & Identify the report as a scoping review. & 33 \\
\hline \multicolumn{4}{|l|}{ Abstract } \\
\hline $\begin{array}{l}\text { Structured } \\
\text { summary }\end{array}$ & 2 & $\begin{array}{l}\text { Provide a structured summary that includes } \\
\text { (as applicable): background, objectives, } \\
\text { eligibility criteria, sources of evidence, } \\
\text { charting methods, results, and conclusions } \\
\text { that relate to the review questions and } \\
\text { objectives. }\end{array}$ & 34/Abstract \\
\hline \multicolumn{4}{|l|}{ Introduction } \\
\hline Rationale & 3 & $\begin{array}{l}\text { Describe the rationale for the review in the } \\
\text { context of what is already known. Explain } \\
\text { why the review questions/objectives lend } \\
\text { themselves to a scoping review approach. }\end{array}$ & $35-36$ \\
\hline Objectives & 4 & $\begin{array}{l}\text { Provide an explicit statement of the } \\
\text { questions and objectives being addressed } \\
\text { with reference to their key elements (e.g., } \\
\text { population or participants, concepts, and } \\
\text { context) or other relevant key elements } \\
\text { used to conceptualize the review questions } \\
\text { and/or objectives. }\end{array}$ & 36 \\
\hline \multicolumn{4}{|l|}{ Methods } \\
\hline $\begin{array}{l}\text { Protocol and } \\
\text { registration }\end{array}$ & 5 & $\begin{array}{l}\text { Indicate whether a review protocol exists; } \\
\text { state if and where it can be accessed (e.g., a } \\
\text { Web address); and if available, provide } \\
\text { registration information, including the } \\
\text { registration number. }\end{array}$ & 36 \\
\hline $\begin{array}{l}\text { Eligibility } \\
\text { criteria }\end{array}$ & 6 & $\begin{array}{l}\text { Specify characteristics of the sources of } \\
\text { evidence used as eligibility criteria (e.g., } \\
\text { years considered, language, and publication } \\
\text { status), and provide a rationale. }\end{array}$ & $37-38$ \\
\hline $\begin{array}{l}\text { Information } \\
\text { sources }\end{array}$ & 7 & $\begin{array}{l}\text { Describe all information sources in the } \\
\text { search (e.g., databases with dates of } \\
\text { coverage and contact with authors to } \\
\text { identify additional sources), as well as the } \\
\text { date the most recent search was executed. }\end{array}$ & $37-38$ \\
\hline Search & 8 & $\begin{array}{l}\text { Present the full electronic search strategy } \\
\text { for at least one database, including any } \\
\text { limits used, such that it could be repeated. }\end{array}$ & 37-38, Appendix B-D \\
\hline
\end{tabular}




\begin{tabular}{|c|c|c|c|}
\hline $\begin{array}{l}\text { Selection of } \\
\text { sources of } \\
\text { evidence }\end{array}$ & 9 & $\begin{array}{l}\text { State the process for selecting sources of } \\
\text { evidence (i.e., screening and eligibility) } \\
\text { included in the scoping review. }\end{array}$ & 38 \\
\hline $\begin{array}{l}\text { Data charting } \\
\text { Process }\end{array}$ & 10 & $\begin{array}{l}\text { Describe the methods of charting data from } \\
\text { the included sources of evidence (e.g., } \\
\text { calibrated forms or forms that have been } \\
\text { tested by the team before their use, and } \\
\text { whether data charting was done } \\
\text { independently or in duplicate) and any } \\
\text { processes for obtaining and confirming data } \\
\text { from investigators. }\end{array}$ & 38, Appendix $E$ \\
\hline Data items & 11 & $\begin{array}{l}\text { List and define all variables for which data } \\
\text { were sought and any assumptions and } \\
\text { simplifications made. }\end{array}$ & Appendix $E$ \\
\hline $\begin{array}{l}\text { Critical } \\
\text { appraisal of } \\
\text { individual } \\
\text { sources of } \\
\text { evidence }\end{array}$ & 12 & $\begin{array}{l}\text { If done, provide a rationale for conducting a } \\
\text { critical appraisal of included sources of } \\
\text { evidence; describe the methods used and } \\
\text { how this information was used in any data } \\
\text { synthesis (if appropriate). }\end{array}$ & Not applicable \\
\hline $\begin{array}{l}\text { Synthesis of } \\
\text { results }\end{array}$ & 13 & $\begin{array}{l}\text { Describe the methods of handling and } \\
\text { summarizing the data that were charted. }\end{array}$ & 38 \\
\hline $\begin{array}{l}\text { Selection of } \\
\text { sources of } \\
\text { evidence }\end{array}$ & 14 & $\begin{array}{l}\text { Give numbers of sources of evidence } \\
\text { screened, assessed for eligibility, and } \\
\text { included in the review, with reasons for } \\
\text { exclusions at each stage, ideally using a } \\
\text { flow diagram. }\end{array}$ & Figure 1 \\
\hline $\begin{array}{l}\text { Characteristics } \\
\text { of sources of } \\
\text { Evidence }\end{array}$ & 15 & $\begin{array}{l}\text { For each source of evidence, present } \\
\text { characteristics for which data were charted } \\
\text { and provide the citations. }\end{array}$ & $38-66$ \\
\hline $\begin{array}{l}\text { Critical } \\
\text { appraisal within } \\
\text { sources of } \\
\text { evidence }\end{array}$ & 16 & $\begin{array}{l}\text { If done, present data on critical appraisal of } \\
\text { included sources of evidence (see item 12). }\end{array}$ & Not applicable \\
\hline $\begin{array}{l}\text { Results of } \\
\text { Individual } \\
\text { sources of } \\
\text { evidence }\end{array}$ & 17 & $\begin{array}{l}\text { For each included source of evidence, } \\
\text { present the relevant data that were charted } \\
\text { that relate to the review questions and } \\
\text { objectives. }\end{array}$ & $38-66$ \\
\hline $\begin{array}{l}\text { Synthesis of } \\
\text { results }\end{array}$ & 18 & $\begin{array}{l}\text { Summarize and/or present the charting } \\
\text { results as they relate to the review } \\
\text { questions and objectives. }\end{array}$ & $38-66$ \\
\hline
\end{tabular}




\begin{tabular}{|c|c|c|c|}
\hline \multicolumn{4}{|l|}{ Discussion } \\
\hline $\begin{array}{l}\text { Summary of } \\
\text { Evidence }\end{array}$ & 19 & $\begin{array}{l}\text { Summarize the main results (including an } \\
\text { overview of concepts, themes, and types of } \\
\text { evidence available), link to the review } \\
\text { questions and objectives, and consider the } \\
\text { relevance to key groups. }\end{array}$ & $66-69$ \\
\hline Limitations & 20 & $\begin{array}{l}\text { Discuss the limitations of the scoping } \\
\text { review process. }\end{array}$ & $68-69$ \\
\hline Conclusions & 21 & $\begin{array}{l}\text { Provide a general interpretation of the } \\
\text { results with respect to the review questions } \\
\text { and objectives, as well as potential } \\
\text { implications and/or next steps. }\end{array}$ & 69 \\
\hline \multicolumn{4}{|l|}{ Funding } \\
\hline Funding & 22 & $\begin{array}{l}\text { Describe sources of funding for the included } \\
\text { sources of evidence, as well as sources of } \\
\text { funding for the scoping review. Describe the } \\
\text { role of the funders of the scoping review. }\end{array}$ & Table 1 \\
\hline
\end{tabular}

Note. Adapted from www. prisma-statement.org/Extensions/ScopingReviews.

Appendix B - Search Strings for PubMed

\begin{tabular}{|c|c|}
\hline Behavior & Search terms \\
\hline $\begin{array}{l}\text { Dietary } \\
\text { behavior }\end{array}$ & $\begin{array}{l}\text { (("Decision Support Techniques"[Mesh] OR "Decision Support Techniques" [tiab] } \\
\text { OR "Decision model" [tiab] OR "decision aid*" [tiab]) AND ("Diet"[Mesh] OR } \\
\text { "Diet*"[tiab] OR "dietary behavio*"[tiab] OR "Eating"[Mesh] OR "Eating"[tiab] OR } \\
\text { "food restriction"[tiab] OR "Weight Loss"[Mesh] OR "Weight Loss"[tiab] OR } \\
\text { "Weight Gain"[tiab] OR "Diet, Food, OR Nutrition"[Mesh] OR "food"[tiab] OR } \\
\text { "weight reduction plan"[tiab] OR "weight reduction"[tiab])) NOT ("Policy } \\
\text { Making"[Mesh] OR "Policy Making"[tiab] OR "Public Policy"[Mesh] OR "Public } \\
\text { Policy"[tiab] OR "Health Policy"[tiab]) }\end{array}$ \\
\hline $\begin{array}{l}\text { Physical } \\
\text { activity }\end{array}$ & $\begin{array}{l}\text { (("Decision Support Techniques"[Mesh] OR "decision aid*"[tiab] OR "Decision } \\
\text { Support Techniques" [tiab] OR "Decision model" [tiab]) AND ("Exercise"[Mesh] OR } \\
\text { "Exercise*"[tiab] OR "Physical Activity"[tiab] OR "movement"[tiab] or "sport*"[tiab] } \\
\text { or "active behavio*"[tiab] OR "fitness"[tiab])) NOT ("Policy Making"[Mesh] OR } \\
\text { "Policy Making"[tiab] OR "Public Policy" [Mesh] OR "Public Policy"[tiab] OR "Health } \\
\text { Policy"[tiab]) }\end{array}$ \\
\hline $\begin{array}{l}\text { Sleep- } \\
\text { related } \\
\text { behaviors }\end{array}$ & $\begin{array}{l}\text { (("Decision Support Techniques"[Mesh] OR "decision aid*"[tiab] OR "Decision } \\
\text { Support Techniques" [tiab] OR "Decision model"[tiab]) AND ("Sleep"[Mesh] OR } \\
\text { "Sleep*"[tiab] OR "Sleep hygiene"[MeSH] OR "Sleep hygiene"[tiab] OR "Sleep } \\
\text { habit"[tiab] OR "Rest"[Mesh] OR "Rest*"[tiab])) NOT ("Policy Making"[Mesh] OR } \\
\text { "Policy Making"[tiab] OR "Public Policy" [Mesh] OR "Public Policy"[tiab] OR "Health } \\
\text { Policy"[tiab]) }\end{array}$ \\
\hline
\end{tabular}




\section{Chapter 3}

\begin{tabular}{|l|l}
\hline $\begin{array}{l}\text { Substance } \\
\text { use }\end{array}$ & (("Decision Support Techniques"[Mesh] OR "decision aid*"[tiab] OR "Decision \\
& Support Techniques" [tiab] OR "Decision model"[tiab]) AND ("Alcohol \\
& Drinking"[Mesh] OR "Alcohol Drinking"[tiab] OR "alcohol*"[tiab] OR \\
& "Alcoholism"[Mesh] OR "Ethanol"[MeSH] OR "Ethanol"[tiab] OR "Alcoholic \\
& Beverages"[MeSH] OR "Alcoholic Beverages" [tiab] OR "Smoking"[Mesh] OR \\
& "Smok*"[tiab] OR "Smoking Cessation"[Mesh] OR "Smoking Cessation"[tiab] OR \\
& "Smoking Reduction"[Mesh] OR "Smoking Reduction"[tiab] OR "Tobacco Use \\
& Cessation Products"[Mesh] OR "Tobacco Use Cessation Products"[tiab] OR \\
& "Smoking Devices"[Mesh] OR "Smoking Devices"[tiab] OR "Tobacco"[Mesh] OR \\
& "Tobacco Use"[Mesh] OR "Tobacco Use"[tiab] OR "Tobacco Use Cessation"[Mesh] \\
& OR "Tobacco"[tiab] OR "cigarette*"[tiab] OR "e-cigarette*"[tiab] OR "Drug \\
& Misuse"[Mesh] OR "Drug*"[tiab] OR "Substance-Related Disorders"[Mesh])) NOT \\
& ("Policy Making"[Mesh] OR "Policy Making"[tiab] OR "Public Policy"[Mesh] OR \\
& "Public Policy"[tiab] OR "Health Policy"[tiab])
\end{tabular}

Appendix C - Search Strings for PsycINFO and CINAHL

\begin{tabular}{|c|c|}
\hline Behavior & Search terms \\
\hline $\begin{array}{l}\text { Dietary } \\
\text { behavior }\end{array}$ & $\begin{array}{l}\text { (SU Decision Support Systems OR TI "Decision Support Systems" OR AB "Decision } \\
\text { Support Systems" OR TI "decision aid*" OR AB "decision aid*" OR TI "Decision } \\
\text { Support Technique*" OR AB "Decision Support Technique*" OR TI "Decision } \\
\text { model" OR AB "Decision model") AND (SU Diets OR TI "Diet*" OR AB "Diet*" OR SU } \\
\text { Eating Behavior OR TI "Eat*" OR AB "Eat*" OR SU Food Intake OR SU Food OR TI } \\
\text { "food" OR AB "food" OR SU Nutrition OR SU Weight Control OR SU Weight Gain OR } \\
\text { TI "Weight Gain" OR AB "Weight Gain" OR SU Weight Loss OR TI "Weight Loss" OR } \\
\text { AB "Weight Loss" OR TI "dietary behavio*" OR AB "dietary behavio*" OR TI "eat*" } \\
\text { OR AB "eat*" OR TI "food restriction" OR AB "food restriction" OR TI "weight } \\
\text { reduction plan" OR AB "weight reduction plan") NOT (SU Policy Making OR TI } \\
\text { "Policy Making" OR AB "Policy Making" OR TI "public policy" OR AB "public policy" } \\
\text { OR TI "health policy" OR AB "health policy") }\end{array}$ \\
\hline $\begin{array}{l}\text { Physical } \\
\text { activity }\end{array}$ & $\begin{array}{l}\text { (SU Decision Support Systems OR TI "Decision Support Systems" OR AB "Decision } \\
\text { Support Systems OR TI "decision aid*" OR AB "decision aid*" OR TI "Decision } \\
\text { Support Technique*" OR AB "Decision Support Technique*" OR TI "Decision } \\
\text { model" OR AB "Decision model") AND (SU Physical Activity OR SU Physical Fitness } \\
\text { OR TI Exercise* OR AB Exercise* OR SU Physical Activity OR TI Physical Activity OR } \\
\text { AB Physical Activity OR TI movement OR AB movement OR SU Sports OR TI Sport* } \\
\text { OR AB Sport* OR TI active behavio* OR AB active behavio* OR TI fitness OR AB } \\
\text { fitness) NOT (SU Policy Making OR TI "Policy Making" OR AB "Policy Making" OR TI } \\
\text { "public policy" OR AB "public policy" OR TI "health policy" OR AB "health policy") }\end{array}$ \\
\hline $\begin{array}{l}\text { Sleep- } \\
\text { related } \\
\text { behaviors }\end{array}$ & $\begin{array}{l}\text { (SU Decision Support Systems OR TIX "Decision Support Systems" OR AB "Decision } \\
\text { Support Systems OR TI "decision aid*" OR AB "decision aid*" OR TI "Decision } \\
\text { Support Technique*" OR AB "Decision Support Technique*" OR TI "Decision } \\
\text { model" OR AB "Decision model") AND (SU Sleep OR TI Sleep* OR AB Sleep* OR TI } \\
\text { Rest* OR AB Rest*) NOT (SU Policy Making OR TI "Policy Making" OR AB "Policy }\end{array}$ \\
\hline
\end{tabular}




\begin{tabular}{|l|l|}
\hline & $\begin{array}{l}\text { Making" OR TI "public policy" OR AB "public policy" OR TI "health policy" OR AB } \\
\text { "health policy") }\end{array}$ \\
\hline $\begin{array}{l}\text { Substance } \\
\text { use }\end{array}$ & $\begin{array}{l}\text { (SU Decision Support Systems OR TIX "Decision Support Systems" OR AB "Decision } \\
\text { Support Systems OR TI "decision aid*" OR AB "decision aid*" OR TI "Decision } \\
\text { Support Technique*" OR AB "Decision Support Technique*" OR TI "Decision } \\
\text { model" OR AB "Decision model") AND (SU Drinking Behavior OR TI Alcohol* OR AB } \\
\text { Alcohol* OR SU Drug Usage OR SU Ethanol OR TI Ethanol OR AB Ethanol OR SU } \\
\text { Alcoholic Beverages ORTI Smok* OR AB Smok* OR SU Smoking Cessation OR SU } \\
\text { Nicotine OR TI Tobacco* OR AB Tobacco* OR TI Nicotine* OR AB Nicotine* OR SU } \\
\text { Drug Withdrawal OR TI Cigarette* OR AB Cigarette* OR TI e-cigarette* OR AB e- } \\
\text { cigarette* OR TI drug*OR AB drug* OR TI substance*OR AB substance*) NOT (SU } \\
\text { Policy Making OR TI "Policy Making" OR AB "Policy Making" OR TI "public policy" } \\
\text { OR AB "public policy" OR TI "health policy" OR AB "health policy") }\end{array}$ \\
\hline
\end{tabular}

Appendix D - Search Strings for Google Scholar

\begin{tabular}{|l|l|}
\hline Behavior & Search terms \\
\hline $\begin{array}{l}\text { Dietary } \\
\text { behavior }\end{array}$ & $\begin{array}{l}\text { (("Decision Support Techniques" OR "Decision model" OR "decision aid*") AND } \\
\text { ("Diet*" OR "Eat* OR "food*" OR "Weight*")) -policy }\end{array}$ \\
\hline $\begin{array}{l}\text { Physical } \\
\text { activity }\end{array}$ & $\begin{array}{l}\text { (("Decision Support Techniques" OR "Decision model" OR "decision aid*") AND } \\
\text { ("Exercise*" OR "Physical Activity" OR "move*" or "sport*" or "active behavio*" } \\
\text { OR "fitness")) -policy }\end{array}$ \\
\hline $\begin{array}{l}\text { Sleep-related } \\
\text { behaviors }\end{array}$ & $\begin{array}{l}\text { (("Decision Support Techniques" OR "Decision model" OR "decision aid*") AND } \\
\text { ("Sleep*" OR "Rest*")) -policy }\end{array}$ \\
\hline $\begin{array}{l}\text { Substance use } \\
\text { (("Decision Support Techniques" OR "Decision model" OR "decision aid*") AND } \\
\text { ("alcohol*" OR "Ethanol" OR "Ethanol" OR "Smok*" OR "Tobacco*" OR } \\
\text { "cigarette*" OR "e-cigarette*" OR "Drug*" OR "Substance*)) -policy }\end{array}$ \\
\hline
\end{tabular}

Appendix E - Extracted Information

\begin{tabular}{|l|l|}
\hline Questions & Sub questions \\
\hline $\begin{array}{l}\text { On which behavior did the decision aid (DA) (under study) } \\
\text { focus, both in general (e.g., dietary behavior) and specifically } \\
\text { (e.g., weight loss)? }\end{array}$ & \\
\hline How was the (studied) DA delivered to the user? & \\
\hline How long did it take to complete the DA (under study)? & $\begin{array}{l}\text { Did the DA contain information } \\
\text { provision elements? }\end{array}$ \\
\hline Of which elements did the DA (under study) consist? & $\begin{array}{l}\text { Did the DA contain elements to } \\
\text { clarify values and preferences? }\end{array}$ \\
\hline
\end{tabular}




\begin{tabular}{|c|c|}
\hline & $\begin{array}{l}\text { Were those elements explicit or } \\
\text { implicit? } \\
\text { Which other intervention } \\
\text { elements were employed? }\end{array}$ \\
\hline $\begin{array}{l}\text { Was the DA (under study) scientifically published (and was } \\
\text { certain necessary information described)? }\end{array}$ & $\begin{array}{l}\text { If it was, what was/were the: } \\
\text { Study design and methodology? } \\
\text { Study population? } \\
\text { Aims/purposes? } \\
\text { Origin/country of origin? } \\
\text { Author(s)? } \\
\text { Year of publication? }\end{array}$ \\
\hline How were theories used? & $\begin{array}{l}\text { Specifically: } \\
\text { Has a theory been used at all? } \\
\text { Which theory has been used } \\
\text { specifically and how was it used? }\end{array}$ \\
\hline What were the effects on the attributes of the choice made? & $\begin{array}{l}\text { Specifically, the effects on: } \\
\text { Knowledge? } \\
\text { Accurate risk perceptions? } \\
\text { Value congruency? } \\
\text { Regret? }\end{array}$ \\
\hline $\begin{array}{l}\text { What were the effects on the attributes of the decision- } \\
\text { making process? }\end{array}$ & $\begin{array}{l}\text { Specifically, the effects on: } \\
\text { Decisional conflict? } \\
\text { Proportion undecided? } \\
\text { Decisional satisfaction? } \\
\text { Patient-practitioner } \\
\text { communication, if applicable? } \\
\text { Participation in decision making, if } \\
\text { applicable? }\end{array}$ \\
\hline
\end{tabular}




\begin{tabular}{|c|c|}
\hline What were the effects on behavior? & $\begin{array}{l}\text { Specifically, the effects on: } \\
\text { (Actual) behavior after the choice } \\
\text { has been made? } \\
\text { Adherence to chosen option (time } \\
\text { of adherence)? }\end{array}$ \\
\hline What were the results regarding economic impact? & $\begin{array}{l}\text { Specifically, the effects on: } \\
\text { Costs? } \\
\text { Cost-effectiveness? }\end{array}$ \\
\hline What were the effects on health outcomes? & $\begin{array}{l}\text { Specifically, the effects on: } \\
\text { Health status? } \\
\text { Quality of life? } \\
\text { Anxiety? } \\
\text { Depression? } \\
\text { Emotional distress? }\end{array}$ \\
\hline
\end{tabular}




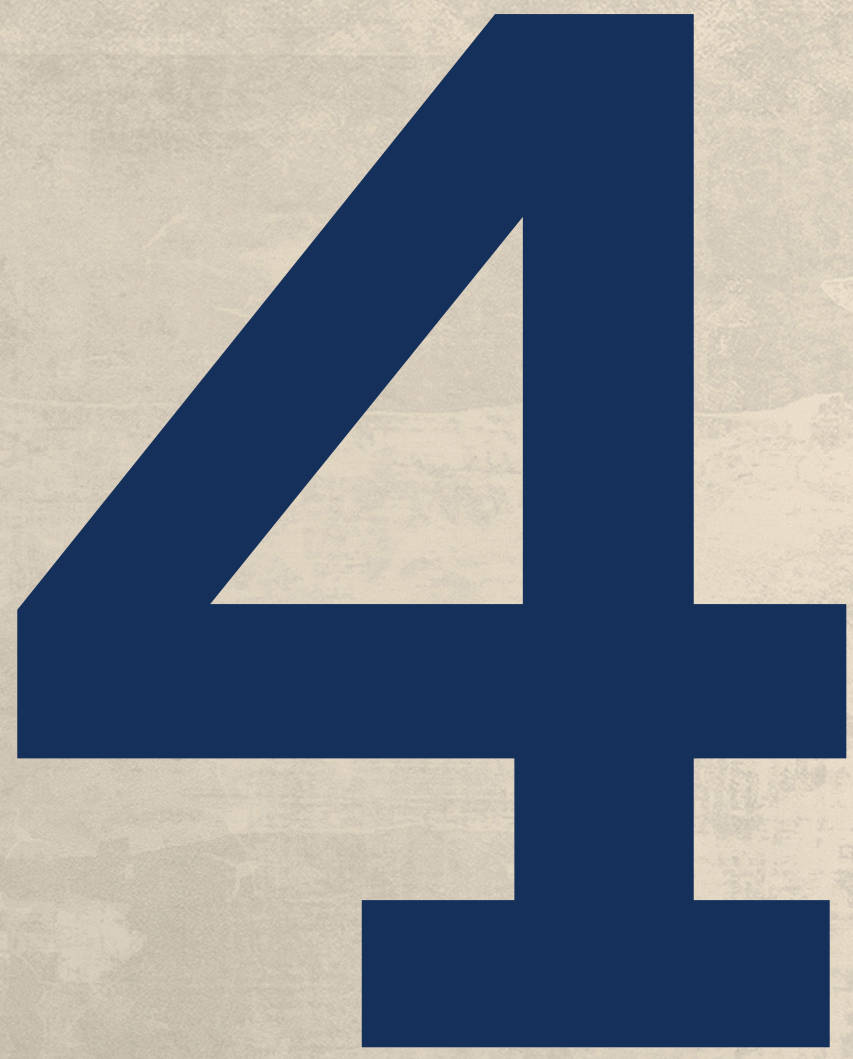




\section{chapter 4}

\section{smoker profiles and their influence on smokers' intention to use a digital decision aid aimed at the uptake of evidence-based smoking cessation tools: an explorative study}

This chapter has been published as:

Gültzow, T., Smit, E. S., Hudales, R., Dirksen, C. D., \& Hoving, C. (2020). Smoker profiles and their influence on smokers' intention to use a digital decision aid aimed at the uptake of evidence-based smoking cessation tools: An explorative study. DIGITAL HEALTH, 6, 2055207620980241. https://doi.org/10.1177/2055207620980241 


\section{Abstract}

Objectives: Evidence-based smoking cessation support tools (EBSTs) can double the quitting chances, but uptake among smokers is low. A digital decision aid (DA) could help smokers choose an EBST in concordance with their values and preferences, but it is unclear which type of smokers are interested in a digital DA. We hypothesized that smokers' general decision-making style (GDMS) could be used to identify early adopters. This study therefore aimed to identify smoker profiles based on smokers' GDMS and investigate these profiles' association with intention to use a digital DA.

Design: A cross-sectional dataset ( $N=200$ smokers intending to quit) was used to perform a hierarchical cluster analysis based on smokers' GDMS scores.

Methods: Clusters were compared on demographic and socio-cognitive variables. Mediation analyses were conducted to see if the relationship between cluster membership and intention was mediated through socio-cognitive variables (e.g., attitude).

Results: Two clusters were identified; "Avoidant Regretters" ( $n=134)$ were more avoidant, more regretful, and tended to depend more on others in their decision making, while "Intuitive Non-regretters" ( $n=66)$ were more spontaneous and intuitive in their decision making. Cluster membership was significantly related to intention to use a DA, with "Avoidant Regretters" being more interested. Yet, this association ceased to be significant when corrected for socio-cognitive variables (e.g., attitude). This indicates that cluster membership affected intention via socio-cognitive variables.

Conclusions: The GDMS can be used to identify smokers who are interested in a digital DA early on. As such, the GDMS can be used to tailor recruitment and DA-content. 


\section{Introduction}

Tobacco smoking continues to be the leading cause of preventable diseases and premature death [20]. It is estimated that $16 \%$ of all deaths in Europe and the Americas can be attributed to smoking [153]. In the Netherlands, smoking-related mortality is even higher (21\%) [153]. Evidence-based smoking cessation support tools (EBSTs) have been shown to be effective in facilitating quitting and maintaining smoking abstinence, i.e., pharmacotherapy, such as nicotine replacement therapy (NRT) [26]; and behavioral support, such as counselling by health professionals [104]. Such EBSTs more than double successful cessation rates [23], but are severely underused [60] and in the Netherlands uptake of nonevidence based support tools (e.g., acupuncture) is only slightly below that of EBSTs [42]. EBST uptake could therefore be significantly improved.

However, even if smokers are interested in using an EBST, they still have to choose between all the different EBSTs. It is known that individuals that face health-related decisions often encounter uncertainty about what to choose, which can result in a feeling of discomfort $[81,154]$. Offering a decision support system (often called decision aid, DA) to smokers that helps them choose from the multitude of options could help reduce this discomfort [13]. DAs are specifically designed to facilitate informed decision making between different healthcare options, by providing information and helping users to define their own values and preferences regarding these different options $[13,79]$. Nowadays, many DAs are delivered online as this allows for a broad and sustainable dissemination $[56,155,156]$. DAs have been used a lot concerning treatment or screening decisions [13], and have in a few instances also been used for smoking cessation [52]. For example, Willemsen et al. [42] developed a paperbased DA, which was effective in promoting quitting attempts and abstinence. However, it failed to increase uptake of EBSTs and resulted in higher dropout rates compared to the control group. Early dropout may cause problems for smokers, as they will not benefit from the intervention [157], and for researchers, as this makes it difficult to evaluate the effects [158]. Designing a digital DA instead of a paper-based DA could potentially already reach more people [155] and, particularly targeting a group that is planning to use a digital DA for EBSTs before it is available (i.e., early adopters) [57,159], could lead to a more attractive intervention which could prevent high dropout rates. Such a digital DA aimed at facilitating informed decision making between different EBSTs could be of particular interest for smokers that (1) are planning to stop smoking within the foreseeable future (otherwise, they would probably not be interested in smoking cessation to begin with) and (2) for smokers that hold favorable attitudes towards EBSTs. However, acceptance of or interest in using a DA for lifestyle behavior decisions, such as smoking cessation, by their intended audience has never been assessed (as opposed to interest in using EBSTs, for an example see [23]). DAs especially target processes traditionally linked to deliberate decision making; e.g., the clarification of values through deliberation [32,34], which makes them different from 
traditional health promotion interventions [13]. However, as people differ in terms of their response patterns to decision-making situations (often called decision-making style) $[160,161]$, their interest in using an online tool that facilitates deliberative processes could potentially also differ. Therefore, we hypothesize that smokers' decision-making style may influence their interest in a DA for EBSTs, as not all people value the activation of said cognitive processes targeted by DAs. This information could potentially be used to identify the aforementioned early adaptors. Also, this information could ultimately be used to design a digital DA that is attractive and beneficial to all adopter categories. Researchers usually distinguish five archetypical decision-making styles [160,161]: (1) The rational style is characterized by a thorough search for all information; (2) the intuitive style is characterized by a tendency to base decisions on emotions; (3) the dependent style is characterized by a tendency to ask for advice and guidance from others; (4) the avoidant style is characterized by a general aversion to decision making; and (5) the spontaneous style is characterized by people having the tendency go through the decision-making process as quickly as possible [161]. Other researchers [162] have added a style which is characterized by post-decisional regret after a decision has been made (i.e., (6) the regret style). In general, people do not rely on one style, but rather use a combination of styles [160]. Therefore, the styles should not be analyzed in isolation. One way to examine the styles and their joint effect on intention to use a smoking cessation DA would be to first identify groups (or clusters) based on decision-making styles and, secondly, to examine whether these groups differ in their intention to use such a DA. As this, to the best of our knowledge, has never been attempted before, we decided to take an explorative approach, leading to our first two research questions (RQ):

RQ1: Is it possible to identify specific groups (or clusters) of smokers planning to quit based on their decision-making styles?

RQ2: How do these groups (or clusters) differ in their intention to use a digital DA for EBSTs?

In order to further cross-sectionally validate and compare the identified groups (or clusters), [163], we decided to compare them demographically and on the basis of smoking (cessation) behavior and other factors that could be associated with the intention of using a smoking cessation DA. Next to the aforementioned favorable attitudes we hypothesize that the following factors could be associated with the intention of using a smoking cessation DA: (1) a smoker's health locus of control [164], as smokers that attribute events in their personal life to either their own or external control as opposed to chance may be more likely to use assistance to achieve their goal [165] and (2) motivation, as smokers that are planning to stop smoking because it is intrinsically valuable to them (i.e., smokers that are autonomously motivated to stop smoking as defined in the Self-Determination Theory (SDT) [166]) might be more interested in using assistance to achieve said goal (similar patterns have been 
identified in others domains, e.g., [167-169]). Autonomous motivation to stop smoking on the other hand has been shown to be related to feelings of perceived competence and perceived autonomy support [170]. Since no hypotheses could, however, be formulated with regard to differences in clusters in terms of demographics and smoking (cessation) behavior, we formulated the following third research question:

RQ3: How do these groups (or clusters) differ in respect to other characteristics, i.e., demographic factors, smoking (cessation) behavior, attitude towards EBSTs, health locus of control, motivation as defined in SDT and factors associated with motivation (i.e., perceived competence and perceived autonomy support)?

\section{Methods}

A cross-sectional study was conducted in January 2018. Participants were recruited through the internet research agency Flycatcher [171]. Panel members were preselected, based on their smoking status and if they were planning to stop smoking within six months. All preselected participants received an online questionnaire that lasted an average of 15.5 minutes. The questionnaire included an informed consent form, information about EBSTs, and information about DAs in general. Participants were excluded if they were younger than 18 , did not provide informed consent, or were not planning to quit smoking within six months. In total 250 smokers were invited. Due to the explorative nature of this study, no power analysis has been performed. Therefore, the sample size was based on the experience of the research team. Participants received $€ 1.40$ from the internet research agency to complete the questionnaire, which would equal about $\$ 1,54$.

\section{Measurements}

A Dutch questionnaire was developed and pretested among eight experts and five (ex)smokers. Pretesting took place with both native and second language Dutch speakers. If possible, validated scales were used that had been used in a Dutch context before. If this was not possible, English validated scales were initially translated by professional translators (forward translation), followed by back-translation by the research team (backward translation) [172].

Demographics

Gender identity ( 0 = woman; 1 = man; 2 = other) and country of birth were measured with one question each, age was measured continuously. Education was measured via three questions. The first question was asked to identify which degree had already been awarded, the second question was asked to identify respondents who were currently enrolled in a 
degree granting program ( $0=$ no; $1=$ yes), and the third to identify which degree granting program they are currently following. Three variables were later transformed: country of origin ( 0 = Dutch, 1 = non-Dutch), first and last educational question ( $0=$ low; 1 = medium; 2 = high). No, primary, and vocational education were regarded as a low educational attainment; secondary vocational education and a high school degree were regarded as a medium educational attainment; and higher vocational education, college, and university degrees were regarded as high educational attainment.

Smoking Behavior and Smoking-Related Cognitions

Smoking behavior assessment was based on the Dutch version of the Fagerström test for nicotine dependence [71,173]. However, it was adapted to include all smoking behavior, e.g., the original "How soon after you wake up do you smoke your first cigarette?" was changed to "How soon after you wake up do you smoke?" to reflect that not all smokers smoke cigarettes. Vapers (i.e., e-cigarette users) were considered smokers for this study, which was reflected in all measuring instruments, as e-cigarettes are not treated as EBSTs in the Netherlands. The Fagerström test for nicotine dependence had a sufficient level of internal consistency within our sample $(\alpha=.75)$. Additionally, used nicotine products ("Which products do you smoke regularly? Multiple answers are possible.", 0 = cigarette; 1 = handrolled cigarette; 2 = e-cigarette; 3 = pipe; 4 = other product) as well as previous cessation attempts (e.g., "How many times have you made a serious quit attempt? By this we mean that you have not smoked for at least 24 hours.", could be answered on a continuous scale) were examined with four questions based on Mudde et al. [174] as common in Dutch studies focused on tobacco use/smoking (e.g., [71,123]).

\section{Decision-Related Cognitions}

A modified version of the general decision-making style (GDMS) questionnaire [160-162] was used to assess respondents' decision-making styles in order to perform a hierarchical cluster analysis. The modified version included questions to measure the regret style mentioned in the introduction. Thus, the scale consists of six subscales each measuring a decision-making style: rational ( $\alpha=.70)$, intuitive $(\alpha=.77)$, dependent $(\alpha=.81)$, avoidant ( $\alpha$ $=.86)$, spontaneous $(\alpha=.67)$, and regret $(\alpha=.79)$. Answering categories were given on a 5 point scale, ranging from 1 = "strongly disagree" to 5 = "strongly agree".

\section{Other Cognitions}

The treatment self-regulation questionnaire (TSRQ) was used to assess the degree to which respondents' motivation for a future cessation attempt is relatively autonomous (autonomous-regulated style) or determined by external control (controlled-regulated style) 
[175]. The scale consisted of two scales; one measuring the autonomous-regulated style (1 = "not at all true", 7 = "very true"; $\alpha=.87$ ), one the controlled-regulated style ( 1 = "not at all true", 7 = "very true"; $\alpha=.76)$. Secondly, the perceived competence scale (PCS) [176] was used to measure how confident respondents felt in their ability to quit smoking [177] (1 = "not at all true", 7 = "very true"; $\alpha=.90)$. Thirdly, the health care climate questionnaire (HCCQ) [176], was used to capture respondents' perceptions of the degree to which their healthcare providers were autonomy-supportive regarding smoking cessation ( 1 = "not at all true", $7=$ "very true"; $\alpha=.92$ ), i.e., whether they support behaviors that their patients want to do of their own volition. The HCCQ was only administered to respondents that had contact with a healthcare professional in the past 12 months.

To assess health locus of control, the multidimensional health locus of control scale (MHLC) was applied to measure the extent to which people believed that they, their physician, or chance have direct control over their own health $[164,178]$. The Dutch version of the MHLC consists of three subscales: internal health locus of control (ILOC; $\alpha=.77$ ), physicians' health locus of control (PLOC; $\alpha=.78$ ), and chance health locus of control (CLOC; $\alpha=.76$ ). Answering categories were given on 6-point scales as originally validated, ranging from 1 = "strongly disagree" to 6 = "strongly agree" [164].

Attitude towards EBSTs was measured with 10 items (five measuring attitude towards pharmacological support, five measuring attitude towards behavioral support) based on Rogers' Diffusion of Innovation Theory [57] using a 5-point scale, ranging from 1 = "strongly disagree" to 5 = "strongly agree". For example, respondents were asked "If I use these tools in a new cessation attempt, I will increase my chances of successfully quitting smoking" for both categories of EBSTs (pharmacological and behavioral support) to reflect Rogers' relative advantage [57]. As said items were developed for this study specifically, principal component analyses were conducted before scales were computed. These revealed that one item (based on Roger's concept of complexity) seemed to measure a different construct. Closer inspection of said item, revealed that it was the only one that was phrased negatively. Recoding of said item, did not change this, which is why we only used the other four items to compute the attitude scales. Both scales showed sufficient alphas (attitude pharmacological support; $\alpha=.78$, and attitude behavioral support; $\alpha=.79$ ). In-depth results of the principal component analyses can be provided on demand.

Finally, we measured intention to use an EBST DA via three items by asking the respondents whether a hypothetical DA would be used if it was available using a 7-point scale ( $\alpha=.96)$, e.g., "I want to use an online DA during a next quit attempt (if available)." 


\section{Statistical Analysis}

All analyses were conducted using SPSS 24.0 [179], $P$-values lower than .05 were considered significant. Firstly, descriptive analyses were conducted to assess sample characteristics. Secondly, cluster analyses were performed based on the GDMS subscales. Ward's hierarchical method with Euclidean distance as metric distance was used as clustering algorithm with GDMS scores standardized into z-scores [180]. Multiple methods were employed to investigate the number of clusters within the sample: (1) Cluster profiles were visually inspected to assess shape, level, scatter, and interpretability; (2) inverse scree tests were employed to indicate the optimal number of clusters; and (3) analyses were replicated within three random subsamples to investigate the stability of the cluster solutions $[163,181]$.

Thirdly, identified clusters were externally validated by comparing them on all measured variables using Mann-Whitney $\mathrm{U}$ tests, Kruskal-Wallis $\mathrm{H}$ tests, or chi-squared tests. For this (and all other analyses that follow) we created a new variable named "cluster membership" based on the cluster analysis described above. Nonparametric tests were used as most variables showed heterogeneity of variance and a substantial number of outliers. For categorical variables chi-square tests were conducted.

Fourthly, we investigated whether cluster membership was associated with intention to use a DA (main outcome variable) by conducting both a simple linear regression (unadjusted) and multiple linear regression (adjusted for other psychological constructs). As no previous studies have investigated the topic at hand before, variables were added to the adjusted model if their respective theoretical backgrounds (e.g., SDT) linked them to decision making and if they were statistically significantly associated with both intention (main outcome variable) and cluster membership (independent variable). A log transformation was applied to the scale measuring the controlled regulated style to not violate the linearity assumption.

Fifthly, mediation analyses were conducted post hoc with the technique as described by Preacher and Hayes [182] to test for possible mediation to explain the results from the linear regressions. Testing took place for all variables that clusters significantly differed on and/or appeared to be significant in the previous regressions. We tested each possible mediator separately. Results were interpreted using bootstrapped analyses [182]. As we carried out these mediation analyses in order to better understand the results from the analyses that were planned a priori, they do not correspond to any of the RQs mentioned in the introduction. 
Results

Of the 250 invited smokers, 200 completed the questionnaire and provided informed consent (response rate $=80 \%$ ). Table 1 displays all sample characteristics.

Table 1. Sample Characteristics.

\begin{tabular}{|c|c|}
\hline \multicolumn{2}{|l|}{ Gender identity } \\
\hline Women; $n(\%)$ & $102(51.0 \%)$ \\
\hline Men; $n(\%)$ & $98(49.0 \%)$ \\
\hline Not listed; $n(\%)$ & $0(0.0 \%)$ \\
\hline \multicolumn{2}{|l|}{ Age } \\
\hline Mean (SD) & $49.01(14.25)$ \\
\hline \multicolumn{2}{|l|}{ Country of birth } \\
\hline The Netherlands; $n(\%)$ & $191(95.5 \%)$ \\
\hline Outside The Netherlands; $n$ (\%) & $9(4.5 \%)$ \\
\hline \multicolumn{2}{|l|}{ Education level } \\
\hline Low (Completed); $n(\%)$ & $14(7.0 \%)$ \\
\hline Medium (Completed); $n(\%)$ & $135(67.5 \%)$ \\
\hline High (Completed); $n$ (\%) & $51(25.5 \%)$ \\
\hline Currently studying for a degree; $n(\%)$ & $28(14.0 \%)$ \\
\hline \multicolumn{2}{|l|}{ Smoking behavior and smoking-related cognitions } \\
\hline FTCD; Mean (SD) & $3.86(2.52)$ \\
\hline People that had attempted to quit (for at least 24 hours); $n(\%)$ & $181(90.5 \%)$ \\
\hline $\begin{array}{l}\text { Number of cessation attempts (lasting at least } 24 \text { hours); Mean } \\
\text { (SD) }\end{array}$ & $3.66(4.37)$ \\
\hline $\begin{array}{l}\text { Time in days since last cessation attempt (lasting at least } 24 \\
\text { hours); Mean (SD) }\end{array}$ & $957.27(1528.01)$ \\
\hline $\begin{array}{l}\text { Duration in days last cessation attempt (lasting at least } 24 \\
\text { hours); Mean (SD) }\end{array}$ & $253.33(676.13)$ \\
\hline Cigarette users; $n(\%)$ & $154(77.0 \%)$ \\
\hline Hand-rolled cigarette users; $n(\%)$ & $70(35.0 \%)$ \\
\hline E-cigarette users; $n(\%)$ & $24(12.0 \%)$ \\
\hline Pipe users; $n(\%)$ & $0(0.0 \%)$ \\
\hline Other products users; $n$ (\%) & $7(3.5 \%)$ \\
\hline \multicolumn{2}{|l|}{ Decision-related cognitions (GDMS) } \\
\hline Rational style; Mean (SD) & $3.79(0.61)$ \\
\hline Intuitive style; Mean (SD) & $4.03(0.56)$ \\
\hline Dependent style; Mean (SD) & $3.15(0.82)$ \\
\hline Avoidant style; Mean (SD) & $2.86(0.95)$ \\
\hline Spontaneous style; Mean (SD) & $3.38(0.65)$ \\
\hline Regret style; Mean (SD) & $3.01(0.89)$ \\
\hline
\end{tabular}




\begin{tabular}{|l|r|}
\hline Other cognitions & \\
\hline TSRQ: controlled regulatory style; Mean (SD) & $3.63(1.33)$ \\
\hline TSRQ: autonomous regulatory style; Mean (SD) & $5.55(1.13)$ \\
\hline PCS; Mean (SD) & $3.98(1.37)$ \\
\hline HCCQ; Mean (SD) & $4.41(1.18)$ \\
\hline Attitude pharmacological support; Mean (SD) & $3.21(0.98)$ \\
\hline Attitude behavioral support; Mean (SD) & $3.09(0.99)$ \\
\hline Internal (health) locus of control; Mean (SD) & $18.53(4.31)$ \\
\hline Chance (health) locus of control; Mean (SD) & $20.66(4.45)$ \\
\hline Physician (health) locus of control; Mean (SD) & $22.98(4.74)$ \\
\hline Intention to use a digital DA for cessation support tools in the & $3.82(1.76)$ \\
future; Mean (SD) & \\
\hline
\end{tabular}

Note. Time since last attempt, and duration of the last attempt variables only include people that remembered and tried to quit smoking at least once; FTCD = Fagerström test for cigarette dependence; $T S R Q=$ treatment self-regulation questionnaire; $P C S$ = perceived competence scale; GDMS = general decision-making style; $D A=$ decision aid. 


\section{Cluster Analysis}

Visual inspection of both the whole and random subsamples' dendrograms indicated that two clusters were formed, but that those two clusters seemed to split into smaller clusters. Inverse scree tests indicated that the two-cluster solution was best supported by the data, while both the three- and four-cluster-solution seemed feasible. In all three possible solutions, participants differed significantly on most of the GDMS subscales. Comparing the cluster analysis of the whole sample to the cluster analyses of the subsamples showed that clusters replicated most consistently in the two-cluster solution. Based on all available tests, we therefore selected the two-cluster solution for further analysis.

\section{External Validation}

The two clusters differed statistically significantly on all of the clustering variables except the rational style. Respondents in cluster $1(n=134)$ indicated that they were more avoidant, dependent, and regretful in or after decision-making situations, while respondents in cluster $2(n=66)$ reported a greater tendency to be spontaneous and intuitive. Therefore, cluster 1 was termed "Avoidant Regretters", while cluster 2 was termed "Intuitive Non-regretters". Additionally, "Avoidant Regretters" scored significantly higher on the intention to use the digital DA in the future, controlled regulatory style, and both attitude scales. There were also significantly less Dutch people in the "Avoidant Regretters" cluster, however 95.5\% of the whole sample were born in the Netherlands (therefore, 'Country of birth' was not used in further analyses). Examination of the other possible cluster solutions (i.e., three- and fourcluster solutions) revealed that the cluster named "Intuitive Non-regretters" remained stable in all of them, while the "Avoidant Regretters" seemed to diverge into multiple subclusters in the other solutions -indicating a more heterogeneous cluster (specific data is available on demand). Table 2 shows the characteristics of the two clusters found in the two-cluster solution. 


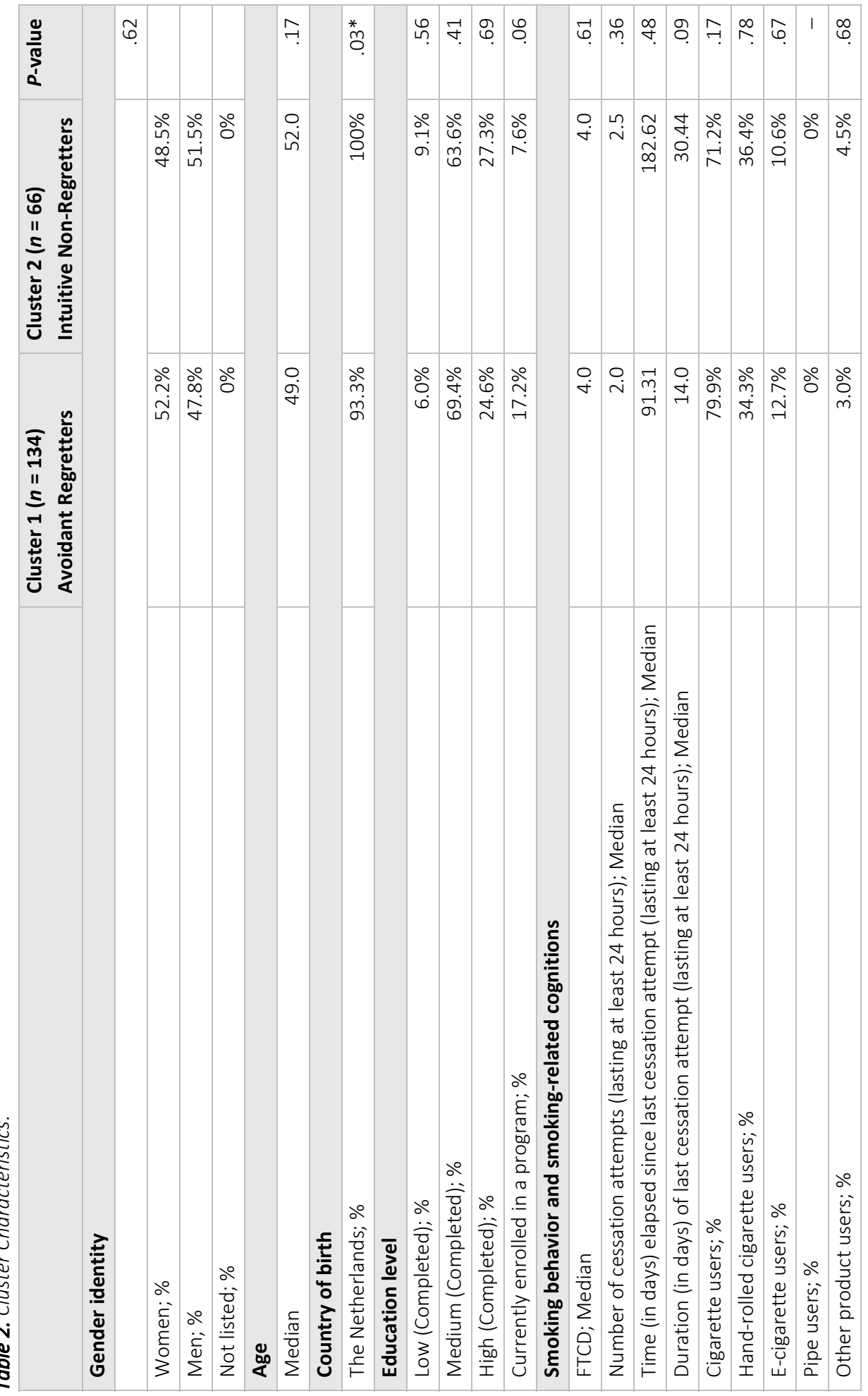




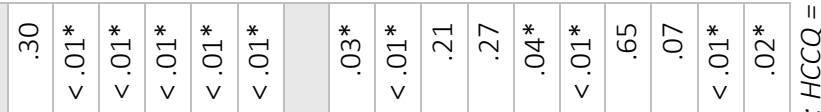

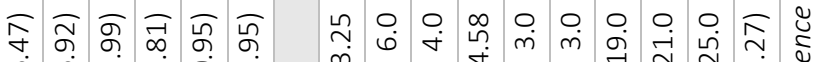

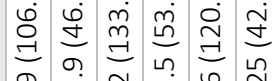

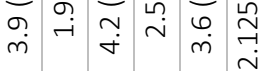

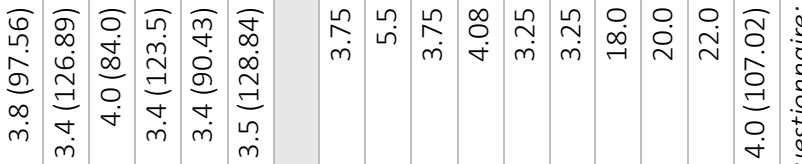

ஸे है

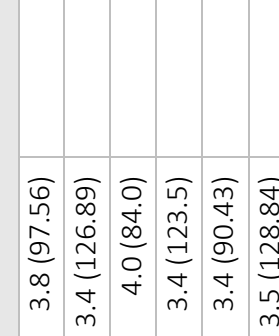

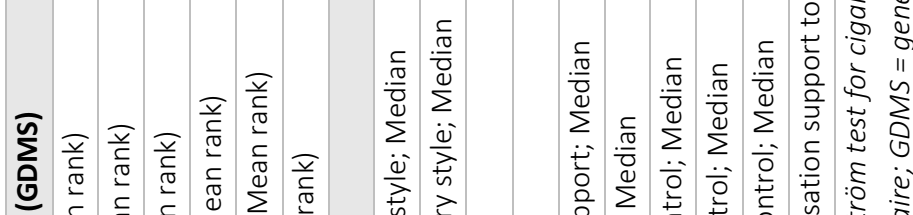

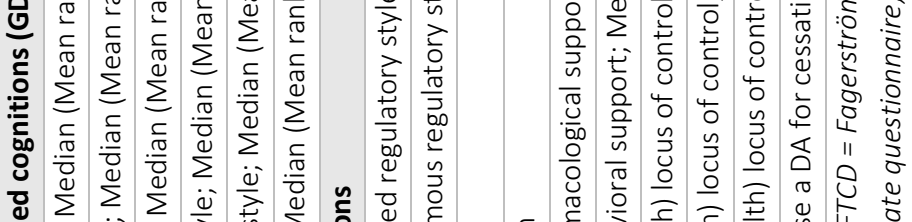

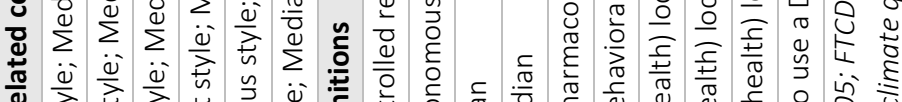

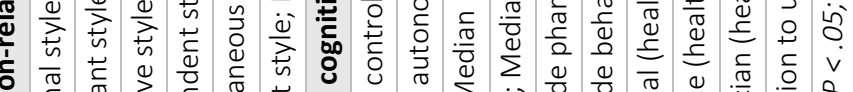

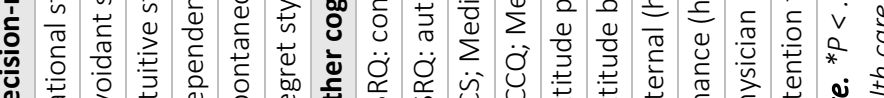

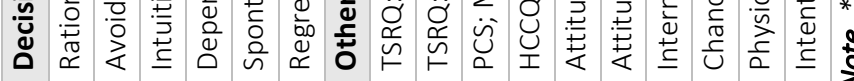




\section{Direct Effects of Cluster Membership}

Cluster membership (i.e., if participant belonged to either the "Avoidant Regretters" or "Intuitive Non-regretters" cluster) statistically significantly predicted intention to use a digital DA to choose EBSTs, $F(1,198)=5.06, P=.026$, accounting for $2.5 \%$ of the variation with adjusted R2 $=2.0 \%$. "Avoidant Regretters" reported an intention score that was 0.59 points higher than the score of "Intuitive Non-regretters", 95\% Cl [0.073, 1.108]. In the adjusted multiple regression, cluster membership ceased to statistically significantly predict intention to use a digital DA to choose EBSTs in the future. Table 3 shows the regression model. 

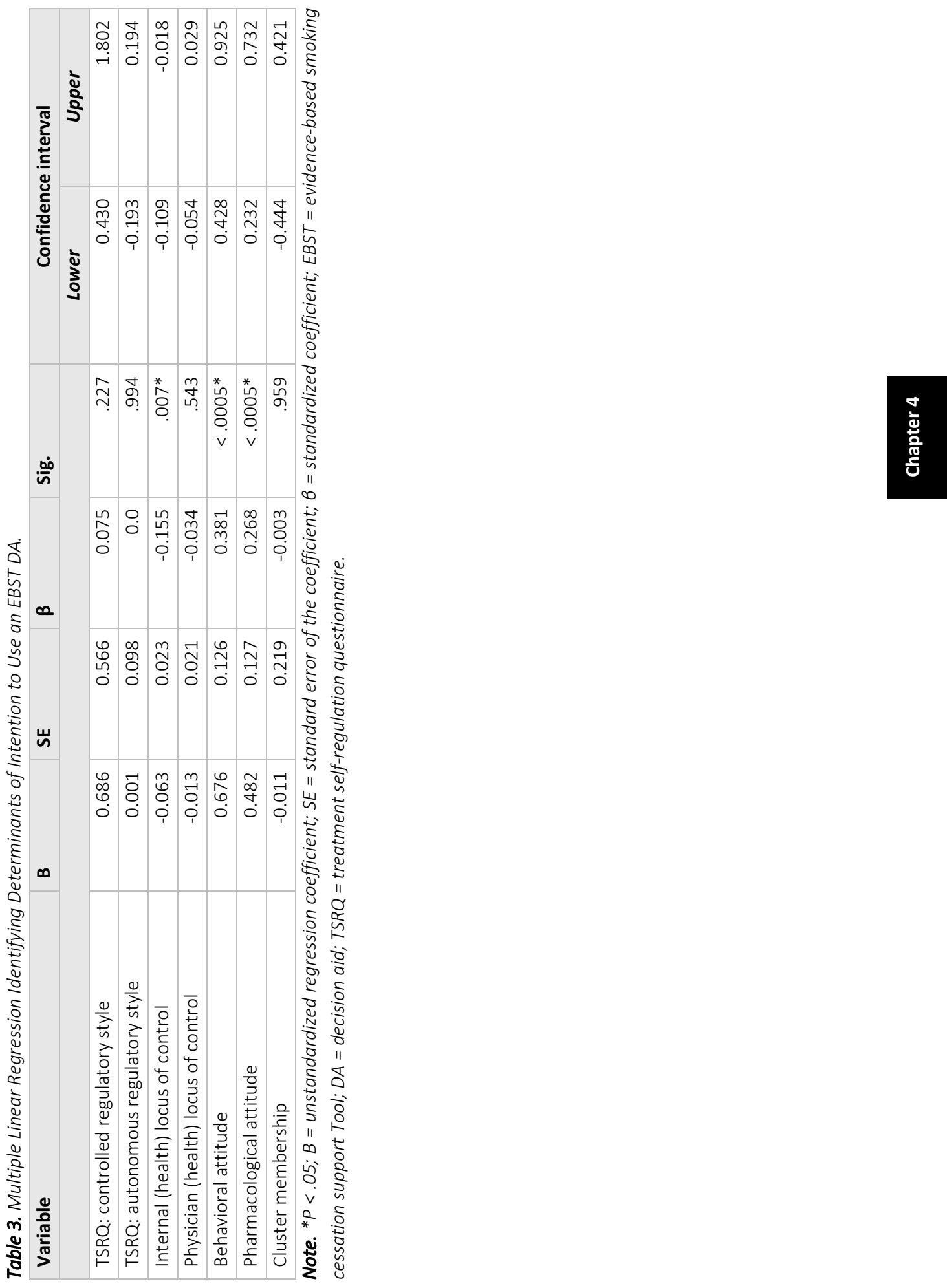
Indirect Effects of Cluster Membership

Indications were found that attitude and the controlled regulatory style seemed to fully mediate the effect of cluster membership, while the autonomous regulatory style seemed to mediate the effect only partially. No such indications were found for the internal and physician locus of control scales. Table 4 shows the results of the mediation analyses. 


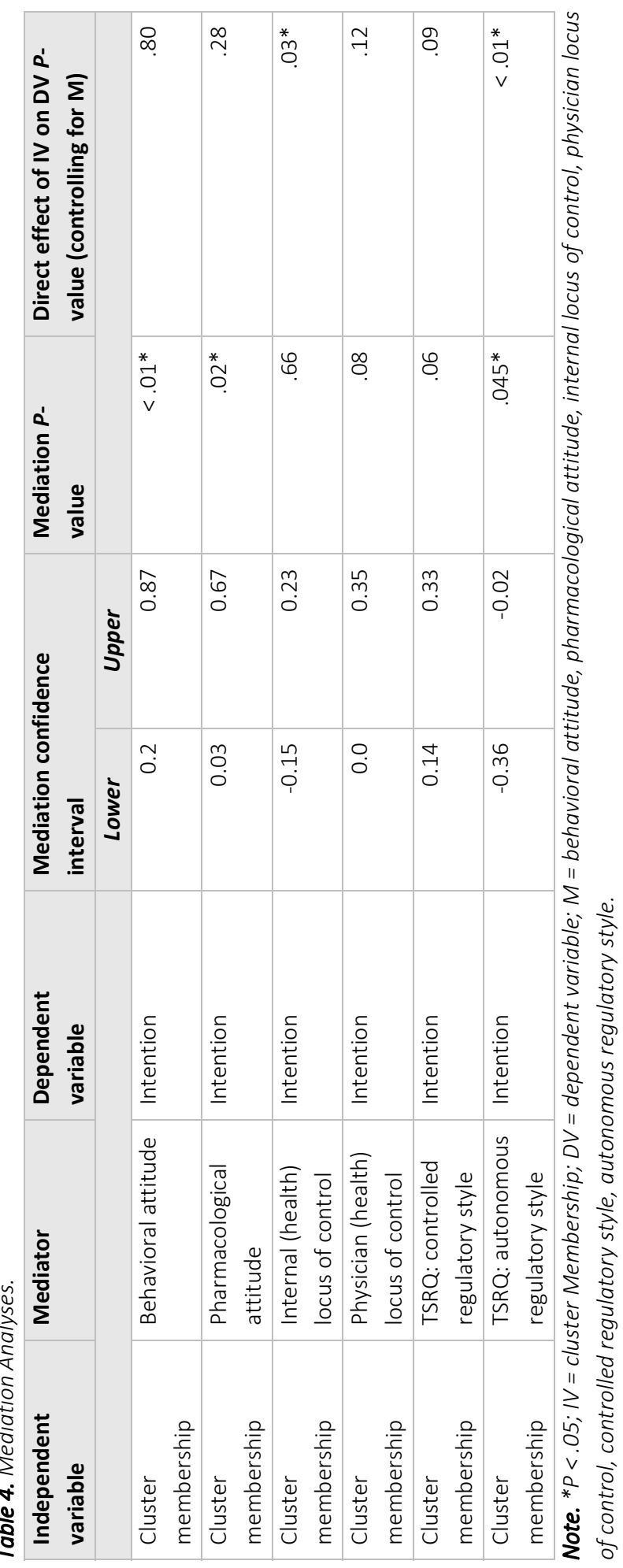




\section{Discussion}

In this study, it was investigated if clusters of smokers could be identified based on their decision-making styles and how those clusters differed to identify people that were interested in a digital DA for EBSTs.

Two distinct clusters were identified. "Avoidant Regretters" were characterized by their avoidant and dependent tendencies, as well as their tendency to regret decisions once those have been made. "Intuitive Non-regretters" on the other hand showed a tendency to be spontaneous and intuitive. Although this was the first cluster analysis based on GDMS, correlations between the styles found in earlier studies are comparable to the clusters we found in this study-especially regarding studies that also included regret styles [161,162]. For example, Dewberry et al. [162] found moderate to strong correlations between the regret (called brooding in their study), avoidant, and dependent styles and a moderate correlation between the spontaneous and intuitive styles. Furthermore, the authors found no significant correlation between the dependent and the intuitive style [162].

Additionally, it was found that "Avoidant Regretters" showed a significantly higher intention to use a digital DA aimed at facilitating the process of choosing an EBST_corresponding with their prevailing characteristics. Smokers belonging to this cluster showed a higher tendency to avoid decision making in general and also tended to regret decisions after they have been made. DAs are interventions specifically designed to assist decision making [13] and might consequently be especially appealing to the smokers who might have difficulties with decision making. In addition to their tendency to avoid and regret decisions, "Avoidant Regretters" also showed to be more dependent on others when they face decisions. "Avoidant Regretters" might perceive a digital DA as an external entity that supports them in their decision making. In line with this reasoning, research by Dewberry et al. [162] has shown that the dependent, avoidant, and regret style are all correlated with the tendency to look for the best option and not to choose the first option that is acceptable (i.e., maximization). While this was not measured in this current study, this might indicate that "Avoidant Regretters" prefer strategies aiming at finding the optimal solution, such as using a digital DA.

On the other hand, "Intuitive Non-regretters" were characterized by their spontaneity and impulsivity in decision-making situations. Again, two traits that seem to be linked $[161,162]$. Our results indicate that individuals in this cluster tend to use emotions to guide decisions and that they prefer quick decisions. Both traits could potentially make traditional DAs less interesting for them, given that they take time to use [13] and tend to be focused on deliberative thinking [183]. In line with this reasoning, both styles have not been shown to be linked to maximizing strategies in the study from Dewberry et al. [162]. Therefore, it might 
be possible that this cluster is less interested to invest time and energy in an intervention that helps them to find the most optimal solutions. And while intuition is sometimes linked to more favorable outcomes such as satisfaction [184], previous research has shown the effectiveness of intuition is largely dependent on the level on expertise a person has [185]. While knowledge or similar constructs were not measured, the two clusters did not differ in terms of their education or their number of cessation attempts. In other words, there is no evidence in this study that "Intuitive Non-Regretters" have more smoking cessation or health-related expertise than "Avoidant Regretters". Therefore, even though this type of quitters might be less interested in using a digital DA in the future, they are still likely to profit from it. And in fact, findings indicate that this cluster is interested in the proposed DA, even if they are less interested than "Avoidant Regretters" (with an average of 3.5 on a 7-point scale).

Based on the aforementioned findings it could be argued that both clusters could be targeted with a digital DA developed to facilitate choosing an EBST, but that it might be smart to tailor either both the DA itself and/or the recruitment strategy to smokers' cluster membership. For example, advertisements for the EBST DA could highlight that DAs have been proven to reduce decisional regret. This would probably be especially appealing for "Avoidant Regretters". On the other hand, measures could be taken to make the DA more appealing to "Intuitive Non-Regretters", e.g., by limiting the time needed to use the DA. Integrating intuitive elements $[63,183]$ in recruitment materials and DA-content could also increase the appeal of the DA for "Intuitive Non-Regretters", whereas incorporating proven strategies to reduce regret could increase the attractiveness for "Avoidant Regretters".

While the results of the multivariable regression indicate that the differences in intention may be explained by other variables than cluster membership (such as attitude), mediation analyses show that there is a possibility that the effect of cluster membership on intention is in fact mediated through said variables. While this has not been tested before, this is in line with integrated models that are used to predict behavior (change) in contemporary behavioral science $[186,187]$. Those models commonly state that personality characteristics are one of the most distal variables that influence behavior, (partly) mediated through other socio-cognitions. This possibly suggests that belonging to a particular group of decision makers has an impact on socio-cognitions, which in turn influences one's intention to use a DA.

Implications for Future Research

Since our study was explorative in nature and could rather be described as hypothesisgenerating (as opposed to hypothesis-testing), efforts should be made to test whether the identified results can be replicated. Ideally, to this end researchers should use a longitudinal 
design, as our post-hoc results indicate a mediation path may exist from decision-making styles through socio-cognitive variables (e.g., attitude) to intention to use a digital DA. Given the cross-sectional nature of our study, we were unfortunately unable to confirm this mediation path based on the data collected. In view of the so-called "intention-behavior gap" [95], it would also be particularly interesting to test whether the identified paths also apply to the actual uptake of a digital DA and not only to the intention to do so. In the discussion we also highlighted a number of ways in which our results can be used to inform either the recruitment of DA-participants (e.g., in recruitment materials it could be highlighted that DAs are known to reduce regret) or the (digital) DA-design itself (e.g., by including intuitive elements). Researchers could investigate whether suggested recruitment strategies and potential improving alterations to the DA have positive effects (e.g., on DA-uptake or on the quality of the decision-making process [85]) and how these effects relate to decision-making styles-e.g., based on our results, one could assume that highlighting regret-reducing in recruitment materials would be especially attractive and effective for "Avoidant Regretters".

\section{Strength and Limitations}

This study was mainly limited due to its cross-sectional and explorative nature. As conclusions regarding causality cannot be drawn based on our cross-sectional data, findings should be confirmed by longitudinal data (see also Implications for Future Research). Moreover, due to the explorative nature of our study we were unable to conduct a formal a priori power analysis, limiting confidence in our findings (especially due to our reliance on non-parametric tests which generally require more power). To elaborate, as the sample was relatively small, there is a possibility that we might have missed clusters that would have been identified given a bigger sample. Future research that aims to replicate the findings presented would benefit from conducting an a priori power analysis and recruit a sample of a size required for testing the hypotheses that can be formulated based on these findings. However, despite this limitation we are confident that our findings are valuable, as they reflect modern models and theories, and as our findings are in line with findings from comparable research [162] and as, generally, the sample sizes required to perform the main analysis of this study (i.e., cluster analysis) do not have to be too large [188].

\section{Conclusion}

Our explorative study has shown that it is indeed possible to identify smoker clusters based on their decision-making styles and that cluster membership has at least some impact on smokers' intention to use a digital EBSTs DA. Future studies in the field of digital DAs would therefore benefit from including decision-making style in their design, and it would be interesting to see if our findings could be replicated in longitudinal studies. In addition, it would be interesting to see how decision-making style could be used in digital DA- 
development, as well as recruitment of participants for a study on digital DA effectiveness. Practitioners and developers of digital DAs might use our findings to initially tailor their recruitment strategies and identify early adopters, who might be able to contribute to the development of more attractive interventions that are ultimately appealing for smokers from all adopter categories.

Abbreviations

CLOC - chance health locus of control

DA - decision aid

EBST - evidence-based smoking cessation support tool

GDMS - general decision-making style

$\mathrm{HCCQ}$ - health care climate questionnaire

ILOC - internal health locus of control

MHLC - multidimensional health locus of control scale

NRT - nicotine replacement therapy

PCS - perceived competence scale

PLOC - physicians' health locus of control

$\mathrm{RQ}$ - research question

SDT - Self-Determination Theory

TSRQ - treatment self-regulation questionnaire 


$$
5
$$




\section{chapter 5}

informed decision making on the uptake of evidence-based smoking cessation assistance by individuals motivated to quit smoking: a needs assessment among potential end users and experts to inform decision aid development

This chapter has been published as:

Gültzow, T., Smit, E. S., Hudales, R., Dirksen, C. D., \& Hoving, C. (2022). Informed Decision Making on the Uptake of Evidence-Based Smoking Cessation Assistance by Individuals Motivated to Quit Smoking: A Needs Assessment Among Potential End Users and Experts to Inform Decision Aid Development. European Journal of Health Communication, 3(1), 1-30. https://doi.org/10.47368/ejhc.2022.101 


\section{Abstract}

Evidence-based cessation assistance increases cessation rates. Activating preferences during decision making could improve effectiveness further. Decision aids (DAs) facilitate deciding by taking preferences into account. To develop effective DAs, potential end users' (i.e., individuals motivated to quit) needs and experts' viewpoints should be considered. Therefore, the aim of this needs assessment was: (1) to explore end users' needs and (2) to obtain consensus among smoking cessation counselors and scientific experts to develop a self-administered DA to support end users in choosing cessation assistance. Data was gathered via two approaches: (1) twenty semi-structured interviews with potential end users and (2) two three-round Delphi studies with 61 counselors and 44 scientific experts. Interview data and the first Delphi rounds were analyzed qualitatively, the other Delphi rounds were analyzed quantitatively. Potential end users acquired information in different ways, e.g., via own experiences. Important characteristics to decide between tools varied, however effectiveness and costs were commonly reported. Experts reached consensus on 38 and 40 statements, e.g., tools should be appropriate for users' addiction level. Although some trends emerged, due to the variation among stakeholders, a 'one size fits all'-approach is undesirable. This heterogeneity should be considered, e.g., by enabling users to customize the DA. 


\section{Introduction}

Worldwide tobacco smoking continues to be one of the leading causes of preventable diseases and premature death [189]. Evidence-based cessation assistance tools used by individuals during their smoking cessation attempts are known to increase successful smoking cessation chances. Examples of such evidence-based cessation assistance tools are nicotine replacement therapy [190] and smoking cessation counselling [105]. Zhu et al. [23] reported that cessation assistance tools double the chances of attaining a smoke-free status. However, evidence-based cessation assistance tools are currently underused [191]. Increasing the uptake of said tools would therefore likely result in more individuals achieving smoking abstinence, which can lead to improved population health, as well as a decrease in healthcare costs $[192,193]$.

One commonly described barrier to using evidence-based cessation assistance tools is incorrect knowledge regarding their safety and efficacy [29], while adequate knowledge is one of the prerequisites for making informed decisions $[1,16]$. Conversely, this means that increasing individuals' knowledge using health communication interventions about evidence-based smoking cessation assistance tools could lead to more informed decisions and potentially result in more individuals using said assistance during a quit attempt. However, even if individuals are knowledgeable and do choose to seek additional help in the form of cessation assistance tools, the variety of effective tools (e.g., $[26,103,104,194]$ ) means that they still have to make a decision between the different effective tools available. As making choices based on personal preferences has been shown to positively influence the effectiveness of clinical treatments [61], incorporating personal preferences when deciding on which cessation assistance support tool to use could be beneficial for smoking cessation as well. In addition to providing information to expand knowledge, it could, therefore, be useful to help individuals to identify what is important to them personally (i.e., help them to clarify their values) and support them in choosing a cessation aid that fits their personal preferences-another prerequisite for making informed decisions $[1,16]$.

Decision aids (DAs) are interventions specifically meant to enable unbiased communication of relevant health(care) information, in order to increase knowledge among people making health(care) decisions (e.g., patients) and to support these users in choosing a health(care) option that best reflects their personal values and preferences [13]. In other words: DAs support informed decision making among DAs' end users. DAs have predominantly been developed to support people in making decisions about medical treatments and screening programs [13], but are also increasingly used to help individuals make informed decisions regarding preventive health-related behaviors, such as smoking cessation $[52,195]$. In various studies it has been shown that DAs can have positive effects on smoking cessation outcomes (e.g., $[42,53,135])$, however, as far as we are aware, in all those studies 
assessments of needs and viewpoints of potential end users in initial phases of DAdevelopment were not reported. This is in contrast to the criteria of the International Patient Decision Aid Standards (IPDAS) Collaboration [31] that explicitly recommend this assessment in the developmental process of any DA. This is unfortunate, as including potential end users early in the development process may lead to increased end user acceptance and improved long-term implementation and effectiveness of DAs $[59,196,197]$. Within the IPDAS' development guidelines, the assessment of clinicians' (such as physicians' and nurses') viewpoints is also advocated [59]. This is mainly a reflection of the fact that DAs are traditionally employed in clinical settings $[13,197]$. Given that decisions on health promotion and public health issues (such as smoking cessation [60]) are commonly made without the involvement of clinicians, the question is whether different or additional experts (such as scientific experts) should play a role in the development of DAs that serve a health promotion or public health goal. There are currently no guidelines on how to develop such DAs and, consequently, there is no consensus on which experts should be involved in the development process. Therefore, we chose to expand the commonly accepted approach to include clinicians (in our specific case, smoking cessation counselors) with the inclusion of additional experts, i.e., scientific experts in the field of smoking cessation.

Therefore, the aim of this needs assessment was to explore the needs and viewpoints regarding the development of a self-administered DA to support informed decision making within the context of smoking cessation from the perspective of three main stakeholders: (1) potential end users (i.e., individuals motivated to quit smoking), (2) smoking cessation counselors, and (3) scientific experts. In order to optimally explore the needs and viewpoints of the different stakeholder groups, two different approaches were applied in three studies: (1) In-depth, semi-structured interviews were conducted to identify the smokers' needs and wishes in relation to the decision-making process on how to quit smoking and the content and format of an online self-administered DA that aims to support them in this process; (2) two three-round Delphi studies among (a) smoking cessation counselors and (b) scientific experts, to gather their viewpoints and experiences regarding smoking cessation decision making and to obtain consensus on the required content of an online self-administered smoking cessation DA. The result from these studies were directly applied by the research team to develop a self-administered DA that individuals motivated to quit smoking can use themselves to prepare their cessation attempt [96]. However, the results of the three studies also offer new insights into the decision-making process that smokers go through when they decide how they want to quit smoking-thereby contributing to the health communication literature. 


\section{Material and Methods}

As indicated in the introduction, this needs assessment was part of a larger project with the ultimate goal of developing a self-administered online DA to support individuals motivated to quit smoking in making an informed decision about the use of smoking cessation assistance tools [96]. This larger project is funded by the Dutch Cancer Society (UM20157744). Evaluation of this project by the Medical Ethics Committee METC Z (16-N-227) revealed that this project did not require medical ethics approval under the rules of the Medical Research Involving Human Subjects Act (WMO). All materials that participants received, interview guides, questionnaires, and SPSS syntaxes can be found on the

Open Science Framework (OSF), https://osf.io/6dg7r/?view_only=98e511e58fcd4870a 583c2071430e526. For the interview study, we only produced materials in Dutch, however, to facilitate understanding we provide approximate translations.

\section{In-Depth Semi-Structured Interviews With Individuals Motivated to Quit Smoking}

Participants and Procedure

For the interview study, we recruited among members from a national research panel [171] that participated in an earlier study about smoking cessation assistance decision making [198]. We only included individuals motivated to quit smoking (or that quitted smoking recently) and chose not to include other individuals, such as those who need to stop smoking while not wanting to (e.g., due to health reasons). We chose to do this as the selfadministered smoking cessation DA that was developed as part of the overarching project was meant for end users with an active interest in smoking cessation. Individuals not interested in quitting smoking presumably would not be interested in a self-administered smoking cessation DA and might in fact need another form of intervention before they use a DA, e.g., motivational interviewing [199]. We employed purposeful sampling to invite a heterogenic sample using the following four characteristics: (1) intention to use the proposed online DA [73], (2) decision-making style as measured by the General DecisionMaking Style measurement [160] (i.e., we aimed for a balance between participants who were more regretful, avoidant, and dependent in their decision making and participants who tended to make spontaneous and intuitive decisions; for more information see [198]), (3) geographical location, and (4) reported gender identity (i.e., man, woman, and non-binary participants). Interviews were conducted until data saturation was reached [200], i.e., when three consecutive interviews did not generate new knowledge related to the research objectives.

All interviews were conducted by telephone and recorded, for which participants gave their consent after a brief explanation about the study and the procedure. Prior to the actual interview, participants received: (1) a description of the different decision-making styles 
based on the aforementioned earlier study (see [198] for more information), (2) information about which cessation assistance tools are evidence based (e.g., behavioral support) and which are not (e.g., acupuncture), and (3) DA mock-up screenshots to give an indication of what a self-administered online DA to support people in making an informed decision about the use of cessation assistance could look like. During the interviews, participants were asked whether they had actually read and viewed these materials and if not, were given the opportunity to do so on the spot or were given a verbal summary of all materials (depending on the circumstances). Also, the participants were able to ask questions regarding the materials. Participants received a $€ 30$ (\$34.79) gift card for their participation.

Interview Guide

A semi-structured interview guide was developed which was used to gather information for the larger project [96] and this study, and therefore also included questions that were beyond the scope and objectives of this study (see the entire interview guide on the OSF, https://osf.io/6dg7r/?view_only=98e511e58fcd4870a583c2071430e526). The relevant questions for this study were related to: (1) knowledge (e.g., capability to accurately distinguish between evidence-based and non-evidence-based forms of assistance), (2) values related to cessation assistance tools (e.g., characteristics participants would pay attention to if they were to choose a cessation assistance tool), and (3) the proposed DA (e.g., personal needs regarding functional aspects of the DA). Two nearly identical interview guides were developed; one for participants that were still smoking and one for smokers who recently quit (e.g., questions were posed about the last quit attempt instead of future attempts).

Data Analysis

Data was analyzed using the Framework Method [201] using Nvivo 12 [202]. After interviews were transcribed verbatim by $\mathrm{RH}, \mathrm{TG}$ and $\mathrm{RH}$ together created a codebook in an iterative process. TG and RH used the final version to code all interviews (TG) and $10 \%(n=2, \mathrm{RH})$ respectively, resulting in a high intercoder reliability of 0.81 (Cohen's Kappa) with $99.5 \%$ of agreement. After intercoder reliability was ensured, data was interpreted by making use of a framework matrix to look for patterns in relation to knowledge, values, and the proposed online DA. In a framework matrix, qualitative data is summarized and analyzed. This enables easy comparison between the participants to identify patterns [201]. Additionally, we exploratively analyzed if patterns could be observed based on gender identity, age, and level of education-all factors that were chosen a priori. As those analyses were purely explorative in nature, we chose to focus on a relatively small set of subgroups based on demographics. Subgroup analyses based on other variables (e.g., nicotine dependence) fell outside of the scope of this article. Results regarding those explorative analyses were only reported if any 
patterns could in fact be observed. We would like to emphasize that numerical terms used to describe the findings from the interviews (apart from the sample description) are not intended to imply that quantitative analyses have taken place, but merely to shed light on our findings. Sample characteristics were examined using SPSS [203].

\section{Three-Round Delphi Studies Among Smoking Cessation Counselors and Scientific Experts} Participants and Procedure

For the Delphi studies, we used different recruitment approaches for the two expert groups. Counselors were included if they offered smoking cessation counseling services. We mainly drew from our professional networks, relevant Dutch organizations, newsletters, and we employed snowball recruitment (i.e., participants were asked to name up to three additional experts).

Scientific experts' recruitment occurred through a literature search, mass media channels (e.g., newsletters and social media), and (again) snowballing. We used database searches in PubMed to identify relevant papers using the following search terms: "(smoking cessation OR tobacco cessation) AND (intervention OR program*)". The search was limited to the past five years and to journal articles written in English or Dutch. First, second, and last authors of papers relevant to the development, testing, and/or implementation of smoking cessation interventions were invited by email to participate in our study. In order to ensure that participants had enough relevant expertise five inclusion criteria (based on Davis et al. [204]) were used: Participants had to (1) have published at least two journal articles/book chapters or one book on smoking (cessation) and/or smoking cessation interventions within the last 5 years, (2) have been awarded a grant within the last five years in the area of smoking (cessation) and/or smoking cessation interventions, (3) have been involved in at least one development process of a smoking cessation intervention within the last 5 years, and (4) have at least five years of experience in the field of smoking (cessation) and/or smoking cessation interventions. Participants had to fulfil at least two criteria to be included in the first round (as the answers from the first round form the basis for the following rounds - see The Questionnaires) and at least one to be included in the other rounds.

The Questionnaires

The questionnaires of all rounds consisted of questions pertaining to (1) the professional function and background of participants, (2) the inclusion criteria (see Participants and Procedure), and (3) participants' email addresses. Starting with round two, experts were also asked if they have been involved in the development of at least one DA. Additionally, five open-ended questions were posed in the first round (Table 1) and one in the second and third round ("If you have any additions or comments, please feel free to add them here."). 
Table 1. Questions First Round.

\section{Question}

1. Based on your expertise, which characteristics of smoking cessation support tools are important for smokers when a choice between different tools is made?

2. Based on your expertise, what do you think are important elements of an online decision aid aimed at helping smokers make an informed choice about the use of a smoking cessation support tool?

3. Based on your expertise, how do you think visual aids can best be utilized in an online decision aid for smokers?

4. Based on your expertise, what other design elements should be considered when developing an online decision aid for smokers?

5. Based on your expertise, how would you advise to use such an online decision aid within existing services and/or evidence-based intervention strategies?

The questions of the first round were deliberately designed to align with the questions asked to the end users to facilitate comparison between the different groups. The questionnaire of the second round included 75 statements and was based on the answers of the first round (and other literature for two statements to supplement the experts' answers) regarding (1) cessation assistance tools' characteristics, (2) online DA-functions, (3) visual aids, (4) and embedment of the DA in the current, clinical context. After answering statements regarding cessation assistance tools' characteristics, participants were shown DA mock-up screenshots to give them an indication of what an online self-administered DA could look like based on input from the first round to provide them with context before answering the rest of the statements. All statements were scored on a 7-point Likert scale (see Table 4 for more information).

The questionnaire of the third round included only those statements that smoking cessation counselors and scientific experts failed to reach consensus on in the second round (63 and 60 statements, respectively). Participants received feedback on how much experts agreed with the statements (importance) and each other (consensus).

Questionnaires for the smoking cessation counselors were formulated in Dutch, while the questionnaires for the scientific experts were formulated in English.

\section{Data Analysis}

The data of the first rounds were analyzed using Nvivo 12 [202]. Again, TG and RH created a code tree in an iterative process and used it to code all answers separately and reached a substantial intercoder reliability of 0.67 (Cohen's Kappa) and 99.24\% (percentage of agreement). 
The data of the second and third rounds were analyzed with SPSS [203]. Respondents' answers were included if respondents answered at least $80 \%$ of the questions. Frequencies were used to examine the demographics, medians were calculated to examine which importance participants (as a group) attributed to individual statements (a median of $\geq 6$ was regarded as cut-off point), and interquartile ranges (IQR) were used to examine the consensus reached for every statement (an IQR of $\leq 1$ was regarded as cut-off point). Cut-off points were based on similar studies (e.g., [205]). The two expert groups were analyzed separately.

Results

In-Depth Semi-Structured Interviews

Twenty individual interviews were conducted. Nine (45\%) of the interviewees identified as women, while the other 11 (55\%) identified as men. The majority ( $n=12,60 \%$ ) held a medium level of education and on average interviewees undertook three previous smoking cessation attempts that lasted for 178 days on average (Table 2).

Table 2. Descriptive Characteristics of Participants $(N=20)$.

\begin{tabular}{|l|r|r|}
\hline Descriptive characteristics & M & \\
\hline Age in years & 50.3 & 13.5 \\
\hline Smoking behaviour & 3.0 & 2.1 \\
\hline Number of quit attempts & 177.8 & 445 \\
\hline Duration of quit attempt in days & 4.2 & 2.0 \\
\hline Intention to use the decision aid & \\
\hline & $n$ & \\
\hline Gender $\quad$ & 9 & $45 \%$ \\
\hline Identified as women (\%) & 11 & $55 \%$ \\
\hline Identified as men (\%) & 0 & $0 \%$ \\
\hline Identified as another gender/non-binary (\%) & & \\
\hline Education & \\
\hline Low (\%) & 1 & $5 \%$ \\
\hline Medium (\%) & 12 & $60 \%$ \\
\hline High (\%) & 7 & $35 \%$ \\
\hline
\end{tabular}

Note. ${ }^{a}$ Intention: 1 = low intention; $7=$ high intention. ${ }^{b}$ Education: Low $=$ no, primary, and vocational education; Medium = secondary vocational education and a high school degree; High = higher vocational education, college, and university degrees. 
Knowledge

Overall, the level of cessation assistance tool knowledge varied considerably between individuals. Interviewees tended to be aware of some facts (e.g., tools' side effects) without being aware of other facts (e.g., insurance reimbursement). The terms (non-)evidence-based also were not always clear and some interviewees were not aware of the distinction between evidence-based and non-evidence-based tools. Interviewees also sometimes equated believing and knowing, for example by expressing that they did not believe in the distinction between evidence-based and non-evidence-based tools. Interestingly, some interviewees displayed a discrepancy between perceived and objective knowledge, i.e., they regarded themselves as being informed while also lacking information.

Multiple information sources were described to acquire knowledge: (1) other people's personal experiences, such as colleagues or neighbors; (2) one's own previous experience(s); and (3) the media. Sometimes interviewees described shortcoming of these strategies without necessarily realizing them, e.g., someone considered an evidence-based tool to be ineffective in general because it was ineffective for them personally; and a few people described that one source was more important than the other, e.g., personal experiences as opposed to other people's experiences.

Values Related to Cessation Assistance

Values and beliefs were notably very heterogenous and often linked to personal circumstances. Interviewees also described aspects that directly influenced decision making for them that were not inherent to the tools, e.g., one's personal experiences with a tool. Despite the variety, one pattern emerged: Effectiveness was referred to the most, followed by costs. The same pattern also emerged when the interviewees were asked about the characteristics that were the most important to them. Interviewees often made references to long-term success and some used the term in a broader way (e.g., a tool has to fit personally in order to be effective). Also, valuing effectiveness as important did not seem to be related to educational level, although this idea was conveyed by one of the highly educated interviewees. Other particularly important values were: (1) that the tool is covered by health insurance; (2) that the tool is offered by $a(n)$ (professional) expert (e.g., general practitioners); (3) side effects; (4) safety; (5) personal contact with another person; (6) personal fit; and (7) practical considerations, such as length of treatment, number of doses/sessions, and the location. The only characteristic that was almost universally accepted as being unimportant was whether tools were advertised. Other characteristics were alternately framed as advantages or disadvantages, e.g., some interviewees appreciated personal contact, while others did not. Interviewees also clearly made tradeoffs during the interviews, e.g., by describing that certain characteristics were important but 
less so than others. For example, short-term side effects that were considered as unimportant and long-term side effects that were considered as important. Temporal dynamics of values were also acknowledged by describing that the evaluations of certain characteristics depend on the smoking cessation phase one is in, e.g., someone indicated that personal contact would become important after the period in which they were able to not smoke on their own.

\section{Decision Aid}

Interviewees often referred to the materials we sent before the interview. Those were regarded as pleasant or useful and interviewees appreciated that the differences between the different cessation tools became apparent by naming the tools' advantages and disadvantages - this could be used to compare tools to make a decision. Also, some interviewees seemed to expect that they would receive advice from the DA. The majority of the interviewees were at least somewhat interested in using the proposed DA. The interviewees that were either not interested or less interested in the DA had different reasons, e.g., mistrust in online information, that the information could also be acquired somewhere else (e.g., through a face-to-face meeting with a healthcare professional), or not being interested in cessation (assistance) at the moment. Other interviewees did not fully grasp the concept of a DA at the beginning and needed more explanation. No clear patterns emerged based on gender identity and level of education. However, fewer younger interviewees $(<40)$ talked about mistrust in online information or that information could be acquired elsewhere.

Personal Needs Regarding Functional Aspects of the Decision Aid

Interviewees often indicated that they liked the table with all options that we presented as preparation for the interviews. A number of interviewees also indicated that they would like to hear about others' choices and experiences. The content of the offered information was discussed as well, with information that often related to earlier described values (see Values Related to Cessation Assistance). Interestingly, no-one indicated that the inclusion of certain functions would make the DA uninteresting for them, rather they indicated that they would skip the function or that they would use the function passively. Functions were often linked to personal circumstances and/or preferences. Overall, interviewees often liked the idea that they could have control over the amount of information in the DA.

Personal Needs Regarding Device Compatibility and Usage Duration of the Decision Aid

Multiple possible devices (e.g., personal computers) to use the DA were named, as were various time spans. The majority wanted to use the DA on their computer and only a few 
mentioned mobile phones-only one interviewee wanted to use the DA as a mobile phone app. Interestingly, no-one from the oldest age group (> 69) and only one person from the age group 30-39 indicated that they would like to use the DA on their mobile phone and people with a medium level of education seemed to prefer mobile phones more. That said, only one interviewee had a low level of education, limiting conclusions that can be drawn from this finding.

Preferred time spans ranged from five minutes to an unlimited time span, however, some were unable to name a time span at all. Older interviewees ( $>50)$ did not indicate a clear time frame, with one interviewee (70-year-old) stressing the amount of time at their disposal. A few interviewees who preferred short time spans indicated that they would be willing to spend more time if they were in doubt or if they wanted to receive extra information. Some interviewees indicated that they would want to use the DA multiple times.

Personal Needs Regarding the Layout of the Decision Aid

Interviewees overall indicated that they would like the DA to be neutral, functional, simple, positive, and motivating, however quite a few interviewees found the question difficult to answer. Several interviewees expressed that they rather not see any negative or off-putting imagery, such as used on cigarette packs. Regarding visual aids, some interviews indicated that they could be supportive but should only be used if they were of added value (e.g., pictures of different tools to recognize them), while others indicated that they would not be needed for them at all. People who disfavored visual aids tended to identify as women between 40-59 (most 40-49) years old with a medium level of education. Overall, an intuitive and easy to navigate layout seemed to be preferred.

\section{Three-Round Delphi Study}

First Round

Nine scientific experts and eight smoking cessation counselors participated in the first round. The majority of the scientific experts were either associate $(n=1,11.1 \%)$ or full professors ( $n=4,44.4 \%)$, while the biggest group of the smoking cessation counselors were practice nurses $(n=6,75 \%)$. Answers fell into nine categories (Table 3 ). 
Table 3. Answer Categories and Examples of the First Round.

\begin{tabular}{|c|c|c|}
\hline & Answer categories & Answer examples \\
\hline 1. & Advertising the decision aid & $\begin{array}{l}\text { If a patient comes to a GP for a } \\
\text { consultation [...] point out this possibility } \\
\text { [the decision aid] to the patient. }\end{array}$ \\
\hline 2. & $\begin{array}{l}\text { Characteristics of smoking cessation } \\
\text { assistance tools }\end{array}$ & Effectiveness \\
\hline 3. & Characteristics of users & $\begin{array}{l}\text { Previous cessation attempts and } \\
\text { experience }\end{array}$ \\
\hline 4. & $\begin{array}{l}\text { Content of the decision aid regarding } \\
\text { evidence-based cessation tools }\end{array}$ & $\begin{array}{l}\text { If it includes non-evidence-based tools } \\
\text { this has to be clearly stated }\end{array}$ \\
\hline 5. & Decision aid attributes & Accessibility \\
\hline 6. & Decision aid functions & $\begin{array}{l}\text { Possibility to obtain in-depth info when } \\
\text { needed }\end{array}$ \\
\hline 7. & Layout considerations & Match with target audience \\
\hline 8. & $\begin{array}{l}\text { Use of the decision aid in the current } \\
\text { clinical context }\end{array}$ & $\begin{array}{l}\text { Healthcare providers could show it [the } \\
\text { decision aid] to patients and they can use } \\
\text { the decision aid together. }\end{array}$ \\
\hline 9. & Other & $\begin{array}{l}\text { Actors need to be appealing to the end- } \\
\text { users. Tip: Involve the end-user in the } \\
\text { development of the tool and especially in } \\
\text { the development of the visual aids to } \\
\text { ensure these appeal to them and match } \\
\text { their needs and wants. }\end{array}$ \\
\hline
\end{tabular}

Note. GP = general practitioner.

Second and Third Round

Sixty-one smoking cessation counselors and 44 scientific experts participated in the second round; $62.3 \%(n=38)$ of the smoking cessation counselors and $54.5 \%$ of the scientific experts ( $n=24$ ) returned to fill in the third round. In both rounds, the majority of the smoking cessation counselors were practice nurses in both the second $(n=43,70.5 \%)$ and third round ( $n=29,76.3 \%)$, while the majority of the scientific experts were either associate $(n=9,20.5 \%$ in the second round; and $n=4,16.7 \%$ in the third round) or full professors ( $n=16,36.4 \%$ in the second round; and $n=8,33.3 \%$ in the third round). More details regarding the samples of the second and third rounds can be found in Appendix $A$.

Consensus and Importance

The smoking cessation counselors reached consensus (IQR $\leq 1$ ) on 12 statements ( $16 \%$ of all statements) in the second round and on 26 statements (34.7\% of all statements, $41.3 \%$ of all statements in the third round) in the third round. In the second round, they reached 
consensus on six statements regarding cessation assistance tools' characteristics, on six statements regarding online DA-functions, and on no statements regarding visual aids and how to embed the DA in the current, clinical context. Of those 12 statements only two statements were regarded as unimportant (i.e., importance score < 6). In the third round, they reached consensus on seven statements regarding cessation assistance tools' characteristics, on nine statements regarding online DA-functions, on eight statements regarding visual aids, and on two statements regarding how to embed the DA in the current, clinical context. Of those 24 statements 11 statements were regarded as unimportant. The scientific experts reached consensus on 15 statements (20\% of all statements) in the second round and on 25 statements (33.3\% of all statements, $41.7 \%$ of all statements in the third round) in the third round. In the second round, they reached consensus on four statements regarding cessation assistance tools' characteristics, on six statements regarding online DAfunctions, on four statements regarding visual aids, and on one statement regarding how to embed the DA in the current, clinical context. Of those 15 statements only three statements were regarded as unimportant. In the third round, they reached consensus on seven statements regarding cessation assistance tools' characteristics, on 10 statements regarding online DA-functions, on eight statements regarding visual aids, and on no statements regarding how to embed the DA in the current, clinical context. Of those 25 statements nine statements were regarded as unimportant. See Table 4 for all statements and the respective scores for importance and consensus. 


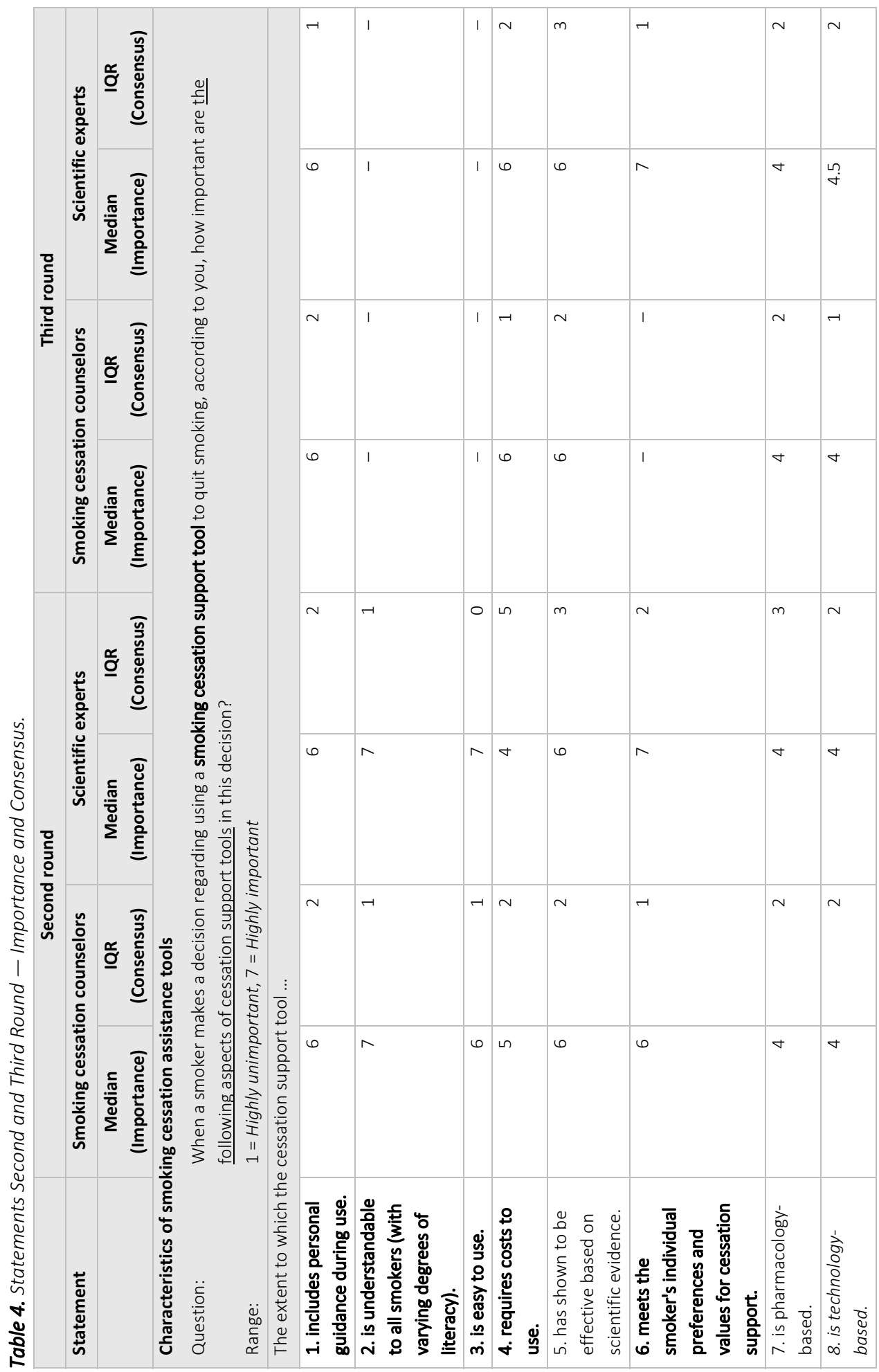




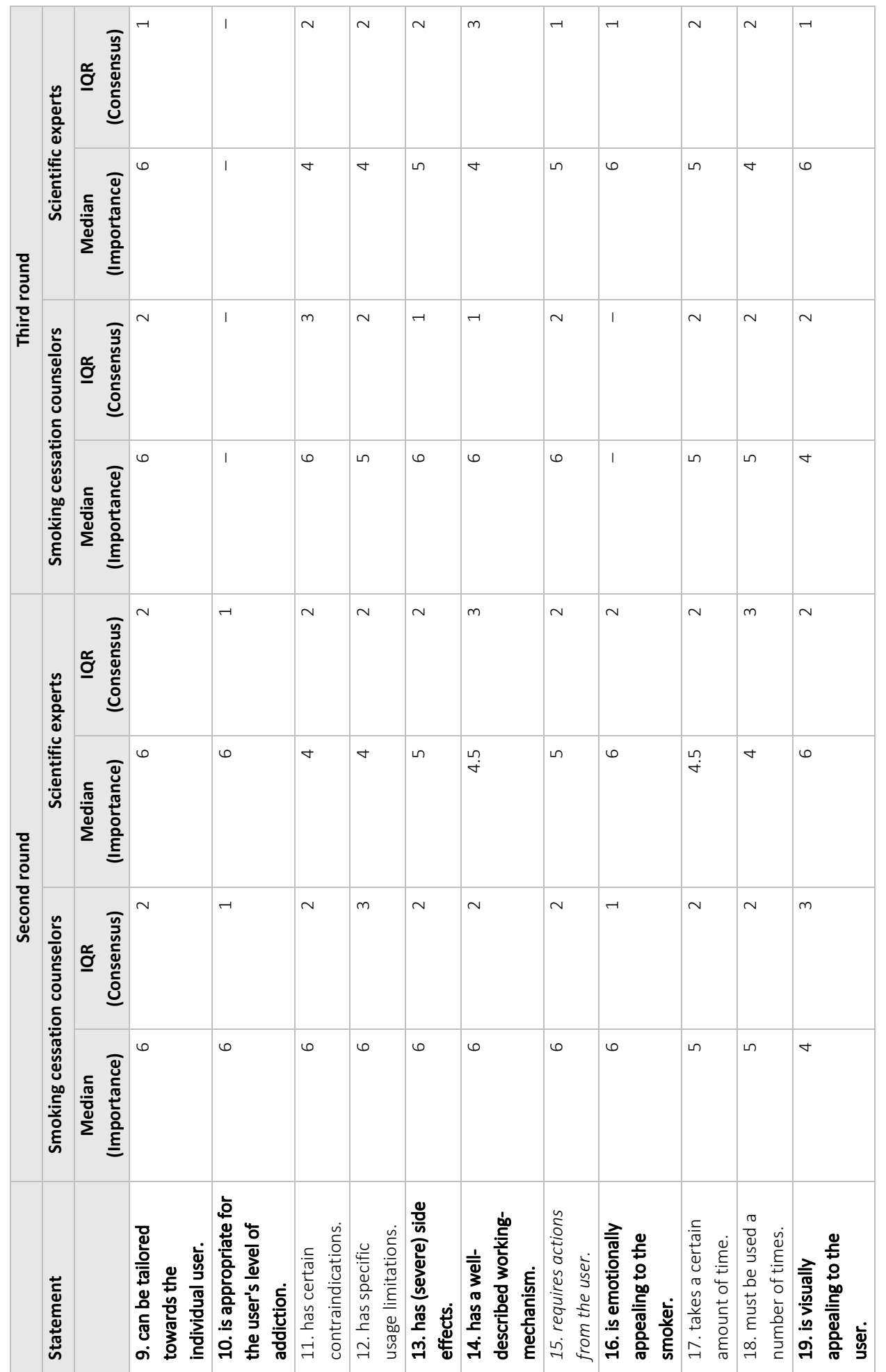




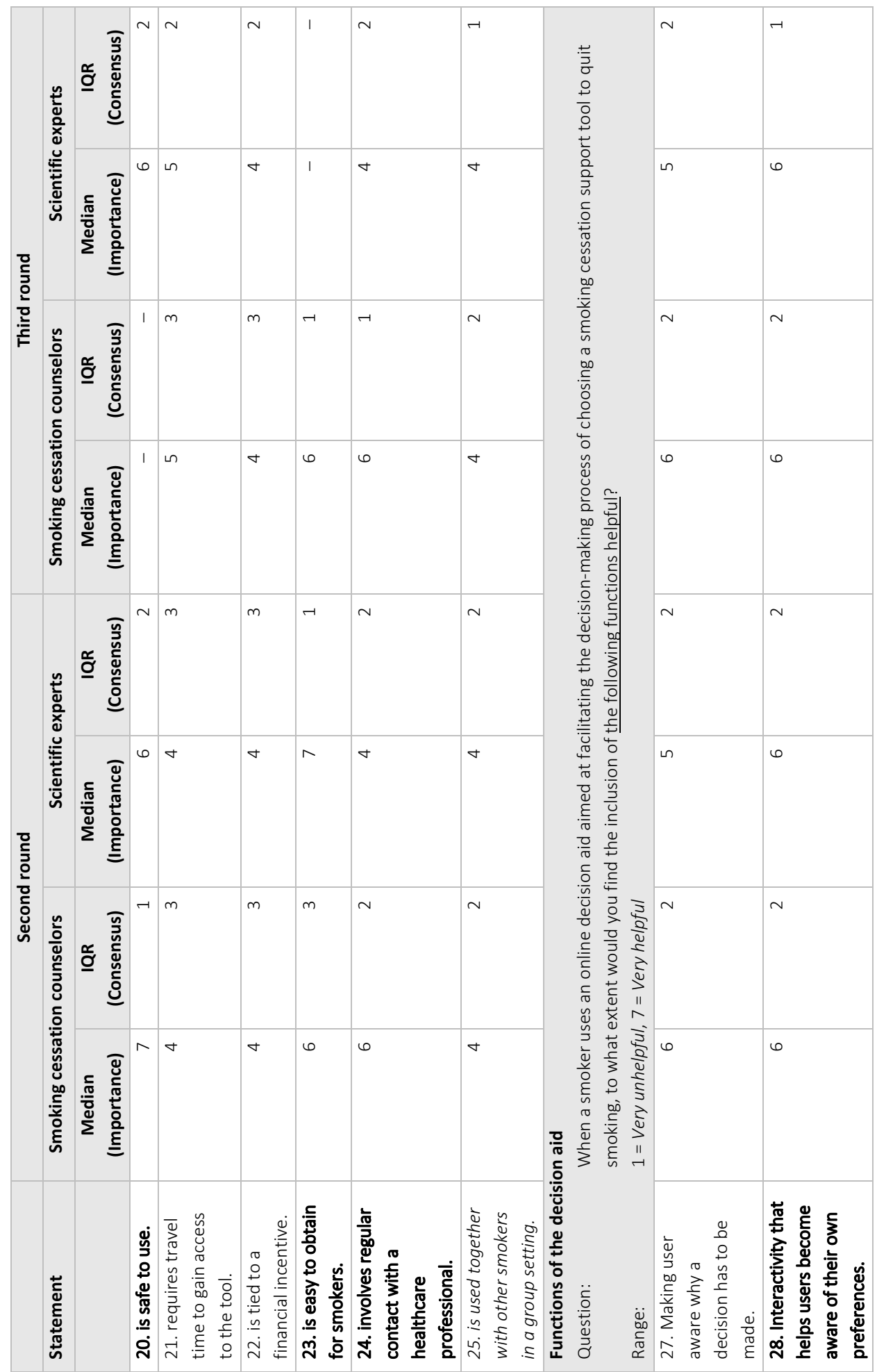




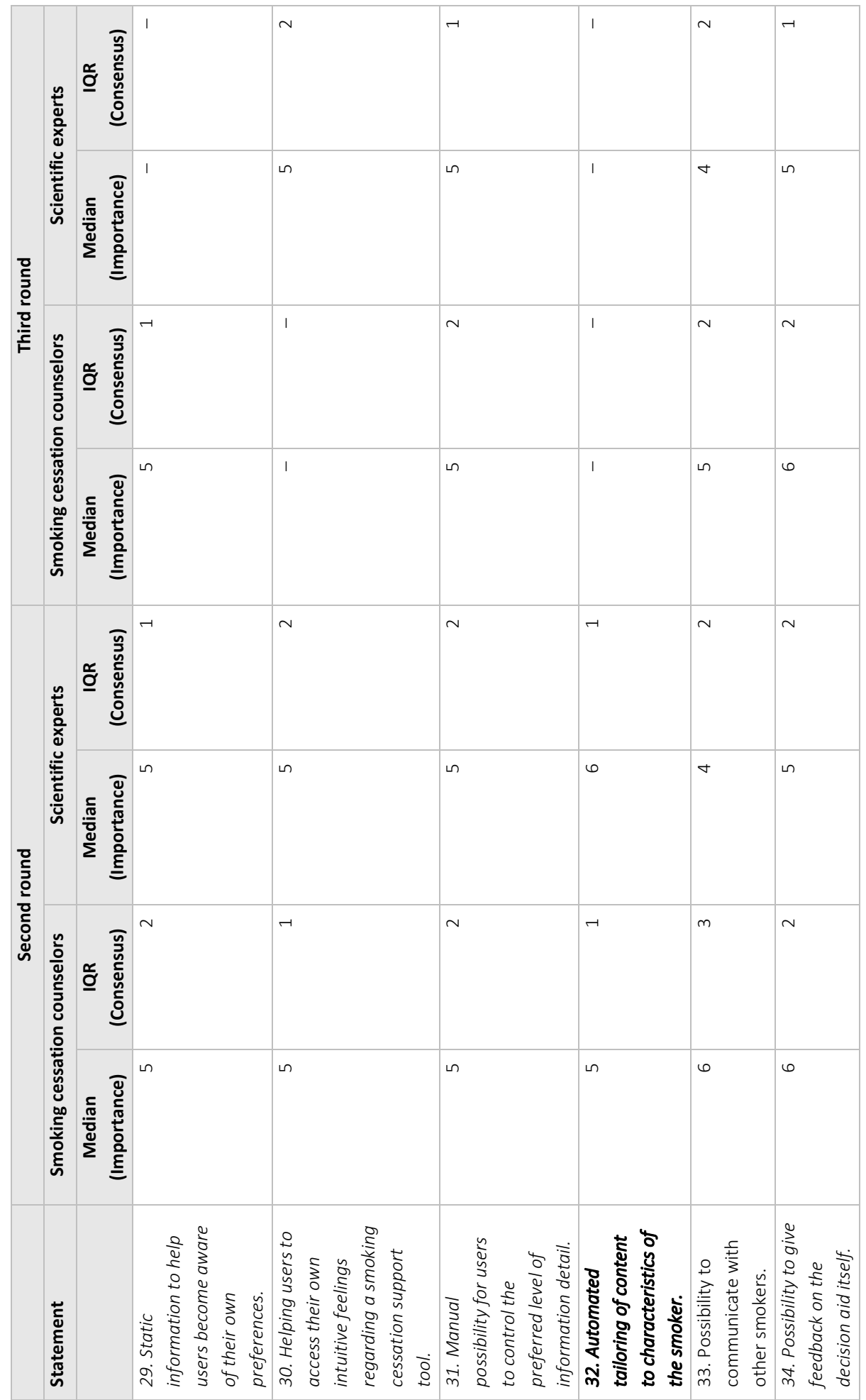




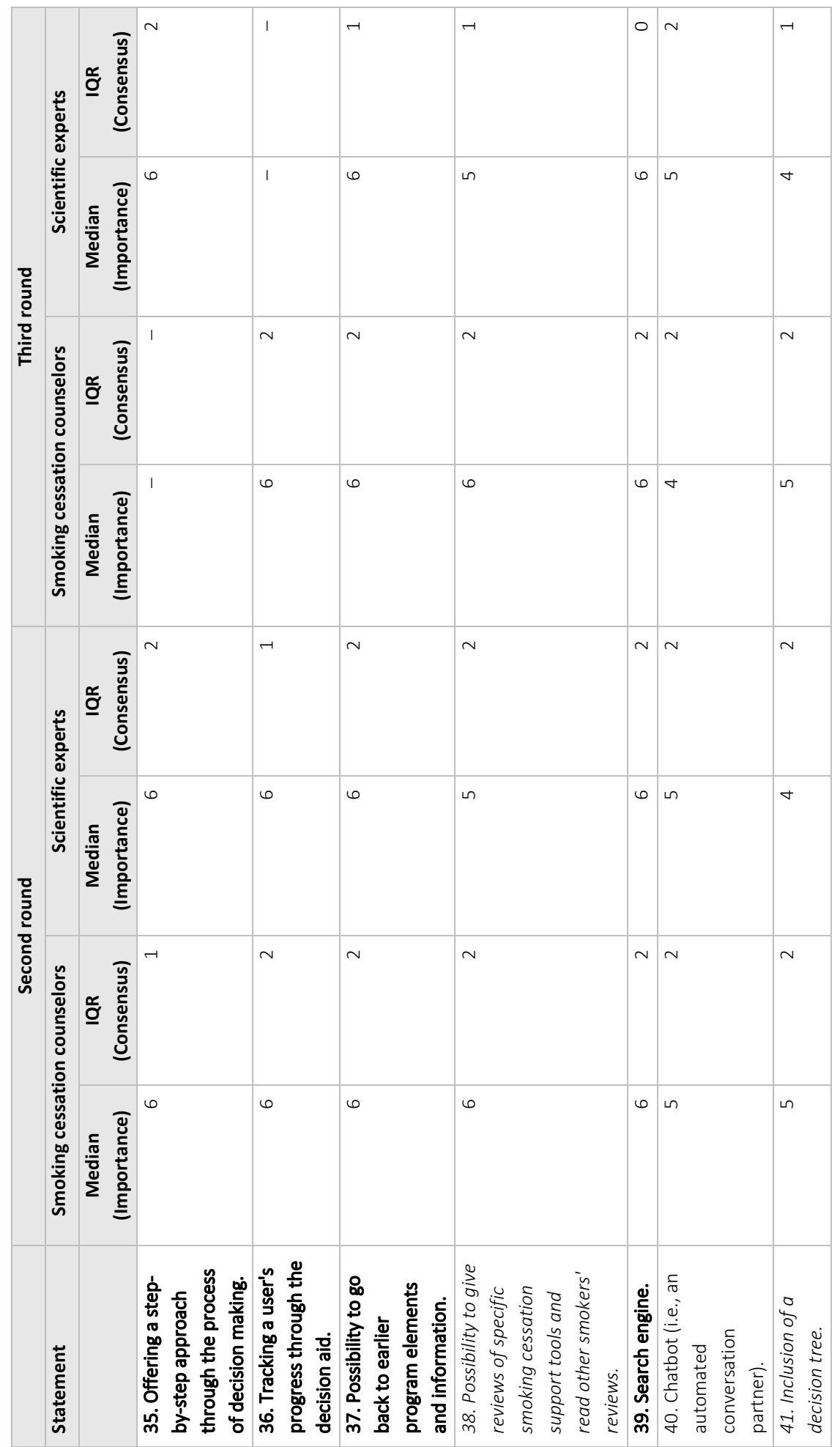




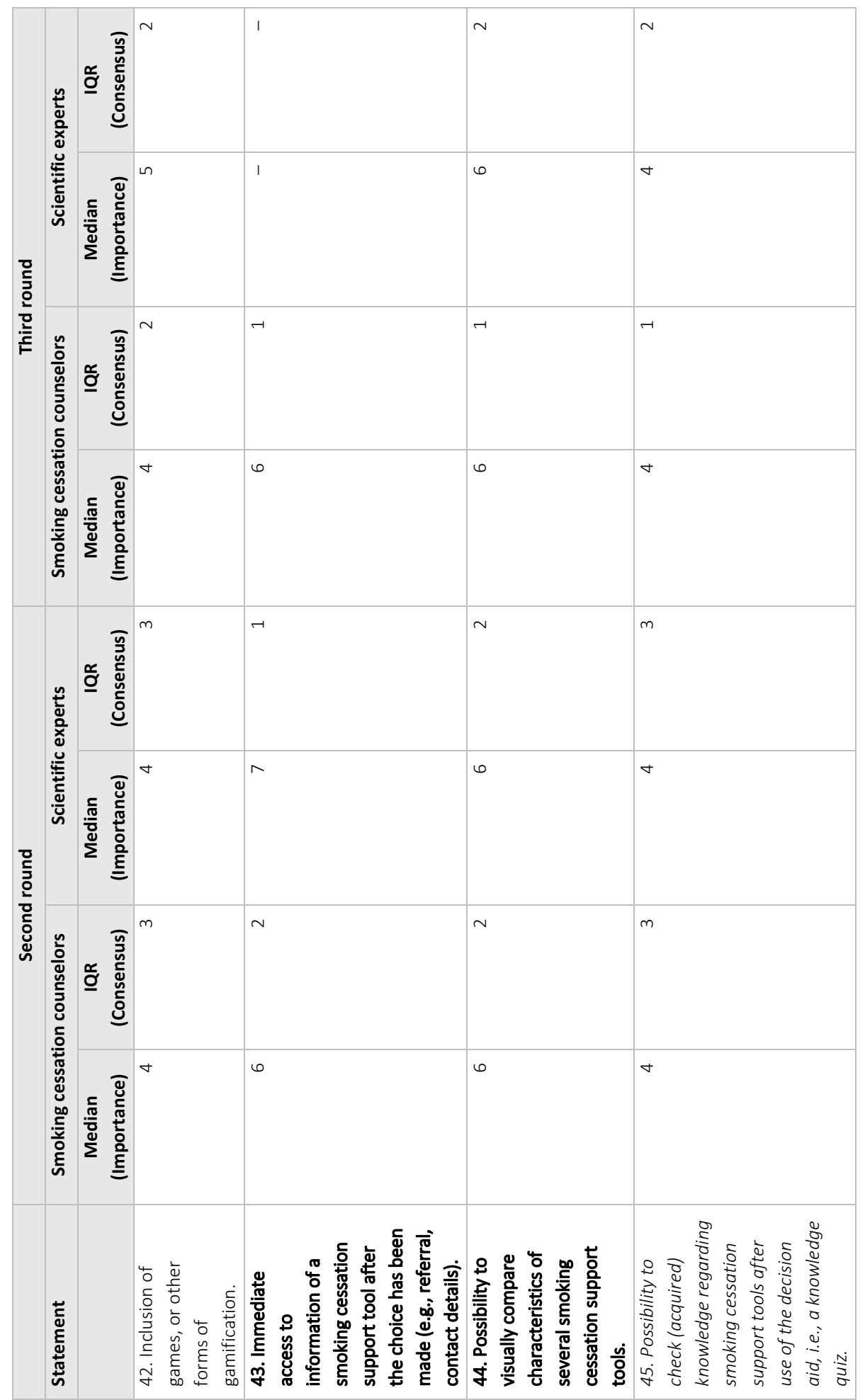




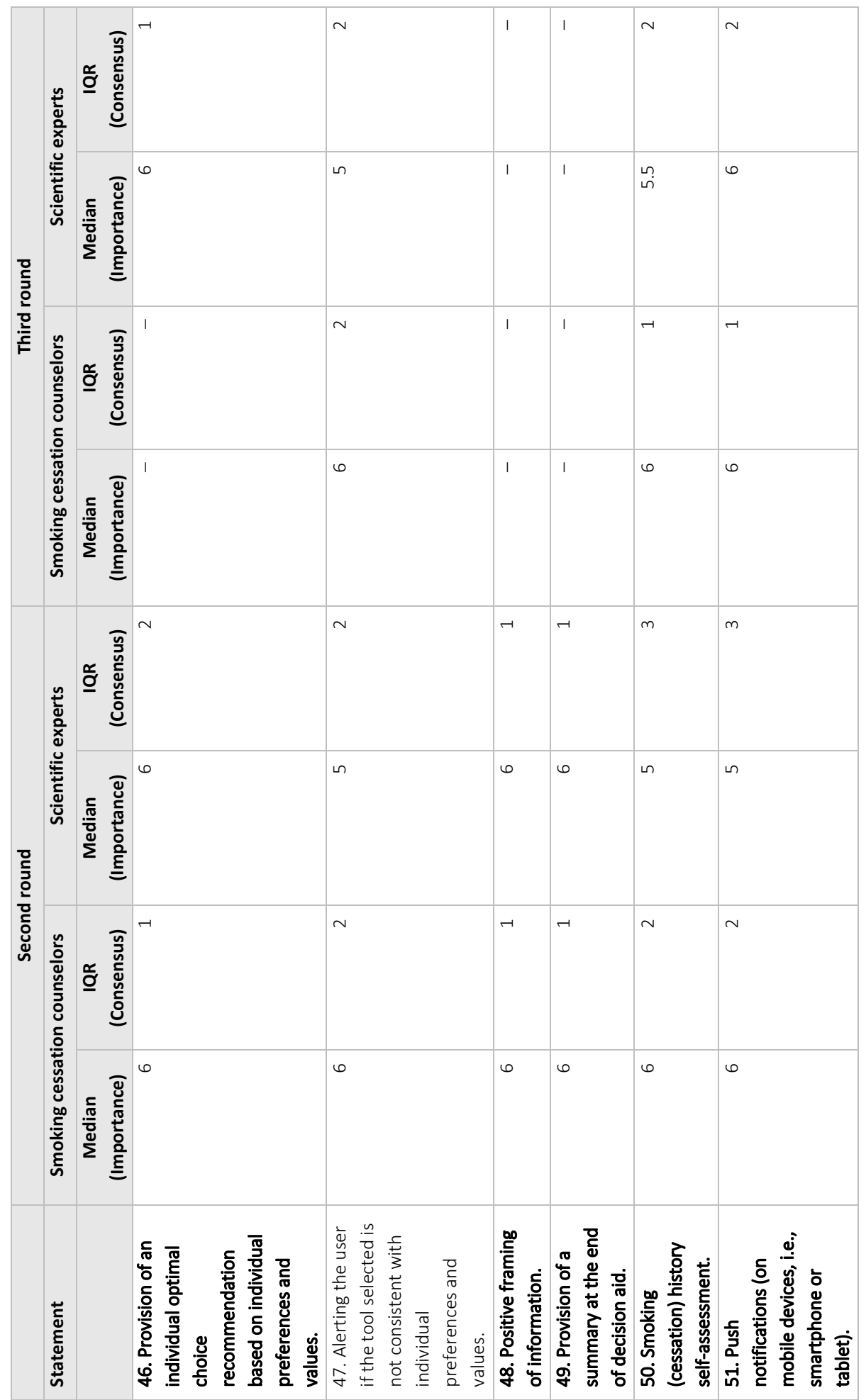




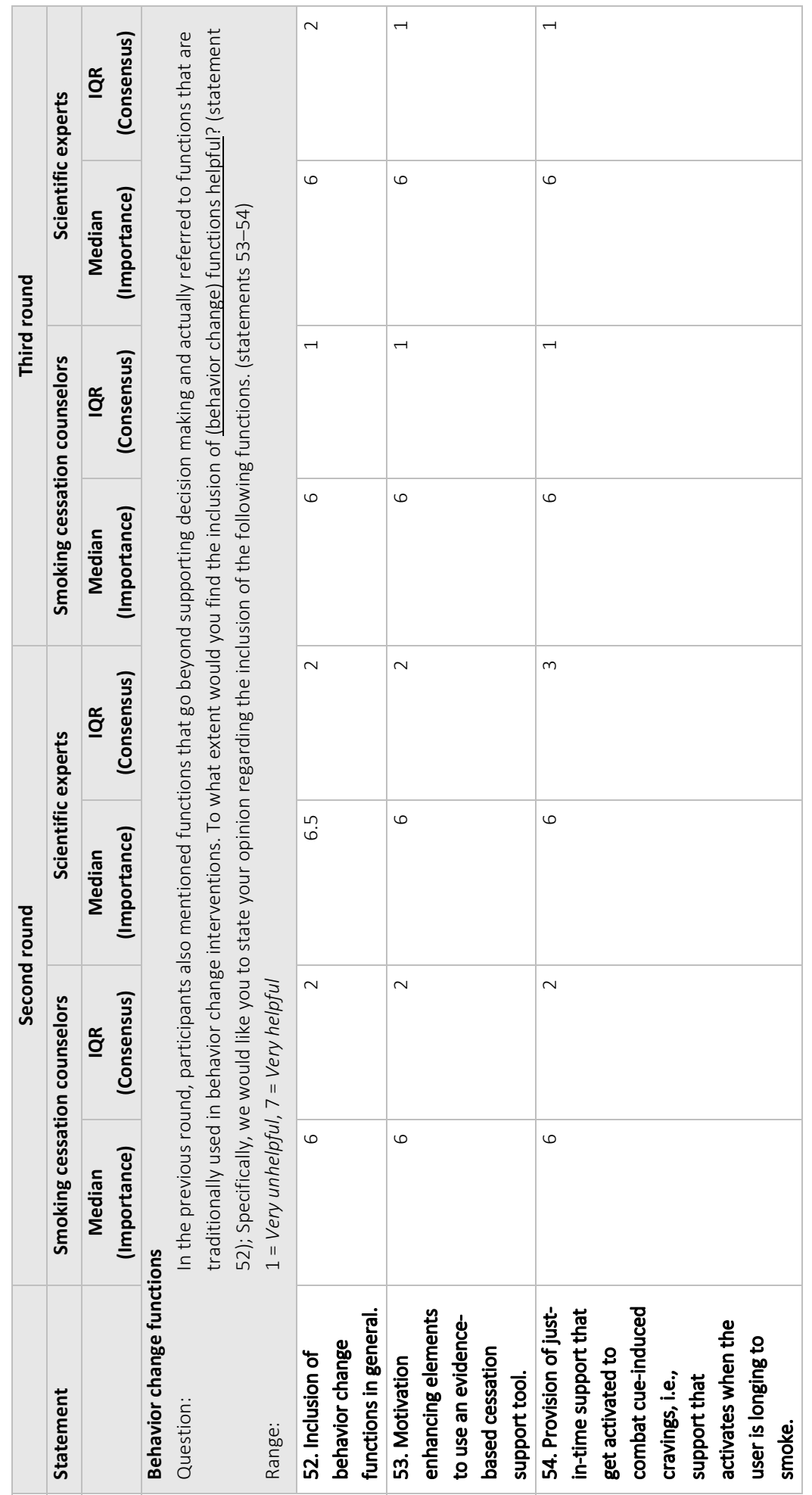




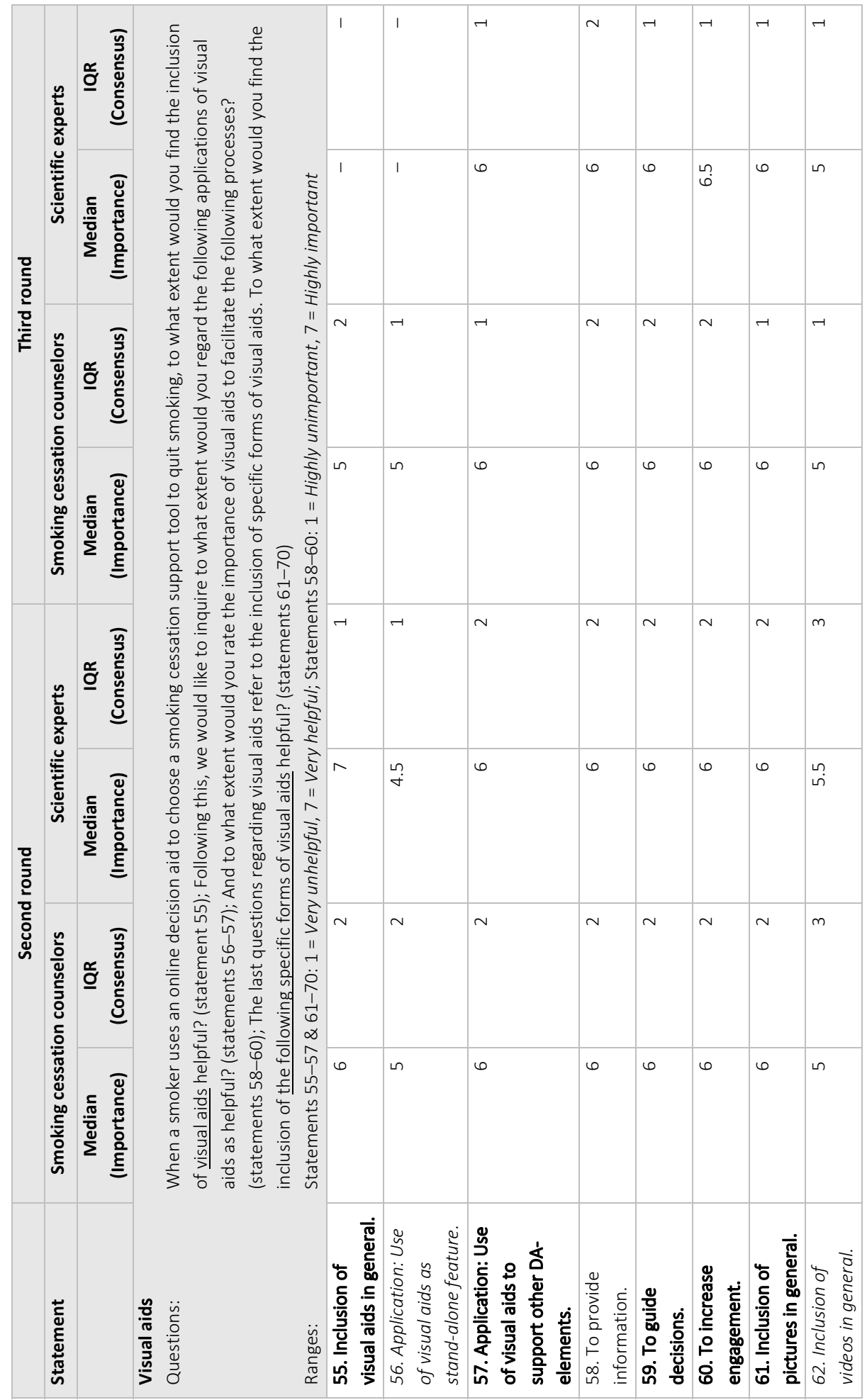




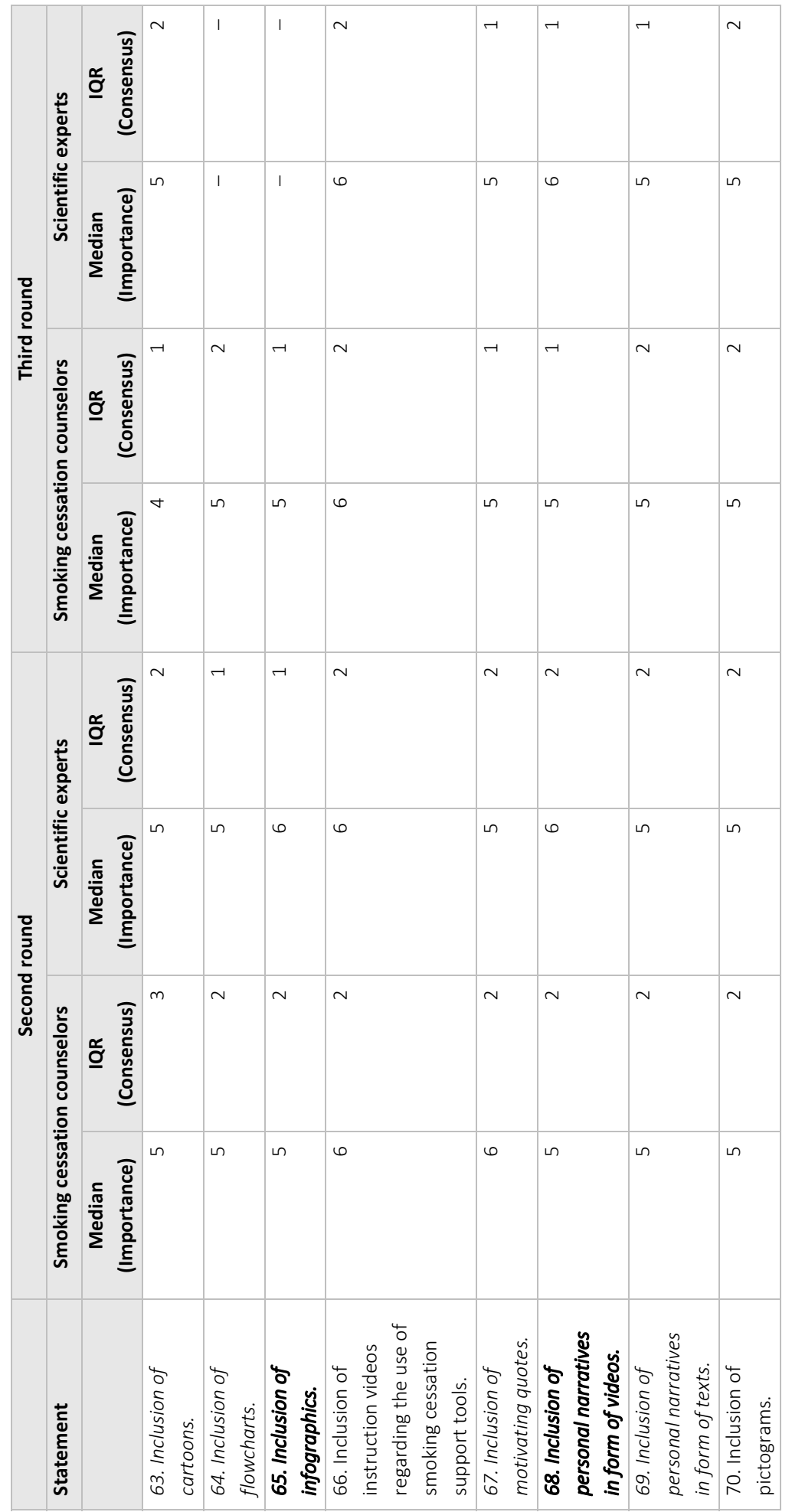




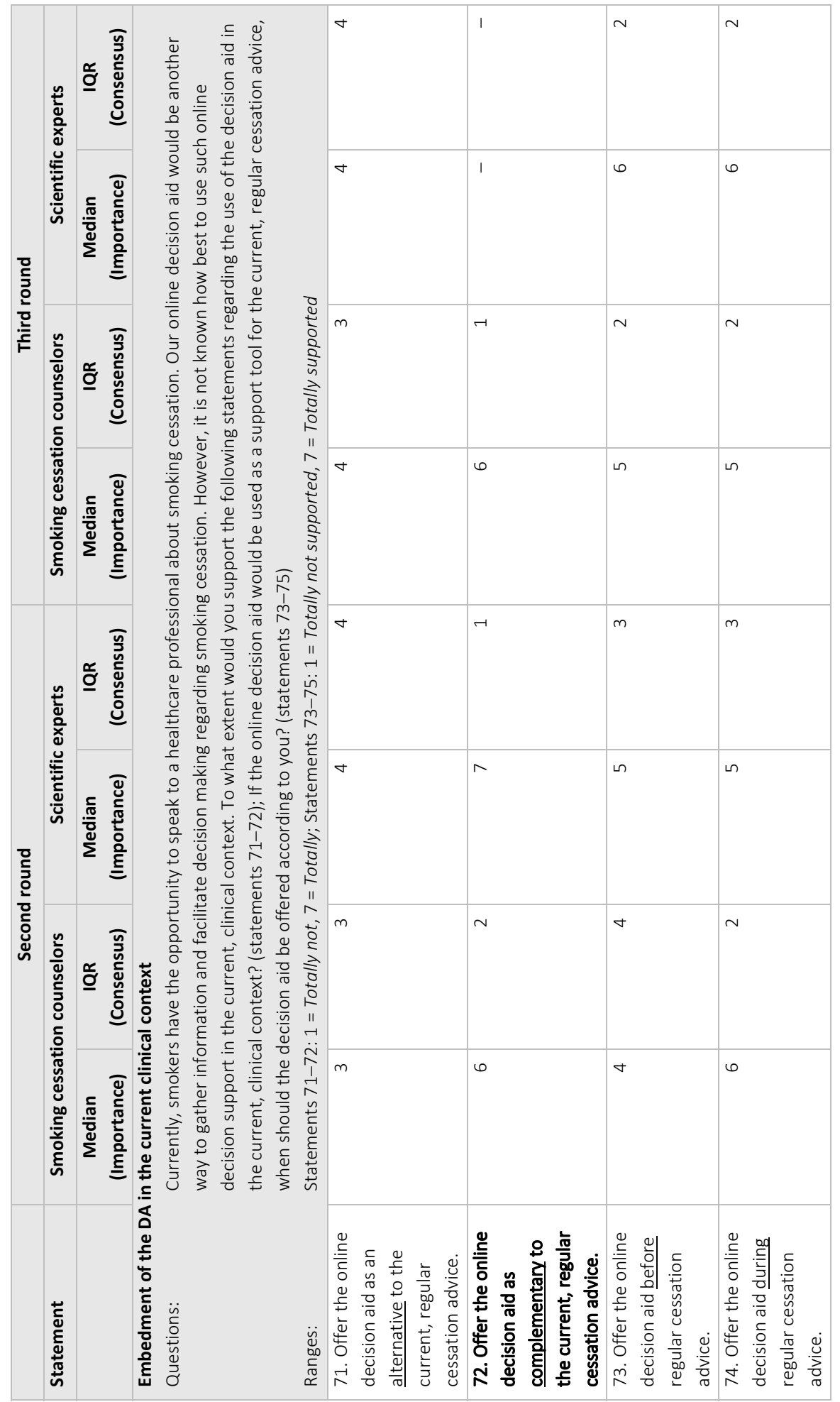




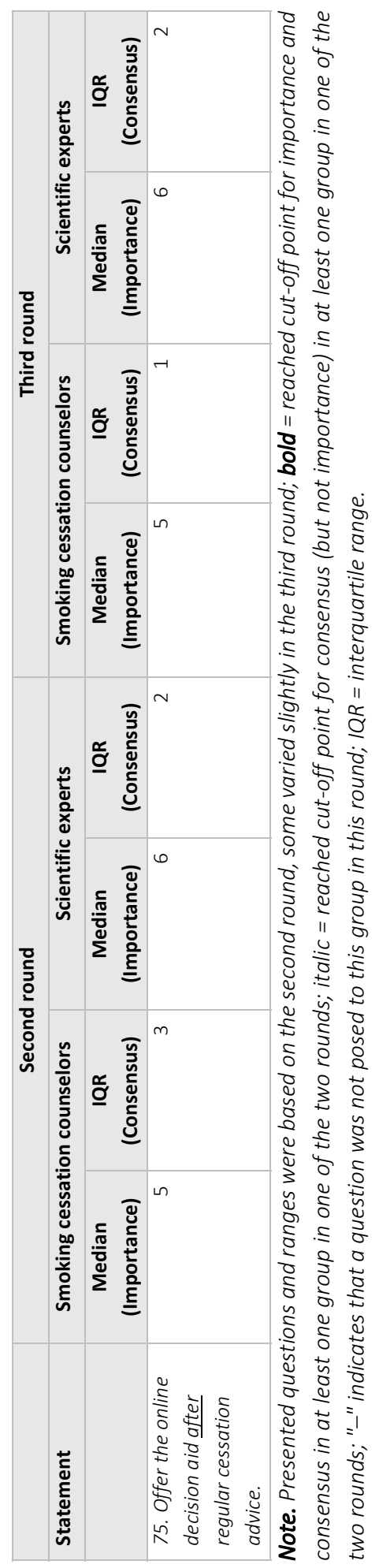




\section{Discussion}

The aim of this needs assessment was to explore the needs and viewpoints regarding supporting informed decision making within the context of smoking cessation from the perspective of three stakeholders: (1) potential end users (i.e., individuals motivated to quit smoking), (2) smoking cessation counselors, and (3) scientific experts.

\section{Potential End Users (i.e., Individuals Motivated to Quit Smoking)}

Most importantly the interview study showed that potential end users are not a completely homogenous group-especially not in terms of knowledge, but also to a certain degree in terms of values or when queried about the desired characteristics of a potential DA. We will reflect on all three aspects in the following.

Especially the first two aspects (i.e., heterogeneity in knowledge and values) highlight that DAs might be valuable health communication interventions to support individuals during smoking cessation attempts as their two core elements-information provision [33] and socalled value clarification methods [34]-are designed to support users in acquiring more knowledge and using this knowledge to identify what is important to them personally. Also, the literature shows that they are effective in doing just that [13]. Our results indicate that individuals motivated to quit smoking especially regard the effectiveness of smoking cessation assistance as important and, therefore, should be provided with information about this aspect of said assistance. Interestingly, however, these end users seemingly understand the concept of effectiveness differently from experts. This becomes clear when one looks at the way end users acquire and interpret information around effectiveness. For example, end users consider smoking cessation assistance to be effective if someone in their environment has stopped while using assistance and consider a tool to be ineffective if they themselves have used it once and ultimately have not quit smoking. This indicates that laypersons understand information about effectiveness on a personal or individual level, while scientists interpret the effectiveness of a tool by comparing different groups (e.g., one group receives an evidence-based smoking cessation assistance tool, while the other group receives 'careas-usual') and examining whether additional effects arise on top of what you would expect anyway. Therefore, evidence-based tools are not 100 percent effective, but simply more effective than the comparator (e.g., 'care-as-usual'). Lay people motivated to quit smoking do not seem to be aware of this crucial information possibly leading them to rather not use an evidence-based tool in the future-whereby, they unknowingly decrease their own cessation chances. It therefore seems important to provide end users with accurate information regarding effectiveness in an accessible manner to prevent them from not using a specific tool or evidence-based tools in general after a failed quit attempt with smoking cessation assistance. One way to achieve this could be to use icon arrays. These are graphical 
representations consisting of icons that symbolize individuals within a group who are affected by a certain event [206]. Such icon arrays are mostly used to convey risk information and have been shown to increase accuracy of understanding [206]. Based on the results from the needs assessment described in this paper and on previous findings from risk communication studies, we therefore decided to make icon arrays for our DA (using the website Iconarray.com [207]) to convey information regarding the effectiveness of different smoking cessation assistance tools. More information on the application of this method can be found in our paper describing the DA [96].

The third aspect that made the end user group rather heterogenous (i.e., varying needs regarding DA-characteristics) shows that smoking cessation DAs should be more flexible in terms of functionalities to appeal to a wide audience and should be adaptable to-and potentially by-the end users themselves. This most likely means that digital DAs should be offered to individuals wishing to quit smoking as digital tools allow for more adaptability [155]. Health psychologist and experts in health communication often acknowledge two different forms of adaption to users' characteristics: (1) system-driven tailoring, or the automatized adaption of intervention materials to individual users' characteristics [45] and (2) customization, or enabling users to adapt the intervention materials themselves (i.e., via a non-automated process) [208]. While system-driven tailoring has shown promising results in smoking cessation interventions (e.g., [209]), theoretical [177], and empirical insights [56] suggest that customization is better suited for DAs compared to system-driven tailoring. In fact, allowing users the possibility to customize DAs to a certain degree may lead to greater chances for autonomous behavior change [208]. However, developers have to ensure that 'core functions' and the most important information that the DA aims to convey should be retained. We ourselves have used various options to deal with this in the self-administered smoking cessation DA developed as part of the associated broader project [96], e.g., we have chosen to enable users (1) to receive in-depth information regarding options that they are interested in while ensuring that all users receive the necessary information to make a decision and (2) to skip certain functionalities that are not regarded as necessary but as attractive by some users (e.g., a knowledge quiz). Having said all that, our explorative subgroup analyses also show that tailoring to end users' characteristics might be a worthwhile avenue to explore as we found some differences between different demographic groups. Interestingly, the differences we found revolved around modalities (e.g., that younger interviewees seemed to prefer shorter DAs). However, due to our article's scope we also only examined a few subgroups. Future research should also invest in examining other subgroup differences (e.g., based on nicotine dependence) and should test whether tailoring to these characteristics could be useful when applied to smoking cessation DAs. Of course, again, another possible approach, could be to simply offer multiple modalities (e.g., longer and abbreviated versions of the same DAs) and have end users choose-akin to customization. 
The two Delphi studies resulted in a breadth of information that can be used to support DA development for smoking cessation or other preventive health-related behaviors. Interestingly, the two groups only disagreed on a few statements during the last two rounds, i.e., the adoption of (system-driven) tailoring strategies and the inclusion of infographics and personal narratives in the form of videos were deemed important by the scientific experts, but not by the smoking cessation counselors. This could reflect the composition of the group of scientists whose expertise has been mainly behavioral intervention (development), i.e., experts who often apply behavior change techniques, such as system-driven tailoring. Here it should be acknowledged that the statements that the experts rated were mostly global in nature, i.e., they mostly referred to smoking cessation DAs overall and not to specific section of DAs. It is also possible to integrate experts' viewpoints and use tailoring strategies for different purposes, while not using them throughout the entire DA. To illustrate, tailoring in general has been shown to negatively impact on quality of decision making when used in digital DAs [56]. On the other hand, very specific tailoring in the form of tailored advice based on identified values might help DA-users to make value-consistent choices [40]. In our own DA, we therefore aimed for a synergistic approach. Throughout the majority of the DA, users were able to customize the information and functions they received. For example, users could choose to receive in-depth information regarding a cessation assistance tool they were interested in or could choose to do a knowledge quiz (or not). However, we also provided them with system-tailored advice at the end of the DA [96]. This shows that it is not only possible to integrate dissenting expert opinions in one DA, but also to integrate two seemingly 'opposing' functions, such as customization and system-driven tailoring in one and the same DA.

It should also be noted that both expert groups agreed on several statements, e.g., that cessation assistance should be appropriate for users' level of addiction. This specific statement can also help to illustrate how the information from these two Delphi studies can be used to inform DA-development: Developers, often scientific experts, of a smoking cessation DA could include a smoking assessment to provide end users with information about which tools are appropriate for their level of addiction-a function which has also been deemed helpful by the smoking cessation counselors. Again, this is something we have implemented in our own DA [96]: End users were asked to indicate if they smoke more than 10 cigarettes a day or if they had multiple unsuccessful smoking cessation attempts in the past. Subsequently, end users that showed these characteristics were advised to use a combination of both behavioral as well as pharmacological cessation assistance-as indicated by Dutch smoking cessation guidelines [210]. Another example would be that both expert group groups agreed that smoking cessation assistance should meet individuals' preferences and values for cessation support. Which is why we are currently testing if a 
smoking cessation DA is more effective if users are supported in identifying smoking cessation assistance tools that reflect their values and preferences [96].

\section{Integration of the Results in Different Stakeholders}

Our results show that it is of great importance to include multiple stakeholders during the needs assessment, as different stakeholders' opinions do not always align; e.g., important values that were identified among potential end users differed from the one's identified among (professional) experts. That being said, one should always consider which stakeholders should be included. The original IPDAS' development process advocates to include both potential end users as well as clinicians (during the design phase) as the main focus of the IPDAS collaboration is on DAs that are used within (or around) clinical encounters [59]. The studies presented in this article show that the inclusion of other stakeholder groups is potentially beneficial for other types of DAs, such as DAs with a health promotion focus and/or self-administered DAs. Based on our insights, future DA developers should therefore consider which stakeholders are of importance for their specific DA and not 'simply' follow the IPDAS' advice. In some cases, one might even consider not to include clinicians, e.g., regarding the decision whether to eat more fruit. Including too many stakeholder groups might overcomplicate things and, for instance, create too many issues about which no consensus can be reached, as illustrated by our finding that different stakeholders' opinions do not always align.

This is illustrated most clearly when one looks at the aspect of cessation assistance's effectiveness: Potential end users clearly valued information on effectiveness and largely agreed with each other, while experts failed to establish consensus regarding the value of this type of information. This discrepancy may be due to experts' and laypersons' different conceptualizations of the 'effectiveness' concept as explained earlier-see Potential End Users (i.e., Individuals Motivated to Quit Smoking). Other researchers have shown that individuals motivated to quit smoking often do not choose to use smoking cessation assistance tools that have shown to be effective [191]. Our interviews may give proof to the claim that this might not be due to a disinterest in effectiveness, but that they simply lack the knowledge on how experts conceptualize the term effectiveness. Providing them with this information in an accessible manner (as explained earlier) might thus enable them to choose tools with a greater effectiveness. This specific example shows that integrating the views of different stakeholders can be difficult at first but can also generate valuable insights and that there is no single approach to integrate all discrepancies. In this specific case, it could be sensible to meet the clear informational needs of the end users, who clearly indicated that effectiveness is very important to them. In other cases (e.g., which other characteristics to include in a DA), however, it may be better to take a more balanced approach and combine the insights of the different stakeholder groups. For example, aside 
from the 'effectiveness-factor', we have mostly chosen to only include information when more than one stakeholder group indicated the importance of a factor or used the information provided by the end users to deepen the information presented in the selfadministered smoking cessation DA that was developed by our team [96].

\section{Strengths and Limitations}

Including three different groups of participants in this needs assessment provided us with a breath of information that can be used to inform the development of (self-administered) smoking cessation DAs. Also, were we generally able to include a diverse group of participants. However, we were unable to include end users identifying as non-binary. Given that gender specifically was not the main focus of this study we do not regard this as a major issue, yet future studies could make extra efforts to include non-binary individuals, especially because gender and sexuality minority groups are assumed to be provided with less possibilities to engage in high-quality decision making [211]. Likewise, respondents in the group of the smoking cessation counselors mainly were practice nurses. Yet, as practice nurses increasingly provide smoking cessation counselling in the Netherlands [212,213], we believe our sample still accurately reflects the viewpoints of counselors in our Dutch context. We also acknowledge that it could be viewed as a limitation that only Dutch smoking cessation counselors were involved. However, we assume that our results could be broadly generalizable to other Western countries as Western countries often share similarities in healthcare systems (e.g., the Dutch healthcare systems shows similarities to other Central and Northern European countries, such as the United Kingdom [214]). Finally, due to the two different study designs and because we treated the two Delphi studies as separate studies (especially after the first round), results are not directly comparable. However, the different study designs and not being able to directly compare the Delphi studies also reflect the choices that we made during the process of the studies to choose a study design that was most fitting for the relevant stakeholder groups and because we preferred to provide the experts only with feedback from their specific peers. That being said, it is technically possible to include end users in Delphi studies as well (e.g., [205]) and future studies could attempt to do this in order to directly compare needs and viewpoints of the different groups which could facilitate comparative studies. At this point, however, it is unclear if this would be of any added value.

\section{Conclusion}

The results from the three studies reported in this article complement the general DAliterature and can be used as input for smoking cessation DA-development, especially selfadministered DAs. In fact, we have used the results to develop a digital self-administered smoking cessation DA [96]. Yet, the results of the three studies described in this article can 
also be used to inform the development of other DAs, related to smoking cessation or other preventive health-related behaviors. For example, other researchers and/or DA-developers could use the findings presented in this article to develop an alpha version of an DA that is then tested among their respective target group. Several ideas on how to achieve this are outlined in this article; for example, that icon arrays can be used to make information about effectiveness more accessible to people who want to quit smoking as it was found that this groups understands this information differently than (professional) experts. Overall, given the variation in the needs and wishes revealed among different stakeholders, the combination of these studies highlights that a 'one size fits all'-approach is not desirable for (smoking cessation) DAs. In the development of future DAs, heterogeneity should certainly be taken into account, for example by enabling users to customize a DA based on their personal preferences while safeguarding essential elements.

Abbreviations

DA - decision aid

GP - general practitioner

IPDAS - International Patient Decision Aid Standards

IQR - interquartile range

OSF - Open Science Framework 


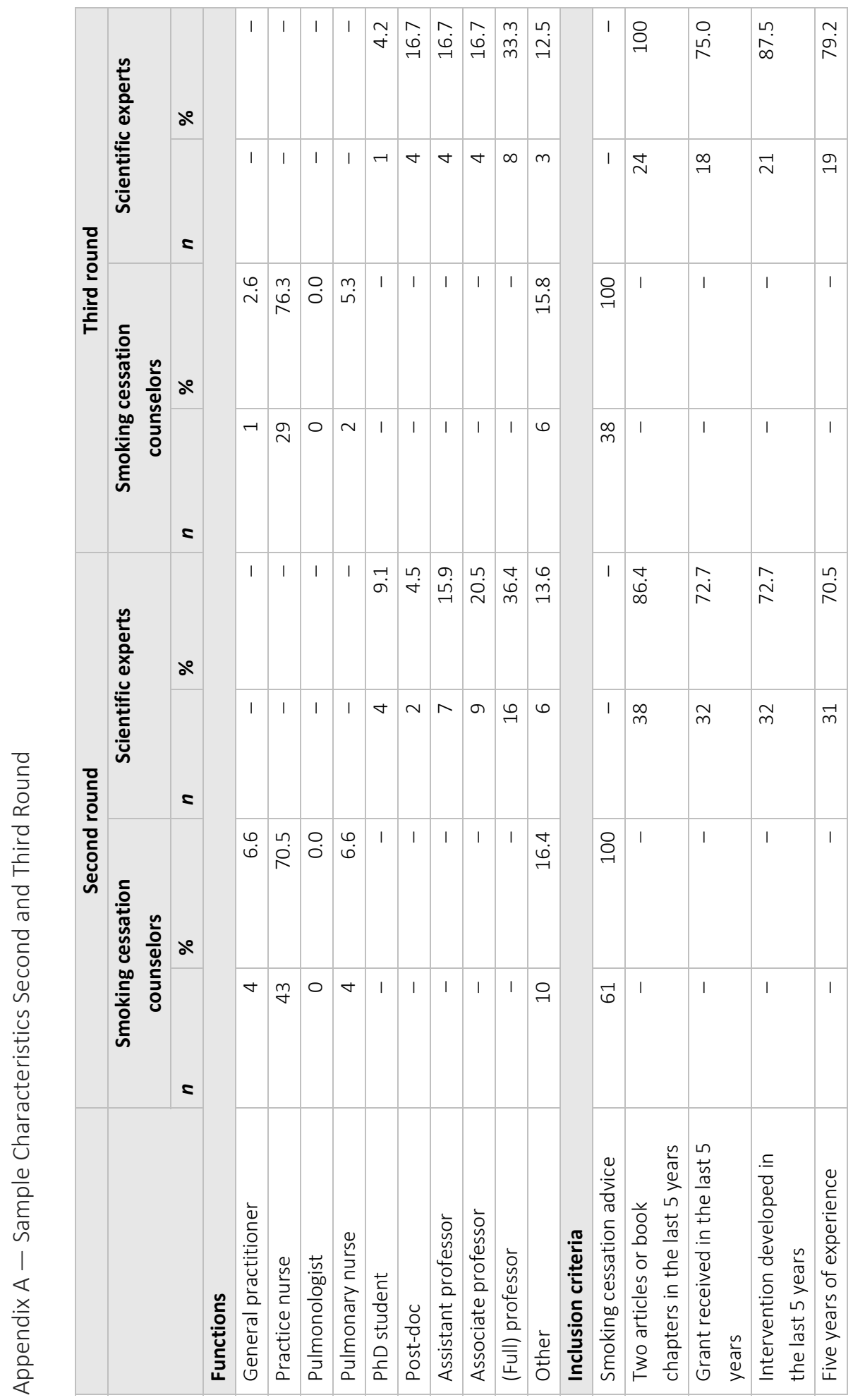




$$
D
$$




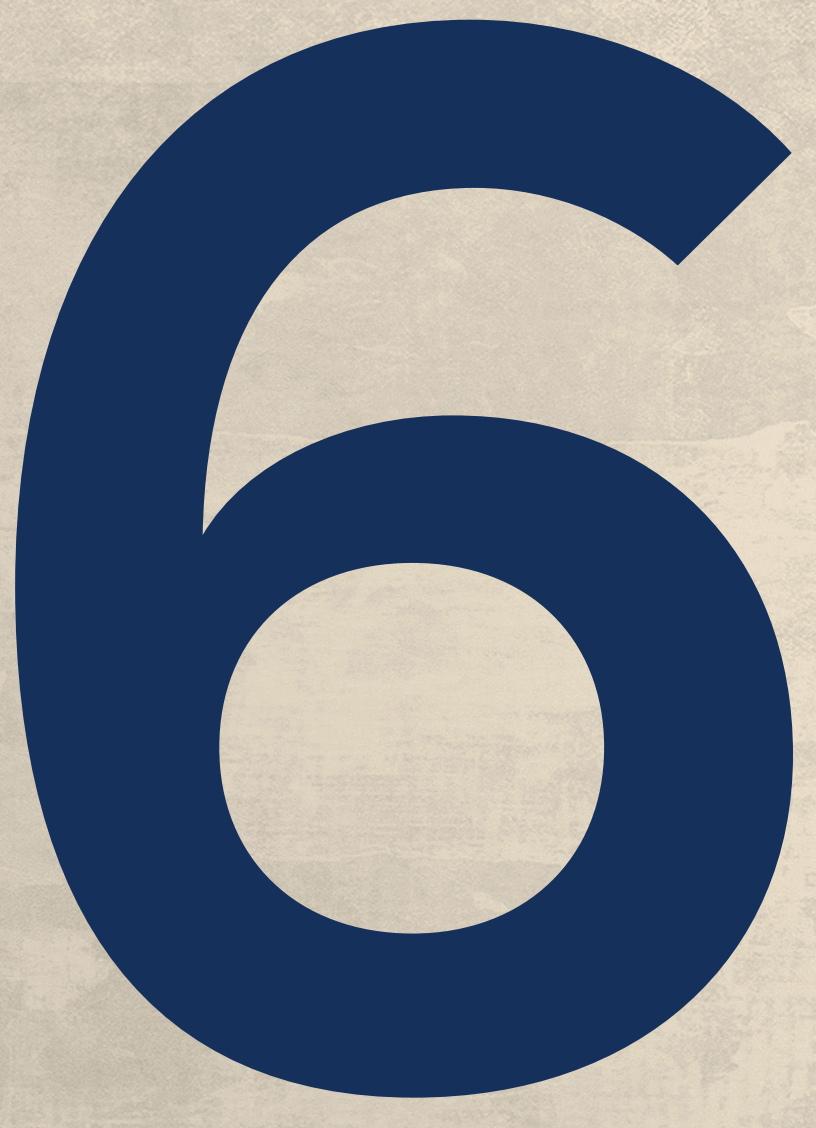




\section{chapter 6}

an autonomy-supportive online decision aid to assist smokers in choosing evidence-based cessation assistance: development process and protocol of a randomized controlled trial

This chapter has been published as:

Gültzow, T., Smit, E. S., Hudales, R., Knapen, V., Rademakers, J., Dirksen, C. D., \& Hoving, C. (2020). An Autonomy-Supportive Online Decision Aid to Assist Smokers in Choosing Evidence-Based Cessation Assistance: Development Process and Protocol of a Randomized Controlled Trial. JMIR Research Protocols, 9(12), e21772. https://doi.org/10.2196/21772 
Abstract

Background: Decision aids (DAs) may be used to facilitate an autonomous, informed decision to cease smoking and promote the uptake of evidence-based cessation assistance (i.e., behavioral support, nicotine replacement therapy, or prescription medication). However, knowledge is lacking regarding their effective elements and (cost-)effectiveness.

Objective: We describe the development process of an online DA (called VISOR) that helps smokers to choose evidence-based cessation assistance. Additionally, we provide a description of the protocol of an ongoing randomized controlled trial in which the DA containing an explicit value clarification method (VCM) and tailored advice is compared with a DA without an explicit VCM and tailored advice.

Methods: The development of VISOR was based on the International Patient Decision Aid Standards guidelines. Viewpoints of end users (collected through 20 interviews with smokers) and clinical and scientific experts (assessed using two Delphi studies with 24 scientists and 38 clinicians) were assessed regarding cessation tool decision making and preferred DA-content. These findings, together with principles from the Self-Determination Theory, served as input for the development of the online DA. A first DA-prototype was alpha-tested in September 2019 and beta-tested for usability in December 2019; feedback was incorporated and resulted in a final version. The final DA contains (1) an information section, (2) an optional knowledge quiz, (3) a brief smoking assessment, (4) intuitive decision, (5) intermediate advice, (6) an explicit VCM, (7) tailored advice, and (8) access information. A randomized controlled trial is currently being conducted to assess the DA's (cost)effectiveness compared to a DA that does not include the explicit VCM and the tailored advice; specifically, the DA's effect on smoking abstinence, uptake of evidence-based cessation assistance, smoking abstinence mediated through uptake of evidence-based cessation assistance, and decisional conflict are investigated. Participants are randomly allocated to receive access to one of the two DAs and are asked to complete five questionnaires (including the baseline questionnaire) over a period of 12 months. To evaluate the effects of the DA on the outcome measures, logistic and linear regression analyses as well as mediation analyses will be carried out. An economic evaluation will be performed to assess the cost-effectiveness.

Results: Data regarding the effect of the VISOR DA are currently being collected, and data collection is expected to be concluded in 2021.

Conclusions: By making use of an iterative process that integrated different stakeholders' perspectives (including end users), we were able to systematically design an evidence-based DA. The study will contribute to the current knowledge regarding smoking cessation DA 
application, the added value of explicit VCMs, and the effect of behavioral and informed decision-making outcomes. 


\section{Introduction}

Smoking continues to kill, both on a global level [20] and in the Netherlands [215]. According to predictions, more than eight million deaths per year will be caused worldwide by tobacco use by 2030 if evidence-based smoking cessation interventions are not put into use [216]. One way to reduce tobacco-related deaths is to promote the uptake of evidence-based cessation assistance, such as pharmacological support (e.g., nicotine replacement therapy [NRT]) [26], and behavioral support (e.g., counselling [24]). Unlike non-evidence-based cessation assistance, such as acupuncture [27], evidence-based cessation assistance can greatly increase successful smoking cessation [23]. However, uptake of evidence-based cessation assistance is low in the Netherlands [60]. And, even if people are interested in using evidence-based cessation assistance, they still have to choose between the multitude of options that exist in order to make an informed decision. Making an informed decision requires people to gather and review information regarding cessation assistance options and weigh up all the advantages and disadvantages of those options [13] -tasks that can be difficult for unsupported lay people [106].

Decision aids (DAs) are interventions that are specifically designed to help users with those difficult decisional processes. DAs aim to facilitate informed decision making between different health or healthcare options by providing information and helping users to become aware of their own values in relation to those options [13]. Often, DAs are used when people have to choose between different medical treatment options or if they are considering whether to participate in a screening program [13]. However, a systematic review regarding DAs for smoking cessation has also shown positive results in terms of increased quit attempts [52]. Insights from the Self-Determination Theory (SDT) also suggest that the offering of choice (as DAs explicitly do) enables individuals to engage in long-term behavior change, such as smoking cessation, by supporting their need for autonomy [91,92]. Nonetheless, only one DA for smoking cessation has previously been developed and tested in the Dutch setting [42]. And, while this DA was effective in promoting quit attempts and smoking abstinence, no effects were found regarding the use of cessation assistance [42]. As increased cessation assistance uptake could further improve cessation outcomes [23], we propose several limitations of the earlier DA that might need to be overcome to increase cessation assistance uptake and subsequent smoking cessation behavior. First, the earlier DA was paper-based and sent to people by mail, thereby limiting widespread dissemination. Offering a smoking cessation DA online could potentially reach many more people [217], especially given that the Dutch are likely to search for health-related information online nowadays [218]. Second, offering the DA online allows for a more flexible and interactive design that can be particularly interesting for people with a high need for autonomy [208]. And third, the aforementioned DA did not explicitly include methods that help users to become aware of 
their own values, even though explicitly including smokers' values in their decisional process could potentially improve cessation assistance uptake rates [61,219].

So-called value clarification methods (VCMs, also referred to as value clarification exercises) can support users to "evaluate the desirability of options or attributes of options within a specific decision context, in order to identify which option he/she prefers" as defined by the International Patient Decision Aid Standards (IPDAS) [34]. The underlying belief is that users with clarified values (i.e., users who know what is important to them) will be more likely to choose an option that reflects their own preferences, which is regarded as a prerequisite of high-quality decision making [13,34]. VCMs can either be explicit or implicit; the former refers to methods that involve the user actively engaging in an activity (e.g., scoring certain statements), while the latter refers to the provision of information that is specifically linked to the decision at hand (similar to the aforementioned paper-based DA [42]), with the underlying belief that users will engage in cognitive processes themselves to reach a decision [37]. There is some evidence that shows that explicit VCMs are more effective than implicit VCMs (in terms of decisional processes) [32], especially in the long run [37], and when people are supported in understanding the implications of their clarified values for the decision $[39,40]$. One way to help users understand these implications is to show participants which options fit their clarified values [40] (i.e., tailored advice following the VCMs based on the answers that were provided). Interestingly, the feasibility study of a digital DA for smoking cessation that did include an explicit VCM has shown promising results, both in terms of evidence-based cessation assistance uptake and smoking cessation outcomes [135]. Thus, explicit VCMs could potentially be used to enhance the effectiveness of DAs for smoking cessation. However, the feasibility study's design (pretest and posttest assessment without a control group) limits the interpretability of the feasibility study's findings [135], and it remains unclear whether such an explicit VCM results in significant changes in cessation rates and decisional outcomes.

Based on these limitations and the existing literature, an online DA, called VISOR containing an explicit evidence-based VCM and tailored advice was developed that helps smokers to choose evidence-based cessation assistance tools. This DA was systematically developed and is currently being tested in a national randomized controlled trial (RCT) for its effect on individual decisional processes and smoking cessation attempts, as well as smoking abstinence compared to a DA without the explicit VCM component. Specifically, we aim to test the following hypotheses.

$\mathrm{H}_{1 \mathrm{a} / \mathrm{b} / \mathrm{c}}$ is that a DA with an explicit VCM and tailored advice will lead to a statistically significant increase in smoking abstinence after one month $\left(\mathrm{H}_{1 \mathrm{a}}\right)$, six months $\left(\mathrm{H}_{1 \mathrm{~b}}\right)$, and 12 months $\left(\mathrm{H}_{1 \mathrm{c}}\right)$ compared to a DA without an explicit VCM and tailored advice: direct effect on smoking abstinence. 
$\mathrm{H}_{2 \mathrm{a} / \mathrm{b} / \mathrm{c}}$ is that a DA with an explicit VCM and tailored advice will lead to a statistically significant increase in evidence-based cessation assistance use after one month $\left(\mathrm{H}_{2 \mathrm{a}}\right)$, six months $\left(\mathrm{H}_{2 \mathrm{~b}}\right)$, and 12 months $\left(\mathrm{H}_{2 \mathrm{c}}\right)$ compared to a DA without an explicit VCM and tailored advice.

$\mathrm{H}_{3 \mathrm{a} / \mathrm{b}}$ is that the positive effect of a DA with an explicit VCM and tailored advice (vs a DA without an explicit VCM and tailored advice) on smoking abstinence after six months and 12 months will be at least partially mediated by the use of evidencebased cessation assistance at 1-month $\left(\mathrm{H}_{3 a}\right)$ and 6-month $\left(\mathrm{H}_{3 \mathrm{~b}}\right)$ follow-ups, respectively: indirect effect on smoking abstinence.

$\mathrm{H}_{4}$ is that a DA with an explicit VCM and tailored advice will lead to a statistically significant decrease in decisional conflict (state of uncertainty about which course of action to take) right after the DA compared to a DA without an explicit VCM and tailored advice.

In addition, it will also be tested whether the DA is more cost-effective compared to a DA without the explicit VCM component. This paper aims to thoroughly describe the DA VISOR, its development process, and the study protocol to test its (cost-)effectiveness.

\section{Methods}

An RCT is being conducted and will be reported in line with the CONSORT-EHEALTH checklist [220]. Participants are randomly allocated to receive access to either a DA with an explicit VCM and tailored advice (intervention group) or a DA without an explicit VCM and tailored advice (control group). Study materials (such as questionnaires, but not the DA) will be made available on the open science framework website, in line with recommendations in the field of behavioral science [221].

The study does not fall under the scope of the Medical Research Involving Human Subjects Act as indicated by the Medical Ethics Committee Zuyderland, the Netherlands (16-N-227). The development of VISOR and the accompanying studies are funded by the Dutch Cancer Society, UM2015-7744.

\section{Study Population}

Recruitment is predominantly conducted online to reflect the online nature of the DA. We make use of various methods: Mainly, we are recruiting participants through project social media accounts (e.g., [222]) and paid social media advertisements. We decided to make use of four big social media platforms (i.e., Facebook, Instagram, Twitter, and Linkedln) as they 
cater to different target groups [223]. In addition, our recruitment messages are being shared on the social media accounts of the project members and their institutions. Also, we promote the RCT through the project's website, and we asked various relevant Dutch institutes and organizations (e.g., the Dutch institute for addiction and mental health and municipal health services) to promote the study. Finally, we advertise the study in online newspapers. All recruitment materials include a link to enroll in the RCT, if possible, to simplify the process for potential participants as much as possible. Study inclusion criteria are: (1) participants are currently smoking, (2) participants are motivated to stop smoking within six months, (3) participants are 18-100 years old, (4) participants are able to understand Dutch, and (5) participants have access to the internet and have the necessary internet literacy skills to use the intervention. People are excluded if they only use ecigarettes; however, dual users can participate. The rationale behind the inclusion and exclusion criteria is described in Table 1.

Table 1. Overview of the Randomized Controlled Trial Inclusion and Exclusion Criteria, Including Rationale.

\begin{tabular}{|c|c|}
\hline Criteria & Rationale \\
\hline \multicolumn{2}{|l|}{ Inclusion criteria } \\
\hline Participants are currently smoking & $\begin{array}{l}\text { To test the primary hypothesis that the } \\
\text { decision aid (DA) will have a positive effect on } \\
\text { smoking cessation, it has to be tested among } \\
\text { smokers }\end{array}$ \\
\hline $\begin{array}{l}\text { Participants are motivated to stop smoking } \\
\text { within six months }\end{array}$ & $\begin{array}{l}\text { As smokers unmotivated to quit cannot be } \\
\text { expected to be willing to consider the use of } \\
\text { cessation assistance }\end{array}$ \\
\hline Participants are $18-100$ years old & As the DA was developed for adults \\
\hline Participants are able to understand Dutch & $\begin{array}{l}\text { To ensure that participants understand all } \\
\text { provided information }\end{array}$ \\
\hline $\begin{array}{l}\text { Participants have access to the internet and } \\
\text { have the necessary internet literacy (skills) to } \\
\text { use the intervention }\end{array}$ & $\begin{array}{l}\text { As the DA is fully online, participants need both } \\
\text { access to the internet and the necessary } \\
\text { internet literacy (skills) to access and use the } \\
\text { DA }\end{array}$ \\
\hline \multicolumn{2}{|l|}{ Exclusion criteria } \\
\hline Participants exclusively use e-cigarettes & $\begin{array}{l}\text { As there is no consensus on whether cessation } \\
\text { assistance tools can be used to cease e- } \\
\text { cigarette use }\end{array}$ \\
\hline
\end{tabular}

The power calculation was based on the dichotomous outcome measure of smoking abstinence (primary outcome: seven-day point prevalence abstinence). The only previous $\mathrm{RCT}$ in the Netherlands testing the effect of a DA for smoking cessation on this outcome 
measure showed a significant effect $(20.2 \%$ vs $13.6 \%)$ at six months [42]. To be able to significantly ( $\alpha=.05 ; \beta=.20$ ) detect the same effect in a one-sided test, 398 smokers per arm are necessary at the end of the trial (796 in total). Considering $50 \%$ attrition over the intervention period, we aim to include 1592 smokers at baseline.

\section{The Intervention}

Initial DA-Development

The development process of the DA was based on the IPDAS guidelines for DA-development [59]. In line with these guidelines, both end users' (i.e., smokers) and clinicians' needs were assessed. In addition, we asked scientific smoking cessation experts for extra input as smoking cessation is often done without consulting clinicians-especially in the Netherlands [60]. In the end user needs assessment, 20 interviews were conducted to assess end users' needs regarding potential cessation assistance's characteristics that should be described in the DA and DA-functions (e.g., a knowledge quiz). The input of the experts was gathered through two Delphi studies (24 scientists and 38 clinicians completed the final round). The input of these three qualitative studies was used to inform the design and content of the DA, supplemented by the IPDAS background papers (e.g., [224]) as well as other relevant literature in the field (e.g., [40]) and in consultation with established experts in the field. The SDT served as the theoretical framework. After this, a first prototype was developed that was alpha-tested in September 2019 with potential end users $(n=3)$ and Dutch smoking cessation experts $(n=8)$. During the alpha test, participants were asked to focus on the content of the DA. The end users alpha-tested parts of the DA, while the smoking cessation experts tested the whole first version. Before the beta test, the DA was evaluated by one of the co-authors (JR) with regard to the comprehensibility of the text for people with limited health literacy. On the basis of that evaluation, some sections and sentences were rephrased or simplified.

Usability Testing

In December 2019, after the alpha test, the DA was beta-tested among 15 experts and end users: five smoking cessation counselors, five eHealth experts (of whom, two had digital DAexperience), and five potential end users (i.e., people motivated to stop smoking). Respondents were given access to the DA to assess the DA's usability using the heuristic evaluation method (smoking cessation counselors and eHealth experts) and the think-aloud method (potential end users) [225].

Using the heuristic evaluation method, smoking cessation counselors and eHealth experts were asked to evaluate the DA against a list of recognized usability principles: (1) use simple and natural dialogue, (2) speak the user's language, (3) minimize memory load, (4) be 
consistent, (5) provide feedback, (6) provide clearly marked exits, (7) provide shortcuts, (8) provide good error messages, (9) prevent errors, and (10) provide help and documentation. The smoking cessation counselors and eHealth experts were asked to use predetermined scenarios (e.g., whether cessation attempts have been undertaken before) during the evaluation.

With the think-aloud method, end users were asked to complete the DA while verbalizing their thoughts. The think-aloud method is considered particularly useful in understanding processes of cognition and is considered to be of high value in evaluating an intervention's design on usability flaws [225].

Data gathered through these tests were compared and compiled into a summary describing the usability flaws of the DA. Based on these results, adjustments to the DA were identified, which can be seen in Textbox 1.

Textbox 1. Overview of Alterations to the Decision Aid (DA).

- More information was added on how to use the DA: for example, "Now click on 'next' to start with the information."

- The language and complexity level have been simplified.

- Certain subparts (e.g., results of the knowledge quiz) were rewritten in a more positive tone.

- $\quad$ Content was shortened if possible (e.g., by combining pages or by removing too many details).

- $\quad$ Some content was added (e.g., required duration of prescription medication use).

- More visuals were added (e.g., icon arrays to showcase cessation assistance's effectiveness).

- Information regarding cessation assistance's effectiveness was adapted to make it more accessible.

- The order of the given information was changed (e.g., information regarding nonevidence-based cessation assistance was moved to the beginning as testers found it confusing that this information was mentioned only at the end of the information section).

- A function was added to enlarge visuals and tables.

- $\quad$ The layout was changed to make the DA more accessible to users with visual impairments (e.g., we added more lines and changed the color of the text).

- All abbreviations were removed, as were references to terms that were used in other parts of the DA.

- All hyperlinks were removed.

- Two possible options (i.e., combination of behavioral support and nicotine replacement therapy; combination of behavioral support and prescription medication) were added.

- A ranking of the evidence-based cessation assistance tools based on the value clarification method was added. 
Description of the DA

An interactive Dutch DA with an explicit VCM and tailored advice that facilitates the process of choosing an evidence-based cessation assistance was developed. The DA follows a stepwise approach and is designed to encourage a decision that is autonomous and consistent with participant's values and smoking behavior. The DA consists of eight sequential sections: (1) information section, (2) optional knowledge quiz, (3) brief smoking assessment, (4) intuitive decision, (5) intermediate advice, (6) explicit VCM, (7) tailored advice, and (8) access information.

In step 1, the information section explains the decision at hand, as well as all the available cessation assistance in the Netherlands. The following topics are addressed: (1) smoking cessation with and without evidence-based cessation assistance, (2) non-evidence-based cessation assistance, and (3) evidence-based cessation assistance.

For the first topic, differences in smoking cessation outcomes are described between people using and not using evidence-based cessation assistance using simple frequency formats (e.g., "By using one or more of these stop methods, 15 out of 100 people are still smoke-free after one year.").

For the second topic, a list of various non-evidence-based cessation assistance tools (i.e., acupuncture, laser therapy, hypnotherapy, mindfulness, smartphone apps, self-help books, and e-cigarettes) are described, and a remark is made that the use of these tools is not advised.

For the third topic, evidence-based cessation assistance is described (Table 2), including behavioral support in general, in which users can choose to read more detailed information about all possible options (i.e., face-to-face counseling, counseling over the phone, group coaching, and eHealth); NRT in general, in which users can choose to read more detailed information about all possible options (i.e., nicotine patches, nicotine gum, nicotine lozenge, nicotine mouth spray, and nicotine inhaler); prescription medication in general, in which users can choose to read more detailed information about all possible options (i.e., varenicline, bupropion, and nortriptyline); and cessation assistance during pregnancy and breastfeeding. Users can compare all cessation assistance options using an option grid and are informed about combinations of multiple cessation assistance options. In addition, costs and reimbursement are described, and sources used to inform the information section content (i.e., reports, scientific manuscripts, websites) are provided. 
Table 2. Overview of Cessation Assistance Characteristics Included in the Decision Aid (DA).

\begin{tabular}{|c|c|c|}
\hline $\begin{array}{l}\text { Cessation assistance } \\
\text { characteristic }\end{array}$ & Rationale for inclusion in the DA & $\begin{array}{l}\text { This characteristic is } \\
\text { included in the } \\
\text { statements }\left(\mathrm{VCM}^{\mathrm{a}}\right) \text { for: }\end{array}$ \\
\hline $\begin{array}{l}\text { Effectiveness of the cessation } \\
\text { assistance options }\end{array}$ & $\begin{array}{l}\text { During the interviews with } \\
\text { potential end users, this was both } \\
\text { the most mentioned characteristic } \\
\text { and considered the most important } \\
\text { characteristic. }\end{array}$ & $\begin{array}{l}\text { Behavioral support, } \\
\text { prescription medication }\end{array}$ \\
\hline $\begin{array}{l}\text { Probability of nausea and } \\
\text { dizziness when the cessation } \\
\text { assistance option is used }\end{array}$ & \multirow{5}{*}{$\begin{array}{l}\text { Probability of side effects was the } \\
\text { third most often mentioned } \\
\text { characteristic during the interviews } \\
\text { with potential end users and was } \\
\text { also considered to be of } \\
\text { importance by the clinicians. The } \\
\text { first three side effects were } \\
\text { specifically named as important by } \\
\text { potential end users, while the } \\
\text { fourth was added, as this is a } \\
\text { common side effect. Other side } \\
\text { effects were included if they are } \\
\text { regarded as being very likely to } \\
\text { occur. }\end{array}$} & \multirow[t]{5}{*}{$\begin{array}{l}\text { Nicotine replacement } \\
\text { therapy, prescription } \\
\text { medication }\end{array}$} \\
\hline $\begin{array}{l}\text { Probability of mood changes } \\
\text { when the cessation assistance } \\
\text { option is used }\end{array}$ & & \\
\hline $\begin{array}{l}\text { Probability of headaches when } \\
\text { the cessation assistance option } \\
\text { is used }\end{array}$ & & \\
\hline $\begin{array}{l}\text { Probability of sleeping } \\
\text { problems when the cessation } \\
\text { assistance option is used }\end{array}$ & & \\
\hline $\begin{array}{l}\text { Probability of other side effects } \\
\text { that are very likely to occur } \\
\text { when the cessation assistance } \\
\text { option is used }\end{array}$ & & \\
\hline $\begin{array}{l}\text { Extent and type of contact } \\
\text { someone has with a } \\
\text { professional when the } \\
\text { cessation assistance option is } \\
\text { used }\end{array}$ & $\begin{array}{l}\text { Both scientists and potential end } \\
\text { users indicated that this was of } \\
\text { great importance. Clinicians } \\
\text { indicated that required contact } \\
\text { with a healthcare professional was } \\
\text { of great importance. }\end{array}$ & Behavioral support \\
\hline $\begin{array}{l}\text { How the cessation assistance } \\
\text { option is used }\end{array}$ & $\begin{array}{l}\text { This was integrated after it had } \\
\text { been decided that Dutch health } \\
\text { insurance companies would cover } \\
\text { evidence-based cessation } \\
\text { assistance use, as that left too few } \\
\text { features to distinguish between the } \\
\text { different nicotine replacement } \\
\text { therapies and prescription } \\
\text { medications. }\end{array}$ & $\begin{array}{l}\text { Nicotine replacement } \\
\text { therapy, prescription } \\
\text { medication }\end{array}$ \\
\hline
\end{tabular}

Note. ${ }^{a}$ VCM: value clarification method.

In step 2, users can choose to complete the knowledge quiz or skip the knowledge quiz and proceed to the following step. If they complete the knowledge quiz, they will receive the 
results, see the correct answers to the questions that they answered incorrectly or could not answer (seeing the correct answers can also be skipped even if a respondent opts to do the knowledge quiz), and proceed to the following step.

The brief smoking assessment in step 3 consists of two closed (yes/no) questions: "To ensure that our advice suits you best, we would like to ask you if you smoke more than 10 cigarettes (or other tobacco products) on a normal day?" and "To ensure that our advice suits you best, we would like to ask you if you have ever made one or more smoking cessation attempts in the past that were unsuccessful?"

In step 4, an intuitive decision occurs between different clusters of cessation assistance tools: behavioral support; NRT; combination of behavioral support, NRT, and prescription medication; combination of behavioral support and NRT; combination of behavioral support and prescription medication; other, non-evidence-based cessation assistance; and no cessation assistance at all.

In step 5, intermediate advice is provided for users to reevaluate their intuitive decision in step 4 when they chose behavioral support only or NRT only, while also affirming at least one of the two questions in step 3 (indicating that they smoke more than 10 cigarettes on a normal day or have made one or more smoking cessation attempts in the past): They are advised to consider using a combination of behavioral and pharmacological cessation assistance tools. Intermediate advice is also given to users who chose non-evidence-based cessation assistance or no cessation assistance at all regardless of their answers in step 3: They are advised to consider using one or more evidence-based cessation assistance tools.

The explicit VCM in step 6 is provided for users that chose evidence-based cessation assistance tools in steps 4 or 5; users are asked to rate certain statements regarding cessation assistance characteristics (e.g., "I prefer a stop method that works better, even if that means that I have to leave the house."). These statements are also described in Table 2. Users only rate statements for options that belong to the cluster of cessation assistance options they selected in the previous step.

Step 7 is tailored advice based on the explicit VCM and an optional ranking of all options. The advice is given only when it is possible to give clear advice (i.e., if users' scores do not suggest that more than two cessation assistance tools are suitable based on their indicated values).

Step 8 involves accessing information on how to obtain the chosen cessation assistance (e.g., nicotine patches). 
Above and beyond these eight steps, framing throughout the DA is positive and autonomysupportive [226] (in line with the SDT as theoretical framework) to support users' need for autonomy (e.g., we refrained from using fear appeals [227] and controlling language $[92,226])$. We also clearly communicate that users can make their own choice at the end and included 'cues' to support this process (again, in order to support users' need for autonomy; e.g., by stating that the advice is only a recommendation and not a command and that it might be a good idea to discuss this with either the social environment, a healthcare provider, or both). A flow chart of the DA can be seen in Figure 1. The digital DA is hosted by the Dutch company OverNite Software Europe BV, and we make use of their product "TailorBuilder" [228]. Translated screenshots of different sections can be seen in Figure 2 (example of the information section) and Figure 3 (example of the explicit VCM). 


\section{Chapter 6}

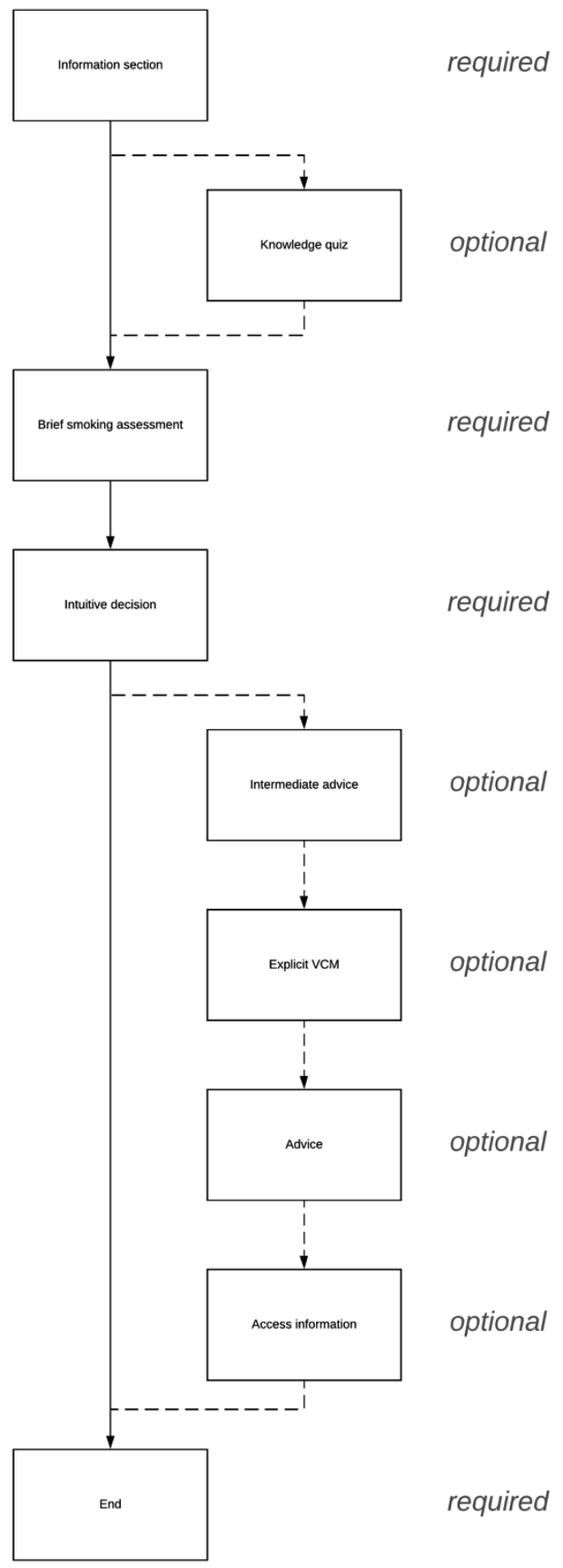

Figure 1. Sections of the Decision Aid. VCM: Value Clarification Method. 


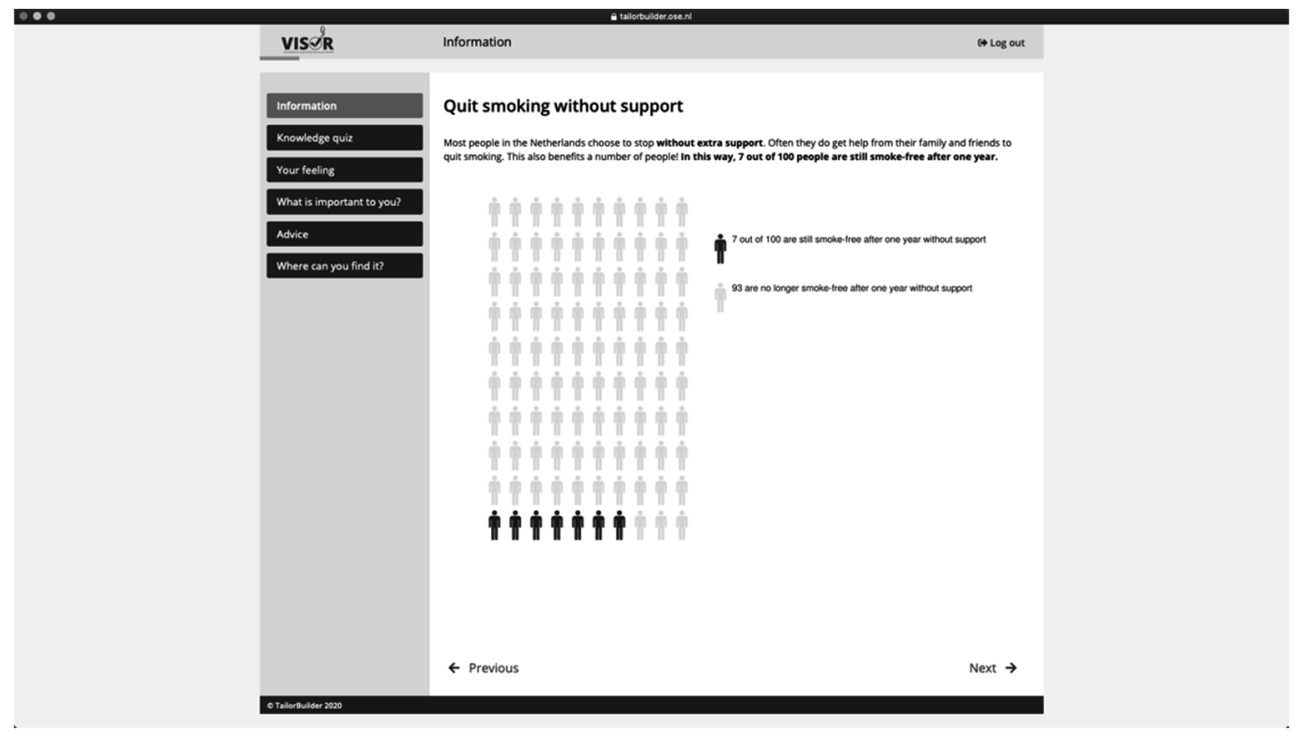

Figure 2. Screenshot of the Information Section in the Decision Aid (Original Text Translated From Dutch).

\section{Statements behavioral support}

\section{The following statements are about behavioral support.}

I prefer a stop method that works better, even if that means that I have to leave the house.

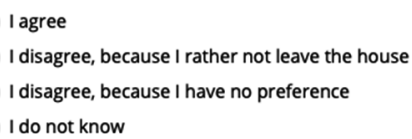

I prefer not to leave the house for a stop method, even though it means I can only use stop methods that work less well.

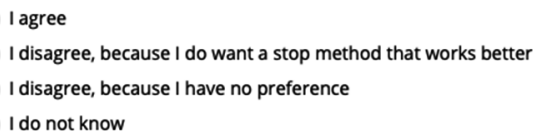

I would rather use a stop method with a trained health-care provider or stop coach than a stop method without a trained health-care provider or stop coach.

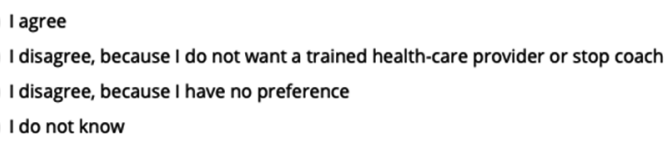

Figure 3. Screenshot of the Value Clarification Method (VCM) in the Decision Aid (Original Text Translated From Dutch). 
Theoretical Background of the Different Sections

An information section was predominantly included as relevant knowledge is needed to engage in high-quality decision making [229] and as it is regarded as an active ingredient by the IPDAS [31,33]. Given this fact, and as potential end users indicated that they would like a knowledge quiz within the DA, a knowledge quiz was included at the end of the information section. Users are thus able to check for themselves if they have the necessary knowledge to engage in the decision process and can obtain additional information if desired. The content of the information section was mainly based on Dutch guidelines (e.g., [210]), supplemented by various Cochrane reviews (e.g., [190]), online information from the Dutch National Health Care Institute [230], and the Dutch Medicines Evaluation Board [231].

An intuitive decision to limit the choice set for the explicit VCM was included to both prevent choice overload [232] and to include intuitive decision-making processes in the DA. Choice overload describes a scenario in which the difficulty of the decision to be made is greater than the cognitive means of the person faced with the decision, due to too many options. Many DAs focus on a rather small set of available options [38] (e.g., deciding between intensive or less intensive aftercare after breast cancer treatment [63]); yet, a cessation assistance DA has to include more options to present all available possibilities. Considering that the comparison of too many characteristics of too many options can lead to choice overload, which can in turn lead to worse decisional outcomes [232], in step 4 of the DA we decided to have users intuitively choose to go on with a limited choice set (e.g., only behavioral support). Another benefit of this approach is that users are encouraged to make use of both deliberative and intuitive decision-making processes. While many DAs focus on facilitating deliberation, de Vries et al. [229] have argued that combining those two strategies can lead to improved decisional outcomes. Importantly, however, users receive information about all possible options before they intuitively make a first, broad decision as knowledge is regarded as a necessary prerequisite for high-quality intuitive decision making [229].

As the Dutch smoking cessation guideline indicates that a combination of both behavioral and pharmacological support should be considered if smokers smoke $>10$ cigarettes per day or if many of their past cessation attempts failed [210], users that indicated in step 3 that this applies to them are provided with information on the improved effectiveness of a behavioral and pharmacological combination. Of course, even then, they can autonomously decide to continue with another cessation assistance set-in line with their preference and the SDT. In case smokers choose to use either no cessation assistance or no evidence-based cessation assistance, they are also reminded that evidence-based cessation assistance will probably lead to more successful cessation outcomes, but if they choose not to change their mind, they are directed to the last step (i.e., access information) if they decided to use no 
cessation assistance or directly to the end if they decided to use no evidence-based cessation assistance.

We decided to use an explicit VCM in the intervention group as VCMs are regarded as active ingredients by the IPDAS [31,34] and while conflicting evidence exists [143], an overwhelming amount of research has shown that interactive methods to clarify people's values seem to result in better decisional outcomes [32], especially in the long run [37] and if people are supported in understanding the implications of their clarified values $[39,40]$. This is why we decided to integrate individual tailored advice that is supplemented with information on how to obtain the different cessation assistance options to remove barriers for the users.

\section{Study Comparator Group}

Participants in the control group receive the same DA without an explicit VCM and tailored advice; thus, the DA includes the following steps: (1) information section, (2) optional knowledge quiz, (3) brief smoking assessment, (4) intuitive decision, (5) intermediate advice, and (8) access information. Steps 6 and 7 are skipped. The only other difference is that participants in the control group are not immediately directed towards the end after they have chosen to use no evidence-based cessation assistance to also provide them with a chance to reevaluate their choice. More information about the individual steps can be found under Description of the DA.

\section{Trial Flow and Measurement Instruments}

As participants register for the study via an online form, which includes their provision of informed consent and the creation of an account, participants are automatically randomized into one of the two groups (intervention or control group), allocating approximately $50 \%$ of respondents to either group. After this, they are asked to fill in the first part of the baseline questionnaire $(t=0)$ consisting of 22-94 items (depending on the respective answers; e.g., if the participants state that they have never attempted to cease smoking, no follow-up questions are posed), in which general demographic information (e.g., age), smoking behavior, nicotine dependence, productivity loss, healthcare utilization, quality of life, and stages of decision making are assessed. Participants are excluded from the study if they indicate that they are $<18$ years old, do not smoke, are not motivated to stop smoking within six months, or only use e-cigarettes. Participants immediately receive access to one of the two DAs after being randomized, as this process is fully automated. After having accessed the DA, the second part of the baseline questionnaire is made available $(t=1)$. The second part consists of 53-56 items regarding stages of decision making, the decision, the decision- 
making process, knowledge, perceived autonomy support, perceived competence, user evaluation, and recruitment channels.

One month after the baseline questionnaire $(t=2)$, users are asked to fill in a short followup questionnaire consisting of 14-21 items regarding cessation assistance utilization, smoking cessation status, stages of decision making, and knowledge.

After six months $(t=3)$ and 12 months $(t=4)$, users are asked to fill in a longer follow-up questionnaire that consists of 16-88 items relating to cessation assistance utilization, smoking abstinence (seven-day point prevalence abstinence and prolonged abstinence), and questions regarding smoking behavior for those that did not achieve smoking abstinence. Healthcare utilization, productivity losses, quality of life, and decisional regret are also assessed (again). During $t=4$, two qualitative items are included to assess users' experience of having used the DA during their cessation attempt.

All included measures are based on our theoretical background (i.e., SDT $[233,234]$ ) the IPDAS guidelines on establishing effectiveness of DAs [85], Dutch guidelines on health economic evaluation [235], and expert knowledge regarding smoking cessation outcomes $[77,174]$. For a more detailed overview of all included constructs, measurements, and their respective sources, see Table 3.

Table 3. Overview of Included Constructs, Measurements, and Sources.

\begin{tabular}{|c|c|c|}
\hline Constructs & Measurements and sources & Purpose \\
\hline \multicolumn{3}{|c|}{ Baseline $(t=0)-$ directly before the decision aid } \\
\hline Demographics & Age, gender, education [174] & $\begin{array}{l}\text { Sample description, attrition } \\
\text { analyses, covariate(s) in } \\
\text { (main) analyses }\end{array}$ \\
\hline Smoking behavior & $\begin{array}{l}\text { Smoking status, motivation to quit, } \\
\text { type of tobacco products, amount of } \\
\text { tobacco consumption, amount of past } \\
\text { cessation attempts, and cessation } \\
\text { assistance utilization in the past six } \\
\text { months [174] }\end{array}$ & $\begin{array}{l}\text { Sample description, attrition } \\
\text { analyses }\end{array}$ \\
\hline Nicotine dependence & $\begin{array}{l}\text { Revised Fagerström Test for Nicotine } \\
\text { Dependence (FTND-R) [236] }\end{array}$ & $\begin{array}{l}\text { Sample description, attrition } \\
\text { analyses, covariate in (main) } \\
\text { analyses }\end{array}$ \\
\hline Productivity loss & $\begin{array}{l}\text { iMTA Productivity Cost Questionnaire } \\
\text { (iPCQ) [237] }\end{array}$ & Economic evaluation \\
\hline Healthcare utilization & $\begin{array}{l}\text { Contacts with healthcare professionals } \\
\text { (plus frequency) in the past six } \\
\text { months, cessation assistance }\end{array}$ & Economic evaluation \\
\hline
\end{tabular}




\begin{tabular}{|c|c|c|}
\hline & $\begin{array}{l}\text { utilization (plus frequency) in the past } \\
\text { six months [238] }\end{array}$ & \\
\hline Quality of life & $\begin{array}{l}\text { ICEpop CAPability measure for Adults } \\
\text { (ICECAP-A) [239] }\end{array}$ & Economic evaluation \\
\hline $\begin{array}{l}\text { Stages of decision } \\
\text { making }\end{array}$ & Stage of Decision Making [240] & $\begin{array}{l}\text { Sample description, attrition } \\
\text { analyses, process information, } \\
\text { covariate in (main) analyses }\end{array}$ \\
\hline \multicolumn{3}{|c|}{ Follow-up $(t=1)-$ directly after the decision aid } \\
\hline $\begin{array}{l}\text { Stages of decision } \\
\text { making }\end{array}$ & Stage of Decision Making [240] & $\begin{array}{l}\text { Sample description, process } \\
\text { information }\end{array}$ \\
\hline Decision & $\begin{array}{l}\text { Decision after having used the decision } \\
\text { aid (DA), not yet implemented }\end{array}$ & $\begin{array}{l}\text { Sample description, process } \\
\text { information }\end{array}$ \\
\hline $\begin{array}{l}\text { Decision-making } \\
\text { process }\end{array}$ & $\begin{array}{l}\text { Decisional conflict scale [4], first item } \\
\text { from the Preparation for Decision } \\
\text { Making Scale [241] }\end{array}$ & $\begin{array}{l}\text { Hypothesis testing, } \mathrm{H}_{4} \\
\text { (decisional conflict scale [4]); } \\
\text { process information and } \\
\text { additional studies (see Data } \\
\text { Analysis) }\end{array}$ \\
\hline Knowledge & Self-developed knowledge scale [242] & $\begin{array}{l}\text { Additional studies (see Data } \\
\text { Analysis) }\end{array}$ \\
\hline $\begin{array}{l}\text { Perceived autonomy } \\
\text { support }\end{array}$ & $\begin{array}{l}\text { The Virtual Care Climate Questionnaire } \\
\text { [233] }\end{array}$ & $\begin{array}{l}\text { Additional studies (see Data } \\
\text { Analysis) }\end{array}$ \\
\hline Perceived competence & Perceived competence scale [234] & $\begin{array}{l}\text { Additional studies (see Data } \\
\text { Analysis) }\end{array}$ \\
\hline Evaluation questions & $\begin{array}{l}\text { Regarding attention, clarity, } \\
\text { satisfaction, and one open question }\end{array}$ & $\begin{array}{l}\text { Additional studies (see Data } \\
\text { Analysis) }\end{array}$ \\
\hline Recruitment channels & $N / A^{a}$ & $\begin{array}{l}\text { Recruitment monitoring and } \\
\text { analyses }\end{array}$ \\
\hline \multicolumn{3}{|c|}{ Follow-up $(t=2)-$ one month after baseline } \\
\hline $\begin{array}{l}\text { Decision } \\
\text { implementation }\end{array}$ & $\begin{array}{l}\text { Implemented decision (choice) after } \\
\text { having used the DA }\end{array}$ & $\begin{array}{l}\text { Hypothesis testing, } \mathrm{H}_{2 \mathrm{a}} \text { and } \\
\mathrm{H}_{3 \mathrm{a}}\end{array}$ \\
\hline $\begin{array}{l}\text { Smoking (cessation) } \\
\text { behavior }\end{array}$ & $\begin{array}{l}\text { Prolonged abstinence; seven-day point } \\
\text { prevalence abstinence; for users that } \\
\text { did not successfully stop: type of } \\
\text { tobacco products and amount of } \\
\text { tobacco consumption [174] }\end{array}$ & $\begin{array}{l}\text { Hypothesis testing, } \mathrm{H}_{1 \mathrm{a}} \\
\text { (seven-day point prevalence } \\
\text { abstinence [174]); additional } \\
\text { studies (see Data Analysis) }\end{array}$ \\
\hline $\begin{array}{l}\text { Stages of decision } \\
\text { making }\end{array}$ & Stage of Decision Making [240] & $\begin{array}{l}\text { Sample description, process } \\
\text { information }\end{array}$ \\
\hline Knowledge & Self-developed knowledge scale [242] & $\begin{array}{l}\text { Additional studies (see Data } \\
\text { Analysis) }\end{array}$ \\
\hline
\end{tabular}




\begin{tabular}{|c|c|c|}
\hline \multicolumn{3}{|c|}{ Follow-up $(t=3)-$ six months after baseline } \\
\hline $\begin{array}{l}\text { Decision } \\
\text { implementation }\end{array}$ & $\begin{array}{l}\text { Implemented decision (choice) after } \\
\text { having used the DA }\end{array}$ & $\begin{array}{l}\text { Hypothesis testing, } \mathrm{H}_{2 b} \text { and } \\
\mathrm{H}_{3 b}\end{array}$ \\
\hline $\begin{array}{l}\text { Smoking (cessation) } \\
\text { behavior }\end{array}$ & $\begin{array}{l}\text { Prolonged abstinence; seven-day point } \\
\text { prevalence abstinence; for users that } \\
\text { did not successfully stop: type of } \\
\text { tobacco products and amount of } \\
\text { tobacco consumption [174] }\end{array}$ & $\begin{array}{l}\text { Hypothesis testing, } \mathrm{H}_{1 b} \text { and } \\
\mathrm{H}_{3 a} \text { (seven-day point } \\
\text { prevalence abstinence [174]); } \\
\text { additional studies (see Data } \\
\text { Analysis) }\end{array}$ \\
\hline Productivity loss & iPCQ [237] & Economic evaluation \\
\hline Healthcare utilization & $\begin{array}{l}\text { Contacts with healthcare professionals } \\
\text { (plus frequency) in the past six } \\
\text { months, cessation assistance } \\
\text { utilization (plus frequency) in the past } \\
\text { six months [238] }\end{array}$ & Economic evaluation \\
\hline Quality of life & ICECAP-A [239] & Economic evaluation \\
\hline Decisional regret & Decision regret scale [243] & $\begin{array}{l}\text { Additional studies (see Data } \\
\text { Analysis) }\end{array}$ \\
\hline \multicolumn{3}{|c|}{ Follow-up $(t=4)-$ twelve months after baseline } \\
\hline $\begin{array}{l}\text { Decision } \\
\text { implementation }\end{array}$ & $\begin{array}{l}\text { Implemented decision (choice) after } \\
\text { having used the DA }\end{array}$ & Hypothesis testing, $\mathrm{H}_{2 c}$ \\
\hline $\begin{array}{l}\text { Smoking (cessation) } \\
\text { behavior }\end{array}$ & $\begin{array}{l}\text { Prolonged abstinence; seven-day point } \\
\text { prevalence abstinence; for users that } \\
\text { did not successfully stop: type of } \\
\text { tobacco products and amount of } \\
\text { tobacco consumption [174] }\end{array}$ & $\begin{array}{l}\text { Hypothesis testing, } \mathrm{H}_{1 \mathrm{c}} \text { and } \\
\mathrm{H}_{3 \mathrm{~b}} \text { (seven-day point } \\
\text { prevalence abstinence [174]); } \\
\text { additional studies (see Data } \\
\text { Analysis) }\end{array}$ \\
\hline Productivity loss & iPCQ [237] & Economic evaluation \\
\hline Healthcare utilization & $\begin{array}{l}\text { Contacts with healthcare professionals } \\
\text { (plus frequency) in the past six } \\
\text { months; cessation assistance } \\
\text { utilization (plus frequency) in the past } \\
\text { six months [238] }\end{array}$ & Economic evaluation \\
\hline Quality of life & ICECAP-A [239] & Economic evaluation \\
\hline Decisional regret & Decision regret scale [243] & $\begin{array}{l}\text { Additional studies (see Data } \\
\text { Analysis) }\end{array}$ \\
\hline Evaluation questions & Regarding perceived decision support & Process information \\
\hline
\end{tabular}

Note. ${ }^{a} \mathrm{~N} / \mathrm{A}$ : not applicable.

Participants are invited to fill in each follow-up questionnaire if they made use of the entire DA, even when they skipped one of the other follow-up questionnaires. To avoid high dropout rates, participants receive either one automatic reminder after a week (if they have not filled in the follow-up questionnaires at all) or two after two days and a week (if they already started filling in at least parts of the follow-up questionnaires). Participants that started using either the DA or started filling in the baseline questionnaire $(t=0)$ without 
finishing it also receive two automatic reminders (after two days and a week). In addition, participants who took part in the last measurement receive $€ 10$ (US \$11.84). A visual representation of the trial flow can be seen in Figure 4. 


\section{Chapter 6}

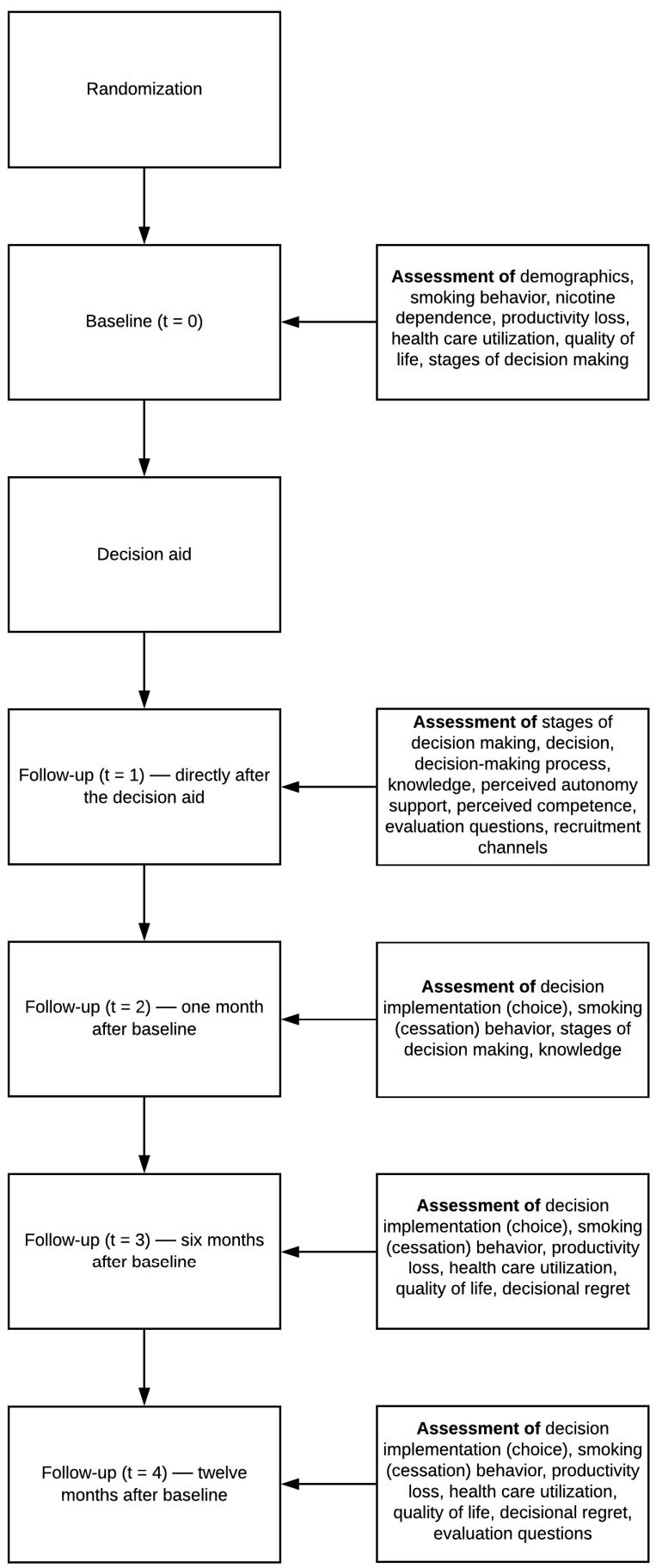

Figure 4. Trial Flow. 


\section{Data Analysis}

Before conducting any analyses, we will assess the scale quality of all measurement instruments where appropriate, following the two steps proposed by Crutzen \& Peters [244]: (1) conformation of intended structure of the measurement instrument by means of exploratory factor analyses and (2) calculation of omega [245] as a less biased alternative to (Cronbach) $\alpha$. Compared to $\alpha$, omega has more realistic assumptions regarding variances of and covariance between items [246]. Omegahierarchical is based upon the sum of the squared loadings of items on the general factor and reduces the risks of misjudging the internal consistency of scientific scales [247]. Values will be calculated with R [248], making use of the integrated development environment RStudio [249].

Analysis of Primary and Secondary Outcomes

To assess the effects of the DA on dichotomous and continuous (primary and secondary) outcome measures, logistic and linear regression analyses will be conducted, respectively. Mediation analyses will be conducted to determine whether the effects of the DA on smoking abstinence are mediated through cessation assistance use. All analyses will include covariates that were selected a priori (as recommended by Gruijters [250] and De Boer et al. [251]), if these are also associated with the outcome measures as described in our hypotheses (i.e., smoking abstinence, evidence-based cessation assistance use, and decisional conflict) within our sample. Demographic factors (i.e., age, gender, and education) were selected for all three outcome measures. The Revised Fagerström Test for Nicotine Dependence was also selected for the smoking-related outcome measures, whereas stage of decision making was selected for decisional conflict. More information on the rationale for selecting those covariates can be found in Appendix $A$. Results will be reported from both the fully adjusted as well as crude analyses [250]. These analyses as well will be done with $\mathrm{R}$ [248], making use of the integrated development environment RStudio [249]. To test the robustness of the results, all analyses will be conducted according to three different approaches (if applicable): analyses based on (1) worst-case scenario (dropout respondents are considered not to have changed), (2) multiple imputations, and (3) complete cases only. We will also test whether selective dropout has occurred in order to subsequently minimize the bias that this can cause.

\section{Economic Evaluation}

An economic evaluation will be performed from a societal perspective with a time horizon of 12 months. Both a cost-effectiveness analysis and a cost-utility analysis will be conducted. For the cost-effectiveness analysis, two incremental cost-effectiveness ratios will be calculated, based on the cost per abstinent respondent and the cost per individual who uses 
evidence-based cessation assistance. For the cost-utility analysis, an incremental cost-utility ratio will be calculated, based on the ICEpop CAPability measure for Adults, as proposed by Smit et al. [252]. Uncertainty will be accounted for by bootstrapping and several univariate and multivariate sensitivity analyses. Cost-effectiveness acceptability curves will be constructed, showing for varying willingness-to-pay thresholds the probability that the DA with an explicit VCM and tailored advice is cost-effective compared to the DA without an explicit VCM and tailored advice.

\section{Additional Studies}

An additional study using the data collected during the RCT as described in this protocol will be conducted in the future to test the cognitive processes (e.g., the clarification of one's values) that may underlie the effects of the DA on primary (i.e., smoking abstinence) and secondary (e.g., decisional regret) outcomes activated by the DAs by making use of Structural Equation Modelling in R [248] with the lavaan package [253].

Results

The RCT started in January 2020, and at this writing, 1248 users created an account, of which 519 finished the DA. Data collection is ongoing and will be conducted until September 2021.

\section{Discussion}

This paper describes the systematic development of an autonomy-supportive DA to assist smokers in choosing evidence-based cessation assistance and the intended study design to test its (cost-)effectiveness. In order to systematically develop an evidence-based online DA for a lifestyle behavior, we applied the IPDAS development process guideline [59]. The DA described in this article does not require the assistance of a healthcare professional and is intended for all adults that want to quit smoking in the near future (i.e., within the coming six months). Therefore, we explicitly try to reach a broad range of potential users by focusing our recruitment strategy on social media platforms and other, more traditional media outlets. This enables us to reach a target group interested in making a decision on smoking cessation support independently from more traditional channels, such as their healthcare provider. This is especially interesting, as Dutch smokers often do not engage in smoking cessation discussions with their healthcare provider and instead most often turn to the internet for smoking cessation advice [60]; therefore, we believe this recruitment strategy is most suitable for reaching this rather general target group and could expose an additional group of smokers motivated to quit to evidence-based cessation assistance. 
The results of this RCT can be used to improve our understanding of decision-making processes (especially in the context of smoking cessation) and to provide new insights into effective elements of DAs, to support not only informed decision making but also subsequent behavioral change. Formative studies (i.e., the aforementioned interviews and beta/usability tests) have shown that potential end users are interested in the DA as they want to achieve long-term behavior change (i.e., smoking abstinence). However, most RCTs testing the effects of DAs focus on decisional outcomes alone [13]. Our RCT will thus be of added value to the field and might provide unique insights that have remained unexplored so far.

Furthermore, if the DA will be proven to be (cost-)effective, it can be implemented nationwide and will thus help to reduce tobacco-related diseases and deaths. Two of three unique characteristics of this DA that were mentioned in the Introduction to overcome the limitation of the paper-based DA that was tested in the Netherlands before [42] could make a nationwide implementation particularly interesting: The online nature will (1) allow for a wider reach and (2) enable a more flexible and interactive approach.

\section{(Potential) Strengths of the Study}

The study shows several potential strengths. First, we are conducting an RCT to assess the DA's impact on behavior change and decision-making outcomes, with randomization being done automatically. The fact that participants are being blinded (as the control group receives a DA as well) further strengthens this study. DAs aimed at smoking cessation with explicit VCMs have been tested before, but without following an RCT protocol (e.g., [135]). Testing the DA in a longitudinal RCT design will allow for stronger conclusions about the DA's impact. Following participants for a longer period of time is especially interesting as explicit VCMs have been shown to result in long-term benefits but not necessarily in short-term benefits [37].

Second, recruited end users are only included if they are motivated to stop smoking in the near future, meaning they actually have to decide how they wish to stop smoking. A previous study that tested the effects of an explicit VCM found no effects [143]; however, they made use of a hypothetical decision, which hampers interpretation of their findings. As both primary (i.e., smoking abstinence) and secondary (e.g., decisional conflict) outcomes relate to 'real-life' phenomena and affect, testing the DA in a nonhypothetical and 'natural' context will also allow for stronger conclusions about the DA's impact.

Third, and as previously mentioned, the DA is tested not only for outcomes at the decisional level but also at the behavioral level. Above and beyond this, the DA will also be tested regarding its cost-effectiveness. This is especially interesting as experts strongly urge to test eHealth applications not only for their effectiveness but for their cost-effectiveness as well 
[254]. However, these practices are not commonly applied. Testing the DA's costeffectiveness will enable decision makers to make evidence-based recommendations regarding the widespread implementation of the cessation assistance DA-which is particularly interesting given the scarcity of healthcare resources.

(Potential) Limitations of the Study

The study also has a few potential limitations. First, as it was decided to include two, and not three, study arms in our RCT, it will not be possible to assess the effects of the explicit VCM and the tailored advice separately. However, including a third arm would have required an even bigger sample, which was deemed not feasible.

Second, the online nature of both the DA and our recruitment strategy exclude potential participants that either have no access to the internet or lack the digital skills needed to use the DA. However, given that the Netherlands is one of the two countries with the highest percentage of households with internet access in the European Union [255], we expect this to be a relatively minor limitation.

\section{Conclusion}

DAs that assist smokers in choosing evidence-based cessation assistance offer a potential approach to both counteract the public health effects of smoking and facilitate individual's smoking cessation attempts. However, knowledge regarding effective elements and (costleffectiveness is lacking. Our study is therefore expected to contribute significantly to the current knowledge regarding smoking cessation DA-application, the added value of explicit VCMs within such DAs, and the effect on behavioral and informed decision-making outcomes.

\section{Abbreviations}

DA - decision aid

FTND-R - Revised Fagerström Test for Nicotine Dependence

ICECAP-A - ICEpop CAPability measure for Adults

iPCQ - iMTA Productivity Cost Questionnaire

IPDAS - International Patient Decision Aid Standards

NRT - nicotine replacement therapy

RCT - randomized controlled trial

SDT - Self-Determination Theory

VCM - value clarification method 


\section{Appendix A - Selection of Covariates}

All analyses will include covariates that were selected a priori (as recommended by Gruijters [250] and De Boer et al. [251]), if these are also associated with the three outcome measures as described in our hypotheses (i.e., smoking abstinence, evidence-based cessation assistance use, and decisional conflict) within our sample. Demographic factors (i.e., age, gender, and education) were selected for all three outcome measures. The Revised Fagerström Test for Nicotine Dependence was also selected for the smoking-related outcome measures, whereas stages of decision making was selected for decisional conflict.

\begin{tabular}{|c|c|c|}
\hline Outcome of interest & Covariates selected & Rationale \\
\hline Smoking abstinence & $\begin{array}{l}\text { Revised Fagerström Test for } \\
\text { Nicotine Dependence (FTND- } \\
\text { R) and demographic factors } \\
\text { (age, gender, and education) }\end{array}$ & $\begin{array}{l}\text { Nicotine dependence is the } \\
\text { only consistent predictor of } \\
\text { successful smoking cessation } \\
\text { in adult general populations as } \\
\text { shown in a systematic review } \\
\text { [256] which was also found in } \\
\text { a review about clinical } \\
\text { populations [257]. And while } \\
\text { demographic factors on the } \\
\text { other hand have not been } \\
\text { shown to play a consistent role } \\
\text { in smoking cessation success } \\
\text { [256,257]-especially not in } \\
\text { general populations [256], } \\
\text { other reviews [257] do report } \\
\text { that certain demographic } \\
\text { factors (e.g., age) can } \\
\text { influence successful smoking } \\
\text { cessation. }\end{array}$ \\
\hline $\begin{array}{l}\text { Evidence-based cessation } \\
\text { assistance use }\end{array}$ & $\begin{array}{l}\text { Revised Fagerström Test for } \\
\text { Nicotine Dependence (FTND- } \\
\text { R) and demographic factors } \\
\text { (age, gender, and education) }\end{array}$ & $\begin{array}{l}\text { Previous research }[23,258,259] \\
\text { has shown that both 'heavy } \\
\text { smokers' and certain } \\
\text { demographic groups are more } \\
\text { likely to use cessation } \\
\text { assistance (e.g., women are } \\
\text { seemingly more likely to use } \\
\text { cessation assistance [23]), Zhu } \\
\text { et al. [23] also showed that } \\
\text { smoking behavior can } \\
\text { influence cessation assistance } \\
\text { uptake-however, as the } \\
\text { FTND-R already captures }\end{array}$ \\
\hline
\end{tabular}




\section{Chapter 6}

\begin{tabular}{|l|l|l|}
\hline & $\begin{array}{l}\text { smoking behavior [236], it was } \\
\text { decided to only include the } \\
\text { FTND-R. }\end{array}$ \\
\hline Decisional conflict & $\begin{array}{l}\text { Stages of decision making and } \\
\text { demographic factors (age, } \\
\text { gender, and education) }\end{array}$ & $\begin{array}{l}\text { Predictors of decisional } \\
\text { conflict are not commonly } \\
\text { studied as far as we are aware, } \\
\text { however, it has been shown } \\
\text { that someone's stage of } \\
\text { decision making is associated } \\
\text { with decisional conflict [240], } \\
\text { also one's ability to } \\
\text { understand health information } \\
\text { also appears to be related to } \\
\text { decisional conflict [260], which } \\
\text { in turn is often shown to be } \\
\text { associated with demographic } \\
\text { factors [261,262]. }\end{array}$ \\
\hline
\end{tabular}




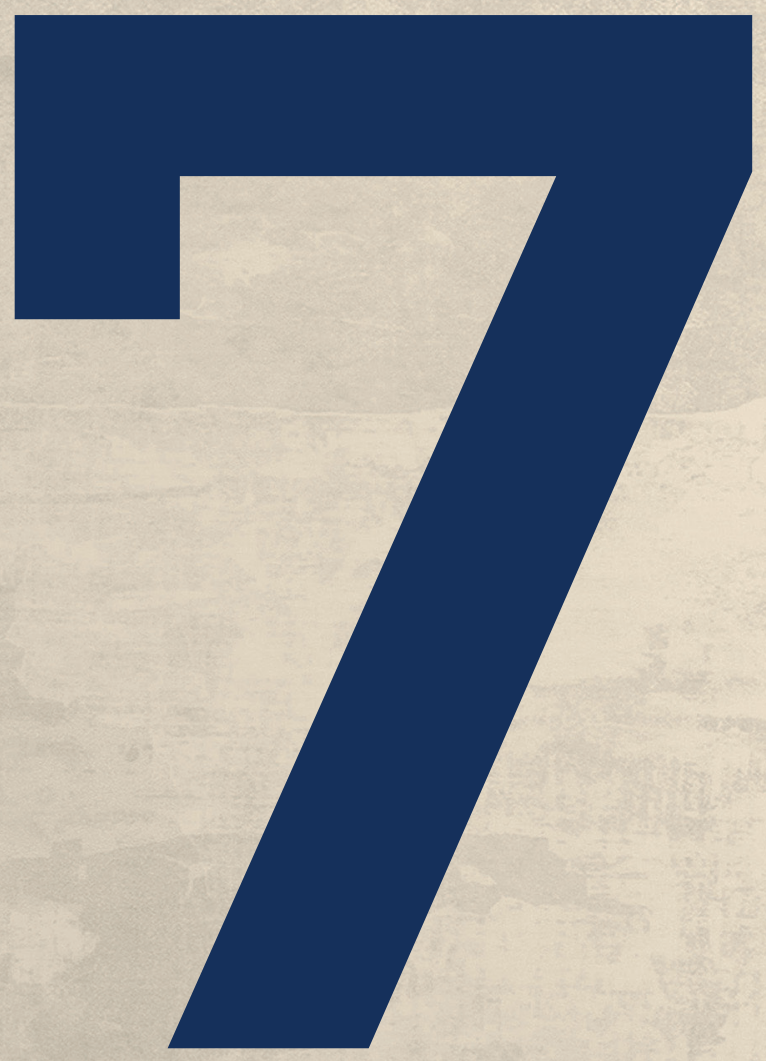




\section{chapter 7}

\section{does an explicit value clarification method with computer-tailored advice increase the effectiveness of a web- based decision aid to assist smokers in choosing evidence-based cessation assistance? findings from a randomized controlled trial}

This chapter has been submitted to the Journal of Medical Internet Research and has been published (as a preprint) at:

Gültzow, T., Smit, E. S., Crutzen, R., Jolani, S., Hoving, C., \& Dirksen, C. D. (2021). Does an Explicit Value Clarification Method with ComputerTailored Advice Increase the Effectiveness of a Web-based Decision Aid to Assist Smokers in Choosing Evidence-Based Cessation Assistance? Findings From a Randomized Controlled Trial. JMIR Preprints. https:// doi.org/10.2196/preprints.34246 
Abstract

Background: Smoking continues to be a driver of mortality. Various forms of evidence-based cessation assistance exist, but their use is limited. The decision between them may also induce decisional conflict. Offering decision aids (DAs) may be beneficial, but insights into their effective elements are lacking.

Objective: To test the added value of an effective element (i.e., an 'explicit value clarification method' [VCM] paired with computer-tailored advice) of a web-based DA focusing on cessation assistance. The computer-tailored advice indicated the most fitting cessation assistance. The primary outcome measure was seven-day point prevalence abstinence six months post baseline $(t=3)$. Secondary outcome measures were seven-day point prevalence abstinence one month post baseline $(t=2)$, evidence-based cessation assistance use $(t=2$ and $t=3$ ), and decisional conflict (immediately post $D A, t=1$ ).

Methods: A randomized controlled trial (RCT) was conducted. The intervention group received a DA with an explicit VCM with computer-tailored advice, the control group received the same DA without these elements. Participants were mainly recruited online (e.g., social media). All data was self-reported. Logistic and linear regression analyses (crude and adjusted for covariates) were performed to assess the outcomes. To test the robustness, analyses were conducted following two (decisional conflict) and three (smoking cessation outcomes) different scenarios: (1) complete cases, (2) worst-case scenario (dropout respondents are considered to smoke, smoking outcomes only), and (3) multiple imputations. According to an a priori sample size calculation $(\alpha=.05 ; \beta=.20), 796$ participants were needed.

Results: 2375 participants were randomized ( $n=1164$ intervention), 599 participants completed the DAs ( $n=275$ intervention), 276 ( $n=143$ intervention), 97 ( $n=54$ intervention), and 103 ( $n=56$ intervention) participants completed $t=1, t=2$ and $t=3$, respectively. Effects in favor of the intervention group on the primary outcome were only observed in the worst-case scenario ( $P=.02$ [crude]; $P=.04$ [adjusted]). Effects on the secondary outcomes were only observed regarding smoking abstinence after one month $(P$ $=.02$ in the crude and adjusted model), cessation assistance uptake after one month (only in the crude model, $P=.04$ ) and after six months ( $P=.01$ [crude]; $P=.02$ [adjusted]), but also only in the worst-case scenario. Non-usage attrition was $34.19 \%$ higher in the intervention group than in the control group $(P<.001)$.

Conclusions: We cannot confidently recommend the inclusion of explicit VCMs and computer-tailored advice at this point. In fact, they might result in higher attrition rates during DA completion, thereby limiting their potential. However, because a lack of statistical 
power may influenced our findings regarding the outcomes, we recommend replicating this study, taking our lessons learned into account. For example, we found indications that a stronger emphasis on usage times is justified in relation to digital DAs. 


\section{Introduction}

Smoking continues to be a major driver of global mortality [189], a trend that is mirrored in the Netherlands $[263,264]$, showcasing that smoking prevention is imperative. However, as $21.7 \%$ of the adult Dutch population still smokes according to recent figures [263], it is also of the utmost importance to invest in effective and evidence-based cessation assistance. Several cessation assistance tools are currently recognized as being effective in aiding individuals to successfully achieve smoking abstinence. They range from behavioral interventions (e.g., counselling [24]) to nicotine replacement therapy (NRT) [190] and prescription medication [26]. Unfortunately, evidence-based cessation assistance is known to be underutilized in various countries, including the Netherlands [60].

A common barrier to cessation assistance utilization is incorrect knowledge in relation to the safety and efficacy of cessation assistance [29]. Increasing individuals' knowledge may therefore be a worthwhile avenue to explore when it comes to successfully increasing individuals' cessation rates. Especially, as being uninformed can lead to a state of uncertainty about which course of action to take-better known as decisional conflict [4]-which in turn is known to increase the chances of decision delay [4,8]. Individuals that have decided to stop smoking, yet are uninformed about the possibilities of cessation assistance, might therefore delay the decision on how to stop smoking or simply decide to use the most common approach: attempting to quit smoking without the use of cessation assistance [191]. That said, knowledge provision alone is often insufficient to facilitate behavior change. This is commonly referred to as the knowledge-behavior gap in health promotion research (e.g., $[265,266])$. Knowledge provision alone might thus not be enough to support smokers motivated to quit smoking in their decision around smoking cessation assistance. However, providing individuals who are motivated to stop smoking with decision support that also includes accurate information (next to other decision support elements) about cessation assistance tools might decrease decisional conflict. That in turn, might facilitate smoking cessation efforts and ultimately increase chances of long-term smoking abstinence.

Such decision support can be provided in the form of so-called decision aids (DAs), interventions specifically designed to facilitate decisional processes [13]. A recent systematic review by Moyo et al. [52] has shown that DAs can be beneficial for smoking cessation even though traditionally DAs have most often been applied to treatment and screening rather than lifestyle-related decisions [13]. However, information about the effective elements of smoking cessation DAs is currently lacking and the only smoking cessation DA that has been studied in a Dutch context before [42] has shown a number of limitations: It (1) was largely paper-based, thereby limiting widespread dissemination; (2) lacked an interactive design, even though interactivity has been shown to positively influence factors such as information comprehensibility and attitudinal beliefs $[55,267]$ and; (3) did not explicitly include methods 
to help end users to become aware of what is important to them personally (in the DAliterature this is often referred to as value clarification [36]), even though this is regarded as an active DA-element [34,35]. Moreover, interestingly, this DA had a positive effect on smoking cessation success, but not on the uptake of cessation assistance [42]. Improving cessation assistance uptake might further increase the effectiveness of smoking cessation DAs on smoking cessation and overcoming the aforementioned limitations could play a promising role in achieving this.

To illustrate, given that a lack of knowledge is considered to be a barrier to cessation assistance utilization, adding interactive elements to a smoking cessation DA might be particular helpful as interactive elements can improve information comprehensibility and positively influence individuals' beliefs $[55,267]$. In addition to the use of interactive elements, explicitly devoting attention to smokers' personal values could also positively influence smoking cessation DAs' effects. The International Patient Decision Aid Standards (IPDAS) Collaboration regards so-called value clarification methods (VCMs) as an active DAelement $[34,35]$. Recently, scholars have started to pay more attention to studying the added value of so-called explicit (as opposed to implicit) VCMs. Explicit VCMs refer to exercises that actively engage users in an activity to clarify what is important to them personally (e.g., scoring certain statements), whereas implicit VCMs refer to the provision of static information that is specifically linked to the decision at hand-in other words, explicit VCMs include an element of interactivity that implicit VCMs lack. Previous studies showed that explicit VCMs seem to be more effective than implicit VCMs in terms of decision-making processes [32], especially in the long run [37] and when people are supported in understanding the implications of their clarified values $[39,40]$. One approach to facilitate understanding of the implication of clarified values is to show participants which options fit their clarified values best [40], e.g., by providing computer-tailored advice based on answers provided in the explicit VCM. However, so far it has not been studied if the addition of explicit VCMs paired with such advice will also positively impact on smoking cessation outcomes. To advance our understanding of the effectiveness of smoking cessation DAs and to support more people in the Netherlands to quit smoking successfully, we have developed a webbased smoking cessation DA (called VISOR) that includes interactivity and an explicit VCM paired with computer-tailored advice and studied its effects in a randomized controlled trial (RCT).

The Smoking Cessation DA VISOR

In accordance with the IPDAS guidelines for DA-development [59,96], VISOR was developed by a steering team ( $T G, E S, C D$, and $C H$ ) that lead a development process involving both professional experts and potential end users, e.g., by assessing their needs and opinions 
before the initial development, and by conducting usability tests - this development process is described in detail elsewhere [96].

Moreover, we used the Self-Determination Theory (SDT) [86] as theoretical background. The SDT resolves around the formation of motivation and posits that three psychological needs (i.e., the need for autonomy, relatedness, and competence) are essential to develop autonomous motivation, i.e., motivation that emanates from one self and is not primarily externally motivated $[86,166]$. Autonomous motivation is assumed to have a bigger influence on long-term behavior change compared to more controlled forms of motivation [91]. The SDT is particularly well-suited to support DA-developers due to its focus on perceived autonomy as opposed to theories that are often used to develop more persuasive interventions. DAs are similarly geared towards personal autonomy. The SDT therefore supported us in developing a DA that not only provided accurate information, but also helped to motivate end users, e.g., by framing the information in the DA autonomy-supportively. In the end, VISOR took a stepwise approach to facilitate autonomous decision making that is concordant with personal values and smoking behavior. VISOR consisted of eight sections that are described in Textbox 1. 
Textbox 1. Overview of VISOR's Sections.

1. Information section explaining the decision at hand, as well as all the cessation assistance available in the Netherlands.

2. Optional knowledge quiz.

3. Brief smoking assessment.

4. Intuitive decision between different clusters of cessation assistance tools:

- Behavioral support.

- $\quad$ NRTa

- Combination of behavioral support, NRT, and prescription medication.

- Combination of behavioral support and NRT.

- Combination of behavioral support and prescription medication.

- $\quad$ Other (non-evidence based) cessation assistance.

- No cessation assistance at all.

5. Intermediate advice to use a combination of behavioral and pharmacological cessation assistance tools, for users that:

- Chose behavioral support only, or NRT only, while also indicating that they smoke more than 10 cigarettes on a normal day, and/or have made one or more smoking cessation attempt(s) in the past (in step 3).

- Chose non-evidence-based cessation assistance or no cessation assistance at all in step 4 regardless of their answers in step 3.

6. Explicit VCM ${ }^{b}$ for users that chose evidence-based cessation assistance tools in step four or five, in which users are asked to rate certain statements regarding cessation assistance characteristics (e.g., "I prefer a stop method that works better, even if that means that I have to leave the house.").

- Users only rated statements for options that belong to the cluster of cessation assistance options they selected in the previous step(s).

7. Computer-tailored advice based on the explicit VCM, including an optional ranking of all options. Only when it was possible to give clear advice, i.e., if users' scores did not suggest that more than two cessation assistance tools were equally suitable based on their indicated values.

8. Access information on how to obtain the chosen cessation assistance (e.g., nicotine patches).

${ }^{a}$ NRT: nicotine replacement therapy, ${ }^{b}$ VCM: value clarification method

VISOR was a stand-alone, one-time intervention meant to support adult smokers in the general population in their decision to use smoking cessation assistance and did not have to be used together with a healthcare professional. That being said, VISOR could be used to prepare patients/clients for a healthcare consultation about smoking (cessation) and certain cessation assistance options (e.g., prescription medication) did require a healthcare provider to prescribe said options. Therefore, if VISOR-users chose to use a cessation assistance option that required prescription by a healthcare provider, they were advised to contact their healthcare provider to gain access to this specific option. More in-depth information on VISOR-including the specific theoretical underpinnings of each step-can be found 
elsewhere [96]. Examples of the information section and the explicit VCMs can be found in Figure 1 (information section) and Figure 2 (explicit VCMs). The screenshots have been translated from the original Dutch to English to facilitate understanding.

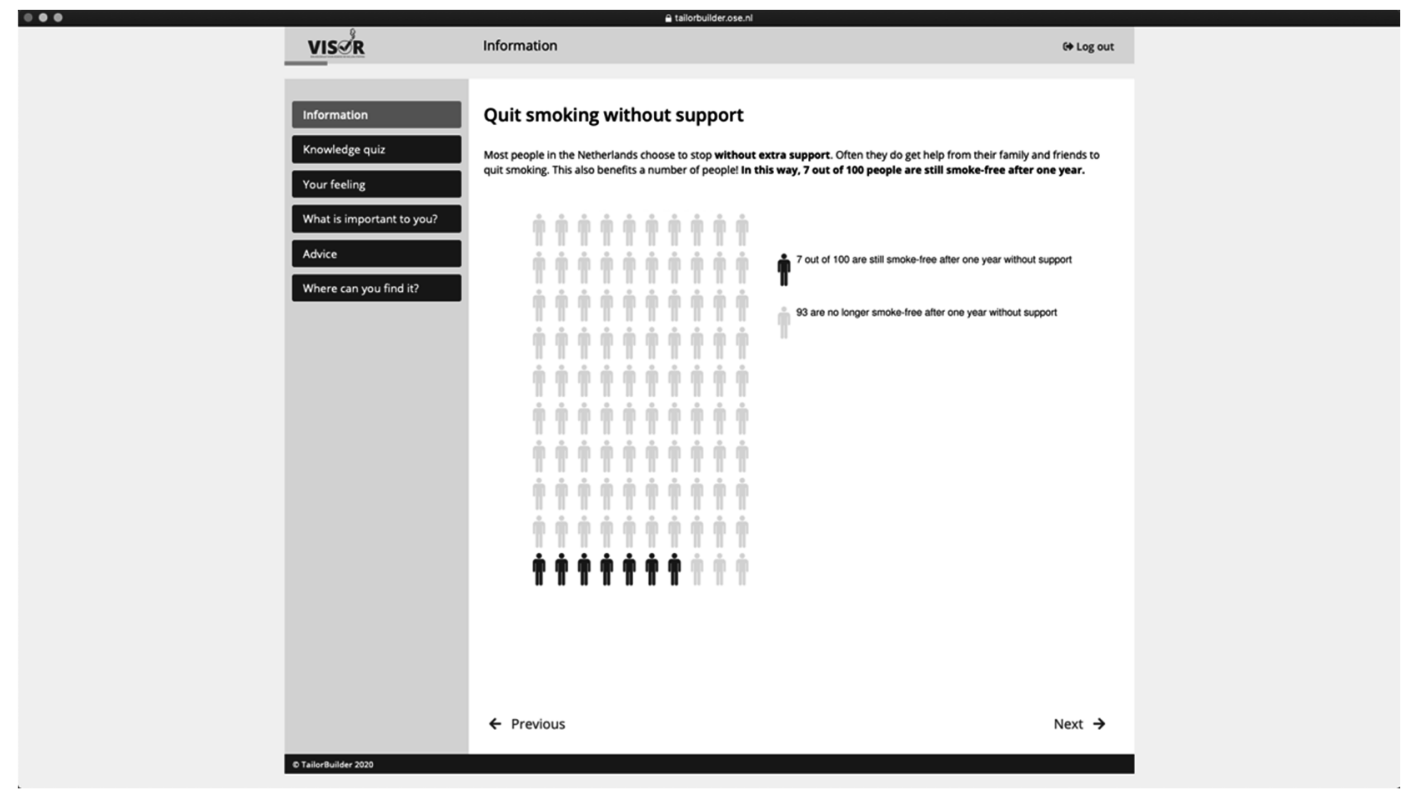

Figure 1. Screenshot of the Information Section in the Decision Aid (Original Text Translated From Dutch); the Displayed Icon Array Has Been Created Using IconArray.com [207]. 


\section{Statements behavioral support}

\section{The following statements are about behavioral support.}

I prefer a stop method that works better, even if that means that I have to leave the house.

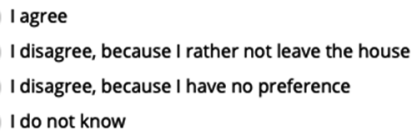

I prefer not to leave the house for a stop method, even though it means I can only use stop methods that work less well.

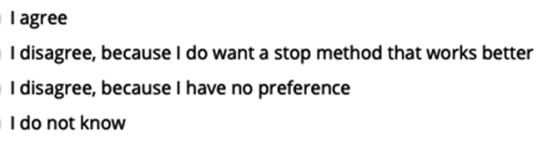

I would rather use a stop method with a trained health-care provider or stop coach than a stop method without a trained health-care provider or stop coach.

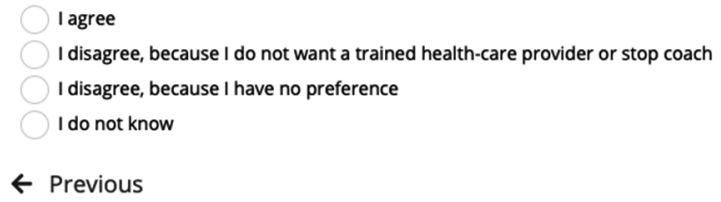

Figure 2. Screenshot of a Part of the Value Clarification Method (VCM) in the Decision Aid (Original Text Translated From Dutch).

\section{Study Goals and Hypotheses}

In this article, the behavioral (i.e., smoking abstinence and cessation assistance use) and decisional effects (i.e., decisional conflict) of an explicit VCM paired with computer-tailored advice (within a smoking cessation DA) will be reported. An RCT was carried out to investigate these effects. Specifically, we set out to test the following hypotheses (as described in the study protocol [96] and the Netherlands Trial Register [NL8270]):

$\mathrm{H}_{1 \mathrm{a} / \mathrm{b}}$ is that a DA with an explicit VCM and computer-tailored advice will lead to a statistically significant increase in smoking abstinence after one month $\left(\mathrm{H}_{\mathrm{a}}\right)$ and six months $\left(\mathrm{H}_{\mathrm{b}}\right)$ compared to a DA without an explicit VCM and computer-tailored advice.

$\mathrm{H}_{2 \mathrm{a} / \mathrm{b}}$ is that a DA with an explicit VCM and computer-tailored advice will lead to a statistically significant increase in evidence-based cessation assistance use after one month $\left(\mathrm{H} 2_{\mathrm{a}}\right)$ and six months $\left(\mathrm{H} 2_{\mathrm{b}}\right)$ compared to a DA without an explicit VCM and computer-tailored advice. 
$\mathrm{H}_{3}$ is that a DA with an explicit VCM and computer-tailored advice will lead to a statistically significant decrease in decisional conflict (state of uncertainty about which course of action to take) right after the DA compared to a DA without an explicit VCM and computer-tailored advice.

\section{Methods}

An RCT in line with the CONSORT-EHEALTH checklist was conducted [220]. However, it should be noted that we deviated from the checklist in one respect: Participants were only invited for the follow-up measurements if they completed the DA(s), that is, if they completed the intervention until the end. This was done as we wanted to ensure that the participants, that we included for the analysis, received the additional intervention elements-as those were the focus of our RCT.

The study did not fall under the scope of the Medical Research Involving Human Subjects Act as indicated by the Medical Ethics Committee Zuyderland, the Netherlands (16-N-227), and the development of VISOR and the accompanying studies (e.g., the aforementioned needs assessment [268]) were funded by the Dutch Cancer Society, UM2015-7744. Study materials (including the underlying data and statistical scripts) can be found on the on the open science framework (OSF) website [269].

\section{Changes From the Study Protocol}

In the original study protocol, and as such described in the Netherlands Trial Register, it was planned to contact participants four times after having used VISOR (i.e., directly after the DA, after one month, six months, and 12 months). Unfortunately, this was not possible as we had to extend our recruitment period due to the Coronavirus disease 2019 (COVID-19) pandemic and consequently, the recruitment period lasted for approximately 12 months, i.e., six months longer than initially planned. Consequently, given the maximum project duration funded by the Dutch Cancer Society, we had to drop the 12-month follow-up measurement. Therefore, the original primary outcome (i.e., seven-day point prevalence after 12 months) had to be adjusted and, ultimately, seven-day point prevalence after six months was used as the primary outcome.

Furthermore, due to a technical mistake, some participants who completed VISOR, but had not completed $t=1$ (directly after the decision aid), did not receive an automatic invite for the other follow-ups. At the time this mistake was discovered, some participants were already lost to follow-up. We dealt with this situation in two different ways: (1) People who had already missed $t=2$ but completed the DA in the three months prior to the discovery of the mistake received the invite to participate in $t=2$ regardless $(n=38$ received this 
invitation, ultimately, $n=3$ made use of this); (2) people who already missed $t=3$ were still invited to participate in $\mathrm{t}=3$ but not $\mathrm{t}=2(n=130$ received the invitation, ultimately, $n=5$ made use of this).

Lastly, we had originally planned to only adjust our analyses for covariates that were selected a priori if those were actually associated with the outcomes. However, based on the advice of the involved statistician (SJ), we decided to adjust our analyses for all selected covariates a priori (as described in the study protocol [96]).

\section{Primary and Secondary Outcomes}

The primary outcome of this study was (as described in Changes from the study protocol) seven-day point prevalence after six months (corresponding to $\mathrm{H}_{1 \mathrm{~b}}$ ). Secondary outcomes were seven-day point prevalence after one month (corresponding to $\mathrm{H}_{1 \mathrm{a}}$ ), evidence-based cessation assistance use after one month and six months (corresponding to $\mathrm{H}_{2 \mathrm{a} / \mathrm{b}}$ ), and decisional conflict directly after the decision aid (corresponding to $\mathrm{H}_{3}$ ).

\section{Sample Size}

An a priori power calculation was conducted based on the primary outcome measure of seven-day point prevalence abstinence (after 12 months originally, see Changes From the Study Protocol) and the only other RCT in the Netherlands testing the effect of a smoking cessation DA in which a significant effect (20.2\% vs $13.6 \%)$ at six months was found [42]. To be able to significantly $(\alpha=.05 ; \beta=.20$ ) detect the same effect in a one-sided test, 398 smokers per arm were necessary at the end of the trial (796 in total). Considering 50\% attrition over the study period, we had aimed to include 1592 smokers at baseline.

\section{Study Population}

Participants could be included if they were (1) currently smoking, (2) motivated to stop smoking within six months, (3) between 18-100 years old, (4) able to understand Dutch, and (5) had access to the internet and the necessary internet literacy (skills) to use the DA. This last inclusion criterion was not actively screened for but was deemed inherent to participation given the fully online set-up of VISOR and the RCT. Participants were excluded if they did not meet these inclusion criteria and/or if they exclusively used e-cigarettes. As described in the study protocol [96] participants were mainly recruited online to reflect the online nature of VISOR and the entire trial was web-based (i.e., there were no offline contacts with the study team). Recruitment took mainly place by making use of paid social media advertisements and 'regular' social media posts on project accounts (e.g., [222]) that were also shared on accounts of the team members, their respective institutions, and other 
relevant organizations within the Netherlands (e.g., via Twitter's retweet-function). Additionally, VISOR was also featured in regional media (e.g., a newspaper interview) and we used a project website that provided the direct access point to VISOR via a clickable button that was placed prominently on the website. Due to a too small influx of participants (especially after the start of the COVID-19 pandemic), we decided to deploy other additional recruitment activities as well, e.g., a study call/information in a relevant Dutch journal for general practitioners in which readers were informed about VISOR and the accompanying RCT [270] and using student pools at the University of Amsterdam and Maastricht University. All (potential) participants received information about the content of VISOR, the duration of the study (including the amount of follow-up measurements) and the compensation that they received for completing the last measurement ( $€ 10$, which approximately equates to US \$12.17 at the time of writing). Throughout the entire study, participants also received information on the duration of VISOR and the questionnaires. Participants received no information regarding the differences between the intervention group and the control group as to not bias the results of the trial. University of Amsterdam students received research credits instead of a monetary compensation. All recruitment materials (e.g., the project website) included a display of the project team's institutional affiliations in some form.

\section{Intervention and Comparator}

Participants in the intervention group received the DA as described in the introduction (see The Smoking Cessation DA VISOR), whereas participants in the control group received the same DA excluding the explicit VCM and computer-tailored advice, i.e., for them steps 6 and 7 described in Textbox 1 were skipped. The only other (small) difference was that participants in the intervention group were immediately directed towards the end after they had chosen to use no evidence-based cessation assistance (i.e., step 8-access information-was skipped), which was not the case for the control group. This way both groups had a chance to reevaluate their choice as the intervention group was offered a chance to reevaluate their choice during the additional elements. Neither the DA received by the intervention group, nor the DA received by the control group changed throughout the trial. More information can be found in the study protocol [96].

\section{Trial Flow and Measurement Instruments}

In total, the study consisted out of four fully automated and web-based contact moments: $\mathrm{t}=0$ the baseline questionnaire and VISOR, $\mathrm{t}=1$ directly after participants had used VISOR, $\mathrm{t}=2$ after one month, and $\mathrm{t}=3$ after six months. Participants were asked to fill in each followup questionnaire if they made use of the entire DA, even when they did not fill in one of the other follow-up questionnaires. To avoid high attrition rates, participants received either one automatic reminder after a week (if they had not filled in a follow-up questionnaire at all) or 
two after two days and a week (if they already started filling in at least part of a follow-up questionnaire). Participants that started using VISOR or started filling in the baseline questionnaire $(t=0)$ without finishing it also received two automatic reminders (after two days and a week). In the last reminder for $t=3$, participants were also offered the option to only share their answer regarding the primary outcome (i.e., seven-day point prevalence abstinence) with the research team. All data were self-assessed. If available, we used previously validated measurements (e.g., [4]) and measurements that were used in a Dutch context before (e.g., [174]), if possible we used measurements that were used in selfadministered online studies before $[46,71]$. In the case of psychological constructs that were collected using multiple items (e.g., decisional conflict) we assessed scale quality as proposed by Crutzen \& Peters [244] using the Rosetta Stats package in R [248,271] in two steps: (1) investigating scale structure by exploratory factor analysis, and (2) calculation of omega $(\Omega)$ [245] as a less biased alternative to (Cronbach's) alpha ( $\alpha$ ).

Participants registered for the study via an online form, which included their provision of informed consent and the creation of an account. Before the account creation participants were automatically randomized into either the intervention or the control group by the online platform on which questionaries and VISOR were hosted, allocating approximately $50 \%$ of the respondents to either group. End users were blinded to which group they were allocated to. Immediately after their registration, participants were asked to fill in the baseline questionnaire.

Baseline Measurements: Directly Before the DA

Demographic information was collected based on three criteria: age, gender, and education. Smoking behavior was collected regarding tobacco products used, amount of tobacco consumption per product per day, past cessation attempts, amount of past cessation attempts (for people that ever attempted to stop smoking), and cessation assistance utilization in the past six month. If participants indicated that they have utilized cessation assistance they were also asked what has been used. More information regarding demographic information and smoking behavior can be found in Appendix A.

Nicotine dependence was measured with the Revised Fagerström Test for Nicotine Dependence (FTND-R) which has shown better psychometric properties than the unrevised version [236] and was verified to be unidimensional within our sample $(\Omega=.76$, more information can be found on the OSF [269]). We changed the wordings of the items slightly (e.g., "smoking moment" instead of "cigarette") to include other tobacco products as well and initially left out the item relating to the amount of cigarette consumption. Rather we created a composite score based on participants' answers to the item about amount of tobacco products - expressed as number of cigarettes, with one hand-rolled cigarette, and 
one other, or cannabis product equaling one cigarette, one pipe equaling two and a half cigarettes, and one cigar equaling four cigarettes [174,272]. Afterwards, this composite score was recoded in line with the FTND-R $(0=0-10$ cigarettes, $1=11-20$ cigarettes, $2=21-$ 30 cigarettes, $3=>30$ cigarettes). We had to exclude e-cigarette usage for this composite score as it was unclear how the answer categories of the e-cigarette usage item relate to the amount of cigarette consumption. Afterwards, a composite score was created by summing this item with the other five FTND-R items resulting in one FTND-R score per individual ranging from $0-16$, with 0 indicating no dependence.

Finally, stage of decision making was measured with one item ("Have you thought about how to quit smoking at this point? Choose the answer that best suits your situation.; 1 = I haven't begun to think about the choices, $2=$ I haven't begun to think about the choices, but am interested in doing so, $3=1$ am considering the options now, $4=1$ am close to selecting an option, 5 = I have already made a decision, but am still willing to reconsider, 6 = I have already made a decision and am unlikely to change my mind") [240].

Follow-up t $=1$ : Directly After the DA

After the DA, we measured decisional conflict (secondary outcome) using the decisional conflict scale [4] which was also verified to be unidimensional in our sample ( $\Omega=.98$, more information can be found on the OSF [269]). We employed all 16 items using the original statement format with five response categories ( $0=$ strongly agree, $1=$ agree, $2=$ neither agree or disagree, 3 = disagree, 4 = strongly disagree) and created a composite score as described in the user manual provided by O'Connor [7]: Individuals' scores were (1) summed, (2) divided by 16 , and (3) multiplied by 25 . Thus, every participant had a score ranging from 0 (no decisional conflict) to 100 (extremely high decisional conflict). Scores exceeding 37.5 are generally regarded to be associated with decision delay or being unsure about decision implementation [7].

Follow-ups $t=2$ and $t=3$ : One and Six Month(s) After Baseline

After one month and six months, participants were queried regarding the choice made (i.e., the implemented decision; secondary outcome) and if they were able to abstain from smoking in the previous seven days (i.e., seven-day point prevalence abstinence; primary outcome).

\section{Data Analysis}

All analyses were carried out in R [248] using the integrated development environment RStudio [249]. First, descriptive analyses were conducted to assess sample characteristics. 
Second, in order to find out which factors influenced non-usage attrition (i.e., attrition during the intervention; using a variable denoting that the end of the intervention has been reached as an indicator) and dropout attrition (i.e., not returning to the follow-ups; using the same principle as for non-usage attrition) [273] we first compared participants at baseline that did not finish VISOR with the participants that finished VISOR using Wilcoxon rank sum test (for continuous and ordinal non-normally distributed data with equal variances between groups), Mood's median test (for continuous and ordinal non-normally distributed data without equal variances between groups), chi-squared tests (for categorical variables where all expected numbers were greater than five), or Fisher's Exact Tests (for categorical variables where not all expected numbers were greater than five). Afterwards we checked the results of these univariate analyses, by employing logistic regression with non-usage attrition as the outcome and all significant variables from the univariate analyses as predictors. Regarding dropout attrition we then compared participants at $t=1, t=2$, and $t=3$ to the participants lost to follow-up (after having used the intervention) using the same approach as described above.

Third, to test $\mathrm{H}_{1 \mathrm{a} / \mathrm{b}}$ and $\mathrm{H}_{2 \mathrm{a} / \mathrm{b}}$ logistic regressions were employed. First, we conducted crude analyses in which we only included the allocated group (i.e., intervention group compared to the control group) as a predictor and seven-day point prevalence abstinence as the outcome, followed by fully adjusted analyses in which we corrected for age, gender, education, and the FTND-R [96,251]. All covariates were selected a priori $[96,250,251]-$ however, due to multicollinearity issues in some of the adjusted models testing $\mathrm{H}_{2 \mathrm{a} / \mathrm{b}}$ we needed to recode the educational variable into low and high, instead of low, medium, and high (as for the other analyses) - this was only done for the models where this was the case. Also, due to the size of two very small gender (identity) groups (i.e., non-binary participants, and participants who preferred not to state their gender) we were unable to include participants belonging to these groups in all adjusted models. We therefore decided to include the data of those participants in the crude models, but not in the adjusted models. To test the robustness of the results, the logistic regressions were conducted according to three different approaches: (1) complete cases only, (2) worst-case scenario (dropout respondents are considered to still smoke, i.e., penalized imputation), and (3) multiple imputations (MI) using the mice package [274]. Variables that were included in the imputation model can be found in Appendix B. In the MI-models we accounted for the technical mistake described in Changes From the Study Protocol. As we had directional hypotheses we conducted one-sided tests as planned a priori [96]. To calculate the $P$-values, we used the following formulas: (1) $P$-value/ 2 if the effect moved into the hypothesized direction and (2) 1-( $P$-value/2) if the effect moved into the other direction. Similarly, we swapped the upper bound of the calculated confidence intervals with infinity. This was only done for the intervention effect as we had no specific directional hypotheses regarding the covariates. 
Fourth, $\mathrm{H}_{3}$ was tested by employing linear regression. Again, by starting with crude analyses using the allocated group as a predictor and the decisional conflict scale as the outcome, followed by fully adjusted analyses corrected for covariates selected a priori (i.e., age, gender, education, and stage of decision making) $[96,250,251]$. Again, we had to exclude certain participants from the analyses due to them belonging to too small gender (identity) groups. Regarding $\mathrm{H}_{3}$ we only used (1) complete cases only and (2) Ml using the mice package [274]. Variables that were included in the imputation model can be found in Appendix $C$. Again, we calculated the values for the one-sided tests as described above, but as we expected a negative effect, we swapped the lower bound of the calculated confidence intervals with infinity. In complete case analyses all participants were included that filled in the respective outcome measure (e.g., everyone that filled in the entire decisional conflict scale) even when said participants did not finish the entire follow-up questionnaire.

\section{Results}

Sample

The total sample consisted out of 2375 participants that were randomized of which 1164 participants completed the baseline questionnaire. Subsequently, 599 participants completed the DA(s), 276 participants then filled in $t=1$ completely, 97 participants filled in $\mathrm{t}=2$ completely, and 103 participants filled in $\mathrm{t}=3$ completely. The entire trial flow can be seen in Figure 3. The characteristics of participants that finished the baseline questionnaire can be seen in Table 1. 


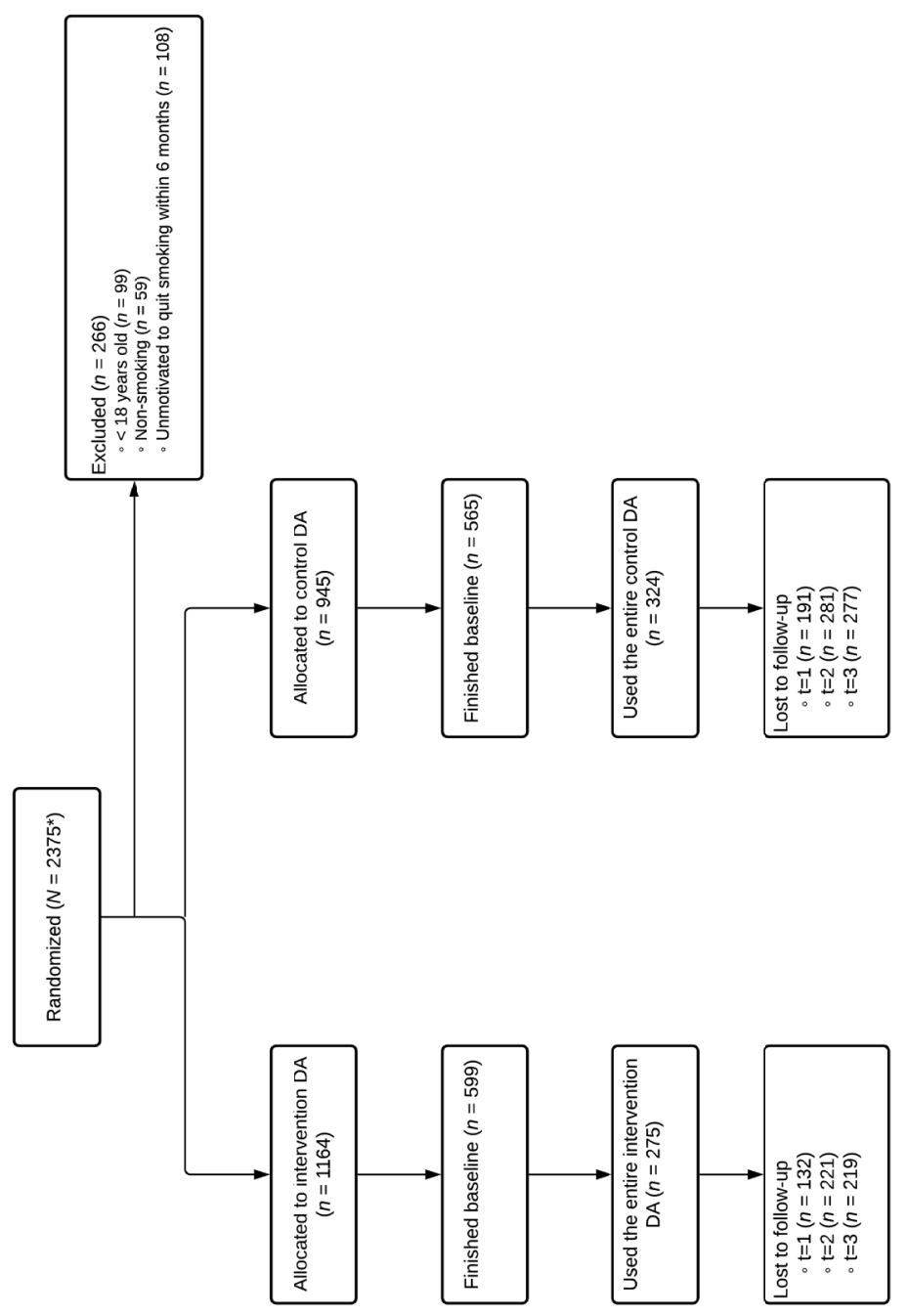

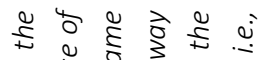

$\leq$ गे

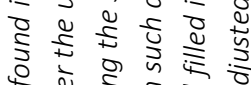

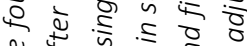

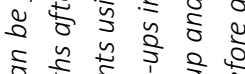

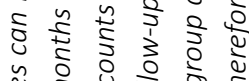

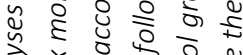

वे क⿺辶一兀)

ठ 11 胥

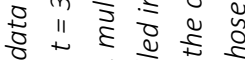

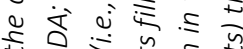

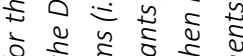

के है के ई

ㅇำ 0 के

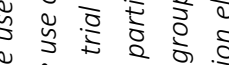

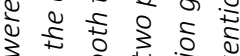

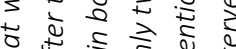

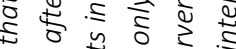

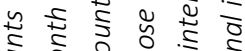

ठै वे

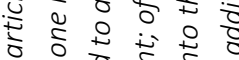

व.

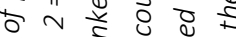

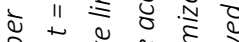

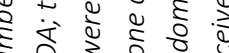

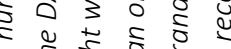

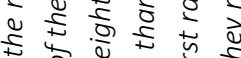

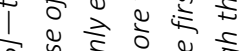

रू जे है है के

ㄱำ

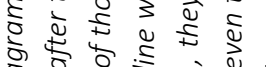

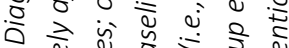

उ के

प्रे के के के के के

व है

11

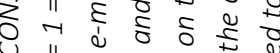

० $\leftarrow$ है

స के

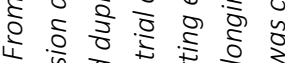

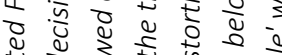

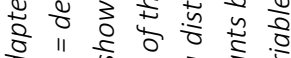

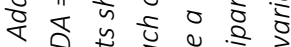

उे iो

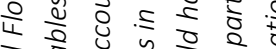

के

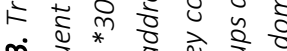

mं

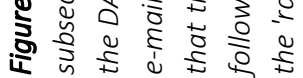




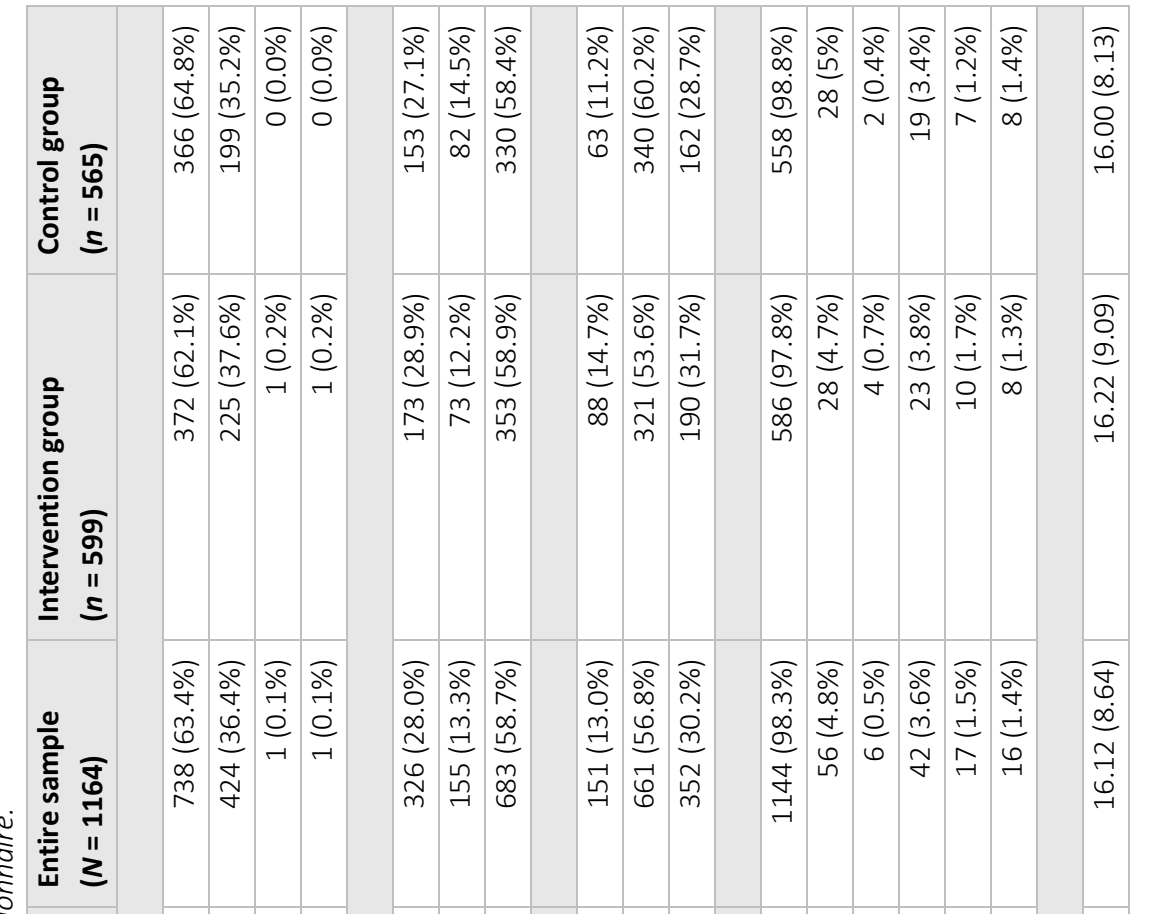




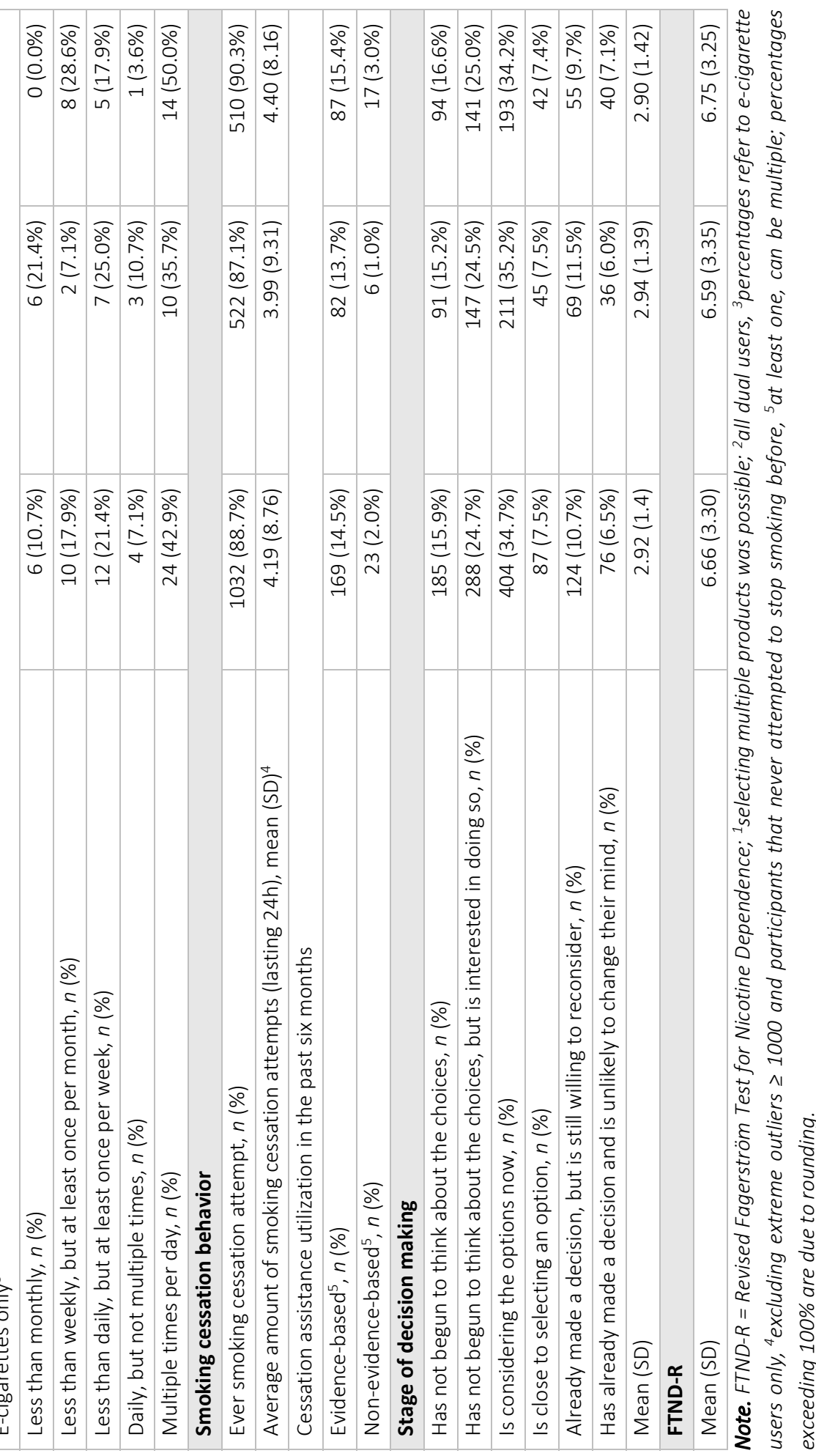




\section{Attrition}

Non-Usage Attrition

Comparisons of the participants who did not complete the DA(s) and those who did showed significant differences in group allocation, $X^{2}(1, N=1164)=15.22, P<.001$; participants' age, $X^{2}(2, N=1164)=78.32, P<.001$; level of education, $X^{2}(2, N=1164)=12.48, P=.002$; if people used other tobacco products, $X^{2}(1, N=1164)=4.55, P=.03$; the average amount of cessation attempts ( $W=121312, P=.02)$; and participants' stage of decision making at baseline ( $\mathrm{W}=153400, P=.004)$. All comparisons can be found in Appendix $D$. In the logistic regression model only having been allocated to the intervention group (OR $=1.65,95 \% \mathrm{Cl}$ $1.28-2.14, P<.001$ ), being between $24-29$ years (compared to $18-23$ years) old ( $O R=0.60$, $95 \% \mathrm{Cl} 0.38-0.93, P=.02$ ), being between 30-100 years (compared to 18-23 years) old (OR $=0.29,95 \% \mathrm{Cl} 0.21-0.39, P<.001)$, and a high (compared to a low) level of education ( $\mathrm{OR}=$ $0.64,95 \% \mathrm{Cl} 0.42-0.99, P=.046$ ) remained significant; i.e., participants in the intervention group were more likely not to complete VISOR, while those that were 24-100 years (compared to 18-23 years) old, and those with a high (compared to a low) level of education were more likely to complete VISOR.

\section{Dropout Attrition}

Comparisons of the participants who did not complete $t=1$ and those who did showed significant differences in group allocation, $X^{2}(1, N=599)=7.18, P=.01$; gender, $X^{2}(1, N=$ $598)=5.85, P=.02$; and stage of decision making, $W=37942, P=.001$. All comparisons can be found in Appendix E. All variables remained significant in the logistic regression model: having been allocated to the intervention group $(\mathrm{OR}=0.65,95 \% \mathrm{Cl} 0.47-0.90, P=.01)$, men compared to women $(\mathrm{OR}=0.65,95 \% \mathrm{Cl} 0.46-0.92, P=.01)$, and stage of decision making (OR $=0.82,95 \% \mathrm{Cl} 0.72-0.92, P=.001)$; i.e., participants in the intervention group, men (compared to women), and those in higher stages of decision making were less likely to dropout.

Comparisons of the participants who did not complete $t=2$ and those who did showed significant differences in group allocation, $X^{2}(1, N=599)=4.44, P=.04$; and stage of decision making, $W=21158, P=.03$. All comparisons can be found in Appendix $F$. In the logistic regression model only having been allocated to the intervention group remained significant $(\mathrm{OR}=0.63,95 \% \mathrm{Cl} 0.41-0.98, P=.04)$; i.e., participants in the intervention group were less likely to dropout.

Comparisons of the participants who did not complete $t=3$ and those who did only showed significant differences in stage of decision making, $W=21601, P=.01$, which also remained significant in the logistic regression $(\mathrm{OR}=0.84,95 \% \mathrm{Cl} 0.72-0.97, P=.02)$; i.e., participants in 
higher stages of decision making were less likely to dropout. All comparisons can be found in Appendix G.

As next to the demographical variables (that were already planned as covariates for all analyses) and group allocation, only stage of decision making was most consistently associated with dropout; we decided to include stage of decision making as a covariate for all analyses as well-and not only decisional conflict as planned in the protocol [96].

\section{Hypotheses Testing}

$\mathrm{H}_{1 \mathrm{a}}$ : Smoking Cessation After One Month

While it was observed that more participants stopped smoking in the intervention group ( $n$ $=15 ; 27.3 \%$ of the respondents) than in the control group ( $n=7 ; 15.2 \%$ of the respondents) after one month, the intervention did not result in a significant effect on smoking cessation in the complete case analyses ( $P=.07$ [crude]; $P=.13$ [adjusted]) or the Ml-analyses $(P=.25$ [crude]; $P=.24$ [adjusted]). However, in the worst-case scenario effects in favor of the intervention group could be observed ( $P=.02$ in both the crude model and adjusted model). In two of the three adjusted models (i.e., complete cases and worst-case scenario), the only (other) variable that had a significant effect on smoking cessation was stage of decision making $(P=.004$ [complete cases analysis]; $P=.001$ [worst-case scenario]). In the Mlscenario none of the included variables had a significant effect on smoking cessation rates after one month. $\mathrm{H}_{1}$ could therefore only be confirmed in the worst-case scenario. See Table 2 for all results and more information.

$\mathrm{H}_{1 \mathrm{~b}}$ : Smoking Cessation After Six Months

While it was, again, observed that after six months more participants reported to have stopped smoking in the intervention group ( $n=23 ; 36.5 \%$ of the respondents) than in the control group ( $n=14 ; 26.9 \%$ of the respondents), the intervention did not result in a significant effect on smoking cessation in the complete case analyses ( $P=.14$ [crude]; $P=.30$ [adjusted]) or the Ml-analyses ( $P=.23$ [crude]; $P=.33$ [adjusted]). Similar to the results based on data collected after one month, effects in favor of the intervention group could be observed in the worst-case scenario ( $P=.02$ [crude]; $P=.04$ [adjusted]). In the worst-case scenario, the FTND-R ( $P=.02$, i.e., nicotine dependence) and stage of decision making $(P=$ .01) had a significant effect on smoking cessation rates. However, the FTND-R violated the linearity assumption in this model, dropping it did not change the conclusions in relation to the primary outcome (see Appendix $H$ ). In the complete case analyses and the MI-scenario none of the included variables had a significant effect on smoking cessation rates after six 


\section{Chapter 7}

months. $\mathrm{H}_{1 b}$ could therefore only be confirmed in the worst-case scenario. See Table 2 for all results and more information. 


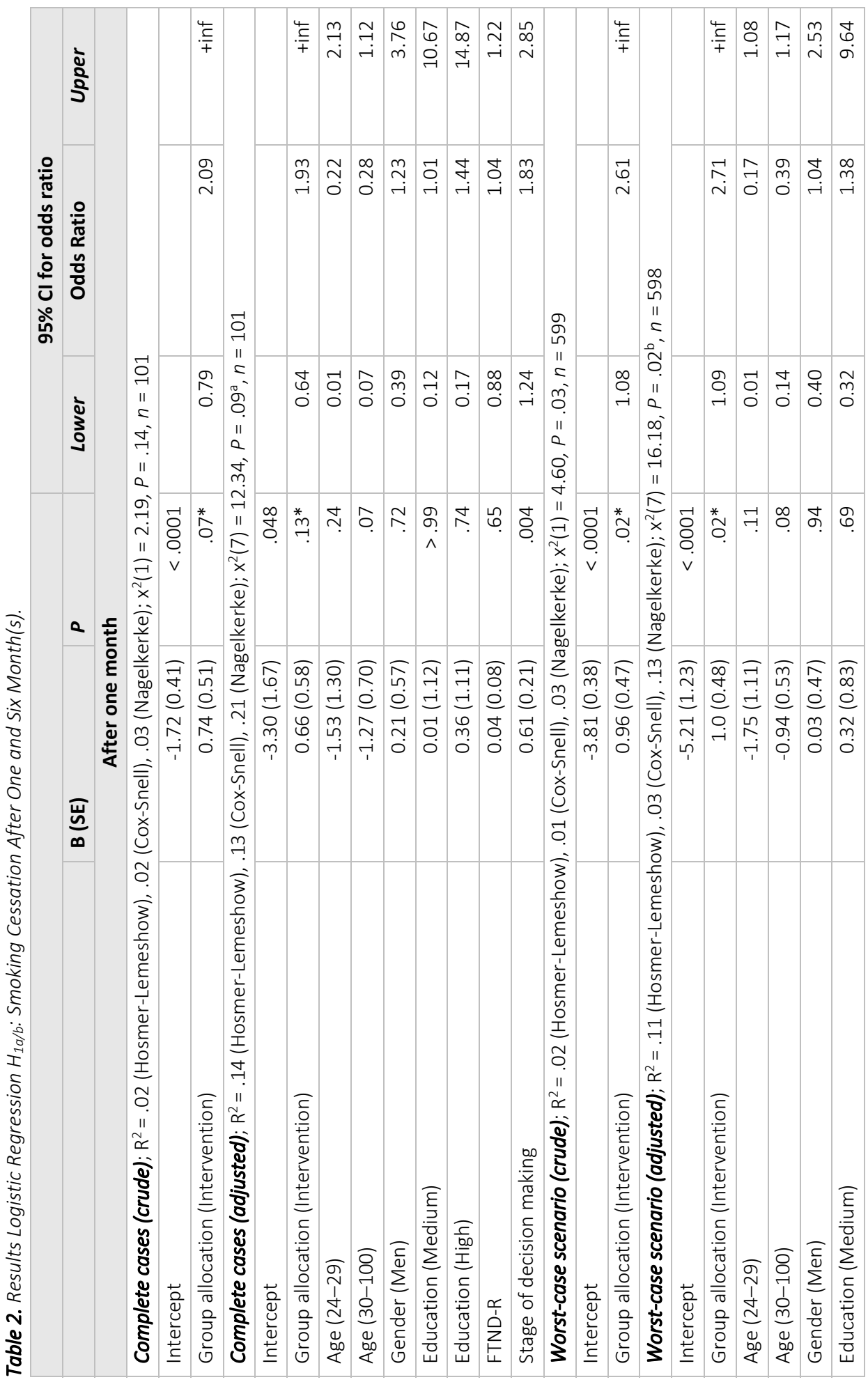




\begin{tabular}{|c|c|c|c|c|}
\hline$\stackrel{\sim}{\sim} \underset{\sim}{\sim} \underset{\sim}{\sim} \underset{N}{N}$ & $\stackrel{4}{+\frac{4}{+}}$ & 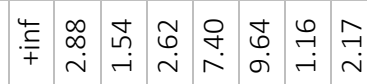 & $\frac{4}{+}$ & 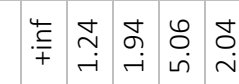 \\
\hline 䒹兽 & 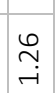 & 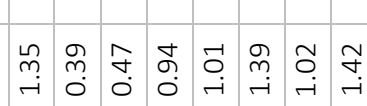 & 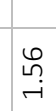 & 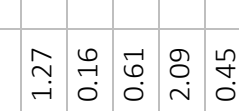 \\
\hline
\end{tabular}

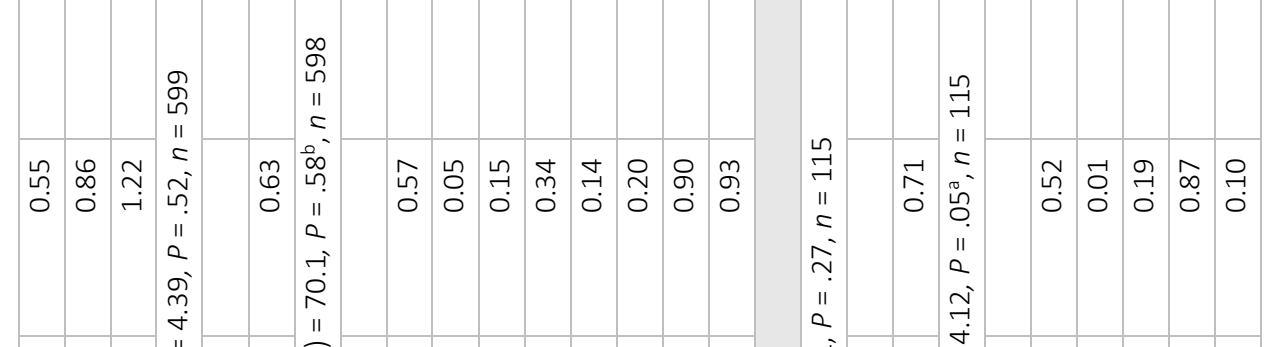

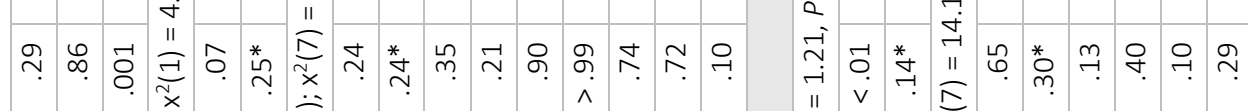

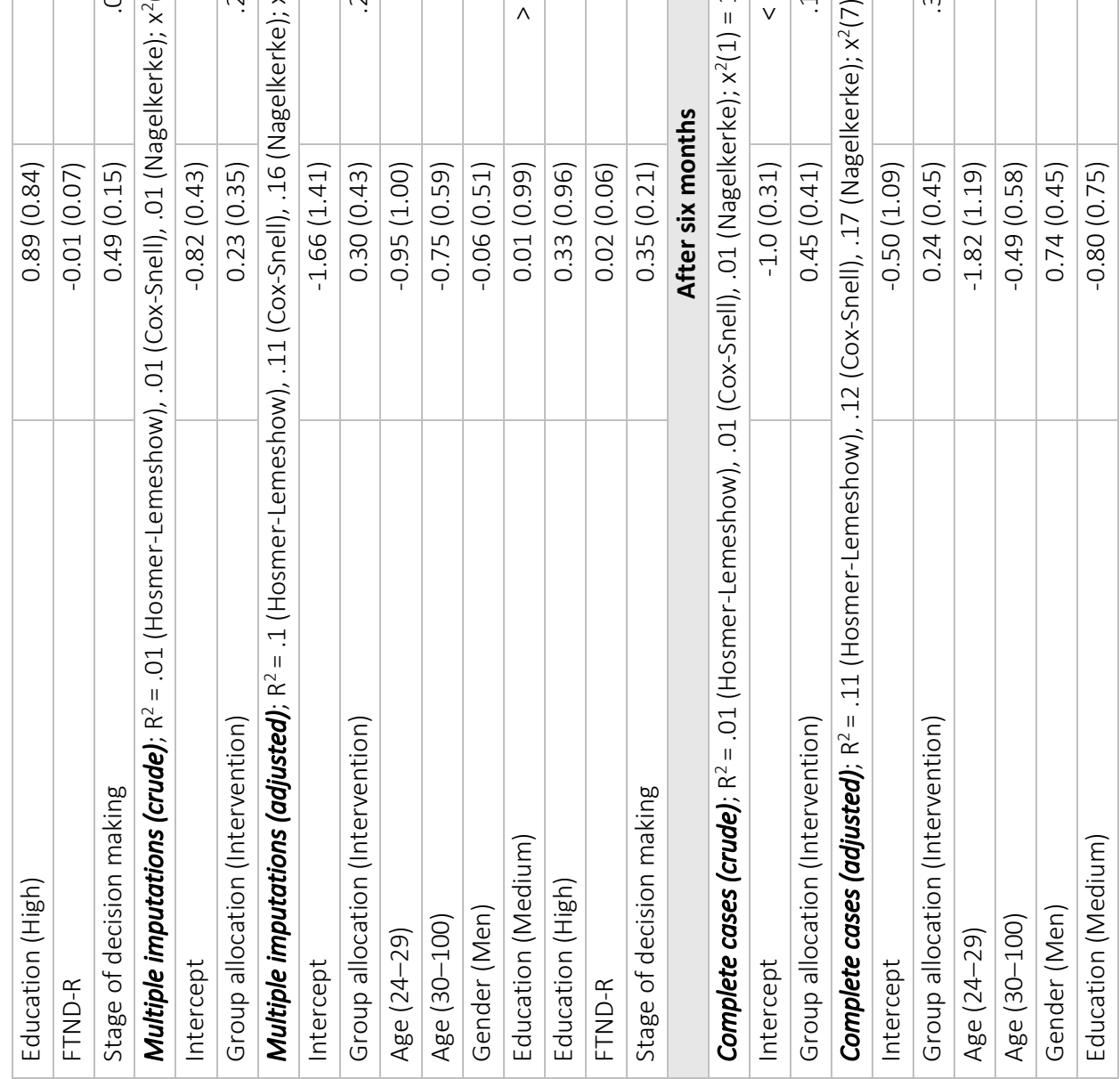




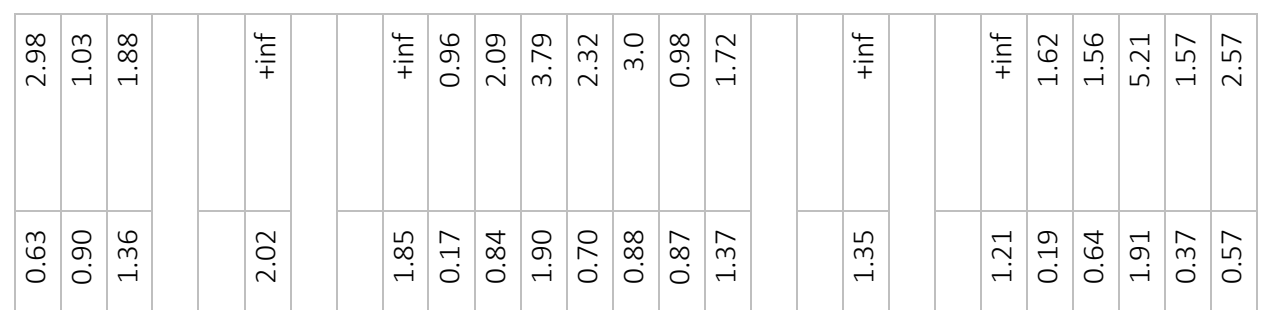
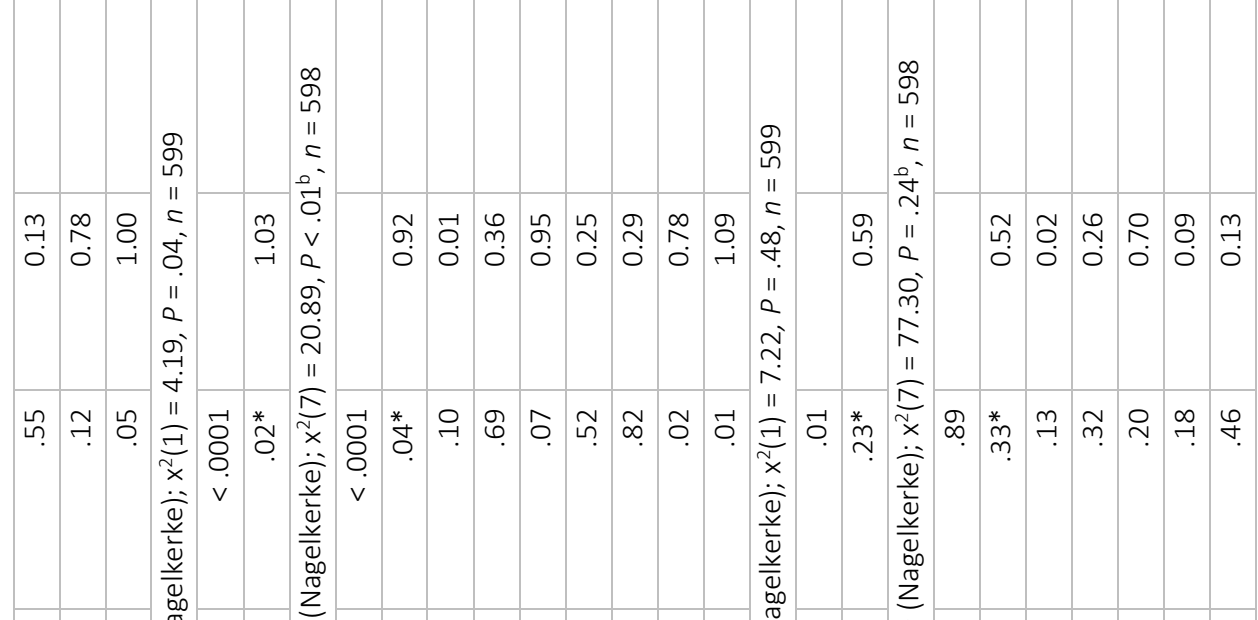

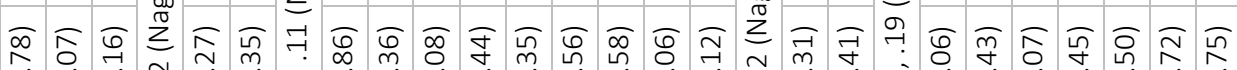

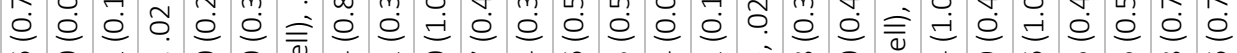

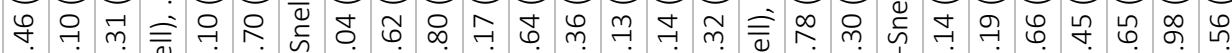

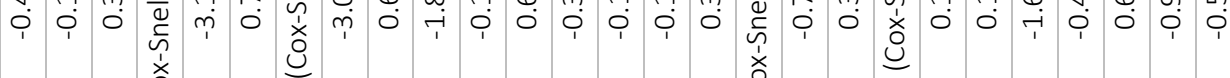

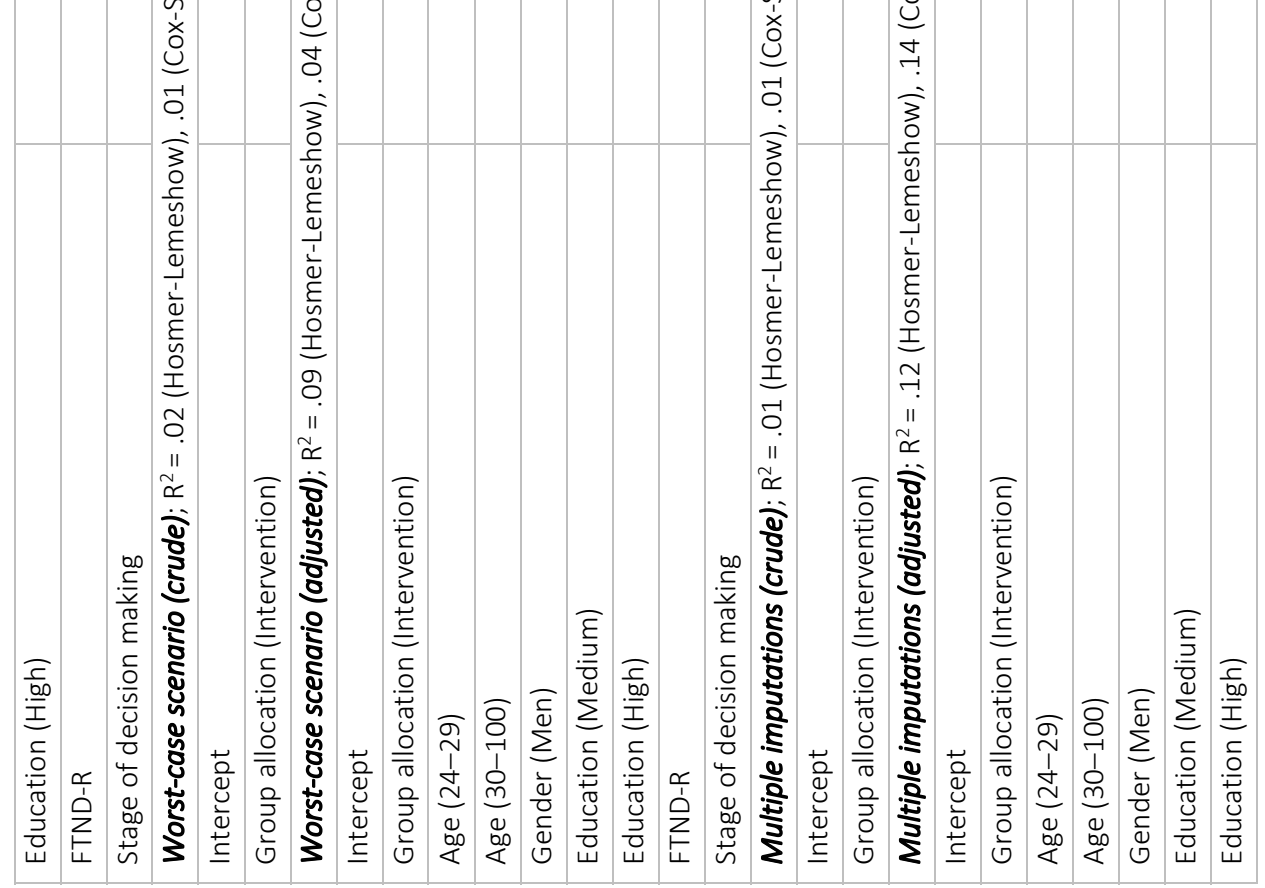




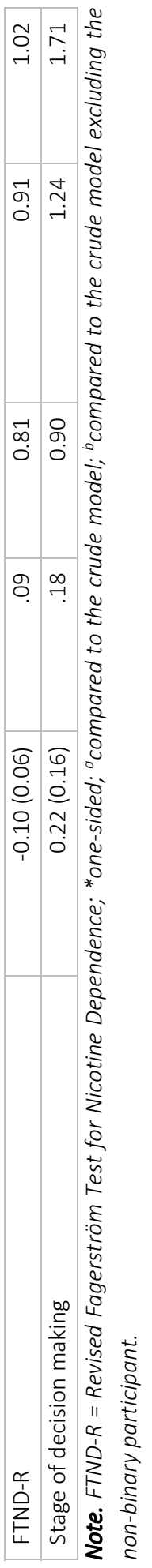


$\mathrm{H}_{2 \mathrm{a}}$ : Use of Evidence-Based Cessation Assistance After One Month

While after one month more people in the intervention group reported to have used an evidence-based cessation assistance tool ( $n=26,46.4 \%$ of the respondents) compared to the control group ( $n=18,38.3 \%$ of the respondents), the intervention did not result in a significant effect on the uptake of evidence-based cessation assistance in the complete case analyses $(P=.20$ [crude]; $P=.21$ [adjusted]) or the Ml-analyses $(P=.23$ [crude]; $P=.20$ [adjusted]). Similar to smoking cessation, effects in favor of the intervention group appeared in the worst-case scenario, but only in the crude model ( $P=.04$ [crude]; $P=.05$ [adjusted]). In the adjusted models, again, only stage of decision making had a significant effect on the outcome $(P=.01$ in the complete cases analysis; $P=.005$ in the worst-case scenario; $P=.02$ in the Ml-analysis). $\mathrm{H}_{2 a}$ could therefore only partially be confirmed in the worst-case scenario. See Table 3 for all results and more information.

$\mathrm{H}_{2 b}$ : Use of Evidence-Based Cessation Assistance After Six Months

Similarly, while after six months more people in the intervention group reported to have used an evidence-based cessation assistance tool ( $n=38,62.3 \%$ of the respondents) compared to the control group ( $n=26,52.0 \%$ of the respondents), the intervention did not result in a significant effect on the uptake of evidence-based cessation assistance in the complete cases analyses $(P=.14$ [crude]; $P=.11$ [adjusted]) or the Ml-analyses $(P=.07$ [crude]; $P=.13$ [adjusted]). However, in the worst-case scenario, effects in favor of the intervention group emerged ( $P=.01$ [crude]; $P=.02$ [adjusted]). In the adjusted models, again, stage of decision making had a significant effect on the outcome, but only in the worstcase scenario $(P=.01) . \mathrm{H}_{2 \mathrm{~b}}$ could therefore only be confirmed in the worst-case scenario. See Table 3 for all results and more information. 


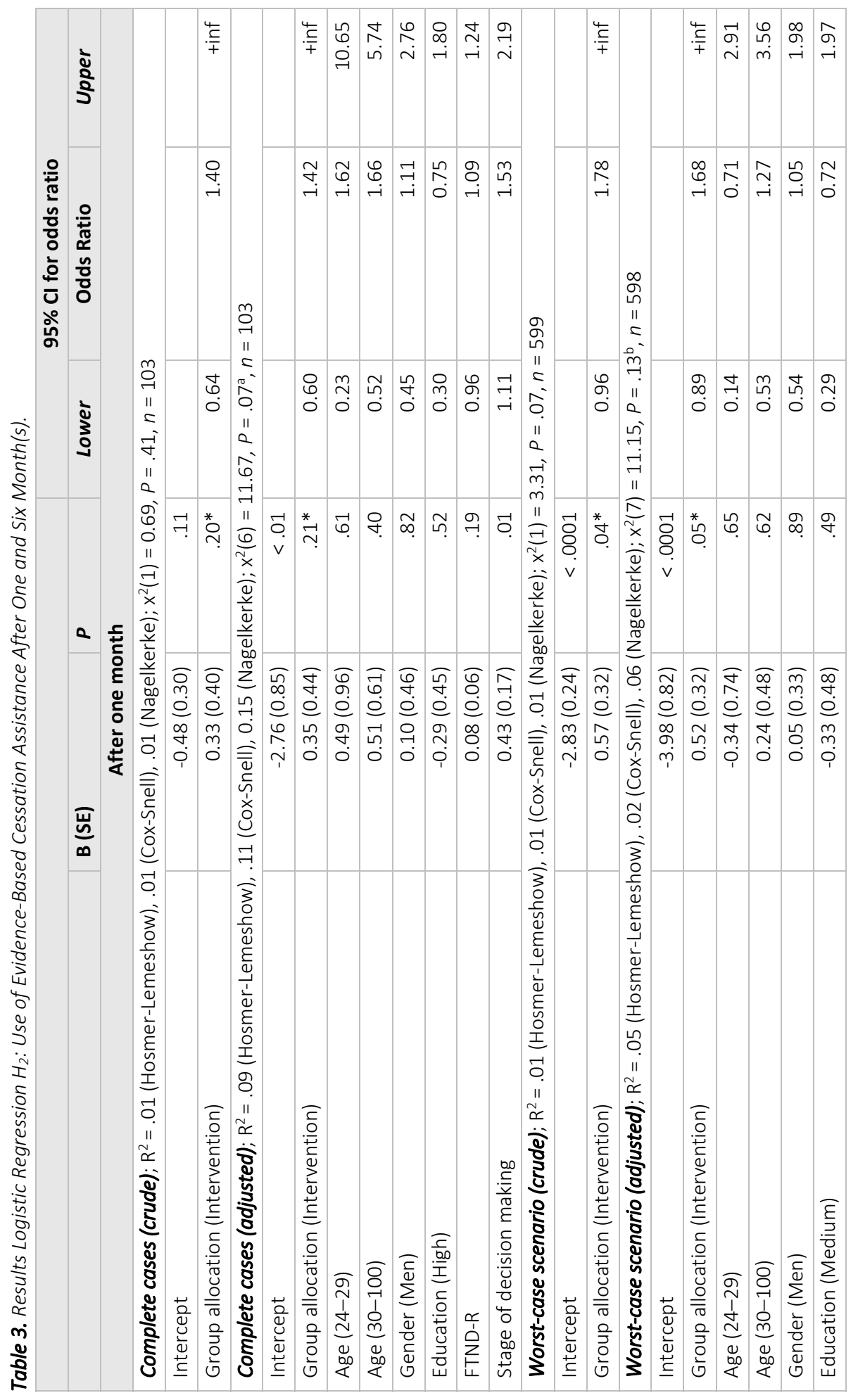




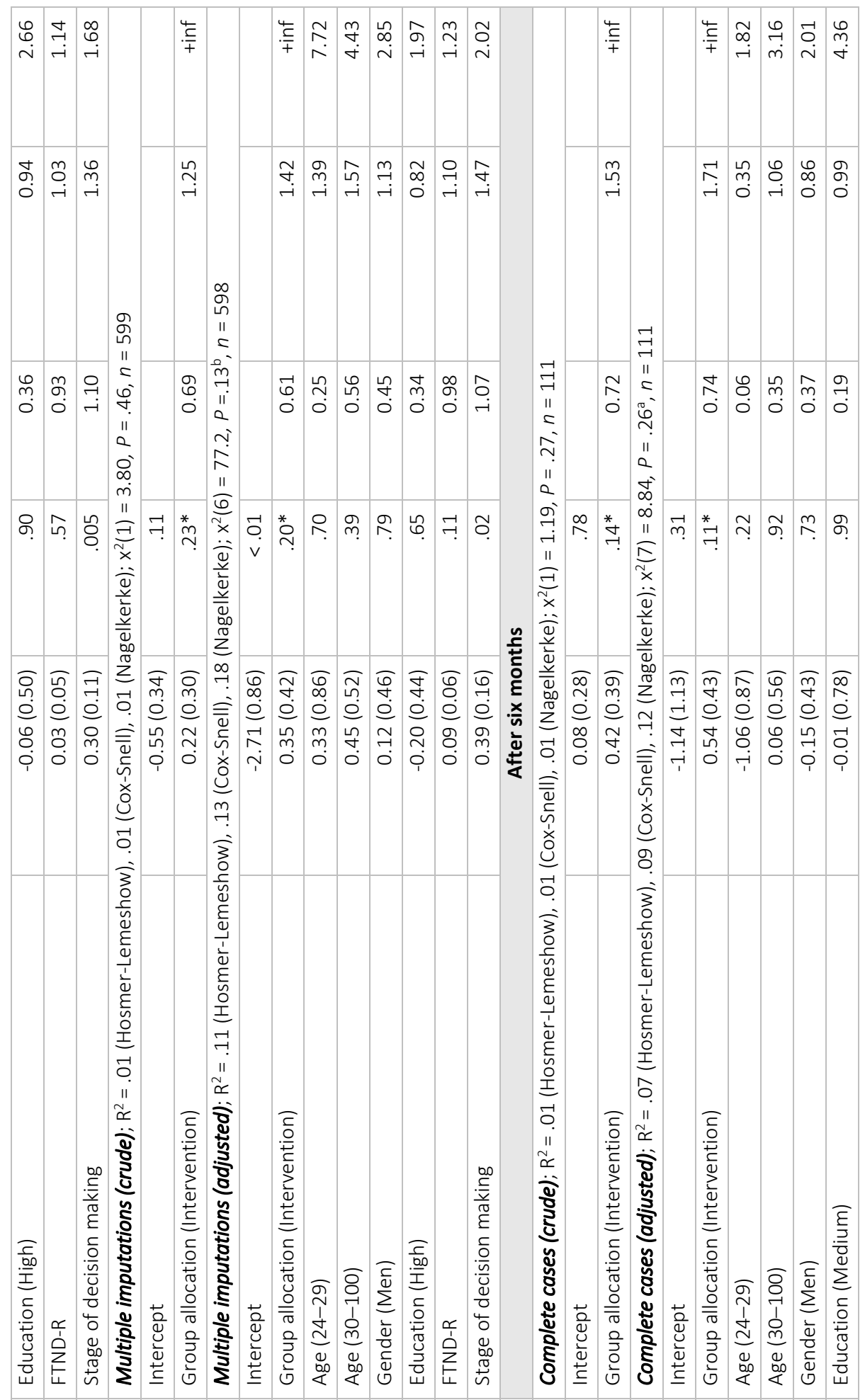




\begin{tabular}{|c|c|c|c|c|}
\hline 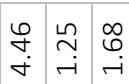 & $\stackrel{4}{+\frac{c}{+}}$ & 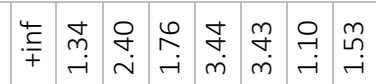 & $\stackrel{4}{+\frac{E}{+}}$ & 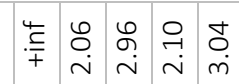 \\
\hline 势 & 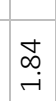 & 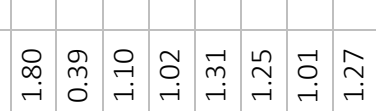 & $\underset{\mathrm{N}}{\mathrm{N}}$ & 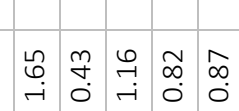 \\
\hline
\end{tabular}

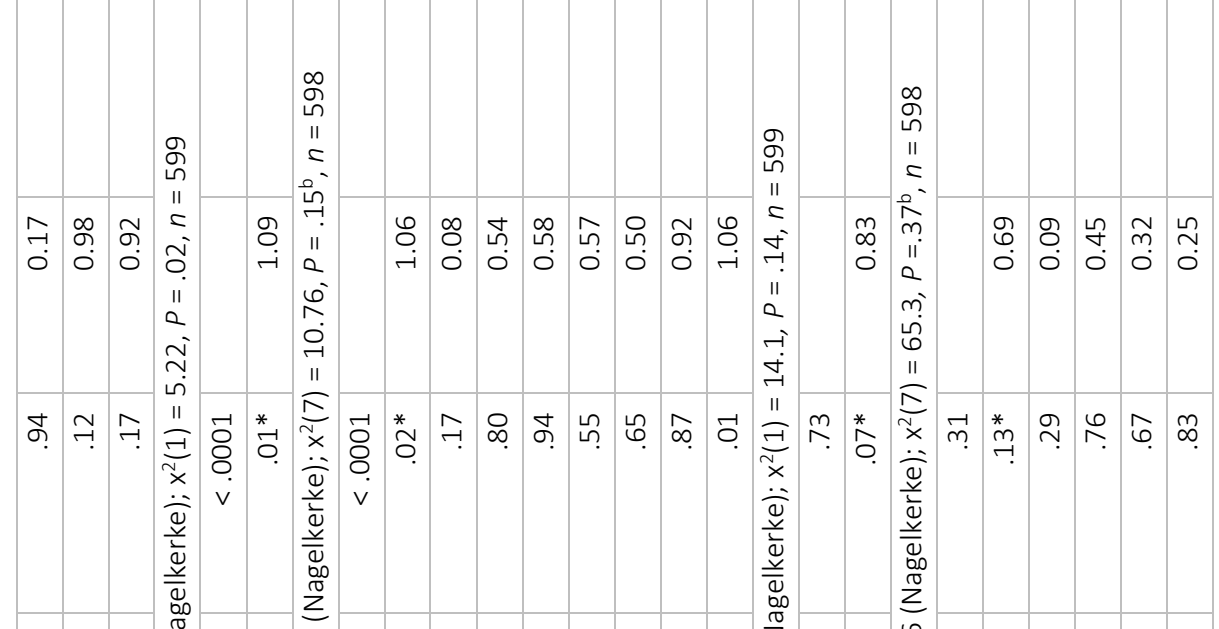

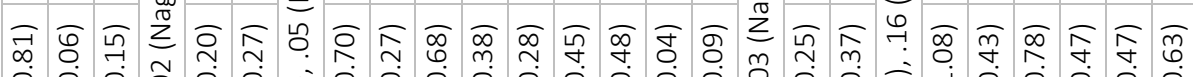

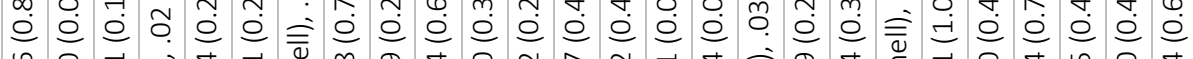

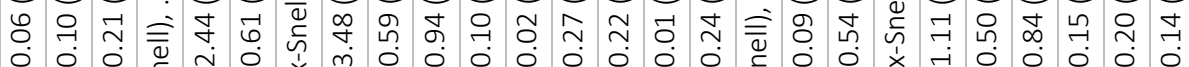

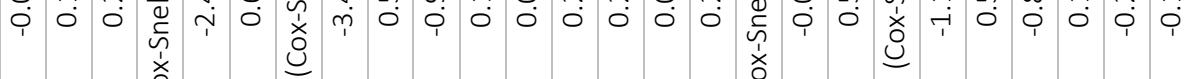

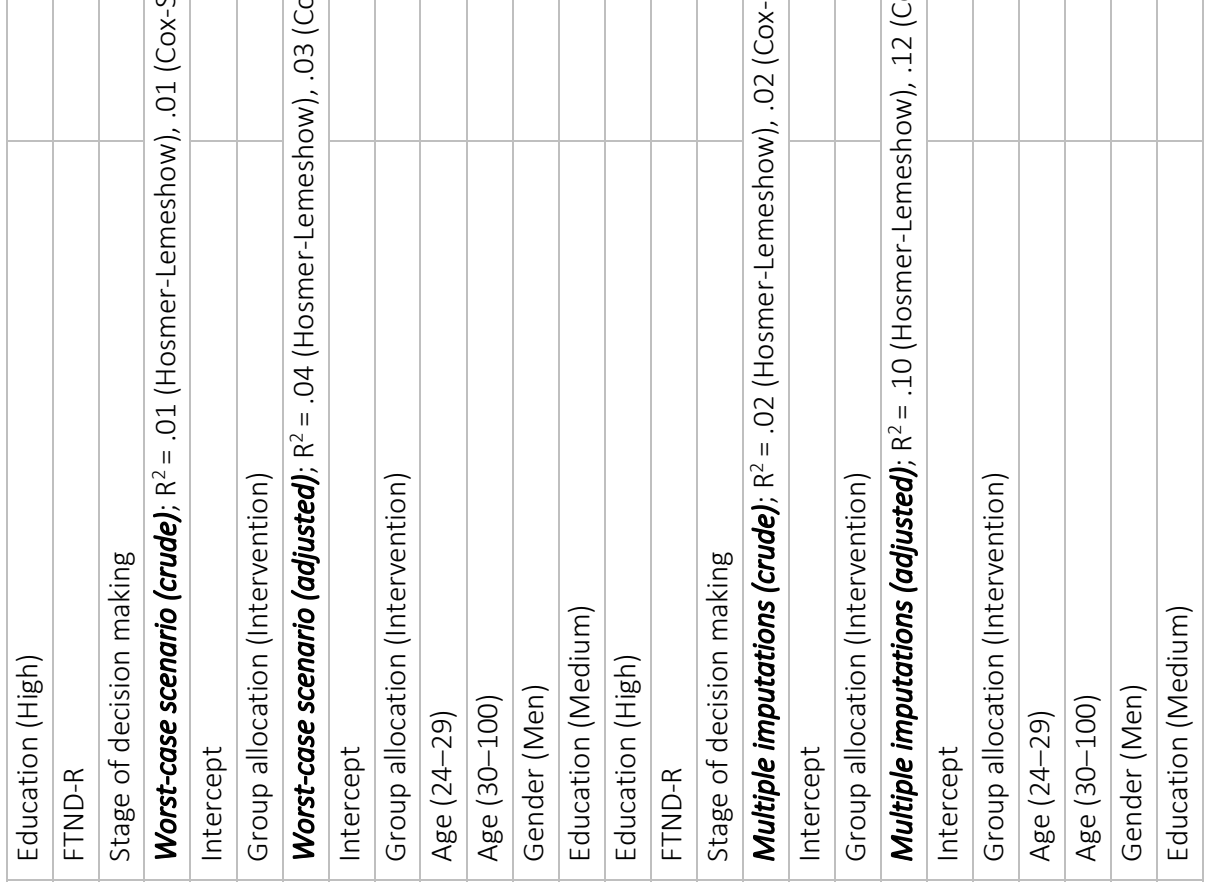




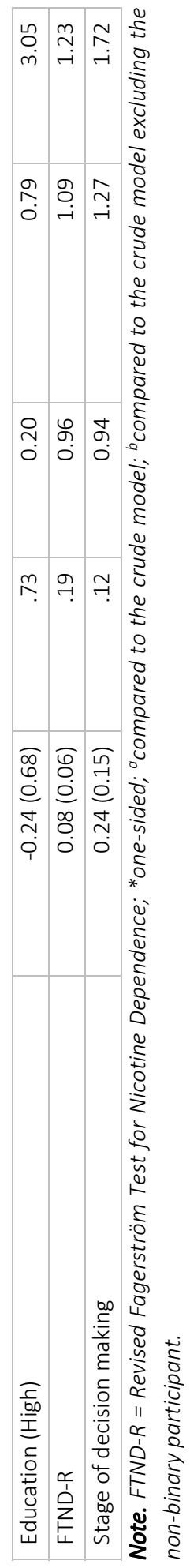


$\mathrm{H}_{3}$ : Decisional Conflict Immediately After the DA

Despite a small difference in averages between the intervention $(M=39.29, S D=25.00)$ and the control group ( $M=41.17, S D=25.23)$, the intervention had no significant effect on decisional conflict in both scenarios. In the adjusted models only stage of decision making ( $P$ $=.005$ in the complete case analysis; $P=.01$ in the Ml-analysis), being between 30-100 years (compared to 18-23 years) old (only in the Ml-analysis, $P=.045$ ), and a high level of education ( $P=.002$ in the complete case analysis; $P=.005$ in the Ml-analysis) had a significant effect on the outcome. All had a negative effect, i.e., people who reported a higher stage of decision making, participants between 30-100 years (compared to 18-23 years) old, and participants with a high level of education (compared to participants with a low level of education) experienced less decisional conflict. $\mathrm{H}_{3}$ could therefore not be confirmed. See Table 4 for all results and more information. 


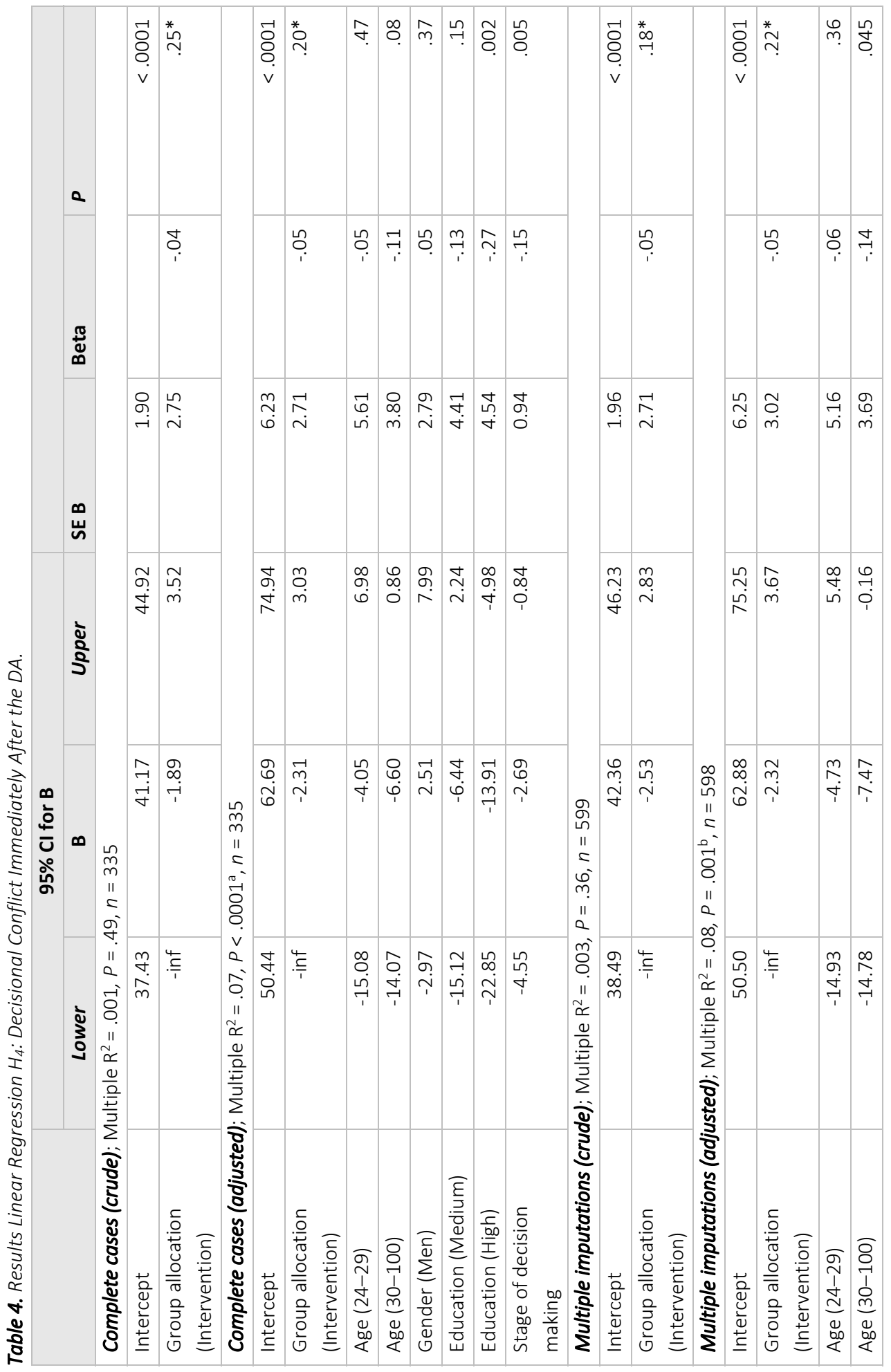




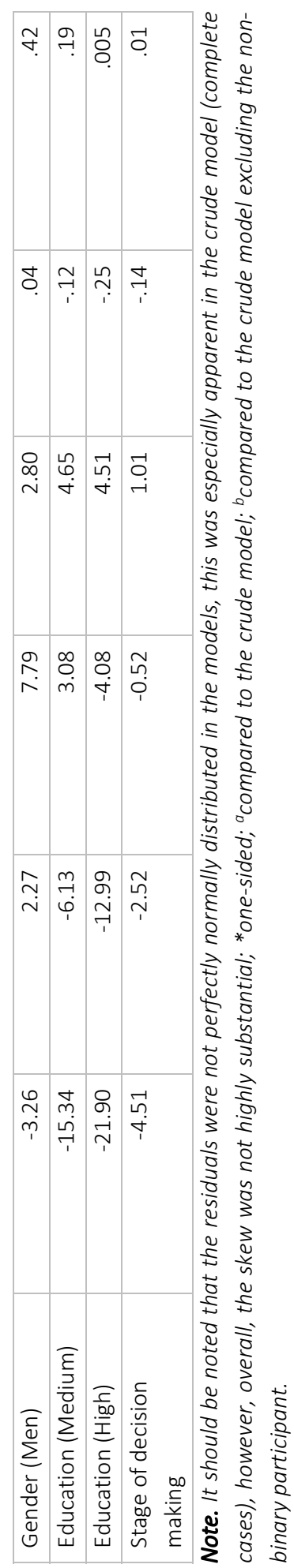




\section{Discussion}

The aim of this article was to report on the effects of adding an explicit VCM with computertailored advice to a smoking cessation DA (called VISOR) on both smoking cessation outcomes and decisional conflict. We hypothesized that end users that received an explicit VCM and computer-tailored advice within a smoking cessation DA (compared to users that received a smoking cessation DA without those elements) would be more likely to successfully stop smoking after one (secondary outcome) and six month(s) (primary outcome) and would be more likely to use evidence-based cessation assistance (secondary outcome). We also expected that participants in the intervention group would show less decisional conflict after having used the DA (secondary outcome). Contrary to our expectations, we did not find any effect on decisional conflict. Also, while the worst-case scenarios might suggest an effect on smoking cessation rates and cessation assistance uptake, this finding was not replicated in the either the complete case analyses or the MIanalyses. Moreover, given the fact that Blankers et al. [276] have shown that analyses based on penalized imputation can be biased when missingness is unbalanced between trial arms (as in our case), we cannot confidently speak of effects on smoking cessation success and cessation assistance uptake, despite the suggestion of effects in the worst-case scenarios. That said, all the significant and non-significant effects found were in the expected direction, i.e., participants in the intervention group showed more smoking cessation, more cessation assistance uptake, and less decisional conflict.

However, based on these findings, it may be too early to declare the addition of explicit VCMs paired with computer-tailored advice to smoking cessation DAs as ineffective. Previous studies have consistently found effects in favor of explicit VCMs on value-congruent decision making in other contexts, especially when combined with computer-tailored advice $[35,40]$. That said, we were the first (to our knowledge) to test this in a smoking cessation context. We would also like to emphasize that our findings do not imply that smoking cessation DAs are ineffective, as we did not compare a smoking cessation DA to usual care, another intervention, or no intervention, but rather compared two different versions of one and the same DA: one with a VCM and paired computer-tailored advice, and one without. In fact, even the control group in this RCT exceeded the smoking cessation rates achieved by the only other Dutch smoking cessation DA described in the literature [42]. However, this study shows that adding explicit VCMs paired with computer-tailored advice might not always be a good idea as non-usage attrition was 34.19\% higher in the intervention than in the control group. Also, our non-significant findings entail some caveats. In this discussion we will focus on the two most likely explanations for the lack of effects we found: (1) we were unable to find an effect due to a lack of power, and (2) there truly is no effect. Additionally, we will also describe the implications of those two explanations and how readers might benefit from the insights generated during our project. 


\section{Lack of Statistical Power}

The most likely explanation for the lack of significant effects is the lack of statistical power. During this trial, we were faced with a relatively high attrition rate (as typical for digital interventions [273]), and ultimately, our trial was widely underpowered as even the MIanalyses were underpowered to conduct our analyses. Based on our power analyses we planned to include 1592 smokers at baseline accounting for 50\% attrition. Ultimately, we randomized 2375 participants but already lost $50.99 \%$ of this group between randomization and the end of the baseline questionnaire. Within this group, subsequently, we lost $48.54 \%$ to non-usage attrition, and among the participants invited to follow-up 53,92\%-83,80\% to dropout attrition. Attrition was therefore much higher than originally anticipated. While a replication of our study with a larger sample is therefore recommended, researchers and DA-developers can learn from the reasons for attrition uncovered in this project.

Of the allocated participants, only $23.63 \%$ of the participants allocated to the intervention group finished VISOR, compared to $34.29 \%$ of the participants allocated to the control group. Interestingly, available data provided by the intervention host indicates that the differences in usage times between the two groups were significantly different. The median usage time of the intervention group was approximately nine minutes, while in the control group it was approximately six minutes (see the OSF for more information [269]). This difference may have driven the differences in non-usage attrition between the intervention and control group. Our other findings regarding non-usage attrition also give credence to this explanation because younger participants were more likely to not complete VISOR. During the needs assessment conducted to develop VISOR, younger participants in particular indicated that relatively short time frames were acceptable for using a smoking cessation DA. Future research should therefore explore whether VCMs and tailored advice can be designed to be delivered in a shorter manner to alleviate this problem. Witteman et al. [40], for example, reported on a digital VCM (including tailored advice) that consisted of dynamic web sliders representing both values and preferences (i.e., the included options). Those web sliders were then linked, meaning that for participants who indicated that a particular value is very important to them, the slider representing the preference moved equally. Such a VCM could potentially be much shorter to use as end users do not have to answer multiple statements. However, at this point this technique is relatively hard to use for decisions involving multiple options, which is why we were unable to use it within VISOR. Based on our findings, however, one might also conclude that it is important to spend more time studying user experience components, especially usage times.

Interestingly, based on our data it can also be concluded that we did not experience a recruitment problem (i.e., 2109 participants were initially randomized and eligible to participate), but rather a problem of retention-only a small amount of the participants 
completed VISOR and even less participants completed the follow-up questionnaires. While this is often observed in digital health(care) interventions [277], it is crucial to find ways to increase actual usage of digital DAs and retention in DA-trials - to ensure benefit for users, but also to reach sufficient statistical power during studies. One way to achieve this would be to embed DAs (such as VISOR) in a counseling pathway as the involvement of a professional has been shown to positively influence time spent on websites aimed at improving healthy lifestyles [277]. Concurrently, this might also positively influence retention rates in studies (i.e., people returning for follow-up measurements) in which digital DAs are evaluated $[278,279]$. There could be multiple ways to accomplish this in the context of VISOR (or other digital DAs for that matter): (1) VISOR could be used together with a healthcare professional (e.g., a practice nurse) or could be sent to participants before or after a healthcare consultation, in which (the result of) VISOR is discussed; or (2) a digital form of counseling could be included into VISOR, e.g., through the form of videocalls [280] or an automated chatbot [281]. The second manner might be especially promising as it would keep the fully digital and automated nature of VISOR intact, thereby, still ensuring optimal reach of the DA. Future research should investigate which modalities are especially beneficial in term of outcomes and retention, and which modalities are preferred by end users themselves.

Related to the problem of retention, an interesting chance finding of our study was that it highlighted the importance of stage of decision making in this regard, as this influenced both non-usage and dropout attrition. Interestingly, this influence seemed to (partially) disappear once we corrected for other dropout predictors. The most plausible explanation for this is that different groups within our sample (e.g., older compared to younger participants) differed in their stage of decision making (see the OSF for more information [269]), leading to stage of decision making becoming insignificant once all predictors were added in the same model. Interestingly, stage of decision making is much less routinely assessed than decisional conflict - to illustrate, the validation article of the original decisional conflict scale [4] has been cited more than 2000 times according to Google Scholar, while the user manual of the stage of decision making scale [240] has only been cited a little over 30 times-and when it is assessed, it is mostly done to include it as a covariate (e.g., [282,283]). Consequently, the role of those decisional stages seems to be much less understood. That said, it is assumed that individuals' stages of decision making influence their receptiveness to DAs and that people who are in active stages of deliberation would benefit the most from DAs, while people who either have not even begun to think about the decision, or are unwilling to reconsider their decision may not benefit from a DA as much [240]. Interestingly, the biggest group (approximately 40\%) within our sample was at an early stage of decision making, which is also reflected in respondents' average score for this variable (i.e., 2.92), which is slightly below active deliberation (i.e., the stage in which individuals start weighing the different options). As such, it might be the case that VISOR (with or without the explicit 
VCM and advice) simply overwhelmed a big part of our sample. The relatively high decisional conflict scores in both groups seems to confirm this. Therefore, tailoring DAs to stage of decision making might be a promising approach to limit attrition more. While demographic variables seem like the more obvious choice based on our data, the one-item scale used to assess stage of decision making has the major advantage of being easy to use and not requiring participants to complete lengthy questionnaires. By tailoring DAs to the stage of decision making (as opposed to other constructs or demographic characteristics), it would be possible to alleviate one of the core problems of most contemporary approaches to computer-tailoring, namely that they often impose a significant burden on participants [284]. That participants' stage of decision making had the most consistent effect on the outcomes among the included predictors, only adds to that. Individuals in initial stages could, for example, be offered interventions that are merely educational and supportive in nature, while individuals in active deliberation stages could be offered 'traditional' DAs and individuals in later stages could be offered support to implement their decision. In other words, insights from behavior change could be used to further improve DAs for specific groups of decision makers [285].

\section{True Absence of an Effect}

Due to the lack of statistical power, we cannot confidently conclude that there truly was no effect of VISOR. Interestingly, however, Sheridan et al. [143] also tested the additional effect of an explicit VCM added to a DA aimed at a prevention-related decision (i.e., heart disease prevention) and also found no effects on outcomes such as decisional conflict or intention to reduce heart disease risk. Yet, most other studies tested DAs (and by extension explicit VCMs) in a treatment context (e.g., between different surgeries [40]), with positive effects reported quite often. Based on this contradiction, we could possibly deduct that preventionrelated decisions are somewhat different and may not be affected by DAs in a similar way as treatment decisions are. Anecdotally, throughout the project in which VISOR was developed and tested, people not involved in the project often stated that prevention-related decisions (such as smoking cessation decisions) seem easier to make compared to other decisions, such as treatment decisions. While this conclusion is to certain degree logical-as treatment decisions often involve much more imminent risks, such as death in the nearer future [40]we found no indications that the participants in this study regarded the decision among different cessation aids to be easy. In fact, participants in both trial arms experienced decisional conflict that scored above the accepted cut-off value of 37.5 [7]. The decisional conflict scores observed in this study were also higher than those averagely reported in the literature [5] which tends to focus on treatment decisions, indicating that prevention-related decisions are not necessarily easier to make than treatment decisions. In other words, there is no indication that prevention-related decisions are perceived differently to treatment decisions (at least not in terms of experienced difficulty), meaning that they can be assumed 
to respond similarly to intervention elements commonly included in DAs (such as VCMs). However, based on our data it cannot be fully excluded that prevention-related decisions differ on another factor than experienced difficulty and therefore do not respond to explicit VCMs as expected. A more viable difference between prevention-related DAs and DAs focused on treatment decisions is that those focused on prevention are often used without a direct involvement of a healthcare professional (e.g., [42,53])-i.e., prevention-related DA are more likely to be self-administered [286] than DAs focused on treatments. Yet, a recent systematic review and meta-analyses from Larsen et al. [286] has shown that selfadministered DAs about colorectal cancer screening can also be beneficial in relation to prevention-related decisions, such as population screenings. Showcasing that this explanation (i.e., the lack of healthcare involvement) is unlikely. In other words, at this point, no convincing argument can be made that prevention-related decision are different from other health(care) decision. Therefore, they should also respond similarly to elements typically used in DAs. However, also given the substantial amount of heterogeneity in the literature about DAs aimed at primary prevention [195], it may be worth investigating further whether prevention-related decisions really respond to DA-elements as one would expect based on the wider DA-literature. For example, by replicating our work with a larger sample.

\section{Strengths and Limitations}

The major strength of our study was that we conducted it in a 'real life' context and that we mainly recruited individuals that were interested in smoking cessation (as opposed to a hypothetical scenario often used to evaluate VCMs [40]). Also, our sample was relatively representative of the Dutch population except for women being overrepresented and participants with a low level of education being slightly underrepresented [287]. Our findings might thus be largely generalizable to the smoking Dutch population with access to the internet. The fact that both groups received a DA also allowed us to blind participants, which strengthened the RCT, as participants were not biased in the sense that they knew to which intervention arm they were allocated and, thus, what the other group was offered. Finally, we also focused our analyses on three outcomes of interest, thereby, decreasing the risk of a Type I error.

However, we were also faced with some limitations. Due to our study design, we might have suffered from a selection bias as we only invited people to complete the follow-ups if they had used the entire intervention. We tried to alleviate the influence of this selection bias by including variables associated with this selection (e.g., age) as covariates in analysis and imputation models. However, it is possible that more motivated smokers completed the intervention and therefore, for example, showed higher quit rates. Also, the trial suffered from such a big attrition that we were underpowered for most of our analyses. That said, the findings from the complete case analyses and findings based on MI lead to the same 
conclusions, making our findings more robust. It might also be a limitation that we relied on self-reported data. However, as previous research showed that self-reported smoking status tends to be accurate [288] and because participants received the reward for study completion regardless of their smoking status we are confident that this has had little to no influence on our findings. Finally, we were unable to test the effects of the explicit VCM and computer-tailored advice separately from each other. This would have required three trial arms and an even bigger sample and was therefore deemed unfeasible. Lastly, this article focused on direct behavioral and decisional effects only, as indirect effects were beyond the scope of this article. The same applies to cost-effectiveness and cost-utility. Investigating indirect effects (as hypothesized in the study protocol [96]) as well as cost-effectiveness and cost-utility might provide additional insights besides the direct effects reported in this article.

\section{Conclusion}

At this point, we cannot confidently recommend the inclusion of explicit VCMs and computer-tailored advice in smoking cessation DAs. In fact, these two elements might result in higher attrition rates during the usage of the DAs, thereby limiting their potential. However, our findings in relation the primary and secondary outcomes might be influenced by a lack of statistical power, and we therefore advocate for the replication of our trial with a bigger sample. Last, researchers should put more emphasis on user experience, especially usage times, and experiment with (innovative) solutions to deal with a (too) high perceived user burden, such as the integration of VISOR within a counseling pathway or tailoring VISOR and other DAs to people's stage of decision making. Finally, researchers should investigate more how prevention-related decisions respond to DA(-elements). 
Abbreviations

COVID-19 - Coronavirus disease 2019

DA - decision aid

FTND-R - Revised Fagerström Test for Nicotine Dependence

IPDAS - International Patient Decision Aid Standards

$\mathrm{MI}$ - multiple imputation

NRT - nicotine replacement therapy

OSF - Open Science Framework

$\mathrm{RCT}$ - randomized controlled trial

SDT - Self-Determination Theory

VCM - value clarification method 
Appendix A - Details Baseline Measurements Demographic Information and Smoking Behavior

\begin{tabular}{|c|c|c|}
\hline Measurements & Answer categories & Other information \\
\hline \multicolumn{3}{|c|}{ Demographic information } \\
\hline Age & $\begin{array}{l}1=18-23 \\
2=24-29 \\
3=30-100\end{array}$ & $\begin{array}{l}\text { Initially collected continuously } \\
\text { but was recoded into three } \\
\text { categories to comply with the } \\
\text { privacy regulations of the } \\
\text { University of Amsterdam. }\end{array}$ \\
\hline Gender & $\begin{array}{l}0=\text { woman } \\
1=\text { man } \\
2=\text { not listed } / \text { non-binary } \\
3=\text { prefer not to say }\end{array}$ & $N / A^{a}$ \\
\hline Education & $\begin{array}{l}0=\text { low } \\
1=\text { medium } \\
2=\text { high }\end{array}$ & $\begin{array}{l}\text { Was originally collected as the } \\
\text { highest educational } \\
\text { qualification achieved to be } \\
\text { recoded into three categories } \\
\text { based on Statistics } \\
\text { Netherlands (the Dutch } \\
\text { Central Agency for Statistics) } \\
\text { [289]: 0 = low (including either } \\
\text { only or no primary education, } \\
\text { and lower vocational } \\
\text { education), } 1 \text { = medium } \\
\text { (including general secondary } \\
\text { education, secondary } \\
\text { vocational education, and } \\
\text { secondary general education) } \\
\text { and } 2 \text { = high (including higher } \\
\text { professional education and } \\
\text { university education). }\end{array}$ \\
\hline \multicolumn{3}{|l|}{ Smoking behavior } \\
\hline Tobacco products used & $\begin{array}{l}0=\text { cigarette } \\
1=\text { hand-rolled cigarette } \\
2=\text { e-cigarette } \\
3=\text { pipe } \\
4=\text { other products }\end{array}$ & $\begin{array}{l}\text { Due to a substantial number } \\
\text { of participants indicating that } \\
\text { they used cannabis products } \\
\text { (e.g., joints) and cigars, we } \\
\text { created variables reflecting } \\
\text { this. If participants only } \\
\text { indicated those options under } \\
\text { "4 = other products", we } \\
\text { manually removed "4 = other } \\
\text { products" from their data to } \\
\text { prevent double counting. }\end{array}$ \\
\hline
\end{tabular}




\begin{tabular}{|c|c|c|}
\hline $\begin{array}{l}\text { Amount of tobacco } \\
\text { consumption per product per } \\
\text { day }\end{array}$ & $\begin{array}{l}\text { Continuously, except for e- } \\
\text { cigarettes which was } \\
\text { measured categorically: } \\
1 \text { = less than monthly } \\
2 \text { = less than weekly, but at } \\
\text { least once per month } \\
3=\text { less than daily, but at least } \\
\text { once per week } \\
4=\text { daily, but not multiple } \\
\text { times per day } \\
5=\text { multiple times per day }\end{array}$ & $N / A^{a}$ \\
\hline Past cessation attempts & $\begin{array}{l}0=\text { yes } \\
1=\text { no }\end{array}$ & $N / A^{a}$ \\
\hline $\begin{array}{l}\text { Amount of past cessation } \\
\text { attempts }\end{array}$ & Continuously & $N / A^{a}$ \\
\hline $\begin{array}{l}\text { Cessation assistance utilization } \\
\text { in the past six month }\end{array}$ & $\begin{array}{l}0=\text { yes } \\
1=\text { no }\end{array}$ & $N / A^{a}$ \\
\hline $\begin{array}{l}\text { Cessation assistance utilization } \\
\text { in the past six month (if } \\
\text { participants indicated that } \\
\text { they have utilized cessation } \\
\text { assistance) }\end{array}$ & $\begin{array}{l}1=\text { varenicline } \\
2=\text { bupropion } \\
3=\text { nortriptyline } \\
4=\text { nicotine patches } \\
5=\text { nicotine gums } \\
6=\text { nicotine lozenge } \\
7=\text { nicotine mouth spray } \\
8=\text { nicotine inhaler } \\
9=\text { face-to-face counseling } \\
10=\text { counseling over the } \\
\text { phone } \\
11=\text { group coaching } \\
12=\text { eHealth } \\
13=\text { something else } \\
14=\text { cessation without } \\
\text { assistance }\end{array}$ & $\begin{array}{l}\text { A substantial group of people } \\
\text { indicated similar options } \\
\text { under "13 = something else" } \\
\text { (i.e., cytisine and options not } \\
\text { clearly described but with a } \\
\text { high chance of being effective } \\
\text { [e.g., general practitioners]). } \\
\text { We, therefore, also chose to } \\
\text { create variables for those two } \\
\text { options (again, we manually } \\
\text { removed "13 = something } \\
\text { else", if those were the only } \\
\text { options that people used). } \\
\text { Both options were regarded as } \\
\text { being evidence-based. } \\
\text { However, it should be said } \\
\text { that cytisine is not legally } \\
\text { available at this point in the } \\
\text { Netherlands-nevertheless, } \\
\text { we chose to regard it as being } \\
\text { evidence-based as it has been } \\
\text { shown to be effective in } \\
\text { individual studies [290] and } \\
\text { meta-analyses [291]. }\end{array}$ \\
\hline
\end{tabular}




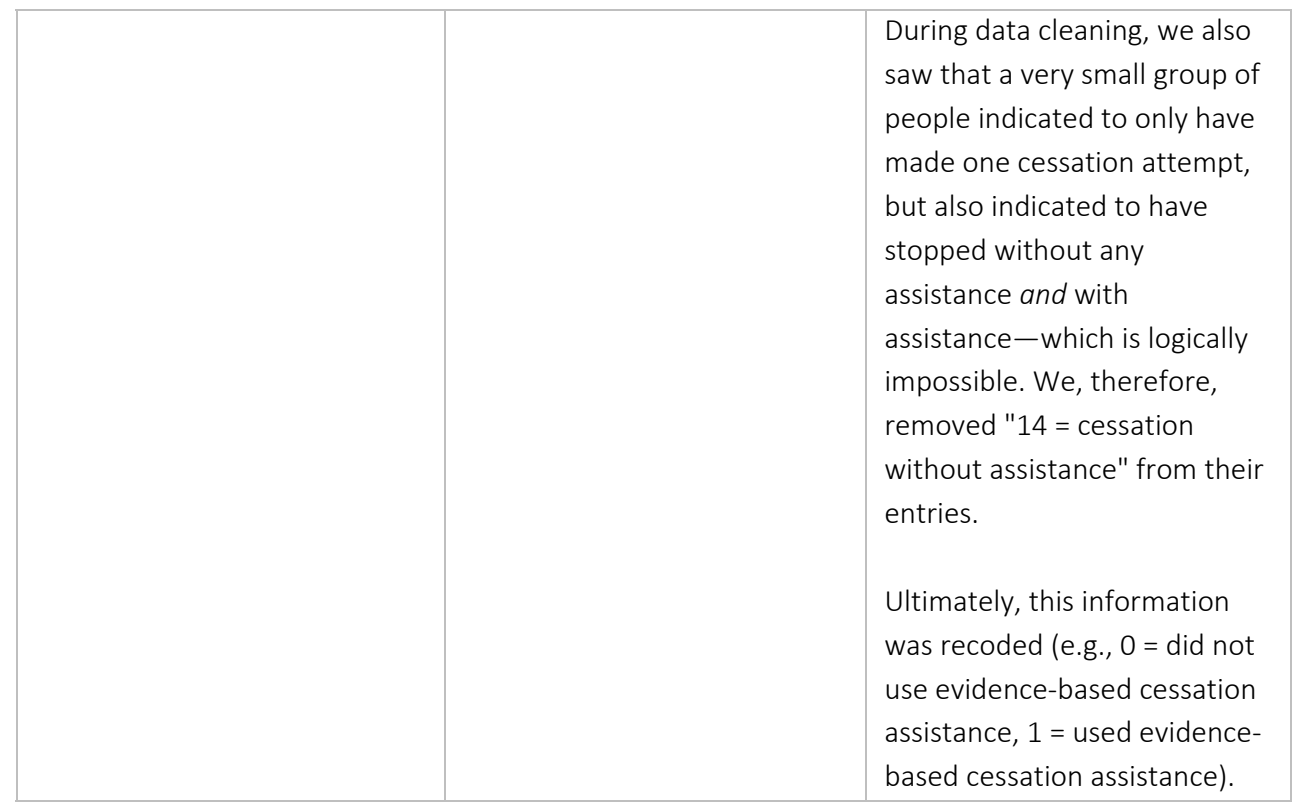

Note. ${ }^{a} N /$ A: not applicable.

Appendix B - Variables Included in the Imputation Model to Test $\mathrm{H}_{1 \mathrm{a} / \mathrm{b}}$ and $\mathrm{H}_{2 \mathrm{a} / \mathrm{b}}$

\begin{tabular}{|l|l|}
$\begin{array}{l}\text { Variables that were also part of the analysis } \\
\text { model }\end{array}$ & $\begin{array}{l}\text { Group allocation, age, gender, education, } \\
\text { Revised Fagerström Test for Nicotine } \\
\text { Dependence (FTND-R), and stage of decision } \\
\text { making. }\end{array}$ \\
\hline $\begin{array}{l}\text { Other additional variables that were also } \\
\text { related to dropout }\end{array}$ & $\begin{array}{l}\text { Whether the invite for } \mathrm{t}=2 \text { was sent (only for } \\
\mathrm{H}_{1 \mathrm{a}} \text { and } \mathrm{H}_{2 \mathrm{a}} \text { ), whether participants only shared } \\
\text { the answer to the primary outcome with the } \\
\text { research team (only for } \mathrm{H}_{1 \mathrm{~b}} \text { and } \mathrm{H}_{2 \mathrm{~b}} \text { ), and } \\
\text { whether the date for } \mathrm{t}=3 \text { had been adjusted } \\
\text { (only for } \mathrm{H}_{1 \mathrm{~b}} \text { and } \mathrm{H}_{2 \mathrm{~b}} \text { ). }\end{array}$ \\
\hline Auxiliary variables & $\begin{array}{l}\text { Evidence-based cessation assistance utilization } \\
\text { in the past six months (at baseline). }\end{array}$ \\
\hline
\end{tabular}

Appendix $\mathrm{C}-$ Variables Included in the Imputation Model to Test $\mathrm{H}_{3}$

\begin{tabular}{|l|l|}
\hline $\begin{array}{l}\text { Variables that were also part of the analysis } \\
\text { model }\end{array}$ & $\begin{array}{l}\text { Group allocation, age, gender, education, and } \\
\text { stage of decision making. }\end{array}$ \\
\hline $\begin{array}{l}\text { Other additional variables that were also } \\
\text { related to dropout }\end{array}$ & $\mathrm{N}^{\mathrm{a}} \mathrm{A}$ \\
\hline \begin{tabular}{l} 
Auxiliary variables \\
\hline
\end{tabular} & $\mathrm{N} / \mathrm{A}^{\mathrm{a}}$ \\
\hline
\end{tabular}

Note. ${ }^{a} \mathrm{~N} / \mathrm{A}$ : not applicable. 


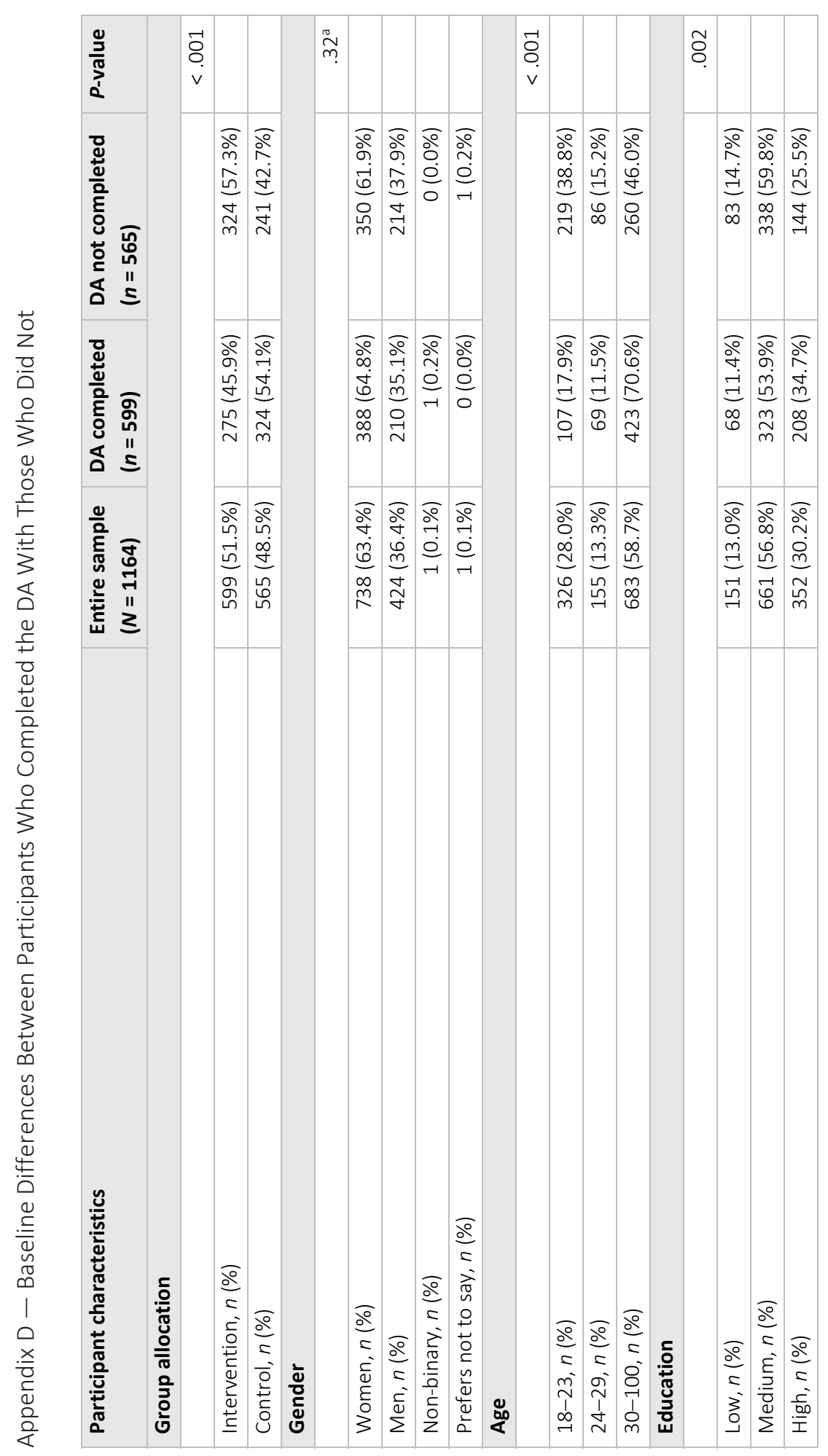




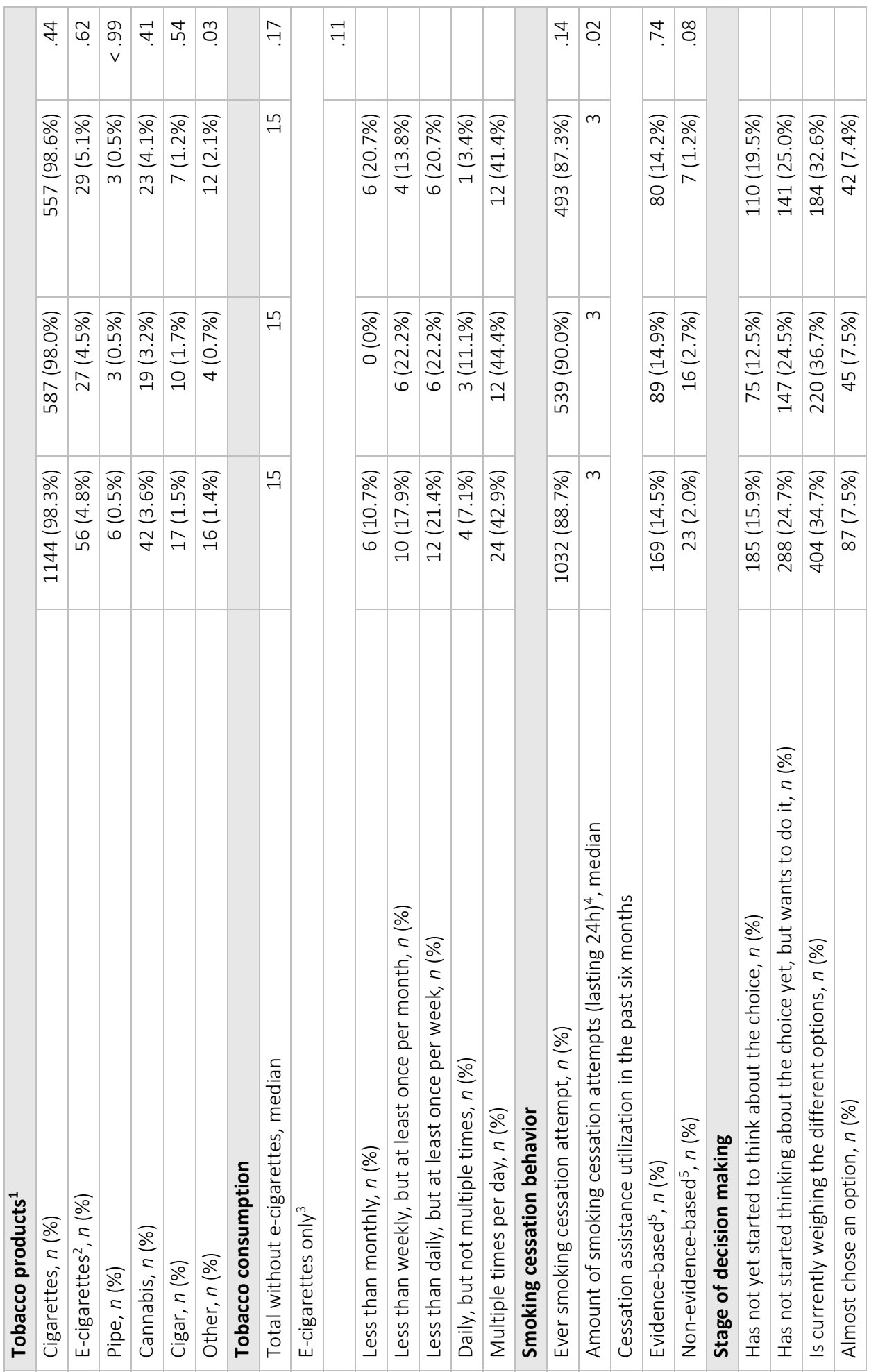




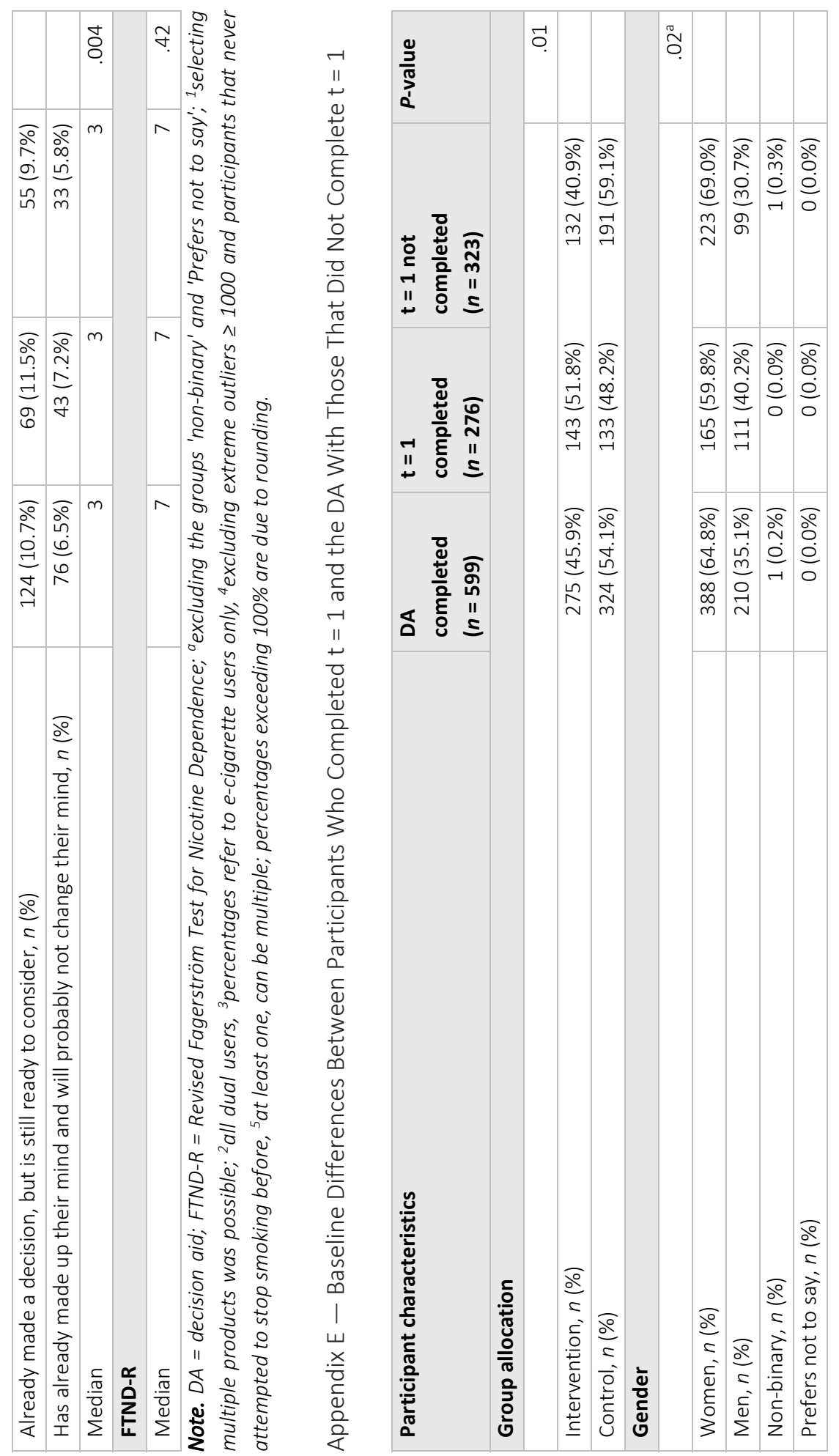




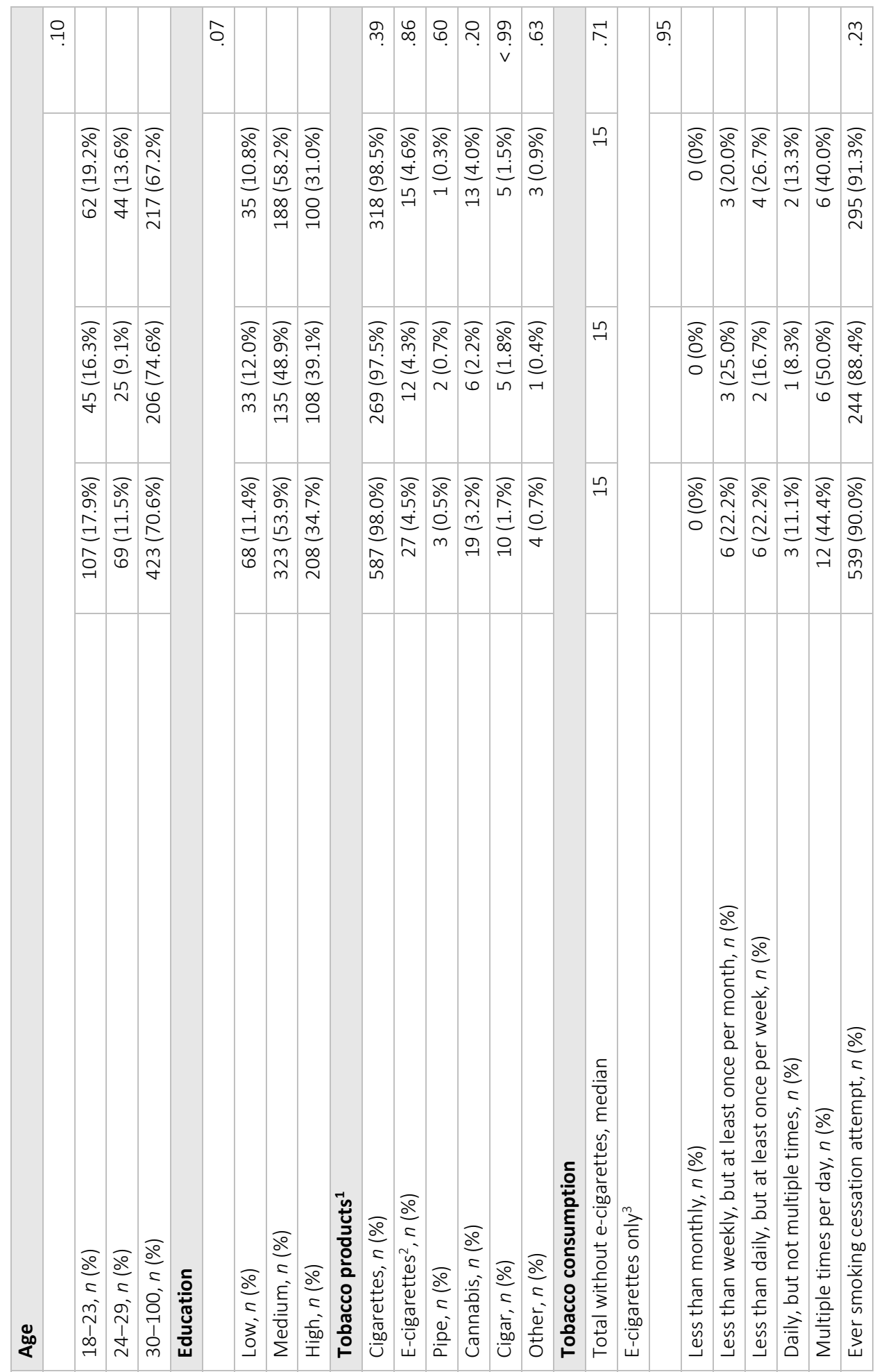




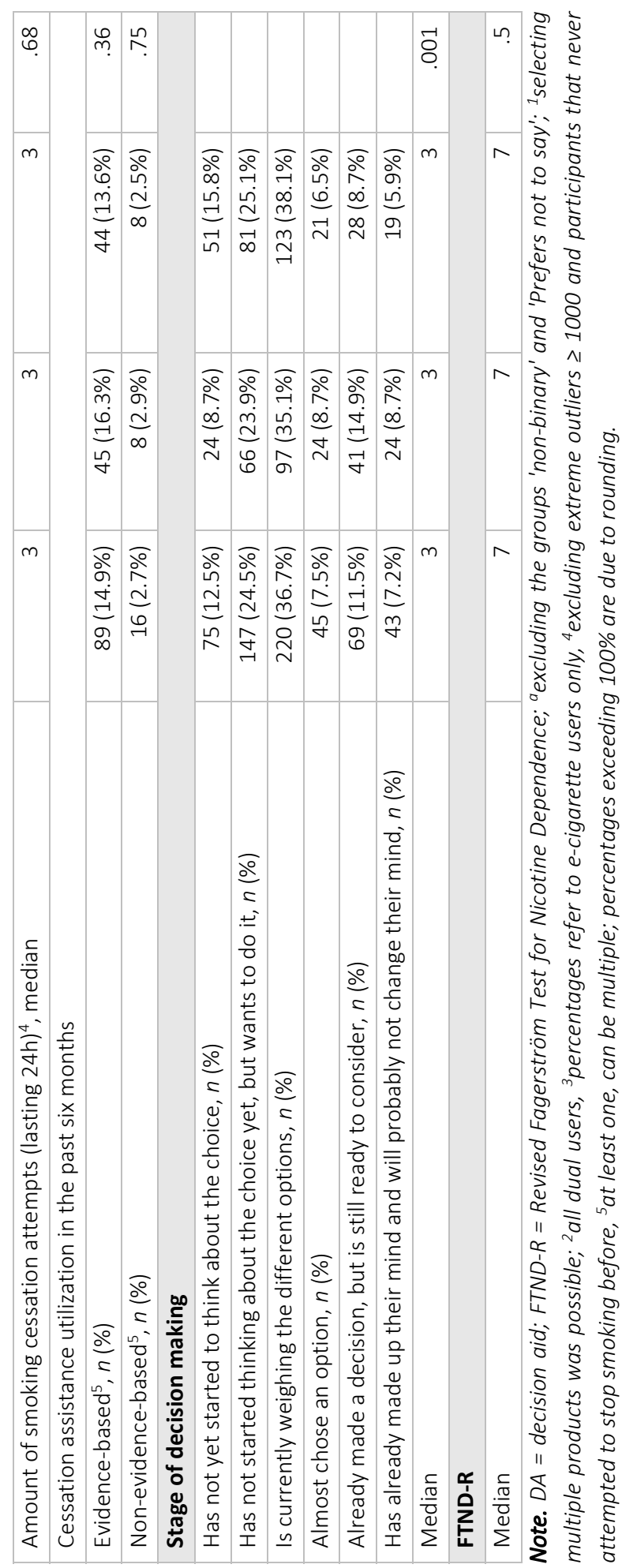




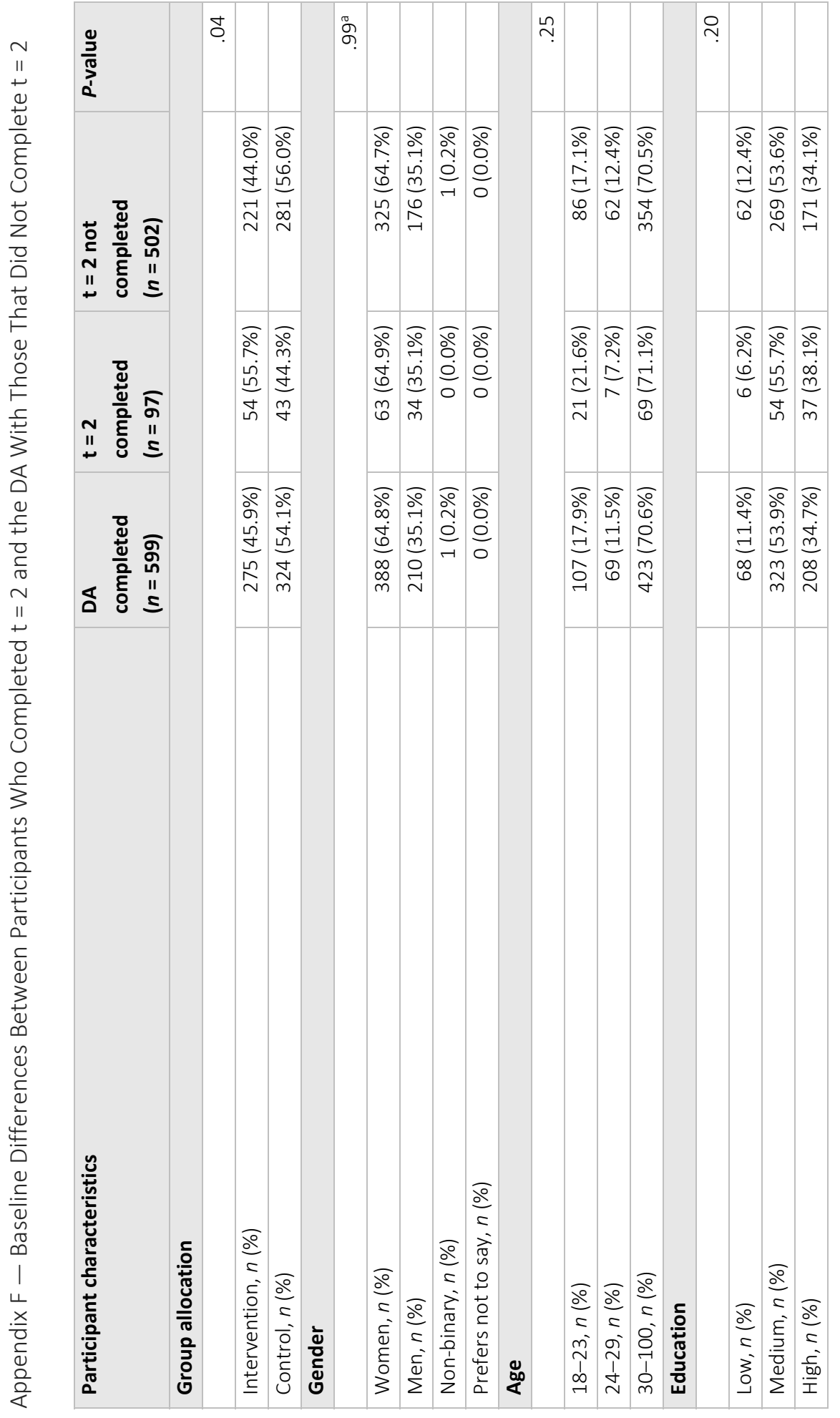




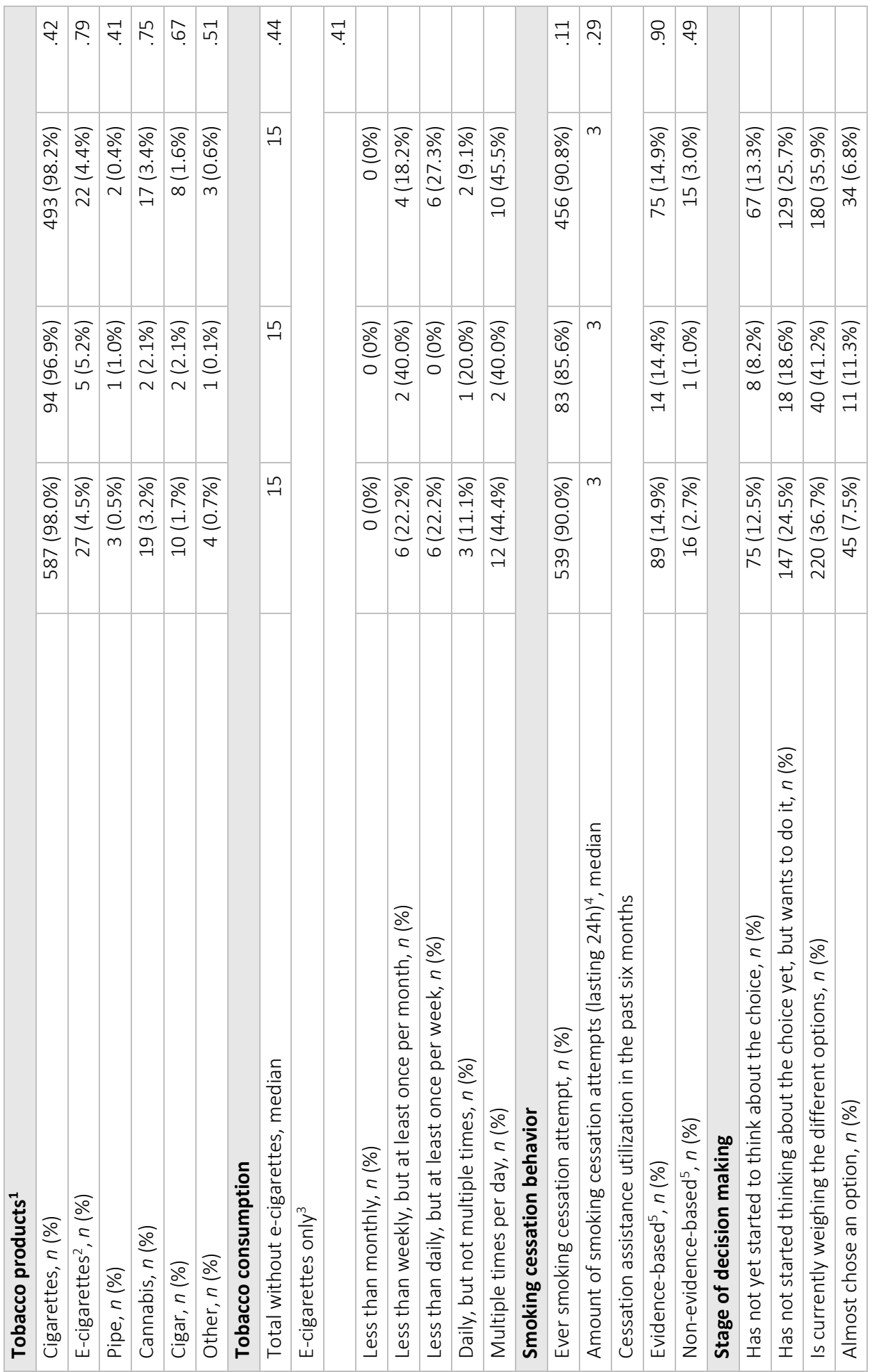




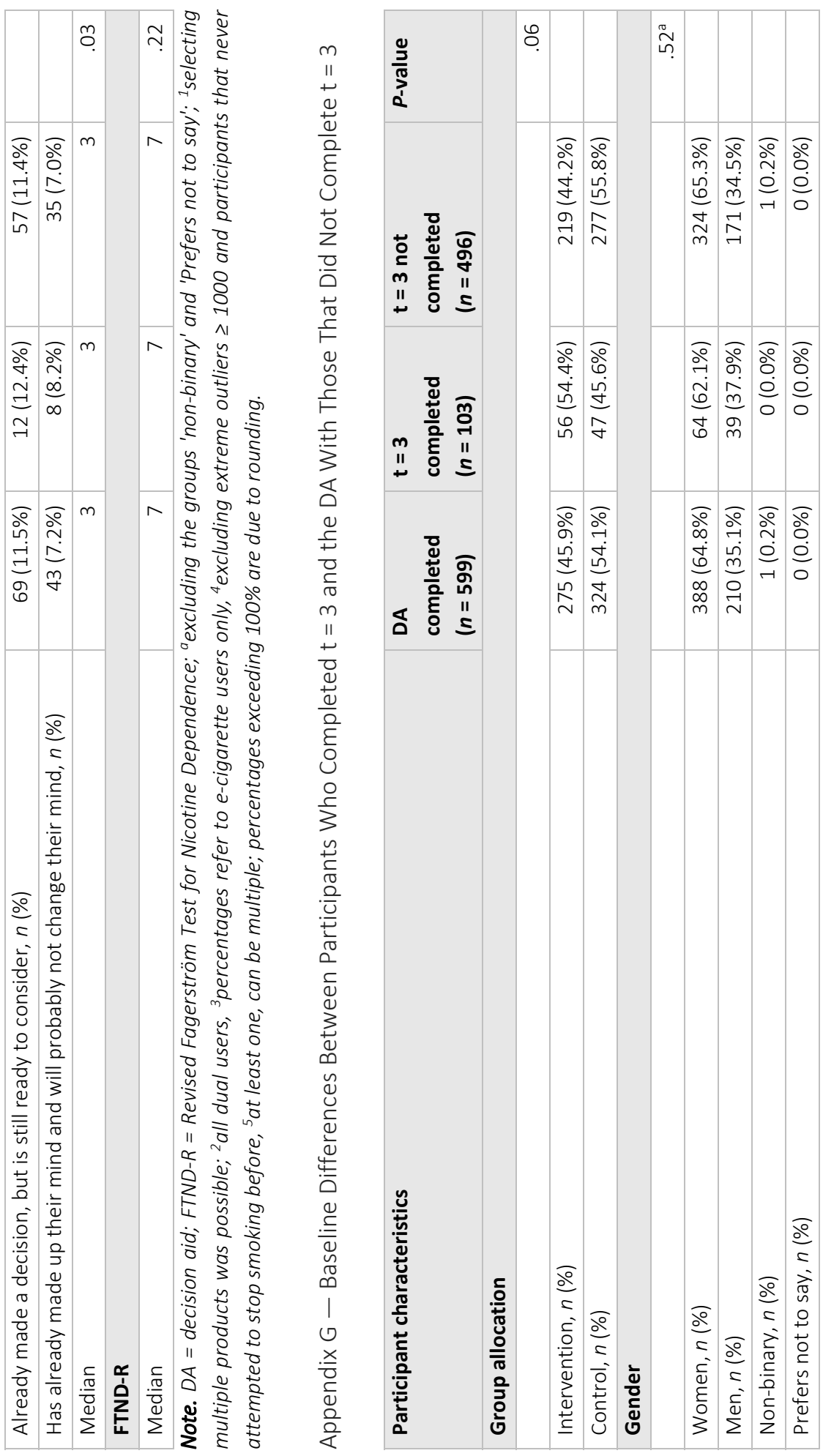




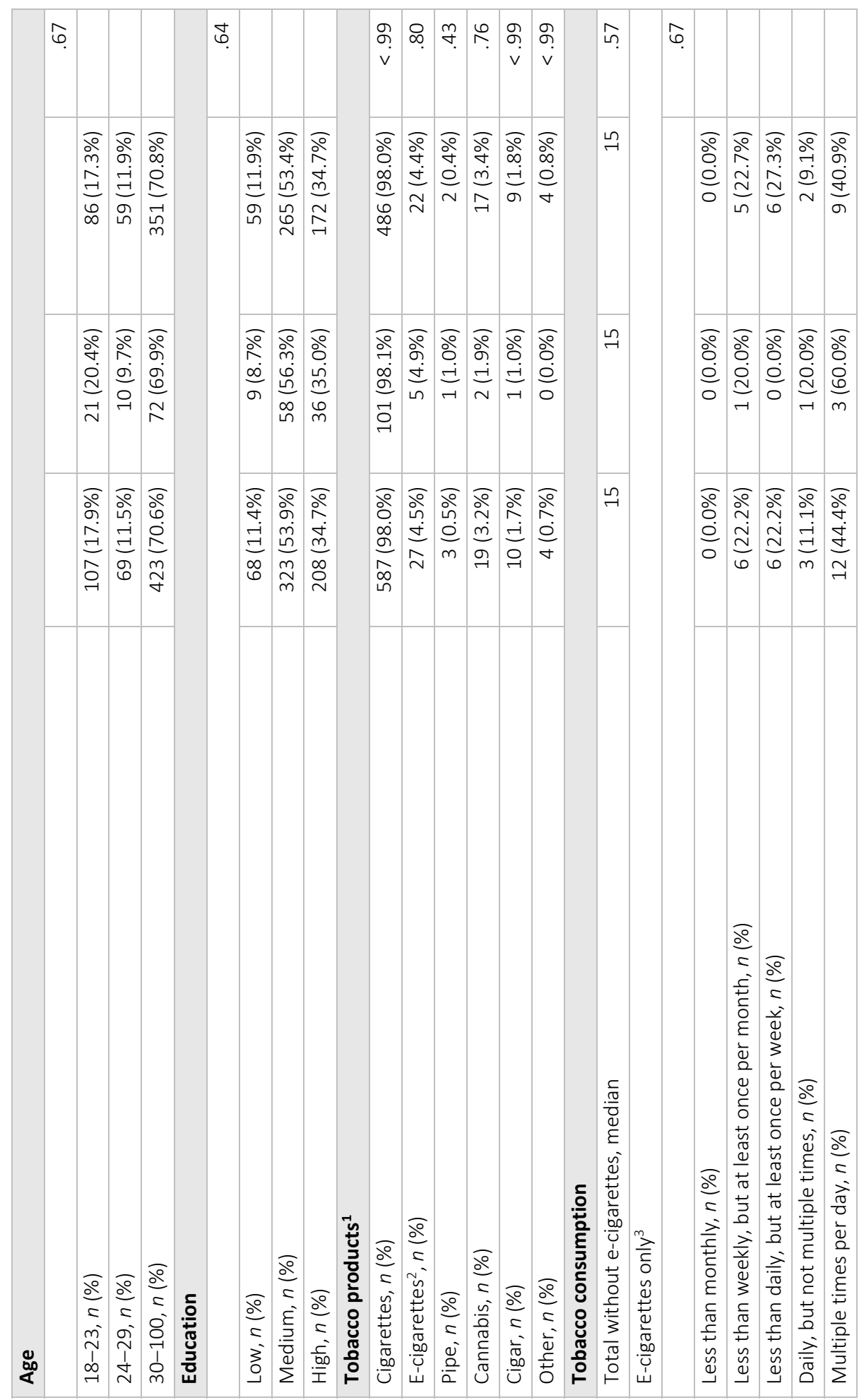




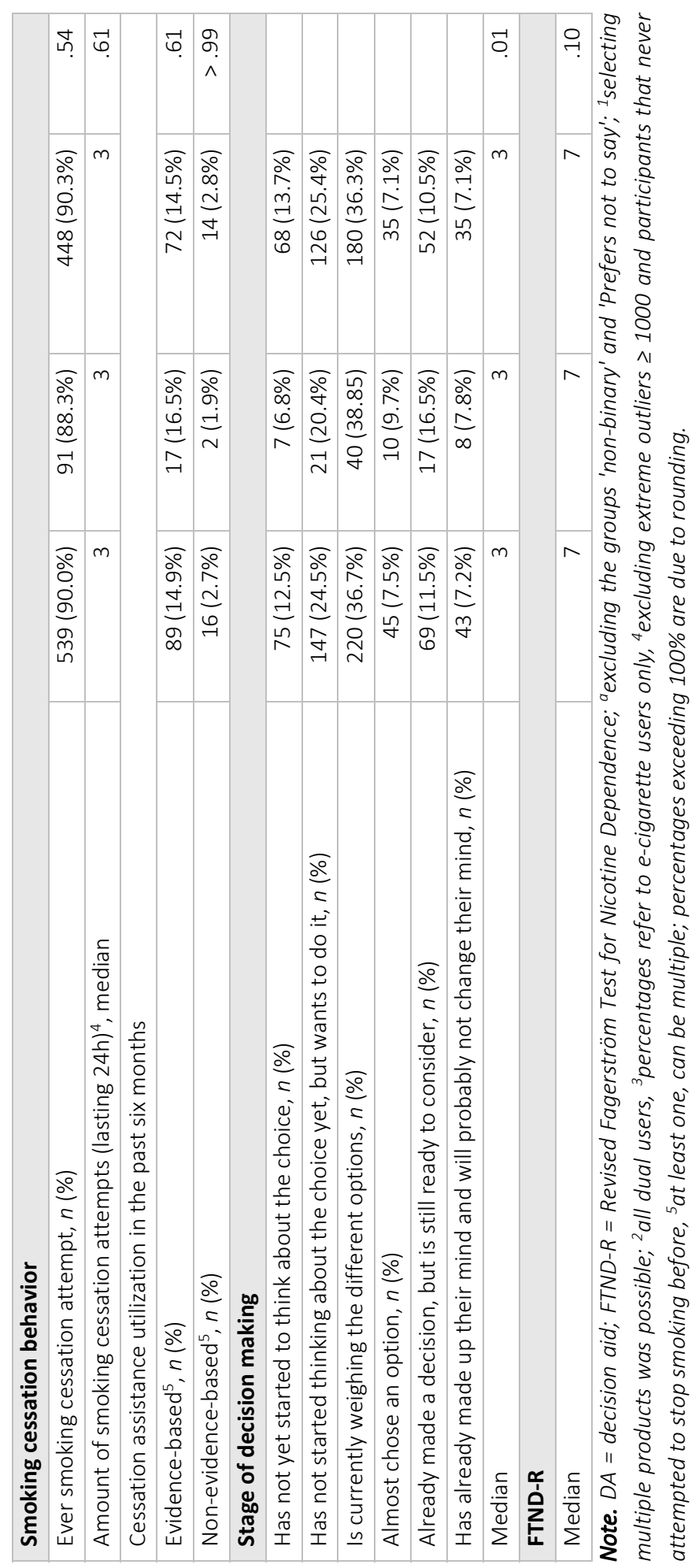




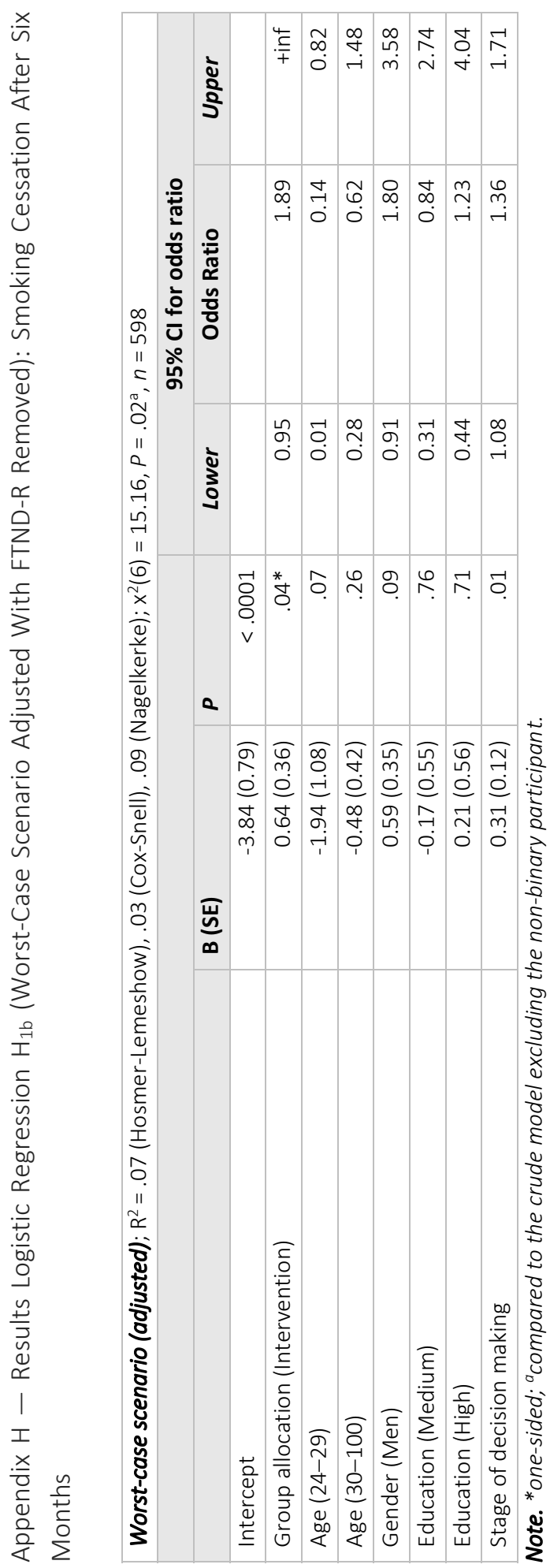




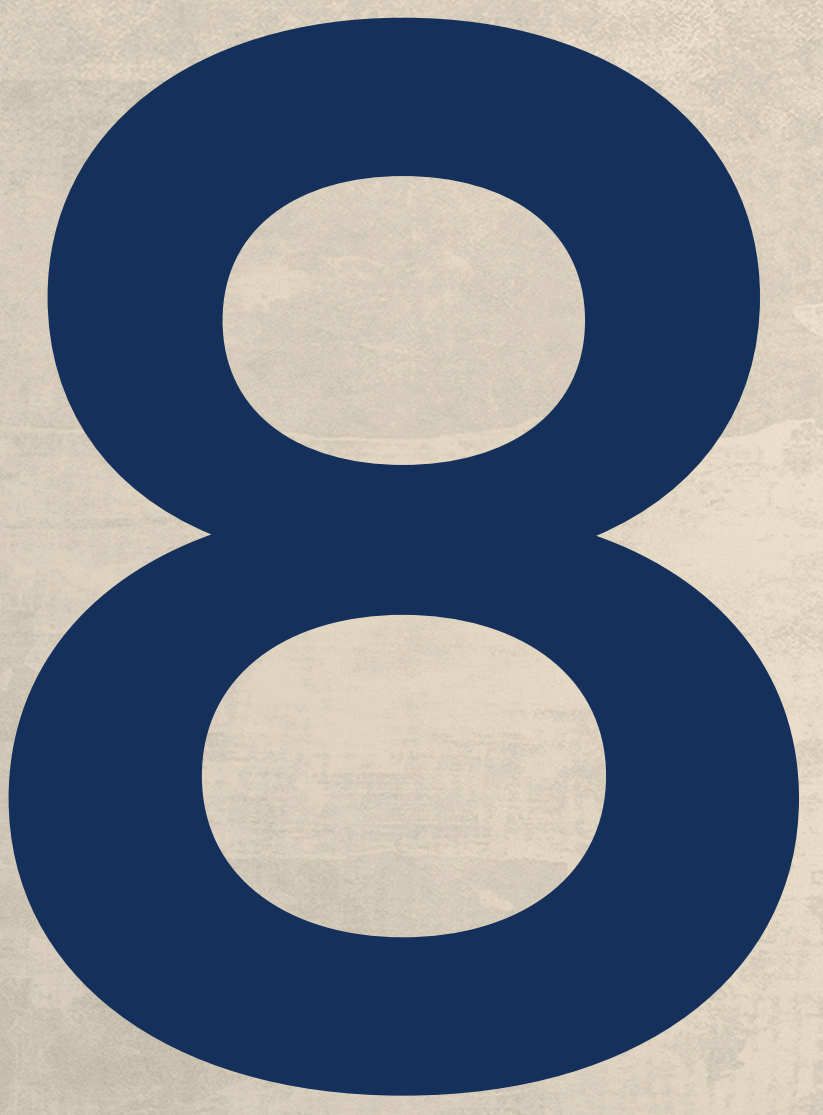




\section{chapter 8}

general discussion 
The aim of this thesis was twofold: (1) to provide a more thorough understanding of decision aids (DAs) applied to health promotion and (2) to describe the work that was undertaken to develop and test the smoking cessation DA VISOR. Therefore, this general discussion will also be divided according to these sub aims. In both sections, first, the main findings from the different chapters will be presented briefly. Second, these findings will be integrated, discussed, and put into a broader context and recommendations will be summarized at the end of both sections. Finally, strengths and weaknesses of the research presented in this thesis will be described.

\section{DAs Applied to Health Promotion (Decisions) Main Findings}

In Chapter 2 theoretical work was presented to show the similarities and differences between behavior change interventions and DAs, while also highlighting room for synergistic effects. The two approaches differed in terms of their (1) goals and foci, (2) theoretical basis, (3) development frameworks, (4) active intervention ingredients, and (5) effect evaluation. Interestingly, the differences identified seemingly stem from the fact that behavior change intervention developers focus on improving behavioral outcomes in their target population, whereas DA-developers tend to focus on improving (deliberate) decisional processes. Despite these differences, room for synergy was also identified. Namely, that knowledge generated by researching DAs and (informed) decision-making processes could be used to help individuals choose goals that are in line with their values and preferences. Insights from researching behavior change interventions, on the other hand, could subsequently be used to facilitate both engagement with and implementation of those goals. In the long run, this integration could increase the benefits for people engaging with their health, healthcare, and health behaviors.

In Chapter 3 a scoping review was presented to reveal the current state of knowledge about DAs that support informed decision making in the area of health promotion, namely diet, physical activity, sleeping, and substance use-focusing on their characteristics, intervention elements, theoretical foundations, and (cost-)effectiveness. Thirty-five scientific articles and four DAs identified from grey literature were included in this scoping review. A clear majority of included sources described DAs in the field of substance use. All DAs offered information, and value and/or preference clarification methods, however in all DAs many other elements were included as well (e.g., goal setting). Overall, DAs' effects on the reported outcomes were mixed, and only few studies used standardized measures, such as decisional conflict, for (effect) evaluation. However, the scoping review also showed some positive behavioral effects due to the health promotion DAs, particularly regarding smoking (especially taken together with other evidence [52]). The included studies, however, reported heterogeneous results and outcomes, impeding knowledge synthesis. I therefore identified areas of 
improvement, e.g., that consensus needs to be reached on which outcomes are relevant for health promotion DAs, so that it can be established which intervention elements are effective regarding health behavior decision making and for which processes.

\section{Optimizing DAs Applied to Health Promotion}

In the theoretical work presented in Chapter 2, ideas were raised as to how behavior change interventions typically used in health promotion can benefit from DA-insights and vice versa. Based on this theoretical discussion and to facilitate the integration of scientific insights from researching behavior change interventions and DAs, I recommended to (1) bring both fields together and promote interprofessional discussions, (2) train (health) professionals to recognize strengths of both approaches, (3) investigate the synergy of the two fields, and (4) be prepared for and try to mitigate a culture shock when the fields start to interact. One of the practical examples of how to achieve synergies described in Chapter 2 is that behavior change techniques, such as implementation intentions [75] (a form of goal setting), could be used to increase DAs' effectiveness by helping people to make plans to implement the goals that have been chosen during the DA. The scoping review in Chapter 3 revealed that in the field of health promotion, DAs often already contain intervention elements that are regularly used in behavior change, such as goal setting, for example by setting a quit date [53,131]. However, our scoping review also showed that the added value of such behavior change techniques is seldomly investigated, as both intervention and control groups often received these intervention elements. Likewise, most of the included studies (with one notable exception [143]) also did not test if elements typically used in DAs had positive effects on behavior and decision making within health promotion DAs. Therefore, it is currently rather unclear which intervention elements are effective within health promotion DAs - the typical DA-elements (e.g., value clarification methods [VCMs]) or the behavior change techniques (e.g., goal setting) that were included-and whether synergy can indeed be achieved when both types of intervention elements are combined. Historically evaluations of DAs have focused on the question "Are DAs effective?" (e.g., [13]), however, based on the insights described in this thesis it might also be important to focus on "Which ingredients make DAs effective?". This will not only help to build a stronger empirical basis but will also help to develop more effective (health promotion) DAs in the future. For example, an experimental study could be set up in which a goal-setting exercise is added to a health promotion DA that is than compared to a DA lacking only this element. This way researchers would avoid what is described as method co-occurrence by Peters et al. [292] in order to acquire a robust evidence base with regard to effective (health promotion) DA-elements.

Next to this, there are two other reasons that make it difficult to find out how health promotion DAs can be optimally used. First, a distinct lack of theoretical work linking informed decision making to behavior change, as was observed in the scoping review 
described in Chapter 3. Theoretical underpinnings of interventions allow researchers to investigate why and under which conditions interventions are effective. This will be illustrated using an example from health promotion. That is, the well-known behavior change technique 'fear appeal' (also known as 'fear arousal'). Fear appeals are often described as being able to change individuals' behavior-if individuals also hold high self-efficacy expectations (i.e., if they believe that they are able to change their behavior) [75]. This means, that the effect of fear arousing communication is not straightforward and always positive but rather depends on another theoretical condition (i.e., self-efficacy expectations) [293]. This has been shown in a meta-analytic test by Peters et al. [293] which was only possible due to the use of theory and the consequent measurement of theoretical constructs. This example shows that theories can be used to gain insight into the conditions under which intervention elements can be optimally used. However, theoretical work as this is rarely being done in relation to DAs [294] and when it is done the descriptions are often superficial in nature [82]. This was also shown in the scoping review conducted as part of this thesis. A relatively high percentage of studies used theories to some extent, e.g., to guide DA-content development, however, this was often described implicitly, and it was not often tested whether theoretical constructs act as moderators or mediators to further our understanding of why and how DAs work. Also, the dual nature of health promotion DAs (i.e., they mostly target both decisional as well as behavioral processes) requires that the theoretical lens that is being used to study health promotion DAs explicitly links informed decision making and behavior (change) to inform DA-optimalization, however (as shown in the scoping review) most studies used either the one or the other approach. Research around health promotion DAs would therefore benefit from making greater use of both the behavioral as well as the decisional sciences to further understand how we can optimize decision support. One possible way to do this would be to use the Self-Determination Theory (SDT) [86]. The SDT is a macro theory of human motivation often used in behavior change, but underused in relation to DAs, although it is well suited due to its focus on personal autonomy. SDT states (among other things) that people are more likely to achieve longlasting behavior (change) when self-determined motivation is facilitated, i.e., a form of motivation that reflects aspects such as personal enjoyment or important values, as opposed to more controlled forms of motivation. This self-determined motivation is achieved by fulfilling three psychological needs: (1) competence, the feeling to be able to perform a behavior; (2) autonomy, the feeling of having agency over one's life; and (3) relatedness, the feeling of being connected. I used the SDT throughout the whole project described in this thesis as a theoretical framework as it is unique among the theories used in the behavioral sciences due to the focus on personal autonomy, which aligns with the core ideas of informed decision making [16]. Also, the SDT has been linked to concepts related to informed decision making before, namely shared decision making [15]. However, due to a lack of time, I was unfortunately unable to test whether the SDT can indeed help to better understand 
the conditions under which health promotion DAs are effective. However, this could be a way to better understand the effects of such DAs.

Second, in our scoping review we found that that not all studies reporting on health promotion DAs followed a randomized controlled trial (RCT) or similar protocol and that reported outcomes were rather heterogenous. Both aspects limit synthesis and interpretation of the findings. It was especially striking that most of the included studies did not measure decisional outcomes, even though DA-scholars strongly advocate that evidence regarding decisional outcomes should be gathered $[85,295]$. Due to the dual nature of health promotion DAs behavioral effects should of course also be assessed, but decisional effects should not be disregarded as researchers and developers otherwise cannot make any claims regarding one of the main goals of health promotion DAs, i.e., to support users in making informed decision. Heterogeneous outcome reporting hinders knowledge syntheses (such as scoping reviews), in this particular case, regarding health promotion DAs' effects on decisional outcomes. Moreover, at this point, it is also rather unclear how most decisional aspects, such as decisional conflict, link to most behavioral outcomes, such as smoking cessation. If it would become more common to assess both, studies could investigate the link between the two more thoroughly. For example, a longitudinal study could assess whether people with higher decisional conflict are less likely to achieve smoking abstinence.

\section{Summary and Recommendations}

In conclusion, future research should invest in three domains: (1) identification of effective (health promotion) DA-elements, (2) theoretical work linking decisional processes and behavior to identify conditions of use (i.e., moderators and mediators) for DA-elements (e.g., by making use of the SDT), and (3) the assessment of decisional and behavioral outcomes in randomized studies. In the long term, this could not only help to answer why certain health promotion DAs are effective while others are not, but also enable DA-researchers and developers alike to offer optimized (health promotion) DAs. Theoretically, this will also allow to develop a taxonomy describing which DA-elements should be used under which conditions to ensure DAs' effectiveness (similarly to taxonomies used in behavior change [75]). This taxonomy could then be used as an accessible 'manual' by DA-researchers and developers to develop highly effective (health promotion) DAs. A taxonomy would not be only more user-friendly but would also ensure consistency and quality to the degree that it is used and allow for comparisons between different DAs. 
VISOR

Main Findings

In Chapter 4 a cross-sectional study was presented that was conducted to identify potential early adopters of a digital smoking cessation DA-such as VISOR-before it is available. I was able to show that individuals' general tendencies to react to decision-making situations (i.e., their general decision-making style) can influence intention to use a smoking cessation DA in the future. Further, I identified two types of decision makers among individuals motivated to quit smoking: (1) "Avoidant Regretters" were more avoidant, more regretful, and tended to depend more on others in their decision making, while (2) "Intuitive Non-regretters" were more spontaneous and intuitive in their decision making. "Avoidant Regretters" also showed a higher interest in the (still) hypothetical DA, yet this effect disappeared when corrected for socio-cognitive variables - hinting at a possible mediation effect of general decision-making style on intention through socio-cognitive variables, such as attitude-an idea that was supported by the subsequent mediation analysis.

In Chapter 5 a needs assessment among potential end users (i.e., people motivated to quit smoking) and smoking cessation experts was presented that was used to inform the development of VISOR. I showed that potential end users' knowledge, values, needs, and opinions regarding a smoking cessation DA were rather heterogenous. However, cessation assistance tools' effectiveness and costs were most often described as the most important characteristics of smoking cessation assistance. Also, I found that at this point (i.e., before the development of VISOR) end users often acquired information via own experiences, people in their environment, or the media. Discrepancies between potential end users were identified that could hint at different conceptualizations used by the end users and professional experts. This was especially clear regarding the concept of 'effectiveness'. Experts did not establish consensus regarding the importance of this characteristic, while the potential end users were quite clear that this was highly important to them. However, the potential end users seemed to regard effectiveness as a rather individualistic concept (i.e., "Is a tool effective for one particular person?") opposed to the more group-orientated definition commonly used by experts.

In Chapter 6 the development process of VISOR was presented. In order to develop a first version of VISOR, the insights from the needs assessment were used together with insights from the SDT. This first prototype was then alpha-tested in September 2019 and beta-tested for usability in December 2019. Ultimately, we developed two versions of VISOR during the development process. The final DA-versions both contained (1) an information section, (2) an optional knowledge quiz, (3) a brief smoking assessment, (4) intuitive decision, (5) intermediate advice, (6) an explicit VCM (only in the intervention version), (7) computertailored advice (only in the intervention version), and (8) access information. Next to the 
development process, I also presented the study protocol that was followed to compare the two versions in Chapter 6 . The primary outcome of this RCT was smoking cessation success after six months, while smoking cessation success after one month, uptake of evidencebased smoking cessation assistance, and decisional conflict were secondary outcomes.

Subsequently, in Chapter 7 the behavioral and decisional effects of VISOR as found in this RCT were presented. Effects on the primary outcome (i.e., smoking cessation success after six months) in favor of the intervention version were only observed in a worst-case scenario (i.e., using penalized imputation in which participants lost to follow-up were considered to still smoke). Effects on the secondary outcomes were only observed regarding smoking abstinence after one month, and cessation assistance uptake after one and six month(s), but also only in the worst-case scenario. No effects on decisional conflict were observed. While many participants signed up for the RCT, attrition during the completion of both versions of VISOR and during the RCT in general were remarkably high, which resulted in an underpowered trial-possible leading to the lack of clear effects. Interestingly, during the completion of VISOR attrition was higher in the intervention group, but at all other points attrition was higher in the control group. A notable finding during the RCT was that participants' stages of decision making (i.e., in which phase of the decision-making phase they were in before the used VISOR) had a rather consistent effect on attrition and all primary and secondary outcomes.

\section{Smoker Recruitment and Retention}

The cluster analysis presented in Chapter 4 showed that general decision-making style might play a role in the interest in smoking cessation DAs. Therefore, this finding was used to design the recruitment strategies (e.g., by naming the reduction of regret as one possible benefit of using VISOR) and VISOR itself (e.g., by including an intuitive element). However, even though I identified types of individuals interested in a smoking cessation DA based on their general decision-making style, and included this knowledge in the RCT recruitment strategies, together with my team I still struggled to include the necessary number of individuals to reach sufficient statistical power to conduct the analyses planned. That said, the major recruitment issue that we faced during the RCT was not related to the initial recruitment, but rather to attrition during completion of the DA (i.e., non-usage attrition [273]) and the follow-up questionnaires (i.e., dropout attrition [273]). Initially, more than 2300 individuals initially signed up for the study - far exceeding the number initially strived for (i.e., 1592). Before I will be focusing on these two types of attrition in more depth, it should be stressed that digital interventions, such as VISOR, are known for high attrition rates [273] and that recruitment started during the hack of Maastricht University (largely covered in national Dutch media [296]) and that the largest part of the RCT took place during the pandemic of 
the Coronavirus disease 2019 (COVID-19), which might both have aggravated the attrition problems.

Non-Usage Attrition

In Chapter 4 I focused on intention to use a digital smoking cessation DA and not on actual behavior, and used this information to design the recruitment strategies for the RCT. However, as it is known that there is a substantial gap between intention and behavior [95], findings from the attrition analysis presented in Chapter 7 might provide better insights into factors that influence actual usage of a smoking cessation DAs as actual behavior (i.e., usage of the DA) was observed. As such, these findings might provide better input to design recruitment strategies in the future. Factors that participants lost to non-usage attrition differed on (compared to participants that completed VISOR) were: their age, level of education, if people used other tobacco products than those included in the questionnaire (e.g., cigarettes), the average amount of cessation attempts, if people were relatively early in their decision-making process (e.g., if they had not yet started thinking about the options), and interestingly, if people were allocated to the intervention version. In the multivariate analysis, only group allocation, age, and the level of education remained as significant predictors of non-usage attrition. That is, participants in the intervention group were more likely not to complete VISOR, while those that were 24-100 years (compared to 18-23 years) old, and those with a high (compared to a low) level of education were more likely to complete VISOR.

Using these findings together with the findings from the interview study presented in Chapter 5 might lead one to conclude that the duration of the DA(s) might have played a role in the high level of non-usage attrition as it was shown in the interview study, that overall younger people favored a shorter DA, while older people seemed to lack a strong opinion about a DA's optimal duration. Given that younger people and those randomized in the intervention group (in which VISOR was longer due to the additional intervention elements) were less likely to finish VISOR, this could indicate that the duration needed to complete VISOR played a role here. And in fact, available data provided by the intervention host indicates that the differences in usage times between the groups were significantly different. This difference possibly drove the differences in non-usage attrition between the intervention and control group as end users might have perceived the intervention as too long which in turn negatively impacted on their user experience. For online healthcommunication programs it has already been shown and described that that positive user experience plays an important role in the completion of said programs (e.g., $[205,297]$ ), however, I am not aware of specific studies focusing on this in relation to DAs. Future research should therefore be conducted to (1) investigate whether user experience also plays an important role in the decision to complete online DAs, for example, by focusing on 
usage times, and (if so) subsequently (2) investigate which user experience components are particularly important. Also, research should find more ways to provide abbreviated forms of DAs, if possible, also for decisions with a large set of options. To illustrate, Witteman et al. [40] already described a digital VCM (including computer-tailored advice) that consisted of dynamic web sliders representing both values and preferences (i.e., the included options). Those web sliders were linked, which meant that participants who indicated that a particular value is very important to them, the slider representing the preference moved equally. While this technique could shorten usage times considerably, at this point this technique is relatively hard to use for decisions involving multiple options, which is why we were unable to use it within VISOR.

Next to this, taken together the attrition analysis, also indicates that there is no such thing as one perfect DA for everyone, but that tailored versions may be the way forward. Tailored or so-called 'intelligent DAs' have recently been proposed in the literature [298]. Motorny et al. [298] recently presented a framework that can be used to individualize DAs based on multiple components, such as one's information needs. While promising, their approach could potentially lead to the same problem recently described in the scientific literature in relation to computer-tailored interventions: that computer-tailoring based on multi-item questionnaires can increase participant burden and thereby non-usage attrition as well [284]. Based on the findings from the RCT, a feasible way to offer computer-tailored DAs without increasing participant burden (much) could be to employ the one-item stage of decision making scale as the scale is much shorter than the scales traditionally used within computer-tailored interventions (e.g., $[46,71,299,300])$. This might be especially promising in the context of smoking cessation as approximately $40 \%$ of the participants in our sample had not even started to think about the choice (i.e., which smoking cessation tool to use) before they started using either version of VISOR. Also, participants' decision-making stage was the only factor that consistently emerged as important in univariate analyses in relation to both non-usage and dropout attrition, further highlighting its importance. It should be noted, however, that these effects on attrition became less consistent once they were corrected for other variables. The most plausible explanation for this is that different groups within our sample (e.g., older compared to younger participants) differed in their stage of decision making, leading to stage of decision making becoming insignificant once all predictors were added in the same model. Overall, research into these decisional stages seems to be limited in general-especially compared to other decisional determinants and/or factors, such as decisional conflict. Our findings suggest that DAs might not be highly suitable for people who are very early in their decision-making process as those in early stages had higher attrition rates. As described before, this is especially problematic in the context of smoking cessation, as a substantial group of people in the Netherlands seems unaware of the many effective tools to aid smoking cessation efforts [301], and thus of the decision at hand (i.e., the decision between different cessation assistance tools). This was 
also reflected in the findings of the interview study presented in Chapter 5 in which participants tended to not be aware of all possible smoking cessation assistance tools. Logically, a substantial numbers of Dutch smokers - and also within the sample of the RCT presented in Chapter 7-are located in the earliest stage of decision making in which people have not started thinking about the decision at all [240]. While research into this area is lacking, it is assumed that DA-users in early stages of decision making are simply not ready for DAs, for example because they are not willing to think about the decision yet or are still in denial [240]. Based on those insights it might thus be the case that even though a substantial part of our sample was interested in decision support regarding smoking cessation (and thus initially signed up), a large group of people experienced VISOR as too overwhelming due to the decision-making stage they were in. This could be alleviated by tailoring DAs - such as VISOR - to participants' stage of decision making. For example, individuals in the early stages might receive interventions that are educational and supportive in nature, while individuals in active deliberation might be offered 'traditional' DAs, and individuals at later stages might receive support to implement their decision. The problem at this point, however, is that research has not yet tested this approach and also does not show suitable strategies for each stage. As this idea is therefore still purely theoretical, it should (1) be explored in future research projects how stage of decision making is related to non-usage attrition (e.g., by making use of the think-aloud method to see how people in earlier stages react to 'traditional' DAs [225] to identify potential areas to intervene on), and (2) (decisional) needs per stage of decision should be identified to design effective and attractive DA strategies for participants in each stage. In the long run, studies could then be set up to see if tailoring DAs to participants' stage of decision making actually leads to more promising retention-and, if more participants are retained-to greater engagement with and effectiveness of the DA. To this end, the earlier identified strategies could be tested in experimental and/or randomized studies for their effects on retention, engagement and, ultimately, effectiveness.

Finally, it was also found that higher educated people completed DA more often than lower educated people. While the association between health literacy and education is not perfect (i.e., even highly educated people can show inadequate health literacy skills [302]), there is a substantial association between the two [302]. One might therefore assume that both versions of VISOR were not fully equipped to be used appropriately by users with lower health literacy-leading to the second possible explanation for the high level of non-usage attrition. This is remarkable insofar as significant changes were made in the course of the iterative development process to ensure optimal usage capabilities for especially this group, e.g., by having an expert in this area check the texts included in VISOR. One could therefore conclude that these changes were not substantial enough. When one critically compares the current versions of VISOR to the updated recommendations from the International Patient Decision Aid Standards (IPDAS) regarding health literacy [303], it stands out that most of 
them have already been applied to a certain extent (e.g., steps were taken to ensure VISOR's readability), except one: Both versions of VISOR heavily relied on written information and did not include (audio-)visual support to increase their accessibility for users with a low level of health literacy. Future versions of VISOR might thus benefit from more (audio-)visual content next to the written content. In the 2021 IPDAS update regarding health literacy and DAs it is also stated that only $11 \%$ of the DAs included in the last Cochrane review [13] focusing on DAs were developed for, or evaluated with, populations who are socially-disadvantaged (including people with low health literacy) [303], highlighting a dire need to focus stronger on these groups. Future research should therefore strive to find new ways to make DAsincluding VISOR-more accessible for users with a low level of health literacy and explore the benefits of using (audio-)visual content. In the end this information could then be used to design DAs that are effective for end users with a low and high level of health literacy as research has shown that audio-visual content can be helpful for both groups [304-306].

\section{Dropout Attrition}

Similar to non-usage attrition, comparing people who dropped out before completing the follow-up questionnaire with people who did not, provides an indication of underlying reasons for dropout attrition. Overall, considerably fewer factors seemed to have played a role in dropout attrition. The group that dropped out directly after completing VISOR differed from those who did not in terms of group allocation, gender, and stage of decision making, while group allocation was also revealed as a significant factor associated with dropout after one month and stage of decision making with dropout after one and six month(s). All those factors remained significant in multivariate analyses, expect for stages of decision making after one month, meaning: (1) Participants in the intervention group were less likely to dropout directly after the DAs and after one month, (2) men (compared to women) were less likely to dropout directly after the DAs, and (3) those in higher stages of decision making were less likely to dropout directly after the DAs and after six months. Interestingly, the differences in group allocation moved in the opposite direction for dropout attrition compared to non-usage attrition, as more people allocated to the intervention version completed the follow-up questionnaires than people allocated to the control version. Thus, while receiving the intervention version resulted in significantly less people finishing VISOR, those who did finish the intervention version were more likely to fill in the follow-up questionnaires compared to those who received the control version. At this point, this difference is hard to explain without further research. However, the information in regard to participants' stage of decision making reinforces the argument that researchers should investigate whether tailoring to stages of decision making could be a worthwhile approach to improve retention in DA-studies, as this factor again emerged as important. 
Another way in which retention during an RCT (or another trial) could be optimized is that DAs (such as VISOR) could be offered in whole or in part in a healthcare setting. Recruitment that involves direct contact with potential participants has been shown to positively influence initial recruitment and retention in RCTs focused on behavioral smoking cessation interventions [279]. Applied to VISOR, for example, patients/clients could use VISOR before a smoking cessation consultation to prepare said consultation. Anecdotally, I know that different healthcare providers at least planned to use VISOR in such a manner during the RCT presented in this thesis. Alternatively, VISOR could be used together during a consultation so that providers and patients/clients could identify suitable and value-consistent smoking cessation options while talking to one another. While potentially promising, the major drawback of this approach would be that the fully online nature of DAs such as VISOR would be partially lost as there would also be some form of 'offline' (i.e., face-to-face) contact, which could limit a DA's potential reach, while also increasing related costs. Also, using VISOR together within a consultation might lengthen the consultation time [13], which might not be desirable from a healthcare provider's perspective. To counteract all of this, healthcare counseling as part of a DA could also be offered online via videocalls [280] or a virtual chatbot [281] that fulfills the role of a healthcare provider. A hybrid model is of course also possible in which the DA is both available online and offered by healthcare providers such as general practitioners. Patients/clients might then be invited to choose between the version that is fully online and the version that is partly offline. This hybrid approach might even increase the potential reach as people could choose between the different counseling modalities depending on their own preferences. To elaborate, the current version of VISOR probably was not attractive to people preferring offline contact, while an adapted offline version could be potentially rejected by people preferring fully online interventions. A hybrid approach, on the other hand, would be appealing to both. Moreover, a hybrid approach might help to counteract that VISOR in its current form excludes people with a low level of digital literacy (or without access to the internet for that matter). Having said all that, the major potential problem with a stronger embedment within healthcare is that implementation of DAs in clinical practice is known to be suboptimal [307]. That said, Joseph-Williams et al. [308] have recently identified strategies to facilitate routine DA-implementation and Zijlstra et al. [309] recently developed an intervention similar to a DA that is meant to support consultations in primary care around evidence-based cessation assistance, showcasing that implementation of such interventions is indeed possible. Should VISOR be adapted for healthcare use, it is important to follow the aforementioned strategies to ensure optimal implementation, i.e., (1) the adaption of VISOR should be coproduced with the healthcare providers meant to adopt it to ensure optimal implementation; (2) entire healthcare teams meant to use VISOR should be trained in using it optimally; (3) VISOR's end users (i.e., individuals motivated to quit smoking) should be prepared and prompted to use VISOR; (4) management should be convinced of the added value of VISOR; and (5) data should be collected to showcase actual improvements due to using VISOR [308]. It should be stressed that healthcare 
implementation is not an 'easy fix' but is a complex process that should be regarded as such. Should implementation of VISOR within healthcare be considered, this means setting up a separate follow-up trajectory and process to ensure optimal implementation.

\section{Development Process of VISOR}

Both versions of VISOR (with and without the additional elements) were developed by following the IPDAS development process [59] which involved a needs assessment among all stakeholder groups-potential end users, smoking cessation counselors, and scientific experts-(Chapter 5), an alpha test in which an early version was tested among all stakeholder groups (Chapter 6 ), and a beta test in the form of a usability test in which the second to last version of VISOR was tested before the RCT (Chapter 6) took place, in which VISOR's final versions were tested (Chapter 7).

However, during the development process we also encountered several issues that highlight future areas of interest. First, the stakeholder groups were broadened throughout VISOR's iterative development process compared to the 2013 IPDAS development process that we followed [59] by also including scientific experts. In this development process the IPDAS focusses on patients (i.e., the direct end users of DAs) and clinicians (especially in the early phase were needs and views are assessed). Here it should be noted that in both the 2013 IPDAS background paper on DA-development [59] as well as in the 2021 update [310] references to 'other' stakeholders are made. That said, their focus is on end users and healthcare professionals. This reflects the fact that the IPDAS tends to focus on treatment and screening DAs that are often offered in clinical settings [13]. However, we decided to also include a third group with relevant expertise (i.e., scientific smoking cessation experts) as health promotion-related decisions (such as smoking cessation) are in fact often made without consulting clinicians (e.g., [60]). As this group provided us with valuable insights (see Chapter 6), it should be considered whether the inclusion of additional stakeholder groups is needed next to the ones strongly advocated by IPDAS. That said, DA-developers also need to align the (sometimes) dissenting opinions and including more academically trained stakeholders could potentially also increase the complexity of DAs. In the case of VISOR, however, I found that that these dissenting opinions can also generate valuable insights, if one takes the time to analyze how and why the opinions of different stakeholders differ. For example, insights regarding the concept 'effectiveness' only emerged after comparing the opinions of different stakeholders, otherwise one might have concluded that 'effectiveness' is (not) important (depending on which stakeholder groups would have been included). Handling dissenting stakeholder opinions will only become more difficult the more stakeholder groups are involved, yet it is my opinion that DA-development processes should reflect that clinicians are not the only professionals with relevant expertise that should be 
included and that DA-developers should therefore reflect critically on which stakeholders to involve in the development and, if necessary, also include 'less traditional' groups.

Second, even after extensive testing of VISOR before the national RCT some technical difficulties during the RCT still occurred (see Chapter 7 for more information). While technical difficulties cannot always be avoided within digital interventions [220], they can lead to a number of later difficulties which, contrary to the magnitude of the actual technical difficulties, can have greater ramifications than expected. In our case, for example, some people who should have been invited to the follow-up measurement after one month were lost due to a small coding error. In 2012, Peters et al. [221] published a commentary in which they called for full disclosure in the behavioral sciences to facilitate scientific advances in a manner that aligns with the 'Open Science' movement [311]. In said article, the authors proposed several guidelines, such as the sharing of all study materials needed to replicate the study at hand. Interestingly, the authors point out humans tend to make mistakes, however, subsequently they do not ask scientists to actively share those mistakes with a broader public even though they do advocate to share other elements more openly. Based on the VISOR-development process and the difficulties I encountered, I propose that technical difficulties within digital health(care) interventions should be reported more, as well as ways to limit these. In Chapter 7, I made an attempt not only to be very open about technical errors that can occur during an RCT, but also to explain very transparently how I dealt with the specific error that occurred during the RCT and how this error influenced the further course of the trial and analyses. In a similar vein, it would be interesting if future studies could be carried out that investigate the influence technical errors can have on studies and participants, e.g., by mimicking such a technical mistake as the one that happened during the RCT presented in this thesis. At the same time, it might also have been useful if additional steps had been taken to prevent errors altogether. Retrospectively, for example, I also could have included a pilot trial of VISOR in between the beta test and the RCT which might have revealed any lingering technical difficulties. Overall, it could be helpful if the behavioral sciences not only developed strategies to communicate technical difficulties more clearly (similar to other materials more openly shared nowadays), but also if research projects were set up to investigate the difficulties' effects more closely and how those could be avoided. Ultimately, this would probably also require a change in scientific culture as I noted that this topic is not routinely brought up in scientific publications.

\section{Effectiveness of VISOR}

As described earlier, I was unable to find effects in favor of the intervention version on either the primary or secondary outcome(s) except for the effects uncovered in the worst-case scenario. In Chapter 7, two different explanations were provided for this: (1) the RCT was underpowered or (2) there truly is no effect. Based on the available data, I can only conclude 
that the first explanation (i.e., that the RCT was underpowered) is true in the sense that the needed sample size that was calculated a priori was not achieved. However, this does not mean that effects would have been found if more participants had been included. That said, since all the effects found point in the expected directions, it is at least somewhat likely that a larger sample would have led to significant effects. However, I am unable to gauge how (un)likely the other explanation (i.e., that there truly is no effect) is. The only other study that tested if an explicit VCM can increase health promotions DAs' effectiveness also failed to show an effect [143], however, the authors tested this in a hypothetical scenario which limits the conclusion that can be taken away from this study. A replication of the RCT presented in this thesis might thus seem crucial. However, before a replication should be conducted, it might be worthwhile to investigate if the potential strategies described throughout this discussion could be feasible approaches to improve DA-usage and study retention. Based on the insights presented in this thesis, for instance, participants' stage of decision making emerged as a potential interesting factor to improve DAs such as VISOR. Also, as it emerged as the most consistent factor impacting on behavioral as well as decisional outcomes. In the future, it might thus be interesting to study if tailoring to participants' stage of decision making would increase DAs' effects. More information about how this could be realized can be found in Interest in a Smoking Cessation DA.

Next to this, it should be stressed that the RCT was not conducted to evaluate if smoking cessation DAs in themselves are effective. Rather it was investigated if specific elements commonly used in DAs (i.e., explicit VCMs paired with computer-tailored advice) are effective in terms of behavioral and decisional effects. Essentially, I thus already tried to counter one of the shortcomings described in Optimizing DAs Applied to Health Promotion. That is, that the effects of specific elements are rarely investigated. Thus, ultimately, a lack of an effect does not indicate that smoking cessation DAs are ineffective-in fact, other research suggests that they are [52] and, interestingly, even within the control group in our RCT a higher percentage of smokers stopped smoking than within the intervention group of the only other tested Dutch smoking cessation DA [42]. What the RCT presented in this thesis does add is that adding explicit VCMs paired with computer-tailored advice to smoking cessation DAs might in fact make them more effective as all effects moved in the expected direction. However, more research (with larger samples) is needed to substantiate this.

\section{Summary and Recommendations}

To conclude, even though I was unable to find clear effects of adding an explicit VCM paired with computer-tailored advice to a smoking cessation DA, important and interesting conclusions can be drawn from the entire development and evaluation process presented in this thesis. These conclusions can be applied to future versions of VISOR, but also have more broader ramifications that can be used in future works related to (health promotion) DAs. 
Based on the insights obtained in relation to attrition, future research should invest in three domains: (1) establishing if user experience components (especially usage times) can influence attrition rates within DAs and DA-studies, and (if so) which ones are particularly important; (2) further investigating the role of tailoring DAs, both with regard to attrition and their effectiveness, e.g., to stages of decision making; and (3) putting a stronger emphasis on making DAs overall more accessible to end users with a low level of health literacy, e.g., by investigating the added value of (audio-)visual content in (health promotion) DAs.

Based on the insights generated during the development process, DA and digital health researchers can mainly take away two lessons from this thesis: (1) DA-development processes can include more stakeholders than (potential) end users and clinicians, and (2) if researchers are more open about sharing and describing (technical) problems during scientific studies, this could help the scientific community, as new ways can be found to prevent said (technical) problems.

When it comes to the practical aspect of implementing VISOR nationally and openly (i.e., not within a trial context) one should not only draw from the domains described above, but also consider whether a stronger embedding in healthcare pathways could be beneficial for a DA as VISOR. Based on our findings, it is also still unclear if the explicit VCM (paired with computer-tailored advice) should be included as we found no clear indications that it does increase effectiveness (although this was probably due to a lack of statistical power), while we found that it may increase non-usage attrition. This means that even if the explicit VCM paired with computer-tailored advice does increase effectiveness it might come at the cost of losing more end users.

For more practical implications of the findings presented in this thesis, I would like to refer to the impact paragraph.

\section{Strengths and Weaknesses}

The work presented in this thesis has strengths and weaknesses. One of its major strengths is that this thesis is not only grounded in quantitative approaches (Chapter 4, Chapter 7) and qualitative approaches (Chapter 5), but also encompasses theoretical work (Chapter 2, Chapter 6), and a knowledge synthesis (Chapter 3). I also was able to use theoretical concepts to understand a practical problem (Chapter 4) and used underlying theory to inform DAdevelopment (Chapter 5). Together with my team I also followed a very systematic DAdevelopment process in the form of the IPDAS development process [59] that included multiple relevant stakeholder groups throughout the entire iterative process presented in this thesis. However, this work also contains some weaknesses, which I will address in the following. 
As common in the field of health promotion my team and I also heavily relied on selfreported data throughout the project described in this thesis. However, this stems from the fact that we tended to research concepts that cannot be naturally observed [152]. To illustrate, in the study reported in Chapter 4 participants were asked to answer several questions that related to their general decision-making style. This is a concept that cannot be directly measured or observed. Even if people would be observed in multiple situations researchers could still not infer totally objective data regarding their decision-making style as the construct in itself is not a natural construct that can be objectively measured (as for example weight). That said, we also relied on self-reported data for outcomes that could have been objectively measured-namely, smoking cessation. However, due to the fact that this would have required more resources (e.g., monetary) and as self-reported smoking data has shown high validity [288], I regarded this limitation as minor. Therefore, I decided to only rely on self-reported data for this outcome. It should also be emphasized that participants themselves did not experience any disadvantage in case they indicated to still smoke during the follow-up measurement, making it unlikely that participants provided certain answers to receive a reward.

Related to this, the primary outcome of the RCT carried out as part of this project (i.e., smoking cessation success) might be perceived as unusual by some, as DAs are mostly evaluated to the extent that they can support users in their decision-making processes. However, given that even smokers themselves indicated throughout the project that they would use VISOR to increase their chances of success in smoking cessation, I would still consider this primary outcome to be the most appropriate. That said, it should be emphasized that I made sure to test the DA both for its behavioral as well as decisional effects which is a major strength of the study-especially in light of the results of the scoping review presented in Chapter 3 in which it was found that RCTs tend to only focus on behavioral outcomes.

Another weakness may be that some studies reported in this thesis rely on cross-sectional data. This becomes especially apparent in Chapter 4 which would have majorly benefitted from a longitudinal design as that would have allowed to draw stronger conclusions from the conducted mediation analysis. However, most of these studies were preliminary in nature and were conducted to inform the development of the two versions of VISOR-which were in fact tested in a longitudinal trial.

Lastly, the RCT might be perceived as being weakened due to its study design. My team and I decided to not fully follow the CONSORT-EHEALTH checklist [220] and only invited participants for follow-up that used the entire DAs. This was done as the focus of our RCT was on capturing the added value of the VCM paired with computer-tailored advice. At the time this decision was made, it was considered the optimal solution as that meant that we 
only collected data from participants that we planned to use in our analyses, which we deemed most ethical and is in line with recommendations within the field of health psychology [312]. However, retrospectively and given the attrition-problem that was encountered during our RCT-that we did not foresee to the extent that it took place-it might be wise to follow a more traditional intention-to-treat-principle in a future replication (or adoption) of our work.

Abbreviations

COVID-19 - coronavirus disease 2019

DA - decision aid

IPDAS - International Patient Decision Aid Standards Collaboration

$\mathrm{RCT}$ - randomized controlled trial

SDT - Self-Determination Theory

VCM - value clarification method 

impact paragraph 
Making decisions can be difficult. Especially when decisions involve risks or when people feel like they do not know enough. But also, when they do not know what is important to them when making decisions, or when they are not supported enough while making decisions. In healthcare, difficult decisions are highly common. Decision aids are meant to help people to make such decisions in an informed way. They include information about all available options without convincing people to choose one option over the other. Traditionally, (research focused on) decision aids focused on supporting people to make decisions between different treatments or different screening programs. Yet, also decisions related to health promotion and disease prevention (in this impact paragraph both will be referred to as health promotion) can be difficult.

To illustrate, this thesis focused on smoking - a prominent health promotion topic and worldwide still the leading cause of preventable disease and early death. In the Netherlands, smoking kills about 20,000 people a year and costs the Dutch health system about $€ 2.4$ billion per year. To reduce (or eliminate) this problem, Dutch smokers have access to many smoking cessation tools: (1) behavioral support (such as counseling by a general practitioner), (2) nicotine replacement therapy (such as nicotine patches), and (3) smoking cessation medication. All these tools have been shown to help smokers to stop smoking. However, Dutch smokers often stop without using any tool-commonly referred to as stopping 'cold turkey'. This is problematic because stopping without any tool means that smokers have a higher risk of starting smoking again. Interestingly, the decision on how to stop smoking can be difficult: People often do not know enough about these tools, and therefore also do not know which tools match their preferences or needs. People motivated to stop smoking might therefore experience difficulties in choosing the right tool, and instead decide not to use any tool. As a result, they unwittingly limit their chances of stopping smoking. This clearly shows that decision aids for a health promotion topic such as smoking cessation should also be developed and made available to a broad audience.

To respond to the problems described above, the work presented in this thesis was carried out. From a more theoretical starting point: How can researchers combine ideas from behavior change and research focused on decision aids, and what health promotion decision aids are there? And also, from a practical starting point: the development and evaluation of a smoking cessation decision aid.

The smoking cessation decision aid (called VISOR) was developed together with smokers, smoking cessation counselors and scientists. I then compared two versions of VISOR within a large national study. One version included an interactive feature designed to help smokers discover what matters to them, followed by tailored advice. The other version lacked these features. In this study, I tested whether VISOR showed greater effects in terms of people stopping smoking and using smoking cessation tools with those feature(s) added. Also, I 
tested if less people were uncertain regarding their decision on how to stop smoking if VISOR included this interactive feature(s). In this study no clear effects were found on the number of people stopping smoking, using smoking cessation tools, or uncertainty. However, I was able to identify factors that influenced if people used VISOR completely and other factors (than VISOR) that influenced the effects. For example, I found that fewer people under the age of 30 and with less than a higher professional education have completed VISOR. The added interactive feature(s) also seemed to result in more people not finishing VISOR. Interestingly, another interesting factor that I identified during this study was the stage of decision making participants were in right before they used VISOR.

\section{Scientific Relevance}

In this thesis, I contributed to a field that has not been covered often in the literature around decision aids: decision aids applied to health promotion, specifically smoking cessation. This thesis - and its parts - therefore not only function(s) as a traditional thesis, but also as a call for action to further this relevant and interesting line of research. For example, I published one of the chapters as a discussion paper to encourage cooperation between experts in (health) behavior change and decision aids. Moreover, this thesis also provides the first broad literature review of health promotion decision aids that has also been published as a scientific paper. Not only does this literature review provide a starting point for further research, but it has also enabled me to formulate recommendations to advance the field of health promotion decision aids. Ultimately, these recommendations can lead to innovations in science, and my literature review provides a first basis for those innovations. Within this thesis, I was also able to shed more light on one specific decision aid element (the interactive feature(s) described earlier). These features helped users figure out what matters to them in relation to smoking cessation (for example, how likely it is that people will stop smoking using a specific tool). And even though, adding this interactive feature did not seem to be overly helpful, my study provides an interesting basis for scientific innovations. For example, I found that participants' stage of decision making might play an interesting role in the field of decision aids. This finding can be used to design attractive and more helpful decision aids or other decision support interventions in the future. For example, interventions could be tailored to individuals' stages of decision making to ensure that users use the entire intervention as intended. These findings can therefore be used to drive interesting innovations in science and ultimately to develop helpful interventions with a wider reach.

Next to this thesis, I used traditional scientific outlets to promote my findings, such as scientific articles, (poster) presentations at (inter)national conferences, and symposia that I organized together with other researchers. As my research was rooted in both behavioral sciences and the research behind decision aids, I specifically focused on conferences that are attended by experts in both areas. 
However, insights were also promoted by making use of more innovative ways to spread knowledge. Almost all articles were published open access (and promoted on social media), and study materials, data, and preprints were made available openly online. In this way, this innovative line of research that I followed during the work presented in this thesis, was made accessible to other scientists more quickly.

Societal Relevance

As the direct focus of this thesis was on individual (smoking cessation) decision making, people motivated to stop smoking are the first and foremost group to benefit from the research presented in this thesis. Logically, I also focused on them the most and they were involved in all development steps of VISOR. Unfortunately, the large national study did not show a clear effect of VISOR on smoking cessation. It should be noted that smokers that used VISOR in the large national study might still have profited from using it, as I tested VISOR among two groups and both groups received a version of VISOR (with or without the interactive feature(s)). And in fact, even the group that received VISOR without the additional elements achieved higher smoking cessation rates than found in the study that tested the only other smoking cessation decision aid in the Netherlands.

Next to this potential impact, I also reached many people motivated to stop smoking through mass media channels. For example, I developed and carried out a social media campaign including posts specifically designed to promote VISOR. This social media campaign made it clear that smokers can decide for themselves how they want to stop smoking and that there are many available tools to help. The needs assessment carried out for the development of VISOR showed that smokers are generally not familiar with all the tools that can help them stop smoking. Since changes in behavior often require awareness, this social media campaign may have already created this awareness, especially due to the wide reach of social media. As all social media posts are still online, this effect can also be realized after this project has been completed.

Also, even smokers that were not directly involved in the studies (or were reached by our recruitment strategies) can benefit from the work presented in this thesis. For example, I took part in multiple science communication initiatives, such as the "Maffe Mensen Podcast". The podcast aims to make the insights from the communication and behavioral sciences more accessible to a broader public. In this podcast, I not only presented my work, but also described easy-to-follow steps to make an informed decision about smoking cessation. People wishing to stop smoking, can easily find this podcast online even after the completion of this project. 
Ultimately, the newly generated knowledge can also be used to directly improve decision support for people motivated to stop smoking in the future. For example, I have found that information about how effective tools are can often be misunderstood by smokers. People motivated to stop smoking often referred to how likely one person is to stop smoking using tools, while experts mostly speak in terms of groups. Helping more people to understand such information better will enable them to make a more informed decision regarding smoking cessation in the future. In VISOR I showed end users images made up of icons representing groups of smokers. The number of smokers who successfully stopped smoking over the long term differed depending on whether they used smoking cessation tools or not. In this way I made sure to deliver the effectiveness information so that it is accessible to the 'average smoker' as well. This is just one of the examples on how I used the input given by the (potential) end users to ensure that the information would be as accessible as possible. In the long run this accessible information may help more smokers to stop smoking. These ideas could be used to improve other health education materials aimed at smoking cessation as well. Therefore, organizations such as the Trimbos Institute (the Dutch institute for mental health and addiction) can use this information that was uncovered to make their own materials more accessible. For this purpose, the VISOR-team is already in contact with the Trimbos Institute, for example.

Moreover, healthcare providers can also benefit from these findings as they often communicate with patients/clients about smoking cessation. Therefore, I included the needs of healthcare providers in my research early on and they were also updated throughout the development process. Not only by traditional outlets (see Scientific Relevance), but, for example, also by hosting an expert meeting at the Trimbos Institute. With this expert meeting I not only collected important input for VISOR, but already spread important information uncovered throughout the project presented in this thesis. Just as organizations, healthcare providers can use my findings to make their communication more accessible. For this it is important that they also find out about these findings. This could be implemented, for example, by including the findings in the teaching of the students. For example, a lecture could be provided to nursing students to provide the newest generation of healthcare providers with this new knowledge. During the project, I was already contacted by students who missed this content, which shows that this is worth exploring.

Policy makers and commercial parties could also benefit by using my findings to improve their smoking cessation offerings. My findings could be used to improve VISOR based on the generated insights and an updated version could be implemented and disseminated nationally after another careful test of its effects.

Finally, helping smokers to stop smoking will also improve public health in the long term and help achieve a smoke-free generation in the future. Smoking has major consequences for 
public health and the costs of healthcare, for example because passive smoking also has a negative impact on people's health. The research presented in this thesis therefore also indirectly benefits the general public-especially because we found that VISOR helped smokers to stop smoking. 



\section{references}


[1] H. Bekker, J.G. Thornton, C.M. Airey, J.B. Connelly, J. Hewison, M.B. Robinson, J. Lilleyman, M. MacIntosh, A.J. Maule, S. Michie, A.D. Pearman, Informed decision making: an annotated bibliography and systematic review, Health Technol. Assess. 3 (1999) 1-156. https://doi.org/10.3310/hta3010.

[2] J.-P. Claessen, S. Bates, K. Sherlock, F. Seeparsand, R. Wright, Designing interventions to improve tooth brushing, Int. Dent. J. 58 (2008) 307-320. https://doi.org/10.1111/j.1875595X.2008.tb00208.x.

[3] M. Cuypers, R.E.D. Lamers, P.J.M. Kil, L.V. van de Poll-Franse, M. de Vries, Impact of a web-based prostate cancer treatment decision aid on patient-reported decision process parameters: results from the Prostate Cancer Patient Centered Care trial, Support. Care Cancer. 26 (2018) 3739-3748. https://doi.org/10.1007/s00520-018-4236-8.

[4] A.M. O'Connor, Validation of a Decisional Conflict Scale, Med. Decis. Making. 15 (1995) 25-30. https://doi.org/10.1177/0272989X9501500105.

[5] M.M. Garvelink, L. Boland, K. Klein, D.V. Nguyen, M. Menear, H.L. Bekker, K.B. Eden, A. LeBlanc, A.M. O'Connor, D. Stacey, F. Légaré, Decisional Conflict Scale Findings among Patients and Surrogates Making Health Decisions: Part II of an Anniversary Review, Med. Decis. Making. 39 (2019) 316-327. https://doi.org/10.1177/0272989X19851346.

[6] F. Légaré, A.C. O'Connor, I. Graham, D. Saucier, L. Côté, M. Cauchon, L. Paré, Supporting patients facing difficult health care decisions: use of the Ottawa Decision Support Framework., Can. Fam. Physician. 52 (2006) 476-477.

[7] A.M. O'Connor, User Manual - Decisional Conflict Scale [document on the Internet], (1993). https://decisionaid.ohri.ca/docs/develop/User_Manuals/UM_Decisional_Conflict.pdf (accessed January 14, 2021).

[8] Q. Sun, Predicting Downstream Effects of High Decisional Conflict: Meta-analyses of the Decisional Conflict Scale, University of Ottawa, 2005. http://hdl.handle.net/10393/27050.

[9] A.M. Knops, A. Goossens, D.T. Ubbink, D.A. Legemate, L.J. Stalpers, P.M. Bossuyt, Interpreting Patient Decisional Conflict Scores: Behavior and Emotions in Decisions about Treatment, Med. Decis. Making. 33 (2013) 78-84. https://doi.org/10.1177/0272989X12453500.

[10] M. Gattellari, J.E. Ward, Will men attribute fault to their GP for adverse effects arising from controversial screening tests? An Australian study using scenarios about PSA screening, J. Med. Screen. 11 (2004) 165-169. https://doi.org/10.1258/0969141042467386.

[11] B.K. Rimer, P.A. Briss, P.K. Zeller, E.C.Y. Chan, S.H. Woolf, Informed decision making: What is its role in cancer screening?, Cancer. 101 (2004) 1214-1228. https://doi.org/10.1002/cncr.20512.

[12] G. Elwyn, D. Frosch, S. Rollnick, Dual equipoise shared decision making: definitions for decision and behaviour support interventions, Implement. Sci. 4 (2009). https://doi.org/10.1186/1748-5908-4-75.

[13] D. Stacey, F. Légaré, K. Lewis, M.J. Barry, C.L. Bennett, K.B. Eden, M. Holmes-Rovner, H. Llewellyn-Thomas, A. Lyddiatt, R. Thomson, L. Trevena, Decision aids for people facing health treatment or screening decisions, Cochrane Database Syst. Rev. (2017). https://doi.org/10.1002/14651858.CD001431.pub5.

[14] A. Fagerlin, I. Lakhani, P.M. Lantz, N.K. Janz, M. Morrow, K. Schwartz, D. Deapen, B. Salem, L. Liu, S.J. Katz, An informed decision?: Breast cancer patients and their knowledge about 
treatment, Patient Educ. Couns. 64 (2006) 303-312.

https://doi.org/10.1016/j.pec.2006.03.010.

[15] G. Elwyn, D. Frosch, R. Thomson, N. Joseph-Williams, A. Lloyd, P. Kinnersley, E. Cording, D. Tomson, C. Dodd, S. Rollnick, A. Edwards, M. Barry, Shared Decision Making: A Model for Clinical Practice, J. Gen. Intern. Med. 27 (2012) 1361-1367. https://doi.org/10.1007/s11606012-2077-6.

[16] M. van den Berg, D.R.M. Timmermans, L.P. ten Kate, J.M.G. van Vugt, G. van der Wal, Informed decision making in the context of prenatal screening, Patient Educ. Couns. 63 (2006) 110117. https://doi.org/10.1016/j.pec.2005.09.007.

[17] M.M. Garvelink, L. Boland, K. Klein, D.V. Nguyen, M. Menear, H.L. Bekker, K.B. Eden, A. LeBlanc, A.M. O'Connor, D. Stacey, F. Légaré, Decisional Conflict Scale Use over 20 Years: The Anniversary Review, Med. Decis. Making. 39 (2019) 301-314. https://doi.org/10.1177/0272989X19851345.

[18] R.H. Thaler, C.R. Sunstein, Nudge: Improving decisions about health, wealth, and happiness, Yale University Press, 2008.

[19] Ministerie van Volksgezondheid, Welzijn en Sport, Nationaal Preventieakkoord: Naar een gezonder Nederland, (2018).

https://www.rijksoverheid.nl/binaries/rijksoverheid/documenten/convenanten/2018/11/23/ nationaal-preventieakkoord/nationaal-preventieakkoord.pdf (accessed January 26, 2021).

[20] U.S. Department of Health and Human Services, The health consequences of smoking - 50 years of progress: a report of the Surgeon General, (2014). https://stacks.cdc.gov/view/cdc/21569/cdc_21569_DS1.pdf.

[21] Rijksinstituut voor Volksgezondheid en Milieu, Volksgezondheid Toekomst Verkenning 2018 Een gezond vooruitzicht: Synthese, (2018). https://www.vtv2018.nl/sites/default/files/201808/112810_010513_VTV_kernboodschappen_TG3_1.pdf (accessed January 26, 2021).

[22] R. Peto, A.D. Lopez, J. Boreham, M. Thun, C. Heath Jr, R. Doll, Mortality from smoking worldwide, Br. Med. Bull. 52 (1996) 12-21. https://doi.org/10.1093/oxfordjournals.bmb.a011519.

[23] S.-H. Zhu, T. Melcer, J. Sun, B. Rosbrook, J.P. Pierce, Smoking cessation with and without assistance: A population-based analysis, Am. J. Prev. Med. 18 (2000) 305-311. https://doi.org/10.1016/S0749-3797(00)00124-0.

[24] T. Lancaster, L.F. Stead, Individual behavioural counselling for smoking cessation, Cochrane Database Syst. Rev. (2017). https://doi.org/10.1002/14651858.CD001292.pub3.

[25] J. Hartmann-Boyce, S.C. Chepkin, W. Ye, C. Bullen, T. Lancaster, Nicotine replacement therapy versus control for smoking cessation, Cochrane Database Syst. Rev. (2018). https://doi.org/10.1002/14651858.CD000146.pub5.

[26] K. Cahill, S. Stevens, R. Perera, T. Lancaster, Pharmacological interventions for smoking cessation: an overview and network meta-analysis, Cochrane Database Syst. Rev. (2013). https://doi.org/10.1002/14651858.CD009329.pub2.

[27] A.R. White, H. Rampes, J.P. Liu, L.F. Stead, J. Campbell, Acupuncture and related interventions for smoking cessation, Cochrane Database Syst. Rev. (2014). https://doi.org/10.1002/14651858.CD000009.pub4. 
[28] M.Y. Elrashidi, J.O. Ebbert, Emerging drugs for the treatment of tobacco dependence: 2014 update, Expert Opin. Emerg. Drugs. 19 (2014) 243-260. https://doi.org/10.1517/14728214.2014.899580.

[29] M.A. Bansal, K.M. Cummings, A. Hyland, G.A. Giovino, Stop-smoking medications: Who uses them, who misuses them, and who is misinformed about them?, Nicotine Tob. Res. 6 (2004) S303-S310. https://doi.org/10.1080/14622200412331320707.

[30] J.M. Royce, K. Corbett, G. Sorensen, J. Ockene, Gender, social pressure, and smoking cessations: The Community Intervention Trial for Smoking Cessation (COMMIT) at baseline, Soc. Sci. Med. 44 (1997) 359-370. https://doi.org/10.1016/S0277-9536(96)00149-9.

[31] M.-A. Durand, J. Witt, N. Joseph-Williams, R.G. Newcombe, M.C. Politi, S. Sivell, G. Elwyn, Minimum standards for the certification of patient decision support interventions: Feasibility and application, Patient Educ. Couns. 98 (2015) 462-468. https://doi.org/10.1016/j.pec.2014.12.009.

[32] P. Abhyankar, H.L. Bekker, B.A. Summers, G. Velikova, Why values elicitation techniques enable people to make informed decisions about cancer trial participation, Health Expect. 14 (2011) 20-32. https://doi.org/10.1111/j.1369-7625.2010.00615.x.

[33] P. Abhyankar, R.J. Volk, J. Blumenthal-Barby, P. Bravo, A. Buchholz, E. Ozanne, D.C. Vidal, N. Col, P. Stalmeier, Balancing the presentation of information and options in patient decision aids: an updated review, BMC Med. Inform. Decis. Mak. 13 (2013). https://doi.org/10.1186/14726947-13-s2-s6.

[34] A. Fagerlin, M. Pignone, P. Abhyankar, N. Col, D. Feldman-Stewart, T. Gavaruzzi, J. Kryworuchko, C.A. Levin, A.H. Pieterse, V. Reyna, A. Stiggelbout, L.D. Scherer, C. Wills, H.O. Witteman, Clarifying values: an updated review, BMC Med. Inform. Decis. Mak. 13 (2013). https://doi.org/10.1186/1472-6947-13-S2-S8.

[35] H.O. Witteman, R. Ndjaboue, G. Vaisson, S.C. Dansokho, B. Arnold, J.F.P. Bridges, S. Comeau, A. Fagerlin, T. Gavaruzzi, M. Marcoux, A. Pieterse, M. Pignone, T. Provencher, C. Racine, D. Regier, C. Rochefort-Brihay, P. Thokala, M. Weernink, D.B. White, C.E. Wills, J. Jansen, Clarifying Values: An Updated and Expanded Systematic Review and Meta-Analysis, Med. Decis. Making. 41 (2021) 801-820. https://doi.org/10.1177/0272989X211037946.

[36] H.A. Llewellyn-Thomas, R.T. Crump, Decision Support for Patients: Values Clarification and Preference Elicitation, Med. Care Res. Rev. 70 (2013) 50S-79S. https://doi.org/10.1177/1077558712461182.

[37] D. Feldman-Stewart, C. Tong, R. Siemens, S. Alibhai, T. Pickles, J. Robinson, M.D. Brundage, The Impact of Explicit Values Clarification Exercises in a Patient Decision Aid Emerges After the Decision Is Actually Made: Evidence From a Randomized Controlled Trial, Med. Decis. Making. 32 (2012) 616-626. https://doi.org/10.1177/0272989X11434601.

[38] H.O. Witteman, L.D. Scherer, T. Gavaruzzi, A.H. Pieterse, A. Fuhrel-Forbis, S. Chipenda Dansokho, N. Exe, V.C. Kahn, D. Feldman-Stewart, N.F. Col, A.F. Turgeon, A. Fagerlin, Design Features of Explicit Values Clarification Methods: A Systematic Review, Med. Decis. Mak. 36 (2016) 453471. https://doi.org/10.1177/0272989x15626397.

[39] H.O. Witteman, T. Gavaruzzi, L.D. Scherer, A.H. Pieterse, A. Fuhrel-Forbis, S. Chipenda Dansokho, N. Exe, V.C. Kahn, D. Feldman-Stewart, N.F. Col, A.F. Turgeon, A. Fagerlin, Effects of Design Features of Explicit Values Clarification Methods: A Systematic Review, Med. Decis. Making. 36 (2016) 760-776. https://doi.org/10.1177/0272989X16634085. 
[40] H.O. Witteman, A.-S. Julien, R. Ndjaboue, N.L. Exe, V.C. Kahn, A. Fagerlin, B.J. Zikmund-Fisher, What Helps People Make Values-Congruent Medical Decisions? Eleven Strategies Tested across 6 Studies, Med. Decis. Making. 40 (2020) 266-278.

https://doi.org/10.1177/0272989X20904955.

[41] M.S. Koelewijn-van Loon, T. van der Weijden, B. van Steenkiste, G. Ronda, B. Winkens, J.L. Severens, M. Wensing, G. Elwyn, R. Grol, Involving patients in cardiovascular risk management with nurse-led clinics: a cluster randomized controlled trial, CMAJ. 181 (2009) E267-E274. https://doi.org/10.1503/cmaj.081591.

[42] M.C. Willemsen, M. Wiebing, A. Van Emst, G. Zeeman, Helping smokers to decide on the use of efficacious smoking cessation methods: a randomized controlled trial of a decision aid, Addiction. 101 (2006) 441-449. https://doi.org/10.1111/j.1360-0443.2006.01349.x.

[43] M.S. Koelewijn-van Loon, B. van Steenkiste, G. Ronda, M. Wensing, H.E. Stoffers, G. Elwyn, R. Grol, T. van der Weijden, Improving patient adherence to lifestyle advice (IMPALA): a clusterrandomised controlled trial on the implementation of a nurse-led intervention for cardiovascular risk management in primary care (protocol), BMC Health Serv. Res. 8 (2008). https://doi.org/10.1186/1472-6963-8-9.

[44] E.S. Smit, A.J. Linn, J.C.M. van Weert, Taking online computer-tailoring forward: The potential of tailoring the message frame and delivery mode of online health behaviour change interventions, Eur. Health Psychol. 17 (2015) 25-31.

[45] H. de Vries, J. Brug, Computer-tailored interventions motivating people to adopt health promoting behaviours: Introduction to a new approach., Patient Educ. Couns. (1999) 99105. https://doi.org/10.1016/S0738-3991(98)00127-X.

[46] M. Altendorf, C. Hoving, J.C.M. Van Weert, E.S. Smit, Effectiveness of Message Frame-Tailoring in a Web-Based Smoking Cessation Program: Randomized Controlled Trial, J. Med. Internet Res. 22 (2020) e17251. https://doi.org/10.2196/17251.

[47] G.-J.Y. Peters, A practical guide to effective behavior change: how to identify what to change in the first place, Eur. Health Psychol. 16 (2014) 142-155.

[48] I. van de Kolk, S.M.P.L. Gerards, L.S.E. Harms, S.P.J. Kremers, A.M.H.S. van Dinther-Erkens, M. Snellings, J.S. Gubbels, Study Protocol for the Evaluation of "SuperFIT", a Multicomponent Nutrition and Physical Activity Intervention Approach for Preschools and Families, Int. J. Environ. Res. Public Health. 17 (2020). https://doi.org/10.3390/ijerph17020603.

[49] H.C. Eyles, C.N. Mhurchu, Does tailoring make a difference? A systematic review of the longterm effectiveness of tailored nutrition education for adults, Nutr. Rev. 67 (2009) 464-480. https://doi.org/10.1111/j.1753-4887.2009.00219.x.

[50] M.M. Schapira, S. Swartz, P.S. Ganschow, E.A. Jacobs, J.M. Neuner, C.M. Walker, K.E. Fletcher, Tailoring Educational and Behavioral Interventions to Level of Health Literacy: A Systematic Review, MDM Policy Pract. 2 (2017). https://doi.org/10.1177/2381468317714474.

[51] I. van de Kolk, S.R.B. Verjans-Janssen, J.S. Gubbels, S.P.J. Kremers, S.M.P.L. Gerards, Systematic review of interventions in the childcare setting with direct parental involvement: effectiveness on child weight status and energy balance-related behaviours, Int. J. Behav. Nutr. Phys. Act. 16 (2019). https://doi.org/10.1186/s12966-019-0874-6.

[52] F. Moyo, E. Archibald, J.T. Slyer, Effectiveness of decision aids for smoking cessation in adults: a quantitative systematic review, JBI Database Syst. Rev. Implement. Rep. 16 (2018) 17911822. https://doi.org/10.11124/JBISRIR-2017-003698. 
[53] N.F. BinDhim, K. McGeechan, L. Trevena, Smartphone Smoking Cessation Application (SSC App) trial: a multicountry double-blind automated randomised controlled trial of a smoking cessation decision-aid 'app', BMJ Open. 8 (2018) e017105. https://doi.org/10.1136/bmjopen-2017-017105.

[54] B. Gaglio, J.A. Shoup, R.E. Glasgow, The RE-AIM Framework: A Systematic Review of Use Over Time, Am. J. Public Health. 103 (2013) e38-e46. https://doi.org/10.2105/AJPH.2013.301299.

[55] M.L.A. Lustria, Can interactivity make a difference? Effects of interactivity on the comprehension of and attitudes toward online health content, J. Assoc. Inf. Sci. Technol. 58 (2007) 766-776. https://doi.org/10.1002/asi.20557.

[56] A. Syrowatka, D. Krömker, A.N. Meguerditchian, R. Tamblyn, Features of Computer-Based Decision Aids: Systematic Review, Thematic Synthesis, and Meta-Analyses, J. Med. Internet Res. 18 (2016) e20. https://doi.org/10.2196/jmir.4982.

[57] E.M. Rogers, Diffusion of Innovations, 5th ed., Free Press, New York, 2003.

[58] F. Frattini, M. Bianchi, A. De Massis, U. Sikimic, The Role of Early Adopters in the Diffusion of New Products: Differences between Platform and Nonplatform Innovations, J. Prod. Innov. Manag. 31 (2014) 466-488. https://doi.org/10.1111/jpim.12108.

[59] A. Coulter, D. Stilwell, J. Kryworuchko, P.D. Mullen, C.J. Ng, T. van der Weijden, A systematic development process for patient decision aids, BMC Med. Inform. Decis. Mak. 13 (2013). https://doi.org/10.1186/1472-6947-13-S2-S2.

[60] R. Borland, L. Li, P. Driezen, N. Wilson, D. Hammond, M.E. Thompson, G.T. Fong, U. Mons, M.C. Willemsen, A. McNeill, J.F. Thrasher, K.M. Cummings, Cessation assistance reported by smokers in 15 countries participating in the International Tobacco Control (ITC) policy evaluation surveys, Addiction. 107 (2012) 197-205. https://doi.org/10.1111/j.13600443.2011.03636.x.

[61] J.E. Brazier, S. Dixon, J. Ratcliffe, The Role of Patient Preferences in Cost-Effectiveness Analysis, PharmacoEconomics. 27 (2009) 705-712. https://doi.org/10.2165/11314840-00000000000000 .

[62] K. Sainsbury, B. Mullan, L. Sharpe, A Randomized Controlled Trial of an Online Intervention to Improve Gluten-Free Diet Adherence in Celiac Disease, Am. J. Gastroenterol. 108 (2013) 811-817. https://doi.org/10.1038/ajg.2013.47.

[63] L.A. Klaassen, C.D. Dirksen, L.J. Boersma, C. Hoving, A novel patient decision aid for aftercare in breast cancer patients: A promising tool to reduce costs by individualizing aftercare, Breast. 41 (2018) 144-150. https://doi.org/10.1016/j.breast.2018.06.015.

[64] N. Mathers, C.J. Ng, M.J. Campbell, B. Colwell, I. Brown, A. Bradley, Clinical effectiveness of a patient decision aid to improve decision quality and glycaemic control in people with diabetes making treatment choices: a cluster randomised controlled trial (PANDAs) in general practice, BMJ Open. 2 (2012). https://doi.org/10.1136/bmjopen-2012-001469.

[65] C. Charles, A. Gafni, T. Whelan, Shared decision-making in the medical encounter: What does it mean? (or it takes at least two to tango), Soc. Sci. Med. 44 (1997) 681-692. https://doi.org/10.1016/S0277-9536(96)00221-3.

[66] J. Crowley, S. O'Connell, A. Kavka, L. Ball, C.A. Nowson, Australian general practitioners' views regarding providing nutrition care: results of a national survey, Public Health. 140 (2016) 713. https://doi.org/10.1016/j.puhe.2016.08.013. 
[67] H. Shahsavari, P. Matourypour, S. Ghiyasvandian, M.R.G. Nejad, Medical Research Council framework for development and evaluation of complex interventions: A comprehensive guidance, J. Educ. Health Promot. 9 (2020) 88. https://doi.org/10.4103/jehp.jehp_649_19.

[68] J.M. Murray, S.F. Brennan, D.P. French, C.C. Patterson, F. Kee, R.F. Hunter, Effectiveness of physical activity interventions in achieving behaviour change maintenance in young and middle aged adults: A systematic review and meta-analysis, Soc. Sci. Med. 192 (2017) 125133. https://doi.org/10.1016/j.socscimed.2017.09.021.

[69] K. Cahill, T. Lancaster, R. Perera, Pharmacological interventions for smoking cessation: an overview of reviews, Cochrane Database Syst. Rev. (2011). https://doi.org/10.1002/14651858.CD009329.

[70] S. Michie, M.M. Van Stralen, R. West, The behaviour change wheel: a new method for characterising and designing behaviour change interventions, Implement. Sci. 6 (2011). https://doi.org/10.1186/1748-5908-6-42.

[71] E.S. Smit, H. de Vries, C. Hoving, Effectiveness of a Web-Based Multiple Tailored Smoking Cessation Program: A Randomized Controlled Trial Among Dutch Adult Smokers, J. Med. Internet Res. 14 (2012) e82. https://doi.org/10.2196/jmir.1812.

[72] J. Côté, G. Godin, P.R. Garcia, M. Gagnon, G. Rouleau, Program development for enhancing adherence to antiretroviral therapy among persons living with HIV, AIDS Patient Care STDS. 22 (2008) 965-975. https://doi.org/10.1089/apc.2008.0124.

[73] I. Ajzen, The theory of planned behavior, Organ. Behav. Hum. Decis. Process. 50 (1991) 179211. https://doi.org/10.1016/0749-5978(91)90020-T.

[74] W. Hardeman, M. Johnston, D. Johnston, D. Bonetti, N. Wareham, A.L. Kinmonth, Application of the theory of planned behaviour in behaviour change interventions: A systematic review, Psychol. Health. 17 (2002) 123-158. https://doi.org/10.1080/08870440290013644a.

[75] G. Kok, N.H. Gottlieb, G.-J.Y. Peters, P.D. Mullen, G.S. Parcel, R.A.C. Ruiter, M.E. Fernández, C. Markham, L.K. Bartholomew, A taxonomy of behaviour change methods: an Intervention Mapping approach, Health Psychol. Rev. 10 (2016) 297-312. https://doi.org/10.1080/17437199.2015.1077155.

[76] L.K.B. Eldredge, C.M. Markham, R.A.C. Ruiter, M.E. Fernández, G. Kok, G.S. Parcel, Planning health promotion programs: an intervention mapping approach, 4th ed., Jossey-Bass, 2016.

[77] K.L. Cheung, D. de Ruijter, M. Hiligsmann, I. Elfeddali, C. Hoving, S.M.A.A. Evers, H. de Vries, Exploring consensus on how to measure smoking cessation. A Delphi study, BMC Public Health. 17 (2017). https://doi.org/10.1186/s12889-017-4902-7.

[78] H.L. Bekker, Genetic Screening: Facilitating Informed Choices, in: ELS, 2006. https://doi.org/10.1002/9780470015902.a0005591.

[79] International Patient Decision Aids Standards (IPDAS) Collaboration, International Patient Decision Aids Standards (IPDAS) Collaboration, (n.d.). http://ipdas.ohri.ca/what.html (accessed November 20, 2020).

[80] H.L. Bekker, J. Hewison, J.G. Thornton, Applying decision analysis to facilitate informed decision making about prenatal diagnosis for Down syndrome: a randomised controlled trial, Prenat. Diagn. 24 (2004) 265-275. https://doi.org/10.1002/pd.851.

[81] I.L. Janis, L. Mann, Decision Making: A Psychological Analysis of Conflict, Choice, and Commitment, Free press, 1977. 
[82] M.-A. Durand, M. Stiel, J. Boivin, G. Elwyn, Where is the theory? Evaluating the theoretical frameworks described in decision support technologies, Patient Educ. Couns. 71 (2008) 125135. https://doi.org/10.1016/j.pec.2007.12.004.

[83] C. Cook, C. Sheets, Clinical equipoise and personal equipoise: two necessary ingredients for reducing bias in manual therapy trials, J. Man. Manip. Ther. 19 (2011) 55-57. https://doi.org/10.1179/106698111X12899036752014.

[84] C. Ammendolia, P. Côté, C. Cancelliere, J.D. Cassidy, J. Hartvigsen, E. Boyle, S. Soklaridis, P. Stern, B. Amick III, Healthy and productive workers: using intervention mapping to design a workplace health promotion and wellness program to improve presenteeism, BMC Public Health. 16 (2016). https://doi.org/10.1186/s12889-016-3843-x.

[85] K.R. Sepucha, C.M. Borkhoff, J. Lally, C.A. Levin, D.D. Matlock, C.J. Ng, M.E. Ropka, D. Stacey, N. Joseph-Williams, C.E. Wills, R. Thomson, Establishing the effectiveness of patient decision aids: key constructs and measurement instruments, BMC Med. Inform. Decis. Mak. 13 (2013). https://doi.org/10.1186/1472-6947-13-s2-s12.

[86] R.M. Ryan, E.L. Deci, Self-determination theory and the facilitation of intrinsic motivation, social development, and well-being, Am. Psychol. 55 (2000) 68-78. https://doi.org/10.1037/0003066X.55.1.68.

[87] H.L. Bekker, Using decision-making theory to inform clinical practice, in: A. Edwards, G. Elwyn (Eds.), Shar. Decis.-Mak. Health Care Achiev. Evid.-Based Patient Choice, 2nd ed., Oxford University Press, 2009: pp. 45-52.

[88] B.M. Loneck, L.A. Kola, Using the conflict-theory model of decision making to predict outcome in the alcoholism intervention, Alcohol. Treat. Q. 5 (1989) 119-136.

https://doi.org/10.1300/J020V05N03_09.

[89] K.K.B. Peetoom, R. Crutzen, R. Verhoeven, J.M.H.A. Bohnen, B. Winkens, G.J. Dinant, J.W.L. Cals, Optimizing decision-making among childcare staff on fever and common infections: cluster randomized controlled trial, Eur. J. Public Health. (2018). https://doi.org/10.1093/eurpub/cky246.

[90] A. O'Cathain, L. Croot, E. Duncan, N. Rousseau, K. Sworn, K.M. Turner, L. Yardley, P. Hoddinott, Guidance on how to develop complex interventions to improve health and healthcare, BMJ Open. 9 (2019) e029954. https://doi.org/10.1136/bmjopen-2019-029954.

[91] R.M. Ryan, H. Patrick, E.L. Deci, G.C. Williams, Facilitating health behaviour change and its maintenance: Interventions based on Self-Determination Theory, Eur. Health Psychol. 10 (2008) 2-5.

[92] Y.-L. Su, J. Reeve, A Meta-analysis of the Effectiveness of Intervention Programs Designed to Support Autonomy, Educ. Psychol. Rev. 23 (2011) 159-188. https://doi.org/10.1007/s10648010-9142-7.

[93] P.J. Teixeira, M.M. Marques, M.N. Silva, J. Brunet, J.L. Duda, L. Haerens, J. La Guardia, M. Lindwall, C. Lonsdale, D. Markland, S. Michie, A.C. Moller, N. Ntoumanis, H. Patrick, J. Reeve, R.M. Ryan, S.J. Sebire, M. Standage, M. Vansteenkiste, N. Weinstein, K. Weman-Josefsson, G.C. Williams, M.S. Hagger, A classification of motivation and behavior change techniques used in self-determination theory-based interventions in health contexts., Motiv. Sci. 6 (2020) 438-455. https://doi.org/10.1037/mot0000172.

[94] A.J. Goossens, K.L. Cheung, E. Sijstermans, R. Conde, J.G. Gonzalez, M. Hiligsmann, A discrete choice experiment to assess patients' preferences for HIV treatment in the rural population 
in Colombia, J. Med. Econ. (2020) 803-811.

https://doi.org/10.1080/13696998.2020.1735398.

[95] F.F. Sniehotta, U. Scholz, R. Schwarzer, Bridging the intention-behaviour gap: Planning, selfefficacy, and action control in the adoption and maintenance of physical exercise, Psychol. Health. 20 (2005) 143-160. https://doi.org/10.1080/08870440512331317670.

[96] T. Gültzow, E.S. Smit, R. Hudales, V. Knapen, J. Rademakers, C.D. Dirksen, C. Hoving, An Autonomy-Supportive Online Decision Aid to Assist Smokers in Choosing Evidence-Based Cessation Assistance: Development Process and Protocol of a Randomized Controlled Trial, JMIR Res. Protoc. 9 (2020) e21772. https://doi.org/10.2196/21772.

[97] EHPS.net | Home of the European Health Psychology Society, (n.d.). https://ehps.net/ (accessed November 9, 2020).

[98] World Health Organization, Global status report on noncommunicable diseases 2014: "Attaining the nine global noncommunicable diseases targets; a shared responsibility", Geneva, 2014. https://apps.who.int/iris/bitstream/handle/10665/148114/9789241564854_eng.pdf;jsession id=72C65AEE09BF394ED9D1A41374F6C129?sequence=1 (accessed December 14, 2020).

[99] M.H. Forouzanfar, A. Afshin, L.T. Alexander, H.R. Anderson, Z.A. Bhutta, S. Biryukov, M. Brauer, R. Burnett, K. Cercy, F.J. Charlson, A.J. Cohen, L. Dandona, K. Estep, A.J. Ferrari, J.J. Frostad, N. Fullman, P.W. Gething, W.W. Godwin, M. Griswold, S.I. Hay, Y. Kinfu, H.H. Kyu, H.J. Larson, X. Liang, S.S. Lim, P.Y. Liu, A.D. Lopez, R. Lozano, L. Marczak, G.A. Mensah, A.H. Mokdad, M. Moradi-Lakeh, M. Naghavi, B. Neal, M.B. Reitsma, G.A. Roth, J.A. Salomon, P.J. Sur, T. Vos, J.A. Wagner, H. Wang, Y. Zhao, M. Zhou, G.M. Aasvang, A.A. Abajobir, K.H. Abate, C. Abbafati, K.M. Abbas, F. Abd-Allah, A.M. Abdulle, S.F. Abera, B. Abraham, L.J. Abu-Raddad, G.Y. Abyu, A.O. Adebiyi, I.A. Adedeji, Z. Ademi, A.K. Adou, J.C. Adsuar, E.E. Agardh, A. Agarwal, A. Agrawal, A.A. Kiadaliri, O.N. Ajala, T.F. Akinyemiju, Z. Al-Aly, K. Alam, N.K.M. Alam, S.F. Aldhahri, R.W. Aldridge, Z.A. Alemu, R. Ali, A. Alkerwi, F. Alla, P. Allebeck, U. Alsharif, K.A. Altirkawi, E.A. Martin, N. Alvis-Guzman, A.T. Amare, A. Amberbir, A.K. Amegah, H. Amini, W. Ammar, S.M. Amrock, H.H. Andersen, B.O. Anderson, C.A.T. Antonio, P. Anwari, J. Ärnlöv, A. Artaman, H. Asayesh, R.J. Asghar, R. Assadi, S. Atique, E.F.G.A. Avokpaho, A. Awasthi, B.P.A. Quintanilla, P. Azzopardi, U. Bacha, A. Badawi, M.C. Bahit, K. Balakrishnan, A. Barac, R.M. Barber, S.L. Barker-Collo, T. Bärnighausen, S. Barquera, L. Barregard, L.H. Barrero, S. Basu, C. Batis, S. Bazargan-Hejazi, J. Beardsley, N. Bedi, E. Beghi, B. Bell, M.L. Bell, A.K. Bello, D.A. Bennett, I.M. Bensenor, A. Berhane, E. Bernabé, B.D. Betsu, A.S. Beyene, N. Bhala, A. Bhansali, S. Bhatt, S. Biadgilign, B. Bikbov, D. Bisanzio, E. Bjertness, J.D. Blore, R. Borschmann, S. Boufous, R.R.A. Bourne, M. Brainin, A. Brazinova, N.J.K. Breitborde, H. Brenner, D.M. Broday, T.S. Brugha, B. Brunekreef, Z.A. Butt, L.E. Cahill, B. Calabria, I.R. Campos-Nonato, R. Cárdenas, D.O. Carpenter, J.J. Carrero, D.C. Casey, C.A. Castañeda-Orjuela, J.C. Rivas, R.E. Castro, F. Catalá-López, J.-C. Chang, P.P.-C. Chiang, M. Chibalabala, O. Chimed-Ochir, V.H. Chisumpa, A.A. Chitheer, J.-Y.J. Choi, H. Christensen, D.J. Christopher, L.G. Ciobanu, M.M. Coates, S.M. Colquhoun, A.G.C. Manzano, L.T. Cooper, K. Cooperrider, L. Cornaby, M. Cortinovis, J.A. Crump, L. Cuevas-Nasu, A. Damasceno, R. Dandona, S.C. Darby, P.I. Dargan, J. das Neves, A.C. Davis, K. Davletov, E.F. de Castro, V. De la Cruz-Góngora, D. De Leo, L. Degenhardt, L.C. Del Gobbo, B. del Pozo-Cruz, R.P. Dellavalle, A. Deribew, D.C.D. Jarlais, S.D. Dharmaratne, P.K. Dhillon, C. Diaz-Torné, D. Dicker, E.L. Ding, E.R. Dorsey, K.E. Doyle, T.R. Driscoll, L. Duan, M. Dubey, B.B. Duncan, I. Elyazar, A.Y. Endries, S.P. Ermakov, H.E. Erskine, 
B. Eshrati, A. Esteghamati, S. Fahimi, E.J.A. Faraon, T.A. Farid, C.S. e S. Farinha, A. Faro, M.S. Farvid, F. Farzadfar, V.L. Feigin, S.-M. Fereshtehnejad, J.G. Fernandes, F. Fischer, J.R.A.

Fitchett, T. Fleming, N. Foigt, K. Foreman, F.G.R. Fowkes, R.C. Franklin, T. Fürst, N.D. Futran, E. Gakidou, A.L. Garcia-Basteiro, T.T. Gebrehiwot, A.T. Gebremedhin, J.M. Geleijnse, B.D. Gessner, A.Z. Giref, M. Giroud, M.D. Gishu, G. Giussani, S. Goenka, M.C. Gomez-Cabrera, H. Gomez-Dantes, P. Gona, A. Goodridge, S.V. Gopalani, C.C. Gotay, A. Goto, H.N. Gouda, H.C. Gugnani, F. Guillemin, Y. Guo, R. Gupta, R. Gupta, R.A. Gutiérrez, J.A. Haagsma, N. HafeziNejad, D. Haile, G.B. Hailu, Y.A. Halasa, R.R. Hamadeh, S. Hamidi, A.J. Handal, G.J. Hankey, Y. Hao, H.L. Harb, S. Harikrishnan, J.M. Haro, M.S. Hassanvand, T.A. Hassen, R. Havmoeller, I.B. Heredia-Pi, N.F. Hernández-Llanes, P. Heydarpour, H.W. Hoek, H.J. Hoffman, M. Horino, N. Horita, H.D. Hosgood, D.G. Hoy, M. Hsairi, A.S. Htet, G. Hu, J.J. Huang, A. Husseini, S.J. Hutchings, I. Huybrechts, K.M. Iburg, B.T. Idrisov, B.V. Ileanu, M. Inoue, T.A. Jacobs, K.H. Jacobsen, N. Jahanmehr, M.B. Jakovljevic, H.A.F.M. Jansen, S.K. Jassal, M. Javanbakht, S.P. Jayaraman, A.U. Jayatilleke, S.H. Jee, P. Jeemon, V. Jha, Y. Jiang, T. Jibat, Y. Jin, C.O. Johnson, J.B. Jonas, Z. Kabir, Y. Kalkonde, R. Kamal, H. Kan, A. Karch, C.K. Karema, C. Karimkhani, A. Kasaeian, A. Kaul, N. Kawakami, D.S. Kazi, P.N. Keiyoro, L. Kemmer, A.H. Kemp, A.P. Kengne, A. Keren, C.N. Kesavachandran, Y.S. Khader, A.R. Khan, E.A. Khan, G. Khan, Y.-H. Khang, S. Khatibzadeh, S. Khera, T.A.M. Khoja, J. Khubchandani, C. Kieling, C. Kim, D. Kim, R.W. Kimokoti, N. Kissoon, M. Kivipelto, L.D. Knibbs, Y. Kokubo, J.A. Kopec, P.A. Koul, A. Koyanagi, M. Kravchenko, H. Kromhout, H. Krueger, T. Ku, B.K. Defo, R.S. Kuchenbecker, B.K. Bicer, E.J. Kuipers, G.A. Kumar, G.F. Kwan, D.K. Lal, R. Lalloo, T. Lallukka, Q. Lan, A. Larsson, A.A. Latif, A.E.B. Lawrynowicz, J.L. Leasher, J. Leigh, J. Leung, M. Levi, X. Li, Y. Li, J. Liang, S. Liu, B.K. Lloyd, G. Logroscino, P.A. Lotufo, R. Lunevicius, M. Maclntyre, M. Mahdavi, M. Majdan, A. Majeed, R. Malekzadeh, D.C. Malta, W.A.A. Manamo, C.C. Mapoma, W. Marcenes, R.V. Martin, J. Martinez-Raga, F. Masiye, K. Matsushita, R. Matzopoulos, B.M. Mayosi, J.J. McGrath, M. McKee, P.A. Meaney, C. Medina, A. Mehari, F. Mejia-Rodriguez, A.B. Mekonnen, Y.A. Melaku, Z.A. Memish, W. Mendoza, G.B.M. Mensink, A. Meretoja, T.J. Meretoja, Y.M. Mesfin, F.A. Mhimbira, A. Millear, T.R. Miller, E.J. Mills, M. Mirarefin, A. Misganaw, C.N. Mock, A. Mohammadi, S. Mohammed, G.L.D. Mola, L. Monasta, J.C.M. Hernandez, M. Montico, L. Morawska, R. Mori, D. Mozaffarian, U.O. Mueller, E. Mullany, J.E. Mumford, G.V.S. Murthy, J.B. Nachega, A. Naheed, V. Nangia, N. Nassiri, J.N. Newton, M. Ng, Q.L. Nguyen, M.I. Nisar, P.M.N. Pete, O.F. Norheim, R.E. Norman, B. Norrving, L.

Nyakarahuka, C.M. Obermeyer, F.A. Ogbo, I.-H. Oh, O. Oladimeji, P.R. Olivares, H. Olsen, B.O. Olusanya, J.O. Olusanya, J.N. Opio, E. Oren, R. Orozco, A. Ortiz, E. Ota, M. PA, A. Pana, E.-K. Park, C.D. Parry, M. Parsaeian, T. Patel, A.J.P. Caicedo, S.T. Patil, S.B. Patten, G.C. Patton, N. Pearce, D.M. Pereira, N. Perico, K. Pesudovs, M. Petzold, M.R. Phillips, F.B. Piel, J.D. Pillay, D. Plass, S. Polinder, C.D. Pond, C.A. Pope, D. Pope, S. Popova, R.G. Poulton, F. Pourmalek, N.M. Prasad, M. Qorbani, R.H.S. Rabiee, A. Radfar, A. Rafay, V. Rahimi-Movaghar, M. Rahman, M.H.U. Rahman, S.U. Rahman, R.K. Rai, S. Rajsic, M. Raju, U. Ram, S.M. Rana, K. Ranganathan, P. Rao, C.A.R. García, A.H. Refaat, C.D. Rehm, J. Rehm, N. Reinig, G. Remuzzi, S. Resnikoff, A.L. Ribeiro, J.A. Rivera, H.S. Roba, A. Rodriguez, S. Rodriguez-Ramirez, D. RojasRueda, Y. Roman, L. Ronfani, G. Roshandel, D. Rothenbacher, A. Roy, M.M. Saleh, J.R. Sanabria, L. Sanchez-Riera, M.D. Sanchez-Niño, T.G. Sánchez-Pimienta, L. Sandar, D.F. Santomauro, I.S. Santos, R. Sarmiento-Suarez, B. Sartorius, M. Satpathy, M. Savic, M. 
Sawhney, J. Schmidhuber, M.I. Schmidt, I.J.C. Schneider, B. Schöttker, A.E. Schutte, D.C. Schwebel, J.G. Scott, S. Seedat, S.G. Sepanlou, E.E. Servan-Mori, G. Shaddick, A. Shaheen, S. Shahraz, M.A. Shaikh, T.S. Levy, R. Sharma, J. She, S. Sheikhbahaei, J. Shen, K.N. Sheth, P. Shi, K. Shibuya, M. Shigematsu, M.-J. Shin, R. Shiri, K. Shishani, I. Shiue, M.G. Shrime, I.D. Sigfusdottir, D.A.S. Silva, D.G.A. Silveira, J.I. Silverberg, E.P. Simard, S. Sindi, A. Singh, J.A. Singh, P.K. Singh, E.L. Slepak, M. Soljak, S. Soneji, R.J.D. Sorensen, L.A. Sposato, C.T. Sreeramareddy, V. Stathopoulou, N. Steckling, N. Steel, D.J. Stein, M.B. Stein, H. Stöckl, S. Stranges, K. Stroumpoulis, B.F. Sunguya, S. Swaminathan, B.L. Sykes, C.E.I. Szoeke, R. Tabarés-Seisdedos, K. Takahashi, R.T. Talongwa, N. Tandon, D. Tanne, M. Tavakkoli, B.W. Taye, H.R. Taylor, B.A. Tedla, W.M. Tefera, T.K. Tegegne, D.Y. Tekle, A.S. Terkawi, J.S. Thakur, B.A. Thomas, M.L. Thomas, A.J. Thomson, A.L. Thorne-Lyman, A.G. Thrift, G.D. Thurston, T. Tillmann, R. Tobe-Gai, M. Tobollik, R. Topor-Madry, F. Topouzis, J.A. Towbin, B.X. Tran, Z.T. Dimbuene, N. Tsilimparis, A.K. Tura, E.M. Tuzcu, S. Tyrovolas, K.N. Ukwaja, E.A. Undurraga, C.J. Uneke, O.A. Uthman, A. van Donkelaar, J. van Os, Y.Y. Varakin, T. Vasankari, J.L. Veerman, N. Venketasubramanian, F.S. Violante, S.E. Vollset, G.R. Wagner, S.G. Waller, J.L. Wang, L. Wang, Y. Wang, S. Weichenthal, E. Weiderpass, R.G. Weintraub, A. Werdecker, R. Westerman, H.A. Whiteford, T. Wijeratne, C.S. Wiysonge, C.D.A. Wolfe, S. Won, A.D. Woolf, M. Wubshet, D. Xavier, G. Xu, A.K. Yadav, B. Yakob, A.Z. Yalew, Y. Yano, M. Yaseri, P. Ye, P. Yip, N. Yonemoto, S.-J. Yoon, M.Z. Younis, C. Yu, Z. Zaidi, M.E.S. Zaki, J. Zhu, B. Zipkin, S. Zodpey, L.J. Zuhlke, C.J.L. Murray, Global, regional, and national comparative risk assessment of 79 behavioural, environmental and occupational, and metabolic risks or clusters of risks, 1990-2015: a systematic analysis for the Global Burden of Disease Study 2015, Lancet. 388 (2016) 1659-1724. https://doi.org/10.1016/S0140-6736(16)31679-8.

[100] A. Tamakoshi, K. Tamakoshi, L. Yingsong, K. Yagyu, S. Kikuchi, JACC Study Group, Healthy lifestyle and preventable death: findings from the Japan Collaborative Cohort (JACC) Study, Prev. Med. 48 (2009) 486-492. https://doi.org/10.1016/j.ypmed.2009.02.017.

[101] World Health Organization, WHO | Risk factors, (2018). http://www.who.int/gho/ncd/risk_factors/en/ (accessed June 25, 2018).

[102] Y. Wu, L. Zhai, D. Zhang, Sleep duration and obesity among adults: a meta-analysis of prospective studies, Sleep Med. 15 (2014) 1456-62. https://doi.org/10.1016/j.sleep.2014.07.018.

[103] G.M.J. Taylor, M.N. Dalili, M. Semwal, M. Civljak, A. Sheikh, J. Car, Internet-based interventions for smoking cessation, Cochrane Database Syst. Rev. (2017). https://doi.org/10.1002/14651858.CD007078.pub5.

[104] V.H. Rice, L. Heath, J. Livingstone-Banks, J. Hartmann-Boyce, Nursing interventions for smoking cessation, Cochrane Database Syst. Rev. (2017). https://doi.org/10.1002/14651858.CD001188.pub5.

[105] W. Matkin, J.M. Ordóñez-Mena, J. Hartmann-Boyce, Telephone counselling for smoking cessation, Cochrane Database Syst. Rev. (2019). https://doi.org/10.1002/14651858.CD002850.pub4.

[106] V.M. Montori, A. LeBlanc, A. Buchholz, D.L. Stilwell, A. Tsapas, Basing information on comprehensive, critically appraised, and up-to-date syntheses of the scientific evidence: a quality dimension of the International Patient Decision Aid Standards, BMC Med. Inform. Decis. Mak. 13 (2013). https://doi.org/10.1186/1472-6947-13-S2-S5. 
[107] P. Abhyankar, R.J. Volk, J. Blumenthal-Barby, P. Bravo, A. Buchholz, E. Ozanne, D.C. Vidal, N. $\mathrm{Col}, \mathrm{P}$. Stalmeier, Balancing the presentation of information and options in patient decision aids: an updated review, BMC Med. Inform. Decis. Mak. 13 (2013) S6. https://doi.org/10.1186/1472-6947-13-s2-s6.

[108] L. Trenaman, S. Bryan, N. Bansback, The cost-effectiveness of patient decision aids: A systematic review, Healthcare. 2 (2014) 251-257. https://doi.org/10.1016/j.hjdsi.2014.09.002.

[109] H. Arksey, L. O'Malley, Scoping studies: towards a methodological framework, Int. J. Soc. Res. Methodol. 8 (2005) 19-32. https://doi.org/10.1080/1364557032000119616.

[110] M.D.J. Peters, C. Godfrey, P. McInerney, C. Baldini Soares, H. Khalil, D. Parker, Chapter 11: Scoping Reviews, in: E Aromataris, Z. Munn (Eds.), Joanna Briggs Inst. Rev. Man., JBI, 2017.

[111] A.C. Tricco, E. Lillie, W. Zarin, K.K. O'Brien, H. Colquhoun, D. Levac, D. Moher, M.D.J. Peters, T. Horsley, L. Weeks, S. Hempel, E.A. Akl, C. Chang, J. McGowan, L. Stewart, L. Hartling, A. Aldcroft, M.G. Wilson, C. Garritty, S. Lewin, C.M. Godfrey, M.T. Macdonald, E.V. Langlois, K. Soares-Weiser, J. Moriarty, T. Clifford, Ö. Tunçalp, S.E. Straus, PRISMA Extension for Scoping Reviews (PRISMA-ScR): Checklist and Explanation, Ann. Intern. Med. 169 (2018) 467-473. https://doi.org/10.7326/M18-0850.

[112] T. Gültzow, D. Zijlstra, C. Bolman, H. de Vries, C. Dirksen, S. Jongen, J.W.M. Muris, E.S. Smit, C. Hoving, Study Design, (2018). https://osf.io/umf4a/ (accessed March 5, 2020).

[113] Decision Aid Library Inventory - Patient Decision Aids - Ottawa Hospital Research Institute, (n.d.). https://decisionaid.ohri.ca/cochinvent.php (accessed March 5, 2020).

[114] R.J. Volk, H. Llewellyn-Thomas, D. Stacey, G. Elwyn, Ten years of the International Patient Decision Aid Standards Collaboration: evolution of the core dimensions for assessing the quality of patient decision aids, BMC Med. Inform. Decis. Mak. 13 (2013). https://doi.org/10.1186/1472-6947-13-S2-S1.

[115] M.E. Cupples, J.A. Cole, N.D. Hart, N. Heron, M.C. McKinley, M.A. Tully, Shared decisionmaking (SHARE-D) for healthy behaviour change: a feasibility study in general practice, BJGP Open. 2 (2018) bjgpopen18X101517. https://doi.org/10.3399/bjgpopen18X101517.

[116] K.S. Geller, I.D. Mendoza, J. Timbobolan, H.L. Montjoy, C.R. Nigg, The Decisional Balance Sheet to Promote Healthy Behavior Among Ethnically Diverse Older Adults, Public Health Nurs. 29 (2012) 241-246. https://doi.org/10.1111/j.1525-1446.2011.00987.x.

[117] O. Hirsch, H. Keller, C. Albohn-Kühne, T. Krones, N. Donner-Banzhoff, Satisfaction of Patients and Primary Care Physicians With Shared Decision Making, Eval. Health Prof. 33 (2010) 321 342. https://doi.org/10.1177/0163278710376662.

[118] O. Hirsch, H. Keller, T. Krones, N. Donner-Banzhoff, Acceptance of shared decision making with reference to an electronic library of decision aids (arriba-lib) and its association to decision making in patients: an evaluation study, Implement. Sci. 6 (2011). https://doi.org/10.1186/1748-5908-6-70.

[119] O. Hirsch, H. Keller, C. Albohn-Kühne, T. Krones, N. Donner-Banzhoff, Pitfalls in the statistical examination and interpretation of the correspondence between physician and patient satisfaction ratings and their relevance for shared decision making research, BMC Med. Res. Methodol. 11 (2011). https://doi.org/10.1186/1471-2288-11-71.

[120] O. Hirsch, H. Keller, T. Krones, N. Donner-Banzhoff, Arriba-lib: association of an evidencebased electronic library of decision aids with communication and decision-making in patients 
and primary care physicians, Int. J. Evid. Based Healthc. 10 (2012) 68-76. https://doi.org/10.1111/j.1744-1609.2012.00255.x.

[121] O. Hirsch, H. Keller, T. Krones, N. Donner-Banzhoff, Arriba-lib: evaluation of an electronic library of decision aids in primary care physicians, BMC Med. Inform. Decis. Mak. 12 (2012). https://doi.org/10.1186/1472-6947-12-48.

[122] O. Hirsch, E. Szabo, H. Keller, L. Kramer, T. Krones, N. Donner-Banzhoff, arriba-lib: Analyses of user interactions with an electronic library of decision aids on the basis of log data, Inform. Health Soc. Care. 37 (2012) 264-276. https://doi.org/10.3109/17538157.2012.654841.

[123] M.S. Koelewijn-van Loon, T. van der Weijden, G. Ronda, B. van Steenkiste, B. Winkens, G. Elwyn, R. Grol, Improving lifestyle and risk perception through patient involvement in nurseled cardiovascular risk management: A cluster-randomized controlled trial in primary care, Prev. Med. 50 (2010) 35-44. https://doi.org/10.1016/j.ypmed.2009.11.007.

[124] T. Krones, H. Keller, A. Sönnichsen, E.-M. Sadowski, E. Baum, K. Wegscheider, J. Rochon, N. Donner-Banzhoff, Absolute Cardiovascular Disease Risk and Shared Decision Making in Primary Care: A Randomized Controlled Trial, Ann. Fam. Med. 6 (2008) 218-227. https://doi.org/10.1370/afm.854.

[125] T. Krones, H. Keller, A. Becker, A. Sönnichsen, E. Baum, N. Donner-Banzhoff, The theory of planned behaviour in a randomized trial of a decision aid on cardiovascular risk prevention, Patient Educ. Couns. 78 (2010) 169-176. https://doi.org/10.1016/j.pec.2009.06.010.

[126] S.L. Sheridan, L.B. Draeger, M.P. Pignone, P.D. Sloane, C. Samuel-Hodge, E.A. Finkelstein, Z. Gizlice, M.B. Vu, D.P. Gitterman, S.I. Bangdiwala, K.E. Donahue, K. Evenson, A.S. Ammerman, T.C. Keyserling, Designing and implementing a comparative effectiveness study of two strategies for delivering high quality CHD prevention: Methods and participant characteristics for the Heart to Health study, Contemp. Clin. Trials. 36 (2013) 394-405. https://doi.org/10.1016/j.cct.2013.07.013.

[127] T.C. Keyserling, S.L. Sheridan, L.B. Draeger, E.A. Finkelstein, Z. Gizlice, E. Kruger, L.F. Johnston, P.D. Sloane, C. Samuel-Hodge, K.R. Evenson, M.D. Gross, K.E. Donahue, M.P. Pignone, M.B. Vu, E.A. Steinbacher, B.J. Weiner, S.I. Bangdiwala, A.S. Ammerman, A Comparison of Live Counseling With a Web-Based Lifestyle and Medication Intervention to Reduce Coronary Heart Disease Risk: A Randomized Clinical Trial, JAMA Intern. Med. 174 (2014) 1144-1157. https://doi.org/10.1001/jamainternmed.2014.1984.

[128] I. Tinsel, A. Siegel, C. Schmoor, A. Buchholz, W. Niebling, DECADE-pilot: decision aid, action planning, and follow-up support for patients to reduce the 10-year risk of cardiovascular diseases - a protocol of a randomized controlled pilot trial, Pilot Feasibility Stud. 3 (2017). https://doi.org/10.1186/s40814-017-0172-5.

[129] I. Tinsel, A. Siegel, C. Schmoor, I. Poguntke, A. Maun, W. Niebling, Encouraging selfmanagement in cardiovascular disease prevention: A randomized controlled study of a structured advice and patient activation intervention in primary care, Dtsch. Ärztebl. Int. 115 (2018) 469-476. https://doi.org/10.3238/arztebl.2018.0469.

[130] B. van Steenkiste, T.M. van der Weijden, J.H.E.H. Stoffers, R.P.T.M. GROL, Patients' responsiveness to a decision support tool for primary prevention of cardiovascular diseases in primary care, Patient Educ. Couns. 72 (2008) 63-70. https://doi.org/10.1016/j.pec.2008.02.009. 
[131] N.F. BinDhim, K. McGeechan, L. Trevena, Assessing the effect of an interactive decision-aid smartphone smoking cessation application (app) on quit rates: a double-blind automated randomised control trial protocol, BMJ Open. 4 (2014) e005371.

https://doi.org/10.1136/bmjopen-2014-005371.

[132] M.F. Brunette, J.C. Ferron, G.J. McHugo, K.E. Davis, T.S. Devitt, S.M. Wilkness, R.E. Drake, An Electronic Decision Support System to Motivate People With Severe Mental IIlnesses to Quit Smoking, Psychiatr. Serv. 62 (2011) 360-366.

https://doi.org/10.1176/ps.62.4.pss6204_0360.

[133] M.F. Brunette, J.C. Ferron, R.E. Drake, T.S. Devitt, P.T. Geiger, G.J. McHugo, J.A. Jonikas, J.A. Cook, Carbon monoxide feedback in a motivational decision support system for nicotine dependence among smokers with severe mental illnesses, J. Subst. Abuse Treat. 45 (2013) 319-324. https://doi.org/10.1016/j.jsat.2013.04.005.

[134] M.F. Brunette, W. Gunn, H. Alvarez, P.C. Finn, P. Geiger, J.C. Ferron, G.J. McHugo, A pre-post pilot study of a brief, web-based intervention to engage disadvantaged smokers into cessation treatment, Addict. Sci. Clin. Pract. 10 (2015). https://doi.org/10.1186/s13722-0150026-5.

[135] A.P. Cupertino, K. Richter, L.S. Cox, S. Garrett, R. Ramirez, F. Mujica, E.F. Ellerbeck, Feasibility of a Spanish/English Computerized Decision Aid to Facilitate Smoking Cessation Efforts in Underserved Communities, J. Health Care Poor Underserved. 21 (2010) 504-517. https://doi.org/10.1353/hpu.0.0307.

[136] J.C. Ferron, M.F. Brunette, G.J. McHugo, T.S. Devitt, W.M. Martin, R.E. Drake, Developing a quit smoking website that is usable by people with severe mental illnesses., Psychiatr. Rehabil. J. 35 (2011) 111-116. https://doi.org/10.2975/35.2.2011.111.116.

[137] J.C. Ferron, M.F. Brunette, S.R. McGurk, H. Xie, R. Frounfelker, J.A. Cook, J.A. Jonikas, G.J. McHugo, Do Symptoms and Cognitive Problems Affect the Use and Efficacy of a Web-Based Decision Support System for Smokers With Serious Mental Illness?, J. Dual Diagn. 8 (2012) 315-325. https://doi.org/10.1080/15504263.2012.723316.

[138] P.J. Hollen, V.L. Tyc, S.F. Donnangelo, S.V. Shannon, M.C. O'Laughlen, I. Hinton, M.E. Smolkin, G.R. Petroni, A Substance Use Decision Aid for Medically at-Risk Adolescents: Results of a Randomized Controlled Trial for Cancer-Surviving Adolescents, Cancer Nurs. 36 (2013) 355367. https://doi.org/10.1097/NCC.0b013e31827910ba.

[139] J.E. Lee, D.W. Shin, B. Suh, S. Chun, Y.-S. Nam, B. Cho, Development and application of culturally appropriate decision aids for smoking cessation in Korea: a pragmatic clustered randomization crossover trial, Patient Prefer. Adherence. 10 (2016) 1929-1936. https://doi.org/10.2147/PPA.S114387.

[140] K.K. McDonnell, L.F. Bullock, B.D. Kozower, P.J. Hollen, J. Heath, V. Rovnyak, A Decision Aid to Improve Smoking Abstinence for Families Facing Cancer, Oncol. Nurs. Forum. 41 (2014) 649658. https://doi.org/10.1188/14.ONF.649-658.

[141] K.K. McDonnell, P.J. Hollen, J. Heath, J.O. Andrews, Recruiting family dyads facing thoracic cancer surgery: Challenges and lessons learned from a smoking cessation intervention, Eur. J. Oncol. Nurs. 20 (2016) 199-206. https://doi.org/10.1016/j.ejon.2015.08.006.

[142] H. Rhee, P.J. Hollen, M.J. Belyea, M.A. Sutherland, Decision-Making Program for Rural Adolescents With Asthma: A Pilot Study, J. Pediatr. Nurs. 23 (2008) 439-450. https://doi.org/10.1016/j.pedn.2008.01.079. 
[143] S.L. Sheridan, J.M. Griffith, L. Behrend, Z. Gizlice, J. Cai, M.P. Pignone, Effect of Adding a Values Clarification Exercise to a Decision Aid on Heart Disease Prevention: A Randomized Trial, Med. Decis. Making. 30 (2010) E28-E39. https://doi.org/10.1177/0272989X10369008.

[144] S.L. Sheridan, L.B. Draeger, M.P. Pignone, T.C. Keyserling, R.J. Simpson Jr, B. Rimer, S.I. Bangdiwala, J. Cai, Z. Gizlice, A randomized trial of an intervention to improve use and adherence to effective coronary heart disease prevention strategies, BMC Health Serv. Res. 11 (2011). https://doi.org/10.1186/1472-6963-11-331.

[145] S.L. Sheridan, L.B. Draeger, M.P. Pignone, B. Rimer, S.I. Bangdiwala, J. Cai, Z. Gizlice, T.C. Keyserling, R.J. Simpson Jr, The effect of a decision aid intervention on decision making about coronary heart disease risk reduction: secondary analyses of a randomized trial, BMC Med. Inform. Decis. Mak. 14 (2014). https://doi.org/10.1186/1472-6947-14-14.

[146] D.O. Warner, A. LeBlanc, S. Kadimpati, K.S. Vickers, Y. Shi, V.M. Montori, Decision Aid for Cigarette Smokers Scheduled for Elective Surgery, Anesthesiology. 123 (2015) 18-28. https://doi.org/10.1097/ALN.0000000000000704.

[147] B. van Steenkiste, T. van der Weijden, H.E.J.H. Stoffers, A.D.M. Kester, D.R.M. Timmermans, R. Grol, Improving cardiovascular risk management: a randomized, controlled trial on the effect of a decision support tool for patients and physicians, Eur. J. Cardiovasc. Prev. Rehabil. 14 (2007) 44-50. https://doi.org/10.1097/01.hjr.0000239475.71805.1e.

[148] Healthwise Staff, Quitting Smoking: Should I Use Medicine? | Kaiser Permanente, (n.d.). https://healthy.kaiserpermanente.org/health-wellness/health-encyclopedia/he.quittingsmoking-should-i-use-medicine.zw1124 (accessed April 16, 2020).

[149] Healthwise Staff, Obesity: Should I Use a Diet Plan to Lose Weight?, (n.d.). https://www.lancastergeneralhealth.org/healthwise-library/condition-categories/weightmanagement?lang=en-us\&Documentld=ud3826 (accessed April 16, 2020).

[150] Healthwise Staff, Weight Management: Should I Use Over-the-Counter Diet Aids? | Kaiser Permanente, (n.d.). https://healthy.kaiserpermanente.org/health-wellness/healthencyclopedia/he.weight-management-should-i-use-over-the-counter-diet-aids.z×3344 (accessed April 16, 2020).

[151] Healthwise Staff, Sleep Apnea: Should I Have a Sleep Study?, (n.d.). https://www.cardiosmart.org/healthwise/aa71/651/aa71651 (accessed April 16, 2020).

[152] G.-J.Y. Peters, R. Crutzen, Pragmatic nihilism: how a Theory of Nothing can help health psychology progress, Health Psychol. Rev. 11 (2017) 103-121. https://doi.org/10.1080/17437199.2017.1284015.

[153] World Health Organization, WHO global report: mortality attributable to tobacco., Geneva, 2012. https://apps.who.int/iris/bitstream/handle/10665/44815/9789241564434_eng.pdf?sequenc $\mathrm{e}=1$ \&isAllowed=y.

[154] P. Thompson-Leduc, S. Turcotte, M. Labrecque, F. Légaré, Prevalence of clinically significant decisional conflict: an analysis of five studies on decision-making in primary care, BMJ Open. 6 (2016) e011490. https://doi.org/10.1136/bmjopen-2016-011490.

[155] A.S. Hoffman, R.J. Volk, A. Saarimaki, C. Stirling, L.C. Li, M. Härter, G.R. Kamath, H. LlewellynThomas, Delivering patient decision aids on the Internet: definitions, theories, current evidence, and emerging research areas, BMC Med. Inform. Decis. Mak. 13 (2013). https://doi.org/10.1186/1472-6947-13-s2-s13. 
[156] K. Reumkens, M.H.E. Tummers, J.J.G. Gietel-Habets, S.M.J. van Kuijk, C.M. Aalfs, C.J. van Asperen, M.G.E.M. Ausems, M. Collée, C.J. Dommering, C.M. Kets, L.E. van der Kolk, J.C. Oosterwijk, V.C.G. Tjan-Heijnen, T. van der Weijden, C.E.M. de Die-Smulders, L.A.D.M. van Osch, The development of an online decision aid to support persons having a genetic predisposition to cancer and their partners during reproductive decision-making: a usability and pilot study, Fam. Cancer. 18 (2019) 137-146. https://doi.org/10.1007/s10689-018-00924.

[157] M.J. Stark, Dropping out of substance abuse treatment: A clinically oriented review, Clin. Psychol. Rev. 12 (1992) 93-116. https://doi.org/10.1016/0272-7358(92)90092-M.

[158] O. Siddiqui, B.R. Flay, F.B. Hu, Factors Affecting Attrition in a Longitudinal Smoking Prevention Study, Prev. Med. 25 (1996) 554-560. https://doi.org/10.1006/pmed.1996.0089.

[159] J.W. Dearing, Applying Diffusion of Innovation Theory to Intervention Development, Res. Soc. Work Pract. 19 (2009) 503-518. https://doi.org/10.1177/1049731509335569.

[160] S.G. Scott, R.A. Bruce, Decision-Making Style: The Development and Assessment of a New Measure, Educ. Psychol. Meas. 55 (1995) 818-831. https://doi.org/10.1177/0013164495055005017.

[161] P. Thunholm, Decision-making style: habit, style or both?, Personal. Individ. Differ. 36 (2004) 931-944. https://doi.org/10.1016/S0191-8869(03)00162-4.

[162] C. Dewberry, M. Juanchich, S. Narendran, The latent structure of decision styles, Personal. Individ. Differ. 54 (2013) 566-571. https://doi.org/10.1016/j.paid.2012.11.002.

[163] E.S. Smit, S. Brinkhues, H. de Vries, C. Hoving, Subgroups Among Smokers in Preparation: A Cluster Analysis Using the I-Change Model, Subst. Use Misuse. 53 (2018) 400-411. https://doi.org/10.1080/10826084.2017.1334062.

[164] K.A. Wallston, B. Strudler Wallston, R. DeVellis, Development of the Multidimensional Health Locus of Control (MHLC) Scales, Health Educ. Monogr. 6 (1978) 160-170. https://doi.org/10.1177/109019817800600107.

[165] B.L. Bennett, C.M. Goldstein, E.C. Gathright, J.W. Hughes, J.D. Latner, Internal health locus of control predicts willingness to track health behaviors online and with smartphone applications, Psychol. Health Med. 22 (2017) 1224-1229. https://doi.org/10.1080/13548506.2017.1317354.

[166] M.S. Hagger, S.J. Hardcastle, A. Chater, C. Mallett, S. Pal, N.L.D. Chatzisarantis, Autonomous and controlled motivational regulations for multiple health-related behaviors: between- and within-participants analyses, Health Psychol. Behav. Med. 2 (2014) 565-601. https://doi.org/10.1080/21642850.2014.912945.

[167] K.L. Morton, S.J.H. Biddle, M.R. Beauchamp, Changes in self-determination during an exercise referral scheme, Public Health. 122 (2008) 1257-1260. https://doi.org/10.1016/j.puhe.2007.11.006.

[168] M.J. Eynon, C. O'Donnell, L. Williams, Assessing the impact of autonomous motivation and psychological need satisfaction in explaining adherence to an exercise referral scheme, Psychol. Health Med. 22 (2017) 1056-1062. https://doi.org/10.1080/13548506.2016.1274041.

[169] R.J. Rahman, C. Thogersen-Ntoumani, J. Thatcher, J. Doust, Changes in need satisfaction and motivation orientation as predictors of psychological and behavioural outcomes in exercise 
referral, Psychol. Health. 26 (2011) 1521-1539.

https://doi.org/10.1080/08870446.2010.538849.

[170] G.C. Williams, H.A. McGregor, D. Sharp, C. Levesque, R.W. Kouides, R.M. Ryan, E.L. Deci, Testing a self-determination theory intervention for motivating tobacco cessation: Supporting autonomy and competence in a clinical trial, Health Psychol. 25 (2006) 91-101. https://doi.org/10.1037/0278-6133.25.1.91.

[171] Flycatcher Internet Research, Home - Flycatcher, (2018). https://www.flycatcher.eu/nl/.

[172] S. Tsang, C.F. Royse, A.S. Terkawi, Guidelines for developing, translating, and validating a questionnaire in perioperative and pain medicine, Saudi J. Anaesth. 11 (2017) 80-89. https://doi.org/10.4103/sja.SJA_203_17.

[173] T.F. Heatherton, L.T. Kozlowski, R.C. Frecker, K.-O. Fagerström, The Fagerström Test for Nicotine Dependence: a revision of the Fagerström Tolerance Questionnaire, Addiction. 86 (1991) 1119-1127. https://doi.org/10.1111/j.1360-0443.1991.tb01879.x.

[174] A.N. Mudde, M.C. Willemsen, S.P.J. Kremers, H. De Vries, Meetinstrumenten voor onderzoek naar stoppen met roken, Stivoro, The Hague, 2000.

[175] C.S. Levesque, G.C. Williams, D. Elliot, M.A. Pickering, B. Bodenhamer, P.J. Finley, Validating the theoretical structure of the Treatment Self-Regulation Questionnaire (TSRQ) across three different health behaviors, Health Educ. Res. 22 (2007) 691-702. https://doi.org/10.1093/her/cyl148.

[176] G.C. Williams, C.P. Niemiec, H. Patrick, R.M. Ryan, E.L. Deci, The Importance of Supporting Autonomy and Perceived Competence in Facilitating Long-Term Tobacco Abstinence, Ann. Behav. Med. 37 (2009) 315-324. https://doi.org/10.1007/s12160-009-9090-y.

[177] R.M. Ryan, E.L. Deci, Self-determination theory and the role of basic psychological needs in personality and the organization of behavior., in: O.P. John, R.W. Robins, L.A. Pervin (Eds.), Handb. Personal. Theory Res., 3rd ed., The Guilford Press, New York, 2008: pp. 654-678.

[178] P. Norman, P. Bennett, C. Smith, S. Murphy, Health Locus of Control and Health Behaviour, J. Health Psychol. 3 (1998) 171-180. https://doi.org/10.1177/135910539800300202.

[179] IBM Corp, BM SPSS Statistics for Macintosh, Version 24.0, IBM Corp, Armonk, NY, 2016.

[180] A. Field, Postgraduate Statistics: Cluster Analysis, (2000). http://webcitation.org/68DVVTUDf.

[181] L.J. Cronbach, G.C. Gleser, Assessing similarity between profiles, Psychol. Bull. 50 (1953) 456473. https://doi.org/10.1037/h0057173.

[182] K.J. Preacher, A.F. Hayes, SPSS and SAS procedures for estimating indirect effects in simple mediation models, Behav. Res. Methods Instrum. Comput. 36 (2004) 717-731. https://doi.org/10.3758/BF03206553.

[183] A.H. Pieterse, M. de Vries, On the suitability of fast and frugal heuristics for designing values clarification methods in patient decision aids: a critical analysis, Health Expect. 16 (2013) e73-e79. https://doi.org/10.1111/j.1369-7625.2011.00720.x.

[184] A. Dijksterhuis, M.W. Bos, L.F. Nordgren, R.B. Van Baaren, On Making the Right Choice: The Deliberation-Without-Attention Effect, Science. 311 (2006) 1005-1007. https://doi.org/10.1126/science.1121629.

[185] E. Dane, K.W. Rockmann, M.G. Pratt, When should I trust my gut? Linking domain expertise to intuitive decision-making effectiveness, Organ. Behav. Hum. Decis. Process. 119 (2012) 187194. https://doi.org/10.1016/j.obhdp.2012.07.009. 
[186] H. De Vries, An Integrated Approach for Understanding Health Behavior; The I-Change Model as an Example, Psychol. Behav. Sci. Int. J. 2 (2017).

http://dx.doi.org/10.19080/PBSIJ.2017.02.555585.

[187] M. Fishbein, A Reasoned Action Approach to Health Promotion, Med. Decis. Making. 28 (2008) 834-844. https://doi.org/10.1177/0272989X08326092.

[188] E.S. Dalmaijer, C.L. Nord, D.E. Astle, Statistical power for cluster analysis, ArXiv E-Prints. (2020) arXiv:2003.00381.

[189] C.J.L. Murray, A.Y. Aravkin, P. Zheng, C. Abbafati, K.M. Abbas, M. Abbasi-Kangevari, F. AbdAllah, A. Abdelalim, M. Abdollahi, I. Abdollahpour, K.H. Abegaz, H. Abolhassani, V. Aboyans, L.G. Abreu, M.R.M. Abrigo, A. Abualhasan, L.J. Abu-Raddad, A.I. Abushouk, M. Adabi, V. Adekanmbi, A.M. Adeoye, O.O. Adetokunboh, D. Adham, S.M. Advani, G. Agarwal, S.M.K. Aghamir, A. Agrawal, T. Ahmad, K. Ahmadi, M. Ahmadi, H. Ahmadieh, M.B. Ahmed, T.Y. Akalu, R.O. Akinyemi, T. Akinyemiju, B. Akombi, C.J. Akunna, F. Alahdab, Z. Al-Aly, K. Alam, S. Alam, T. Alam, F.M. Alanezi, T.M. Alanzi, B. wassihun Alemu, K.F. Alhabib, M. Ali, S. Ali, G. Alicandro, C. Alinia, V. Alipour, H. Alizade, S.M. Aljunid, F. Alla, P. Allebeck, A. AlmasiHashiani, H.M. Al-Mekhlafi, J. Alonso, K.A. Altirkawi, M. Amini-Rarani, F. Amiri, D.A. Amugsi, R. Ancuceanu, D. Anderlini, J.A. Anderson, C.L. Andrei, T. Andrei, C. Angus, M. Anjomshoa, F. Ansari, A. Ansari-Moghaddam, I.C. Antonazzo, C.A.T. Antonio, C.M. Antony, E. Antriyandarti, D. Anvari, R. Anwer, S.C.Y. Appiah, J. Arabloo, M. Arab-Zozani, F. Ariani, B. Armoon, J. Ärnlöv, A. Arzani, M. Asadi-Aliabadi, A.A. Asadi-Pooya, C. Ashbaugh, M. Assmus, Z. Atafar, D.D. Atnafu, M.M.W. Atout, F. Ausloos, M. Ausloos, B.P. Ayala Quintanilla, G. Ayano, M.A. Ayanore, S. Azari, G. Azarian, Z.N. Azene, A. Badawi, A.D. Badiye, M.A. Bahrami, M.H. Bakhshaei, A. Bakhtiari, S.M. Bakkannavar, A. Baldasseroni, K. Ball, S.H. Ballew, D. Balzi, M. Banach, S.K. Banerjee, A.B. Bante, A.G. Baraki, S.L. Barker-Collo, T.W. Bärnighausen, L.H. Barrero, C.M. Barthelemy, L. Barua, S. Basu, B.T. Baune, M. Bayati, J.S. Becker, N. Bedi, E. Beghi, Y. Béjot, M.L. Bell, F.B. Bennitt, I.M. Bensenor, K. Berhe, A.E. Berman, A.S. Bhagavathula, R. Bhageerathy, N. Bhala, D. Bhandari, K. Bhattacharyya, Z.A. Bhutta, A. Bijani, B. Bikbov, M.S. Bin Sayeed, A. Biondi, B.M. Birihane, C. Bisignano, R.K. Biswas, H. Bitew, S. Bohlouli, M. Bohluli, A.S. Boon-Dooley, G. Borges, A.M. Borzi, S. Borzouei, C. Bosetti, S. Boufous, D. Braithwaite, N.J.K. Breitborde, S. Breitner, H. Brenner, P.S. Briant, A.N. Briko, N.I. Briko, G.B. Britton, D. Bryazka, B.R. Bumgarner, K. Burkart, R.T. Burnett, S. Burugina Nagaraja, Z.A. Butt, F.L. Caetano dos Santos, L.E. Cahill, L.L.A. Cámera, I.R. Campos-Nonato, R.

Cárdenas, G. Carreras, J.J. Carrero, F. Carvalho, J.M. Castaldelli-Maia, C.A. Castañeda-Orjuela, G. Castelpietra, F. Castro, K. Causey, C.R. Cederroth, K.M. Cercy, E. Cerin, J.S. Chandan, K.-L. Chang, F.J. Charlson, V.K. Chattu, S. Chaturvedi, N. Cherbuin, O. Chimed-Ochir, D.Y. Cho, J.Y.J. Choi, H. Christensen, D.-T. Chu, M.T. Chung, S.-C. Chung, F.M. Cicuttini, L.G. Ciobanu, M. Cirillo, T.K.D. Classen, A.J. Cohen, K. Compton, O.R. Cooper, V.M. Costa, E. Cousin, R.G. Cowden, D.H. Cross, J.A. Cruz, S.M.A. Dahlawi, A.A.M. Damasceno, G. Damiani, L. Dandona, R. Dandona, W.J. Dangel, A.-K. Danielsson, P.I. Dargan, A.M. Darwesh, A. Daryani, J.K. Das, R. Das Gupta, J. das Neves, C.A. Dávila-Cervantes, D.V. Davitoiu, D. De Leo, L. Degenhardt, M. DeLang, R.P. Dellavalle, F.M. Demeke, G.T. Demoz, D.G. Demsie, E. Denova-Gutiérrez, N. Dervenis, G.P. Dhungana, M. Dianatinasab, D. Dias da Silva, D. Diaz, Z.S. Dibaji Forooshani, S. Djalalinia, H.T. Do, K. Dokova, F. Dorostkar, L. Doshmangir, T.R. Driscoll, B.B. Duncan, A.R. Duraes, A.W. Eagan, D. Edvardsson, N. El Nahas, I. El Sayed, M. El Tantawi, I. Elbarazi, I.Y. 
Elgendy, S.I. El-Jaafary, I.R. Elyazar, S. Emmons-Bell, H.E. Erskine, S. Eskandarieh, S. Esmaeilnejad, A. Esteghamati, K. Estep, A. Etemadi, A.E. Etisso, J. Fanzo, M. Farahmand, M. Fareed, R. Faridnia, A. Farioli, A. Faro, M. Faruque, F. Farzadfar, N. Fattahi, M. Fazlzadeh, V.L. Feigin, R. Feldman, S.-M. Fereshtehnejad, E. Fernandes, G. Ferrara, A.J. Ferrari, M.L. Ferreira, I. Filip, F. Fischer, J.L. Fisher, L.S. Flor, N.A. Foigt, M.O. Folayan, A.A. Fomenkov, L.M. Force, M. Foroutan, R.C. Franklin, M. Freitas, W. Fu, T. Fukumoto, J.M. Furtado, M.M. Gad, E. Gakidou, S. Gallus, A.L. Garcia-Basteiro, W.M. Gardner, B.S. Geberemariyam, A.A.A.A. Gebreslassie, A. Geremew, A. Gershberg Hayoon, P.W. Gething, M. Ghadimi, K. Ghadiri, F. Ghaffarifar, M. Ghafourifard, F. Ghamari, A. Ghashghaee, H. Ghiasvand, N. Ghith, A. Gholamian, R. Ghosh, P.S. Gill, T.G.G. Ginindza, G. Giussani, E.V. Gnedovskaya, S. Goharinezhad, S.V. Gopalani, G. Gorini, H. Goudarzi, A.C. Goulart, F. Greaves, M. Grivna, G. Grosso, M.I.M. Gubari, H.C. Gugnani, R.A. Guimarães, R.A. Guled, G. Guo, Y. Guo, R. Gupta, T. Gupta, B. Haddock, N. Hafezi-Nejad, A. Hafiz, A. Haj-Mirzaian, A. Haj-Mirzaian, B.J. Hall, I. Halvaei, R.R. Hamadeh, S. Hamidi, M.S. Hammer, G.J. Hankey, H. Haririan, J.M. Haro, A.I. Hasaballah, M.M. Hasan, E. Hasanpoor, A. Hashi, S. Hassanipour, H. Hassankhani, R.J. Havmoeller, S.I. Hay, K. Hayat, G. Heidari, R. Heidari-Soureshjani, H.J. Henrikson, M.E. Herbert, C. Herteliu, F. Heydarpour, T.R. Hird, H.W. Hoek, R. Holla, P. Hoogar, H.D. Hosgood, N. Hossain, M. Hosseini, M. Hosseinzadeh, M. Hostiuc, S. Hostiuc, M. Househ, M. Hsairi, V.C. Hsieh, G. Hu, K. Hu, T.M. Huda, A. Humayun, C.K. Huynh, B.-F. Hwang, V.C. Iannucci, S.E. Ibitoye, N. Ikeda, K.S. Ikuta, O.S. Ilesanmi, I.M. Ilic, M.D. Ilic, L.R. Inbaraj, H. Ippolito, U. Iqbal, S.S.N. Irvani, C.M.S. Irvine, M.M. Islam, S.M.S. Islam, H. Iso, R.Q. Ivers, C.C.D. Iwu, C.J. Iwu, I.O. Iyamu, J. Jaafari, K.H. Jacobsen, H. Jafari, M. Jafarinia, M.A. Jahani, M. Jakovljevic, F. Jalilian, S.L. James, H. Janjani, T. Javaheri, J. Javidnia, P. Jeemon, E. Jenabi, R.P. Jha, V. Jha, J.S. Ji, L. Johansson, O. John, Y.O. John-Akinola, C.O. Johnson, J.B. Jonas, F. Joukar, J.J. Jozwiak, M. Jürisson, A. Kabir, Z. Kabir, H. Kalani, R. Kalani, L.R. Kalankesh, R. Kalhor, T. Kanchan, N. Kapoor, B. Karami Matin, A. Karch, M.A. Karim, G.M. Kassa, S.V. Katikireddi, G.A. Kayode, A. Kazemi Karyani, P.N. Keiyoro, C. Keller, L. Kemmer, P.J. Kendrick, N. Khalid, M. Khammarnia, E.A. Khan, M. Khan, K. Khatab, M.M. Khater, M.N. Khatib, M. Khayamzadeh, S. Khazaei, C. Kieling, Y.J. Kim, R.W. Kimokoti, A. Kisa, S. Kisa, M. Kivimäki, L.D. Knibbs, A.K.S. Knudsen, J.M. Kocarnik, S. Kochhar, J.A. Kopec, V.A. Korshunov, P.A. Koul, A. Koyanagi, M.U.G. Kraemer, K. Krishan, K.J. Krohn, H. Kromhout, B. Kuate Defo, G.A. Kumar, V. Kumar, O.P. Kurmi, D. Kusuma, C. La Vecchia, B. Lacey, D.K. Lal, R. Lalloo, T. Lallukka, F.H. Lami, I. Landires, J.J. Lang, S.M. Langan, A.O. Larsson, S. Lasrado, P. Lauriola, J.V. Lazarus, P.H. Lee, S.W.H. Lee, K.E. LeGrand, J. Leigh, M. Leonardi, H. Lescinsky, J. Leung, M. Levi, S. Li, L.-L. Lim, S. Linn, S. Liu, S. Liu, Y. Liu, J. Lo, A.D. Lopez, J.C.F. Lopez, P.D. Lopukhov, S. Lorkowski, P.A. Lotufo, A. Lu, A. Lugo, E.R. Maddison, P.W. Mahasha, M.M. Mahdavi, M. Mahmoudi, A. Majeed, A. Maleki, S. Maleki, R. Malekzadeh, D.C. Malta, A.A. Mamun, A.L. Manda, H. Manguerra, F. MansourGhanaei, B. Mansouri, M.A. Mansournia, A.M. Mantilla Herrera, J.C. Maravilla, A. Marks, R.V. Martin, S. Martini, F.R. Martins-Melo, A. Masaka, S.Z. Masoumi, M.R. Mathur, K. Matsushita, P.K. Maulik, C. McAlinden, J.J. McGrath, M. McKee, M.M. Mehndiratta, F. Mehri, K.M. Mehta, Z.A. Memish, W. Mendoza, R.G. Menezes, E.W. Mengesha, A. Mereke, S.T. Mereta, A. Meretoja, T.J. Meretoja, T. Mestrovic, B. Miazgowski, T. Miazgowski, I.M. Michalek, T.R. Miller, E.J. Mills, G. Mini, M. Miri, A. Mirica, E.M. Mirrakhimov, H. Mirzaei, M. Mirzaei, R. Mirzaei, M. Mirzaei-Alavijeh, A.T. Misganaw, P. Mithra, B. Moazen, D.K. Mohammad, Y. 
Mohammad, N. Mohammad Gholi Mezerji, A. Mohammadian-Hafshejani, N.

Mohammadifard, R. Mohammadpourhodki, A.S. Mohammed, H. Mohammed, J.A.

Mohammed, S. Mohammed, A.H. Mokdad, M. Molokhia, L. Monasta, M.D. Mooney, G.

Moradi, M. Moradi, M. Moradi-Lakeh, R. Moradzadeh, P. Moraga, L. Morawska, J. Morgadoda-Costa, S.D. Morrison, A. Mosapour, J.F. Mosser, S. Mouodi, S.M. Mousavi, A. Mousavi Khaneghah, U.O. Mueller, S. Mukhopadhyay, E.C. Mullany, K.I. Musa, S. Muthupandian, A.F. Nabhan, M. Naderi, A.J. Nagarajan, G. Nagel, M. Naghavi, B. Naghshtabrizi, M.D. Naimzada, F. Najafi, V. Nangia, J.R. Nansseu, M. Naserbakht, V.C. Nayak, I. Negoi, J.W. Ngunjiri, C.T. Nguyen, H.L.T. Nguyen, M. Nguyen, Y.T. Nigatu, R. Nikbakhsh, M.R. Nixon, C.A. Nnaji, S. Nomura, B. Norrving, J.J. Noubiap, C. Nowak, V. Nunez-Samudio, A. Oţoiu, B. Oancea, C.M. Odell, F.A. Ogbo, I.-H. Oh, E.W. Okunga, M. Oladnabi, A.T. Olagunju, B.O. Olusanya, J.O. Olusanya, M.O. Omer, K.L. Ong, O.E. Onwujekwe, H.M. Orpana, A. Ortiz, O. Osarenotor, F.B. Osei, S.M. Ostroff, N. Otstavnov, S.S. Otstavnov, S. Øverland, M.O. Owolabi, M. P A, J.R. Padubidri, R. Palladino, S. Panda-Jonas, A. Pandey, C.D.H. Parry, M. Pasovic, D.K. Pasupula, S.K. Patel, M. Pathak, S.B. Patten, G.C. Patton, H. Pazoki Toroudi, A.E. Peden, A. Pennini, V.C.F. Pepito, E.K. Peprah, D.M. Pereira, K. Pesudovs, H.Q. Pham, M.R. Phillips, C. Piccinelli, T.M. Pilz, M.A. Piradov, M. Pirsaheb, D. Plass, S. Polinder, K.R. Polkinghorne, C.D. Pond, M.J. Postma, H. Pourjafar, F. Pourmalek, A. Poznańska, S.I. Prada, V. Prakash, D.R.A. Pribadi, E. Pupillo, Z. Quazi Syed, M. Rabiee, N. Rabiee, A. Radfar, A. Rafiee, A. Raggi, M.A. Rahman, A. Rajabpour-Sanati, F. Rajati, I. Rakovac, P. Ram, K. Ramezanzadeh, C.L. Ranabhat, P.C. Rao, S.J. Rao, V. Rashedi, P. Rathi, D.L. Rawaf, S. Rawaf, L. Rawal, R. Rawassizadeh, R. Rawat, C. Razo, S.B. Redford, R.C. Reiner Jr, M.B. Reitsma, G. Remuzzi, V. Renjith, A.M.N. Renzaho, S. Resnikoff, N. Rezaei, N. Rezaei, A. Rezapour, P.-A. Rhinehart, S.M. Riahi, D.C. Ribeiro, D. Ribeiro, J. Rickard, J.A. Rivera, N.L.S. Roberts, S. Rodríguez-Ramírez, L. Roever, L. Ronfani, R. Room, G. Roshandel, G.A. Roth, D. Rothenbacher, E. Rubagotti, G.M. Rwegerera, S. Sabour, P.S. Sachdev, B. Saddik, E. Sadeghi, M. Sadeghi, R. Saeedi, S. Saeedi Moghaddam, Y. Safari, S. Safi, S. Safiri, R. Sagar, A. Sahebkar, S.M. Sajadi, N. Salam, P. Salamati, H. Salem, M.R.R. Salem, H. Salimzadeh, O.M. Salman, J.A. Salomon, Z. Samad, H. Samadi Kafil, E.Z. Sambala, A.M. Samy, J. Sanabria, T.G. Sánchez-Pimienta, D.F. Santomauro, I.S. Santos, J.V. Santos, M.M. Santric-Milicevic, S.Y.I. Saraswathy, R. Sarmiento-Suárez, N. Sarrafzadegan, B. Sartorius, A. Sarveazad, B. Sathian, T. Sathish, D. Sattin, S. Saxena, L.E. Schaeffer, S. Schiavolin, M.P. Schlaich, M.I. Schmidt, A.E. Schutte, D.C. Schwebel, F. Schwendicke, A.M. Senbeta, S. Senthilkumaran, S.G. Sepanlou, B. Serdar, M.L. Serre, J. Shadid, O. Shafaat, S. Shahabi, A.A. Shaheen, M.A. Shaikh, A.S. Shalash, M. Shams-Beyranvand, M. Shamsizadeh, K. Sharafi, A. Sheikh, A. Sheikhtaheri, K. Shibuya, K.D. Shield, M. Shigematsu, J.I. Shin, M.-J. Shin, R. Shiri, R. Shirkoohi, K. Shuval, S. Siabani, R. Sierpinski, I.D. Sigfusdottir, R. Sigurvinsdottir, J.P. Silva, K.E. Simpson, J.A. Singh, P. Singh, E. Skiadaresi, S.T.S. Skou, V.Y. Skryabin, E.U.R. Smith, A. Soheili, S. Soltani, M. Soofi, R.J.D. Sorensen, J.B. Soriano, M.B. Sorrie, S. Soshnikov, I.N. Soyiri, C.N. Spencer, A. Spotin, C.T. Sreeramareddy, V. Srinivasan, J.D. Stanaway, C. Stein, D.J. Stein, C. Steiner, L. Stockfelt, M.A. Stokes, K. Straif, J.L. Stubbs, M.B. Sufiyan, H.A.R. Suleria, R. Suliankatchi Abdulkader, G. Sulo, I. Sultan, Ł. Szumowski, R. Tabarés-Seisdedos, K.M. Tabb, T. Tabuchi, A. Taherkhani, M. Tajdini, K. Takahashi, J.S. Takala, A.T. Tamiru, N. Taveira, A. Tehrani-Banihashemi, M.-H. Temsah, G.A. Tesema, Z.T. Tessema, G.D. Thurston, M.V. Titova, H.R. Tohidinik, M. Tonelli, R. Topor-Madry, F. Topouzis, A.E. Torre, M. Touvier, M.R.R. Tovani- 
Palone, B.X. Tran, R. Travillian, A. Tsatsakis, L. Tudor Car, S. Tyrovolas, R. Uddin, C.D. Umeokonkwo, B. Unnikrishnan, E. Upadhyay, M. Vacante, P.R. Valdez, A. van Donkelaar, T.J. Vasankari, Y. Vasseghian, Y. Veisani, N. Venketasubramanian, F.S. Violante, V. Vlassov, S.E. Vollset, T. Vos, R. Vukovic, Y. Waheed, M.T. Wallin, Y. Wang, Y.-P. Wang, A. Watson, J. Wei, M.Y.W. Wei, R.G. Weintraub, J. Weiss, A. Werdecker, J.J. West, R. Westerman, J.L. Whisnant, H.A. Whiteford, K.E. Wiens, C.D.A. Wolfe, S.S. Wozniak, A.-M. Wu, J. Wu, S. Wulf Hanson, G. Xu, R. Xu, S. Yadgir, S.H. Yahyazadeh Jabbari, K. Yamagishi, M. Yaminfirooz, Y. Yano, S. Yaya, V. Yazdi-Feyzabadi, T.Y. Yeheyis, C.S. Yilgwan, M.T. Yilma, P. Yip, N. Yonemoto, M.Z. Younis, T.P. Younker, B. Yousefi, Z. Yousefi, T. Yousefinezhadi, A.Y. Yousuf, C. Yu, H. Yusefzadeh, T. Zahirian Moghadam, M. Zamani, M. Zamanian, H. Zandian, M.S. Zastrozhin, Y. Zhang, Z.-J. Zhang, J.T. Zhao, X.-J.G. Zhao, Y. Zhao, M. Zhou, A. Ziapour, S.R.M. Zimsen, M. Brauer, A. Afshin, S.S. Lim, Global burden of 87 risk factors in 204 countries and territories, 1990-2019: a systematic analysis for the Global Burden of Disease Study 2019, Lancet. 396 (2020) 12231249. https://doi.org/10.1016/S0140-6736(20)30752-2.

[190] L.F. Stead, R. Perera, C. Bullen, D. Mant, J. Hartmann-Boyce, K. Cahill, T. Lancaster, Nicotine replacement therapy for smoking cessation, Cochrane Database Syst. Rev. (2012). https://doi.org/10.1002/14651858.CD000146.pub4.

[191] V.E. Cokkinides, E. Ward, A. Jemal, M.J. Thun, Under-use of smoking-cessation treatments: Results from the National Health Interview Survey, 2000, Am. J. Prev. Med. 28 (2005) 119122. https://doi.org/10.1016/j.amepre.2004.09.007.

[192] J.W. Kahende, B.R. Loomis, B. Adhikari, L. Marshall, A Review of Economic Evaluations of Tobacco Control Programs, Int. J. Environ. Res. Public. Health. 6 (2009). https://doi.org/10.3390/ijerph6010051.

[193] J.P. Ruger, C.M. Lazar, Economic Evaluation of Pharmaco- and Behavioral Therapies for Smoking Cessation: A Critical and Systematic Review of Empirical Research, Annu. Rev. Public Health. 33 (2012) 279-305. https://doi.org/10.1146/annurev-publhealth-031811-124553.

[194] L.F. Stead, D. Buitrago, N. Preciado, G. Sanchez, J. Hartmann-Boyce, T. Lancaster, Physician advice for smoking cessation, Cochrane Database Syst. Rev. (2013). https://doi.org/10.1002/14651858.CD000165.pub4.

[195] T. Gültzow, D.N. Zijlstra, C. Bolman, H. de Vries, C.D. Dirksen, J.W.M. Muris, E.S. Smit, C. Hoving, Decision aids to facilitate decision making around behavior change in the field of health promotion: A scoping review, Patient Educ. Couns. 104 (2021) 1266-1285. https://doi.org/10.1016/j.pec.2021.01.015.

[196] T. Hooiveld, J.M. Molenaar, C.M. van der Heijde, F.J. Meijman, T.P. Groen, P. Vonk, End-user involvement in developing and field testing an online contraceptive decision aid, SAGE Open Med. 6 (2018). https://doi.org/10.1177/2050312118809462.

[197] G. Vaisson, T. Provencher, M. Dugas, M.-È. Trottier, S. Chipenda Dansokho, H. Colquhoun, A. Fagerlin, A.M.C. Giguere, H. Hakim, L. Haslett, A.S. Hoffman, N.M. Ivers, A.-S. Julien, F. Légaré, J.-S. Renaud, D. Stacey, R.J. Volk, H.O. Witteman, User Involvement in the Design and Development of Patient Decision Aids and Other Personal Health Tools: A Systematic Review, Med. Decis. Making. 41 (2021) 261-274. https://doi.org/10.1177/0272989X20984134.

[198] T. Gültzow, E.S. Smit, R. Hudales, C.D. Dirksen, C. Hoving, Smoker profiles and their influence on smokers' intention to use a digital decision aid aimed at the uptake of evidence-based 
smoking cessation tools: An explorative study, Digit. Health. 6 (2020) 2055207620980241. https://doi.org/10.1177/2055207620980241.

[199] F. Almusharraf, J. Rose, P. Selby, Engaging Unmotivated Smokers to Move Toward Quitting: Design of Motivational Interviewing-Based Chatbot Through Iterative Interactions, J. Med. Internet Res. 22 (2020) e20251. https://doi.org/10.2196/20251.

[200] M. Mason, Sample Size and Saturation in PhD Studies Using Qualitative Interviews, Forum Qual. Sozialforschung. 11 (2010). https://doi.org/10.17169/fqs-11.3.1428.

[201] N.K. Gale, G. Heath, E. Cameron, S. Rashid, S. Redwood, Using the framework method for the analysis of qualitative data in multi-disciplinary health research, BMC Med. Res. Methodol. 13 (2013). https://doi.org/10.1186/1471-2288-13-117.

[202] QSR International, NVivo, 2018.

[203] IBM Corp, BM SPSS Statistics for Macintosh, Version 25.0, IBM Corp, Armonk, NY, 2017.

[204] S. Davis, A. Huebner, F. Piercy, L. Shettler, P.S. Meszaros, J. Matheson, Female Adolescent Smoking: A Delphi Study on Best Prevention Practices, J. Drug Educ. 34 (2004) 295-311. https://doi.org/10.2190/M8C4-HF1G-153K-TM6E.

[205] F. Schneider, L. van Osch, H. de Vries, Identifying Factors for Optimal Development of HealthRelated Websites: A Delphi Study Among Experts and Potential Future Users, J. Med. Internet Res. 14 (2012) e18. https://doi.org/10.2196/jmir.1863.

[206] M. Galesic, R. Garcia-Retamero, G. Gigerenzer, Using icon arrays to communicate medical risks: Overcoming low numeracy., Health Psychol. 28 (2009) 210-216. https://doi.org/10.1037/a0014474.

[207] Risk Science Center and Center for Bioethics and Social Sciences in Medicine, University of Michigan, Iconarray.com, (n.d.). http://www.iconarray.com/ (accessed January 1, 2020).

[208] N. Bol, N.M. Høie, M.H. Nguyen, E.S. Smit, Customization in mobile health apps: explaining effects on physical activity intentions by the need for autonomy, Digit. Health. 5 (2019). https://doi.org/10.1177/2055207619888074.

[209] S.C. Wangberg, O. Nilsen, K. Antypas, I.T. Gram, Effect of Tailoring in an Internet-Based Intervention for Smoking Cessation: Randomized Controlled Trial, J. Med. Internet Res. 13 (2011) e121. https://doi.org/10.2196/jmir.1605.

[210] N. Chavannes, T. Drenthen, L. Wind, M. Van Avendonk, M. Van den Donk, M. Verduijn, NHGBehandelrichtlijn Stoppen met roken, (2017). https://richtlijnen.nhg.org/behandelrichtlijnen/stoppen-met-roken.

[211] R.H. DeMeester, F.Y. Lopez, J.E. Moore, S.C. Cook, M.H. Chin, A Model of Organizational Context and Shared Decision Making: Application to LGBT Racial and Ethnic Minority Patients, J. Gen. Intern. Med. 31 (2016) 651-662. https://doi.org/10.1007/s11606-016-36083.

[212] T. Freund, C. Everett, P. Griffiths, C. Hudon, L. Naccarella, M. Laurant, Skill mix, roles and remuneration in the primary care workforce: Who are the healthcare professionals in the primary care teams across the world?, Int. J. Nurs. Stud. 52 (2015) 727-743. https://doi.org/10.1016/j.ijnurstu.2014.11.014.

[213] Nederlands Huisartsen Genootschap, Landelijke Huisartsen Vereniging, NHG/LHV-Standpunt: Het (ondersteunend) team in de huisartsen-voorziening, 2011. 
[214] P.L. Ferreira, A.I. Tavares, C. Quintal, P. Santana, EU health systems classification: a new proposal from EURO-HEALTHY, BMC Health Serv. Res. 18 (2018). https://doi.org/10.1186/s12913-018-3323-3.

[215] L. Springvloet, J. Bommelé, M. Willemsen, M. van Laar, Kerncijfers roken 2017, (2018). https://assets.trimbos.nl/docs/cdade14e-20dc-462a-9f63-1b71ee995a84.pdf (accessed January 24, 2019).

[216] World Health Organization, WHO report on the global tobacco epidemic, 2009: implementing smoke-free environments, (2009). https://www.who.int/tobacco/mpower/2009/en/.

[217] K.L. Cheung, B. Wijnen, H. de Vries, A Review of the Theoretical Basis, Effects, and Cost Effectiveness of Online Smoking Cessation Interventions in the Netherlands: A MixedMethods Approach, J. Med. Internet Res. 19 (2017) e230. https://doi.org/10.2196/jmir.7209.

[218] T.H. Van de Belt, L.J.L.P.G. Engelen, S.A.A. Berben, S. Teerenstra, M. Samsom, L. Schoonhoven, Internet and Social Media For Health-Related Information and Communication in Health Care: Preferences of the Dutch General Population, J. Med. Internet Res. 15 (2013) e220. https://doi.org/10.2196/jmir.2607.

[219] C.R. Brewin, C. Bradley, Patient preferences and randomised clinical trials, BMJ. 299 (1989) 313. https://doi.org/10.1136/bmj.299.6694.313.

[220] G. Eysenbach, CONSORT-EHEALTH Group, CONSORT-EHEALTH: Improving and Standardizing Evaluation Reports of Web-based and Mobile Health Interventions, J. Med. Internet Res. 13 (2012) e126. https://doi.org/10.2196/jmir.1923.

[221] G.-J.Y. Peters, C. Abraham, R. Crutzen, Full disclosure: doing behavioural science necessitates sharing, Eur. Health Psychol. 14 (2012) 77-84.

[222] Keuzehulp Visor (@keuzehulp_visor) •Instagram photos and videos, (n.d.). https://www.instagram.com/keuzehulp_visor/?hl=en (accessed March 24, 2020).

[223] Pew Research Center, Social Media Use in 2018, 2018. https://www.pewresearch.org/internet/wpcontent/uploads/sites/9/2018/02/PI_2018.03.01_Social-Media_FINAL.pdf.

[224] D. Feldman-Stewart, M.A. O'Brien, M.L. Clayman, B.J. Davison, M. Jimbo, M. Labrecque, R.W. Martin, H. Shepherd, Providing information about options in patient decision aids, BMC Med. Inform. Decis. Mak. 13 (2013). https://doi.org/10.1186/1472-6947-13-s2-s4.

[225] M.W.M. Jaspers, A comparison of usability methods for testing interactive health technologies: Methodological aspects and empirical evidence, Int. J. Med. Inform. 78 (2009) 340-353. https://doi.org/10.1016/j.ijmedinf.2008.10.002.

[226] M.B. Altendorf, J.C. van Weert, C. Hoving, E.S. Smit, Should or could? Testing the use of autonomy-supportive language and the provision of choice in online computer-tailored alcohol reduction communication, Digit. Health. 5 (2019). https://doi.org/10.1177/2055207619832767.

[227] J.E. Maddux, R.W. Rogers, Protection motivation and self-efficacy: A revised theory of fear appeals and attitude change, J. Exp. Soc. Psychol. 19 (1983) 469-479. https://doi.org/10.1016/0022-1031(83)90023-9.

[228] OverNite Software Europe, TailorBuilder | Create online questionnaires with personal feedback, (n.d.). https://www.tailorbuilder.com/en/home.html (accessed November 16, 2020). 
[229] M. de Vries, A. Fagerlin, H.O. Witteman, L.D. Scherer, Combining deliberation and intuition in patient decision support, Patient Educ. Couns. 91 (2013) 154-160. https://doi.org/10.1016/j.pec.2012.11.016.

[230] Zorginstituut Nederland, Home | National Health Care Institute, (n.d.). https://english.zorginstituutnederland.nl/ (accessed August 30, 2020).

[231] College ter Beoordeling van Geneesmiddelen, Home - Medicines Evaluation Board, (n.d.). https://english.cbg-meb.nl/ (accessed August 30, 2020).

[232] A. Chernev, U. Böckenholt, J. Goodman, Choice overload: A conceptual review and metaanalysis, J. Consum. Psychol. 25 (2015) 333-358. https://doi.org/10.1016/j.jcps.2014.08.002.

[233] E.S. Smit, A.L. Dima, S.A.M. Immerzeel, B. van den Putte, G.C. Williams, The Virtual Care Climate Questionnaire: Development and Validation of a Questionnaire Measuring Perceived Support for Autonomy in a Virtual Care Setting, J. Med. Internet Res. 19 (2017) e155. https://doi.org/10.2196/jmir.6714.

[234] G.C. Williams, Z.R. Freedman, E.L. Deci, Supporting Autonomy to Motivate Patients With Diabetes for Glucose Control, Diabetes Care. 21 (1998) 1644-1651. https://doi.org/10.2337/diacare.21.10.1644.

[235] Zorginstituut Nederland, Guideline for economic evaluations in healthcare, (2016). https://english.zorginstituutnederland.nl/publications/reports/2016/06/16/guideline-foreconomic-evaluations-in-healthcare.

[236] K.J. Korte, D.W. Capron, M. Zvolensky, N.B. Schmidt, The Fagerström Test for Nicotine Dependence: Do revisions in the item scoring enhance the psychometric properties?, Addict. Behav. 38 (2013) 1757-1763. https://doi.org/10.1016/j.addbeh.2012.10.013.

[237] C. Bouwmans, M. Krol, H. Severens, M. Koopmanschap, W. Brouwer, L. Hakkaart-van Roijen, The iMTA Productivity Cost Questionnaire: A Standardized Instrument for Measuring and Valuing Health-Related Productivity Losses, Value Health. 18 (2015) 753-758. https://doi.org/10.1016/j.jval.2015.05.009.

[238] D. de Ruijter, C. Hoving, S. Evers, R. Hudales, H. de Vries, E. Smit, An economic evaluation of a computer-tailored e-learning program to promote smoking cessation counseling guideline adherence among practice nurses, Patient Educ. Couns. 102 (2019) 1802-1811. https://doi.org/10.1016/j.pec.2019.07.015.

[239] H. Al-Janabi, T.N. Flynn, J. Coast, Development of a self-report measure of capability wellbeing for adults: the ICECAP-A, Qual. Life Res. 21 (2012) 167-176. https://doi.org/10.1007/s11136011-9927-2.

[240] A.M. O'Connor, Stage of Decision Making [document on the Internet], (2000). https://decisionaid.ohri.ca/docs/develop/User_Manuals/UM_Stage_Decision_Making.pdf (accessed March 24, 2020).

[241] C. Bennett, I.D. Graham, E. Kristjansson, S.A. Kearing, K.F. Clay, A.M. O'Connor, Validation of a Preparation for Decision Making scale, Patient Educ. Couns. 78 (2010) 130-133. https://doi.org/10.1016/j.pec.2009.05.012.

[242] A. O'Connor, User Manual - Knowledge [document on the Internet], (2000). http://decisionaid.ohri.ca/docs/develop/User_Manuals/UM_Knowledge.pdf (accessed January 1, 2019). 
[243] J.C. Brehaut, A.M. O'Connor, T.J. Wood, T.F. Hack, L. Siminoff, E. Gordon, D. Feldman-Stewart, Validation of a Decision Regret Scale, Med. Decis. Making. 23 (2003) 281-292. https://doi.org/10.1177/0272989x03256005.

[244] R. Crutzen, G.-J.Y. Peters, Scale quality: alpha is an inadequate estimate and factor-analytic evidence is needed first of all, Health Psychol. Rev. 11 (2017) 242-247. https://doi.org/10.1080/17437199.2015.1124240.

[245] R.P. McDonald, Test theory: A unified treatment, Psychology Press, 2013.

[246] G.-J.Y. Peters, The alpha and the omega of scale reliability and validity: Why and how to abandon Cronbach's alpha and the route towards more comprehensive assessment of scale quality, Eur. Health Psychol. 16 (2014) 56-59.

[247] T.J. Dunn, T. Baguley, V. Brunsden, From alpha to omega: A practical solution to the pervasive problem of internal consistency estimation, Br. J. Psychol. 105 (2014) 399-412. https://doi.org/10.1111/bjop.12046.

[248] R Development Core Team, R: A language and environment for statistical computing, (2020). https://www.R-project.org/.

[249] RStudio Team, RStudio: Integrated Development for R, (2020). http://www.rstudio.com.

[250] S.L.K. Gruijters, Baseline comparisons and covariate fishing: Bad statistical habits we should have broken yesterday, Eur. Health Psychol. 18 (2016) 205-209.

[251] M.R. De Boer, W.E. Waterlander, L.D.J. Kuijper, I.H.M. Steenhuis, J.W.R. Twisk, Testing for baseline differences in randomized controlled trials: an unhealthy research behavior that is hard to eradicate, Int. J. Behav. Nutr. Phys. Act. 12 (2015). https://doi.org/10.1186/s12966015-0162-z.

[252] E.S. Smit, H. de Vries, E.J.M. Oberjé, S.M.A.A. Evers, Easier Said Than Done: Overcoming Challenges in the Economic Evaluation of Internet-Based Lifestyle Interventions, Eur. Health Psychol. 17 (2015) 39-44.

[253] Y. Rosseel, lavaan: An R Package for Structural Equation Modeling, J. Stat. Softw. 48 (2012) 136.

[254] D.K. Ahern, J.M. Kreslake, J.M. Phalen, What Is eHealth (6): Perspectives on the Evolution of eHealth Research, J. Med. Internet Res. 8 (2006) e4. https://doi.org/10.2196/jmir.8.1.e4.

[255] Eurostat, Internet access and use statistics - households and individuals, (2016). https://ec.europa.eu/eurostat/statisticsexplained/index.php?title=Archive:Internet_access_and_use_statistics__households_and_individuals\&oldid=379591.

[256] E. Vangeli, J. Stapleton, E.S. Smit, R. Borland, R. West, Predictors of attempts to stop smoking and their success in adult general population samples: a systematic review, Addiction. 106 (2011) 2110-2121. https://doi.org/10.1111/j.1360-0443.2011.03565.x.

[257] P. Caponnetto, R. Polosa, Common predictors of smoking cessation in clinical practice, Respir. Med. 102 (2008) 1182-1192. https://doi.org/10.1016/j.rmed.2008.02.017.

[258] A.L. Smith, S. Chapman, S.M. Dunlop, What do we know about unassisted smoking cessation in Australia? A systematic review, 2005-2012, Tob. Control. 24 (2015) 18-27. https://doi.org/10.1136/tobaccocontrol-2013-051019.

[259] F.T. Filippidis, V. Gerovasili, C.I. Vardavas, I.T. Agaku, Y. Tountas, Determinants of use of smoking cessation aids in 27 European countries, Prev. Med. 65 (2014) 99-102. https://doi.org/10.1016/j.ypmed.2014.05.004. 
[260] K.E. Pecanac, R.L. Brown, H.B. Kremsreiter, Decisional Conflict During Major Medical Treatment Decision-making: a Survey Study, J. Gen. Intern. Med. (2021) 55-61. https://doi.org/10.1007/s11606-020-06125-1.

[261] S.N. Davis, J.W. Wischhusen, S.K. Sutton, S.M. Christy, E.A. Chavarria, M.E. Sutter, S. Roy, C.D. Meade, C.K. Gwede, Demographic and psychosocial factors associated with limited health literacy in a community-based sample of older Black Americans, Patient Educ. Couns. 103 (2020) 385-391. https://doi.org/10.1016/j.pec.2019.08.026.

[262] J.L. Oliffe, D.R. McCreary, N. Black, R. Flannigan, S.L. Goldenberg, Canadian Men's Health Literacy: A Nationally Representative Study, Health Promot. Pract. (2019). https://doi.org/10.1177/1524839919837625.

[263] J. Bommelé, M. Willemsen, Kerncijfers roken 2019, (2020). https://www.trimbos.nl/docs/452115a7-4a93-4bd4-877a-ae4a7a4d1257.pdf (accessed September 17, 2020).

[264] M.W. van Laar, E.M.T. Beenakkers, A.A.N. Cruts, A.P.M. Ketelaars, M.C. Kuin, R.F. Meijer, C.J.A. van Miltenburg, A. Mujcic, L. Strada, Jaarbericht Nationale Drug Monitor 2020, (2021). https://www.trimbos.nl/?act=winkeldl.download\&prod=1400 (accessed October 8, 2021).

[265] R.N. Rimal, Closing the Knowledge-Behavior Gap in Health Promotion: The Mediating Role of Self-Efficacy, Health Commun. 12 (2000) 219-237. https://doi.org/10.1207/S15327027HC1203_01.

[266] F.X. Sligo, A.M. Jameson, The knowledge-behavior gap in use of health information, J. Am. Soc. Inf. Sci. 51 (2000) 858-869. https://doi.org/10.1002/(SICI)10974571(2000)51:9<858::AID-ASI80>3.0.CO;2-Q.

[267] H. Kim, P.A. Stout, The Effects of Interactivity on Information Processing and Attitude Change: Implications for Mental Health Stigma, Health Commun. 25 (2010) 142-154. https://doi.org/10.1080/10410230903544936.

[268] T. Gültzow, E.S. Smit, R. Hudales, C.D. Dirksen, C. Hoving, Informed decision making on the uptake of evidence-based smoking cessation assistance: A needs assessment among end users and experts to inform decision aid development, MedRxiv. (2021). https://doi.org/10.1101/2021.04.09.21255012.

[269] T. Gültzow, E.S. Smit, R. Crutzen, S. Jolani, C. Hoving, C.D. Dirksen, Effect study, (2021). https://osf.io/7kcpm/ (accessed October 12, 2021).

[270] T. Gültzow, C. Hoving, Nieuw onderzoek naar keuzehulp bij stoppen-met-roken, Huisarts Wet. 64 (2021). https://doi.org/10.1007/s12445-020-0962-5.

[271] The Rosetta Stats Authors, Rosetta Stats, 2020. https://sci-ops.gitlab.io/rosetta-stats/ (accessed March 25, 2021).

[272] Module 4 - Working out Cigarette Equivalents, (n.d.). http://www.smoking2.nes.scot.nhs.uk/module4/working-out-cigarette-equivalents.html (accessed May 6, 2021).

[273] G. Eysenbach, The Law of Attrition, J. Med. Internet Res. 7 (2005) e11. https://doi.org/10.2196/jmir.7.1.e11.

[274] S. van Buuren, K. Groothuis-Oudshoorn, G. Vink, R. Schouten, A. Robitzsch, P. Rockenschaub, L. Doove, S. Jolani, M. Moreno-Betancur, I. White, P. Gaffert, F. Meinfelder, B. Gray, V. ArelBundock, mice: Multivariate Imputation by Chained Equations, 2021. https://CRAN.Rproject.org/package=mice (accessed March 25, 2021). 
[275] Consort - The CONSORT Flow Diagram, (n.d.). http://www.consort-statement.org/consortstatement/flow-diagram (accessed June 9, 2021).

[276] M. Blankers, E.S. Smit, P. van der Pol, H. de Vries, C. Hoving, M. van Laar, The Missing=Smoking Assumption: A Fallacy in Internet-Based Smoking Cessation Trials?, Nicotine Tob. Res. 18 (2016) 25-33. https://doi.org/10.1093/ntr/ntv055.

[277] W. Brouwer, W. Kroeze, R. Crutzen, J. de Nooijer, N.K. de Vries, J. Brug, A. Oenema, Which Intervention Characteristics are Related to More Exposure to Internet-Delivered Healthy Lifestyle Promotion Interventions? A Systematic Review, J. Med. Internet Res. 13 (2011) e2. https://doi.org/10.2196/jmir.1639.

[278] E.S. Smit, C. Hoving, V.C.M. Cox, H. de Vries, Influence of recruitment strategy on the reach and effect of a web-based multiple tailored smoking cessation intervention among Dutch adult smokers, Health Educ. Res. 27 (2012) 191-199. https://doi.org/10.1093/her/cyr099.

[279] A. Bricca, Z. Swithenbank, N. Scott, S. Treweek, M. Johnston, N. Black, J. Hartmann-Boyce, R. West, S. Michie, M. de Bruin, Predictors of recruitment and retention in randomized controlled trials of behavioural smoking cessation interventions: a systematic review and meta-regression analysis, Addiction. (2021). https://doi.org/10.1111/add.15614.

[280] F. Tzelepis, C.L. Paul, C.M. Williams, C. Gilligan, T. Regan, J. Daly, R.K. Hodder, E. Byrnes, J. Byaruhanga, T. McFadyen, J. Wiggers, Real-time video counselling for smoking cessation, Cochrane Database Syst. Rev. (2019). https://doi.org/10.1002/14651858.CD012659.pub2.

[281] O. Perski, D. Crane, E. Beard, J. Brown, Does the addition of a supportive chatbot promote user engagement with a smoking cessation app? An experimental study, Digit. Health. 5 (2019). https://doi.org/10.1177/2055207619880676.

[282] R.A. Bailey, M. Pfeifer, A.C. Shillington, Q. Harshaw, M.M. Funnell, J. VanWingen, N. Col, Effect of a patient decision aid (PDA) for type 2 diabetes on knowledge, decisional self-efficacy, and decisional conflict, BMC Health Serv. Res. 16 (2016). https://doi.org/10.1186/s12913-0161262-4.

[283] W. Osaka, K. Nakayama, Effect of a decision aid with patient narratives in reducing decisional conflict in choice for surgery among early-stage breast cancer patients: A three-arm randomized controlled trial, Patient Educ. Couns. 100 (2017) 550-562. https://doi.org/10.1016/j.pec.2016.09.011.

[284] C. Short, E.S. Smit, R. Crutzen, Measuring psychological constructs in computer-tailored interventions: novel possibilities to reduce participant burden and increase engagement, PsyArXiv. (2021). https://doi.org/10.31234/osf.io/hz593.

[285] T. Gültzow, C. Hoving, E.S. Smit, H.L. Bekker, Integrating behaviour change interventions and patient decision aids: How to accomplish synergistic effects?, Patient Educ. Couns. 104 (2021) 3104-3108. https://doi.org/10.1016/j.pec.2021.04.007.

[286] M.B. Larsen, R. Stokholm, P. Kirkegaard, H.S. Laursen, P. Gabel, B. Andersen, Making decisions on your own: Self-administered decision aids about colorectal cancer screening - A systematic review and meta-analyses, Patient Educ. Couns. (2021). https://doi.org/10.1016/j.pec.2021.07.035.

[287] Centraal Bureau voor de Statistiek, CBS, (n.d.). https://www.cbs.nl/ (accessed September 2, 2021). 
[288] D.L. Patrick, A. Cheadle, D.C. Thompson, P. Diehr, T. Koepsell, S. Kinne, The validity of selfreported smoking: a review and meta-analysis., Am. J. Public Health. 84 (1994) 1086-1093. https://doi.org/10.2105/ajph.84.7.1086.

[289] Centraal Bureau voor de Statistiek, Opleidingsniveau, (n.d.). https://www.cbs.nl/nlnl/nieuws/2019/33/verschil-levensverwachting-hoog-en-laagopgeleidgroeit/opleidingsniveau (accessed April 21, 2021).

[290] R. West, W. Zatonski, M. Cedzynska, D. Lewandowska, J. Pazik, P. Aveyard, J. Stapleton, Placebo-Controlled Trial of Cytisine for Smoking Cessation, N. Engl. J. Med. 365 (2011) 11931200. https://doi.org/10.1056/NEJMoa1102035.

[291] P. Hajek, H. McRobbie, K. Myers, Efficacy of cytisine in helping smokers quit: systematic review and meta-analysis, Thorax. 68 (2013) 1037-1042. https://doi.org/10.1136/thoraxjnl-2012203035.

[292] G.-J.Y. Peters, M. de Bruin, R. Crutzen, Everything should be as simple as possible, but no simpler: towards a protocol for accumulating evidence regarding the active content of health behaviour change interventions, Health Psychol. Rev. 9 (2015) 1-14. https://doi.org/10.1080/17437199.2013.848409.

[293] G.-J.Y. Peters, R.A.C. Ruiter, G. Kok, Threatening communication: a critical re-analysis and a revised meta-analytic test of fear appeal theory, Health Psychol. Rev. 7 (2013) S8-S31. https://doi.org/10.1080/17437199.2012.703527.

[294] G. Elwyn, M. Stiel, M.-A. Durand, J. Boivin, The design of patient decision support interventions: addressing the theory-practice gap, J. Eval. Clin. Pract. 17 (2011) 565-574. https://doi.org/10.1111/j.1365-2753.2010.01517.x.

[295] L. Trenaman, J. Jansen, J. Blumenthal-Barby, M. Körner, J. Lally, D. Matlock, L. Perestelo-Perez, M. Ropka, C. Stirling, K. Valentine, H. Vo, C.E. Wills, R. Thomson, K. Sepucha, Are We Improving? Update and Critical Appraisal of the Reporting of Decision Process and Quality Measures in Trials Evaluating Patient Decision Aids, Med. Decis. Making. 41 (2021) 954-959. https://doi.org/10.1177/0272989X211011120.

[296] Algemeen Nederlands Persbureau, Cyberaanval Universiteit Maastricht duurt mogelijk tot na kerstvakantie, (2019). https://nos.nl/l/2316373 (accessed September 20, 2021).

[297] J.V. 't Riet, R. Crutzen, H. de Vries, Investigating Predictors of Visiting, Using, and Revisiting an Online Health-Communication Program: A Longitudinal Study, J. Med. Internet Res. 12 (2010) e1345. https://doi.org/10.2196/jmir.1345.

[298] S. Motorny, S. Sarnikar, C. Noteboom, Design of an Intelligent Patient Decision aid Based on Individual Decision-Making Styles and Information Need Preferences, Inf. Syst. Front. (2021). https://doi.org/10.1007/s10796-021-10125-9.

[299] D.N. Schulz, S.P.J. Kremers, C. Vandelanotte, M.J.G. van Adrichem, F. Schneider, M.J.J.M. Candel, H. de Vries, Effects of a Web-Based Tailored Multiple-Lifestyle Intervention for Adults: A Two-Year Randomized Controlled Trial Comparing Sequential and Simultaneous Delivery Modes, J. Med. Internet. Res. 16 (2014) e26. https://doi.org/10.2196/jmir.3094.

[300] N. Stanczyk, C. Bolman, M. van Adrichem, M. Candel, J. Muris, H. de Vries, Comparison of Text and Video Computer-Tailored Interventions for Smoking Cessation: Randomized Controlled Trial, J. Med. Internet. Res. 16 (2014) e69. https://doi.org/10.2196/jmir.3016.

[301] R.A. Willems, M.C. Willemsen, E.S. Smit, G.E. Nagelhout, E. Janssen, H. de Vries, Which smoking cessation aids are proven effective according to smokers who want to quit 
smoking? A report from the Netherlands, Tob. Control. 23 (2013) 525-526. https://doi.org/10.1136/tobaccocontrol-2013-051076.

[302] I. van der Heide, J. Wang, M. Droomers, P. Spreeuwenberg, J. Rademakers, E. Uiters, The Relationship Between Health, Education, and Health Literacy: Results From the Dutch Adult Literacy and Life Skills Survey, J. Health Commun. 18 (2013) 172-184. https://doi.org/10.1080/10810730.2013.825668.

[303] D.M. Muscat, J. Smith, O. Mac, T. Cadet, A. Giguere, A.J. Housten, A.T. Langford, S. Smith, M.A. Durand, K. McCaffery, Addressing Health Literacy in Patient Decision Aids: An Update from the International Patient Decision Aid Standards, Med. Decis. Making. 41 (2021) 848-869. https://doi.org/10.1177/0272989X211011101.

[304] K.L. Cheung, I. Schwabe, M.J.L. Walthouwer, A. Oenema, L. Lechner, H. De Vries, Effectiveness of a Video-Versus Text-Based Computer-Tailored Intervention for Obesity Prevention after One Year: A Randomized Controlled Trial, Int. J. Environ. Res. Public. Health. 14 (2017). https://doi.org/10.3390/ijerph14101275.

[305] N.E. Stanczyk, H. de Vries, M.J.J.M. Candel, J.W.M. Muris, C.A.W. Bolman, Effectiveness of video- versus text-based computer-tailored smoking cessation interventions among smokers after one year, Prev. Med. 82 (2016) 42-50. https://doi.org/10.1016/j.ypmed.2015.11.002.

[306] C.S. Meppelink, J.C.M. van Weert, C.J. Haven, E.G. Smit, The Effectiveness of Health Animations in Audiences With Different Health Literacy Levels: An Experimental Study, J. Med. Internet. Res. 17 (2015) e11. https://doi.org/10.2196/jmir.3979.

[307] D. Stacey, V. Suwalska, L. Boland, K.B. Lewis, J. Presseau, R. Thomson, Are Patient Decision Aids Used in Clinical Practice after Rigorous Evaluation? A Survey of Trial Authors, Med. Decis. Making. 39 (2019) 805-815. https://doi.org/10.1177/0272989X19868193.

[308] N. Joseph-Williams, P. Abhyankar, L. Boland, P. Bravo, A.T. Brenner, S. Brodney, A. Coulter, A. Giguère, A. Hoffman, M. Körner, A. Langford, F. Légaré, D. Matlock, N. Moumjid, S. Munro, K. Dahl Steffensen, C. Stirling, T. van der Weijden, What Works in Implementing Patient Decision Aids in Routine Clinical Settings? A Rapid Realist Review and Update from the International Patient Decision Aid Standards Collaboration, Med. Decis. Making. 41 (2021) 907-937. https://doi.org/10.1177/0272989X20978208.

[309] D.N. Zijlstra, J.W.M. Muris, C. Bolman, J.M. Elling, V.E.R.A. Knapen, H. de Vries, A referral aid for smoking cessation interventions in primary care: study protocol for a randomized controlled trial, Prim. Health Care Res. Dev. 22 (2021) e22. https://doi.org/10.1017/S1463423621000244.

[310] H.O. Witteman, K.G. Maki, G. Vaisson, J. Finderup, K.B. Lewis, K. Dahl Steffensen, C. Beaudoin, S. Comeau, R.J. Volk, Systematic Development of Patient Decision Aids: An Update from the IPDAS Collaboration, Med. Decis. Making. 41 (2021) 736-754. https://doi.org/10.1177/0272989X211014163.

[311] Open Science | NWO, (n.d.). https://www.nwo.nl/en/open-science (accessed September 20, 2021).

[312] R. Crutzen, G.-J.Y. Peters, C. Mondschein, Why and how we should care about the General Data Protection Regulation, Psychol. Health. 34 (2019) 1347-1357. https://doi.org/10.1080/08870446.2019.1606222. 



\section{summary}


Decision making, including health-related decision making, is an integral part of people's daily living. Such health-related decision making can be relatively simple (for example, the decision to brush one's teeth), but can also be more complex, involve substantial uncertainty, and have a big impact on people's lives (for example, the decision for or against a certain cancer treatment). Especially as decision making becomes more complex, it is important that the decision is informed, as people generally experience less ambivalence about their decision if they can make an informed (as opposed to an uninformed) decision. Making an informed decision means two things: first, one has the information needed to make a decision, and second, one can use this information to determine what is personally perceived as important in relation to the decision in question.

Informed decision making has become increasingly important since the 1990s, but is mainly applied to treatment (for example, cancer treatment) and population screening (for example, cancer screening) decisions. Health promotion decisions have so far received little attention in the informed decision-making literature, even though such decisions can also evoke ambivalent feelings. This can be seen, for example, in relation to smoking cessation: People who are motivated to quit smoking often lack the information needed to make an informed decision between the different smoking cessation support tools. This also makes them less able to determine what is important to them regarding this decision. Consequently, smokers may find it difficult to make an informed decision between the different smoking cessation aids available, which can lead to ambivalent feelings regarding the decision.

Decision aids can be used to avoid such ambivalent feelings and to support smokers in making an informed decision. Decision aids often have two components: (1) balanced information provision and (2) so-called value clarification methods that can help determine what is personally experienced as important regarding the decision in question. These value clarification methods can be explicit (including interactivity) or implicit (without interactivity). However, decision aids (and by extension value clarification methods) have so far been mainly studied in treatment and screening contexts and not much in the context of health promotion.

Based on the introduction above, this thesis had two objectives: (1) to gain a good understanding of the decision aids used in health promotion and (2) to describe the work that was undertaken to develop and test the smoking cessation decision aid VISOR. This goal as well as the background to this goal are described in more detail in Chapter 1. 


\section{Decision Aids Applied to Health Promotion}

The aim of Chapter 2 was to critically review behavior change interventions (often used in health promotion) and decision aids (described in this chapter as patient decision aids). Similarities and differences were explored and the potential for integration was identified to ultimately increase the benefits for the users of both interventions. We found that behavior change interventions and decision aids mainly differ in terms of (1) goals and foci, (2) theoretical basis, (3) development frameworks, (4) active ingredients, and (5) effect evaluation. Integration can be achieved by using the knowledge generated by decision aid research to facilitate decision-making processes that enable patients to choose goals that align with their personal values and preferences. On the other hand, findings from research on behavior change interventions can be used to support the implementation of these chosen goals. To facilitate the integration of scientific insights from studies of behavior change interventions and decision aids, the following recommendations are made in Chapter 2: (1) bring both fields together and promote interprofessional discussions, (2) train (health) professionals to recognize strengths of both approaches, (3) investigate the synergy of the two fields, and (4) be prepared for and try to mitigate a culture shock when the fields start to interact.

The aim of Chapter 3 was to review the existing literature on decision aids that support health promotion decision making. The focus was on diet, exercise, sleep, and substance use. Thirtyfive scientific articles and four decision aids (grey literature) are included in this 'scoping review', of which 29 were on substance use. All decision aids included information, value clarification methods, and methods to clarify personal preferences. However, there were also many other elements, such as goal setting. This made it difficult to determine which of these elements helped users (and through which processes) and which did not. While most studies used theories to some extent, little was done to integrate insights from behavior change and (informed) decision making. Although some positive behavioral effects (e.g., related to smoking) were reported, the effects of decision aids were generally mixed. Also, studies did not always follow a randomized study design and little use was made of standardized outcome measures. This made knowledge synthesis difficult. Based on this scoping review, several areas for improvement could be identified: (1) researchers should establish which intervention elements are effective regarding health promotion decision making, and for which processes, (2) the integration between theoretical insights from behavior change and (informed) decision making should be strengthened, by either using a flexible theoretical approach or by designing a framework that integrates behavior change and (informed) decision making, and (3) more randomized trials should be conducted to enable other reviews to draw stronger conclusions regarding behavioral and decisional outcomes and how those relate to one another. In conclusion, our literature review shows 
that decision aids may potentially be helpful in helping people change their health behavior, especially when it comes to smoking cessation.

\section{The Smoking Cessation Decision Aid VISOR}

The aim of Chapter 4 was to use a so-called cluster analysis to identify early adopters of a smoking cessation decision aid, such as VISOR, based on the general decision-making style of 200 smokers. The clusters of smokers found were compared on demographic and sociocognitive variables, such as attitude towards smoking cessation aids. Follow-up analyses were performed to see whether cluster membership affected the intention to use a smoking cessation decision aid. Ultimately, two groups were identified; "Avoidant Regretters" were more avoidant, more likely to regret their decisions, and more dependent on others in their decision making, while "Intuitive Non-regretters" were more spontaneous and intuitive in their decision making. Cluster membership was significantly related to intention to use a decision aid, with "Avoidant Regretters" being more interested in a decision aid, such as VISOR. However, this relationship disappeared when adjusting for social-cognitive variables, suggesting that cluster membership may influence this intention via these social-cognitive variables. In general, it was concluded that the general decision-making style can be used to identify smokers at an early stage who may be interested in a digital decision aid. As such, decision-making style can be used to tailor recruitment materials and decision aid content.

The aim of Chapter 5 was to explore the needs and viewpoints of potential users of smoking cessation decision aids and experts in smoking cessation (decision aids) to inform the development of the decision aid VISOR with their input. The data was collected through two approaches applied in three (sub)studies: (1) twenty semi-structured interviews with potential users and (2) two studies with 61 smoking cessation counselors and 44 scientific experts in which consensus was sought. At the time of the interviews, the potential users indicated that they obtain information about smoking cessation aids in various ways: through their own experiences, through their social environment, and through the media. Key characteristics for choosing between smoking cessation aids also varied, but effectiveness and cost were often named as important. The smoking cessation counselors agreed on 38 statements and the scientific experts agreed on 40 statements about the important features of smoking cessation aids and their views on a smoking cessation decision aid. For example, statements such as that a tool should match the addiction level of the user were considered important. However, there was some variation in the needs and wishes between the (different) stakeholders. This indicates that a 'one size fits all'-approach is not desirable within a smoking cessation decision aid. This should be taken into account during the development of such a decision aid, for example by allowing users to adapt a decision aid according to their personal preferences, while safeguarding essential elements. 
The aim of Chapter 6 was to present the systematic development of VISOR. We developed two versions of VISOR: (1) The intervention version contained an explicit value clarification method in combination with computer-tailored advice, while (2) these two elements were missing in the control version. Chapter 6 also describes the protocol of the randomized controlled trial in which the effects of the intervention version of VISOR were compared with the effects of the control version. The development of VISOR was based on the guidelines of the international decision aid organization (that is, the International Patient Decision Aid Standards (IPDAS) Collaboration), the findings from the needs assessment (see Chapter 5), and principles from the so-called 'Self-Determination Theory'. A first prototype of the decision aid was tested in September 2019 and a second version was tested for usability in December 2019; the results from these two tests were then processed and this resulted in the two final versions of VISOR. These final versions include: (1) an information section, (2) an optional knowledge quiz, (3) a brief smoking assessment, (4) an intuitive decision, (5) intermediate advice, (6) an explicit value clarification method (only within the intervention version), (7) tailored advice (only within the intervention version), and (8) information about the availability of smoking cessation aids. In the randomized controlled trial, the effects of these two versions on long-term (primary outcome, after six months) and short-term (after one month) smoking abstinence, on the use of evidence-based smoking cessation aids, and on ambivalence regarding the decision between different smoking cessation aids were compared.

The aim of Chapter 7 was to present the findings from this randomized controlled trial. In the end, 2375 participants randomly received one of the two versions, 599 participants then used it completely, 276 participants completed the follow-up questionnaire immediately after using the decision aid, 97 participants completed the follow-up questionnaire after one month, and 103 participants did so after six months. Statistical analyses were performed to assess the effects on all outcome measures, with and without adjustment for other factors, such as education level. Effects were tested using three scenarios: (1) only with participants who completed the entire experiment, (2) a worst-case scenario assuming that respondents who did not complete the follow-up questionnaires still smoke, and (3) using multiple imputations, in which missing data are imputed on the basis of the available data. Effects in favor of the intervention version on the primary outcome (that is, smoking abstinence after six months) were only found in the worst-case scenario. Effects on the other outcomes were only found regarding smoking abstinence after one month and the use of smoking cessation aids (again only in the worst-case scenario). In conclusion, the inclusion of explicit value clarification methods and computer-tailored advice within a smoking cessation decision aid cannot be recommended with confidence. Adding these elements may even lead to more users not using the decision aid fully, since in our sample more people dropped out during the intervention version than during the control version. However, because too small a sample size may have influenced our findings regarding the outcomes, replication of these 
findings is recommended, taking into account our lessons learned. For example, we found that more attention should be paid to the required usage times of digital decision aids.

The aim of Chapter 8 was to put all chapters and findings in perspective and to compare them with other literature, as well as to make scientific and practical recommendations. 



\section{samenvatting}


Besluitvorming, ook gezondheidsgerelateerde besluitvorming, is een integraal onderdeel van het dagelijks leven van mensen. Dergelijke gezondheidsgerelateerde besluitvorming kan relatief eenvoudig zijn (bijvoorbeeld rondom de beslissing om tanden te poetsen), maar kan ook complexer zijn, grote onzekerheid met zich meebrengen en een grote impact hebben op het leven van mensen (bijvoorbeeld rondom de beslissing voor of tegen een bepaalde kankerbehandeling). Vooral als besluitvorming complexer wordt, is het belangrijk dat het besluit geïnformeerd is, aangezien mensen over het algemeen minder ambivalentie ervaren rondom hun beslissing als ze een geïnformeerde (in tegentelling tot een ongeïnformeerde) beslissing kunnen nemen. Als men een geïnformeerde beslissing neemt, houdt dat twee dingen in: ten eerste heeft men de benodigde informatie om een beslissing te kunnen nemen en ten tweede kan men deze informatie gebruiken om te bepalen wat persoonlijk als belangrijk wordt ervaren met betrekking tot de beslissing in kwestie.

Geïnformeerde besluitvorming is sinds de jaren negentig steeds belangrijker geworden, maar wordt vooral toegepast bij beslissingen over behandelingen (bijvoorbeeld kankerbehandelingen) en rondom bevolkingsonderzoek (bijvoorbeeld kankerscreening). Beslissingen rondom gezondheidsbevordering hebben tot nu toe weinig aandacht gekregen in de literatuur over geïnformeerde besluitvorming. Dit terwijl dergelijke beslissingen ook ambivalente gevoelens kunnen oproepen. Dit kan bijvoorbeeld worden gezien in verband met het stoppen-met-roken: Mensen die gemotiveerd zijn om te stoppen met roken missen vaak de benodigde informatie om een geïnformeerde beslissing te kunnen nemen tussen de verschillende stoppen-met-roken hulpmiddelen. Hierdoor zijn ze ook minder in staat om te bepalen wat voor hen belangrijk is met betrekking tot deze beslissing. Bijgevolg kunnen rokers het moeilijk vinden om een geïnformeerde beslissing te nemen tussen de verschillende beschikbare stoppen-met-roken hulpmiddelen, wat kan leiden tot ambivalente gevoelens met betrekking tot dit besluit.

Om dergelijke ambivalente gevoelens te vermijden en rokers te ondersteunen bij het nemen van een geïnformeerde beslissing, kunnen keuzehulpen worden gebruikt. Keuzehulpen hebben vaak twee componenten: (1) evenwichtige informatievoorziening en (2) zogenaamde waardeverhelderingsmethoden die kunnen helpen om te bepalen wat persoonlijk als belangrijk wordt ervaren met betrekking tot de beslissing in kwestie. Deze waardeverhelderingsmethoden kunnen expliciet (inclusief interactiviteit) of impliciet (zonder interactiviteit) zijn. Keuzehulpen (en in het verlengde daarvan waardeverhelderingsmethoden) zijn tot nu toe echter vooral bestudeerd in behandelingsen screeningcontexten en niet veel in het kader van gezondheidsbevordering.

Gebaseerd op de inleiding hierboven had dit proefschrift twee doelstellingen: (1) het verkrijgen van een goed begrip van de keuzehulpen die worden toegepast binnen de gezondheidsbevordering en (2) het beschrijven van het werk dat is ondernomen om de 
stoppen-met-roken keuzehulp VISOR te ontwikkelen en te testen. Dit doel als ook de achtergrond van deze doelstelling worden in Hoofdstuk 1 nader beschreven.

\section{Keuzehulpen Toegepast Binnen de Gezondheidsbevordering}

Het doel van Hoofdstuk 2 was om kritisch te reflecteren op gedragsveranderingsinterventies (vaak gebruikt binnen de gezondheidsbevordering) en keuzenhulpen (in dit hoofdstuk beschreven als patiëntenkeuzehulpen). Overeenkomsten en verschillen werden onderzocht en het potentieel voor integratie werd geïdentificeerd om zo uiteindelijk de voordelen voor de gebruikers van beide interventies te kunnen vergroten. We ontdekten dat gedragsveranderingsinterventies en keuzehulpen voornamelijk verschillen in termen van (1) doel en focus, (2) theoretische basis, (3) ontwikkelingskaders, (4) actieve componenten en (5) evaluatie van effecten. Integratie kan worden bereikt door de kennis die is gegenereerd door onderzoek naar keuzehulpen te gebruiken om besluitvormingsprocessen te vergemakkelijken die patiënten in staat stellen doelen te kiezen die aansluiten bij hun persoonlijke waarden en voorkeuren. Aan de andere kant kunnen bevindingen uit onderzoek naar gedragsveranderingsinterventies worden gebruikt om de implementatie van deze gekozen doelen te ondersteunen. Om de integratie van wetenschappelijke inzichten uit onderzoeken naar gedragsveranderingsinterventies en keuzehulpen te vergemakkelijken, zijn in Hoofdstuk 2 de volgende aanbevelingen gedaan: (1) breng beide velden samen en bevorder interprofessionele discussies, (2) (gezondheids)professionals opleiden om de sterke punten van beide benaderingen te erkennen, (3) synergiën van interprofessionele samenwerking tussen de twee velden verder onderzoeken en (4) wees voorbereid op een cultuurschok wanneer de velden met elkaar gaan interacteren en probeer deze te verzachten.

Het doel van Hoofdstuk 3 was om de bestaande literatuur over keuzehulpen die de besluitvorming rondom gezondheidsbevordering ondersteunen te bestuderen. De focus lag hierbij op dieet, lichaamsbeweging, slaap en middelengebruik. Vijfendertig wetenschappelijke artikelen en vier keuzehulpen (grijze literatuur) zijn opgenomen in deze 'scoping review', waarvan 29 onderzoeken naar middelengebruik. Alle keuzehulpen omvatten informatie, waardeverhelderingsmethoden en methoden om persoonlijke voorkeuren te verduidelijken. Er waren echter ook veel andere elementen, zoals het stellen van doelen. Hierdoor was het moeilijk om te bepalen welke van deze elementen gebruikers hielpen (en via welke processen) en welke niet. Hoewel de meeste studies tot op zekere hoogte theorieën gebruikten, werd er weinig gedaan om inzichten uit gedragsverandering en (geïnformeerde) besluitvorming te integreren. Hoewel er enkele positieve gedragseffecten (bijvoorbeeld met betrekking tot roken) werden gemeld, waren de effecten van keuzehulpen over het algemeen gemengd. Ook volgden studies niet altijd een gerandomiseerde onderzoeksopzet en werd er weinig gebruik gemaakt van 
gestandaardiseerde uitkomstmaten. Dit maakte kennissynthese moeilijk. Op basis van deze scoping review konden verschillende verbeterpunten worden geïdentificeerd: (1) onderzoekers moeten bepalen welke interventie-elementen effectief zijn in de besluitvorming in gezondheidsbevordering en voor welke processen, (2) de integratie tussen theoretische kennis uit gedragsverandering en (geïnformeerde) besluitvorming moet worden versterkt door ofwel een flexibele theoretische benadering of door het ontwerpen van een theoretisch kader dat gedragsverandering en (geïnformeerde) besluitvorming integreert, en (3) meer gerandomiseerde studies zouden moeten worden uitgevoerd om andere literatuurstudies in staat te stellen sterkere conclusies te trekken met betrekking tot gedrags- en beslissingsresultaten en hoe deze zich tot elkaar verhouden. Concluderend blijkt uit ons literatuuronderzoek dat keuzehulpen mogelijk behulpzaam kunnen zijn om mensen te helpen hun gezondheidsgedrag te veranderen, vooral als het gaat om stoppen-met-roken.

\section{De Stoppen-Met-Roken Keuzehulp VISOR}

Het doel van Hoofdstuk 4 was om een zogenaamde clusteranalyse te gebruiken om 'early adopters' van een stoppen-met-roken keuzehulp, zoals VISOR, te identificeren op basis van de algemene besluitvormingsstijl van 200 rokers. De gevonden clusters van rokers werden vergeleken op demografische en sociaal-cognitieve variabelen, zoals attitude tegenover stoppen-met-roken hulpmiddelen. Vervolganalyses werden uitgevoerd om te zien of clusterlidmaatschap de intentie om een stoppen-met-roken keuzehulp te gebruiken beïnvloedde. Uiteindelijk werden twee groepen geïdentificeerd; "Avoidant Regretters" waren meer vermijdend, hadden vaker spijt van hun beslissingen en waren afhankelijker van anderen in hun besluitvorming, terwijl "Intuitive Non-regretters" spontaner en intuïtiever waren in hun besluitvorming. Clusterlidmaatschap was significant gerelateerd aan de intentie om een keuzehulp te gebruiken, waarbij "Avoidant Regretters" meer geïnteresseerd waren in een keuzehulp, zoals VISOR. Deze relatie verdween echter wanneer gecorrigeerd werd voor sociaal-cognitieve variabelen, wat er op wijst dat clusterlidmaatschap deze intentie mogelijk beïnvloedt via deze sociaal-cognitieve variabelen. Over het algemeen werd geconcludeerd dat de algemene besluitvormingsstijl kan worden gebruikt om in een vroeg stadium rokers te identificeren die mogelijk geïnteresseerd zijn in een digitale keuzehulp. Als zodanig kan besluitvormingsstijl worden gebruikt om wervingsmaterialen en keuzehulpinhoud op maat te maken.

Het doel van Hoofdstuk 5 was om de behoeften en standpunten van mogelijke gebruikers van stoppen-met-roken keuzehulpen en experts in stoppen-met-roken (keuzehulpen) te onderzoeken, om met hun input de ontwikkeling van de keuzehulp VISOR te informeren. De gegevens zijn verzameld via twee benaderingen die zijn toegepast in drie (deel)onderzoeken: (1) twintig semigestructureerde interviews met potentiële gebruikers en (2) twee onderzoeken met 61 stoppen-met-rokencounselors en 44 wetenschappelijke experts waarin 
getracht werd consensus te bereiken. Op het moment van de interviews gaven de potentiële gebruikers aan op verschillende manieren informatie te verkrijgen over stoppen-met-roken hulpmiddelen: via eigen ervaringen, via hun sociale omgeving en via de media. De belangrijkste kenmerken voor het kiezen tussen stoppen-met-roken hulpmiddelen liepen ook uiteen, maar effectiviteit en kosten werden vaak als belangrijk genoemd. De stoppenmet-rokencounselors waren het eens over 38 stellingen en de wetenschappelijke experts over 40 stellingen over de in hun ogen belangrijke kenmerken van stoppen-met-roken hulpmiddelen en over hun standpunten over een stoppen-met-roken keuzehulp. Zo werden stellingen als dat een hulpmiddel moet passen bij het verslavingsniveau van de gebruiker belangrijk gevonden. Er was echter enige variatie in de behoeften en wensen tussen de (verschillende) belanghebbenden. Dit wijst erop dat een 'one size fits all'-aanpak niet wenselijk is binnen een stoppen-met-roken keuzehulp. Hiermee moet rekening worden gehouden tijdens de ontwikkeling van zo'n keuzehulp, bijvoorbeeld door gebruikers in staat te stellen een keuzehulp aan te passen op basis van hun persoonlijke voorkeuren, terwijl essentiële elementen worden beschermd.

Het doel van Hoofdstuk 6 was om de systematische ontwikkeling van VISOR te presenteren. We ontwikkelden twee versies van VISOR: (1) De interventieversie bevatte een expliciete waardeverhelderingsmethode in combinatie met advies op maat, terwijl (2) deze twee elementen in de controleversie ontbraken. Hoofdstuk 6 beschrijft ook het protocol van het gerandomiseerde gecontroleerde experiment waarin de effecten van de interventieversie van VISOR werden vergeleken met de effecten van de controleversie. De ontwikkeling van VISOR was gebaseerd op de richtlijnen van de internationale keuzehulporganisatie (d.w.z. de International Patient Decision Aid Standards (IPDAS) Collaboration) de bevindingen uit het behoefteonderzoek (zie Hoofdstuk 5) en principes uit de zogenaamde 'Self-Determination Theory'. Een eerste prototype van de keuzehulp werd in september 2019 getest en in december 2019 werd een tweede versie getest op bruikbaarheid; de resultaten uit deze twee tests werden vervolgens verwerkt en dit resulteerde in de twee definitieve versies van VISOR. Deze definitieve versies bevatten: (1) een informatiesectie, (2) een optionele kennisquiz, (3) een korte rook-assessment, (4) een intuïtieve beslissing, (5) tussentijds advies, (6) een expliciete waardeverhelderingsmethode (alleen binnen de interventieversie), (7) advies op maat (alleen binnen de interventieversie) en (8) informatie over de verkrijgbaarheid van hulpmiddelen. In het gerandomiseerde gecontroleerde experiment werden de effecten van deze twee versies op rookabstinentie op de lange termijn (primaire uitkomst, na zes maanden) en de korte termijn (na één maand), op het gebruik van bewezen effectieve stoppen-met-roken hulpmiddelen en op ambivalentie met betrekking tot de keuze tussen verschillende hulpmiddelen onderling vergeleken.

Het doel van Hoofdstuk 7 was om de bevindingen uit dit gerandomiseerde gecontroleerde experiment te presenteren. Uiteindelijk ontvingen 2375 deelnemers willekeurig een van de 
twee versies, 599 deelnemers gebruikten deze vervolgens helemaal, 276 deelnemers vulden direct na het gebruik van de keuzehulp de vervolgvragenlijst in, 97 deelnemers vulden de vervolgvragenlijst na een maand in en 103 deelnemers deden dit na zes maanden. Er zijn statistische analyses uitgevoerd om de effecten op alle uitkomstmaten te beoordelen, met en zonder correctie voor andere factoren, zoals opleidingsniveau. Effecten zijn getest aan de hand van drie scenario's: (1) alleen met deelnemers die het gehele experiment volledig dorlopen hebben, (2) een worstcasescenario waarin ervan werd uitgegaan dat respondenten die de vervolgvragenlijsten niet hebben ingevuld nog steeds roken en (3) aan de hand van multipele imputaties, waarbij ontbrekende gegevens worden geïmputeerd op basis van de wel beschikbare gegevens. Effecten ten gunste van de interventieversie op de primaire uitkomst (dat wil zeggen, rookabstinentie na zes maanden) werden alleen gevonden in het worstcasescenario. Effecten op de overige uitkomsten werden alleen gevonden met betrekking tot rookabstinentie na één maand en het gebruik van stoppen-met-roken hulpmiddelen (wederom alleen in het worstcasescenario). Concluderend kan het opnemen van expliciete waardeverhelderingsmethoden en advies op maat binnen een stoppen-metroken keuzehulp niet met vertrouwen worden aanbevolen. Het toevoegen van deze elementen kan er zelfs toe leiden dat meer gebruikers de keuzehulp niet volledig gebruiken, aangezien in onze steekproef meer mensen afhaakten tijdens het gebruik van de interventieversie dan tijdens het gebruik van de controleversie. Omdat een te kleine steekproef onze bevindingen met betrekking tot de uitkomsten echter kan hebben beïnvloed, wordt replicatie van deze bevindingen aanbevolen, rekening houdend met onze geleerde lessen. Zo vonden we dat er meer aandacht moest komen voor de benodigde gebruiksduur van digitale keuzehulpen.

Het doel van Hoofdstuk 8 was om alle hoofdstukken en bevindingen in perspectief te plaatsen en te vergelijken met andere literatuur, als ook aanbevelingen te doen voor wetenschap en praktijk. 



\section{zusammenfassung}


Entscheidungsfindung, einschließlich der gesundheitsbezogenen Entscheidungsfindung, ist ein wesentlicher Bestandteil des täglichen menschlichen Lebens. Solche gesundheitsbezogene Entscheidungsfindung kann relativ einfach sein (z. B. bei der Entscheidung zum Zähneputzen), kann aber auch komplexer sein, mit erheblicher Unsicherheit verbunden sein und einen großen Einfluss auf das Leben von Menschen haben (z. B. bei der Entscheidung für oder gegen eine bestimmte Krebsbehandlung). Gerade bei komplexer Entscheidungsfindung, ist es wichtig, dass die Entscheidung fundiert (oder informiert) ist, da Menschen im Allgemeinen weniger Ambivalenz gegenüber ihrer Entscheidung erleben, wenn sie eine fundierte (im Gegensatz zu einer nicht fundierten) Entscheidung treffen können. Trifft man eine fundierte Entscheidung, so beinhaltet dies zwei Dinge: zum einen verfügt man über die nötigen Informationen zur Entscheidungsfindung und zweitens kann man anhand dieser Informationen feststellen, was persönlich als wichtig erfahren wird bei der jeweiligen Entscheidung.

Die fundierte Entscheidungsfindung hat seit den 1990er Jahren an Bedeutung gewonnen, sie wird jedoch hauptsächlich auf Entscheidungen zu Behandlungen (z.B. Krebsbehandlungen) und Bevölkerungsscreenings (z.B. in der Krebsvorsorge) angewendet. Entscheidungen zur Gesundheitsförderung wurden in der Literatur zur Entscheidungsfindung bislang wenig beachtet. Dabei können solche Entscheidungen auch ambivalente Gefühle hervorrufen. Dies ist beispielsweise im Zusammenhang mit der Rauchentwöhnung erkennbar: Personen, die motiviert sind mit dem Rauchen aufzuhören, fehlen oft erforderliche Informationen, um eine fundierte Entscheidung zwischen den verschiedenen Unterstützungsmitteln zur Rauchentwöhnung treffen zu können. Dies führt wiederum zu einer Einschränkung der Fähigkeit zu wissen, was in Bezug auf die Entscheidung wichtig ist. Folglich kann es Raucher:innen schwer fallen eine fundierte Entscheidung zwischen den verschiedenen verfügbaren Unterstützungsmitteln zur Rauchentwöhnung zu treffen, woraus sich mögliche ambivalente Gefühle bezüglich der Entscheidung ergeben können.

Um solche ambivalente Gefühle zu vermeiden und Raucher:innen zu unterstützen eine fundierte Entscheidung treffen zu können, können Entscheidungshilfen eingesetzt werden. Entscheidungshilfen weisen oft zwei Bestandteile auf: (1) ausgewogene Informationsbereitstellung und (2) sogenannte Wertklärungsmethoden, die dabei helfen können, festzustellen, was im Hinblick auf die jeweilige Entscheidung persönlich als wichtig erlebt wird. Diese Wertklärungsmethoden können entweder explizit (einschließlich Interaktivität) oder implizit (ohne Interaktivität) sein. Entscheidungshilfen (und im weiteren Sinne Wertklärungsmethoden) wurden jedoch auch oft nur in Behandlungs- und Screeningkontexten untersucht und nicht viel im Kontext der Gesundheitsförderung.

Folgend aus der obigen Einführung hatte diese Dissertation zweierlei Ziele: (1) die Vermittlung eines umfassenden Verständnisses über Entscheidungshilfen in der 
Gesundheitsförderung und (2) die Beschreibung der wissenschaftlichen Entwicklung, sowie der Testung der Rauchentwöhnungsentscheidungshilfe VISOR. Kapitel 1 dient einer ausführlichen Beschreibung des Hintergrundes und der Zielsetzung dieser Dissertation.

\section{Entscheidungshilfen in der Gesundheitsförderung}

Das Ziel von Kapitel 2 war sich kritischen mit Verhaltensänderungsinterventionen (wie häufig in der Gesundheitsförderung eingesetzt) und Entscheidungshilfen (in diesem Kapitel als Patient:innenentscheidungshilfen beschrieben) auseinander zu setzen. Gemeinsamkeiten und Unterschiede wurden untersucht und Integrationspotenziale identifiziert, um letztlich die Vorteile für die Nutzer:innen beider Interventionen zu steigern. Wir stellten fest, dass sich Verhaltensänderungsinterventionen und Entscheidungshilfen hauptsächlich in Bezug auf (1) Ziele und Fokusse, (2) theoretische Grundlagen, (3) Entwicklungsrahmen, (4) aktive Bestandteile und (5) Evaluation der Effekte unterscheiden. Integration kann erreicht werden, indem das Wissen aus der Entscheidungshilfeforschung genutzt wird, um Entscheidungsprozesse zu erleichtern, die es Patient:innen ermöglichen, Ziele zu wählen, die ihren persönlichen Werten und Vorlieben entsprechen. Andererseits könnten Erkenntnisse aus der Forschung zu Verhaltensänderungsinterventionen genutzt werden, um die Umsetzung dieser gewählten Ziele zu unterstützen. Um die Integration wissenschaftlicher Erkenntnisse aus Studien zu Verhaltensänderungsinterventionen und Entscheidungshilfen zu erleichtern, wurden in Kapitel 2 die folgenden Empfehlungen ausgesprochen: (1) beide Felder zusammenbringen und interprofessionelle Diskussionen fördern, (2) (Gesundheits)Fachkräfte schulen um die Stärken beider Ansätze zu erkennen, (3) Synergien der interprofessionellen Zusammenarbeit zwischen den zwei Feldern weiter erforschen und (4) antizipieren, dass es zu ,Kulturschocks' in der Zusammenarbeit zwischen den zwei Feldern kommt, sodass diese Schocks abgemildert werden können.

Das Ziel von Kapitel 3 war es, die vorhandene Literatur zu Entscheidungshilfen, die die Entscheidungsfindung in der Gesundheitsförderung unterstützen, zu überprüfen. Der Fokus lag hierbei auf Ernährung, Bewegung, Schlaf und Substanzkonsum. Fünfunddreißig wissenschaftliche Artikel und vier Entscheidungshilfen (graue Literatur) sind in diesem ,Scoping Review' aufgenommen, davon 29 Studien zum Substanzkonsum. Alle in diesen Studien beschriebenen Entscheidungshilfen beinhalteten Informationen, Werteklärungsmethoden und Methoden zur Präferenzklärung. Es gab jedoch auch noch viele andere Elemente, wie z. B. das Setzen von Zielen. Dies machte es schwierig zu bestimmen, welche dieser Elemente den Nutzern:innen (und durch welche Prozesse) geholfen haben und welche nicht. Während die meisten Studien in gewissem Maße Theorien verwendeten, wurde wenig unternommen, um Erkenntnisse aus Verhaltensänderungen und (fundierter) Entscheidungsfindung zu integrieren. Obwohl einige positive Verhaltenseffekte (z.B. bezüglich des Rauchens) berichtet wurden, war die Auswirkungen von 
Entscheidungshilfen im Allgemeinen nicht eindeutig. Außerdem folgten die Studien nicht immer einem randomisierten Studiendesign und standardisierte Zielkriterien wurden wenig verwendet. Basierend auf diesem Scoping Review konnten mehrere Verbesserungsbereiche in Bezug auf die Literatur identifiziert werden: (1) Forschende sollten feststellen, welche Interventionselemente bei der Entscheidungsfindung in der Gesundheitsförderung wirksam sind und für welche Prozesse, (2) die Integration zwischen theoretischen Erkenntnissen aus Verhaltensänderungen und (fundierter) Entscheidungsfindung sollte gestärkt werden, indem entweder ein flexibler theoretischer Ansatz verwendet wird oder durch die Gestaltung eines theoretischen Ansatzes, der Verhaltensänderungen und (fundierte) Entscheidungsfindung integriert, und (3) sollten mehr randomisierte Studien durchgeführt werden, damit andere Literaturrecherchen stärkere Schlussfolgerungen zu Verhaltens- und Entscheidungsergebnissen und deren Beziehung zueinander ziehen können. Schlussfolgernd zeigte unsere Literaturrecherche, dass Entscheidungshilfen möglicherweise hilfreich sein könnten, um Menschen dabei zu unterstützen, ihr Gesundheitsverhalten zu ändern, insbesondere bezüglich der Rauchentwöhnung.

Die Rauchentwöhnungsentscheidungshilfe VISOR

Ziel von Kapitel 4 war es, basierend auf dem allgemeinen Entscheidungsfindungsstil von 200 Rauchern:innen mithilfe einer sogenannten Clusteranalyse frühe Anwender:innen einer Rauchentwöhnungsentscheidungshilfe wie VISOR zu identifizieren. Die gefundenen Rauchercluster wurden nach demografischen und sozialkognitiven Variablen wie der Einstellung zu Unterstützungsmitteln zur Rauchentwöhnung verglichen. Folgeanalysen wurden durchgeführt, um zu sehen, ob die Clusterzugehörigkeit die Absicht beeinflusst, eine Entscheidungshilfe zur Raucherentwöhnung zu verwenden. Letztendlich wurden zwei Gruppen identifiziert; "Avoidant Regretters", welche in ihrer Entscheidungsfindung eher vermeidend, reumütiger und abhängiger von Anderen waren, während "Intuitive Nonregretters" spontaner und intuitiver in ihrer Entscheidungsfindung waren. Die ClusterMitgliedschaft stand in signifikantem Zusammenhang mit der Absicht, eine Entscheidungshilfe zu verwenden, wobei "Avoidant Regretters" mehr an einer Entscheidungshilfe wie VISOR interessiert waren. Dieser Zusammenhang verschwand jedoch bei der Anpassung an sozialkognitive Variablen, was darauf hindeutet, dass die Clusterzugehörigkeit diese Absicht über diese sozialkognitiven Variablen beeinflussen kann. Generell wurde geschlussfolgert, dass der allgemeinen Entscheidungsfindungsstil genutzt werden kann, um Raucher:innen zu identifizieren, die frühzeitig an einer digitalen Entscheidungshilfe interessiert sein könnten. Daher kann der Entscheidungsfindungsstil verwendet werden, um Rekrutierungsmaterialien und die Inhalte einer Entscheidungshilfe anzupassen. 
Ziel von Kapitel 5 war es, die Bedürfnisse und Ansichten potenzieller Nutzer:innen von Entscheidungshilfen zur Raucherentwöhnung und Experten:innen für (Entscheidungshilfen zur) (die) Raucherentwöhnung zu untersuchen, um mit ihren Beiträgen die Entwicklung der Entscheidungshilfe VISOR zu unterstützen. Die Daten wurden mittels leitfadengestützter Interviews mit potenziellen Nutzer:innen, sowie mit zwei Studien mit 61 Raucherentwöhnungsberater:innen und 44 wissenschaftlichen Experten:innen, in denen ein Konsens angestrebt wurde zu erreichen, erhoben. Zum Zeitpunkt der Interviews gaben die potenziellen Nutzer:innen an, sich auf unterschiedliche Weise zu informieren bezüglich Unterstützungsmitteln zur Rauchentwöhnung: durch eigene Erfahrungen, ihr soziales Umfeld und die Medien. Auch die Schlüsselmerkmale für die Wahl zwischen Unterstützungsmitteln zur Rauchentwöhnung variierten, aber Wirksamkeit und Kosten wurden oft als wichtig angeführt. Die Raucherentwöhnungsberater:innen einigten sich auf 38 Aussagen und die wissenschaftlichen Experten:innen auf 40 Aussagen zu den aus ihrer Sicht wichtigen Merkmalen von Unterstützungsmitteln zur Rauchentwöhnung und zu ihren Ansichten zu einer Rauchentwöhnungsentscheidungshilfe. Beispielsweise wurden Aussagen, wie dass ein Unterstützungsmittel für den Suchtgrad der Nutzer:innen geeignet sein sollte als wichtig empfunden. Allerdings gab es bei den (verschiedenen) Interessenträgern:innen Unterschiede in den Bedürfnisse und Ansichten. Dies deutet darauf hin, dass ein einheitlicher Ansatz für eine Rauchentwöhnungsentscheidungshilfe nicht wünschenswert ist. Dies sollte bei der Entwicklung einer solchen Entscheidungshilfe berücksichtigt werden, beispielsweise indem es Nutzer:innen ermöglicht wird, eine Entscheidungshilfe an ihre persönlichen Vorlieben anzupassen, während wesentliche Elemente geschützt werden.

Ziel von Kapitel 6 war es, die systematische Entwicklung von VISOR vorzustellen. Zwei Versionen von VISOR wurden entwickelt: (1) Die Interventionsversion enthielt eine explizite Wertklärungsmethode, wie oben beschrieben, in Kombination mit einem individuell angepassten Ratschlag, (2) in der Kontrollversion fehlten diese zwei Elemente. Kapitel 6 beschreibt auch das Protokoll des randomisierten kontrollierten Experiments, in dem die Effekte der Interventionsversion von VISOR mit den Effekten der Kontrollversion verglichen wurden. Die Entwicklung von VISOR basierte auf den Leitlinien der internationalen Entscheidungshilfeorganisation (d.h. der International Patient Decision Aid Standards (IPDAS) Collaboration), den Erkenntnissen aus Kapitel 5 und Prinzipien aus der sogenannten ,Self-Determination Theory'. Ein erster Prototyp der Entscheidungshilfe wurde im September 2019 einem Test unterzogen und im Dezember 2019 wurde eine zweite Version einem Test bezüglich der Benutzer:innenfreundlichkeit unterzogen; das Feedback wurde dann verarbeitet und führte zu zwei endgültigen Versionen. Die endgültigen Versionen enthalten: (1) einen Informationsteil, (2) ein optionales Wissensquiz, (3) eine kurzes Rauchassessment, (4) eine intuitive Entscheidung, (5) einen Zwischenratschlag, (6) eine explizite Werteklärungsmethode (nur innerhalb der Interventionsversion), (7) einen individuellen angepassten Ratschlag (nur innerhalb der Interventionsversion) und (8) 
Informationen über die Verfügbarkeit von Unterstützungsmitteln zur Rauchentwöhnung. In dem randomisierten kontrollierten Experiment wurden die Effekte dieser beiden Versionen auf die langfristige (primärer Endpunkt, nach sechs Monaten) und kurzfristige (nach einem Monat) Raucherabstinenz, auf die Verwendung von nachweislich wirksamen Unterstützungsmitteln zur Rauchentwöhnung und auf die Ambivalenz hinsichtlich der Wahl zwischen verschiedenen Unterstützungsmitteln verglichen.

Ziel von Kapitel 7 war es, die Ergebnisse dieses randomisierten kontrollierten Experiments vorzustellen. Am Ende erhielten 2375 Teilnehmer:innen nach dem Zufallsprinzip eine der beiden Versionen, 599 Teilnehmer:innen nutzten diese anschließend vollständig, 276 Teilnehmer:innen füllten unmittelbar nach Nutzung der Entscheidungshilfe den Folgefragebogen aus, 97 Teilnehmer:innen füllten den Folgefragebogen nach einem Monat aus und 103 Teilnehmer:innen taten dies nach sechs Monaten. Statistische Analysen wurden durchgeführt, um die Auswirkungen auf alle Endpunkte zu bewerten, mit und ohne Anpassung an andere Faktoren, wie beispielsweise das Bildungsniveau. Effekte wurden anhand von drei Szenarien getestet: (1) nur bei Teilnehmer:innen, die das gesamte Experiment abgeschlossen haben, (2) ein Worst-Case-Szenario unter der Annahme, dass Teilnehmer:innen, die die Folgefragebogen nicht ausgefüllt haben, immer noch rauchen, und (3) ,Multiple Imputations', bei denen fehlende Daten auf der Grundlage anderer verfügbarer Daten imputiert werden. Effekte zugunsten der Interventionsversion auf den primären Endpunkt (d. h. Rauchabstinenz nach sechs Monaten) fanden sich nur im Worst-CaseSzenario. Effekte auf die anderen Endpunkte zeigten sich nur hinsichtlich der Raucherabstinenz nach einem Monat und der Nutzung von Unterstützungsmitteln zur Rauchentwöhnung wieder (wiederum nur im Worst-Case-Szenario). Zusammenfassend lässt sich sagen, dass die Einbeziehung von expliziten Wertklärungsmethoden und individuellen angepassten Ratschlägen in eine Rauchentwöhnungsentscheidungshilfe nicht mit Zuversicht empfohlen werden kann. Das Hinzufügen dieser Elemente kann sogar dazu führen, dass mehr Nutzer:innen die Entscheidungshilfe nicht vollständig nutzen, da in unserer Stichprobe mehr Menschen die Interventionsversion abbrachen als die Kontrollversion. Da jedoch eine zu kleine Stichprobe unsere Ergebnisse zu den Endpunkten beeinflusst haben könnte, wird empfohlen, diese Ergebnisse unter Berücksichtigung unserer gewonnenen Erkenntnisse zu replizieren. Wir haben beispielsweise gefunden, dass die erforderliche Nutzungsdauer digitaler Entscheidungshilfen mehr Aufmerksamkeit geschenkt werden sollte.

Ziel von Kapitel 8 war es, alle Kapitel und Ergebnisse abschließend zu diskutieren und mit anderer Literatur zu vergleichen sowie wissenschaftliche und praktische Empfehlungen zu geben. 



\section{dankwoord}


"Carry out a random act of kindness, with no expectation of reward, safe in the knowledge that one day someone might do the same for you."

- Diana, Princess of Wales

Finally, the part I've been looking forward to writing since the first day of my PhD: "het dankwoord". It has been said a thousand times, but a PhD thesis is not written without acts of kindness of other people. And I was lucky enough to receive many such kindnesses.

Mijn team, oftewel "de dames", zoals ik jullie vaker noemde om te laten zien hoe tof ik het vond om begeleid te worden door drie sterke, inspirerende en grappige vrouwen. Ik weet zeker dat ik zonder jullie niet zo ver was gekomen en vandaag niet dezelfde Thomas zou zijn, want jullie hebben me niet alleen een geweldige kans gegeven, maar jullie hebben me de afgelopen vier jaar ook zoveel geleerd. Ik kan jullie hier niet genoeg voor bedanken, maar ik ga het toch proberen.

Ik las onlangs in het proefschrift van een voormalige collega dat ze niet goed wist wie ze op de eerste plaats moest zetten van haar twee copromotoren en dat sprak me erg aan, aangezien ik ook niet echt zou weten wie ik als eerste moest noemen: Ciska en Eline, Eline en Ciska ... jullie twee hebben me zoveel geleerd en waren zo belangrijk voor mijn promotietraject dat ik eerlijk gezegd niet zou weten wie ik op de eerste plaats zou moeten zetten binnen dit dankwoord en daarom doe ik het gewoon chronologisch en beschouw dit als Ciska én Eline.

Ciska, we kennen elkaar al een tijdje omdat ik mijn masterscriptie al onder jouw begeleiding heb gedaan vóór mijn PhD en sindsdien denk ik de hele tijd: Wauw, wat heb ik een geluk dat ze deze gekke Duitser deze kans biedt, of kansen moet ik eigenlijk zeggen. Ik weet dat ik niet de meest voor de hand liggende kandidaat was toen je me aannam, aangezien mijn Nederlandse taalvaardigheid beperkt was tot "hoi" en "hoor". Voor het project was het onder andere nodig om interviews in het Nederlands te doen, en ook een digitale interventie in het Nederlands te creëren. Daarom was en ben ik nog steeds enorm dankbaar dat je me niet alleen deze kans hebt gegeven om mee te beginnen, maar dat je altijd in me hebt geloofd. We verschilden misschien wel eens van mening, maar één ding is nooit veranderd: Ik kon nog zo onzeker zijn, je bleef maar zeggen dat ik na vier jaar zal verdedigen met een boek in mijn handen. Je had gelijk, en daar ben ik je erg dankbaar voor. Je leerde me kritisch te zijn op mijn eigen werk zonder dit altijd op mezelf te betrekken (oké, laten we eerlijk zijn, hier werk ik steeds nog aan) en dat de meeste dingen echt wel goed komen met een goede planning in handen. Ik ben ook erg dankbaar dat je me hebt geleerd dat een zeer theoretische benadering van gezondheidsbevordering niet betekent dat praktische problemen genegeerd moeten worden. Ik had altijd het gevoel dat je uitblonk in de combinatie van de twee 'werelden' en daar heb ik echt van geleerd. Je gaf me altijd de ruimte 
om te groeien met betrekking tot beide en steunde me in de duizend dingen die ik wilde doen: PhD-vertegenwoordiger, UMPride, wetenschapscommunicatie, onderzoeksbezoek in Leeds om er maar een paar te noemen. Er was niets waar je me niet in steunde zolang ik kon laten zien dat ik het in mijn werk kon inpassen. En hiervoor kan ik niet dankbaarder zijn. Dank je, Ciska. Zonder jou zou ik niet de wetenschapper zijn die ik nu ben-en laten we eerlijk zijn, ik zou niet zoveel gezegdes in het Nederlands kennen zonder jou. En hier ben ik toch ook erg trots op.

Eline, ik gebruik het woord 'held' veel te vaak, maar als er één persoon is die mijn held was tijdens mijn promotietraject, dan ben jij het wel. Vier jaar later weet ik nog steeds niet hoe je het allemaal voor elkaar krijgt, maar ik ben nog steeds onder de indruk van hoe goed je bent als onderzoekster, terwijl je ook uitblinkt als mentor en gewoon een heel aardig en grappig persoon bent. De afgelopen vier jaar heb je me niet alleen geleerd hoe goede wetenschap wordt bedreven, je hebt me echt het verschil laten zien tussen iemand die jou begeleidt en iemand die je mentor is. En ik denk nog steeds dat dat het belangrijkste is dat je me hebt laten zien. Mensen hebben wel eens grappen gemaakt over hoe blij ik was na meetings met jou en dat ik nooit moe werd om je te prijzen. Wat dit nog grappiger maakt, is dat ik in het begin erg nerveus was om je te ontmoeten omdat ik jou zo indrukwekkend vond. Ondertussen vind ik je nog steeds net zo indrukwekkend, maar ben ik niet meer nerveus je te spreken. Je leerde me hoe belangrijk een kritische blik is, maar dat waardering ook nooit kwaad kan. Dat om hulp vragen niet erg is, maar dat ik ook op mezelf kan vertrouwen. Dat het goed is om erg gedreven te zijn en van je werk te houden, maar dat dit niet betekent dat ik mijn vrije tijd niet moest koesteren en beschermen. Ik ben nu en dan nog steeds een stresskip, maar je hebt me geleerd dat met een goede planning, af en toe een "Nee." of "Dat doe ik wel volgende week, oké?" het echt wel goed komt en dat ik de skills in huis heb om op mezelf te vertrouwen. Bedankt voor je mentorschap, Eline, en ik hoop dat nog veel promovendi van je kunnen leren-omdat ik vind dat iedere promovendus en promovenda een 'Eline' verdient.

Carmen, last but not least. Ik vond je indrukwekkend sinds we elkaar voor het eerst ontmoetten en ik ben nog steeds een beetje zenuwachtig als ik je ontmoet. Ik denk dat er maar weinig mensen zijn die zoveel expertise hebben als jij. Er zijn ook maar weinig mensen die zo goed zijn als jij en die niet bang zijn om te zeggen wanneer ze een keer minder weten dan iemand anders in de kamer en dan de andere persoon gewoon aan het woord laten. Ik denk dat ik hierin net zoveel van je heb geleerd als van gezondheidseconomie en besluitvorming. Je leerde me ook dat het goed is om kritisch en nauwkeurig te zijn, maar dat het soms ook net zo belangrijk is om even een stapje terug te doen, even te wachten en het grote geheel te zien. Ik weet dat je het ongelooflijk druk hebt, maar ik stel het erg op prijs dat je altijd klaar stond om me advies te geven. Je zegt altijd dat ik met de tijd meer ontspannen zal zijn met betrekking tot mijn werk, en als jij een indicatie in dat opzicht bent, 
dan weet ik dat het goed komt, want ik ken niet veel mensen die zo rustig en 'effortless' overkomen in hun werk. Dankje Carmen, voor al je adviezen, rust en kennis die je met mij hebt gedeeld.

Ook wil ik de leden van mijn leescommissie, Trudy, Bas, Esther en Marc, bedanken. Jullie raakten er vooral aan het einde bij betrokken, maar ik stel jullie inbreng zeer op prijs en kijk ernaar uit om mijn werk met jullie verder te bespreken.

Natuurlijk wil ik ook even stilstaan bij het feit dat zoveel andere wetenschappers met mij hebben gewerkt aan de artikelen die ik in dit proefschrift mag presenteren. Raesita, ik ben nog steeds zo blij dat je de eerste onderzoeksassistente van mijn project was. Zonder jou was VISOR nooit een succes geworden, hadden we vanaf het begin niet zoveel deelnemers gevonden, was het een stuk chaotischer en minder gezellig geweest en zou mijn Nederlands nu een stuk minder netjes zijn. Dank voor je steun (ook nadat je officieel niet meer bij GB werkte!) en alles wat je voor mij en het project hebt gedaan. Ik waardeer het enorm en wens je het allerbeste! Vera, de tweede onderzoeksassistente van VISOR. En laten we eerlijk zijn, zonder jou zou er geen VISOR zijn-wat heb je veel codeerwerk gedaan. En wat heb je me veel geleerd over Tailor Builder. Dank hiervoor! Je bent zo'n gedreven persoon en ik weet dat er een geweldige carrière op je wacht. Daniëlle, door Corona was je eigenlijk de enige GB-kamergenoot die meer dan twee dagen achter elkaar een kantoor met mij deelde. En ook al vond je me $90 \%$ van de tijd volgens mij te druk, ik ben erg dankbaar dat we onze PhDs samen hebben gedaan. Zonder jou had ik niet zo goed kunnen inburgeren (\#kruidnoten), had ik in het begin veel meer problemen gehad (shout-out naar je vader die heeft geholpen bij het aanmelden van mijn auto!), zou ik nu veel minder Nederlandse scheldwoorden kennen en zou ik nooit hebben geleerd dat af en toe een beetje water bij de wijn prima kan. Bedankt dat je een geweldige kamergenoot was en wat leuk dat we samen een artikel hebben kunnen schrijven. Rik, ik denk niet dat er veel andere mensen zijn die ik zo vaak heb gemaild met "Ik heb een vraagje ..." en er zijn nog minder mensen die altijd zo snel op mijn vragen hebben gereageerd. Je hebt me de afgelopen vier jaar echt veel meer geleerd dan dat je beseft denk ik, en daarom vind ik het zo fijn dat we aan het einde van mijn promotietraject samen nog een artikel hebben kunnen schrijven. Shahab, you taught me so much about statistics, $\mathrm{R}$, and especially mice. It was really nice working with you, thank you! Catherine, Hein en Jean ook van jullie mocht ik veel leren tijdens het uitvoeren en schrijven van onze gezamenlijke scoping review. Veel dank hiervoor. Jany, ik wil je bedanken voor het leren van meer toegankelijke taal en het meewerken aan mijn protocolartikel. Last but not least, Hilary, I've never been so happy that someone had a discussion in front of one of my posters, because that led straight to me visiting you in Leeds. Not only did I learn a lot about decision aids and informed decision making during my visit, but I also had a great time and I honestly think there are few academic hosts who make as sure that their guest feels 
comfortable and has a good time as you did during my visit. Thank you for your time and for writing an article together with me!

Er waren natuurlijk ook andere collega's zonder wie mijn promotietraject niet zo leuk zou zijn geweest. Ik wil graag de hele GB-afdeling bedanken, ik vond het geweldig om bij jullie mijn PhD te doen en ik had niet voor een andere afdeling willen kiezen. Francine, wat heb ik veel van jou geleerd over onderwijs en de academische wereld, maar ook buiten het werk was het altijd gezellig met je-grappig genoeg vooral sinds de eerste lockdown. Dank voor alles wat ik van jou heb geleerd en vooral de gezelligheid. Dat ga ik zeker niet missen want we houden gewoon contact. Adriana, zonder jou was het werken op kantoor zeker minder leuk maar ook moeilijker voor mij geweest. Je had altijd een luisterend oor voor me, vooral toen ik me meer dan twee jaar geleden zo 'down' voelde. Bedankt dat je er altijd voor me was. En bedankt voor het hosten van het verjaardagsfeestje voor mijn 30 e verjaardag. Je bent een geweldige collega en nog belangrijker een geweldige vriendin. Rosa, ich bin froh, dass wir noch Kolleg:innen sein konnten, auch wenn es viel zu kurz war. Ich glaube, es gibt wenige Menschen, mit denen ich so viele tiefgehende Gespräche auf und außerhalb der Arbeit geführt habe, gell? Danke, Rosalein! Anke en Merel, mijn twee Brabo's op kantoor. Bedankt voor jullie gezelligheid en bedankt voor de ontvoering naar Londen, dat blijft toch echt een geweldig verjaardagscadeau. Kim, Daisy, Denise, Leon en Patricia, jullie hebben dat vast al duizend keer gehoord maar jullie houden de hele vakgroep draaiende en daar ben ik jullie al enorm dankbaar voor, maar daarnaast waren jullie altijd ontzettend gezellig collega's die ik niet had willen missen. Ik wil vooral Kim en Patricia bedanken voor hun open deuren en luisterende oren in de tijd dat ik me vaak niet lekker voelde op het werk, zonder jullie zou dit allemaal wat moeilijker zijn geweest-bedankt, dames! Stef, je was een erg betrokken en gezellige vakgroepvoorzitter. Succes met je nieuwe uitdaging. Lisa, mijn 'fellow-ESC lover', hiervoor heb je al een plaatsje in mijn dankwoord verdiend, maar ook daarbuiten was je altijd een superleuke collega. Wie weet ontmoet ik je toch nog een keer als buurman (even serieus, hoe kan het dat we elkaar nooit tegenkomen?). Marion, we zijn bijna samen begonnen en daarom vond ik het ook erg leuk samen met jou een schrijfclubje te hebben aan het einde. Dank voor je steun. Karlijn, we hadden altijd een gezellige tijd samen op kantoor en we konden altijd interessante gesprekken voeren onder het genot van een kopje koffie. Ook mis ik nu al onze gezamenlijke uitstapjes naar de CaRe-dagen waar we een borrel deden ... of twee. Je was een geweldige collega en een geweldige luisteraar. Dankjewel, Karlijn! Gido, je kwam er pas aan het einde bij, maar ik vind het leuk dat we een tijdje hebben samengewerkt en ik vind het geweldig dat we nu samen aan een artikel werken. Lotte en Nicole, ik zou het heel vreemd vinden om jullie niet samen te bedanken, want elke keer als ik het even moeilijk had, stormde ik gewoon bij jullie binnen om met jullie te lachen, om samen een suffe video te kijken of om gewoon samen lekker te zeuren. Hiervoor wil ik jullie al bedanken. Lotte, het was ook een genoegen om je prins te zijn, bedankt voor deze ervaring. Nicole, je ging ook elke week met mij sporten, ook hiervoor wil ik je bedanken. Speaking of exercise, I also want 
to thank you, Daša, for making club power much more fun, en jij, Lieke, want binnen en buiten UMSports was het altijd supergezellig met je! Jacqueline, ook al zat je niet op onze afdeling, het voelde toch vaak alsof dat wel zo was. Je maakte mijn promotietraject veel gezelliger en vooral ons tripje naar Londen zal ik niet zo snel vergeten! I also want to thank my new colleagues - especially Sarah, Karlijn, Karen, Kai, Catia, Tuğçe, Hanne, and the rest of the HIV lab-for making me feel so welcome at my new department. Ook wil ik iedereen van CAPRHI heel erg bedanken, ik voelde me altijd heel erg aan CAPHRI verbonden en ben blij met de kansen die mij door CAPRHI werden geboden. Anne, Vivian en de rest van het 'ResearchTalent program'-clubje, dank jullie wel voor de gezellige en leerzame tijd. Hannerieke, Karin, en Esther wil ik ook bedanken voor de gezellige en leerzame tijd samen. Esther, vooral jij was iemand die nauw verweven was met mijn hele PhD-ervaring, en laten we eerlijk zijn, mijn hele inburgering. Je maakte voor mij de eerste keer hutspot, leerde mij 't kofschip en samen deden we de ene of andere borrel. Je was de perfecte persoon om samen PhD-vertegenwoordiger mee te zijn en je was altijd een geweldige en leuke vriendin. Dankje, Es! Maria, danke für deine großartige Hilfe beim Schreiben der deutschen Zusammenfassung und für all die schönen Abende in A'dam! Last but not least, my fellow Germanz. Danke, Steffi, Karin und Carolin, dass ihr mir am Anfang geholfen habt mich in Maastricht und auf der Abteilung zurechtzufinden. Vor allem möchte ich dir, Caro, meinem Ruhrpöttchen, für deine Unterstützung in der Zeit, in der es mir nicht gut ging, danken. Ohne dich hätte ich die Zeit nicht so gut überstanden, vor allem nicht auf der Arbeit. Ich weiß nicht, wie viel Arbeitszeit du durch mich verloren hast, aber es war eigentlich viel zu viel. Anfangs hätte ich es nicht gedacht, aber ich bin so froh, dass du mein 'zweverige Freundin' geworden bist, mit der ich mich über Meditation, Gefühle, Achtsamkeit, vegetarisches Essen und vieles mehr austauschen kann. Danke, Caro!

Kiki, ich habe $\mathbf{B 3}$ damals den dritten Platz in meiner Danksagung versprochen und wie du siehst habe ich mich hier drangehalten. Danke für die tolle Zeit auf B3 und dafür, dass ich auf B3 meine Begeisterung für Gesundheitsverhalten, -erziehung und -förderung entdecken durfte. Darüber hinaus war es auch eine großartige Zeit, die ich nicht missen möchte und aus der ich sicherlich viel gelernt habe, was mir während meiner Promotionszeit sehr nützlich war. Besonderer Dank gilt Timo, Steffi, Ina, Tina, Christina, Barbara, Isabel, Nina, Kiki, Joaquina, Uta, André, Marina, Sandra und Roman.

I also want to thank all my UMPride peeps not only for making the UM during my PhD a bit more colorful, but for also making it a lot more fun. I especially want to thank my 'researchand-academia-crew', you are an awesome bunch, and I truly learnt a lot from every single one of you. However, there is one person I would like to thank in particular: Ro, you are a wonderful person and you have honestly taught me more than you realize. Most importantly, you celebrated every PhD-achievement that I had (I got a "WORK" message while I was writing this) and what would I be without my pindabaas-sweater? Thank you, Ro! 
Ik wil ook Marie-Jeanne en Virgine bedanken voor hun steun tijdens mijn promotietraject.

I am also privileged to have an incredible number of friends who are all incredibly important to me. I would like to thank some of them here for the small or large role they played in my PhD-trajectory. Tjitske, een 'fellow-HEPper' die ons werkveld (denk ik) net zo boeiend vindt als al die andere duizend dingen waar we vaak over filosoferen, of het nu in Maastricht, Den Haag, Den Bosch, Maasland of een ander deel van Nederland is (we moeten steeds nog naar Friesland gaan!). Bedankt dat je zo'n geweldige vriendin bent, een geweldige luisteraar, een geweldige supporter en bedankt voor alle kaarten die je hebt gestuurd om mijn successen te vieren of om me te troosten als werk of privéleven niet zo meezaten. Tankewol, Tjits. Charlotte, nog een fellow-HEPper op dit lijstje-ook al moet ik toegeven dat ik vooral na onze studie heb ervaren wat voor een geweldige persoon jij bent. Of het nu ging over hoe inclusief ons werk(veld) wel of niet is of hoeveel we van alle soorten dieren houden. Tijdens mijn PhD keek ik er altijd naar uit om bij mijn buurvrouw Charlotte langs te gaan om een leuke avond te hebben en om te ontspannen. Bedankt voor jouw steun, de gezelligheid, en dat ik dankzij jou niet de enige in mijn omgeving ben die tijdens Koningsdag tv wil kijken met een tompouce erbij. Gavin, Gavinsito, I think you have the 'privilege' of being my first gay friend and it seemed to me that due to that you've already deserved to be in my acknowledgments. I really liked that we did our PhD so close to each other in terms of distance (even though I still don't understand the topic of your PhD, sorry) and that we could always walk to each other's office or text each other when needed at work or privately. Dankie, Gavin. Jeanine, I have no idea whether I should thank you in English or Dutch. So, I just decided to do what we always do and do both: Thank you, Jeanine. Heel erg bedankt, Jeanine. You were one of my first science mentors I had and I'm so happy we continue working together and that you make sure that I never have nothing to work on. I've learned so much from you, especially how important it is to keep working on things you love and cherish, and that "we're all smart here, let's be nice too." Thank you for being a wonderful mentor and friend, Jeanine. Here's hoping to have more Vrijthof with you and Chris in the future again! You both rock. Stephi, ich glaube, die Leute denken oft, ich mache einen Witz, wenn ich sage, dass ich dich schon ewig kenne, ohne zu merken, dass ich dich wirklich schon ewig kenne. Buchstäblich. Danke, dass du meine älteste Freundin bist, dass du all meine Erfolge jedes Mal feierst, und am wichtigsten, dass du es immer schaffst mich abzulenken, wenn ich mal wieder viel zu gestresst bin. Du bist eine großartige Freundin und ich bin so froh, dich zu kennen. Sandra und Christina, danke, dass ihr mich immer zum Lachen bringen könnt und eure Familie immer so bereitwilligt mit mir teilt. Ich bin froh euch zu kennen! De Prall Botze, die ganze Bande, ihr habt mich nicht nur immer den Stress vergessen lassen, wenn ich auf der einen oder anderen Feier mit euch in Bergheim war, ihr habt mir auch immer das Gefühl gegeben, zu Hause zu sein und habt mit mir eine meiner Lieblingszeiten gefeiert: Karneval. Vielen, vielen Dank an euch alle! Das gilt auch für die Truppe aus der Ausbildung, vor allem Laura, Julia, Rebecca, Jutta, Anoya, Jana und Kristin danke fürs Ablenken und super sein! Yil en Inez, 
jullie dachten wellicht al dat ik jullie was vergeten boven bij de collega's maar jullie staan expres hier pas vermeld want jullie zijn inmiddels vooral vrienden geworden. Inez, zonder jou zou ik nu niet alleen minder Vlaamse woorden kennen, ik zou vooral minder sterk in mijn eigen schoenen staan. Je bent een onvermoeibare steun en benadrukt steeds dat mijn 'imposter syndrome' nergens voor nodig is. Je bent een geweldige mens, Inez, en ik ben trots dat ik jou mijn vriendin mag noemen. Dank voor al je steun, de gezellige avonden, discussies en alles wat je mij hebt geleerd over de laatste jaren. Ik kijk zo uit naar jouw verdediging. Mercikes, Inez. Yil, Yilleke, Jill, wat ben ik blij jou te hebben leren kennen. Zo voel ik mij nu niet alleen maar beter ingeburgerd in Maastricht (\#rijstartel), en minder alleen als stresskip, met jou had ik eindelijk iemand die net zoveel van keuzehulpen en geïnformeerde besluitvorming hield als ik toen je kwam werken op de afdeling. Maar dat vervaagt allemaal als ik het vergelijk met waar ik het meest dankbaar voor ben: dat je gewoon een van de belangrijkste vrienden van mij hier in Maastricht bent geworden. Of het nu ging om het afleren om voor alles sorry te zeggen, mij te steunen als ik onzeker werd, of voor mij hamburgers te kopen als ik verdrietig in Landgraaf stond-jij stond altijd klaar om mij te steunen. Daanke, Yil, gewoon omdat je een geweldige vriendin en een geweldig persoon bent. Ik denk dat hier dan ook de plaats is om dank te zeggen aan Wizzy, Sieb en Karma. En bedankt, Yil en Inez, dat jullie jullie huisdieren altijd met mij lieten knuffelen en spelen als ik een beetje te gestrest was.

Dieses Kapitel neigt sich langsam dem Ende zu, was bedeutet, dass jetzt der Teil kommt mit den Menschen, von denen ich noch mehr habe als Freunde: Familienmitglieder. Leute machen oft Witze, dass meine Familie schwer zu verstehen ist, da ich einfach so viele Familienmitglieder habe. Ich würde es nicht anders wollen. Ihr seid alle verrückt, lustig, liebevoll, anders als die meisten, aber am wichtigsten ist, dass ihr mich alle immer unterstützt und ihr Bergheim und Umgebung für immer mein Zuhause sein lasst. Danke, Alina, Maxim, Cheyenne, Rambo, Claudia, Bettina, Ralf, Madeline, Onkel Heinz und vielen Dank an Lukas, Papa, Anja, Leana und alle anderen Gültzows und co. Ein paar Menschen möchte ich aber noch mehr Worte widmen. Nadja und Caro, ich weiß nicht, wie viele Menschen das Glück haben, Freundinnen zu haben, die sich wie Familie anfühlen, aber ich habe gleich zwei davon. Danke, für alles was ihr für mich getan habt und immer noch tut. Es würde den Rahmen dieses Kapitel völlig sprengen alles aufzuzählen, aber ich bin für jedes bisschen was ihr für mich getan habt dankbar und ohne euch wäre es gar nicht erst dazu gekommen, dass ich angefangen habe zu promovieren. Danke! Nadja, Nadija, oder wie mein Handy sagt NADJA, eigentlich verdienst du deine ganz eigene Danksagung, weil wie oft hab ich gesagt, dass ich aufhören will und du hast einfach „Nein." gesagt und ich habe weiter gemacht. Danke für jeden "Nein-Moment" ohne die wäre das ganze deutlich schwieriger geworden. Wahrscheinlich sagt das jeder von seiner besten Freundin, aber du bist definitiv die beste Freundin, die man sich wünschen kann. Danke, dass du immer für mich da bist, immer irgendwas für mich bastelst, Schränke aufbaust oder andere Dinge reparierst. Danke, dass 
du alle meine gestressten Sprachnachrichten hörst, mein Deutsch immer noch probierst zu retten und all die hundert Dinge, die du tust, die ich jetzt gerade bestimmt vergessen habe. Ich möchte dir auch dafür danken, dass du deine ganze Familie (und ich meine die ganze Familie) mit mir teilst, bei der ich mich immer wie zu Hause fühle. Ich weiß wir sind beide nicht die besten Menschen im Teilen von Gefühlen, aber ich denke, du weißt, wie wichtig du bist für mich und wie wichtig du warst, sodass diese Doktorarbeit endlich fertig geworden ist. Danke, Nadja! Caro, Carolicious, und doch noch immer ein bisschen, Lutti. Ich vermisse immer noch, dass wir nicht mehr zusammenarbeiten und ich bin so dankbar, dass wir uns auf B3 kennengelernt haben. Ohne dich wäre es definitiv nicht so weit gekommen, dass ich promovieren konnte, da du mich in der Zeit davor so sehr unterstützt hast, dass ich dafür bereit war. Danke, dass du immer ruhig bleibst, wenn ich mal wieder gestresst bin. Danke, dass ich mich bei dir immer wieder wie zu Hause fühle und mit dir über alles sprechen kann. Danke, für all das Essen was du für mich kochst und danke für alle Haushaltstipps. Auch du teilst deine Familie immer bereitwillig mit mir und auch dafür bin ich dir sehr dankbar. Vor allem deine Kinder zeigen mir doch immer wieder, dass es wichtigeres gibt als Arbeitsstress. Danke, Rosa und Oskar. Danke, Rosa und Elias, dass ich euer Patenonkel sein darf und dass ihr die besten Patenkinder seid, die man sich vorstellen kann. Ich freue mich immer euch zu sehen, Geschenke für euch auszusuchen und zu erkennen, dass es nicht das Wichtigste auf Erden ist, stundenlang hinter dem Laptop zu sitzen. Danke euch beiden, dass ihr großartig, süß und schlau seid. Ich hab euch lieb! Danke, Diana und Jörg, dass ich Elias Patenonkel sein darf und immer bei euch zu Hause quatschen darf über Gott und die Welt und mich wie zu Hause fühle. Elianne, mijn Nederlandse tante, zonder jouw hulp en steun was het mij allemaal niet zo makkelijk afgegaan. Je hielp mij vanaf dag één zonder twijfel. Je hielp mij een huis te vinden, Nederlands te leren, me thuis te voelen in Nederland en was van mijn hele familie het meest betrokken bij mijn hele promotietraject. Je kwam naar praatjes, je vroeg hoe het ging en wilde altijd alles weten over mijn PhD, mijn collega's en wat ik nu eigenlijk allemaal deed. En laten we eerlijk zijn, hier en daar, gaf je mij ook een schop onder de kont. Heel veel dank voor alles wat je deed en nog steeds doet. Ik ben heel blij dat je mijn Nederlandse tante bent! Ik wil je ook bedanken dat je Marijke voor mij hebt 'gevonden'. Marijke, zonder jou zou ik dit niet in het Nederlands hebben kunnen schrijven. Bedankt dat je de beste Nederlandse lerares was waar deze Duitse jongen op had kunnen hopen! Miriam, Kiki, René, en Joop dank dat jullie halverwege mijn PhD als gezellige Limburgse schoonfamilie erbij kwamen kijken en mij met open armen ontvingen. Vooral Miriam wil ik bedanken die zo vaak in de lockdown-tijd haar tuin aan mij afstond zodat ik lekker rustig kon schrijven. Ik geniet altijd van onze etentjes en dat ik dan even werk werk kan laten zijn-dank jullie wel! En Kiki, bedankt veur $t$ checken van mien dankwoord en de Limburgs-tips. Dirk, Max und Tante Alex, ich bin froh, dass ich mit euch den besten Onkel, Cousin und Tante habe, die man sich wünschen kann. Egal welches Problem während meiner Promotion auftauchte, ich wusste immer, dass ihr für mich da seid. Und euer Zuhause fühlt sich für mich immer wie ein zweites Zuhause an. Danke für alles, was ihr für mich tut. Von Finanzberatung bis zum 
Umzug. Ich bin dankbar, euch zu haben! Tante Margit, du warst und bist immer, wie eine dritte Oma für mich gewesen und du weißt, wie keine andere mich zurück auf den Boden der Tatsachen zurückzuholen, wenn ich mal wieder völlig gestresst bin von etwas. Danke, dafür und, dass du immer für mich da bist. Oma Agnes und Opa Herbert, danke, dass ihr immer für mich da gewesen seid. Danke, dass ihr mir immer das Gefühl gegeben habt, zu Hause zu sein und dafür, dass ihr großartige Großeltern wart und seid, die ihren Enkeln und Enkelinnen gezeigt haben, was wahre Hingabe ist. Oma Ninchen und Opa Toni, ich habe es jetzt schon so oft gesagt, aber ohne euch hätte es sicher nie eine Promotion gegeben. Ihr habt so viel für mich und meine Schwester aufgegeben und dafür gesorgt, dass wir zwei anständige, gut erzogene, geliebte Menschen werden, und dafür kann ich euch nicht dankbar genug sein. Danke, dass ihr die perfekten Großeltern wart und danke Oma, dass dein Zuhause immer noch mein Zuhause ist. Ohne dich wäre ich nie so weit gekommen. Johannes und Mama, ich habe jetzt schon so oft geschrieben, dass Leute mich wie zu Hause fühlen lassen, aber ihr zwei habt dafür gesorgt, dass ich wirklich ein Zuhause hatte und für immer habe. Danke, dass ihr mich immer unterstützt habt, egal, ob ihr verstanden habt, was ich gerade wieder machen wollte. Danke, dass ihr mir Bücher gekauft habt, um meinen Wissensdurst zu stillen und immer dafür bereit seid, wenn ich mal wieder einfach vorbeikomme. Kalli, Oma erzählt immer, wie ich als Kind gesagt habe, dass ich nicht nur einen Vater habe, sondern zwei. Danke, dass ich dein Kind sein darf und danke, dass du mich bei allem unterstützt und dass ich durch dich jemanden zu Hause habe der sich mit mir über interessante Nachrichten unterhält. Mama, früher haben uns die Leute immer als eine Einheit gesehen und auch jetzt weiß ich noch, dass du bereit bist, alles aufzugeben, um mich zu unterstützen und mir zu helfen. Danke, dass du mir beigebracht hast, was harte Arbeit ist, dass du mir beigebracht hast, Menschen immer zu unterstützen, und danke, dass du mir gezeigt hast, wie ich für mich selbst einstehen kann. Ich habe das Gefühl, dass ich jetzt, da ich älter bin, dir ähnlicher werde, und darauf bin ich stolz.

Sjors, ik heb zoveel mensen bedankt voor de rust die ze me geven, maar als er één persoon in mijn leven is die me altijd rust geeft, dan ben jij het. Ik ben je zo dankbaar. Voor alles wat je voor me doet. Voor alle taken die je hebt overgenomen zodat ik dit proefschrift echt kon afmaken. Als er één persoon is zonder wie dit boek niet tot stand zou zijn gekomen, dan ben jij het wel. Je laat me altijd zien wat rust en positiviteit is, hoe je open kunt zijn over gevoelens en toegewijd kunt zijn. Ik kijk uit naar meer musicals, boeken, tv-shows en films, leuke discussies en zoveel meer zonder PhD-stress samen met jou. Ich haaj van dich. Danke, dass du dich für mich entschieden hast. 
Dankwoord 



\section{publication list}


Gültzow, T., Hoving, C., Smit, E. S., \& Bekker, H. L. (2021). Integrating behaviour change interventions and patient decision aids: How to accomplish synergistic effects? Patient Education and Counseling, 104(12), 3104-3108. https://doi.org/10.1016/j.pec.2021.04.007

Gültzow, T., Zijlstra, D. N., Bolman, C., de Vries, H., Dirksen, C. D., Muris, J. W. M., Smit, E. S., \& Hoving, C. (2021). Decision aids to facilitate decision making around behavior change in the field of health promotion: A scoping review. Patient Education and Counseling, 104(6), 1266-1285. https://doi.org/10.1016/j.pec.2021.01.015

Gültzow, T., Smit, E. S., Hudales, R., Dirksen, C. D., \& Hoving, C. (2020). Smoker profiles and their influence on smokers' intention to use a digital decision aid aimed at the uptake of evidence-based smoking cessation tools: An explorative study. DIGITAL HEALTH, 6, 2055207620980241. https://doi.org/10.1177/2055207620980241

Gültzow, T., Smit, E. S., Hudales, R., Dirksen, C. D., \& Hoving, C. (2022). Informed Decision Making on the Uptake of Evidence-Based Smoking Cessation Assistance by Individuals Motivated to Quit Smoking: A Needs Assessment Among Potential End Users and Experts to Inform Decision Aid Development. European Journal of Health Communication, 3(1), 1-30. https://doi.org/10.47368/ejhc.2022.101

Gültzow, T., Smit, E. S., Hudales, R., Knapen, V., Rademakers, J., Dirksen, C. D., \& Hoving, C. (2020). An Autonomy-Supportive Online Decision Aid to Assist Smokers in Choosing Evidence-Based Cessation Assistance: Development Process and Protocol of a Randomized Controlled Trial. JMIR Research Protocols, 9(12), e21772. https://doi.org/10.2196/21772

Gültzow, T., Smit, E. S., Crutzen, R., Jolani, S., Hoving, C., \& Dirksen, C. D. (2021). Does an Explicit Value Clarification Method with Computer-Tailored Advice Increase the Effectiveness of a Web-based Decision Aid to Assist Smokers in Choosing Evidence-Based Cessation Assistance? Findings From a Randomized Controlled Trial. JMIR Preprints. https://doi.org/10.2196/preprints.34246

Other Publications

Gültzow, T., Guidry, J. P. D., Schneider, F., \& Hoving, C. (2020). Male Body Image Portrayals on Instagram. Cyberpsychology, Behavior, and Social Networking, 23(5), 281-289. https://doi.org/10.1089/cyber.2019.0368 
Gültzow, T., Smit, E. S., Dirksen, C. D., \& Hoving, C. (2021). Keuzehulp helpt roker aan geschikte stopmethode. Pharmaceutisch Weekblad.

Gültzow, T., \& Hoving, C. (2021). Nieuw onderzoek naar keuzehulp bij stoppen-met-roken. Huisarts en wetenschap, 64(1), 10. https://doi.org/10.1007/s12445-020-0962-5

Severijns, Y., de Die-Smulders, C. E. M., Gültzow, T., de Vries, H., \& van Osch, L. A. D. M. (2021). Hereditary diseases and child wish: exploring motives, considerations, and the (joint) decision-making process of genetically at-risk couples. Journal of Community Genetics, 12(3), 325-335. https://doi.org/10.1007/s12687-021-00510-x

\section{Other Preprints}

Peters, G.-J., Kwasnicka, D., Crutzen, R., ten Hoor, G., Varol, T., Berry, E., van Beurden, S., Beyene, K. A., Campbell, K., Eito, L. C., Chan, A. H. Y., Cipolletta, S., DeSmet, A., Dewi, T. K., Dima, A., Encantado, J., Epton, T., Gebretekle, G. B., Gérain, P., ... Gültzow, T., ... Roozen, S. (2021). The Your COVID-19 Risk Assessment Tool and the Accompanying Open Access Data and Materials Repositories. OSF Preprints. https://doi.org/10.31219/osf.io/b8n5g

Sproesser, G., Aulbach, M., Gültzow, T., \& König, L. M. (2022). Do nutrition knowledge, food preferences, and habit strength moderate the association between Preference for Intuition and Deliberation in Eating Decision-making and dietary intake?. PsyArXiv. https://doi.org/10.31234/osf.io/s9bta

\section{Conference Contributions}

Oral

Gültzow, T., Smit, E. S., Hudales, R., Dirksen, C., \& Hoving, C. (2018). Does smoker profile determine adoption of a decision aid to improve evidence-based cessation support uptake?. CaRe Days, 's-Hertogenbosch, 16-17 May.

Gültzow, T., Smit, E. S., Knapen, V., Dirksen, C., Rademakers, J., \& Hoving, C. (2020). An autonomy-supportive decision aid to support smokers in choosing an evidence-based smoking-cessation tool: Development \& usability testing. $18^{\text {th }}$ Biennial European Conference of Society for Medical Decision Making, Berlin, 14-16 June. (Accepted, conference was cancelled due to the COVID-19 pandemic) 
Gültzow, T., Smit, E., Hudales, R., Knapen, V., Dirksen, C., \& Hoving, C. (2020). Een stoppenmet-roken keuzehulp om de waargenomen autonomie te vergroten: een zelfbeschikkingstheorie-gebaseerde aanpak. Amsterdam Center for Health Communication symposium: Digital Health: weerstand of motivatie?, Online event, 17 November.

Gültzow, T., Smit, E. S., Crutzen, R., Jolani, S., Dirksen, C. D, \& Hoving, C. (2021). An autonomysupportive web-based decision aid to assist smokers in choosing evidence-based cessation assistance: Short-term findings from a randomized controlled trial. European Conference on Health Communication 2021, Online event, 4-5 November.

Poster

Gültzow, T., \& Helmbold, A. (2014). Selbstpflege bei Jugendlichen mit Insulinpumpe. Erstellung einer Patientenbroschüre auf der Grundlage einer Literaturanalyse. 5. DGPHochschultag der Deutschen Gesellschaft für Pflegewissenschaft, Lingen, 14 November.

Gültzow, T., Smit, E., Hudales, R., Dirksen, C., \& Hoving, C. (2018). Does Smoker Profile Determine Adoption of a Decision Aid to Improve Evidence-Based Cessation Support Uptake?. $17^{\text {th }}$ Biennial European Conference of Society for Medical Decision Making, Leiden, 10-12 June.

Gültzow, T., Smit, E., Hudales, R., Dirksen, C., \& Hoving, C. (2018). Consensus on the content of a decision aid to improve cessation support uptake: Preliminary results. $32^{\text {nd }}$ Annual Conference of the European Health Psychology Society, Galway, 21-25 August.

Gültzow, T., Smit, E. S., Hudales, R., Dirksen, C., \& Hoving, C. (2018). Does smoker profile determine adoption of a decision aid to improve evidence-based cessation support uptake?. $32^{\text {nd }}$ Annual Conference of the European Health Psychology Society, Galway, 21-25 August.

Gültzow, T., Zijlstra, D., Bolman, C., de Vries, H., Dirksen, C., Muris, J. W. M., Smit, E. S., \& Hoving, C. (2020). The reach, use and effectiveness of decision aids for people facing lifestyle changes in the field of health promotion: a scoping review. $18^{\text {th }}$ Biennial European Conference of Society for Medical Decision Making, Berlin, 14-16 June. (Accepted, conference was cancelled due to the COVID-19 pandemic)

Guidry, J. P. D., Gültzow, T., Nowack, V., Carlyle, K. E. (2020). Pinning about \#HIV/\#AIDS: Filtering information or facilitating conversation?. American Public Health Association Annual Meeting and Expo 2020, Online event, 24-28 October. 
Symposia

Elling, M., Gültzow, T., Smit, E. S., Zijlstra, D., \& Hoving, C. (2020). Never change a winning team? Of blijft het belangrijk om nieuwe manieren te zoeken om tabaksgebruik aan te pakken?. Nederlands Netwerk voor Tabaksonderzoek (NNVT) Congres, Utrecht, 14 February.

Gültzow, T., Severijns, Y., Bailey, J., Jansen, J., \& Smit, E. S. (2021). Digital decision aids: considerations for design and evaluation. $35^{\text {th }}$ Annual Conference of the European Health Psychology Society, Online event, 23-27 August. 



\section{curriculum vitae}




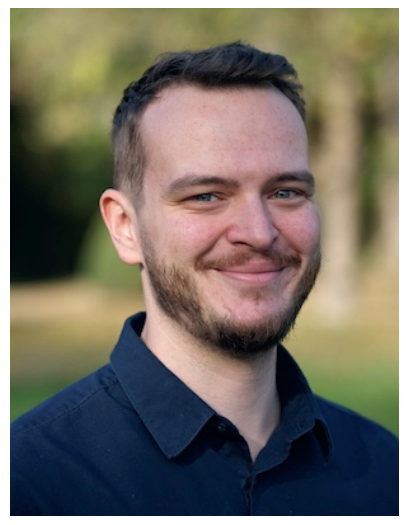

Thomas Gültzow was born in Bergheim on September 27 $7^{\text {th }}$, 1990, where he also went to school. After obtaining his Abitur, he trained as a pediatric nurse at the St. Marien Hospital in Düren. He passed the state examination as a pediatric nurse in 2013. At the same time, he also followed the Dual Course in Nursing at the Catholic University of North Rhine-Westphalia which he finished in 2014 with a Bachelor of Science in Nursing. Already in his bachelor thesis he dealt with a topic of health promotion by developing an information brochure to promote the self-care agency concerning an insulin pump infusion set site change.

He then worked in several healthcare institutions, in particular at the well-known children's hospital Kinderkrankenhaus Amsterdamer Straße in Cologne, where he worked on a ward specialized in the care of chronically ill children and adolescents.

He finished his Master of Science in Health Education and Promotion at Maastricht University with a thesis focusing on male body image on Instagram in 2017 and subsequently started working as a PhD candidate at the Department of Health Promotion, also at Maastricht University. During his PhD, Thomas developed a smoking cessation decision aid called VISOR under the supervision of Dr. Ciska Hoving, Dr. Eline Smit (University of Amsterdam), and Prof. Carmen Dirksen. His PhD was financed by the Dutch Cancer Society. During his PhD, Thomas enjoyed a lot of extracurricular activities and was, for example, $\mathrm{PhD}$ representative at his research school CAPHRI and is currently still chairing the Research and Academia Committee of the LGBT+ network UMPride. He is also a board member of the digital health \& computertailoring special interest group of the European Health Psychology Society. During his PhD, Thomas spent some time at the University of Leeds (hosted by Prof. Hilary Bekker) and greatly enjoyed science communication, both on more traditional channels (for example in the form of an interview in De Limburger) and emerging channels (for example in the form of a podcast episode in De Maffe Mensen Podcast). Also, Thomas was involved in various educational tasks throughout his PhD and successfully obtained his University Teaching Qualification in 2021. In 2021 Thomas was also selected for the CAPHRI Research Talent Program.

Thomas is currently continuing his work at the intersection of informed decision making, health promotion, and (digital) decision support at the Department of Work and Social Psychology, also at Maastricht University. 

